\title{
Characterization of the retinoic acid-induced gene network responsible for pancreas specification in Xenopus laevis
}

\author{
Dissertation for the award of the degree \\ "Doctor rerum naturalium (Dr.rer.nat)" \\ In the GGNB program "Genes and Development" \\ At the Georg August University of Göttingen \\ Faculty of Biology
}

submitted by

Maja B. Gere

born in Hoyerswerda, Germany

Göttingen, January 2016 



\section{Thesis committee member}

Prof. Dr. Tomas Pieler (supervisor and reviewer)

Developmental Biochemistry, Georg August University of Göttingen

Prof. Dr. Herbert Jäckle (reviewer)

Molecular Developmental Biology, MPI for Biophysical Chemistry, Göttingen

Prof. Dr. Andreas Wodarz

Microscopic Anatomy and Molecular Cell Biology, University of Köln

\section{Members of the extended examination board}

Prof. Dr. Ahmed Mansouri

Molecular Cell Differentiation, MPI for Biophysical Chemistry, Göttingen

Prof. Dr. Ernst A. Wimmer

Developmental Biology, Georg August University of Göttingen

Prof. Dr. Matthias Dobbelstein

Molecular Oncology, Georg August University of Göttingen

Date of thesis submission: January 29, 2016

Date of oral examination: March 21, 2016 



\section{Affidavit}

Herewith I declare that I prepared the Doctoral thesis "Characterization of the retinoic acid-induced gene network responsible for pancreas specification in Xenopus laevis" on my own and with no other sources and aids than quoted.

Date of submission:

January 29, 2016

Maja B. Gere 



\section{Table of contents}

Abstract.

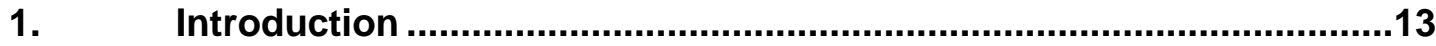

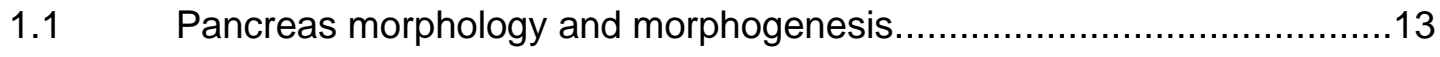

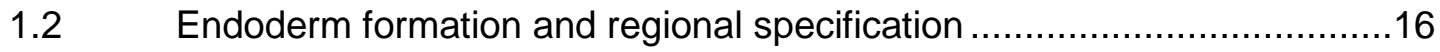

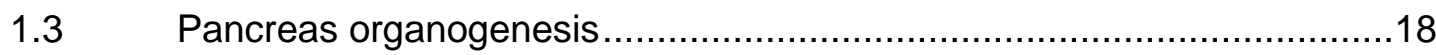

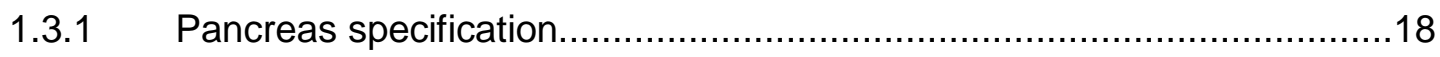

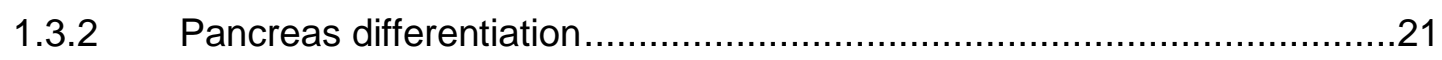

1.4 Role of RA-signaling in pancreas development ..................................24

1.5 Role of Wnt-signaling in pancreas development...............................27

1.6 Potential of organoids in research and clinical applications ....................28

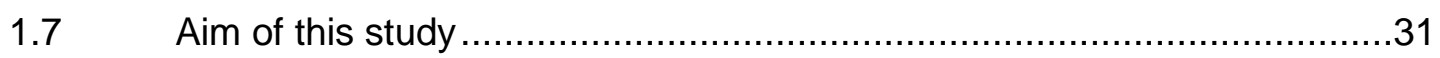

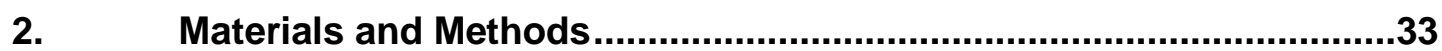

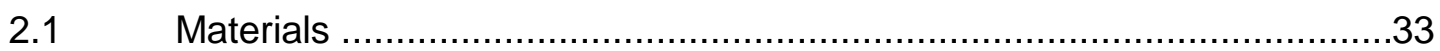

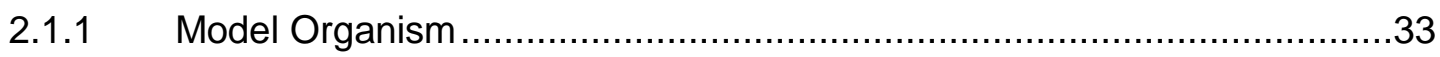

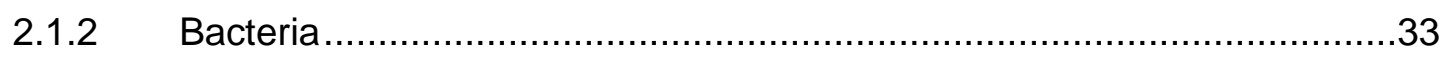

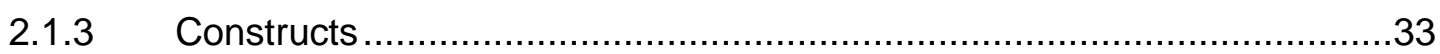

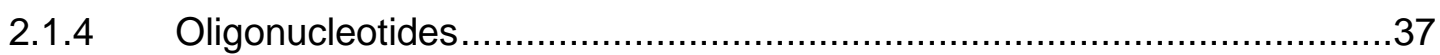

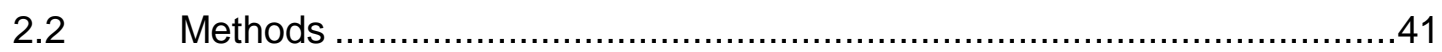

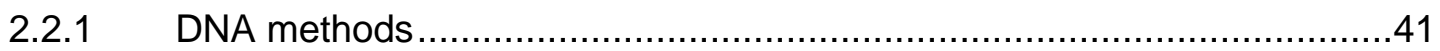

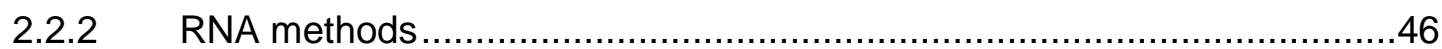

2.2.3 Xenopus laevis embryo culture and micromanipulations .......................51

2.2.4 Whole mount in situ hybridization (WMISH) .........................................54

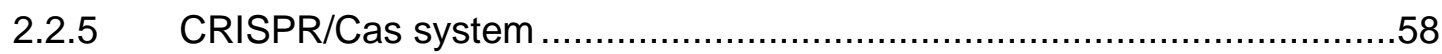

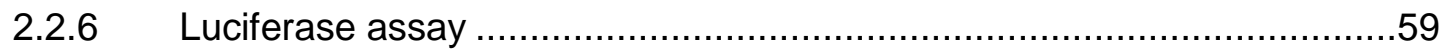

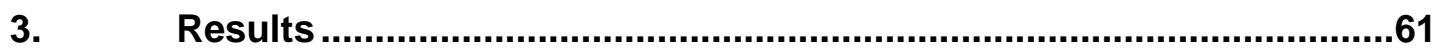

3.1 Formation of pancreatic organoids from Xenopus explants ..................61

3.1.1 RA-dependent induction of pancreatic marker genes in Vegt/Nogginprogramed ectodermal explants

3.1.2 Formation of pancreatic organoids that recapitulate the in vivo program of pancreas development.

3.2 Identification, verification and expression characteristics of early RAresponsive genes.

3.2.1 Induction of direct RA-target gene Cyp26a1 within one hour after RA-

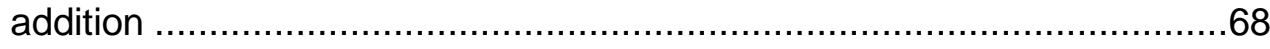

3.2.2 Identification of early RA-target genes by RNA-sequencing ..................70 


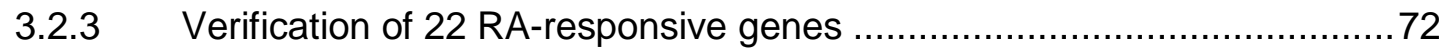

3.2.4 Expression characteristics of 22 verified RA-responsive genes...............73

3.3 The direct RA-target gene Hnf1b is required for pancreas specification .. 75

3.3.1 Hnf1b is RA-responsively expressed in the dorsal endoderm during

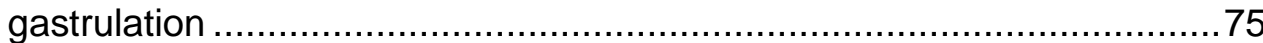

3.3.2 Hnf1b is directly induced by RA in pancreatic organoids .......................76

3.3.3 Hnf1b is required for pancreas specification in vitro ............................ 78

3.3.4 Hnf1b is required for pancreas specification in vivo ............................. 80

3.3.5 Hnf1b alone cannot substitute for RA-activity during pancreas specification 83

3.4 The direct target Fzd4/Fzd4s is required for pancreas specification ........85

3.4.1 Fzd4 and the alternative splice variant Fzd4s are directly induced by RA .. 85

3.4.2 Fzd4/Fzd4s is enriched in the dorsal half of a gastrula embryo including the dorsal endoderm .86

3.4.3 Fzd4/Fzd4s is RA-responsively expressed during gastrulation................87

3.4.4 Fzd4/Fzd4s is required for pancreatic marker gene expression in vitro ... 88

3.4.5 Downregulation of Fzd4/Fzd4s leads to an increase in non-canonical Wntsignaling activity in vitro ............................................................. 91

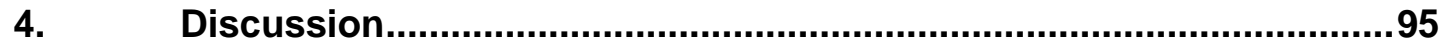

4.1 Pancreatic organoid formation and the requirement of retinoic acid ........95

4.2 Identification of 22 RA-responsive genes ........................................99

4.3 The direct RA-target Hnf1b is required for pancreas development ........ 100

4.4 The direct RA-target Fzd4 is required for pancreas development..........103

$4.5 \quad$ Wnt- and RA-signaling in pancreas development................................. 105

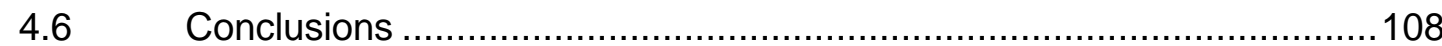

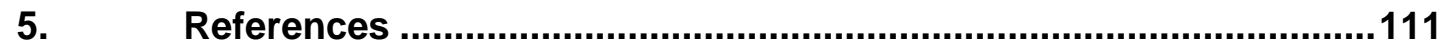

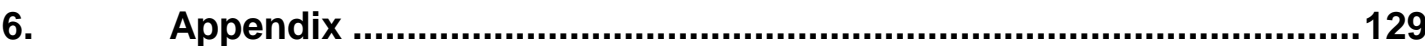

6.1 Formation of pancreatic organoids that recapitulate the in vivo program of pancreas development

6.2 Identification, verification and expression characteristics of early RAresponsive genes

6.3 Expression and functional analysis of the RA-target Hnf1b ...................136

6.4 Expression and functional analysis of RA-target Fzd4/Fzd4s ..............138

6.5 Nanostring analysis data for in vitro generation of pancreatic organoids

6.6 RNA-sequencing data for the identification of RA-target genes 
6.7 Nanostring analysis data for the verification of RA-responsiveness of putative RA-target genes 155

6.8 Nanostring analysis data for expression characteristics of confirmed RAresponsive genes

6.9 Analysis of the Hnf1b-overexpression phenotype 177

6.10 Nanostring code sets.... 180

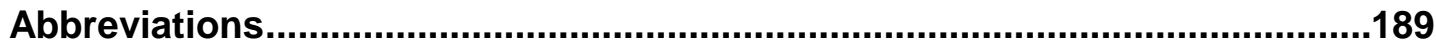

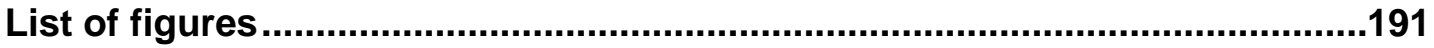

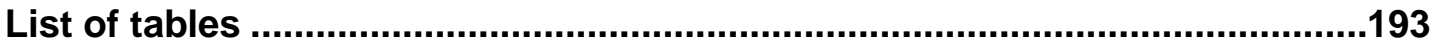

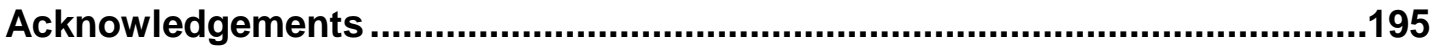

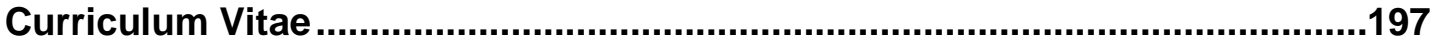




\section{Abstract}

Retinoic acid (RA) is critically required for pancreas specification in Xenopus and other vertebrates. However, the gene network that is directly induced by RAsignaling in this context remains to be defined. We identified 22 RA-target genes through RNA-sequencing of in vitro generated pancreatic organoids. One of these is Hnf1b, which has been shown to be associated with a monogenic form of diabetes in humans and with pancreas hypoplasia in vertebrates. Functional analyses of Hnf1b in pancreatic organoids and whole Xenopus embryos revealed its early requirement for pancreatic progenitor formation. However, we also found that Hnf1b alone is not sufficient to substitute for RA in pancreas specification, indicating a requirement of one or more additional RA-responsive activities. Furthermore, we identified the Wnt-receptor Fzd4 as direct RA-target and novel regulator in pancreas development. Loss-of-function experiments in pancreatic organoids reveal a role of this Wnt-signaling component in pancreas development. Additional experimental data suggest that a modulation of non-canonical Wnt-signaling activity by RA, probably mediated through Fzd4, is required for a proper pancreas specification. 


\section{Introduction}

The pancreas is an organ of the vertebrate digestive system that accomplishes two distinct functions based on its heterogenous composition. It promotes a proper food digestion and maintains glucose homeostasis. The digestive enzyme producing exocrine tissue represents the major component, where clusters of hormone producing endocrine cells are embedded that control glucose homeostasis (Slack, 1995). Pancreas related diseases have encouraged the research on embryonic pancreas development for decades. Several studies showed that the early pancreas development in Xenopus is very similar to that of mice and that the same set of genes used in Xenopus is also involved in mammalian pancreas development (reviewed in Pearl et al., 2009). Lineage tracing experiments in Xenopus revealed that during gastrulation a pancreatic progenitor cell population is specified in the dorsal endoderm (Chalmers and Slack, 2000), considerably earlier than the expression of known pancreatic progenitor markers Ptf1a and Pdx1 (Afelik et al., 2006). It has been demonstrated that signals from the adjacent mesoderm, namely retinoic acid (RA), define a cell population in the dorsal endoderm during gastrulation. Later in development, these cells are capable to respond signals from the notochord which promotes pancreatic fate (Wells and Melton, 2000; Chen et al., 2004; Pan et al., 2007). An overview of the important aspects of pancreas development from endoderm formation over regional specification by mesodermal signals to budding and differentiation is given in the following sections, emphasizing the role of RA- and Wnt-signaling in pancreas development. Furthermore, the progress in research of in vitro organ formation and its relevance for clinical applications will be described.

\subsection{Pancreas morphology and morphogenesis}

The pancreas is $95 \%$ to $99 \%$ an exocrine gland, containing acinar cells that secrete a variety of digestive enzymes. Through a highly branched ductal epithelium, enzymes and bicarbonate ions are transported to the intestine (Slack, 1995). A small percentage of this gland consists of hormone secreting endocrine tissue. Five endocrine cell types, organized in clusters called the islets of Langerhans, are described for mammals, birds, reptiles and amphibians. The main part of the islets is formed by insulin-producing $\beta$-cells. Furthermore glucagon-producing $\alpha$-cells, somatostatin-producing $\delta$-cells, PP-cells that generate pancreatic polypeptide 
(Maake et al., 1998) and $\varepsilon$-cells that secrete ghrelin (Rindi et al., 2004; Wierup et al., 2014) are included in the islets (reviewed in Brereton et al., 2015). Like other organs of the digestive tract, the pancreas originates from the endodermal germ layer. During embryogenesis, the endoderm forms the gut epithelium that is regionalized by various mesodermal signals into overlapping presumptive organ territories from anterior to posterior. The developing pancreas becomes evident as epithelial evaginations of the foregut endoderm.

In Xenopus, one dorsal bud can be detected at stage 35 and two ventral buds at the junction of the liver bud and duodenum at stage 37 (Kelly and Melton, 2000). The earliest known pancreatic markers Ptf1a and Pdx1 are detectable by WMISH before the budding starts at stage 32 (Fig. $1.1 \mathrm{~A}$ ). Ptf1a expression in the endoderm is restricted to the regions of dorsal and ventral pancreatic tissue, whereas $P d \times 1$ is more broadly expressed in the endoderm including the prospective duodenal and stomach tissue. It was shown that epithelium co-expressing these two transcription factors adopts a pancreatic fate (Afelik et al., 2006). During the evagination process, pancreatic progenitor proliferation and differentiation take place. Like in mouse and zebrafish, the Pdx1 expression gets restricted to $\beta$-cells, whereas Ptf1a expression remains only in the exocrine tissue (Krapp et al., 1998; Kawaguchi et al., 2002; Hesselson et al., 2011). In Xenopus, the first endocrine differentiation marker Insulin appears around stage 30 (Fig. 1.1 C) and is detectable only dorsally until stage 45 (Horb and Slack, 2002). Exocrine enzyme Pdia2 gene expression can be detected at stage 39 in both, dorsal and ventral pancreas (Afelik et al., 2004). Pdia2 staining by WMISH is used in Fig. 1.1 B to illustrate the fusion of the three pancreatic primordia at stage 40 caused by gut rotation. The fused pancreas is located predominantly in the left half of the embryo embedded between stomach and duodenum (Fig. 1.1 B panel 5). Transcripts of most of the exocrine marker genes (Amylase, Trypsinogen, Elastase) are first detectable at stage 41 exclusively in the ventral pancreas. Further endocrine markers and a second wave of Insulin expression are visible at stage 46 (reviewed in Pieler and Chen, 2006). 
A

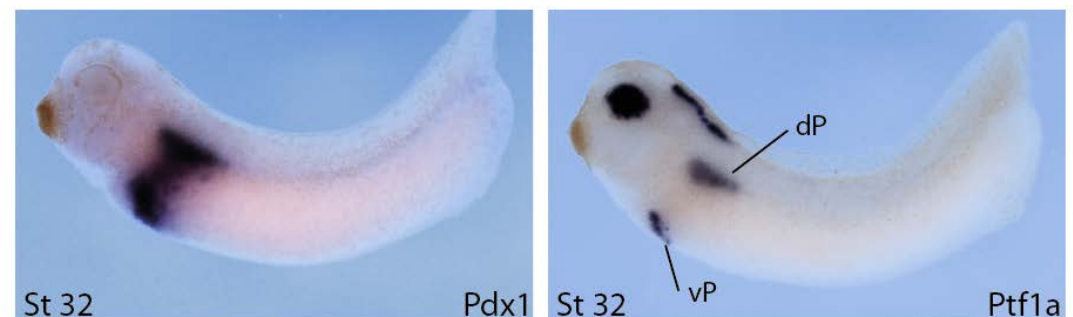

B

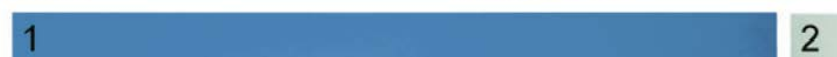

2
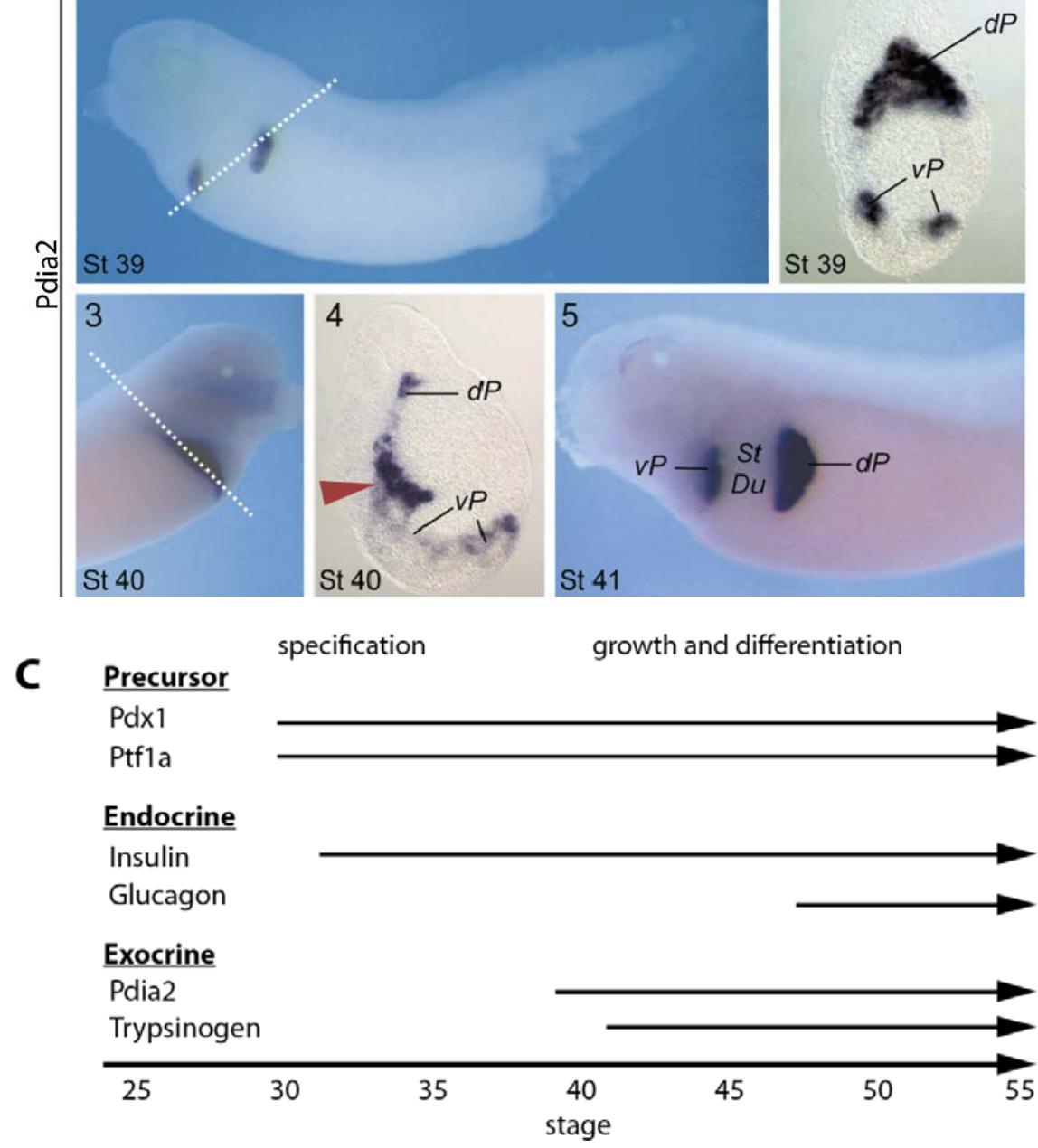

Fig. 1.1 Pancreas organogenesis in Xenopus laevis

(A) Expression of Pdx1 and Ptf1a at stage 32. (B) Pdia2 expression before $(1,2)$ and after (3 to 5) fusion of the dorsal and two ventral pancreatic buds. Panels 2 and 4 show transversal sections across the white dotted line indicated in panels 1 and 3 . (C) Temporal expression profile of pancreatic genes. $\mathrm{dP}=$ dorsal pancreatic bud; Du = duodenum; $\mathrm{St}=$ stomach, $\mathrm{VP}=$ ventral pancreatic buds. $((\mathrm{B})$ and $(\mathrm{C})$ modified from Pieler and Chen, 2006) 


\subsection{Endoderm formation and regional specification}

Although the process of endoderm, mesoderm and ectoderm segregation during gastrulation is different in mice, chicken, fish and frogs, the molecular pathway that directs endoderm formation is evolutionary conserved across these species (reviewed in Zorn and Wells, 2009). Maternal determinants initiate a gene network responsible for endoderm formation. However, these maternal factors differ between the species, while the induced network is conserved (reviewed in Grapin-Botton and Constam, 2007). In Xenopus, the pathway leading to endoderm formation is activated by the maternally provided transcription factor Vegt that is localized to the vegetal cortex of the egg (Fig. 1.2 A) (Hyde and Old, 2000; Xanthos et al., 2001). Vegt is a crucial regulator of endoderm formation in Xenopus as embryos developed from Vegt-depleted eggs lack the endodermal germ layer (Zhang et al., 1998). Vegt induces the expression of early endodermal genes like the transcription factors Sox7 (Zhang et al., 2005), Sox17a (Engleka et al., 2001), several Mix-type homeodomain factors (like Mix.1 and Mixer), GATA-factors (Xanthos et al., 2001) and Nodalrelated genes (Xnrs) (Takahashi et al., 2000) (reviewed in Fukuda and Kikuchi, 2005; Heasman, 2006; Skirkanich et al., 2011).

Xnrs encode for secreted factors of the TGF $\beta$-family, which regulate the gene expression via transmembrane serine-threonine kinase receptors mediated by intracellular Smad proteins. In all vertebrates, the Nodal signaling pathway is essentially required for endoderm development as it induces additional endodermal genes and is capable of restoring the endodermal gene expression in Vegt-depleted embryos (Yasuo and Lemaire, 1999; Xanthos et al., 2001 and reviewed in reviewed in Grapin-Botton and Constam, 2007). Moreover, Nodal signaling also induces mesodermal genes depending on the level of signaling (Clements et al., 1999; Shen 2007). Vegt acts synergistically with dorsally stabilized $\beta$-Catenin for the activation of Xnr genes resulting in a gradient of high Nodal-signaling in the dorsal endoderm and lower levels in the ventral endoderm (Fig. 1.2 B, Agius et al., 2000). Furthermore, Nodal signaling cooperates with $\beta$-catenin/Tcf3-targets Siamois and Twin to induce mesodermal organizer genes like Noggin, Chordin and Follistatin (Brannon et al., 1997; Moon and Kimmelman, 1998; Bae et al., 2011). These secreted organizer molecules act as BMP-inhibitors and establish a dorso-ventral BMP-signaling gradient in all three germ layers (Fig. 1.2 C, Piccolo et al., 1996; Zimmerman et al., 1996). Thereby, dorsal endoderm, from which the pancreatic progenitors will arise, is characterized by low levels of BMP-signaling. In Xenopus, it 
was shown that RA is sufficient to induce pancreatic gene expression in dorsal endodermal explants but not in ventral. Only when BMP-signaling was downregulated by Noggin, ventral endodermal explants expressed pancreasspecific genes in the presence of RA and mesoderm (Pan et al., 2007).
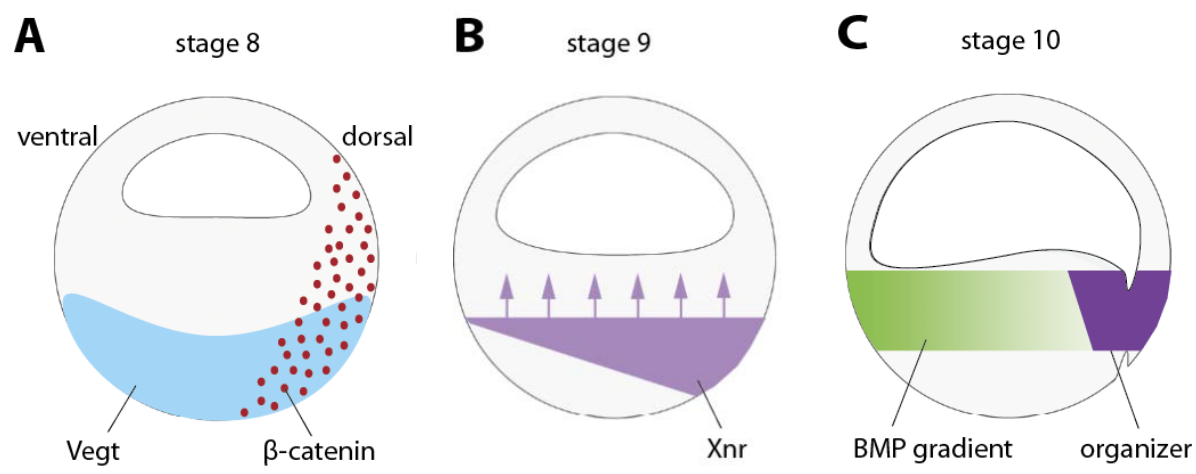

Fig. 1.2 Model for dorsal endoderm patterning, mesoderm induction and organizer formation in Xenopus

(A) At blastula stage, Vegt is localized at the vegetal hemisphere while $\beta$-catenin is stabilized dorsally. (B) At late blastula stage, a Xnr-gradient (Nodal-related) is generated by the synergistic activity of Vegt and $\beta$-catenin. (C) The mesoderm is specified by the Xnr-gradient and at early gastrula stage, the organizer is specified by synergistic activity of $\mathrm{Xnr}$ and $\beta$ catenin-targets Siamois and Twin. A BMP-gradient is generated by BMP-antagonists (for instance Noggin) secreted from the organizer (Adapted from Gilbert and Singer $8^{\text {th }}$ edition 2006; after Agius et al., 2000).

During gastrulation all three germ layers become patterned along the anteriorposterior axis by the organizer. While endoderm and mesoderm involute, the dorsal anterior endoderm is positioned at the prospective ventral foregut whereas the more posterior dorsal endoderm becomes the future dorsal foregut (Fig. 1.3 A, light green and yellow, Keller, 1991). The initial anterior-posterior pattern of the endoderm is not committing as the endoderm remains able to respond to signals released from the overlaying or subjacent mesoderm (Zeynali et al., 2000; Horb and Slack, 2001; McLin et al., 2007 and reviewed in McCracken and Wells, 2012). These include Wnt-, FGF-, BMP- and RA-signaling, which are associated with posteriorizing effects (Dessimoz et al., 2006; Rankin et al., 2011; Sherwood et al., 2011). The resulting gut tube is segmented into foregut, midgut and hindgut domains, whereby Pdx1 is expressed in the posterior foregut and anterior midgut (Wright et al., 1989). However, so far it is not known how FGF-, Wnt-, BMP- and RA-signaling co-operate during endoderm patterning. 


\subsection{Pancreas organogenesis}

\subsubsection{Pancreas specification}

Through cell movements during foregut morphogenesis the dorsal and ventral foregut epithelium are placed into very different environments. This fact already indicates that the extrinsic signals and genetic programs that promote pancreas development in the dorsal and ventral foregut are different. A further difference is the derivation of several organs from the ventral foregut, whereas the dorsal foregut gives rise exclusively to the dorsal pancreas. This suggests that dorsal pancreas formation is a matter of induction, whereas ventral pancreas needs to be segregated from multiple organ lines (reviewed in McCracken and Wells, 2012). Several studies confirmed these assumptions. In chick, Kim and colleagues demonstrated in 1997 that presumptive dorsal pancreatic endoderm when cultivated in isolation showed no pancreatic gene expression, whereas ventral pre-pancreatic endoderm does (Kim at al., 1997). Extensive studies in ectodermal, mesodermal and endodermal explants from Xenopus embryos supplied the evidence that the dorsal pancreatic foregut endoderm initially receives inductive signals from the mesoderm at the onset of gastrulation. Retinoic acid (RA) could be identified as such an inductive signal and the involvement of one or more further signals is assumed (RA-source indicated in Fig. 1.3 A (red)) (Chen at al., 2004; Pan et al., 2007).

During foregut morphogenesis the dorsal foregut epithelium has initially contact with the notochord and subsequently with the dorsal aorta. Both tissues have been shown to be sources of permissive signals for pancreas formation (Kim et al., 1997; Lammert at al., 2001). In chicken, dorsal pancreas development could be prevented by the removal of the notochord at an early stage (Kim et al., 1997). However, the notochord is not able to induce pancreatic gene expression in early gastrula stage or non-pancreatic somite-stage endoderm (Wells and Melton, 2000), which is in accordance with the finding that pre-pancreatic foregut endoderm obtained the ability, at an earlier time, to react to permissive signals from the notochord. These permissive signals were identified as Activin- $\beta B$ and FGF2 (Fig. 1.3 B). Hebrok and colleagues found them as secreted factors from the notochord that suppress the expression of Sonic hedgehog (Shh) in the pancreatic endoderm. Shh is broadly expressed in the endodermal epithelium but is excluded from pancreatic endoderm (Hebrok at al., 1998; Hebrok et al., 2000). The removal of the notochord leads to an ectopic Shh expression that inhibits dorsal pancreas development (Hebrok et al., 2000). It was shown that the repression of Shh in the dorsal endoderm induces 
Pdx1 expression in a dose dependent manner (Hebrok at al., 1998). With continuing embryonal development, the notochord is displaced by fused dorsal aortae that further signals to the dorsal pre-pancreatic endoderm (Lammert at al., 2001). These signals are required for the maintenance of $P d x 1$ expression and for the induction of Ptf1a expression (Yoshitomi and Zaret, 2004). It is assumed that aortic endothelial signals in addition act indirectly on pancreatic endoderm as they promote the survival of dorsal mesenchyme (reviewed in Edlund, 2002). Dorsal mesenchyme secretes FGF10 that is required for the maintenance of Pdx1 and Ptf1a expression (Jacquemin et al., 2006).

The ventral foregut gets in proximity to two mesodermal derivatives, the cardiac mesoderm and septum transversum (Fig. 1.3 B). The posterior region of the ventral foregut was shown to give rise to the ventral pancreas and liver. Explant studies in zebrafish and mouse support the presumption of a common bi-potential cell population in the ventral foregut endoderm that differentiate into liver and ventral pancreas (Deutsch et al., 2001; Bort et al., 2006; Chung et al., 2008). In several studies, signaling pathways have been identified which are involved in the segregation of hepatic and pancreatic fate, most of them appear to repress pancreas formation and promote liver development. FGF-signaling from the cardiac mesoderm (Jung et al., 1999) and BMP-signaling from the septum transversum (Rossi et al., 2001) induce hepatic fate. Deutsch and colleagues found an expression of Pdx1 in cultures of isolated mouse ventral foregut endoderm, whereas the expression of liver marker Hhex required signals from the cardiac mesoderm (Deutsch et al., 2001). These findings suggest the pancreas as a default state of the ventral foregut. Furthermore, a BMP antagonist TGIF $\beta$-induced factor 2 (TGIF2) was found to be expressed in the posterior ventral foregut counteracting the expression of Hhex (Spagnoli and Brivanlou, 2008). Moreover, the assumption of pancreas as a default state is supported by the presence of a pre-existing chromatin modification pattern that promotes pancreas development in the ventral foregut endoderm (Xu et al., 2011; Arnes and Sussel, 2015).

Independent of the way of specification, both dorsal and ventral pancreatic progenitor cell populations are characterized by the co-expression of Pdx1 and Ptf1a (Afelik et al., 2006). During this early phase of pancreatic development, also referred to as primary transition, the pancreatic progenitors proliferate (Pictet et al., 1972). Thus, pancreatic foregut epithelium evaginates and forms the dorsal and two ventral buds. Furthermore, the first Insulin-expressing cells in Xenopus and Glucagon-expressing cells in mouse can be detected. In mouse, the primary transition occurs between E9.5 and E13.5 (Jorgensen et al., 2007). The 
corresponding time period in Xenopus is from stage 28 to stage 38 (Horb and Slack, 2002).

A

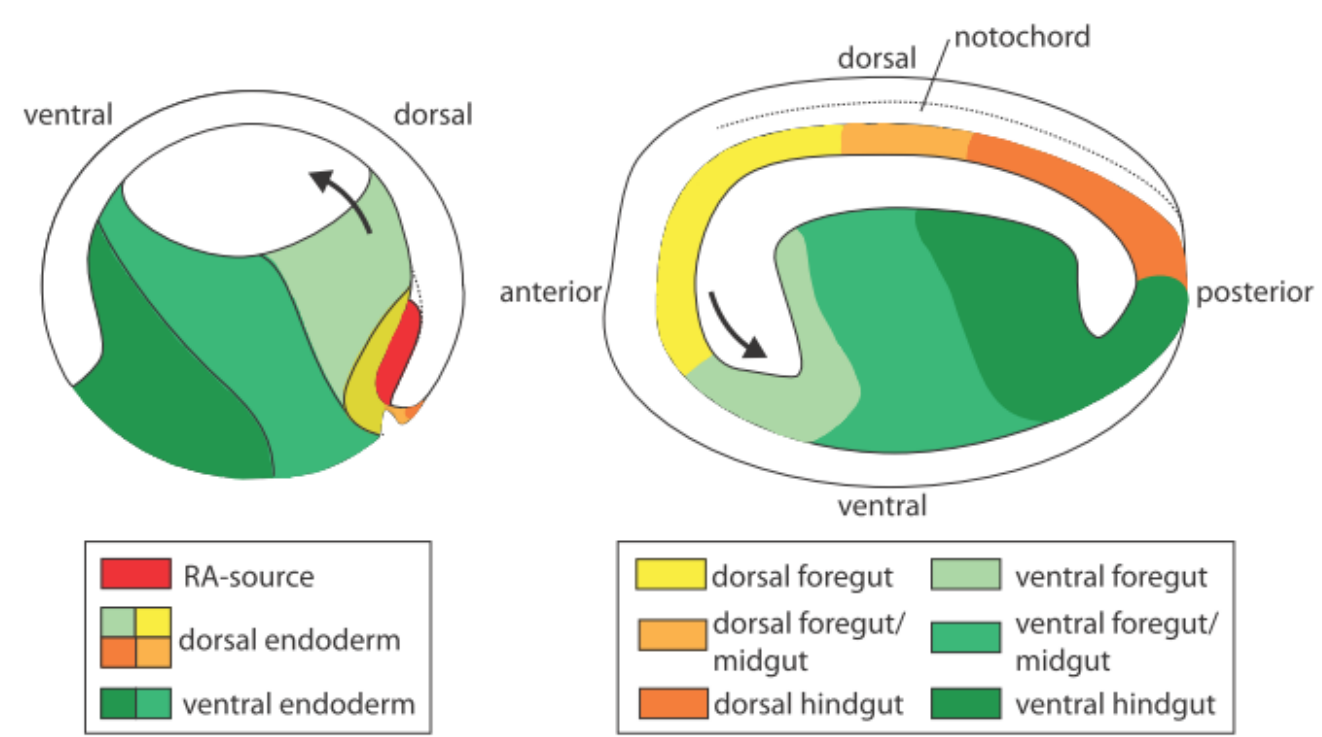

B
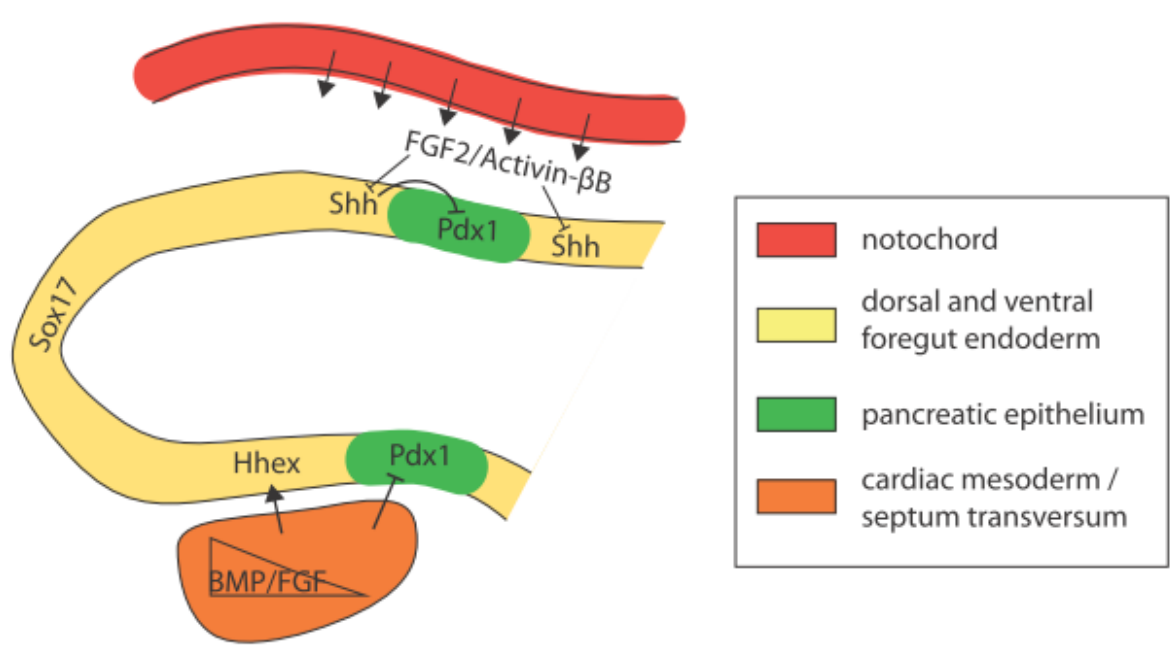

Fig. 1.3 Fate maps of Xenopus endoderm from gastrula to early somite stage and overview of signals involved in pancreas specification

(A) Gastrula stage (left) and early somite stage (right) Xenopus embryos with indicated colored domains that correspond to fate-map studies. The arrows indicate the migration/folding direction of the foregut. (modified after Zorn and Wells, 2009)

(B) Overview of signals involved in pancreas specification in an early somite stage embryo. In the dorsal foregut endoderm, permissive signals from the notochord (FGF2 and Activin$\beta B)$ repress Shh transcription in the pre-pancreatic epithelium and allow Pdx1 expression. FGF- and BMP-signaling from the cardiac mesoderm/septum transversuim promote liver (Hhex) and inhibit pancreas fate $(\mathrm{Pd} \times 1)$ in the ventral foregut epithelium. (modified after McCracken and Wells, 2012) 
Genetic lineage-tracing experiments in mice revealed that all pancreatic cellsubtypes arise from a common pool of multipotent progenitor cells co-expressing Pdx1 and Ptf1a (Gu et al., 2002; Kawaguchi et al., 2002; Burlison et al., 2008). In Xenopus, ectopic co-expression of Ptf1a and Pdx1 is sufficient to convert duodenal precursor cells into pancreatic tissue (Afelik et al., 2006). Furthermore, it was shown that constitutively active forms of $\mathrm{Pdx} 1$ or Ptf1a can push liver progenitors to a pancreatic fate (Horb et al., 2003, Jarikji et al., 2007). As soon as the ventral and dorsal pancreatic domains in the foregut epithelium are specified, a set of transcription factors becomes specifically expressed in these regions (Fig. 1.4 A). Beside Pdx1 and Ptf1a, the earliest transcription factor that marks pancreatic progenitor cells is Sox9 (Lioubinski et al., 2003; Lee and Saint-Jaennet, 2003). In mice, Sox9 was shown to be essential for proliferation and maintenance of pancreatic progenitors (Seymour et al., 2007; Seymour, 2014). Moreover, during the proliferation and expansion of the pancreatic progenitor field, Sox9 regulates a set of additional transcription factors in pancreatic progenitors including Hnf1b (Lynn et al., 2007). Genetic studies in mice revealed that Sox9 acts in cooperation with Pdx1 for the induction of pancreatic fate and the repression of the intestinal lineage differentiation (Shih et al., 2015).

\subsubsection{Pancreas differentiation}

At the end of the primary transition, pancreatic buds are still composed of undifferentiated multipotent progenitors expressing a set of transcription factors including Pdx1, Ptf1a, Sox9, Hnf1b, Nkx6.1 and Gata4 (Fig. 1.4 A) (reviewed in Shih et al., 2013; Seymour, 2014). The next phase of pancreas development, also referred to as secondary transition, is characterized by continuing proliferation and step-wise differentiation. This process starts with the segregation of a tip and trunk domain (Zhou et al., 2007; Villasenor et al., 2010). Lineage tracing experiments in mice showed that endocrine and ductal cells arise from bi-potential cells in the trunk domain whereas the tip regions are restricted to an acinar fate (reviewed in Pan and Wright, 2011). During the separation of tip and trunk, the expression of some transcription factors becomes restricted to only one of the two domains. Trunk specific expressed are Hnf1b, Sox9 and Nkx6.1, whereas Ptf1a and its target Cpa1 become restricted to the tips (Fig. 1.4 B, Zhou et al., 2007; Schaffer et al., 2010; Kopp et al., 2011b). The reciprocal repression between Nkx6.1 and Ptf1a is a critical mechanism through which the progenitors achieve distinct tip or trunk identity (Schaffer et al., 2010). Besides its early role in pancreas fate specification, Ptf1a 
later regulates acinar cell differentiation (Kawaguchi et al., 2002; Masui et al., 2010). Ptf1a acts in a trimeric pancreas transcription factor complex (Ptf1) together with the bHLH-protein E2A and suppressor of hairless RBP-j or its paralog RBP-jl (Obata et al., 2001; Beres et al., 2006). Prior to acinar cell differentiation Ptf1a interacts with RBP-j, which is replaced by RBP-jl during the secondary transition (Masui et al., 2007; Miyatsuka et al., 2007). This complex, termed PTF1-L, directs the activation of acinar-specific genes (Rose et al., 2001; Masui et al., 2010). In Xenopus, the exocrine marker pancreatic protein disulfide isomerase (Pdia2) can be first observed at stage 39, whereas other exocrine markers are not detected before stage 41 (Afelik et al., 2004; Horb and Slack 2002). In the adult exocrine pancreas of mice, lineage-tracing experiments indicate the existence of a low number of Ptf1a-positve multipotent pancreatic cells that can be induced upon injury (Pan et al., 2013).

The trunk domain is bi-potential and gives rise to ductal and endocrine cells (Solar et al., 2009; Kopp et al., 2011a). A subset of progenitor cells within the trunk express the transcription factor Neurogenin 3 (Ngn3) which indicates the onset of endocrine cell differentiation (Gradwohl et al., 2000; Gu et al., 2002). Cells within the trunk that do not activate Ngn3 probably contribute to ductal tissue (Magenheim et al., 2011). Several studies suggest that the Notch-signaling target Hes1 plays an important role in repressing $\mathrm{Ngn} 3$ expression and preventing extensive Ngn3 activation (Apelqvist et al., 1999; Jensen et al., 2000; Lee et al., 2001; Shih et al., 2012) (Fig. 1.4 C). Once $\mathrm{Ngn} 3$ is expressed, progenitors exit the cell cycle and delaminate from the epithelium to form the islet structures (Gouzi et al., 2011). Ngn3 activates the endocrine differentiation program by inducing pro-endocrine transcription factors including NeuroD1 and further factors involved in the delamination process (Huang et al., 2000; Mellitzer et al., 2006; Rukstalis and Habener, 2007; Soyer et al., 2010). Johansson and colleagues have shown in mice that the competence of endocrine precursors to produce different endocrine cell types is temporally controlled (Johansson et al., 2007). In mice the progenitors first differentiate into $\alpha$-cells, then $\beta$ - and $\delta$-cells, and finally PP-cells, each cell-type characterized by a distinct set of transcription factors caused by cross-regulation mechanisms between the single factors (reviewed in Pan and Wright, 2011; Beucher et al., 2012; Napolitano et al 2015). Despite the fact that in Xenopus endocrine progenitors first differentiate into $\beta$-cells (Kelly and Melton, 2000; Horb and Slack, 2002), the mechanism of subtype segregation may proceed in Xenopus similar to mice (Fig. 1.4 D). The formation of islets is completed in mice at E18.5 (Gittes, 2009) whereas in Xenopus a stable islet cell number is not observed before metamorphosis which is completed at stage 66 
(Maake et al., 1998). Moreover, a complex ductal system in the frog pancreas is not observed before the end of metamorphosis (Mukhi et al., 2008).
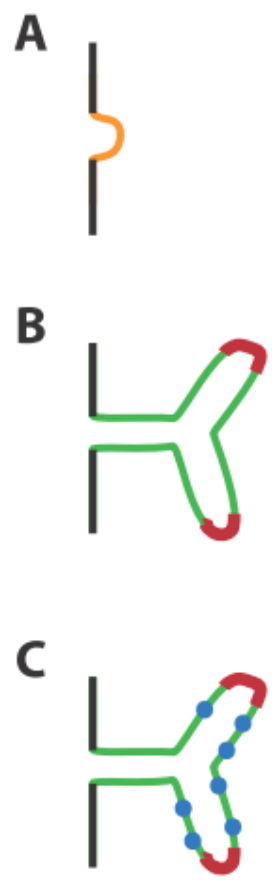

D

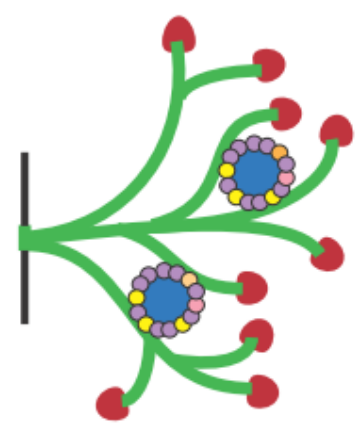

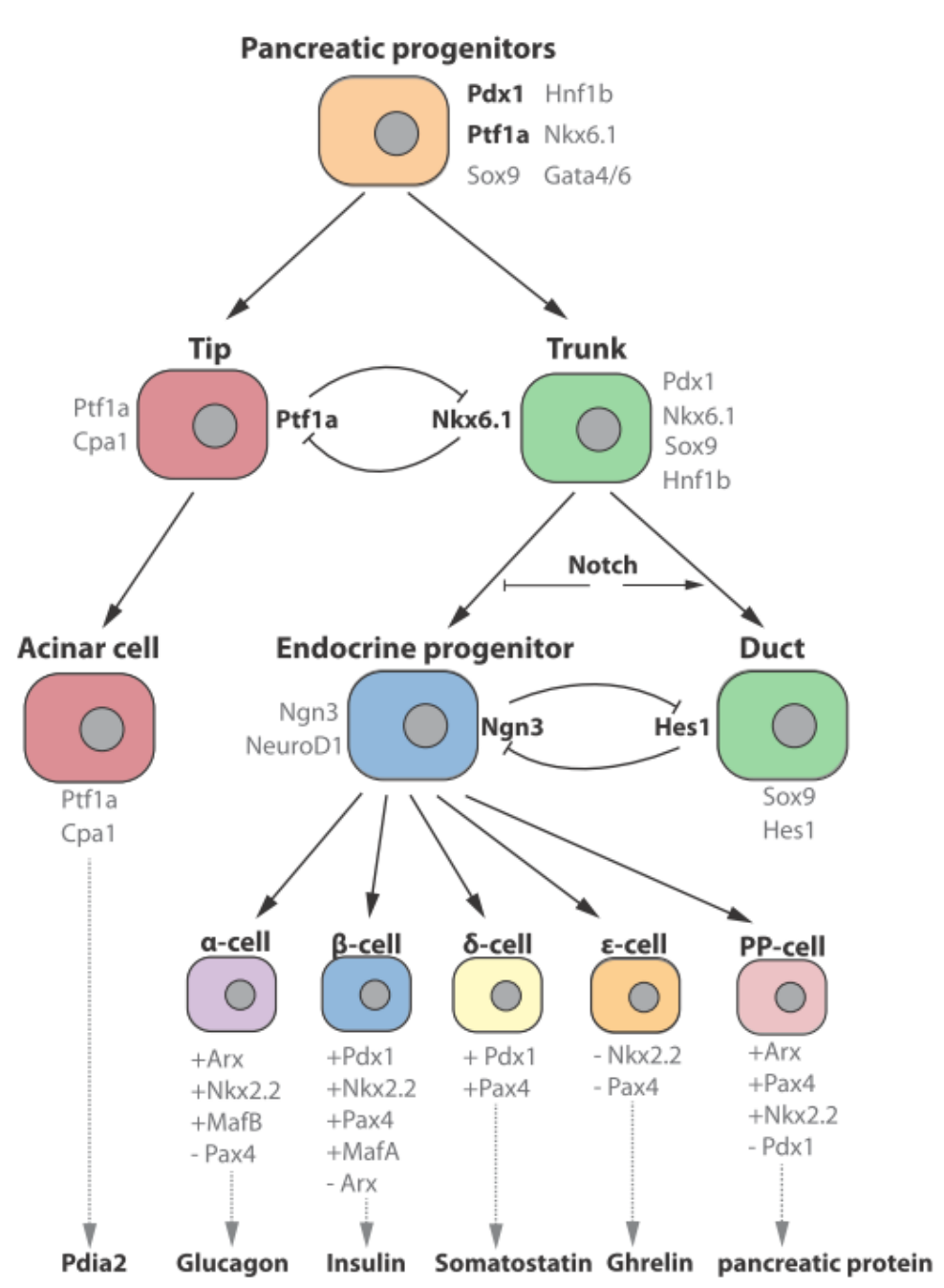

Fig. 1.4 Overview of pancreas organogenesis and lineage decisions

(A) During primary transition, pancreatic progenitors express transcription factors, including Pdx1, Ptf1a, Sox9, Hnf1b, Nkx6.1 and Gata4/6, which mediate proliferation and promote the maintenance of pancreatic identity. Thereby, pancreatic epithelium bulges out from the foregut epithelium to form a bud (orange). (B) At the onset of the secondary transition, the epithelial bud expands and branches. Pancreatic progenitors turn either into tip (red) or trunk (green) cells mediated by the cross-repression between Ptf1a and Nkx6.1. (C) Tip cells are restricted to an acinar fate (red) expressing Ptf1a and later enzymes like Pdia2. Bi-potential trunk cells adopt either ductal (green) or endocrine cell fate (blue). Ductal versus endocrine fate decision is regulated by Notch activity. High Notch signaling mediates ductal fate by the induction of the Ngn3-repressor Hes1. In endocrine progenitors, Ngn3 activates a set of transcription factors including NeuroD1 that regulate the endocrine differentiation.

(D) Endocrine progenitors subsequently delaminate from the trunk epithelium. Subsequently, they differentiate into five endocrine cell subtypes (marked by different colors) expressing distinct sets of transcription factors (+ present, - absent) and hormones. In mouse, at the end of embryogenesis, the pancreas consists of a highly branched ductal tree, connecting acinar cells with the intestine. Endocrine islets are scattered throughout the organ. In Xenopus, these histological structures are not observed before the end of metamorphosis (modified after Pan and Wright, 2011 and Shih, 2013). 


\subsection{Role of RA-signaling in pancreas development}

Retinoic acid (RA) is essential for normal embryogenesis of all vertebrates (reviewed in Rhinn and Dolle, 2012). RA is a vitamin A-derived, small non-peptic, lipophilic molecule. This molecule differs strongly from other signaling factors as it does not need any cell-surface receptors for signal transduction. RA is able to enter the nucleus and binds to target genes through nuclear receptors that recognize RAresponse elements (RAREs), thus switching from repressors to transcriptional activators (Germain et al., 2002). Vitamin A is absorbed from the food, stored in the liver and circulates as its alcohol form Retinol which is bound to a carrier protein, retinol-binding protein 4 (RBP4) (Quadro et al., 1999). During embryogenesis Retinol is maternally provided (Ismadi and Olson, 1982). Retinol-RBP4 enters cells, which is facilitated by the RA-inducible transmembrane protein STRA6 (stimulated by retinoic acid 6) (Kawaguchi et al., 2007) (Fig. 1.5 A). Within the cell, Retinol can be converted to RA in two enzymatic reactions. First, Retinol is reversibly oxidized by retinol dehydrogenases (ROLDH) to Retinal and subsequently irreversibly oxidized by retinal dehydrogenases (RALDH) to retinoic acid (RA) (reviewed in Duester, 2008). Therefore, Retinol is available to all cells of an embryo, but only cells which express one of the RALDHs can generate RA. In vertebrates, three isotypes of RALDH are described, whereby RALDH2 is earliest expressed (Niederreither et al., 1997). RA leaves the RA-generating cells and enters neighbouring cells where it has two main destinies. In cells which express one of the CYP26 genes (cytochrome P450 enzyme) at a high level, RA is converted into inactive metabolites (Ray et al., 1997; White et al., 1996; Hollemann et al., 1998). In cells without or low levels of CYP26, RA enters the nucleus and binds the heterodimeric receptors RAR/RXR (retinoic acid receptor/retinoid $X$ receptor), thereby activating the transcription of genes containing RAREs (reviewed in Mark et al., 2006).

Several early studies in mice and quail using Vitamin A deficiency or RA-receptor mutations indicate the requirement of RA for the development of various organs (Dersch and Zile, 1993; Lohnes et al., 1994; Mendelsohn et al., 1994; Dickman et al., 1997; Clagett-Dame and DeLuca, 2002). Further studies revealed that the requirement of RA for pancreas development is conserved among the vertebrates. Two independent studies in mouse using RALDH2 mutants demonstrated the necessity of RA for dorsal pancreas development (Martin et al., 2005; Molotkov et al., 2005). In zebrafish, additionally to its requirement for dorsal pancreas development, RA is also indispensable for the ventral pancreas and liver (Stafford et 
al., 2002). In Xenopus, studies using the synthetic RA-antagonist BMS453, which binds RA-receptors, show that like in mouse, RA is essential for dorsal pancreas development. Upon the inhibition of RA-signaling, expression of Shh was expanded into the prospective dorsal pancreatic endoderm, thereby repressing pancreatic fate. However, these effects of RA-inhibition were only observed when BMS453 was added before the end of gastrulation, indicating that RA acts at the onset of gastrulation in pancreas specification (Chen et al., 2004). Before that, in 2000 Asashima and colleagues already demonstrated the RA-dependent induction of pancreatic marker gene expression in dorsal lip explants from early gastrula stage Xenopus embryos (Moriya et al., 2000a). The assumption of a RA-gradient within the dorsal endoderm during gastrulation is supported by the expression pattern of RALDH2 and CYP26a1 during Xenopus gastrulation. These two enzyme-encoding genes show a non-overlapping expression in the dorsal mesoderm (Fig. 1.5 B). Thereby, RALDH2 is expressed in the internal involuting mesoderm, directly adjacent to the dorsal endoderm (Hollemann et al., 1998; Chen et al., 2001). Extensive combination experiments with endodermal and mesodermal explants revealed that RA acts directly on the dorsal endoderm as well as indirectly via the dorsal mesoderm (Pan et al., 2007). However, the RA-induced gene network that promotes pancreas fate has not yet been identified. 
A

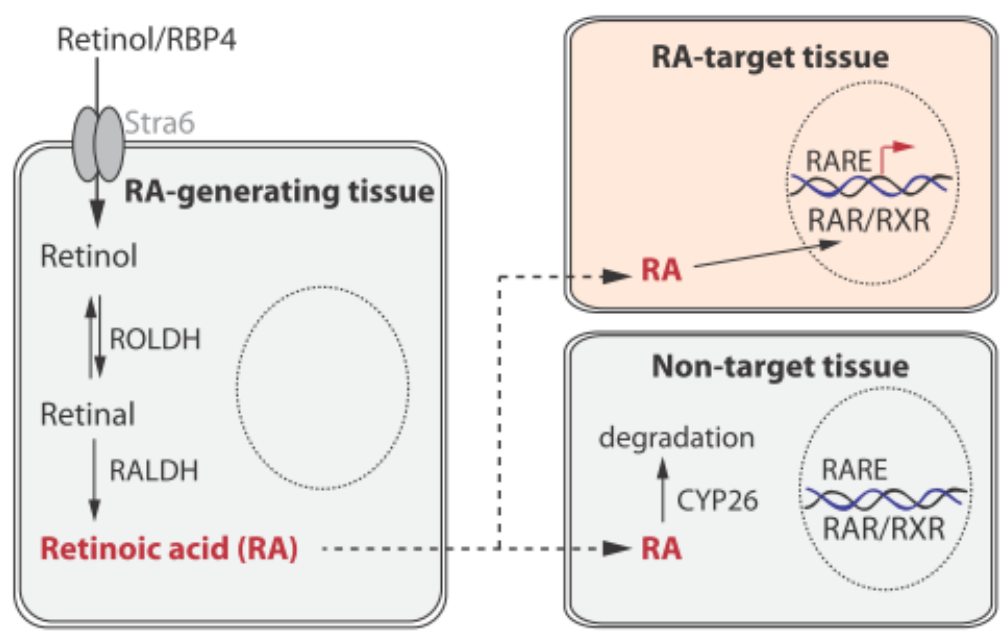

B

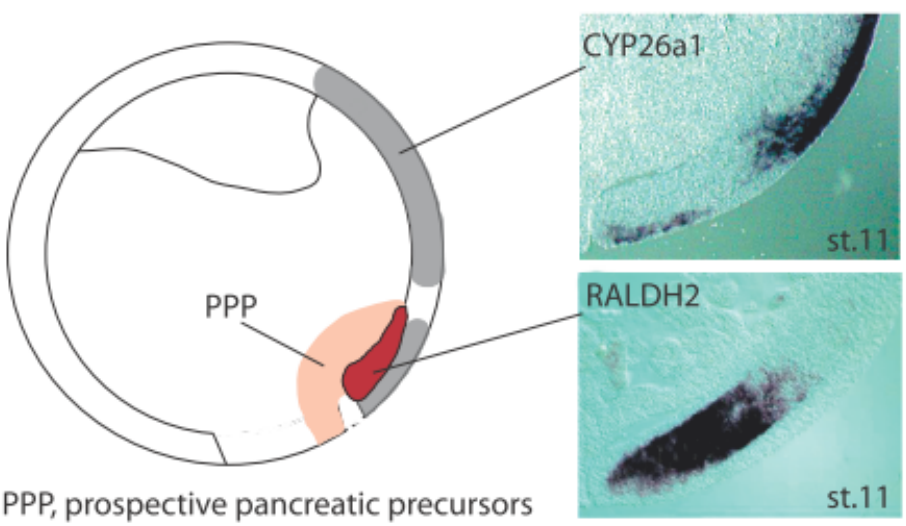

Fig. 1.5 Paracrine RA-signaling and expression of RA-metabolizing enzymes during Xenopus gastrulation

(A) Retinol is transported by the retinol-binding protein (RBP4) and enters the cells via the receptor Stra6 (Stimulated by retinoic acid 6). In RA-generating tissues, ROLDH (retinol dehydrogenases) is expressed which reversibly oxidize Retinol to Retinal. Retinal is further irreversibly oxidized to retinoic acid (RA) by RALDH (retinaldehyde dehydrogenases). RA is released and enters neighboring cells where it either gets oxidized by CYP26 (cytochrome P450) into inactive metabolites or it reaches the nucleus and bind to nuclear RA-receptor dimers RAR/RXR. RAR/RXR dimers are bound to RA-response elements (RARE) within the DNA and act as repressors until they form a complex with RA. The ternary RA-RAR/RXR complex acts as transcriptional activator recruiting further co-activators (modified from Duester, 2008). (B) Expression pattern of CYP26a1 and RALDH2 in a gastrula stage Xenopus embryo by WMISH. The pattern suggests the formation of a RA-gradient within the dorsal endoderm that specifies an area of putative dorsal pancreatic progenitors (modified after Hollemann et al., 1998; Chen et al., 2001). 


\subsection{Role of Wnt-signaling in pancreas development}

During embryonal development in vertebrates, Wnt-signaling has diverse functions. These involve both Wnt-signaling pathways, canonical as well as non-canonical, both initiated by Wnt ligands and Frizzled receptors (Logan and Nusse, 2004). Early in development, maternal $\beta$-catenin is dorsally stabilized, thereby promoting organizer formation and anterior endoderm fate during gastrulation (Zorn et al., 1999; Schier and Talbot, 2005; Rankin et al., 2011). Shortly after gastrulation at early somite stage, zygotic Wnt-signaling has an opposite effect on anterior endoderm. Several studies in Xenopus demonstrated that Wnt-signaling must be inhibited to maintain foregut identity which is essentially required for pancreas development. McLin and colleagues found that foregut gene expression is repressed by Wnt8-overexpression, while in contrast the $\mathrm{Pdx} 1$ domain is expanded by over-expression of Wnt-antagonist Dkk1 (McLin et al., 2007). Furthermore, Li and colleagues identified the secreted Wnt-antagonist Sfrp5 (secreted frizzled-related protein 5) which is expressed in the early foregut epithelium. Embryos with downregulated Sfrp5 developed smaller foregut domains, while ectopic Sfrp5 expression leads to an expanded foregut domain at the expense of the hindgut ( $\mathrm{Li}$ et al., 2008).

However, recent studies strongly indicate that the suggested model of a complete absence of Wnt-signaling for foregut development and high Wnt-signaling levels for hindgut development might be too simple. Two Wnt-signaling components, Wnt11 and Fzd7, were found to be expressed in the foregut endoderm, both mediating canonical as well as non-canonical Wnt-signaling (Wheeler and Hoppler, 1999; Djiane et al., 2000; Medina et al., 2000; Li et al., 2008). The depletion of Fzd7 in the foregut results in liver and pancreas agenesis. Therefore, low levels of Wnt/Fzd7 activity are found to be essential for foregut maintenance (Zhang et al., 2013a). Furthermore, Sfrps are shown to act biphasic on Wnt-signaling in a concentration dependent manner (Mii and Taira, 2009). A moderate dose of Sfrp5 was found to expand the foregut domain, whereas too low or too high concentrations lead to a reduction of foregut endoderm (Zhang et al., 2013a). Moreover, a comparative transcriptome analysis in mouse between liver and pancreas progenitors revealed a pancreas specific expression of Wnt-signaling components (Rodriguez-Seguel et al., 2013). These findings suggest the requirement of low Wnt-signaling activity for foregut maintenance and thereby pancreas development. Later in pancreas development, Wnt-signaling is essential for the expansion of pancreatic epithelium (Wells and Melton, 2000; Murtaugh et al., 2005; Dessimoz et al., 2005). However, 
how Wnt-signaling is regulated during pancreas specification and how it promotes the pancreatic fate remains unclear.

\subsection{Potential of organoids in research and clinical applications}

Organoids are defined as multicellular structures containing multiple organcharacteristic cell types. Moreover, organoids recapitulate the in vivo organogenesis including the temporal program of lineage specification and the expression of celltype specific marker genes (reviewed in Lancaster and Knoblich, 2014; Rookmaaker et al., 2015). Since decades, Amphibians and especially Xenopus were used as model system for in vitro organ formation as they provide a source of pluripotent cells. Already in the 1960s, Nieuwkoop and colleagues demonstrated the pluripotent state of blastocoel roof cells (Nieuwkoop, 1963). Blastocoel roof cells can be cultivated for weeks in a simple salt solution and without any further treatment they form an "atypical epidermis" (Jones, 1985). Thus, they are also named ectodermal explants. These pluripotent cells can be programed by RNA-injections or chemical treatments to differentiate into the derivatives of all three germ layers. In several studies, Asashima and colleagues demonstrated the in vitro formation of various organoids and tissues from Activin A-treated dissociated and re-aggregated ectodermal explants (reviewed in Okabayashi and Asashima, 2006; Asashima et al., 2009). Activin $A$ is a TGFß-family member which mimics nodal signaling in mouse (Conlon et al., 1994; Kubo et al., 2004). Asashima and colleagues found Activin A as concentration-dependent inducer of endodermal and mesodermal tissue (Ariizumi et al., 1991). Thereby, a high Activin A concentration induced the formation of head structures (Ariizumi and Asashima, 1995), whereas a moderate concentration leads to notochord structures and a low concentration to muscle tissue formation (Tamai et al., 1999). They further demonstrated that the additional treatment with RA induces pronephric or pancreatic gene expression (Chan et al., 1999; Moriya et al., 2000b; reviewed in Kurisaki et al., 2010). A later study in our lab applied a refined protocol for the in vitro generation of pancreatic structures from pluripotent ectodermal explants using Vegt and $\beta$-catenin as dorsal endoderm inducers and the BMP-inhibitor Noggin in addition to RA (Chen et al., 2004). A further notable study by Asashima and colleagues is the formation of ectopic beating hearts. Explants were treated with a high concentration of Activin A and started to beat after a few days. These beating structures were transplanted into neurula-stage embryos where they developed into ectopic beating hearts consisting of at least two chambers (Ariizumi et al., 2003; Kurisaki et al., 2010). 
As vertebrate organogenesis is highly conserved between Xenopus and mammals at the molecular level, the knowledge about factors required for the formation of Xenopus-derived organoids were applied to protocols for the in vitro generation of mouse- and human-derived organoids. For the formation of mouse- and humanderived organoids the use of a matrigel was shown to promote organoid formation. The matrigel is an extracellular matrix containing collagen and lamins and it allows the use of the self-organization potential of stem cells. This was first demonstrated for epithelial intestine stem cells (Lgr5 positive) (Li et al., 1987). Cultures of these cells in a three-dimensional matrigel-system lead to the formation of structures comparable to intestinal crypts comprising of cell subtypes found in the intestine in vivo (Sato et al., 2009). This system has been adapted to form other organs including stomach (Barker et al., 2010) as well as organs with low self-renewal potential like prostate (Karthaus et al., 2014). The addition of distinct factors to the culture medium drives lineage determination and differentiation. For example, mouse pancreatic organoids could be generated from pancreatic ductal cells expressing Lgr5 induced by injury and treated with EGF, RSPO1, Noggin, FGF10 and Nicotinamide. These organoids differentiate into duct and endocrine cells upon transplantation (Huch et al., 2013a). The 3D matrigel-system was also applied to form organoids from human cells. Several human adult and embryonic stem-cell derived organoids have been generated including stomach (McCracken et al., 2014), small intestine (Sato et al., 2011), lung (Rock et al., 2009), liver (Huch et al., 2013b) and pancreas (Boj et al., 2015). However, most of the organoids are not "perfect" as they miss characteristic cell types or recapitulate only the earliest stages of organogenesis (reviewed in Willyard, 2015).

As the availability of human adult stem cells is limited and the use of human embryonic stem cells is associated with ethical concerns, several studies used induced pluripotent stem cells (iPSCs) as additional source for the generation of organoids. Human iPSCs have been used to generate kidney- (Takasato et al., 2015) and intestine-organoids (Finkbeiner et al., 2015). The remarkable discovery of in vitro generated "mini brains" was made by Lancaster and colleagues. They found that iPSCs treated with growth factors self-organize into cerebral organoids with distinct forebrain, midbrain and hindbrain regions, cell layers that resemble the cortical layers of a brain and rudiments of eye tissue. These cerebral organoids could be maintained for several months under supply of nutrients (Lancaster et al., 2013).

The ability of in vitro generated organoids to recapitulate the normal organogenesis leads to manifold application possibilities (Fig. 1.6). For clinical applications, biopsy 
material can be cultivated in matrigel to form organoids. These can be used for studies of molecular pathogenesis in cancer-derived organoids as it was done for prostate cancer (Gao et al., 2014). Moreover, patient-derived organoids can serve for drug screens for high efficiency and low toxicity to allow a personalized medication. Furthermore, a recent study with organoids derived from patients with cystic fibrosis demonstrated the potential for gene therapy applications. Schwank and colleagues could repair the CFTR-mutation in patient-derived organoids by the use of CRISPR/Cas system (Schwank et al., 2013). This procedure is a promising method to provide patients with functional tissue. Further applications for research purposes are also conceivable. Some gene knockouts are lethal for mice and could be done in mice-derived organoids to allow functional analysis of these genes. Furthermore, the use of organoids could reduce the number of animals sacrificed for experiments. For instance, conditions can be tested in organoids first and the identified optimal conditions can then be applied to the animal model.

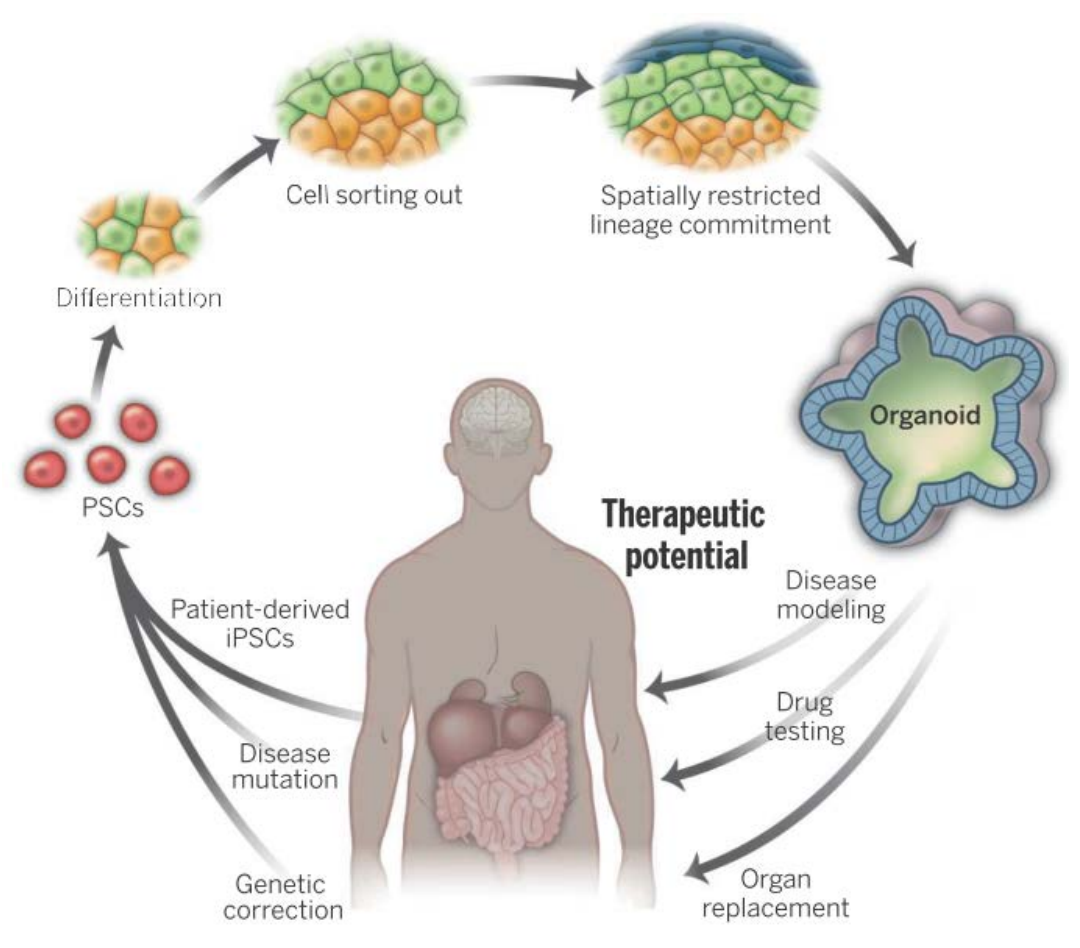

\section{Fig. 1.6 Therapeutic potential of in vitro generated organoids}

Biopsy material or patient-derived iPSCs can be used for in vitro organogenesis. The selforganizing potential through cell sorting and spatial restricted lineage determination promotes to the formation of organoids. These organoids can be used as model system for studies of molecular pathogenesis, drug testing or gene therapeutic applications. The image is adapted from Lancaster and Knoblich, 2014. 


\subsection{Aim of this study}

As described in the previous sections, various studies in Xenopus, zebrafish and mouse revealed that the requirement of RA for pancreas specification is conserved among these species. However, the gene network that is induced by RA and promotes pancreas fate is unknown so far. Therefore, the main goal of this study was the identification of early direct RA-target genes in the endoderm. To reach this aim, pancreatic organoids, in vitro generated from ectodermal explants, were used. For this, pancreatic organoids were first verified for their potential to recapitulate pancreas development. The second goal was the validation of identified RA-target genes for their requirement in pancreas development. 


\section{Materials and Methods}

\subsection{Materials}

\subsubsection{Model Organism}

For experimental studies, the African clawed frog Xenopus laevis was used. The frogs were purchased from Nasco (Ft. Atkinson, USA). The embryonic staging was based on the descriptions of Nieuwkoop and Faber (Nieuwkoop and Faber, 1967).

\subsubsection{Bacteria}

The following E.coli bacteria received from Stratagene $\mathrm{GmbH}$ (Heidelberg, Germany) were used for molecular biology standard methods: XL1-Blue recA1, endA1, gyrA96, thi-1, hsdR17, supE44, relA1, lac[F'proAB, $\operatorname{lacl}^{\mathrm{q}} \mathrm{Z} \Delta \mathrm{M} 15, \operatorname{Tn} 10\left(\right.$ Tet $\left.\left.^{\prime}\right)\right]^{\mathrm{c}}$ (Stratagene).

\subsubsection{Constructs}

\subsubsection{Constructs for sense and anti-sense RNA}

\subsection{Constructs prepared during this study}

\section{Hnf1b-pCS2+}

The open reading frame of Hnf1b was amplified from XHnf1b-pGEM7Z(-) (Vignali et al., 2000) using oligonucleotides MG102 and MG103 and cloned into the Clal and Xbal sites of pCS2+ vector (Rupp et al., 1994; Turner and Weintraub, 1994). This construct served as template for Hnf1b antisense RNA preparation and was linearized with BamHI and transcribed with T7-polymerase.

\section{HNF1b-GRpCS2+}

For the generation of a hormone-inducible Hnf1b for the purpose of gain of function experiments, the open reading frame of Hnf1b was amplified from XHnf1bpGEM7Z(-) (Vignali et al., 2000) without the stop-codon using the oligonucleotides MG102 and MG156. The PCR-product was then cloned into the Clal and Xhol sites of GRpCS2+, a pCS2+ derivate (Gammill and Sive, 1997), containing the sequence of the human glucocorticoid receptor (GR). For sense RNA preparation the construct was linearized with Notl and Hnf1b-GR transcribed with Sp6-polymerase. 


\section{Fzd4s-pCS2+}

For Fzd4-knockdown rescue experiments, the Fzd4s sequence was amplified from cDNA of stage 13 embryos using oligonucleotides MG169 and MG170 and cloned into the $\mathrm{pGem}^{\circledR}-\mathrm{T}$ Easy vector (Promega). The amplified sequence contained the ATG and 920 nucleotides downstream reaching the stop codon within the intron. From this construct the Fzd4s sequence was amplified with oligonucleotides MG173 and MG174 and cloned into the Clal and Xbal sites of pCS2+. For sense RNA preparation, the construct was linearized with Notl and transcribed with Sp6polymerase.

\section{Fzd4_intron-pGEMTeasy}

For specific Fzd4s-RNA detection in WMISH, a 600 nucleotide sequence of the Fzd4-intron was amplified from cDNA of stage 13 embryos using oligonucleotides MG165 and MG170 and cloned into pGem $^{\circledR}-\mathrm{T}$ Easy vector. Antisense RNA was prepared by Sall linearization and transcription with T7-polymerase.

\subsection{Provided constructs for sense and antisense RNA}

Tab. 2.1 Provided constructs for sense RNA

\begin{tabular}{|l|l|l|l|l|l|}
\hline \multirow{2}{*}{ Designation } & \multirow{2}{*}{ Vector } & \multicolumn{1}{|c|}{ Acession } & \multicolumn{2}{c|}{ reference } & \multicolumn{2}{c|}{ sense RNA } \\
\cline { 5 - 6 } Beta-Gal & pCS2+ & NC_000913.3 & $\begin{array}{l}\text { Chitnis et al., } \\
1995\end{array}$ & Notl & Sp6 \\
\hline Cyp26a1 & pBK-CMV & O93323 & $\begin{array}{l}\text { Hollemann et } \\
\text { al., 1998 }\end{array}$ & Mlul & T3 \\
\hline Fzd4-3'MT & MT/pCS2+ & NM_001090453 & $\begin{array}{l}\text { Swain et al., } \\
\text { 2005 }\end{array}$ & Notl & Sp6 \\
\hline GFP & MTpCS2+ & NC_011521.1 & $\begin{array}{l}\text { Rubenstein et } \\
\text { al., 1997 }\end{array}$ & Notl & SP6 \\
\hline Noggin & pGEM5ZF & M98807 & $\begin{array}{l}\text { Smith et al., } \\
1993\end{array}$ & Notl & SP6 \\
\hline Vegt & pCS2+ & AAB93301 & $\begin{array}{l}\text { Zhang and King, } \\
1996\end{array}$ & Notl & SP6 \\
\hline Wnt5a & MTpCS2+ & M55056.1 & $\begin{array}{l}\text { Damianitsch et } \\
\text { al., 2009 }\end{array}$ & Notl & Sp6 \\
\hline Wnt8a & pSP64T & CAA40510 & $\begin{array}{l}\text { Smith and } \\
\text { Harland, 1991 }\end{array}$ & BamHI & Sp6 \\
\hline
\end{tabular}


Tab. 2.2 Provided constructs for anti-sense RNA

\begin{tabular}{|c|c|c|c|c|c|}
\hline \multirow{2}{*}{ Designation } & \multirow{2}{*}{ Vector } & \multirow{2}{*}{ Accession } & \multirow{2}{*}{ referencelsource } & \multicolumn{2}{|c|}{ anti-sense RNA } \\
\hline & & & & Cut & Pol. \\
\hline Cebpd & pCMV-Sport6 & BC093576 & $\begin{array}{l}\text { (Ikuzawa et al., 2005) } \\
\text { Thermo Scientific } \\
\text { (7007767) } \\
\text { cloned with shortened } \\
\text { 3'UTR }\end{array}$ & EcoRI & $\mathrm{T} 7$ \\
\hline Cyp26a1 & pBK-CMV & 093323 & (Hollemann et al., 1998) & Clal & $\mathrm{T} 7$ \\
\hline $\begin{array}{l}\text { Dhrs3- } \\
\text { 3’FLag }\end{array}$ & $\begin{array}{l}\text { pCDNA3.1- } \\
\text { Flag }\end{array}$ & NM_001092373 & (Kam et al., 2010) & EcoRI & SP6 \\
\hline Foxh1 & MT/pCS2+ & $\begin{array}{l}\text { NM_001088351 } \\
\text { (missing first } \\
183 \text { bp from } \\
\text { ATG) }\end{array}$ & (Kofron et al., 2004) & EcoRI & $\mathrm{T7}$ \\
\hline Fst & pCMV-Sport6 & BC068649 & $\begin{array}{l}\text { (Tashiro et al., 1991) } \\
\text { Thermo Scientific } \\
\text { (4406472) }\end{array}$ & Sall & $\mathrm{T} 7$ \\
\hline Fzd4 & pCS2+ & NM_001090453 & (Swain et al., 2005) & $\mathrm{BamHI}$ & $\mathrm{T} 7$ \\
\hline Gbx2 & pGem-Teasy & AF395825 & $\begin{array}{l}\text { (Maczkowiak et al., } \\
\text { 2010) }\end{array}$ & Apal & SP6 \\
\hline Hnf1b & pCS2+ & NM_001089811 & (Vignali et al., 2000) & BamHI & $\mathrm{T} 7$ \\
\hline Hoxa1-b & pCMV-Sport6 & CF28664 & $\begin{array}{l}\text { (Sive and Cheng, 1991) } \\
\text { BioScience } \\
\text { (IRBHp998F2212170Q) }\end{array}$ & Sall & $\mathrm{T} 7$ \\
\hline Hoxb1 & pGEM-3ZF(-) & FJ422584 & (Nieto et al., 1992) & EcoRI & SP6 \\
\hline Hoxd1 & $\mathrm{MT} / \mathrm{pCS} 2+$ & Q08820 & (Sive and Cheng, 1991) & EcoRI & $\mathrm{T} 7$ \\
\hline Hoxd4 & pExpress & BC110765 & $\begin{array}{l}\text { (Klein et al., 2002) } \\
\text { BioScience } \\
\text { (IRBHp990G0190D) }\end{array}$ & EcoRI & $\mathrm{T} 7$ \\
\hline $\operatorname{lgf3}$ & pCMV-Sport6 & AAL06242 & $\begin{array}{l}\text { (Richard-Parpaillon et } \\
\text { al., 2002) }\end{array}$ & Sall & $\mathrm{T} 7$ \\
\hline Ins & pGem-Teasy & P12706 & (Shuldiner et al., 1989) & Not & $\mathrm{T7}$ \\
\hline Lhx1 & pBluescript & NM_001090659 & (Taira et al., 1994) & Xhol & $\mathrm{T} 7$ \\
\hline Meis3a & pSP64T & AF072895 & (Salzberg et al., 1999) & Clal & T3 \\
\hline Nkx6.2 & pBluescript & $\begin{array}{l}\text { NM_001096886 } \\
+270 \text { to }+990\end{array}$ & $\begin{array}{l}\text { (Dichmann and Harland, } \\
\text { 2011) }\end{array}$ & Xhol & $\mathrm{T} 7$ \\
\hline Pdia2 & pBK-CMV & AY351916 & (Sogame et al., 2003) & BamHI & $\mathrm{T} 7$ \\
\hline$P d x 1$ & pGem-Teasy & NM_001172211 & (Wright et al., 1989) & Apal & SP6 \\
\hline Prph & pCMV-Sport6 & BC056020 & $\begin{array}{l}\text { (Sharpe et al., 1989) } \\
\text { ATTC® } \\
(10167281)\end{array}$ & Sall & $\mathrm{T} 7$ \\
\hline Ptf1a & pGem-Teasy & DQ007931 & (Afelik et al., 2006) & Not & $\mathrm{T} 7$ \\
\hline$X I .45046$ & pCMV-Sport6 & CF286593 & $\begin{array}{l}\text { Source BioScience } \\
\text { (IMAGp998J07121170Q } \\
\text { ) }\end{array}$ & Sall & $\mathrm{T} 7$ \\
\hline$X I .47239$ & pCMV-Sport6 & IRAK288Co6 & $\begin{array}{l}\text { Source BioScience } \\
\text { (IRBHp990G0486) }\end{array}$ & Smal & $\mathrm{T} 7$ \\
\hline XI.51509 & pCMV-Sport6 & DY570900 & $\begin{array}{l}\text { Source BioScience } \\
\text { (IMAGp998L119296Q) }\end{array}$ & Sall & $\mathrm{T} 7$ \\
\hline XI.57926 & pCS111 & C0387168 & $\begin{array}{l}\text { Source BioScience } \\
\text { (IMAGp998C1718900Q) }\end{array}$ & Clal & $\mathrm{T7}$ \\
\hline
\end{tabular}




\begin{tabular}{|l|l|l|l|c|c|}
\hline Designation & \multicolumn{1}{|c|}{ Vector } & Accession & \multicolumn{2}{|c|}{ referencelsource } & \multicolumn{2}{|c|}{ anti-sense RNA } \\
\cline { 5 - 6 } Znf703-b & pExpress & DR726975 & $\begin{array}{l}\text { (Hufton et al., 2006) } \\
\text { BioScience } \\
\text { (IRBHp990A1190D) }\end{array}$ & Smal & T7 \\
\hline
\end{tabular}

\subsubsection{Constructs for Luciferase reporter assay}

Tab. 2.3 Luciferase assay constructs

\begin{tabular}{|l|l|l|}
\hline \multicolumn{1}{|c|}{ Designation } & \multicolumn{1}{|c|}{ Vector } & \multicolumn{1}{c|}{ Reference } \\
\hline Atf2-firefly & pGL3B & (van der Sanden et al., 2004) \\
\hline Renilla & pRL-TK & (Promega) \\
\hline Siamois-firefly & pGL3B & (Brannon et al, 1997) \\
\hline
\end{tabular}

\subsubsection{Constructs for CRISPR/Cas system}

Tab. 2.4 CRISPR/Cas system constructs

\begin{tabular}{|c|c|c|c|c|}
\hline \multirow{2}{*}{ Designation } & \multirow{2}{*}{ Vector } & \multirow{2}{*}{ Reference } & \multicolumn{2}{|c|}{ Sense RNA } \\
\hline & & & Cut & Pol. \\
\hline Cas9 & pCasX & (Blitz et al., 2013) & Acc651 & T7 \\
\hline Fzd4-gRNA & pDR274 & section 2.2.5.1 & Dral & T7 \\
\hline
\end{tabular}

\subsubsection{Constructs for real-time RT-PCR standard curves}

Tab. 2.5 Real-time PCR constructs

\begin{tabular}{|l|l|l|}
\hline \multicolumn{1}{|c|}{ Designation } & \multicolumn{1}{|c|}{ Vector } & \multicolumn{1}{c|}{ Reference } \\
\hline Insulin & pGem-T & (Shuldiner et al., 1989) \\
\hline Odc & pGem-T & (Klisch, 2006 PhD) \\
\hline Pdx1 & pGem-T & (Wright et al., 1989) \\
\hline Ptf1a & pGem-T & (Afelik et al., 2006) \\
\hline
\end{tabular}




\subsubsection{Oligonucleotides}

The oligonucleotides for cloning, sequencing and RT-PCR were purchased from SIGMA and dissolved in $\mathrm{dH}_{2} \mathrm{O}$ to $100 \mu \mathrm{M}$ stock solution. The antisense morpholino oligonucleotides were purchased from Gene Tools, LLC (Philomath, USA) and dissolved in RNase-free water to a concentration of $1 \mu \mathrm{M}$ or $2 \mu \mathrm{M}$.

\subsubsection{Cloning oligonucleotides}

\section{Tab. 2.6 Cloning oligonucleotides}

(the digestion enzyme target site is underlined)

\begin{tabular}{|l|l|l|}
\hline \multicolumn{1}{|c|}{ Label } & \multicolumn{1}{|c|}{ oligonucleotide } & \multicolumn{1}{c|}{ Sequence $\mathbf{5}^{\prime} \rightarrow \mathbf{3}^{\prime}$} \\
\hline MG102 & XHNF1b_Clal_fw & ccATCGATggATGGTGTCCAAGCTATCGCC \\
\hline MG103 & XHNF1b_stXba1_rev & GgTCTAGAGCTCACCATGCTTGCAAAGGACACTG \\
\hline MG156 & xHNF1b_Xho_rev & gcgCTCGAGcggCCATGCTTGCAAAGGACACTG \\
\hline MG165 & fz4_intron1_fw & GTTACGCGCAGCAAGTCATT \\
\hline MG169 & fz4S_sense_fw & ATGGGGGCAAGATCGCTGACC \\
\hline MG170 & fz4S_sense_rev & CACAGTCACTTTTTGTGGACG \\
\hline MG173 & fz4S_senseCla_fw & ccATCGATggATGGGGGCAAGATCGCTGACC \\
\hline MG174 & fz4S_senseXba_rev & gcTCTAGAgcCACAGTCACTTTTTGTGGACG \\
\hline MG171 & fz4_5UTRflfw & GATTGTCCGGGAGTGTGCTA \\
\hline MG248 & Fzd4s_P2_rev & GAAAGTAAACCCCCTGTGCTGAG \\
\hline MG273 & fzd7_fw & GTTACGTGGGCATCAACAGC \\
\hline MG274 & fzd7_rev & TTGTAGTTGGGACAGGGCAC \\
\hline MG275 & kremen2_fw & TCACGGTGAATGGGAGAGAC \\
\hline MG294 & kremen2_rev3 & ACAGTTTATTCATAGTGGAAGCTCA \\
\hline MG297 & impad1_fw4 & ATGACCTGAAATGGCTGCCT \\
\hline MG298 & impad1_rev2 & CATGGTCTGCAGTTCGTGATG \\
\hline
\end{tabular}




\subsubsection{Sequencing nucleotides}

Tab. 2.7 Sequencing oligonucleotides

\begin{tabular}{|l|l|}
\hline \multicolumn{1}{|c|}{ Designation } & \multicolumn{1}{c|}{ Sequence $\mathbf{5}^{\prime} \boldsymbol{\rightarrow} \mathbf{3}^{\prime}$} \\
\hline HNF1b_seq1 & GTTCCTGCTGCAAAGGCGATA \\
\hline HNF1b_seq2 & GTGGTGTATCTCCATCTAAAG \\
\hline HNF1b_seq3 & GGGCGTTCCATTGACGTAAATG \\
\hline pCS2_seq & GGGCGTTCCATTGACGTAAATG \\
\hline SP6 & TTAGGTGACACTATAGAATAC \\
\hline T3 & AATTAACCCTCACTAAAGGG \\
\hline T7 $($ pCS2+) & TCTACGTAATACGACTCACTATAG \\
\hline T7 $($ pGem-T) & TAATACGACTCACTATAGGGCGA \\
\hline
\end{tabular}

\subsubsection{Reverse transcriptase (RT) - PCR oligonucleotides}

Tab. 2.8 RT-oligonucleotides and working conditions

\begin{tabular}{|c|c|c|c|c|}
\hline Oligonucleotide & Label & Sequence & $\begin{array}{c}\mathrm{T}_{\text {Annealing }} \\
{\left[{ }^{\circ} \mathrm{C}\right]}\end{array}$ & Cycles \\
\hline Amylase_fw & LP1 & CAAGCTCTGCACTCGTTCTG & \multirow{2}{*}{55} & \multirow{2}{*}{34} \\
\hline Amylase_rev & LP1 & GTTTTCTATTTCGCCATCGC & & \\
\hline Bmp4-F & 338 & GCATGTACGGATAAGTCGATC & \multirow{2}{*}{58} & \multirow{2}{*}{32} \\
\hline Bmp4-R & 339 & GATCTCAGACTCAACGGCAC & & \\
\hline Cer1-F & 009 & TGCCCATGGAAACAAAAGTGC & \multirow{2}{*}{57} & \multirow{2}{*}{28} \\
\hline Cer1-R & 010 & AGCGTCAGGTGGTTCAGGGTAA & & \\
\hline CYP26_2C8_link_F & MG039 & GTCGACCTGTGGATCCAAAGA & \multirow{2}{*}{60} & \multirow{2}{*}{28} \\
\hline CYP26_2C8_link_R & MG040 & GATGCGTCTTGTAGATGCGAC & & \\
\hline CYP26_3'UTR_F & MG025 & CCCGGAGATTCCTCGAGGTT & \multirow{2}{*}{56} & \multirow{2}{*}{30} \\
\hline CYP26_3'UTR_R & MG026 & GACACCACGACCAAGACCCG & & \\
\hline Darmin_RT_fw & MG005 & GGTTACCGATTACTTGGAGG & \multirow{2}{*}{60} & \multirow{2}{*}{33} \\
\hline Darmin_RT_rev & MG006 & AGCATCATCTGGTCCACCAA & & \\
\hline Fzd4s_fw & MG247 & CATCAGGATCACCATGTGCCAG & \multirow{2}{*}{60} & \multirow{2}{*}{28} \\
\hline Fzd4s_rev & MG248 & GAAAGTAAACCСССTGTGCTGAG & & \\
\hline Glucagon_RT_F & LP1 & AGAATTTATTGAGTGGTTGA & \multirow{2}{*}{56} & \multirow{2}{*}{35} \\
\hline Glucagon_RT_R & LP1 & ATCGGCATGTCTTCTGTCC & & \\
\hline H4_RT_fw & 35 & CGGGATAACATTCAGGGTATCACT & \multirow{2}{*}{56} & \multirow{2}{*}{26} \\
\hline H4_RT_rev & 36 & ATCCATGGCGGTAACTGTCTTCCT & & \\
\hline
\end{tabular}




\begin{tabular}{|c|c|c|c|c|}
\hline Oligonucleotide & Label & Sequence & $\begin{array}{c}T_{\text {Annealing }} \\
{\left[{ }^{\circ} \mathrm{C}\right]}\end{array}$ & Cycles \\
\hline HNF1b_E1_fw & MG138 & GGACAGGTGCTCTGGGACAAG & $58 / 56$ & $28 / 31$ \\
\hline HNF1b_E2_rev & MG139 & СССТTTGTTTССТСАСАТАСС & 58 & 28 \\
\hline HNF1b_E3_rev & MG141 & GTCCTTGAACTTGATTTTGCT & 56 & 31 \\
\hline HNF1b_inj_R & MG149 & CGGGGACATGTGCAAGTTCT & 54 & 30 \\
\hline HNF1b_RT_F & MG075 & AAAGGGCAGAAGTGGACAGG & \multirow{2}{*}{58} & \multirow{2}{*}{32} \\
\hline HNF1b_RT_R & MG076 & ATGCAGCACGTTTTTGGGTC & & \\
\hline Hnf4a_RT_fw & MG157 & AGАСТССССААССАТСТССА & \multirow{2}{*}{60} & \multirow{2}{*}{33} \\
\hline Hnf4a_RT_rev & MG158 & CGCTTTCCCAAAGAGGCAAC & & \\
\hline Insulin_RT_F & LP1 & ATGGCTCTATGGATGCAGTG & \multirow[t]{2}{*}{56} & \multirow{2}{*}{$\begin{array}{l}33 \\
33\end{array}$} \\
\hline Insulin_RT_F & LP1 & AGAGAACATGTGCTGTGGCA & & \\
\hline ODC_RT_F & 324 & GCCATTGTGAAGACTCTCTCCATTC & \multirow{2}{*}{56} & \multirow{2}{*}{24} \\
\hline ODC_RT_R & 325 & TTCGGGTGATTCCTTGCCAC & & \\
\hline pCS2_RT_F & PB & CAAGCTACTTGTTCTTTTTTGC & 54 & 30 \\
\hline Pdx1_RT_fw & MG256 & GTCCTCCAGACATCTCACCG & \multirow{2}{*}{60} & \multirow{2}{*}{33} \\
\hline Pdx1_RT_rev & MG257 & AGCATGACTGCCAGCTCTAC & & \\
\hline Pia2_RT_F & LP1 & GGAGGAAAGAGGGACCAA & \multirow{2}{*}{60} & \multirow{2}{*}{33} \\
\hline Pia2_RT_R & LP1 & GCGCCAGGGCAAAAGTG & & \\
\hline Ptf1a_UTR_F & 348 & GTTGTCAGAACGGCCAAAGT & \multirow{2}{*}{60} & \multirow{2}{*}{33} \\
\hline Ptf1a_UTR_R & 349 & GGTACCGAGTGGAACCAAAG & & \\
\hline Sox17a_RT_F & MG063 & CAAGAGACTGGCACAGCAGA & \multirow{2}{*}{60} & \multirow{2}{*}{33} \\
\hline Sox17a_RT_R & MG064 & CTGCTTGGGGTTCCCTGTAG & & \\
\hline Sox2_fw & 266 & GAGGATGGACACTTATGCCCAC & \multirow{2}{*}{58} & \multirow{2}{*}{29} \\
\hline Sox2_rev & 267 & GGACATGCTGTAGGTAGGCGA & & \\
\hline Xfz4_RTE1_fw & MG163 & CCAAAATGCCCAACCTGGTG & \multirow{2}{*}{64} & \multirow{2}{*}{26} \\
\hline Xfz4_RTE2_rev & MG164 & TTGTGGTCATTCTGGGGTGG & & \\
\hline Xfz4S_RT_fw & MG161 & TTGTTGTACCTCCTGTGCTGCCTC & \multirow{2}{*}{60} & \multirow{2}{*}{29} \\
\hline Xfz4S_RT_rev & MG162 & TGGTAGAGTGAAATGCGCAGCAGC & & \\
\hline
\end{tabular}




\subsubsection{Real-time PCR oligonucleotides}

Tab. 2.9 Real-time PCR oligonucleotides

\begin{tabular}{|l|l|l|l|}
\hline Oligonucleotide & Label & Sequence & $\begin{array}{l}\text { target region } \\
\text { (bp) }\end{array}$ \\
\cline { 1 - 2 } ODC_RT_F & 324 & GCCATTGTGAAGACTCTCTCCATTC & \multirow{2}{*}{+222 to +441} \\
\hline ODC_RT_R & 325 & TTCGGGTGATTCCTTGCCAC & \multirow{2}{*}{+1 to +291 } \\
\hline Insulin_RT_F & LP1 & ATGGCTCTATGGATGCAGTG & \multirow{2}{*}{+170 to +551} \\
\hline Insulin_RT_F & LP1 & AGAGAACATGTGCTGTGGCA & \multirow{2}{*}{+522 to +732} \\
\hline Pdx1_fw & MG256 & GTCCTCCAGACATCTCACCG & \\
\hline Pdx1_rev & MG257 & AGCATGACTGCCAGCTCTAC & \\
\hline Ptf1a_fw & MG271 & GGTACAGTCCGATCTGCCGC & \\
\cline { 1 - 3 } Ptf1a_rev & MG272 & GGAGTCCACACTTTGGCCGT &
\end{tabular}

\subsubsection{5 sgRNA oligonucleotides}

\begin{tabular}{|l|l|}
\hline Target site in the Fzd4 gene & GGCACATG[GTGATCCTGATG] \\
\hline Forward (5'Phos) & TAGGCACATGGTGATCCTGATG \\
\hline Reverse (5'Phos) & AAACCATCAGGATCACCATGTG \\
\hline
\end{tabular}

\subsubsection{Morpholino oligonucleotides}

Tab. 2.10 Morpholino oligonucleotides

\begin{tabular}{|l|l|l|}
\hline \multicolumn{1}{|c|}{ Designation } & \multicolumn{1}{|c|}{ Target RNA } & \multicolumn{1}{c|}{ Sequence $\mathbf{5}^{\prime} \rightarrow \mathbf{3}^{\prime}$} \\
\hline HNF1b-MO & $\begin{array}{l}\text { Xenopus laevis HNF1 } \beta \\
\text { intron 1/exon2 boundary }\end{array}$ & ATCCTCGCTGTGAACAAAACACAAA \\
\hline Fzd4-MO & $\begin{array}{l}\text { Xenopus laevis Fzd4 } \\
\text { ATG region (Gorny et al., 2013) }\end{array}$ & ATTATTCTTCTTCTGTTGCCGCTGA \\
\hline Fzd4-mmMO & $\begin{array}{l}\text { mutated Fzd4-MO, which does not } \\
\text { bind target }\end{array}$ & ATTATTaTTaTTCTaTTGCaGCTaA \\
\hline ctr-MO & no target & CCTCTTACCTCAGTTACAATTTATA \\
\hline
\end{tabular}




\subsection{Methods}

\subsubsection{DNA methods}

\subsubsection{Chemical transformation and cultivation of bacterial cells}

LB-medium: $\quad$ Bacto Trypton $10 \mathrm{~g}(1 \%)$; Bacto Yeast Extract $5 \mathrm{~g}(0.5 \%)$; $\mathrm{NaCl} 10 \mathrm{~g}(17.1 \mathrm{mM}) ; \mathrm{dH}_{2} \mathrm{O}$ to $1 \mathrm{l} ; \mathrm{pH}$ to 7.5

LB-agar: $\quad$ Agar $15 \mathrm{~g}(1.5 \%)$ to 1 I LB-medium

Amplicillin: $\quad$ stock solution $100 \mathrm{mg} / \mathrm{ml}$ in $\mathrm{dH}_{2} \mathrm{O}$; stored at $-20^{\circ} \mathrm{C}$; working solution $100 \mu \mathrm{g} / \mathrm{ml}$

Kanamycin: $\quad$ stock solution $100 \mathrm{mg} / \mathrm{ml}$ in $\mathrm{dH}_{2} \mathrm{O}$; stored at $-20{ }^{\circ} \mathrm{C}$; working solution $50 \mu \mathrm{g} / \mathrm{ml}$

In order to increase the amount of DNA for analytical and preparative methods, chemically competent E.coli XL1blue were used. $200 \mu \mathrm{l}$ of bacterial cell suspension were thawed on ice, mixed with $100 \mathrm{ng}$ of plasmid DNA or $5 \mu \mathrm{l}$ of ligation mix, incubated for $30 \mathrm{~min}$ on ice and heat-shocked for $90 \mathrm{sec}$ at $42{ }^{\circ} \mathrm{C}$ and $1 \mathrm{~min}$ on ice. $800 \mu \mathrm{l}$ LB-medium was added and the solution was incubated with mild shaking for $1 \mathrm{~h}$ at $37{ }^{\circ} \mathrm{C}$. The bacterial cells were then pelleted by $30 \mathrm{sec}$ centrifugation at $10,000 \mathrm{rpm}$, re-suspended in $100 \mu \mathrm{l}$ LB-medium and seeded on LB-agar plates supplemented with ampicillin or kanamycin. Colonies were grown over night at 37 ${ }^{\circ} \mathrm{C}$. Single colonies were picked and cultivated in LB-medium containing the appropriate antibiotic (Sambrook and Russel, 2001).

\subsubsection{Plasmid DNA preparations}

For the plasmid preparation in analytical amounts, the "GeneJET" ${ }^{\mathrm{TM}}$ Plasmid Miniprep" kit (Thermo Scientific) was used. For the isolation of plasmid DNA in preparative amounts the "NucleoBond ${ }^{\circledR}$ Xtra Midi" kit (Machery-Nagel) was used according to the manufacturer's instructions. The DNA concentration was measured with the NanoDrop-2000c spectrometer (Thermo Scientific). 


\subsubsection{DNA restriction digestion}

Restriction digests for the purpose of cloning or linearization were carried out with restriction enzymes from Thermo Scientific according to the manufacturer's protocol. The digestions were performed with 2 to $5 \mathrm{U}$ of the appropriate enzyme per $\mu \mathrm{g}$ DNA and incubated at $37^{\circ} \mathrm{C}$ for at least $1 \mathrm{~h}$.

\subsubsection{Agarose gel electrophoresis}

TAE (Tris/Acetat/EDTA): $\quad 40 \mathrm{mM}$ Tris Acetate; $2 \mathrm{mM}$ EDTA; $\mathrm{pH} 8.5$

6x DNA loading dye: $\quad 10 \mathrm{mM}$ Tris-HCL ( $\mathrm{pH} 7.6) ; 0,03 \%$ Bromophenol Blue; $0,03 \%$ Xylene Cyanol FF; $60 \%$ Glycerol; 60 mM EDTA

2x RNA loading dye: $\quad$ 95\% Formamide; 18 mM EDTA; $0.025 \%$ of each SDS, Xylene Cyanol and Bromophenol Blue (Ambion)

The agarose gel electrophoresis was used for the analysis of DNA-restriction or PCR products as well as for the quality control of sense and antisense RNA (Sharp et al., 1973). Agarose was boiled in $1 \times$ TAE buffer to prepare a $0.7 \%$ to $2 \%(\mathrm{w} / \mathrm{v})$ gel, depending on the size of the DNA/RNA fragments. Standard DNA ladders were used to define the size of DNA fragments (Fermentas High, Middle or Low Range). DNA loading dye was added to DNA samples and RNA-samples were mixed with RNA loading dye prior to loading. To visualize the DNA/RNA, ethidium bromide (0.5 $\mu \mathrm{g} / \mathrm{ml}$ ) was added. For the documentation the ChemiDoc video documentation system (EASY view) was used.

\subsubsection{Purification of DNA fragments from agarose gel or digestions}

For the purification of DNA fragments from agarose gels or restriction digestions, the "Invisorb Fragment Cleanup" kit (Invitek) was used according to the manufacturer's instructions. 


\subsubsection{Polymerase chain reaction (PCR)}

The amplification of DNA- or cDNA-fragments was done by PCR (Saiki et al., 1985; Mullis et al., 1986). The reaction needs oligonucleotides complementary to the ends of the sequence that is to be amplified, nucleotides and a DNA-polymerase. The reaction proceeds in three steps that are repeated. Initially, the DNA template is heated to $95^{\circ} \mathrm{C}$ to render it single-stranded (Denaturation). Next, the oligonucleotides bind to the complementary sequence at a lower temperature specific for their size and composition (Annealing). Finally, the bound oligonucleotides are extended by the DNA-polymerase at $72{ }^{\circ} \mathrm{C}$ (Elongation). The DNA-Polymerase as well as the length and composition of the oligonucleotides varied depending on the purpose of amplification.

\subsection{Cloning PCR}

For the purpose of molecular cloning, DNA fragments were amplified by the use of the High Fidelity PCR enzyme Mix (Thermo Scientific). This Mix contains Taq DNA Polymerase and an additional thermostable DNA polymerase that possesses $3^{\prime} \rightarrow 5^{\prime}$ exonuclease "proof-reading" activity. This "proof-reading" activity reduces the occurrence of point mutations during the amplification process.

The PCR reaction mixture contained the following components:

$\begin{array}{lll}\mu \mathrm{l} & \text { final conc. } & \text { component } \\ 1 & 100 \mathrm{ng} & \begin{array}{l}100 \mathrm{ng} / \mu \mathrm{l} \text { template DNA } \\ 10\end{array} \\ 1 \mathrm{x} & \begin{array}{l}5 \mathrm{x} \mathrm{High} \text { Fidelity Buffer with } 15 \mathrm{mM} \\ \mathrm{MgCl}_{2}\end{array} \\ 1 & 0.2 \mathrm{mM} \text { (each) } & \begin{array}{l}10 \mathrm{mM} \text { dNTP Mix (Thermo Scientific) } \\ 10 \mu \mathrm{M} \text { oligonucleotide mix each }\end{array} \\ 0.5 & 0.2 \mu \mathrm{M} & \begin{array}{l}\text { High Fidelity PCR enzyme Mix } \\ \text { (Thermo Scientific) }\end{array} \\ \text { Add water to } 50 \mu \mathrm{l} & 0.02 \mathrm{U} & \end{array}$

The following cycling conditions were used for the amplification:

\begin{tabular}{|c|c|c|c|}
\hline Initial denaturation & $95^{\circ} \mathrm{C}$ & $5 \mathrm{~min}$ & \\
\hline Denaturation & $95^{\circ} \mathrm{C}$ & $45 \mathrm{sec}$ & \\
\hline Annealing & $\mathrm{x}^{\circ} \mathrm{C}$ & $45 \mathrm{sec}$ & 35 cycles \\
\hline Elongation & $72^{\circ} \mathrm{C}$ & $1 \mathrm{~min} / 1 \mathrm{~kb}$ & \\
\hline Final elongation & $72^{\circ} \mathrm{C}$ & $5 \mathrm{~min}$ & \\
\hline
\end{tabular}




\subsection{Semi-quantitative PCR}

For the semi-quantitative PCR, cDNA was used (preparation described in chapter 2.2.2.4). Thus, the semi-quantitative $P C R$ was used to analyze temporal gene expression patterns or alterations of gene expression upon microinjections or chemical treatments. The following components and conditions were used.

$\begin{array}{lll}\mu \mathrm{l} & \text { final conc. } & \text { component } \\ 2.5 & \sim 125 \mathrm{ng} & \sim 50 \mathrm{ng} / \mu \mathrm{l} \mathrm{cDNA} \\ 2.5 & 1 \mathrm{x} & 5 \times \text { Flexi GoTaq buffer with } 25 \mathrm{mM} \\ & & \mathrm{MgCl}_{2} \\ 0.625 & 0.5 \mu \mathrm{M} & \begin{array}{l}10 \mu \mathrm{M} \text { gene specific oligonucleotide } \\ \text { mix }\end{array} \\ 0.1 & 0.5 \mathrm{U} & \text { GoTaq polymerase (Promega) }\end{array}$

Add water to $12.5 \mu \mathrm{l}$

\begin{tabular}{lll|} 
Initial denaturation & $95^{\circ} \mathrm{C}$ & $5 \mathrm{~min}$ \\
\hline Denaturation & $95^{\circ} \mathrm{C}$ & $45 \mathrm{sec}$ \\
Annealing & $\mathrm{x}^{*} \mathrm{C}$ & $45 \mathrm{sec}$ \\
Elongation & $72^{\circ} \mathrm{C}$ & $45 \mathrm{sec}$
\end{tabular} \mid $x^{* *}$ cycles

$x^{*}=$ oligonucleotide specific annealing temperature

$\mathrm{x}^{* *}=$ cycle number depends on the mRNA copy number

\subsection{Quantitative real-time PCR}

For the quantification of gene expression levels, cDNA was applied to the real-time PCR. To detect the amplified DNA the fluorescent dye SYBR Green was used that intercalates into double stranded DNA. The fluorescence is measured each cycle and increases in correlation with the increase in PCR-product. For this purpose, a PCR reaction mix containing SYBR Green was used (Biorad) and the detection took place in the IQ5 Biorad machine. All measurements were performed as duplicates and normalized to the values of ornithine decarboxylase (odc). 


$\begin{array}{lll}\mu \mathrm{l} & \text { final conc. } & \text { component } \\ 2.5 & \sim 250 \mathrm{ng} & \sim 100 \mathrm{ng} / \mu \mathrm{l} \text { cDNA } \\ 10 & 1 \mathrm{x} & 2 \times \mathrm{SYBRG} \text { reen supermix (Biorad) } \\ 0.4 & 0.2 \mu \mathrm{M} & 10 \mu \mathrm{M} \text { gene specific oligonucleotide } \\ & & \text { mix }\end{array}$

Add water to $20 \mu \mathrm{l}$

\begin{tabular}{lcc|c} 
Initial denaturation & $95^{\circ} \mathrm{C}$ & $3 \mathrm{~min}$ & \\
\hline Denaturation & $95^{\circ} \mathrm{C}$ & $10 \mathrm{sec}$ & \\
Annealing & $59^{\circ} \mathrm{C}$ & $15 \mathrm{sec}$ & 60 cycles \\
Elongation & $72^{\circ} \mathrm{C}$ & $30 \mathrm{sec}$ & \\
Melting curve & $56-95^{\circ} \mathrm{C}$ & $+1{ }^{\circ} \mathrm{C} / \mathrm{sec}$ & 39 cycles
\end{tabular}

\subsubsection{DNA sequencing analysis}

To confirm correct DNA sequences, the Dye-termination sequencing method, based on Sanger's chain-termination sequencing, was used (Sanger et al., 1977). The Sequencing was performed with the "Big Dye Terminator Cycle Sequencing" kit (Applied Biosystems). The following sequencing PCR reaction components and conditions were applied.

$\begin{array}{lll}\mu \mathrm{l} & \text { final conc. } & \text { component } \\ 1 & 500 \mathrm{ng} & 500 \mathrm{ng} / \mu \mathrm{l} \mathrm{DNA} \\ 1.5 & & \text { seq mix } \\ 1.5 & 1 \mathrm{x} & \text { seq buffer } \\ 0.625 & 0.625 \mu \mathrm{M} & 10 \mu \mathrm{M} \text { sequencing oligonucleotide }\end{array}$

Add water to $10 \mu \mathrm{l}$

\begin{tabular}{lll|l} 
Denaturation & $95^{\circ} \mathrm{C}$ & $2 \mathrm{~min}$ \\
Denaturation & $95^{\circ} \mathrm{C}$ & $10 \mathrm{sec}$ & \\
Annealing & $\mathrm{x}^{\circ} \mathrm{C}$ & $15 \mathrm{sec}$ & 25 cycles \\
Elongation & $60^{\circ} \mathrm{C}$ & $4 \mathrm{~min}$ &
\end{tabular}

$\mathrm{x}=$ oligonucleotide specific annealing temperature 
The DNA fragments, obtained from the sequencing reaction, were purified by the addition of the following components to the $10 \mu \mathrm{l}$ reaction mix.

$$
\begin{array}{ll}
1 \mu \mathrm{l} & 125 \mathrm{mM} \text { EDTA }(\mathrm{pH} 8.0) \\
1 \mu \mathrm{l} & 3 \mathrm{M} \text { sodium acetate }(\mathrm{pH} 5.4) \\
50 \mu \mathrm{l} & 100 \% \text { ethanol }
\end{array}
$$

The mixture was incubated for $5 \mathrm{~min}$ at room temperature following a centrifugation for $15 \mathrm{~min}$ at $14000 \mathrm{rpm}$. DNA pellets were washed with $70 \mu \mathrm{l} 70 \%$ ethanol. The airdried DNA pellets were dissolved in $15 \mu \mathrm{HiDi}{ }^{\mathrm{TM}}$ buffer (Applied Biosystems). The obtained sequencing PCR products were analyzed by the $\mathrm{ABI} 3100$ Automated Capillary DNA Sequencer (Applied Biosystems).

\subsubsection{RNA methods}

\subsubsection{In vitro synthesis of capped and uncapped sense mRNA}

Capped sense mRNAs for the microinjection into Xenopus embryos were in vitro synthesized using the SP6, T7 or T3 mMessage mMachine Kits ${ }^{\text {TM }}$ (Ambion). $1 \mu \mathrm{g}$ of linearized plasmid DNA template was used in $20 \mu \mathrm{l}$ reaction mixture. For uncapped sgRNA preparation, $2 \mu \mathrm{g}$ of linearized plasmid DNA were used in $25 \mu$ l reaction mixture with components indicated in section 2.2.2.2 devoid of Dig-UTP. After an incubation of 3 hours at $37{ }^{\circ} \mathrm{C}$ the DNA template was removed by $5 \mathrm{U}$ of Turbo DNase I (Ambion) for 30 min at $37^{\circ} \mathrm{C}$. The synthesized RNA was purified by the use of the Illustra TM RNAspin Mini RNA Isolation Kit (GE Healthcare). RNA concentration was measured by the NanoDrop and the quality was analyzed on a 1 $\%$ agarose gel. RNA aliquots were stored at $-80^{\circ} \mathrm{C}$. 


\subsubsection{In vitro synthesis of labeled antisense RNA}

Anti-sense RNA probes were used to detect endogenous transcripts by whole mount in situ hybridization (see chapter 2.2.4.). $1 \mu \mathrm{g}$ of linearized plasmid DNA was used in $25 \mu \mathrm{l}$ reaction. Following components were included in the reaction to generate Dig-labeled anti-sense RNA:

\begin{tabular}{|c|c|}
\hline & \\
\hline 5 & $5 x$ transcription buffer (Fermentas) \\
\hline \multirow[t]{2}{*}{4} & ATP, GTP, CTP, UTP, Dig-UTP (10mM each) \\
\hline & (Boehringer) \\
\hline 1 & DTT $(0.75 \mathrm{M})$ \\
\hline \multirow[t]{2}{*}{1} & 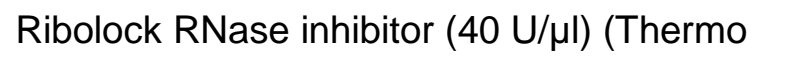 \\
\hline & Scientific) \\
\hline 1.5 & RNA polymerase (20 U/ $\mu$ l Fermentas) \\
\hline
\end{tabular}

The reaction mixture was incubated for $3 \mathrm{~h}$ at $37^{\circ} \mathrm{C}$. The DNA template was removed using $2 \mathrm{U} / \mu \mathrm{l}$ Turbo DNasel (Ambion) for $30 \mathrm{~min}$ at $37^{\circ} \mathrm{C}$. Anti-sense RNA was purified with the RNeasy ${ }^{\circledR}$ Mini Kit (Qiagen). The RNA was eluted in $100 \mu \mathrm{l}$ RNase-free water at $80{ }^{\circ} \mathrm{C}$ for 2 min. The RNA quality was analyzed on a $1 \%$ agarose gel. $1 \mathrm{ml}$ of hybridization mix was added to the prepared anti-sense RNA and stored at $-20^{\circ} \mathrm{C}$.

\subsubsection{RNA isolation from whole embryos and ectodermal explants}

Trizol: $\quad$ peqGOLD TriFast reagent (peQlab)

For total RNA extraction, two to five embryos and 20 to 50 ectodermal explants were fixated in liquid nitrogen and stored at $-80^{\circ} \mathrm{C}$. Total RNA was isolated with trizol. Embryos and explants were macerated in $400 \mu$ l trizol using a sterile Omnican ${ }^{\circledR} 40$ syringe (Braun). Samples were vortexed for $30 \mathrm{sec}, 80 \mu \mathrm{l}$ of Chloroform (Roth) were added and again vortexed for $30 \mathrm{sec}$. To separate the phases, the samples were centrifuged for $10 \mathrm{~min}$ at $4{ }^{\circ} \mathrm{C}$ with maximum speed. The upper phase was transferred into a new tube and mixed with $200 \mu \mathrm{l}$ of Chloroform for $30 \mathrm{sec}$. After a second centrifugation step for 5 min the upper phase was transferred into a new tube and $180 \mu \mathrm{l}$ of 2-propanol (Roth) were added to precipitate nucleic acids over night at $-20^{\circ} \mathrm{C}$. The next day, the nucleic acids were pelleted by a centrifugation for $30 \mathrm{~min}$. The pellet was washed with $75 \%$ ethanol. The air-dried pellet was dissolved in $12.5 \mu \mathrm{l}$ RNase-free water. To remove genomic DNA, the sample was incubated with $1 \mathrm{U} / \mu \mathrm{l}$ DNasel (Thermo Scientific) for $1.5 \mathrm{~h}$ at $37^{\circ} \mathrm{C}$. The DNasel activity was 
inactivated by heating to $70^{\circ} \mathrm{C}$ for $10 \mathrm{~min}$. For the confirmation of complete removal of genomic DNA, a PCR was performed with $1 \mu$ RNA sample (50 $\mathrm{ng} / \mu \mathrm{l})$ described in 2.2.1.6.2 Semi-quantitative PCR using oligonucleotides for housekeeping gene $\mathrm{H} 4$.

\subsubsection{Reverse transcription and PCR}

For cDNA synthesis, 50 to $100 \mathrm{ng}$ total RNA was used for a reaction volume of 10 $\mu \mathrm{l}$. The following additional components were contained in the reaction mix.

$\begin{array}{lll}\mu \mathrm{l} & \text { final conc. } & \text { component } \\ 2 & 1 \mathrm{x} & 5 \times \mathrm{Go} \text { Taq flexi buffer (Promega) } \\ 2 & 5 \mathrm{mM} & 25 \mathrm{mM} \mathrm{MgCl}_{2} \\ 0.5 & 2.5 \mathrm{ng} & 50 \mathrm{ng} \text { random hexamer } \\ & & \text { oligonucleotides (Invitrogen) } \\ 1 & 1 \mathrm{mM} & \text { Ribolock RNase inhibitor } \\ 0.2 & 0.8 \mathrm{U} & \text { MuLV reverse transcriptase (Roche) }\end{array}$

cDNA synthesis was performed under the following conditions.

\begin{tabular}{lll} 
step & temperature $\left({ }^{\circ} \mathrm{C}\right)$ & time $(\mathrm{min})$ \\
\hline 1 & 20 & 20 \\
2 & 42 & 60 \\
3 & 95 & 5
\end{tabular}

From $10 \mu \mathrm{l}$ cDNA reaction, $2.5 \mu \mathrm{l}$ were used for semi-quantitative PCR (see chapter 2.2.1.6.2).

\subsubsection{Quantitative Nanostring analysis}

For the simultaneous quantification of different transcripts, the digital multiplexed expression analysis system Nanostring was used with $600 \mathrm{ng}$ of total RNA. The analyzed genes as well as the target region are shown in the appendix (Tab. 6.25 and Tab. 6.26). For data analysis, the counts were normalized in two steps using the nSolver software program provided by Nanostring. First, the counts were normalized with respect to the mean of positive control counts. Second, the counts were normalized to the geometric mean of the housekeeping gene ornithine carboxylase (odc). Finally, to consider the background, the mean and two-fold of the standard deviation of the eight negative controls were substracted. Negative values were set to 1 . Data from two independent experiments ( $A$ and $B$ ) were used to 
calculate a mean value. Error bars indicate the standard error of the mean (SEM) which results from the division of the standard deviation by square of two.

\subsubsection{RNA-sequencing}

\subsection{RNA isolation}

To isolate total RNA for RNA-sequencing, the trizol-based method, described in chapter 2.2.2.3 (p.47), was used with the following modifications. Ectodermal explants were lysated in $360 \mu \mathrm{l}$ trizol and incubated for $10 \mathrm{~min}$ at RT. Next, $72 \mu \mathrm{l}$ chloroform were added and incubated for additional $5 \mathrm{~min}$ at RT. After a centrifugation step of $20 \mathrm{~min}$ at $4{ }^{\circ} \mathrm{C}$, the upper phase was transferred to a new tube, mixed with 1 volume of chloroform and centrifuged for $10 \mathrm{~min}$. Again, the upper phase was transferred to a new tube, mixed with 1 volume of 2-propanol and incubated over night at $-20{ }^{\circ} \mathrm{C}$. Next day, the nucleic acids were pelleted by centrifugation for $30 \mathrm{~min}$ at $4{ }^{\circ} \mathrm{C}$. The pellet was washed with $500 \mu \mathrm{l} 75 \%$ ethanol, air dried and dissolved in $20 \mu \mathrm{l}$ RNase-free water. To remove the genomic DNA, $1 \mu \mathrm{l}$ DNasel (Thermo Scientific), $0.5 \mu \mathrm{l}$ RNase inhibitor (Thermo Scientific) and $1 \mathrm{x}$ DNase reaction buffer were added to a total volume of $50 \mu \mathrm{l}$ and incubated for $1 \mathrm{~h}$ at $37{ }^{\circ} \mathrm{C}$. To eliminate the DNasel, $100 \mu \mathrm{l}$ RNase-free water and $200 \mu \mathrm{l}$ phenolchloroform-isoamylalcohol were added. After a centrifugation of $20 \mathrm{~min}$ at $4{ }^{\circ} \mathrm{C}$, the upper phase was transferred to a new tube. 1/10 vol. $5 \mathrm{M}$ ammoniumacetate and 1 vol. 2-propanol were added and incubated over night at $-20{ }^{\circ} \mathrm{C}$. Next day, the RNA was pelleted by a centrifugation for $30 \mathrm{~min}$ at $4{ }^{\circ} \mathrm{C}$. The pellet was washed twice with $75 \%$ ethanol, air-dried and dissolved in $12.5 \mu \mathrm{l}$ RNase-free water. RNA quality was analyzed by use of the 2100 Bioanalyzer (Agilent). To confirmation the complete removal of genomic DNA, a PCR was performed with $1 \mu$ RNA sample (100 ng/ $\mu \mathrm{l}$ ) using oligonucleotides for housekeeping gene $\mathrm{H} 4$.

\subsection{Sample preparation and sequencing}

RNA-samples were prepared in two independent experiments. For sequencing the RNA-samples were handled with the "TruSeq RNA Sample Prep Kit v2" according to the manufacturer instructions. The samples were sequenced via HiSeq 2000 (Ilumina). The sequence reads consist of 50 base pairs from the single-end mode and the quality of this reads was checked by FastQC (http://www.bioinformatics.babraham.ac.uk/projects/fastqc/). 


\subsection{Sequence alignment}

The sequence images were transformed to bcl files by the use of the llumina software BaseCaller and were de-multiplexed to fastq files with CASAVA (version 1.8.2). The obtained sequence reads were aligned to the transcript reference sequences of Xenopus tropicalis (kindly provided by Michael J. Gilchrist; Gilchrist et al., 2004). In addition, the reads were also aligned to selected Xenopus laevis transcriptome sequences (UniGene) for genes which were not found in the Xenopus tropicalis transcriptome. The alignment was performed using Bowtie2 (version 2.1.0) in local alignment mode allowing 6 mismatches within 50 bases (Langmead and Salzberg, 2012). The resulting SAM files (Sequence Alignment/Map) were converted to sorted BAM files (binary version of SAM files) and unique hits were filtered. That means reads mapped to more than two genes were removed to facilitate the analysis.

\subsection{Statistical analysis}

The counting of mapped reads was conducted with samtools (Li et al., 2009). The obtained data were pre-processed and analyzed in the R/Bioconductor environment (http://www.bioconductor.org), loading edgeR (Robinson et al., 2010) and biomaRt packages. The counts were normalized to trimmed mean of $\mathrm{M}$-values and the dispersion was estimated. For the detection of differentially expressed genes, a test based on a generalized linear model likelihood ratio assuming negative binominal data distribution was performed via edgeR. Candidate genes were filtered to a minimum of two fold change difference to the control and a FDR-corrected p-value of <0.05. Gene annotation was enriched by data from Xenbase (http://www.xenbase.org), ensemble (mouse) (http://www.ensembl.org) and biomaRt (http://biomart.org). 


\subsubsection{Xenopus laevis embryo culture and micromanipulations}

\subsubsection{Preparation of Xenopus laevis testis}

10x MBS: $\quad 880 \mathrm{mM} \mathrm{NaCl} ; 10 \mathrm{mM} \mathrm{KCl} ; 10 \mathrm{mM} \mathrm{MgSO}$; $25 \mathrm{mM} \mathrm{NaHCO}_{3} ; 50 \mathrm{mM}$ HEPES (pH 7.8)

1x MBS: $\quad$ 1x MBS; $0.7 \mathrm{mM} \mathrm{CaCL}_{2}$

Frog narcotic: $2.5 \%$ Tricaine methanesulfonate in tap water; adjusted to a neutral $\mathrm{pH}$ by $\mathrm{Na}_{2} \mathrm{HPO}_{4}$; reviewed in (Guenette et al., 2013)

For in vitro fertilization of the eggs, a male frog was narcotized and sacrificed. The isolated testis was washed in $1 \times$ MBS and stored at $4{ }^{\circ} \mathrm{C}$. A small part of the testis was minced and diluted in 1x MBS. This solution was stored on ice until it was needed.

\subsubsection{Stimulation of eggs}

HCG: $2000 \mathrm{U} / \mathrm{ml}$ human chorionic gonadotropin (HCG) (SIGMA)

In the evening, the female frogs were stimulated for the next day's egg deposition by the injection of $1000 \cup$ HCG subcutaneously close to the dorsal lymph sac. The frogs were kept at $16{ }^{\circ} \mathrm{C}$ over night so that ovulation started approximately 10 hours later. For a later egg deposition, the frogs were stimulated first with $500 \mathrm{U}$ HCG in the evening and $1000 \mathrm{U}$ HCG in the next morning.

\subsubsection{Fertilization}

Laid eggs were collected and in vitro fertilized with minced testis in 1x MBS. By the addition of water to dilute the testis-salt solution 1 to 10, the sperm movement was activated. $20 \mathrm{~min}$ after the fertilization the clutch of eggs was covered with $0.1 \mathrm{x}$ MBS.

\subsubsection{Embryo culture and microinjections}

Cysteine: $\quad 2 \%$ L-cysteine hydrochloride in $0.1 \times \mathrm{MBS} ; \mathrm{pH} 8.0$

Injection buffer: $\quad 2 \%$ Ficoll in $1 \times$ MBS

To remove the jelly coat, the fertilized eggs/ embryos were treated with $2 \%$ cysteine hydrochloride, $\mathrm{pH}$ 8.0. After the removal of the jelly coat, the embryos were washed 
three times with $0.1 \mathrm{x}$ MBS and cultivated at $12.5{ }^{\circ} \mathrm{C}$ until the microinjections. The injections were performed on a cooling plate at $12.5^{\circ} \mathrm{C}$. The developmental stage as well as the position of injection was dependent on the purpose of the approach. The solutions for the microinjections were loaded into glass needles prepared on a needle puller (PN-30, Science Products $\mathrm{GmbH}$ ). The Microinjector 5242 (Eppendorf) was used to inject $4 \mathrm{nl}$ of injection mixture per blastomere. Injected embryos were kept in injection buffer at $12.5^{\circ} \mathrm{C}$ until the next morning. This allowed the heeling of the injection opening. Afterwards, the embryos were transferred into 0.1x MBS and either prepared for ectodermal explants or further cultivated to the desired developmental stage. The developmental stages were defined according to Nieuwkoop and Faber Table of Xenopus laevis (Nieuwkoop and Faber, 1967).

\subsubsection{Xenopus laevis ectodermal explants}

5x MBS EE: 440 mM NaCl; 5 mM KCl; $4.1 \mathrm{mM} \mathrm{MgSO}_{4} ; 12 \mathrm{mM} \mathrm{NaHCO}_{3}$; $2.05 \mathrm{mM} \mathrm{CaCl}_{2} ; 1.65 \mathrm{mM} \mathrm{Ca}\left(\mathrm{NO}_{3}\right)_{2} ; 50$ mM HEPES; pH 7.8

Agarose dish: $0.7 \%$ agarose in $0.8 x$ MBS EE

First, the vitelline membrane of a micro-manipulated or un-manipulated stage 8 to 9 embryo was removed. Then, the centre of the pigmented animal hemisphere (=blastocoel roof tissue) was isolated by the use of the gastromaster system (Xenotek Engeneering, Bellville, USA). The explants were washed in 0.8x MBS EE on a $0.7 \%$ agarose-coated Petri-dish and cultivated over night at $12^{\circ} \mathrm{C}$. The next day, at the equivalent of stage 11, the explants were treated with RA and/or $\mathrm{CHX}$ or DEX for a defined time span and were subsequently washed in 0,8x MBS EE. The explants were then cultivated in 0,8x MBS EE containing Ampicillin $(100 \mu \mathrm{g} / \mathrm{ml})$, Kanamycin $(10 \mu \mathrm{g} / \mathrm{ml})$ and Gentamycin $(10 \mu \mathrm{g} / \mathrm{ml})$ at 12 to $14{ }^{\circ} \mathrm{C}$ and were daily provided with fresh buffer solution until the control embryos reached the desired developmental stage (Nieuwkoop, 1963; Fukui et al., 2003).

\subsubsection{Xenopus laevis embryo and explant treatments}

\subsection{Dexamethasone (DEX) treatment}

Dexamethasone: stock solution $4 \mathrm{mg} / \mathrm{ml}$ in $100 \%$ ethanol working solution $10 \mu \mathrm{M}$

To induce protein activity at a certain time point during embryonic development, hormone-inducible constructs were injected. Therefore, the ligand-binding domain of 
the glucocorticoid receptor (GR) was fused to the coding regions (Gammill and Sive, 1997). In the absence of the hormone dexamethasone (DEX), the fusion protein is held in an inactive state. This inactive state is caused by complex formation with a chaperone protein (Hutchison et al., 1993). Upon the addition of DEX a conformational change is caused that dissociates the chaperone (Tsai and O'Malley, 1994). Injected embryos or ectodermal explants were cultivated in the dark in the presence of $10 \mu \mathrm{M}$ DEX until the desired stage was reached.

\subsection{Retinoic acid (RA) treatment}

Retinoic acid: $\quad$ stock solution $100 \mathrm{mM}$ in DMSO (stored at $-80^{\circ} \mathrm{C}$, SIGMA); $10 \mathrm{mM}$ in $100 \%$ ethanol (stored at $-20^{\circ} \mathrm{C}$ in the dark) Working solution $5 \mu \mathrm{M}$ in $0.1 \times$ MBS or $0.8 \times$ MBS EE

Ectodermal explants and whole embryos at stage 11 were incubated in $5 \mu \mathrm{M}$ RA (all-trans-RA) in the corresponding buffer for $1 \mathrm{~h}$ at $12{ }^{\circ} \mathrm{C}$ under light protection. After the treatment, embryos and explants were cultivated until the controls reached the desired developmental stage.

\subsection{BMS453 treatment}

BMS453: $\quad$ stock solution $10 \mathrm{mM}$ in DMSO (stored at $-20^{\circ} \mathrm{C}$, gift from Bristol Myers Squibb). working solution $0.25 \mu \mathrm{M}$ in $0.1 \times$ MBS or $0.8 x$ MBS EE

To inhibit endogenous RA-signaling, the synthetic retinoid BMS453 was used. BMS453 specifically binds the RA-receptor RAR $\beta$ thereby blocking the binding of RA (Chen et al., 1995). Whole embryos were incubated with $0.25 \mu \mathrm{M}$ BMS453 in $0.1 \mathrm{x}$ MBS at stage $8 / 9$ until stage 12 . Afterwards, embryos were cultivated in $0.1 \mathrm{x}$ MBS until they reached the desired stage.

\subsection{Cyclohexamide (CHX) treatment}

Cycloheximide: $\quad$ stock solution $100 \mathrm{mg} / \mathrm{ml}$ in DMSO (Sigma-Aldrich) working solution $10 \mu \mathrm{g} / \mathrm{ml}$

To inhibit protein biosynthesis Cycloheximide $(\mathrm{CHX})$ was used. $\mathrm{CHX}$ blocks the translocation step in elongation (Schneider-Poetsch et al., 2010). Ectodermal explants were treated with $10 \mu \mathrm{g} / \mathrm{ml} \mathrm{CHX}$ in $0.8 x$ MBS EE 30 min prior to additional treatments (Perron et al., 1999). 


\subsubsection{Whole mount in situ hybridization (WMISH)}

To visualize the spatial and temporal pattern of endogenous transcripts, a whole mount in situ hybridization (WMISH) was performed. The performance essentially based on (Harland, 1991; Hollemann and Pieler, 1999; Nieber et al., 2009). For the detection, a Digoxygenin-11-UTP (Dig) labeled anti-sense RNA probe was used. The visualization was done by the use of an alkaline phosphatase-coupled anti-Dig antibody. All steps were performed at ambient temperature with mild shaking.

\subsubsection{Fixation and X-Gal staining}

10x MEM: 1 M Mops; 20 mM EGTA; 10 mM MgSO $;$ pH 7.4 (stored light protected)

10x PBS: $\quad 1.75 \mathrm{M} \mathrm{NaCl} ; 1 \mathrm{M} \mathrm{KCl} ; 65 \mathrm{mM} \mathrm{Na}_{2} \mathrm{HPO}_{4} ; 18 \mathrm{mM} \mathrm{KH}_{2} \mathrm{PO}_{4}$; $\mathrm{pH} 7.4$

$\mathrm{K}_{3} \mathrm{FE}(\mathrm{CN})_{6}: \quad 0.5 \mathrm{M}$ in $\mathrm{H}_{2} \mathrm{O}$ (stored light protected)

$\mathrm{K}_{4} \mathrm{FE}(\mathrm{CN})_{6}: \quad 0.5 \mathrm{M}$ in $\mathrm{H}_{2} \mathrm{O}$ (stored light protected)

MEMFA: $\quad 4 \%(v / v)$ formaldehyde $(37 \%)$ in $1 \times$ MEM

X-Gal: $\quad 40 \mathrm{mg} / \mathrm{ml}$ 5-Bromo-4-chloro-3-indolyl-b-D-galactopyranoside in formamide (stored $-20^{\circ} \mathrm{C}$ light protected)

X-Gal staining solution: $\quad 1 \mathrm{mg} / \mathrm{ml} \mathrm{X-Gal;} 5 \mathrm{mM} \mathrm{K}_{3} \mathrm{FE}(\mathrm{CN})_{6} ; 5 \mathrm{mM}$ $\mathrm{K}_{4} \mathrm{FE}(\mathrm{CN})_{6} ; 2 \mathrm{mM} \mathrm{MgCl}_{2}$ in $1 \times$ PBS

Embryos were fixated with MEMFA in glas vials for $1 \mathrm{hr}$ at RT following three washing steps with $100 \%$ ethanol for $5 \mathrm{~min}$. $\beta$-gal injected embryos were fixated in MEMFA for only 25 min following three washing steps with 1x PBS for $10 \mathrm{~min}$. The co-injection of RNA encoding for $\beta$-galactosidase ( $g / b 1$ ) was used as a control for the purpose of knockdown and over-expression studies. To visualize the $\beta$-gal presence, X-Gal staining was performed (Bourguignon et al., 1998). Therefore, the embryos were incubated in X-Gal staining solution under light protection until the desired staining level was achieved. To stop the staining reaction, the embryos were washed three times for $10 \mathrm{~min}$ in $1 x$ PBS and refixated for $25 \mathrm{~min}$ in MEMFA. Finally, the embryos were washed three times for $5 \mathrm{~min}$ in $100 \%$ ethanol and stored at $-20^{\circ} \mathrm{C}$. 


\subsubsection{Rehydration}

PTw: $\quad 0.1 \%$ Tween-20 in $1 x$ PBS

Embryos were rehydrated with an ethanol series (75\%, $50 \%, 25 \%)$ to PTw and washed three times in PTw for 10 min each at RT.

\subsubsection{Proteinase-K treatment}

\section{Proteinase-K: $\quad 10 \mu \mathrm{g} / \mathrm{ml}$ in PTw}

To allow a better penetration of the anti-sense RNA probe, embryos were treated with Proteinase- $\mathrm{K}$. The embryos were incubated in $2 \mathrm{ml}$ of PTw/proteinase- $\mathrm{K}$ solution for a defined time period and temperature depending on the developmental stage.

\begin{tabular}{|c|c|c|}
\hline stage & time [min] & temperature \\
\hline explants & 1 & $\mathrm{RT}$ \\
\hline 10 to12 & 4 & $\mathrm{RT}$ \\
\hline 32 to 35 & 18 & $\mathrm{RT}$ \\
\hline 39 to 41 & 15 & $37^{\circ} \mathrm{C}$ \\
\hline
\end{tabular}

\subsubsection{Acetylation and refixation}

Triethanolamine: $\quad 0.1 \mathrm{M}\left(0.93 \mathrm{~g}\right.$ in $\left.\mathrm{H}_{2} \mathrm{O}\right)$; $\mathrm{pH} 7.5$ (SIGMA)

Acetic anhydride: (SIGMA)

PTw-FA: $\quad 4 \%$ formaldehyde $(v / v)$ in PTw

The proteinase-K treatment was stopped by two washing steps with triethanolamine for $5 \mathrm{~min}$. To prevent an unspecific reaction of the Dig-labeled anti-sense RNA probe, free amino-acid ends were blocked by the treatment with $25 \mu$ acetic anhydride in triethanolamine. Next, the embryos were washed two times with PTw for $5 \mathrm{~min}$ and refixated in PTw-FA for 20 min. Afterwards the embryos were washed 5 times with PTw for 5 min. 


\subsubsection{Hybridization}

Hybridization Mix: $50 \%$ (v/v) formamide; $1 \mathrm{mg} / \mathrm{ml}$ Torula RNA (SIGMA); $100 \mu \mathrm{g} / \mathrm{ml}$ Heparin, 1x Denhardt's; $0.1 \%$ (v/v) Tween20; $0.1 \%(w / v)$ CHAPS; 10 mM EDTA in 5x SSC

100x Denhardt's: $\quad 2 \%$ BSA; 2 \% Polyvinylpyrollidone (PVP); 2 \% Ficoll 400 in $\mathrm{H}_{2} \mathrm{O}(w / v)$

20x SSC:

$3 \mathrm{M} \mathrm{NaCl} ; 0.3 \mathrm{M} \mathrm{NaCitrate} ; \mathrm{pH}$ 7.2-7.4

The Hybridization with the anti-sense RNA probe was preceded by a prehybridization with $1 \mathrm{ml}$ hybridization mix for 5 min followed by incubation with fresh hybridization mix for at least $5 \mathrm{~h}$ at $65^{\circ} \mathrm{C}$. Afterwards the embryos were incubated in $1 \mathrm{ml}$ Dig-labeled anti-sense RNA containing Hybridization mix over night at $65^{\circ} \mathrm{C}$.

\subsubsection{Washing}

20x SSC: $\quad 3 \mathrm{M} \mathrm{NaCl}$; $0.3 \mathrm{M} \mathrm{NaCitrate;} \mathrm{pH}$ 7.2-7.4

MAB: $\quad 100 \mathrm{mM}$ maleic acid; $150 \mathrm{mM} \mathrm{NaCl} ; \mathrm{pH} 7.5$

RNase solution: $\quad 20 \mu \mathrm{g} / \mathrm{ml}$ RNase A and $10 \mathrm{U} / \mathrm{ml}$ RNase T1 (Fermentas) in $2 \times \mathrm{SSC}$

The next day, the anti-sense RNA probe was collected and stored at $-20^{\circ} \mathrm{C}$ for reuse. The embryos were washed in hybridization mix for $10 \mathrm{~min}$ at $65^{\circ} \mathrm{C}$ and three times with $2 \mathrm{x} \mathrm{SSC}$ for $15 \mathrm{~min}$ at $65{ }^{\circ} \mathrm{C}$. To remove the non-hybridized RNA molecules, the embryos were incubated in RNase solution for $1 \mathrm{~h}$ at $37^{\circ} \mathrm{C}$. Afterwards the embryos were washed once in $2 x$ SSC at $37{ }^{\circ} \mathrm{C}$ and twice in $0.2 x$ SSC at $65^{\circ} \mathrm{C}$. Next, the buffer was exchanged to MAB.

\subsubsection{Blocking and antibody incubation}

MAB: $\quad 100 \mathrm{mM}$ maleic acid; $150 \mathrm{mM} \mathrm{NaCl}$; $\quad \mathrm{pH} 7.5$

MAB/BMB: $\quad 2 \% \mathrm{BMB}$ (Bohringer Mannheim blocking reagent) in $1 \mathrm{x}$ MAB

MAB/BMB/HS: $\quad 20 \%$ heat-treated horse serum $(\mathrm{HS})$ in MAB/BMB

MAB/BMB/HS/AK: $1: 5000$ sheep-anti-Dig antibody linked to alkaline phosphatase (AP) in MAB/BMB/HS (SIGMA) 
To minimize unspecific binding of the AP-linked anti-Dig antibody, embryos were blocked in $M A B / B M B$ for 20 min and in $M A B / B M B / H S$ for 40 min. For the detection, the embryos were incubated with anti-Dig antibody containing MAB/BMB/HS/AK for $4 \mathrm{~h}$ at RT. Next, the embryos were extensively washed with MAB, first three times for $10 \mathrm{~min}$ and then overnight at $4{ }^{\circ} \mathrm{C}$.

\subsubsection{Staining reaction}

APB:

$100 \mathrm{mM}$ Tris-HCL; $50 \mathrm{mM} \mathrm{MgCl}_{2} ; 100 \mathrm{mM} \mathrm{NaCl} ; 0.1 \%$

Tween-20; $\mathrm{pH} 9.0$

NBT: $\quad 100 \mathrm{mg} / \mathrm{ml}$ in $70 \%$ Dimethylformamide (light sensitive)

BCIP: $\quad 50 \mathrm{mg} / \mathrm{ml}$ in $100 \%$ Dimthylformamide (light sensitive)

Staining solution: $\quad 0.8 \mu \mathrm{l} \mathrm{NBT}$ and $3.5 \mu \mathrm{lBCIP}$ in $1 \mathrm{ml}$ APB

MEMFA: $\quad 4 \%(v / v)$ formaldehyde $(37 \%)$ in $1 \times$ MEM

The washing with MAB was continued by three times for 10 min with MAB. Then the caps of the glas vials were exchanged and three additional washing steps with MAB followed. Next, the embryos were two times washed in APB for 5 min at $4{ }^{\circ} \mathrm{C}$ and then the staining solution was added. This solution contained NBT and BCIP, substrates for the alkaline phosphatase that were converted to a colored product. The staining reaction was performed in the dark at $4{ }^{\circ} \mathrm{C}$ until the staining was sufficient. To stop the colour reaction and to remove background staining, the embryos were transferred to $100 \%$ methanol. Then the embryos were rehydrated with a methanol series (75 \%, $50 \%, 25 \%)$ to MEMFA and incubated for $30 \mathrm{~min}$.

\subsubsection{Bleaching}

20x SSC:

$3 \mathrm{M} \mathrm{NaCl} ; 0.3$ M NaCitrate; pH 7.2-7.4

MEMFA:

$4 \%(v / v)$ formaldehyde (37\%) in 1x MEM

Bleaching solution: $50 \%$ formamide and $1-2 \% \mathrm{H}_{2} \mathrm{O}_{2}$ in $5 \times \mathrm{SSC}$

To remove the pigments for an easier documentation of the staining, the embryos were first washed twice in 5x SSC and then incubated with the bleaching solution. Afterwards, the embryos were washed again twice in 5x SSC and re-fixated with MEMFA. 


\subsubsection{CRISPR/Cas system}

The CRISPR/Cas system (Clustered Regularly Interspaced Short Palindromic Repeats/CRISPR-associated) was first identified as part of the bacterial adaptive defense mechanism against virus and plasmid DNA (Fineran and Dy, 2014; Hsu et al., 2014; Terns and Terns, 2014). There, the RNA-guided DNA endonuclease Cas9 causes double-strand breaks at the DNA target site that were often imperfectly repaired by non-homologous end-joining (NHEJ). As a result, deletion or insertion mutations occur frequently (reviewed in Waters et al., 2014). Most recently, this system has been successfully applied for genome modification in numerous organisms (Blitz et al., 2013; Nakayama et al., 2013; Guo et al., 2014; Sander and Joung, 2014; Wang et al., 2015). In this study the CRISPR/Cas technology was used for fzd4-gene disruption. The Cas9 protein forms a complex with two short non-coding RNAs, a CRISPR RNA (crRNA) that has complementary sequence to the target DNA and a trans-activating CRISPR RNA (tracrRNA) that base pairs with the crRNA. For genome editing applications, a synthetic guide RNA (sgRNA) was designed by ZiFiT Targeter version 4.1 (Sander et al., 2007; Sander et al., 2010) where the minimal features of both RNAs were combined and the target sequence of $20 \mathrm{bp}$ was added.

\subsubsection{1 sgRNA preparation}

1x annealing buffer: $\quad 40 \mathrm{mM}$ Tris, $20 \mathrm{mM} \mathrm{MgCl}_{2}, 50 \mathrm{mM} \mathrm{NaCl}, 10 \mathrm{mM}$ EDTA; $\mathrm{pH} 8.0$

For the generation of the sgRNA, the DR274 vector (Hwang et al., 2013) was used containing a T7 promotor for in vitro transcription. The pDR274 plasmid (Addgene) was digested with Bsal. Forward and reverse oligonucleotides, containing the sgRNA sequence with overhangs compatible to the Bsal-digestion sites, were annealed (see sequence in section 2.1.4.5, p.40). 100 $\mu \mathrm{M}$ of each oligonucleotide were heated to $95{ }^{\circ} \mathrm{C}$ and cooled $\left(-1^{\circ} \mathrm{C}\right.$ per $\left.30 \mathrm{sec}\right)$ to $4{ }^{\circ} \mathrm{C}$ in a volume of $50 \mu \mathrm{l} 1 \mathrm{x}$ annealing buffer. $15 \mu \mathrm{M}$ of the annealed oligonucleotides were used for the cloning into the pDR274 vector. For the co-injection with cas9 RNA the sgRNA was transcribed as uncapped RNA (described in section 2.2.2.1). 


\subsubsection{Target- and potential off- target-mutation analysis}

For mutation analysis of CRISPR/Cas treated explants, the genomic DNA was isolated by the use of the "DNeasy Blood \& Tissue Kit" (Qiagen). The region around the Fzd4-gRNA target site was amplified from genomic DNA using oligonucleotides MG171 and MG248. The Fzd4 amplicon was cloned into the $\mathrm{pGem}^{\circledR}-\mathrm{T}$ Easy vector and ten to twelve clones were sequenced using the SP6 oligonucleotide. Obtained sequences were aligned to Fzd4 genomic sequence. The CRISPR/Cas target sequence is characterized by twenty nucleotides followed by a protospacer adjacent motif (PAM) (Hsu et al., 2013). Since a stretch of twenty nucleotides can occur multiple times in a genome and mismatches can be accepted by the CRISPR/Cas system depending on their position, it was necessary to predict potential off-targets. For this purpose, the CRISPR/Cas Target online predictor (CCTop) was used (http://crispr.cos.uni-heidelberg.de; Stemmer et al., 2015). The potential off-target sites of Impad1 (MG297/MG298), Kremen2 (MG275/294) and Fzd7 (MG273/MG274) were amplified with the indicated oligonucleotides, cloned and analyzed for mutations.

\subsubsection{Luciferase assay}

For the quantification of Wnt-signaling activities, embryos were either injected with 50 pg Siamois-Luc (Firefly luciferase) (Brannon et al., 1997) or 100 pg atf2-Luc (Firefly luciferase) (Ohkawara and Niehrs, 2011) and 10 pg pRL-TK (Renilla luciferase) (Promega) reporter DNA in combination with respective mRNAs animal at two-cell stage. Ectodermal explants were fixated in liquid nitrogen and stored at $20{ }^{\circ} \mathrm{C}$. Duplicates for each sample were taken using $2 \times 30$ explants. For the preparation of lysates and measurement of Firefly- and Renilla-luciferase activity, the Dual Luciferase Assay Kit (Promega) was used according to the manufacturer's instructions. Measurements were performed with the Centro LB 960 Luminometer (Berthold Technologies). The ratio of Firefly- to Renilla-light units was calculated and was adjusted to 1 for the sample with injected reporter RNA only. The resulting data were presented as relative luciferase activity. Thereby, either the highest activity is set to $100 \%$ or the fold inductions are shown. 


\section{Results}

\subsection{Formation of pancreatic organoids from Xenopus explants}

The aim of this study is the identification of the gene network responsible for pancreas specification. For this purpose, Xenopus laevis was used as model system as it provides a rich source of pluripotent cells which can be programed to differentiate into endodermal, mesodermal and neuro-ectodermal derivatives (reviewed in Borchers and Pieler, 2010). Furthermore, known factors involved in pancreas organogenesis appear to be conserved between Xenopus and mammals (reviewed in Zaret and Grompe, 2008). For the identification of novel key players in pancreas specification, in vitro generated pancreatic organoids were used.

\subsubsection{RA-dependent induction of pancreatic marker genes in Vegt/Noggin- programed ectodermal explants}

A previous study demonstrated that ectodermal explants from Xenopus embryos injected with vegt and $\beta$-catenin RNA express liver specific genes and that the addition of RA at the equivalent of gastrula stage further induces various pancreatic marker genes. Moreover, the co-injection of RNA coding for the BMP-inhibitor Noggin significantly increased the expression of these pancreatic markers at the expense of liver specific markers (Chen at al., 2004). In order to further optimize this procedure, we first asked if a combined Vegt/Noggin activity is able to induce pancreatic markers, so that $\beta$-catenin can be omitted. RNAs coding for vegt and noggin were injected into the animal pole of a two-cell stage embryo. Blastocoel roof tissue was explanted at the late blastula stage and treated with RA for one hour at the equivalent of gastrula stage. Explants were cultivated to the equivalent of stage 30. Total RNA was isolated and analyzed by RT-PCR (Fig. 3.1 A). Indeed, the coinjection of vegt and noggin is sufficient to drive pancreatic gene expression in ectodermal explants. The induction of the pancreatic progenitor markers Ptf1a and $\mathrm{Pdx1}$, the endocrine marker Insulin and the endodermal markers Darmin and Sox $17 \mathrm{a}$ is observed. Moreover, the addition of RA further increases the level of pancreatic gene expression (Fig. 3.1 B). For Noggin it was shown that it induces the expression of the RA-generating enzyme Raldh2 which leads to elevated RA-levels in this system (Pan et al., 2007). Thus, we next asked if endogenous RA-signaling can be blocked in this system in order to allow a temporally controlled activity of RAsignaling in the context of pancreas development. Therefore, we examined if the 
induction of pancreatic genes can be blocked by the use of Cyp26a1, a RAdegrading enzyme (Hollemann et al., 1998). The co-injection of cyp26a1 with vegt and noggin RNA almost completely blocks the expression of pancreatic genes. Importantly, this can be fully rescued by the addition of RA (Fig. 3.1 B). These data show that the used explant system allows a controlled activation of RA-signaling and represents a convenient system to identify RA-target genes.
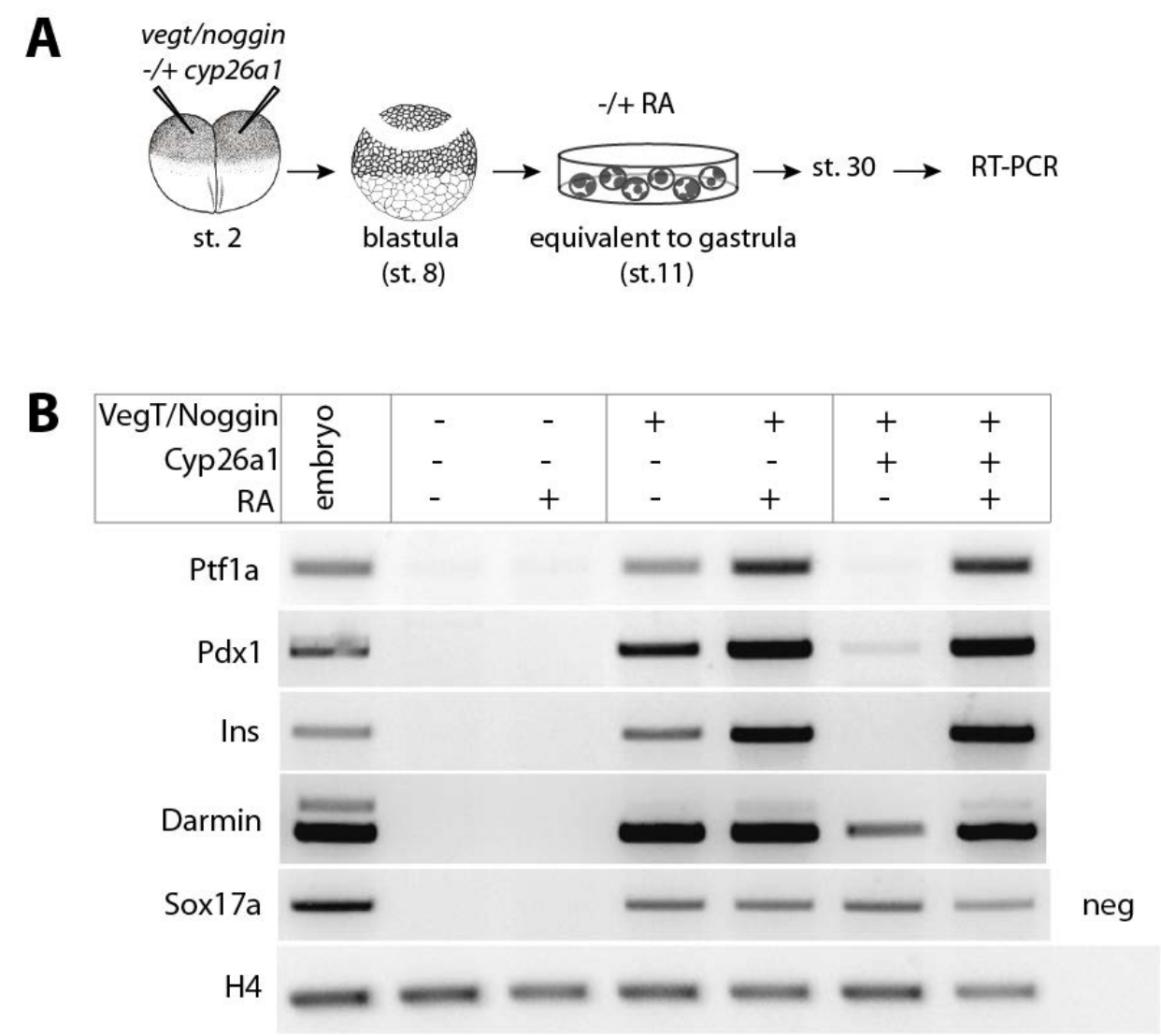

Fig. 3.1 RA-dependent induction of pancreatic marker genes in Vegt/Nogginprogramed explants

(A) Co-injection of vegt (500pg), noggin (500pg) and cyp26a1 (2000pg) RNA into the animal pole of two-cell stage embryos. Tissue of the blastocoel roof was isolated at stage 8, cultivated and treated with $5 \mu \mathrm{M} R A$ at the equivalent of stage 11. Explants were cultivated until stage 30 and extracted RNA was analyzed by RT-PCR. (B) RT-PCR with indicated markers for endoderm (Darmin and Sox17a), pancreatic progenitors (Ptf1a and Pdx1) and endocrine tissue (Ins). 


\subsubsection{Formation of pancreatic organoids that recapitulate the in vivo program of pancreas development}

We could show that ectodermal explants can be programed to express pancreatic marker genes. In Xenopus embryos, the earliest known pancreatic progenitor marker genes can be detected at stage 29 by whole mount in situ hybridization (WMISH). The first endocrine marker is detected shortly after that at stage 32. Further endocrine and first exocrine differentiation marker genes were found at stage 39 and later (reviewed in Pieler and Chen, 2006). Therefore, we asked if our programed explants show the same temporal expression profile of pancreatic progenitor and differentiation marker genes as observed in vivo. For this purpose, Vegt/Noggin/RA-programed explants (pancreatic organoids/PO) were examined for pancreatic gene expression at different time points during development (Fig. 3.2 A). RT-PCR and the quantitative method of Nanostring analysis were used for the detection of selected transcripts. Transcript levels of tested marker genes in the pancreatic organoids were compared to whole embryos (embryo), Vegt/Nogginprogramed explants with blocked RA-signaling (ØRA) and un-programed explants (C) (Fig. 3.2 B). As observed in whole embryos, at the equivalent of stage 17, pancreatic organoids express endodermal marker genes Sox17a (Fig. 3.2 B), Sox17b and Darmin as well as mesodermal marker Xbra (Fig. 6.1). Unlike Sox17a and Sox17b, Darmin expression is RA-dependent enhanced in pancreatic organoids compared to explants with blocked RA-signaling. Transcripts of Gata4, a transcription factor involved in endoderm (Rehorn et al., 1996; Xanthos et al., 2001) and pancreas formation in mouse (Carrasco et al., 2012; Xuan et al., 2012), are detected at stage 17 (Fig. 6.2). Gata4 expression is RA-dependent maintained and further induced in pancreatic organoids at stage 24 together with the start of the expression of the pancreatic progenitor marker genes Ptf1a (Fig. 6.2) and Pdx1 (Fig. 3.2 B). The expression of the pancreatic endocrine progenitor marker Ngn3 (Fig. 6.2) is observed together with the start of Insulin expression (Fig. 3.2 B) at stage 24 in pancreatic organoids. The expression of Ngn3 appears to be transient and is detected only at low levels from stage 24 to 32 . In contrast to pancreatic organoids, in whole embryos, Insulin expression is detected at stage 32 the earliest. Further differentiation marker genes for endocrine (Glucagon) and exocrine (Pdia2) tissue are detected at later stages (Fig. 3.2 B and Fig. 6.2). Moreover, the ventral pancreatic marker gene Tm4sf3, which is involved in the fusion of the ventral pancreatic buds with the dorsal bud, (Jarikji et al., 2009) is expressed together with the late exocrine marker Amylase (Fig. 6.2). 
In summary, the temporal expression profile of analyzed markers in pancreatic organoids correlates with that in whole embryos (Fig. 3.2 C). Interestingly, the levels of endodermal and mesodermal markers detected in embryos and programed explants with blocked RA-signaling are mostly higher than in pancreatic organoids. Furthermore, the pancreatic progenitor markers Pdx1 and Ptf1a as well as the pancreatic differentiation markers Amy2a and Pdia2 are more than 10 fold and Insulin more than 100 fold increased in pancreatic organoids as compared to control embryos. This observation argues for a very efficient conversion of the pluripotent blastocoel roof tissue into a pancreatic fate. Notably, the neuronal progenitor marker Sox2 (Ellis et al., 2004) was also induced in pancreatic organoids (Fig. 6.1) which might reflect the innervation of pancreas by neurons as observed in vivo (Kirchgessner and Gershon, 1990). Detailed Nanostring data sets are shown in the appendix (Tab. 6.1 and Tab. 6.2).

Fig. 3.2 Formation of pancreatic organoids that recapitulate the process of pancreas development

(A) RNAs coding for Vegt (500pg), Noggin (500pg) were co-injected into two-cell stage embryos. In order to block endogenous RA-signaling cyp26a1 RNA (2000pg) was coinjected. Tissue from the blastocoel roof was explanted and cultivated. Explants were treated with $5 \mu \mathrm{M}$ RA at the equivalent of gastrula stage. Explants were fixated at the equivalent of stage 17 to 43 and total RNA was isolated and analyzed by RT-PCR and Nanostring.

(B) Diagrams show the number of counts detected by Nanostring nCounter for the indicated marker genes from two independent experiments. Figures on the right side show the results of the RT-PCR. (C) Summary of the temporal expression profile of indicated marker genes for embryos and pancreatic organoids. $\mathrm{E}=$ embryo; $\mathrm{C}=$ un-programed explants; $\mathrm{PO}=$ pancreatic organoid, ØRA = programed explants with blocked RA-signaling 
A

vegt/noggin -/+ cyp26a1

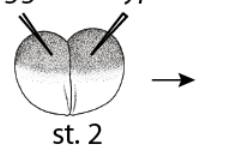

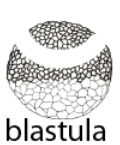

blastula (st. 8)

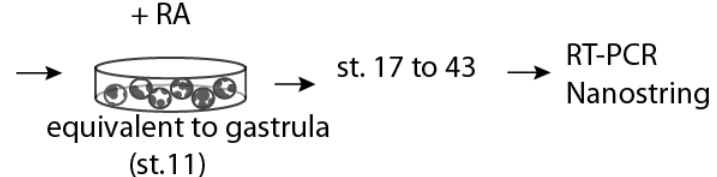

(st.11)
B

embryo

E
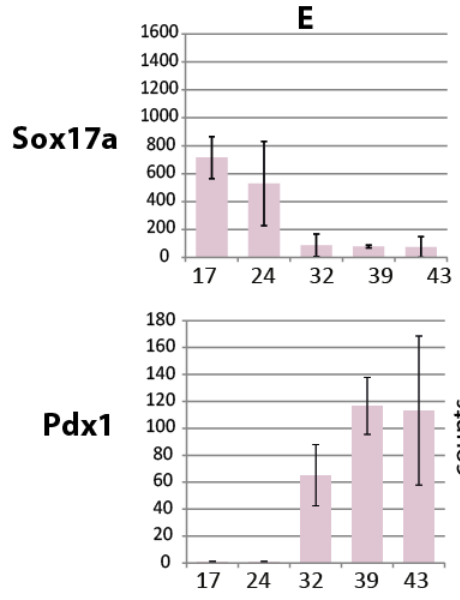

Ins

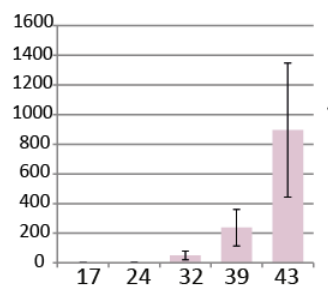

Pdia2

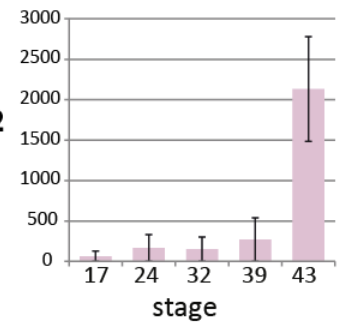

pancreatic organoid
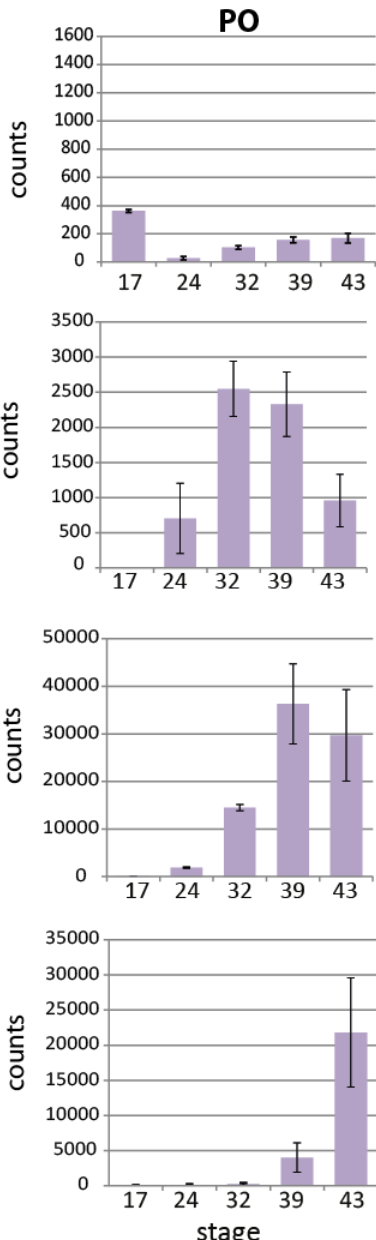

programed explant
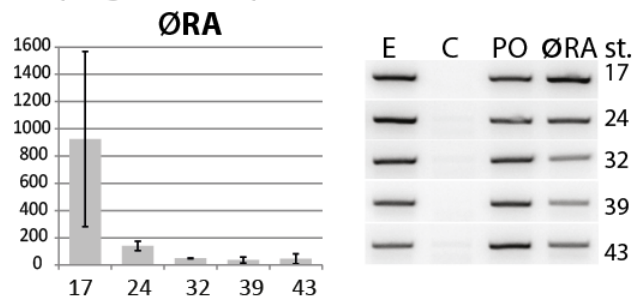

E C PO ØRA st.

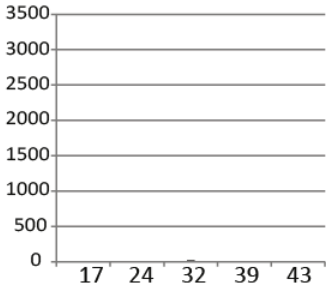

17

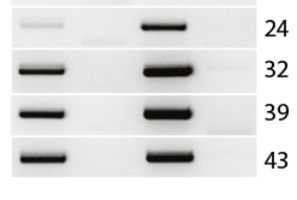

E C PO ØRA st.

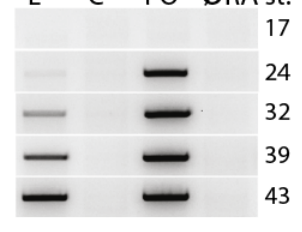

E C PO ØRA st.

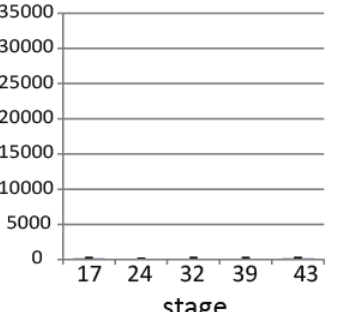

C

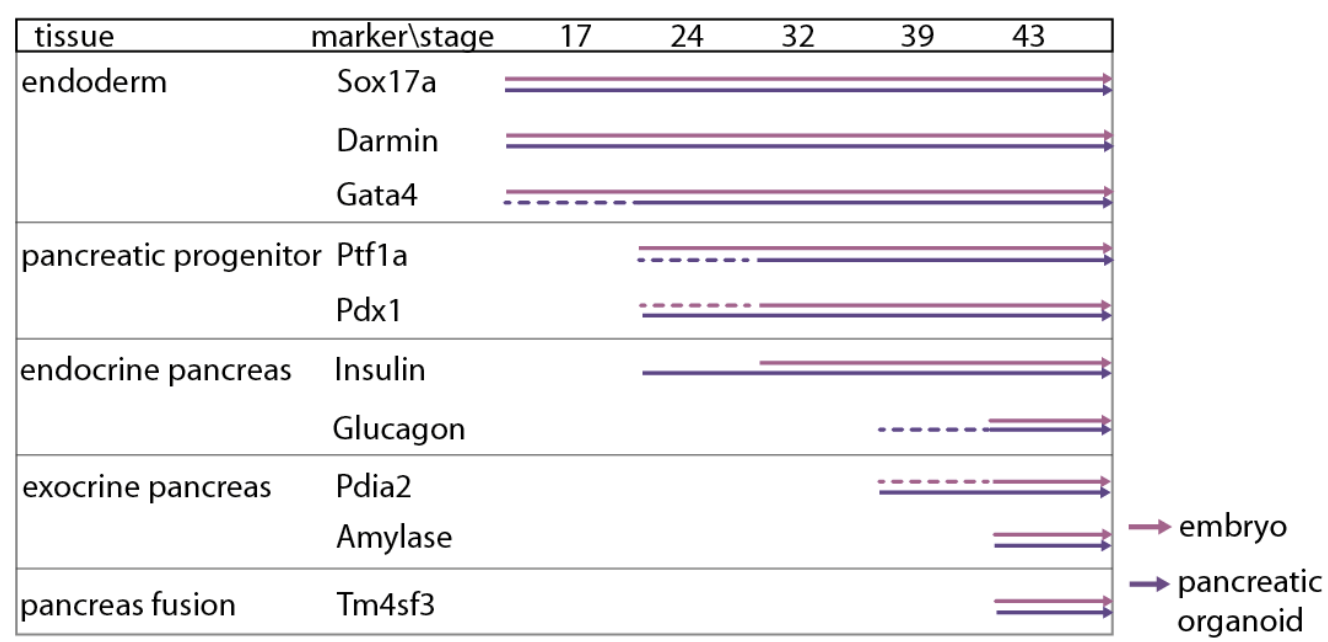

Fig. 3.2 Formation of pancreatic organoids that recapitulate the process of pancreas development 
In order to detect how large the proportion of pancreatic progenitor and endocrine tissue in the organoids is, a WMISH was performed on the programed explants at the equivalent of stage 35. Vegt/Noggin-programed explants were not only treated with $5 \mu \mathrm{M}$ RA but also with $15 \mu \mathrm{M}$ and $30 \mu \mathrm{M}$ RA in order to see if the amount of pancreatic tissue is RA-concentration dependent increased (Fig. 3.3 A). For each RA concentration, explants with a different strength and distribution of pancreatic marker gene expression are observed and were grouped into the three phenotypes, weak, moderate and strong. Transcripts of progenitor marker genes $p t f 1 a$ and $p d x 1$ are detected in larger domains whereas insulin transcripts are detected in single cells scattered within the organoid (Fig. 3.3 B). Indeed, a distinct increase in Ptf1a-, Pdx1- and Insulin-positive cells and percentage of explants showing an expression is observed with $15 \mu \mathrm{M}$ but no further clear increase is detected using $30 \mu \mathrm{M}$ RA. However, already the usually used concentration of $5 \mu \mathrm{M}$ RA leads to positive staining signals of tested marker genes in approximately $30 \%$ to $50 \%$ of the explants (Fig. 3.3 C).

In summary, we could show that Vegt/Noggin-programed ectodermal explants promote pancreatic development in an RA-dependent manner. Therefore, this in vitro system of pancreatic organoids was further used for the identification of RAtarget genes involved in early pancreatic development. 
A

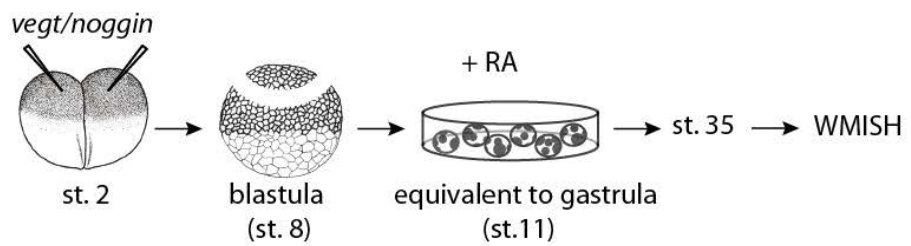

B
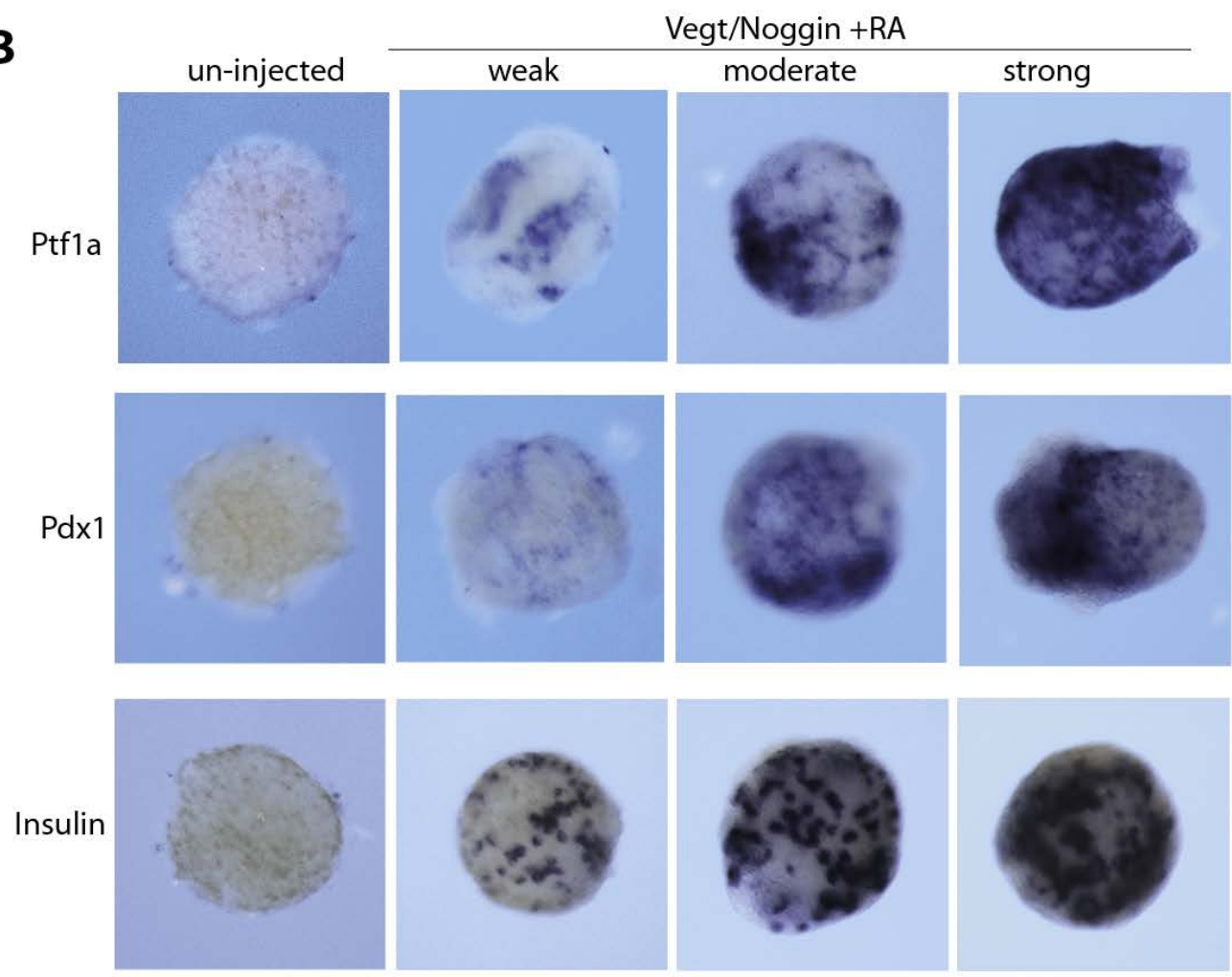

C

$\%$ of pancreatic marker gene expressing explants

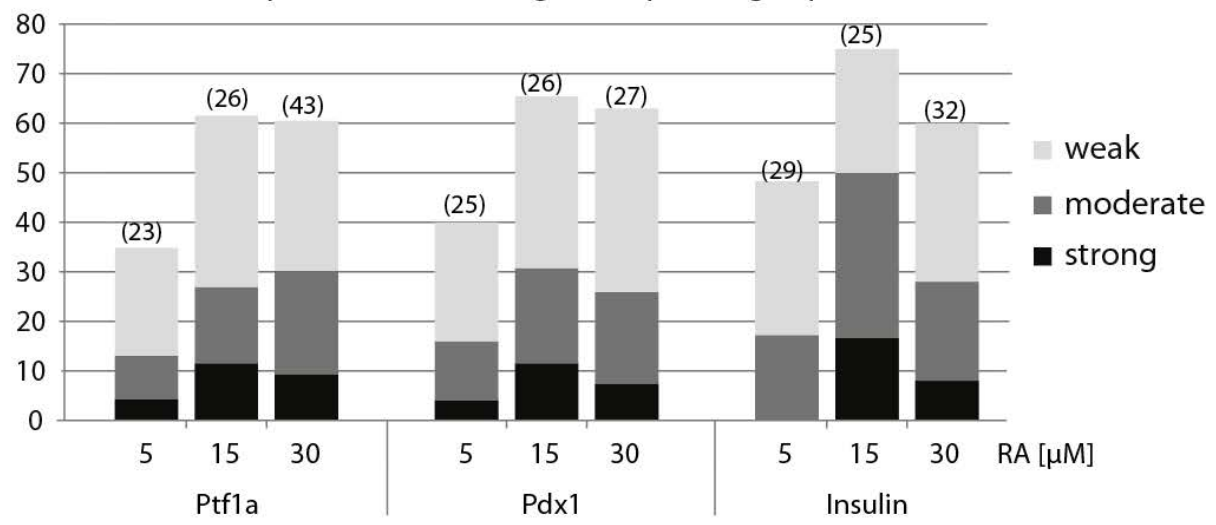

Fig. 3.3 Formation of pancreatic organoids from RA-programed explants (WMISH)

(A) Co-injection of vegt (500pg) and noggin (500pg) RNA into the animal pole of two-cell stage embryos. Tissue of the blastocoel roof was isolated at stage 8 , cultivated and treated with different concentrations of RA $(5 \mu \mathrm{M}$ to $30 \mu \mathrm{M})$ at the equivalent of stage 11 . Explants were cultivated until the equivalent of stage 35 and used for WMISH against Ptf1a, Pdx1 and Insulin transcripts. Explants from un-injected embryos were used as negative control.

(B) Different phenotypes according to the intensity and distribution of pancreatic marker gene expression from weak to strong are presented. (C) The diagram shows the percentage of explants positive for pancreatic marker gene expression with the indicated phenotype. Total number of tested explants is indicated in brackets. 


\subsection{Identification, verification and expression characteristics of early RA-responsive genes}

RA is known to be required at the onset of gastrulation for pancreas development in mice, zebrafish and Xenopus (Stafford and Prince, 2002; Stafford et al., 2004; Chen et al., 2004; Huang et al., 2014a). However, the gene network induced by RA that promotes pancreas development is still unknown. For the identification of RA-target genes in the context of pancreas development, the in vitro system of pancreatic organoids was used.

\subsubsection{Induction of the direct RA-target gene Cyp26a1 within one hour after RA-addition}

It has been shown that RA-activity at gastrula stage is required for pancreas specification in vivo (Chen et al., 2004). This observation correlates with the expression of the RA-generating enzyme Raldh2 in the dorsal involuting mesoderm during gastrulation (Hollemann et al., 1998; Chen et al., 2001). However, the first known pancreatic progenitor markers Pdx1 and Ptf1a are not detected before stage 24 (Fig. 3.2). In order to identify early direct RA-targets that mediate pancreas specification, it was essential to analyze the RA-response in a temporal manner. For this issue, we analyzed the temporal induction of the RA-hydroxylase Cyp26a1, a known direct RA-target gene (Ray et al., 1997; Abu-Abed et al., 1998). For this purpose, embryos were co-injected with RNA coding for Cyp26a1 that effectively blocks endogenous RA-signaling in pancreatic organoids (see above). Ectodermal explants were treated with RA at the equivalent of gastrula stage. In order to detect only direct targets, explants were further treated with the translational inhibitor cycloheximide ( $\mathrm{CHX}) 30$ min before RA-addition. Explants were fixated one and two hours after RA-addition. Endogenous cyp26a1 transcripts were visualized using oligonucleotides targeting the 5'UTR of cyp26a1 RNA. Endogenous cyp26a1 was detected already one hour after RA-addition in the absence as well as in the presence of $\mathrm{CHX}$. Levels of cyp26a1 transcripts are further increased within two hours after RA addition (Fig. 3.4). In conclusion, the known direct RA-target Cyp26a1 is induced by RA within one hour in the Xenopus explant system used here. 


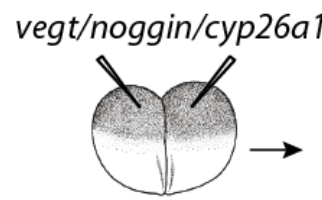

st. 2

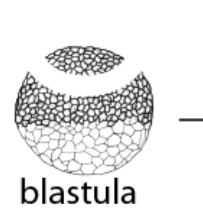

(st. 8)
$-/+\mathrm{RA}$

$-/+\mathrm{CHX}$

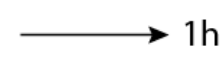

(st.11)

\begin{tabular}{|c|c|c|c|c|c|c|c|c|c|}
\hline \multirow[b]{2}{*}{ Vegt/Noggin/Cyp26a1 } & \multirow{2}{*}{$\begin{array}{c}\text { Oh } \\
-\end{array}$} & \multicolumn{4}{|c|}{$1 \mathrm{~h}$} & \multicolumn{4}{|c|}{$2 \mathrm{~h}$} \\
\hline & & + & + & + & + & + & + & + & + \\
\hline RA & - & - & + & - & + & - & + & - & + \\
\hline $\mathrm{CHX}$ & - & - & - & + & + & - & - & + & + \\
\hline endogenous Cyp26a1 & + & & $\longrightarrow$ & & - & -3 & $\longrightarrow$ & $y$ & \\
\hline $\mathrm{H} 4$ & - & - & - & 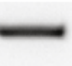 & - & $\longrightarrow$ & & & \\
\hline
\end{tabular}

neg

\section{Fig. 3.4 Direct RA-target gene Cyp26a1 is induced within one hour}

Vegt (500pg), noggin (500pg) and cyp26a1 (2000pg) RNAs were co-injected into the animal pole of a two cell stage embryo. Blastocoel roof tissue was explanted at blastula stage. Explants were treated with RA and cycloheximide $(\mathrm{CHX})$ at the equivalent of gastrula stage. One and two hours after RA addition, total RNA was isolated and analyzed by RT-PCR. Endogenous cyp26a1 is detected one and two hours after RA-addition in the presence and absence of $\mathrm{CHX}$. 


\subsubsection{Identification of early RA-target genes by RNA-sequencing}

As the known direct RA-target Cyp26a1 was shown to be induced within one hour and further increased after two hours, these two time points were chosen for the identification of early RA-target genes in the context of pancreas specification. Pancreatic organoids were programed by the co-injection of RNAs coding for Vegt, Noggin and Cyp26a1. RA and $\mathrm{CHX}$ were added the equivalent of gastrula stage. Samples were taken one hour and two hours after RA-addition. For the identification of RA-target genes, total RNA was extracted and analyzed by RNA-sequencing for two independent experiments (Fig. 3.5 A). About 30.000 transcripts could be detected and at least 10 million sequence reads could be mapped to genomic sequences indicating a good quality of the RNA-sequencing (Fig. 6.3 B). Since the $X$.laevis genome was not completely sequenced, but is highly similar to that of the related species $X$.tropicalis, sequence reads were initially mapped to the Xenopus tropicalis genome and in second instance to the Xenopus laevis genome. Thereby, 102 genes are found to be differentially expressed in pancreatic organoids treated with RA versus untreated. Within one hour, 27 genes and within two hours after RAaddition 96 genes were recorded with an overlap of 21 genes (Fig. 3.5 B, Tab. 6.3). In the presence of the translational inhibitor $\mathrm{CHX}, 61$ genes are differentially expressed (Fig. 3.5 B, Tab. 6.4). Most of the detected genes are up-regulated higher than two-fold and lower than five-fold. Only a few genes show an upregulation higher than five-fold. And even less genes were found to be downregulated (Fig. 6.3 C). Comparison of RA-regulated genes in the absence versus the presence of $\mathrm{CHX}$ was done to detect putative direct RA-targets. Almost half of the detected RA-targets are also differentially expressed during a block of translation (Fig. 6.3 D, Tab. 6.5). Hence, these 49 genes were referred to as candidates for putative direct RA-targets. However, since we want to identify the gene-network that leads to pancreas specification, all 102 detected genes were further analyzed. Detailed information of RNA-sequencing results are listed in the appendix (Tab. 6.6 to Tab. 6.9) 
A

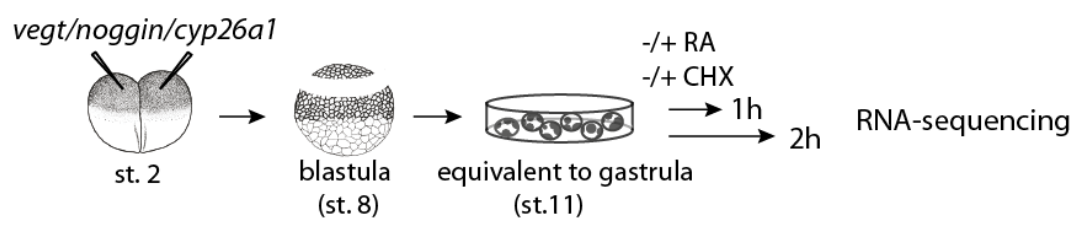

B all targets $(\varnothing \mathrm{CHX})$

direct targets $(+\mathrm{CHX})$
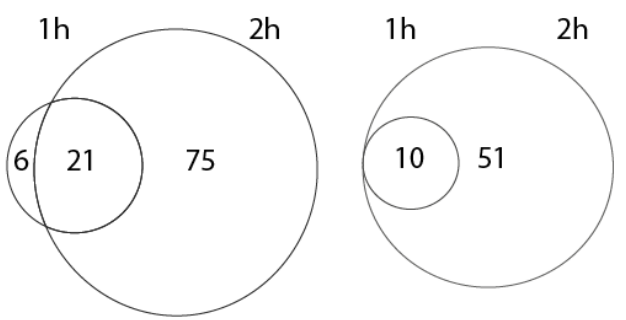

C

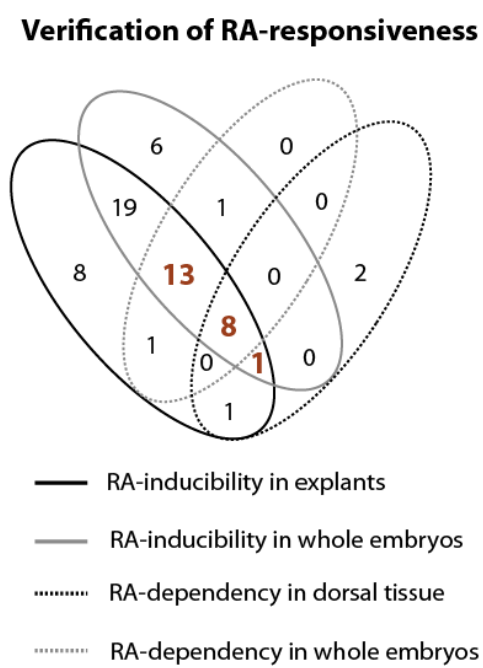

D RA-responsive candidate genes

\begin{tabular}{|c|c|c|}
\hline direct & gene & expression \\
\hline - & $h n f 1 b$ & E \\
\hline \multirow{2}{*}{ - } & \multirow{2}{*}{$f z d 4$} & NEc \\
\hline & & $E$ \\
\hline - & cebpd & $\| \mathrm{M}$ \\
\hline \multirow[t]{3}{*}{ - } & dhrs3 & $\mathrm{IIM}$ \\
\hline & $I h \times 1$ & $\mathrm{IIM}$ \\
\hline & fst & IIM \\
\hline \multirow{2}{*}{ - } & \multirow{2}{*}{ сур26a1 } & NEc \\
\hline & & OIM \\
\hline \multirow{2}{*}{ - } & \multirow{2}{*}{ hoxd4 } & NEC \\
\hline & & OIM \\
\hline \multirow{2}{*}{ • } & \multirow{2}{*}{$g b \times 2.1$} & NEc \\
\hline & & OIM \\
\hline \multirow{2}{*}{ - } & \multirow{2}{*}{ hoxa1-b } & NEC \\
\hline & & OIM \\
\hline \multirow{2}{*}{ - } & \multirow{2}{*}{ hoxd1 } & NEC \\
\hline & & OIM \\
\hline - & znf703 & OIM \\
\hline - & foxh1 & OIM \\
\hline - & meis3 & OIM \\
\hline - & $n k x 6.2$ & OIM \\
\hline - & $X I .45046$ & OIM \\
\hline - & $X 1.51509$ & OIM \\
\hline \multirow[t]{2}{*}{ - } & hoxb1 & OIM \\
\hline & prph & OIM \\
\hline \multirow[t]{3}{*}{ - } & igf3 & OIM \\
\hline & $X 1.47239$ & OIM \\
\hline & $X 1.57926$ & OIM \\
\hline
\end{tabular}

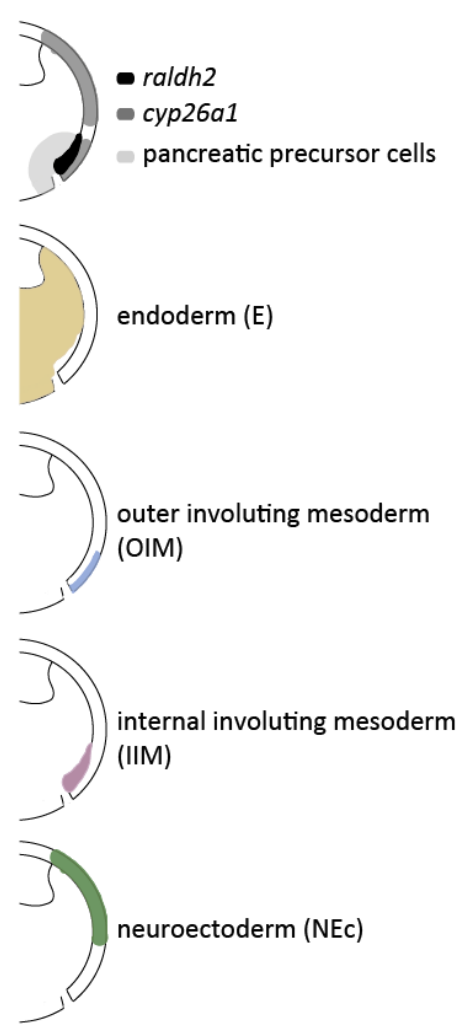

Fig. 3.5 Identification, verification and expression characteristics of early RAresponsive genes in the context of pancreas specification

(A) Vegt (500pg), noggin (500pg) and cyp26a1 (2000pg) RNAs were co-injected into the animal pole of a two cell stage embryo. Blastocoel roof tissue was explanted at blastula stage. Explants were treated with RA and cycloheximide $(\mathrm{CHX})$ at the equivalent of gastrula stage. One and two hours after RA addition samples were taken for RNA-sequencing.

(B) Venn diagrams show the number of differentially expressed genes within one and two hours after RA-addition in the presence or absence of $\mathrm{CHX}$. Genes with less than 50 mapped reads were removed.

(C) Verification of RA-responsive genes. Indicated in red is the number of candidates confirmed for RA-inducibility in pancreatic organoids and/or whole embryos and RA-dependency in dorsal parts and/or whole embryos. 22 genes were confirmed for RA-responsiveness as they show both RAinducibility and -dependency.

(D) 22 confirmed RA-responsive genes and their expression characteristics obtained by WMISH. Indicated with dots are the putative direct RA-target genes. The scheme on the upper right side indicates the expression domains of Raldh2 (RA generating enzyme), Cyp26a1 (RA-degrading enzyme) and the location of prospective pancreatic progenitors during gastrulation. The schemes below indicate the expression domains to which the candidates were assigned. $\mathrm{E}=$ endoderm, IIM = inner involuting mesoderm, NEc = prospective neuroectoderm, OIM = outer involuting mesoderm 


\subsubsection{Verification of 22 RA-responsive genes}

Through RNA-sequencing, 102 putative RA-target genes could be identified. However, not all candidates could be used for further analysis. For 17 genes no sequence for Xenopus laevis was available at this phase of the study. For further three genes no Nanostring probe could be designed (Tab. 6.3). Thus, only 82 candidates were further examined.

In a first step, we re-examined the RA-responsiveness of these 82 presumptive RAtarget genes. For this issue, RA-inducibility was quantitatively analyzed by Nanostring using the ectodermal explant system and whole embryos (Fig. 6.4 A). In the explant system, 51 of the 82 candidates were found to be more than 1.5 fold induced upon RA-addition. For the majority of these candidate genes, the expression is increased between 1.5 and 3 fold one hour after RA-addition compared to RA-negative programed explants. After two hours, half of the induced genes are increased higher than 3 fold. In whole embryos, 48 of the 82 candidate genes were found to be induced more than 1.5 fold upon RA-treatment (Fig. 6.4 A). A comparative analysis revealed that 41 candidates could be confirmed for their RAinducibility in both, the explant system and whole embryos (Fig. 3.5 C).

However, RA-inducibility does not necessarily mean that gene expression is dependent on RA-signaling activity. We further determined the RA-dependency of the 82 candidate genes by the inhibition of RA-signaling in embryos through the injection of cyp26a1 RNA or through treatment with the RA-antagonist BMS453 (Fig. 6.4 B). We examined the gene expression by Nanostring analysis upon the inhibition of RA-signaling in whole embryos as well as in isolated dorsal tissue. We found the expression of 12 out of 82 candidate genes to be reduced more than 1.5 fold in RA-negative compared to untreated dorsal tissue. In whole embryos, 23 of the 82 candidates were found to be reduced more than 1.5 fold upon BMS453 treatment (Fig. 6.4 B).

The comparison of confirmed RA-inducible genes and verified RA-dependent candidates revealed a set of 22 candidate genes that are found to be RA-inducible as well as RA-dependent expressed (Fig. 3.5 C and D). The observed data are summarized in the appendix (Tab. 6.10) and shown in detail in Tab. 6.11 to Tab. 6.18 . 


\subsubsection{Expression characteristics of 22 verified RA-responsive genes}

Identifying genes that control pancreatic specification in response to RA requires not only the determination of RA-responsiveness, but also the exclusion of those candidate genes that are not expressed at the right time and the right place during embryogenesis. RA-target genes involved in pancreas specification are expected to be expressed in the dorsal endoderm and/or the dorsal involuting mesoderm during gastrulation (Pan et al., 2007). Therefore, the expression patterns of the 22 RAresponsive candidate genes were analyzed at gastrula stage by WMISH. Thereby, candidates could be grouped according to their expression pattern (Fig. 3.5 D). We found that almost half of the candidates are expressed exclusively in the outer involuting mesoderm. A second group of five candidates display an additional expression domain in the prospective neuroectoderm and a third group of four candidates shows an expression in the inner involuting mesoderm (Fig. 3.5 D, Fig. 6.5). Two candidate genes, Hnf1b and Fzd4, exhibit an endodermal expression domain. Hnf1b is expressed in the entire endoderm with an enrichment in the dorsal area, whereas Fzd4 expression domain is restricted in the endoderm at the dorsal side. Besides its endodermal expression domain, Fzd4 shows a second expression domain in the prospective neuro-ectoderm (Fig. 6.5).

Pancreatic progenitor cells are known to derive from the dorsal endoderm under the control of RA that is synthesized in the dorsal involuting mesoderm (Pan et al., 2007.) Thus, it was important, especially for Hnf1b, to quantify transcript levels in the dorsal versus ventral endoderm and/or mesoderm. As by the use of the WMISH minor quantitative variations in gene expression cannot be determined, again Nanostring analysis was used for further analysis. Four-cell stage embryos were injected with RNA encoding GFP into the two dorsal blastomeres to facilitate the differentiation between GFP positive dorsal tissue and GFP negative ventral tissue. For the preparation of the endoderm, gastrula stage embryos were bisected form anterior to posterior along the dorso-ventral axis. From both, ectodermal and mesodermal tissue was removed to get pure dorsal and ventral endodermal tissue (Fig. 6.6 A). For the preparation of dorsal tissue surrounding the blastoporus lip, comprising cells of all three germlayers, also gastrula stage embryos were used. 10 preparations each were subjected to Nanostring analysis. Apart from the 22 RAresponsive candidate genes, the expression of marker genes for the three different germlayers was analyzed (Fig. 6.6 B). Candidate genes were grouped according to their expression characteristics into three groups: dorsally enriched, ventrally enriched and equally distributed (Fig. 6.7). Fzd4 shows a clear dorsal enrichment in the endoderm. Hnf1b exhibits high pan-endodermal transcript numbers with a slight 
dorsal enrichment (Fig. 6.7 A). Besides Hnf1b and Fzd4, six more candidates show a dorsal enrichment while eight candidates were found to be ventrally enriched and six candidates are equally distributed (Fig. 6.7 B and C). Data are shown in the appendix (Tab. 6.19 to Tab. 6.21).

As only Hnf1b and Fzd4 exhibit an endodermal expression, these two candidates were chosen for further functional analysis. 


\subsection{The direct RA-target gene Hnf1b is required for pancreas specification}

In our screen, the homeodomain transcription factor Hnf1b was found as RAresponsive gene. We next examined the location of its RA-responsive expression domain during gastrulation. Furthermore, in order to determine the requirement of Hnf1b for pancreas specification, functional analyses were carried out in vitro and in vivo.

\subsubsection{Hnf1b is RA-responsively expressed in the dorsal endoderm during gastrulation}

During gastrulation, Hnf1b is expressed in the entire endoderm (Fig. 6.8 A) with a slight enrichment in the dorsal endoderm (Fig. 6.8 B). In a late neurula stage embryo (stage 18), Hnf1b transcripts are detected in the anterior endoderm that gives rise to foregut derivatives like liver and ventral pancreas. Furthermore, Hnf1b is expressed in the anterior archenteron roof that gives rise to the dorsal pancreatic anlage. An additional Hnf1b expression domain is observed in the neuroectoderm. At stage 23, Hnf1b is expressed in an anterior-posterior gradient within the endoderm including the ventral foregut that will later differentiate into liver and ventral pancreas. Additionally, Hnf1b is detected in the mesoderm derived pronephric anlage. At later stages, Hnf1b transcripts are found in the liver primordium, pronephros and proctodeum (Fig. 6.8 A).

The observed pan-endodermal expression of Hnf1b during gastrulation, with a slight enrichment in the dorsal endoderm, raises the question if differences in RAresponsiveness of endodermal Hnf1b expression domains exist. Therefore, Hnf1b expression in untreated, RA-treated and dorsally cyp26a1-injected gastrula stage embryos was examined by WMISH. We found that an excess of RA leads to a strong increase of Hnf1b expression in the dorsal endoderm, whereas the ventral endoderm remains unaffected (Fig. 6.8 C). Conversely, the degradation of endogenous RA by Cyp26a1 leads to a loss of Hnf1b expression in the dorsal endoderm. However, a strong decrease of Hnf1b in the ventral endoderm is observed as well (Fig. 6.8 C).

Additionally, RA-responsiveness of Hnf1b expression was quantified using Nanostring analysis. Embryos were either treated with RA or BMS453 at blastula stage (Fig. 6.8 D). Compared to untreated embryos, the excess of RA leads to a two-fold increase of Hnf1b transcript levels at stage 11 that is further increased at 
stages 13 and 14. In contrast, the block of RA-signaling by BMS453 results in a twofold decrease in Hnf1b expression compared to untreated embryos.

In summary, Hnf1b is expressed in the dorsal and ventral foregut and we found its endodermal expression to be RA-responsive.

\subsubsection{Hnf1b is directly induced by RA in pancreatic organoids}

Several previous studies in mouse identified RA-responsive elements within the genomic sequence of the hnf1b gene, including a DR1 element in the Hnf1b promotor (Power and Cereghini, 1995) and a second DR5 element within intron 4 (Pouilhe et al., 2007). These findings suggest that Hnf1b is a direct RA-target. However, a direct induction of Hnf1b expression in the endoderm by RA has not been shown so far. RNA-sequencing has revealed that Hnf1b is induced by RA within one hour and its expression is further increased within two hours after RAaddition. Hnf1b transcripts are upregulated upon RA-treatment in the absence as well as in the presence of the translational inhibitor $\mathrm{CHX}$, indicating that Hnf $1 \mathrm{~b}$ is directly induced by RA (Fig. 3.6 A). These data were confirmed by RT-PCR and $\mathrm{Hnf} 1 \mathrm{~b}$ is found to behave in a similar fashion as the known direct RA-target Cyp26a1 (Fig. 3.6 B). However, in contrast to Cyp26a1, Hnf1b shows low but significant expression levels even in the absence of RA. In summary, Hnflb is indeed a direct RA-target gene in Xenopus, but there must be an additional, RAindependent mechanism, regulating the pan-endodermal expression of Hnf1b. 
A
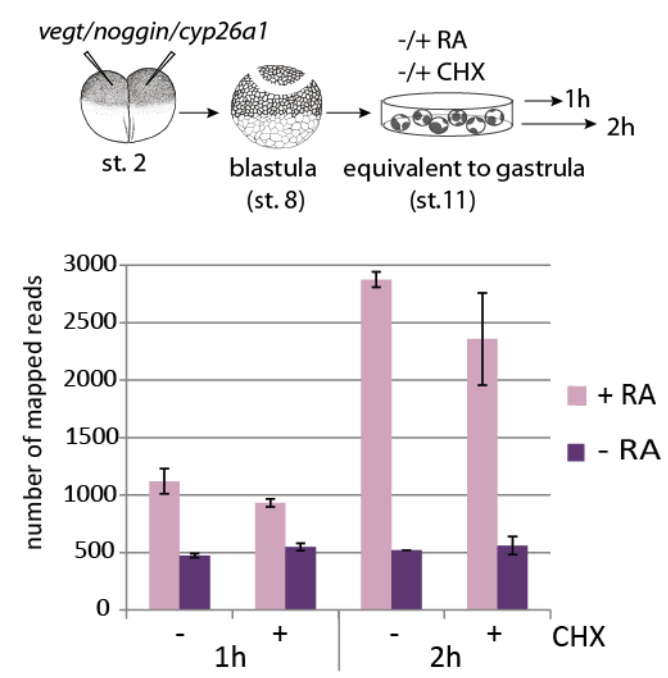

B

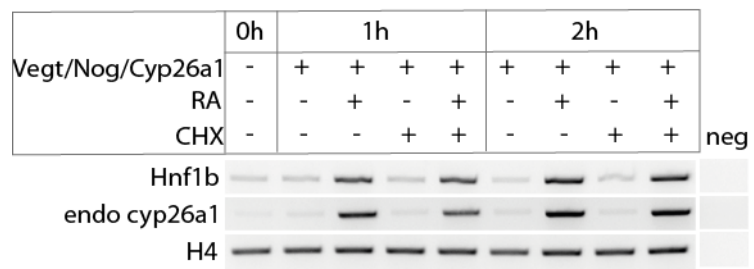

C vegt/noggin $-/+h n f 1 b-m o$ $-/+h n f 1 b-G R$

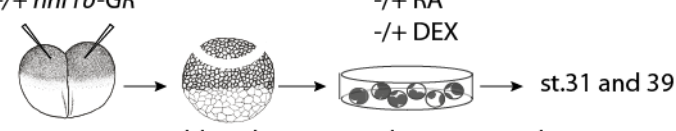

st. 2 blastula equivalent to gastrula (st.11)

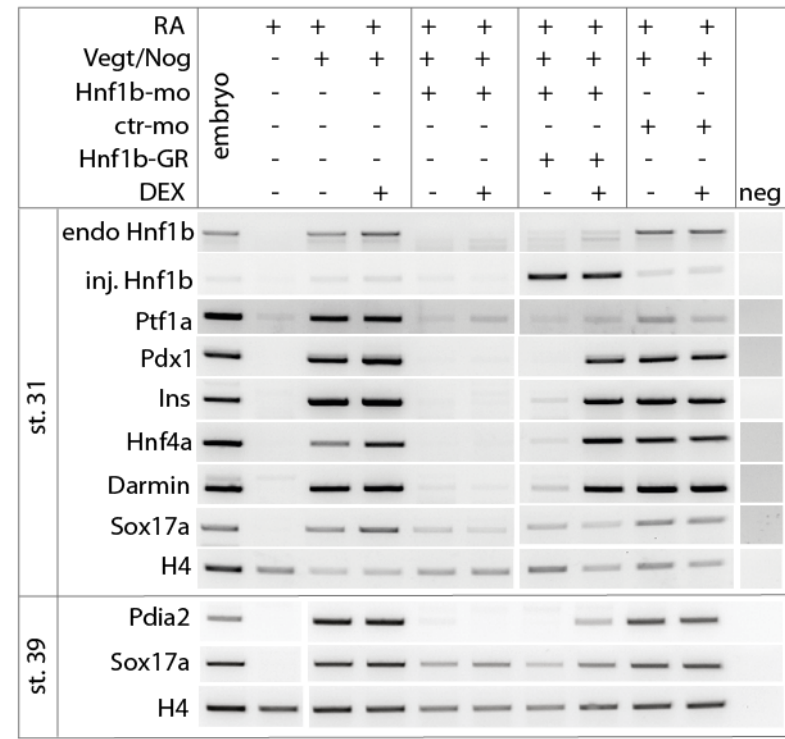

Fig. 3.6 Hnf1b is directly induced by RA and required for pancreas specification in pancreatic organoids

Direct induction of Hnf1b by RA (A) Two cell stage embryos were co-injected with vegt (500pg), noggin (500pg) and cyp26a1 (2000pg) RNA. Blastocoel roof tissue was explanted, cultivated and treated with RA and $\mathrm{CHX}$ at the equivalent of gastrula stage. Samples for RNA-sequencing were taken one and two hours after RA-addition. Hnf1b expression levels are represented by the number of mapped reads. (B) RT-PCR analyzing Hnf1b, Cyp26a1 and the housekeeping gene H4 transcripts for samples of programed explants before and one/two hours after RA addition.

Requirement of Hnf1b for pancreatic gene expression in pancreatic organoids

(C) $55 \mathrm{ng} \mathrm{Hnf1b-morpholino} \mathrm{(Hnf1b-mo)} \mathrm{or} 55 \mathrm{ng}$ control-morpholino (ctr-mo) were co-injected with vegt (500pg) and noggin (500pg) RNA into two cell stage embryos. 800 pg of RNA for a hormoneinducible Hnf1b (Hnf1b-GR) was co-injected and explants were treated with dexamethasone (DEX) and RA at the equivalent of gastrula stage to rescue the morpholino-mediated phenotype. At the equivalent of stages 31 and 39, total RNA was isolated and subjected to RT-PCR. Detection of endogenous (endo) and injected Hnf1b (inj.), pancreatic progenitor markers Pdx1 and Ptf1a, early endocrine differentiation marker Insulin (Ins) and late differentiation marker Pdia2, direct Hnf1b target $\mathrm{Hnf} 4 \mathrm{a}$, endodermal markers Darmin and Sox17a, housekeeping gene H4. 


\subsubsection{Hnf1b is required for pancreas specification in vitro}

In order to determine the requirement of Hnf1b for pancreas specification, Hnf1b protein expression was downregulated by the injection of a morpholino antisense oligonucleotide, targeting the intron1/exon2 boundary of the Hnf1b pre-mRNA (Fig. 6.9 A). Binding of the morpholino to the pre-mRNA should inhibit conventional splicing. In a first step, the functionality of the morpholino was tested in the explant system (Fig. 6.9 B). At the equivalent of neurula stage, the Hnf1b amplicon in Hnf1b-morpholino injected explants is shifted to a smaller size compared to the Hnf1b amplicon of control embryos and control-morpholino injected explants (Fig. $6.9 \mathrm{C})$. Sequencing of the amplicon revealed that indeed exon2 is lost in the presence of the Hnf1b-morpholino (data not shown). Thus, a frameshift occurs and a premature stop codon in exon3 is used that leads to a shortened open reading frame of Hnf1b. As a result, a shortened Hnf1b protein is translated that lacks the functional DNA-binding domains and the transactivation domain (Fig. 6.9 A).

First, we wanted to determine if Hnflb is required for RA-mediated activation of pancreatic marker genes in pancreatic organoids. For this purpose, the morpholino was used to downregulate Hnf1b protein expression in pancreatic organoids (Fig. 3.6 C). At the equivalent of stage 31 , endogenous Hnf1b is only detected in pancreatic organoids and control embryos (Fig. 3.6 C lanes 3 and 4) and is completely abolished upon co-injection of the Hnf1b-morpholino (Fig. 3.6 C lanes 5 and 6) suggesting that the smaller Hnf1b amplicon, observed at late neurula stage, is probably degraded at stage 31 due to nonsense-mediated mRNA decay (Chang et al., 2007). Pancreatic progenitor markers Pdx1 and Ptf1a as well as the early endocrine differentiation marker Insulin and the late exocrine marker Pdia2 are detected in control embryos (lane1) and pancreatic organoids (lanes 3 and 4) but not in un-programed explants (lane2) (Fig. 3.6 C). Furthermore, the direct Hnf1b target gene Hnf4a (Thomas et al., 2001) and endodermal marker genes Darmin and Sox17a are expressed in control embryos and pancreatic organoids. Upon coinjection of the Hnf1b-morpholino, Ptf1a expression is strongly decreased and the expression of Pdx1, Insulin, Pdia2 and Hnf4a are completely lost. In addition, the expression of Darmin is strongly decreased whereas the expression of the other endodermal marker Sox $17 \mathrm{a}$ is only slightly affected. These effects were not observed in the presence of the control-morpholino (lanes 9 and 10) (Fig. 3.6 C). In order to demonstrate that the observed effects are specific for the loss of functional Hnf1b protein, a rescue experiment was performed. For this purpose, RNA encoding a hormone-inducible Hnf1b (Hnf1b-GR) was co-injected. Hnf1b protein function was activated at the equivalent of gastrula stage by dexamethasone (DEX) treatment. 
Indeed, the activation of Hnflb re-establishes the expression of the progenitor marker Pdx1, the direct Hnf1b target gene Hnf4a, the endodermal marker Darmin as well as the differentiation markers Insulin and Pdia2 (Fig. 3.6 C). However, results for Ptf1a expression under rescue conditions are inconsistent. In a few experiments Ptf1a expression could be fully rescued (not shown) and in other experiments it remained strongly decreased.

In summary, Hnf1b is required for RA-mediated expression of pancreatic marker genes in pancreatic organoids. Moreover, Darmin, which was found to be RAinducible expressed in explants, appears to be downstream of Hnf1b. 


\subsubsection{Hnf1b is required for pancreas specification in vivo}

As we could show that Hnf1b is required for pancreatic gene expression in in vitro generated pancreatic organoids, we next asked if Hnf1b is required for pancreas specification in vivo.

\subsubsection{Hnf1b-overexpression leads to an expansion of the pancreatic progenitor field in vivo}

In order to determine the role of Hnf1b for the development of pancreatic progenitor formation in vivo, a gain of function approach was done. RNAs encoding a hormoneinducible Hnf1b (Hnf1b-GR) and $\beta$-galactosidase (glb1) were injected vegetally into the two dorsal blastomeres of a four-cell stage embryo. Hnf1b activity was induced at gastrula stage through dexamethasone-treatment (DEX) until stage 32 and a WMISH was carried out to visualize Pdx1 and Ptf1a expression (Fig. 3.7 A). In control embryos, the progenitor marker $\mathrm{Pdx} 1$ is not exclusively expressed in the pancreas but is also detected in the adjacent duodenum. In Hnf1b-overexpressing embryos, the $\mathrm{Pdx} 1$ expression domain is expanded along the anterior-posterior axis. The second progenitor marker Ptf1a is expressed in the eye, the hindbrain and in the endoderm, where it is restricted to the dorsal and ventral pancreatic buds. Upon Hnf1b-overexpression, the dorsal as well as the ventral pancreatic Ptf1a expression domain is expanded whereas the expression in the eye and the hindbrain is unaffected (Fig. 3.7 A). The effects of Hnf1b-overexpression on Pdx1 and Ptf1a expression, observed by WMISH, were quantified for two independent experiments using ImageJ. At least 20 embryos per experiment were analyzed (Tab. 6.22 and Tab. 6.23). In control embryos on average $9 \%$ of the endodermal area is Pdx1positive with a variation from $4 \%$ to $17 \%$. Upon Hnf1b-overexpression, the average is significantly increased to $13 \% \mathrm{Pdx} 1$ domain in the endoderm with a variation from $8 \%$ to $28 \%$. Ptf1a is expressed in $3 \%$ of the endoderm in control embryos. Hnf1boverexpressing embryos show almost $5 \%$ of the endodermal area positive for Ptf1a with a variation of $2 \%$ to $13 \%$. When dorsal and ventral pancreatic Ptf1a expression is examined individually, we find that the ventral pancreatic domain of Ptf1a is affected more dramatically than the dorsal domain (Fig. 3.7 A). In conclusion, Hnf1b-overexpression in the endoderm leads to the formation of an expanded pancreatic progenitor field. 


\subsubsection{Hnf1b-knockdown causes a decrease in or a complete loss of pancreatic progenitors in vivo}

The observation that the morpholino-mediated knockdown of Hnf1b in pancreatic organoids results in a loss of Ptf1a and Pdx1 expression suggests that Hnf1b is required for pancreas specification. In order to verify this hypothesis, we carried out a morpholino-mediated Hnf1b loss-of-function analysis in vivo. Hnf1b-morpholino injected embryos were cultivated until stage 32 and the expression of Ptf1a and Pdx1 was determined by WMISH. Upon Hnf1b-knockdown, Pdx1 and the endodermal Ptf1a expression are completely lost. However, in control-morpholino injected embryos, Pdx1 and the endodermal Ptf1a expression are also affected as the WMISH signal is decreased (Fig. 3.7 B). Nevertheless, control-morpholino injected embryos do not show a phenotype as severe as caused by the injection of the Hnf1b-morpholino. In order to compare control- and Hnf1b-morpholino effects in more detail, a quantification of pancreatic marker gene expression using real-time PCR was done. The expression levels of Pdx1, Ptf1a and Insulin were calculated with respect to the levels of the housekeeping gene ornithine decarboxylase (odc). In the presence of the Hnf1b-morpholino, the transcript levels of Pdx1, Ptf1a and Insulin are almost un-detectable. In contrast, in the presence of the controlmorpholino, Pdx1 and Insulin transcript levels were decreased to $60 \%$ and $70 \%$, while Ptf1a levels were unaffected compared to control embryos. Hence, the downregulation of Hnf1b in vivo leads to strong decreased expression levels of pancreatic progenitor markers.

In summary, Hnf1b is required for pancreas specification in Xenopus laevis embryos. 
A

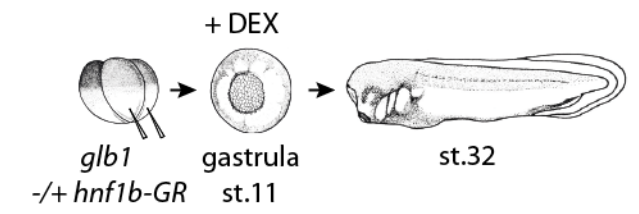

$\mathrm{Pdx} 1$

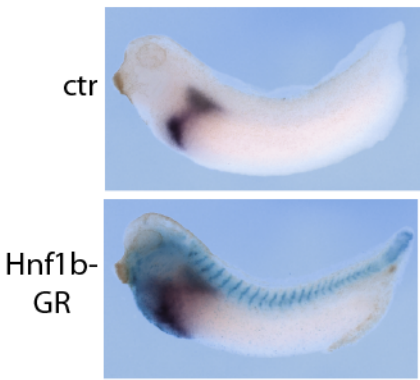

$\%$ of Pdx 1

domain in the endoderm

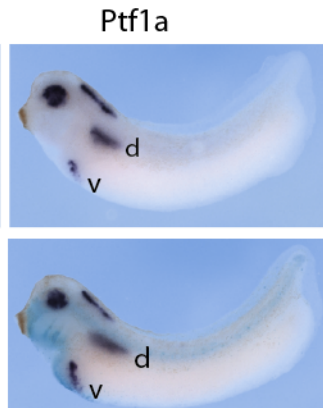

$\%$ of Ptf1a domain in the endoderm
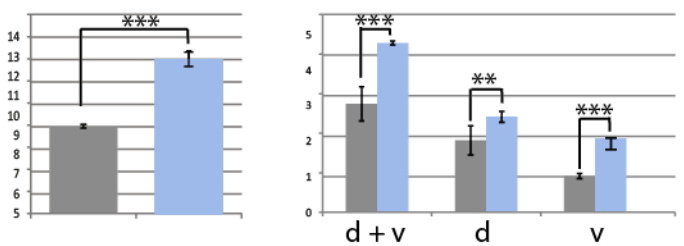

$\square \mathrm{ctr} \square \mathrm{Hnf1b}-\mathrm{GR}$

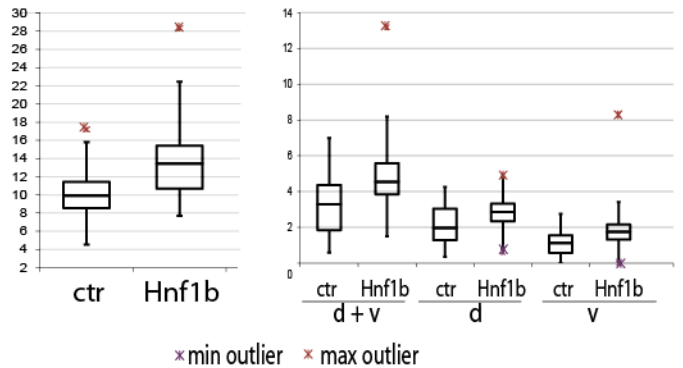

B
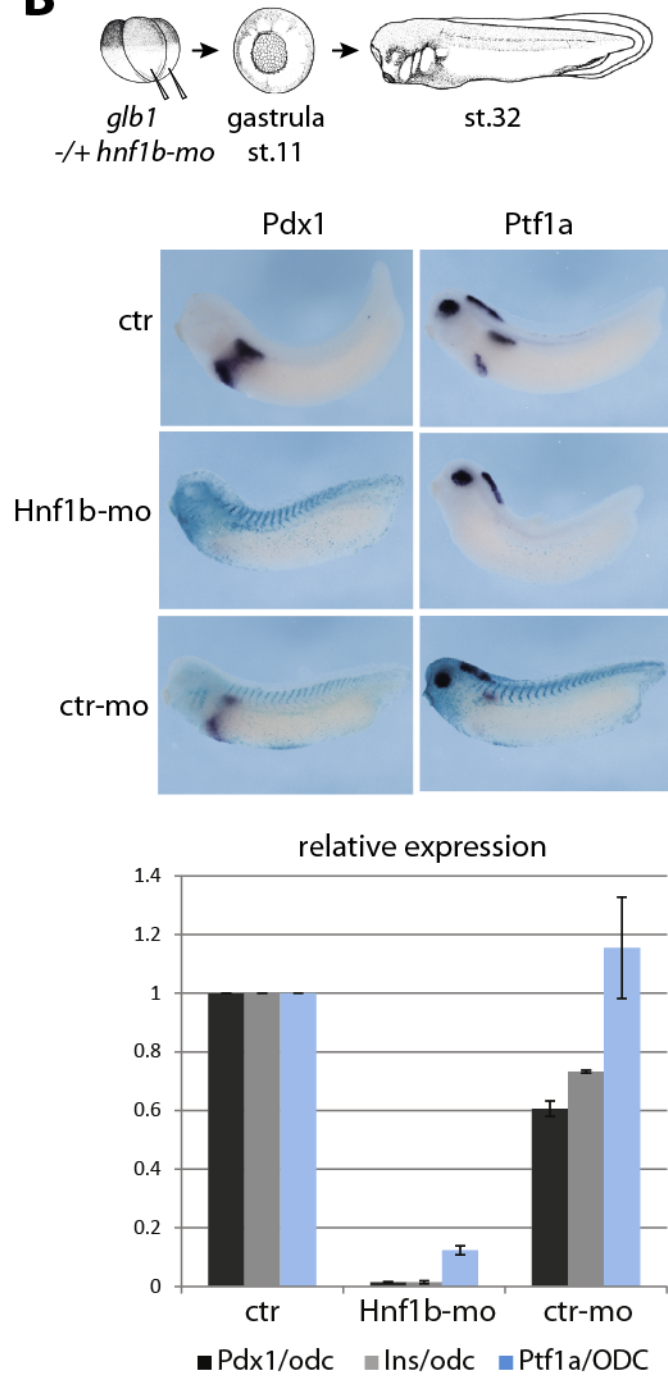

Fig. 3.7 Hnf1b is required for pancreas specification in vivo

Hnf1b-overexpression leads to expanded pancreatic progenitor field. (A) 200pg $\beta$ calactosidase (glb1) and 800pg Hnf1b-GR RNA were co-injected vegetally into the two dorsal blastomeres of a four-cell stage embryo. As control (ctr) served $\beta$-calactosidase RNA injected embryos. At gastrula stage, embryos were treated with dexamethasone (DEX) and cultivated until stage 32. WMISH against Pdx1 and Ptf1a in control and Hnf1boverexpressing embryos is shown. Upper graphs show the percentage of Pdx1 and endodermal Ptf1a domains in the whole endoderm. Lower graphs are boxplots displaying the range of observed data and indicating outliers.

P-values in a Student's $t$-test ${ }^{* *}<0.01,{ }^{* * *}<0.001$.

Hnf1b-downregulation leads to a strong decrease in pancreatic progenitor expression. (B) 200pg $\beta$-calactosidase ( $g / b 1$ ) RNA and 25ng Hnf1b-morpholino or ctr-morpholino were co-injected vegetally into the two dorsal blastomeres of a four-cell stage embryo. Embryos at stage 32 were used for WMISH against Pdx1 and Ptf1a and real-time PCR analysis for Pdx1, Ptf1a and Insulin. Graph indicated the fold changes of tested markers in ratio to ODC. WMISH analysis was performed once and real-time PCR results emerge from two independent biological replicates. 


\subsubsection{Hnf1b alone cannot substitute for RA-activity during pancreas specification}

As we could show that Hnf1b is required for pancreas specification in vitro as well as in vivo, we next asked if Hnflb is the key factor that mediates RA-activity in the context of pancreas specification. The explant system was used to answer that question. RNA coding for the hormone-inducible Hnf1b-GR was co-injected with vegt and noggin RNA. Endogenous RA-signaling was blocked by the co-injection of cyp26a1 RNA (Fig. 3.8 A). In control embryos and explants in the presence of RA, the pancreatic markers Pdx1, Ptf1a and Insulin, the direct Hnf1b target Hnf4a and the endodermal markers Darmin and Sox17a are expressed. If RA-signaling is blocked, only the endodermal marker Sox17a is detected. However, upon the induction of Hnf1b activity (+DEX), pancreatic marker gene expression is still not detectable (Fig. 3.8 B). Interestingly, the endodermal marker Darmin and the direct target Hnf4a are induced by $\mathrm{Hnf} 1 \mathrm{~b}$, indicating the presence of functional Hnf1b protein. Taken together, Hnf1b is not sufficient to substitute for RA-activity in pancreas development. Hence, Hnf1b is not the only RA-responsive gene that is required for pancreas specification in Xenopus. 
A

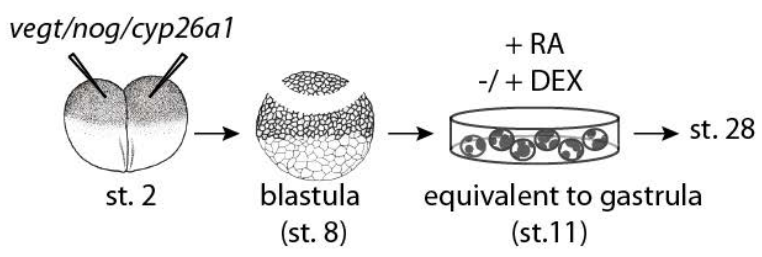

B

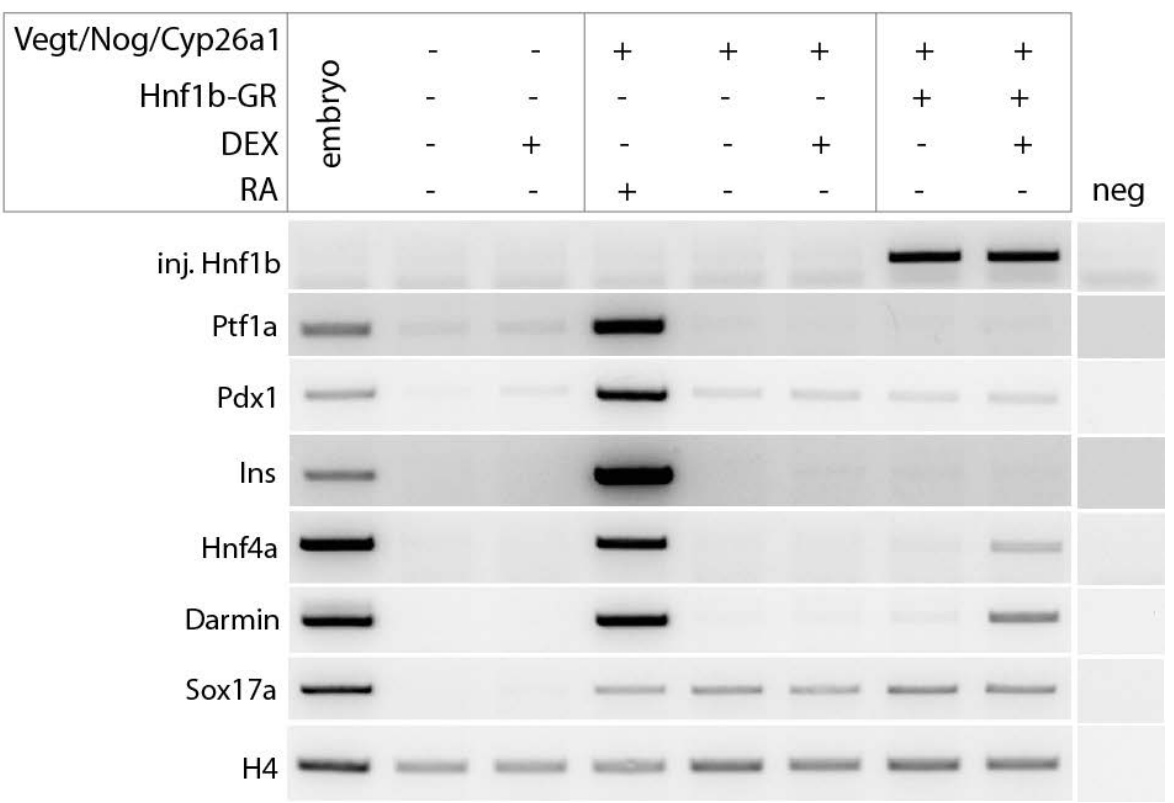

Fig. 3.8 Hnf1b is not sufficient to substitute for RA in pancreas specification in ectodermal explants

(A) Indicated RNAs were co-injected with 800 pg Hnf1b-GR RNA into a two-cell stage embryo. Ectodermal explants were prepared and cultivated. Treatments with dexamethasone (DEX) and RA were performed at the equivalent of gastrula stage. At the equivalent of stage 28 , total RNA was isolated and analyzed by RTPCR. (B) RT-PCR for pancreatic progenitor markers Pdx1 Ptf1a, differentiation marker Insulin (Ins), direct Hnf1b target Hnf4a, endodermal markers Darmin and Sox17a and housekeeping gene histone $\mathrm{H} 4$. 


\subsection{The direct target Fzd4/Fzd4s is required for pancreas specification}

The second promising candidate, potentially involved in pancreas specification, is the Wnt-receptor Fzd4 as it was found to be induced by RA in the RNA sequencing experiment and is expressed in the dorsal endoderm during gastrulation.

\subsubsection{Fzd4 and the alternative splice variant Fzd4s are directly induced by RA}

RNA-sequencing data revealed that Fzd4 is induced by RA even in the presence of the translational inhibitor $\mathrm{CHX}$ (Fig. $3.9 \mathrm{~A}$ ). Moreover, we found that Fzd4 is expressed in the dorsal endoderm during gastrulation, suggesting Fzd4 as a direct RA-target gene potentially involved in pancreas specification.

The Fzd4 gene includes two exons separated by a large intronic region. Through conventional splicing a Fzd4 protein is generated comprising a small extracellular cysteine-rich domain (CRD) for Wnt ligand interaction, a seven transmembrane domain (TM) and an intracellular domain (ICD) (Fig. 6.10). Additionally, an alternative splice variant for Fzd4 that retains the intron was discovered (Yam et al., 2005; Swain et al., 2005). This splice variant retains the Wnt-binding CRD domain, but is missing the transmembrane and the intracellular domain as a premature stop codon within the retained intron is used (Fig. 6.10). As this splice variant was assumed to be secreted it was named Fzd4s (Fzd4 secreted). Fzd4s can act as an activator as well as inhibitor of Wnt-signaling depending on the identidity of the corresponding Wnt-ligand (Swain et al., 2005). The question arises if both Fzd4 variants are induced by RA. The RNA-sequencing data contain sequence reads mainly mapped to exon 1 and exon 2 of the Fzd4 gene locus, but a significant number of reads was mapped to intronic sequences as well (data not shown). Nanostring analysis, used for the verification process, does not give the information which variant is induced by RA as the Nanostring probe against Fzd4 targets the exon2 that is contained in both splice variants (Fig. 6.11 C). Therefore, oligonucleotides were designed either neighbouring the intronic sequence or targeting within the intron to distinguish between the different Fzd4 transcripts. As the intronic sequence is larger than $6 \mathrm{~kb}$, amplicons generated by RT-PCR with oligonucleotides flanking the intron would give only a signal for Fzd4. With the oligonucleotides targeting the intronic region, only Fzd4s transcripts are detected. The RT-PCR results reveal that both variants of Fzd4 are directly induced by RA within two hours after RA-addition (Fig. 3.9 B). 


\subsubsection{Fzd4/Fzd4s is enriched in the dorsal half of a gastrula embryo including the dorsal endoderm}

Fzd4/Fzd4s expression was examined during Xenopus development by WMISH in order to visualize expression domains (Fig. 6.11 A/B). An antisense probe was applied that was already used during the screen for the determination of Fzd4 expression characteristics. This antisense probe targets the complete open reading frame of the Fzd4 transcript. Theoretically, Fzd4s transcripts should also be targeted by this antisense probe as the major part of this probe binds to the sequence derived from exon2 (Fig. 6.11 A). Fzd4/Fzd4s transcripts are detected in the dorsal endoderm and the prospective neuroectoderm at gastrula stage. At neurula stage, Fzd4/Fzd4s transcripts are found in the prospective foregut, in the lateral plate mesoderm and in the forebrain region (Fig. 6.11 A). Later during development, $\mathrm{Fzd} 4 / \mathrm{Fzd} 4 \mathrm{~s}$ is expressed in the head and the regions of pancreas, liver, duodenum and heart primordium. In order to specifically detect transcripts of the alternative splice variant Fzd4s, an antisense probe was designed targeting the intronic-derived sequence (Fig. 6.11 B). During gastrulation, WMISH signals are detected in the dorsal and ventral mesoderm, prospective neuroectoderm and in very low intensity in the entire endoderm. However, in contrast to the first antisense-probe, a clear dorsal enrichment was not observed. At neurula stage, Fzd4s is expressed in the notochord and in the prospective foregut region. Later during development, a moderate expression of Fzd4s is detected in the entire embryo with an enrichment in the head region (Fig. 6.11 B). As low levels of staining are found in the entire embryo for all tested developmental stages it cannot be excluded that the detected signals are unspecific. Thus, the expression of Fzd4 and Fzd4s during gastrulation was tested by RT-PCR using preparations of dorsal and ventral endoderm as well as of dorsal and ventral tissues as described in Fig. 6.6 A. Fzd4 and Fzd4s transcripts could not be detected in endodermal preparations (not shown). This might be due to the preparation technique by which a lot of endodermal tissue gets lost. Nevertheless, for preparations of the tissue surrounding the dorsal and ventral blastopore lip, Fzd4 as well as Fzd4s transcripts are found to be enriched in the dorsal half (Fig. 6.11 C). In order to quantify the level of dorsal enrichment, a Nanostring analysis was carried out, although a distinction between Fzd4 and Fzd4s transcripts is not possible as the Nanostring probe targets the region encoded by exon2. Fzd4/Fzd4s is 1.5-fold enriched in the dorsal compared to ventral endoderm (Fig. 6.7 A) and 4-fold enriched in the dorsal compared to the ventral part around the blastopore lip (Fig. 6.11 C). 
However, neither the expression data from WMISH nor from RT-PCR allow a conclusion about which Fzd4 variant is expressed in the dorsal endoderm during gastrulation. Thus, further following designations concerning Fzd4 are termed as Fzd4/Fzd4s. In conclusion, Fzd4/Fzd4s is enriched in the dorsal tissue of a gastrula embryo including the dorsal endoderm. Later in development, Fzd4/Fzd4s expression is restricted to pancreas, liver, duodenum, heart primordium and head structures.

\subsubsection{Fzd4/Fzd4s is RA-responsively expressed during gastrulation}

In our screen, Fzd4/Fzd4s was identified as direct RA-responsive gene in vitro. In order to verify the RA-responsiveness of Fzd4/Fzd4s expression in vivo during gastrulation, WMISH and Nanostring analyses were performed (Fig. 6.12). Untreated, RA-treated and Cyp26a1-injected embryos were cultivated until gastrula stage and subjected to WMISH analysis using an antisense probe targeting both, Fzd4 and Fzd4s. Upon an excess of RA the expression of Fzd4/Fzd4s in the dorsal endoderm as well as that in the prospective neuro-ectoderm is strongly increased. Conversely, the degradation of endogenous RA by Cyp26a1 leads to a loss of endodermal Fzd4/Fzd4s expression whereas the prospective neuro-ectodermal expression is unaffected (Fig. 6.12 A). Thus, endodermal and neuro-ectodermal Fzd4/Fzd4s expression domains are RA-inducible but only the endodermal expression domain is RA-dependent.

Moreover, quantitative Nanostring analysis of stage 12 to 14 embryos reveals a clear increase of Fzd4/Fzd4s expression from 2 fold up to 2.5 fold in RA-treated embryos compared to untreated embryos. In contrast, upon the inhibition of RAsignaling by BMS453, a reduction in Fzd4/Fzd4s transcript levels up to 2 fold was observed (Fig. 6.12 B). In summary, during gastrulation the endodermal expression domain of Fzd4/Fzd4s is RA-responsive. 


\subsubsection{Fzd4/Fzd4s is required for pancreatic marker gene expression in vitro}

In order to determine if $\mathrm{Fzd} / \mathrm{Fzd} 4 \mathrm{~s}$ is required for pancreas specification, we analyzed the function of Fzd4/Fzd4s in pancreatic organoids. For this issue, two loss-of-function approaches were done, interfere with the translation or impair the gene locus. First, a morpholino was used that targets the start-codon of the $\mathrm{fzd} 4 \mathrm{and}$ fzd4s RNA and thereby blocks the translation of both Fzd4 variants (Fig. 3.9 C) (Gorny et al., 2013). The morpholino was co-injected with vegt and noggin RNA and pancreatic organoids were generated and cultivated until stage 28 and RNA was subjected to RT-PCR. Pancreatic progenitor markers Pdx1 and Ptf1a, the early differentiation marker Insulin as well as the endodermal marker Sox17a were strongly decreased upon Fzd4/Fzd4s-downregulation compared to control embryos or mismatch-morpholino-injected explants. In contrast, the endodermal marker Darmin was not affected (Fig. 3.9 C). These results indicate a requirement of Fzd4 for RA-mediated pancreatic marker gene induction. However, injection of various concentrations of $f z d 4$ and $f z d 4 s$ RNA could not rescue the observed knockdown phenotype (not shown). We suppose that, for pancreatic gene expression, $\mathrm{Fzd} 4 / \mathrm{Fzd} 4 \mathrm{~s}$ protein activity is required during gastrulation and that an earlier activity, caused by RNA injection at early cleavage stage and subsequent translation inhibits pancreatic gene expression. A temporally controlled activation of Fzd4/Fzd4s using the GR/DEX-system, as used for over-expression of Hnf1b, would not function for membrane bound or secreted factors. Due to this reasons, Fzd4 and Fzd4s encoding plasmid DNA was injected, which is not transcribed before MBT (mid blastula transition). However, the Fzd4/Fzd4s-knockdown phenotype of decreased pancreatic marker gene expression could not be restored (not shown). This might be due to an inadequate Fzd4/Fzd4s concentration as the appropriate level of active Fzd4/Fzd4s protein for proper pancreas specification remains to be determined.

In order to confirm the phenotype observed upon morpholino-mediated knockdown of Fzd4/Fzd4s, we went for a second type of loss-of-function approach. The CRISPR/Cas (Clustered Regularly Interspaced Short Palindromic Repeats/CRISPRassociated) is based on the RNA-guided DNA endonuclease Cas9 that can be targeted against a specific genomic region. A guide RNA (Fzd4-gRNA) was designed targeting a twenty nucleotide sequence within exon1 of the $f z d 4$ gene (Fig. 3.9 D). This guide RNA was co-injected with RNAs encoding for Cas9, Vegt and Noggin at one-cell stage. At blastula stage, ectodermal explants were prepared and cultivated as previously described. RA was not added as endogenous RA levels, indirectly induced by Noggin, are sufficient to induce pancreatic marker gene 
expression (Fig. 3.1). Furthermore, a mosaicism of cells carrying wildtype or mutated fzd4 gene in the explants is expected. Therefore, the system was not saturated with exogenous RA in order to detect an effect on pancreatic marker genes upon Fzd4 gene disruption. RT-PCR analysis and genomic DNA extraction for mutation analysis were done using extracts from 50 explants each. Mutation analysis revealed a $100 \%$ mutation rate in total and $66 \%$ effective mutations which result in impaired protein function (Fig. 3.9 D; Fig. 6.13). Upon Fzd4-gRNA coinjection, transcript levels of Ptf1a, Pdx1 and Insulin were strongly reduced as compared to Cas9 over-expressing pancreatic organoids. Furthermore, Darmin transcript levels are decreased whereas Sox17a levels are not affected (Fig. 3.9 D). Since a stretch of twenty nucleotides can be found multiple times in the genome and the CRISPR/Cas system can accept mismatches depending on their position, potential off-targets were predicted using the online tool CCTop (Stemmer et al., 2015). In doing so, eight potential off-target sequences within exonic regions of the $X$. laevis genome were predicted (Tab. 6.24). Three of them were analyzed for mutations in the presence of Fzd4-gRNA. The potential off-target sequence within genomic sequences of Impad1, Kremen2 and Fzd7 contain in total 5 mismatches. However, for Kremen2 and Impad1 only one mismatch is located within protospacer sequence proximal to the PAM (protospacer adjacent motif). A gRNA/Cas9 efficiency study in Xenopus tropicalis revealed that single mismatches within the protospacer completely abolish targeting activity of this RNA/endonuclease complex (Guo et al., 2014). Indeed, mutation analysis of the three potential off-targets revealed that no mutations were introduced into the gene loci. Data for Kremen2 are shown in the appendix (Fig. 6.13).

Taken together, both loss-of-function approaches revealed that Fzd4/Fzd4s is indeed required for pancreatic gene induction in pancreatic organoids, suggesting a role for Fzd4/Fzd4s during pancreas specification. 
A

vegt/noggin/cyp26a1

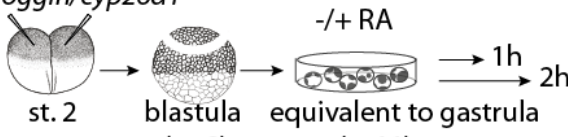

(st. 8) (st.11)

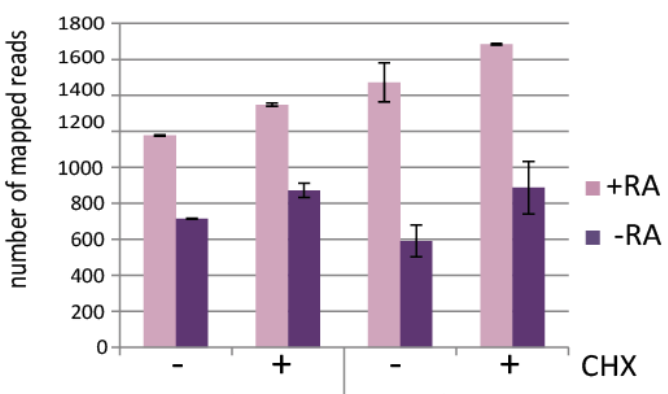

$1 \mathrm{~h}$

C vegt/noggin

$-/+f z d 4-m o$
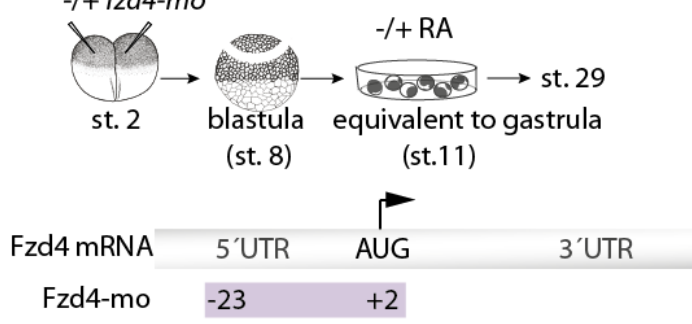

B

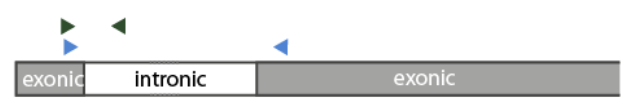

\begin{tabular}{|r|c|ccccc|cccc|}
\hline & \multicolumn{7}{|c|}{ Oh } & \multicolumn{4}{|c|}{$1 \mathrm{~h}$} & \multicolumn{3}{|c|}{$2 \mathrm{~h}$} \\
\cline { 2 - 7 } Vegt/Nog/Cyp26a1 & - & + & + & + & + & + & + & + & + \\
RA & - & - & + & - & + & - & + & - & + \\
CHX & - & - & - & + & + & - & - & + & + \\
\hline
\end{tabular}

$\mathrm{Fzd} 4$

จFzd4s - - - - - - -

endo Cyp26a1

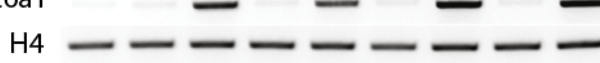

D

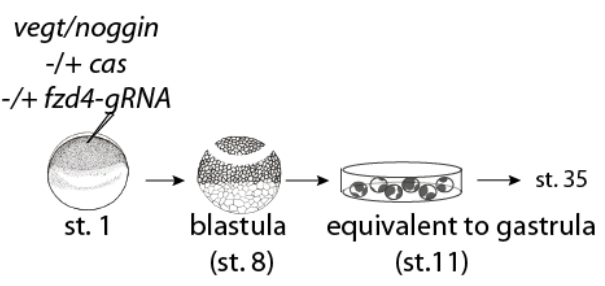

$\begin{array}{llll}\text { Fzd4 gene } & \text { ATG } & & \\ \text { Fzd4 gRNA } & & +98 \quad+118\end{array}$

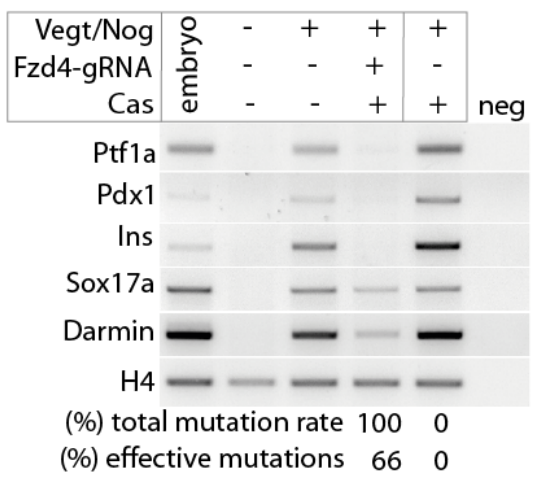

Fig. 3.9 Fzd4 and Fzd4s are directly induced by RA and required for pancreas specification in pancreatic organoids

\section{Direct induction of Fzd4/Fzd4s by RA}

(A) Two cell stage embryos were co-injected with vegt, noggin and cyp26a1 RNA. Blastocoel roof tissue was explanted, cultivated and treated with RA and $\mathrm{CHX}$ at the equivalent of gastrula stage. One and two hours after RA-addition, total RNA was isolated and subjected to RNA-sequencing. Fzd4 expression levels are indicated as number of mapped reads. (B) RT-PCR for splice variants Fzd4 and Fzd4s, for Cyp26a1 as well as the housekeeping gene $\mathrm{H} 4$ in samples of programed explants before and one/two hours after RA addition.

Requirement of Fzd4/Fzd4s for pancreatic gene expression in pancreatic organoids

(C) Fzd4/Fzd4s-knockdown through translational blocking Fzd4-morpholino (Fzd4-mo). 45 ng Fzd4-mo or the corresponding mismatch-morpholino $(\mathrm{mmo})$ were co-injected with vegt and noggin RNA and explants for pancreatic organoid formation were prepared. At the equivalent of stage 28, total RNA was isolated and subjected to RT-PCR. Amplification of pancreatic progenitor markers Pdx1 and Ptf1a, differentiation marker Insulin (Ins), endodermal markers Sox17a and Darmin. (D) Fzd4/Fzd4s knockdown through CRISPR/Cas-technique. Fzd4-gRNA was co-injected with RNAs encoding Cas9, Vegt and Noggin into one-cell stage embryos. Explants were cultivated until the equivalent of stage 35. RT-PCR for pancreatic and endodermal markers was done. Mutation rates are indicated for Cas 9 only or Cas9 with Fzd4-aRNA. 


\subsubsection{Downregulation of Fzd4/Fzd4s leads to an increase in non-canonical Wnt-signaling activity in vitro}

Fzd4/Fzd4s loss-of-function experiments in pancreatic organoids showed that the direct RA-target gene $\mathrm{Fzd} 4 / \mathrm{Fzd} 4 \mathrm{~s}$ is required for pancreatic marker gene expression. Both variants, Fzd4 and Fzd4s, act as Wnt-interacting molecules (Xu et al., 2004; Swain et al., 2005; Mikels and Nusse, 2006). For this reason, we asked if the requirement of Fzd4/Fzd4s for pancreas specification is dependent on its function in Wnt-signaling. We first tested if Wnt-signaling is active in explants programed with Vegt/Noggin and how RA-activity affects this level of Wnt-signaling activity. For this purpose, luciferase reporter assays were carried out using reporter constructs for both Wnt-signaling pathways, canonical as well as non-canonical (Fig. 3.10 A). RNAs coding for Vegt, Noggin and Cyp26a1 were co-injected with plasmids coding for either firefly-luciferase reporters under the control of the Siamois promotor (canonical Wnt-signaling target) or the Atf2 response element (noncanonical Wnt-signaling target). In addition, a plasmid coding for reporter Renillaluciferase was co-injected in order to normalize the levels of Siamois- and Atf2reporter activity. Wnt8a (canonical) and wnt5a (non-canonical) RNA co-injections served as positive controls (Fig. 3.10). Both, canonical as well as non-canonical Wnt-signaling reporter activity is detected in Vegt/Nog-programed explants in the absence of RA-signaling. These activities were set to 100\%. Upon RA-treatment, the level of canonical reporter is decreased to $70 \%$ and the level of the noncanonical reporter below 60\% (Fig. 3.10 B). In conclusion, programed explants exhibit a canonical and non-canonical Wnt-signaling activity that is decreased in the presence of RA-signaling, in which non-canonical Wnt-signaling is slightly stronger affected than canonical Wnt-signaling.

Next, we asked how a knockdown of Fzd4/Fzd4s in these explants would influence the Wnt-signaling activity. For this issue, the Fzd4-morpholino was co-injected. As Noggin indirectly activates endogenous RA-signaling, RA was not applied to the explants. The levels of luciferase activity in the samples injected with the reporters only were set to one (Fig. 3.10 C). Upon Fzd4/Fzd4s-downregulation, the level of canonical reporter activity increases from 10-fold in morpholino-un-injected explants and mismatch-morpholino-injected explants up to 16-fold. However, the high standard deviation between the data of four experiments indicates that the effect on canonical Wnt-signaling is not significant. In contrast, non-canonical reporter activity is significantly increased from two-fold in morpholino-un-injected explants to six-fold 
upon Fzd4/Fzd4s downregulation (Fig. 3.10 C). Taken together, the downregulation of Fzd4/Fzd4s leads to an increase in non-canonical reporter activity. 
A

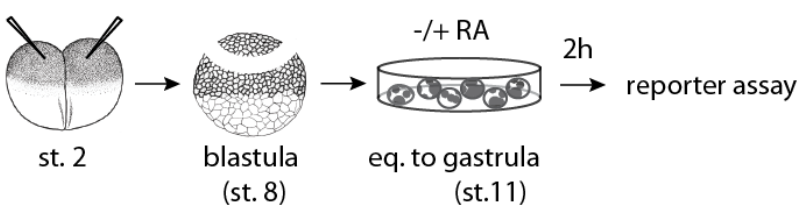

B Wnt-signaling in programed explants
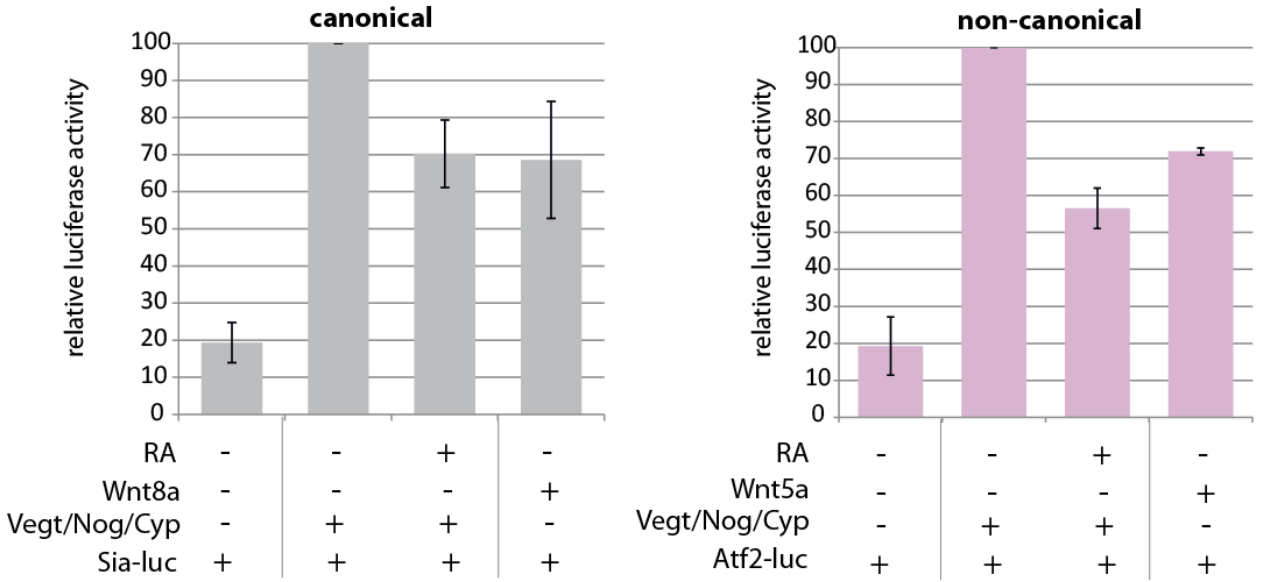

C Wnt-signaling in programed explants with Fzd4-knockdown
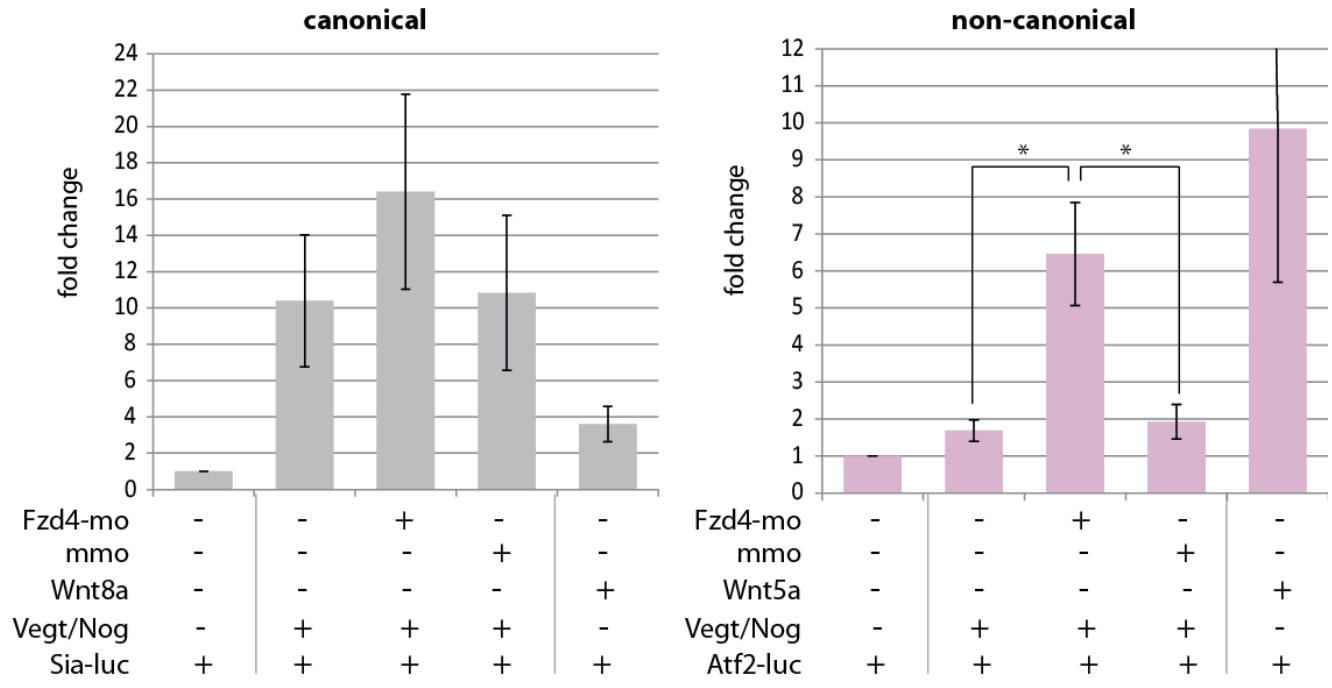

Fig. 3.10 Wnt-signaling in programed explants

(A) RNAs coding for Vegt (500pg), Noggin (500pg) were co-injected with RNAs encoding Cyp26a1 (2000pg), Wnt8a (400 pg) or Wnt5a (500pg). DNAs coding for the canonical reporter Siamois-luciferase $(50 \mathrm{pg})$, non-canonical reporter Atf2-luciferase (100 pg) and Renilla-luciferase $(10 \mathrm{pg})$ were also co-injected. Blastocoel-roof tissue was explanted and treated with RA or kept untreated. Samples for reporter assay were taken as duplicates two hours after RA-addition or at the equivalent of stage 12. (B) Relative luciferase activities of canonical and non-canonical Wnt-reporters in programed explants in the absence or presence of RA-signaling. (C) Fold changes of luciferase activities from canonical and noncanonical Wnt-reporters in Vegt/Noggin-programed explants with downregulated Fzd4/Fzd4s. * P-values in a Student's $t$-test $<0.05$. 


\section{Discussion}

\subsection{Pancreatic organoid formation and the requirement of retinoic acid}

We could demonstrate that pancreatic organoids can be generated in vitro from ectodermal explants of blastula stage Xenopus embryos. In a previous study, it was already shown that ectodermal explants can be programed by Vegt, $\beta$-catenin, Noggin and RA-treatment to form pancreatic tissue (Chen et al., 2004). In comparison to that study, we found that $\beta$-catenin is not essential for the induction of pancreatic marker gene expression. Moreover, we provide clear evidences that our in vitro generated pancreatic organoids recapitulate the in vivo pancreas organogenesis as we detect the same temporal expression profile of pancreatic marker genes as described in vivo.

The co-expression of the two transcription factors Ptf1a and Pdx1 is characteristic for pancreatic progenitor cells (Chiang and Melton, 2003; Afelik et al., 2006). Both transcription factors could be detected starting the expression at stage 24 in our pancreatic organoids, indicating the presence of a pancreatic progenitor population. However, it remains to be evidenced that Ptf1a and Pdx1 are co-expressed in these organoids, what would reflect pancreatic progenitors. Single cell analysis through RNA-sequencing or Nanostring technique would be applicable to show a coexpression of Pdx1 and Ptf1a. Nevertheless, we could detect further genes described to be expressed in pancreatic progenitors. Gata4, which is not exclusively required for pre-pancreatic endoderm formation, but also for the general endoderm formation (Holtzinger and Evans, 2005) was detected in pancreatic organoids and embryos at all tested stages. We further found Gata4 transcript levels increased in pancreatic organoids at the same stage when Ptf1a and Pdx1 were detected. Gata4 was previously shown to be induced by the key endoderm regulator Vegt (Xanthos et al., 2001) what explains the early expression at stage 17. In mouse, Gata4 is further known to be expressed in the early pancreatic epithelium (Decker et al., 2006; Watt et al., 2007) and to be required for early pancreas development (Carrasco et al., 2012; Xuan et al., 2012; Rodriguez-Segui et al. 2012). The endocrine progenitor marker Ngn3 was only slightly and transiently expressed in pancreatic organoids at stages 24 to 32 . This observation is consistent with the described findings in mouse, where Ngn3 was shown to act upstream of endocrine differentiation factors and its expression is turned off prior to final differentiation (Gradwohl et al., 2000; Jensen et al., 2000; Schwitzgebel et al., 2000; Maestro et 
al., 2003). Moreover, in Xenopus, ngn3 transcripts could not been detected in the pancreas at later stages (Nieber et al., 2009).

The earliest endocrine differentiation marker Insulin is detected already at stage 24 together with Ptf1a and Pdx1 in pancreatic organoids which indicates the first wave of Insulin expression during the primary transition phase of pancreas development (Kelly and Melton, 2000; Horb and Slack, 2002; Afelik et al., 2004). Insulin expression was further increased at stage 39 , indicating the formation of $\beta$-cells. However the detection of Insulin expression does not necessarily mean that functional mature ß-cells are formed. In several studies, iPSCs- or hESCs-derived in vitro generated $\beta$-cells have been frequently observed to express additional endocrine hormones together with Insulin. These poly-hormonal Insulin-positive cells were shown to be functional immature as they lack the capacity for Glucosestimulated Insulin release (D'Amour et al., 2006; Basford et al., 2012; Schiesser et al., 2014). So far, we have not tested whether the Insulin-positive cells, found in pancreatic organoids, exclusively express Insulin and no additional hormones. A single cell analysis should bring clarification. Moreover, it remains to be tested if the presence of insulin transcripts reflects the availability of secreted functional Insulin peptide.

Further endocrine and exocrine differentiation markers were detected at later stages. The exocrine differentiation marker Pdia2 is detected at stage 39 and Amylase is expressed at stage 43 in pancreatic organoids comparable with the expression observed in vivo (Horb and Slack 2002; Afelik et al., 2004). The endocrine differentiation marker Glucagon is earlier detected in the organoids than in the embryos (Horb and Slack, 2002). This might be due to the generally observed higher levels of pancreatic marker gene expression in pancreatic organoids compared to embryos. An further evidence that the generated pancreatic organoids recapitulate the in vivo pancreas organogenesis is the detection of Tm4sf3 at stage 43. Tm4sf3 is a transmembrane protein which is described as ventral specific pancreatic marker in Xenopus with a function in the fusion of the two ventral buds with the dorsal bud at stage 40 (Jarikji et al., 2009).

WMISH against the pancreatic marker genes Ptf1a and Pdx1 further revealed that the generated pancreatic organoids exhibit broad expression domains of these markers. Moreover, Insulin-positive cells were detected throughout the organoid indicating that a large portion of the explant turned into pancreatic tissue. Therefore, we used this in vitro system of pancreatic organoids for the identification of RAtarget genes involved in pancreas specification. 
The identification of such RA-dependent regulators of pancreas development could bring us one step closer to the major aim of pancreas research which is the in vitro generation of functional $\beta$-cells for a replacement therapy in order to treat diabetes type 1. Several studies used hESCs (Kroon et al., 2008; Rezania et al., 2012; Schulz et al., 2012; Pagliuca et al., 2014) or iPSCs (Zhang et al., 2009; Schiesser et al., 2014; Shaer et al., 2015) for a successful in vitro generation of $\beta$-cells. All studies applied stepwise differentiation protocols including the application of Activin A, a TGF $\beta$-family member that mimics nodal signaling required for endoderm formation (reviewed in Tam and Loebel, 2007), Noggin and RA. Our identified RAinduced pancreatic regulators could help to optimize these protocols and to increase the amount of generated $\beta$-cells. Moreover, our findings could help to find strategies for the trans-differentiation of patient-derived somatic cells into endocrine pancreatic cells. The trans-differentiation of somatic cells into pancreatic cells would be a promising alternative for ESC-derived pancreatic cells as the potential tumorigenicity of such cells is still a major concern and long-term studies are missing.

\subsection{Identification of 22 RA-responsive genes}

Unlike other signaling molecules, RA enters the cell independent of cell-surface receptors and binds directly to nuclear receptors (Rochette-Egly and Germain, 2009; Huang et al., 2014b). However, no studies are available dealing with the question in which time scale nuclear receptors are activated to induce target gene expression. We could show that the known direct RA-target Cyp26a1 (Loudig et al., 2000) is induced within one hour after RA-addition. Thus, for the identification of early RAtargets, the transcriptome of programed explants was analyzed one and two hours after RA-addition. 102 genes were found to be differentially expressed in the presence of RA-signaling. Almost half of these genes were affected by RA in the presence of $\mathrm{CHX}$, indicating them as putative direct RA-targets. Twelve genes were found to be differentially expressed upon CHX-treatments but not affected by RAsignaling. This might be due to the translational inhibition of transcriptional repressors. Under these conditions, genes are possibly transcribed that are usually repressed. The list of 102 differentially expressed genes arise from the alignment of 50 nucleotide short sequence reads to the genome of two Xenopus species, tropicalis and laevis. The inclusion of the tropicalis genome was necessary as the laevis genome is not fully sequenced. Thus, six mismatches were allowed during the process of alignment to target also the tropicalis genome. Beside the frequently occurring errors using small sequence reads, this further increased the risk of false 
positive candidates (González and Joly, 2013). Thus, several approaches were used to validate the RA-responsiveness of the candidates.

By this, the list of RA-responsive genes was diminished to 22 candidates. This list contains nine homeobox transcription factors (Hnf1b, Lhx1, Gbx2.1, Meis3, Nkx6.2, Hoxd1, Hoxd4, Hoxa1, Hoxb1), three other transcription factors (Znf703, Foxh1, Cebpd), two enzymes (Dhrs3 and Cyp26a1), two signaling components (Fzd4, Igf3), an intermediate filament protein (Prph), an autocrine glycoprotein (Fst) and four candidates with unknown identity. A review by Balmer and Blomhoff in 2002 evaluated published data from in total 1,191 papers covering 532 described RAtarget genes and classified them into categories according to the probability of RAregulation (Balmer and Blomhoff, 2002). However, most of these data were obtained in cell cultures without any developmental aspect. Nevertheless, 13 of the listed 22 RA-responsive genes in our study were already described as RA-regulated genes in the review of Balmer and Blomhoff or in following studies.

The homeobox-containing transcription factor Hnf1b was initially described as RAinducible in a mouse stem cell line (De Simone et al., 1991). Furthermore, Hnf1b was found to be RA-induced in the mouse and zebrafish hindbrain (Hernandez et al., 2004; Pouilhe et al., 2007). It is described in several studies using zebrafish, mouse and Xenopus embryos that an RA-gradient during neural development contributes to the anterior-posterior expression pattern of homeobox-containing genes in the hindbrain (reviewed in Glover et al., 2006). Thus, the RA-inducibility of most of the Hox-genes in our list was previously described including Hoxd4 (Nolte et al., 2003), Hoxa1 (Boylan et al., 1993; Frasch et al., 1995), Hoxb1 (Huang et al., 2002; Ishioka et al., 2012), Hoxd1 (Kolm and Sive, 1994), Gbx2.1 (Bouillet et al., 1995) and Lhx1 (Strate et al., 2009). Furthermore, Fzd4 and Fst were previously shown to be up-regulated by RA in mouse embryonal tumor cells (Hashimoto et al., 1992; Katoh, 2002). For the RA-metabolic enzymes Cyp26a1 (Abu-Abed et al., 1998) and Dhrs3 (Kam et al., 2013), the zinc-finger transcription factor Znf-703 (Mitchell et al., 2015) and the intermediate filament Prph (De Genaro et al., 2013) the RA-inducibility was also shown previously. Beside these known RA-regulated genes detected in our screen, 9 additional candidates are identified as novel RAtarget genes.

Based on the $\mathrm{CHX}$-treatment data, we can distinguish between putative direct and indirect targets. However, the presence of retinoic acid response elements (RARE), as additional indicators for direct RA-regulation, remains to be proven. For Xenopus laevis genes this is a challenging venture as intronic and enhancer sequences are 
not fully sequenced. RAREs are characterized by two direct repeats of the hexameric motif $R G K T S A(R=A / G, K=G / T, S=C / G$ ) separated by either one, two or five nucleotide spacers. RAREs were found to be located between $10 \mathrm{~kb}$ upstream and downstream from the gene locus and were bound by RXR/RAR heterodimers that display distinct motif affinities. Both classes of RA-receptors exist as $\alpha-, \beta-, \gamma^{-}$ isoforms and further numerous isoforms by differential promotor usage or alternative splicing exist, varying in RARE recognition (reviewed in Germain et al., 2006; Lalevee et al., 2011). Several studies in mouse and zebrafish detect RAREs within the gene loci of RA-responsive homeodomain-containing transcription factors. These include Hnf1b (Pouilhe et al., 2007), Hoxd4 (Nolte et al., 2003), Hoxa1 (Langston et al., 1997) and Hoxb1 (Huang et al., 2002).

The expression characteristics of the identified 22 RA-responsive genes during gastrulation gave us further indications about their possible function in pancreas development. Based on their RA-responsive endodermal expression domains, Hnf1b and Fzd4 were selected for functional analysis. Through loss and gain of function approaches, the requirement of Hnf1b for pancreas development could be confirmed and for Fzd4 a function in pancreatic fate determination is strongly suggested. These findings will be discussed in detail in the next sections. However, it remains to be urgently examined if the combined activity of Hnflb and Fzd4 is sufficient to substitute for RA in pancreas specification.

If this is not the case, the list of RA-responsive genes from our screen contains some further interesting candidates. The results of a previous study, using different combinations of dorsal and ventral endodermal and mesodermal explants, suggests that RA-signaling acts simultaneously in both, dorsal endoderm and mesoderm, to promote pancreatic fate (Pan et al., 2007). In our list, four genes were found to be expressed in the dorsal internal involuting mesoderm which corresponds to the Raldh2 expression domain. Cebpd, a transcription factor of the C/EBP family, is linked to $\beta$-cell survival as it has an anti-apoptotic function in rat and human insulinproducing cells (Moore et al., 2012). The enzyme Dhrs3 exhibits a retinal reductase activity that converts retinal to retinol counteracting against RA-generation (Haeseleer et al., 1998). Fst (Follistatin), an autocrine glycoprotein, is an Activin inhibitor (Kogawa et al., 1991). In the context of pancreas development the inhibition of Activin was shown to expand pancreatic epithelium, but decrease the number of differentiated $\beta$-cells. Thus, it is suggested that Fst is required to regulate Activin activity to acquire the homeostasis of growth and differentiation in this context (Zhang et al., 2004). A further candidate, expressed in the dorsal involuting mesoderm, is the transcription factor Lhx1 for which no connection to pancreas 
development is described. Not expressed in the internal involuting mesoderm but linked to pancreas development is Nkx6.2. In mouse, this transcription factor was found to be expressed in pancreatic progenitors regulating pancreatic subtype specification (Henseleit et al., 2005). Therefore, functional studies for the listed candidates appear to be important.

\subsection{The direct RA-target Hnf1b is required for pancreas development}

We identified the transcription factor Hnflb as direct RA-target gene in the dorsal endoderm during gastrulation and could demonstrate that its function is required for pancreas development in Xenopus. In mouse, Hnf1b was found to be expressed in the endodermal germ layer and in endodermal derived structures including pancreas, liver, gallbladder and duodenum (Barbacci et al., 1999; Haumaitre et al., 2003). We could detect Hnf1b transcripts in the entire endoderm during gastrulation with a minor enrichment in the dorsal endoderm. At later stages, Hnf1b expression was observed in the foregut endoderm including the anterior archenteron roof that gives rise to the dorsal pancreatic anlagen. These observations are consistent with findings in mouse where Hnf1b expression was also detected in early proliferating pancreatic progenitor epithelium together with Pdx1 and Ptf1a (Haumaitre et al., 2005). Furthermore, Hnf1b was identified as pancreatic trunk marker in mouse and lineage tracing experiments showed that Hnf1b-positive cells of the pancreatic epithelium are precursors of acinar, duct and endocrine cells (Solar et al., 2009).

We could further show that the dorsal endodermal expression of Hnf1b during gastrulation is RA-responsive. So far, the induction of Hnf1b by RA was solely described for hindbrain tissue (reviewed in Glover et al., 2006) and the only evidence for endodermal RA-inducibility was found in gastric organoids derived from mouse embryonal stem cells (McCracken et al., 2014). We further identified Hnf1b as direct RA-target what is supported by the identification of two RAREs in the promotor sequence and within the fourth intron of the mouse Hnf1b gene locus (Power and Cereghini, 1995; Pouilhe et al., 2007). The RA-responsive expression in the dorsal endoderm strongly indicates a role for Hnf1b in the pre-arrangement of conditions promoting pancreatic fate. Indeed, upon the downregulation of functional Hnf1b in pancreatic organoids almost a complete loss of pancreatic markers was observed. Furthermore, the endodermal marker Darmin was also decreased whereas another endodermal marker Sox17a was only slightly affected. Darmin is described in Xenopus as an endodermal marker with unknown function (Pera et al., 
2003) and no indications for a regulation of Darmin by Hnf1b are found in the literature. Interestingly, Hnflb and Darmin exhibit a similar expression in the entire endoderm in Xenopus blastula embryos and we find both genes to be RA-inducible expressed in programed explants. As we found Darmin downstream of Hnf1b, Darmin could be identified as novel Hnf1b target genes with a possible role in pancreas development. The rescue of most pancreatic markers and Darmin by a hormone-inducible Hnf1b indicates that the observed knockdown-phenotype is specific due to the downregulation of functional Hnf1b. The data observed for the rescue of Ptf1a expression were inconsistent. Some rescue experiments showed a Ptf1a expression (not shown) and others not. The failed rescue of Ptf1a does not reflect a failed rescue of exocrine differentiation as Pdia2 expression could be rescued. We have no convincing explanations for this observation, but we can provide some speculations. Hnf1b and Ptf1a are found to be co-expressed in pancreatic progenitors and later during the segregation of tip and trunk domains Ptf1a is expressed in acinar cells whereas Hnf1b expression is observed in bipotential trunk cells where it promotes endocrine differentiation (reviewed in Pan and Wright, 2011; De Vas et al., 2015). The Hnf1b-GR fusion protein, used for rescue approaches, is probably more stable than endogenous protein and continuous DEX-treatment provides high levels of active Hnf1b. It is possible that high Hnf1b levels promote endocrine fate at the expense of exocrine fate. This would be supported by the low transcript levels of Pdia2 in the rescue. However, we cannot provide evidences for this.

Nevertheless, our data indicate a function of Hnf1b in pancreas development and support and confirm previous studies. Mutations within the Hnf1b gene locus in humans cause a monogenetic form of diabetes, named MODY 5 (maturity onset diabetes of the young) and is further linked to kidney and genital malformations (reviewed in Ryffel, 2001; Wang et al., 2004; Haumaitre et al., 2006). Chimeric mutant mice with a specific loss of Hnf1b in the visceral endoderm exhibit a lack of the ventral pancreas and a hypoblastic dorsal pancreas (Haumaitre et al., 2005). Therefore, Hnflb is considered as putative upstream regulator of pancreatic progenitor markers Pdx1 and Ptf1a. Our findings in pancreatic organoids could be confirmed in whole embryos where the downregulation of Hnf1b leads to $a$ decreased expression of Pdx1, Ptf1a and Insulin. Moreover, upon Hnf1b overexpression, endodermal Ptf1a and $\mathrm{Pdx} 1$ expression domains were significantly expanded. This indicates an early function of $\mathrm{Hnf} 1 \mathrm{~b}$ in promoting pancreas progenitor formation. Further indications for an early function of Hnf1b in pancreas development were previously found by protein-DNA interaction analysis in mouse, 
where Hnf1b was found to directly induce Hnf6 expression (Poll et al., 2006). Hnf6 precedes the expression of $\mathrm{Pdx} 1$ in the foregut-midgut region and is later restricted to the liver and the pancreas (Landry et al., 1997; Rausa et al., 1997). Hnf6 was shown to initiate $\mathrm{Pdx} 1$ expression in mouse and the inactivation of Hnf6 results in a delayed onset of $\mathrm{Pdx} 1$ expression leading to pancreatic hypoplasia (Jacquemin et al., 2003). Therefore, it is suggested that a sequential transcriptional cascade of Hnf1b, Hnf6 and Pdx1 directs endodermal cells into pancreatic progenitors (Poll et al., 2006). However, the fact that the Pdx1 expression in Hnf6 mutants is delayed and not completely missing indicates the requirement of additional factors, possibly further Hnf1b targets.

Besides the indicated early function of Hnflb in progenitor formation, De Vas and colleagues demonstrated in a mouse system the requirement of Hnf1b for endocrine cell specification (De Vas et al., 2015). They inactivated Hnf1b specifically in pancreatic progenitors and observed the absence of Ngn3-positive endocrine precursor cells throughout embryogenesis. Another direct Hnf1b-target Hnf4a is also linked to MODY as $\beta$-cells in patients with Hnf4a mutations exhibit an impaired insulin secretory response to glucose (Yamagata et al., 1996; Hattersley, 1998; Lausen et al., 2000). Pancreatic organoids would be a suitable system for the identification of further direct Hnf1b targets that are possibly involved in pancreas development.

In summary, our data confirm several studies that found Hnf1b as direct RA-target and its requirement for pancreas development. We could provide further evidences for an early endodermal induction of Hnf1b by RA and its early function in pancreatic progenitor formation. However, the gene network induced by Hnf1b that is involved in pancreas development needs to be further investigated. Moreover, we show that Hnf1b is not sufficient to substitute for RA in pancreas development. Hence, Hnf1b is not the only RA-responsive gene that is required for pancreas development. 


\subsection{The direct RA-target Fzd4 is required for pancreas development}

We identified the transmembrane Wnt-receptor Fzd4 and its secreted splice variant Fzd4s as direct RA-target genes required for pancreas development. Fzd4 was previously found to be upregulated together with other Wnt-receptors and Wntligands by RA in human embryonal tumor cells (Katoh, 2002). Furthermore, evidences exist for an interplay of RA-signaling and Wnt-signaling in chondrocytes to regulate cartilage matrix homeostasis (Yasuhara et al., 2010). However, a connection of Fzd4 and RA-signaling in pancreas development is not described so far.

Two studies of Fzd4 in Xenopus describe an expression in the prospective neuroectoderm and later in the head region including the forebrain (Shi and Boucaut, 2000; Zhang et al., 2011). We found an additional Fzd4 RA-responsive expression domain in the dorsal endoderm of gastrula stage embryos. We initially observed this endodermal Fzd4 expression domain in RA-treated embryos only. A prolonged staining of un-treated embryos confirmed a weak endogenous endodermal expression of Fzd4 that have been missed by a too short staining. At later stages, Fzd4 expression was also detected in the foregut. For human and Xenopus Fzd4, an alternative splice variant through intron retention was identified (Sagara et al., 2001; Swain et al., 2005). This Fzd4 variant, named Fzd4s, encodes a small protein missing the transmembrane domain but still containing the Wnt-ligand binding cysteine rich domain (Swain et al., 2005). Therefore, the question appeared which variant is expressed in the dorsal endoderm. The endodermal expression domain was detected by the use of an antisense probe targeting the exonic sequence. This should theoretically detect both variants. Therefore, we additionally used an antisense probe targeting a part of the intronic region that should specifically detect Fzd4s. Steinbeisser and colleagues described an ubiquitous staining of Fzd4s transcripts during gastrulation and at tailbud stages in head region, not identical with Fzd4 but with overlaps in the eye (Swain et al., 2005). In contrast, beside a certain level of staining in the whole embryo, we could detect enriched regions in the mesoderm during gastrulation and later in the notochord and head region. The expression in the notochord was not observed with the probe that targets the exonic regions. Either the probe that targets the exons detects only Fzd4 and not Fzd4s or the probe targeting the intronic region results in an unspecific signal. Thus, we cannot conclude with certainty which variant exhibits the dorsal endodermal expression domain. 
RNA-sequencing and RT-PCR data indicate that both variants of Fzd4 were induced by RA. However, compared to the other direct RA-target genes Hnf1b and Cyp26a1, the Fzd4 variants exhibit only a minor induction by RA. A certain level of Fzd4 and Fzd4s transcripts is detected also in the absence of RA. This probably corresponds to maternally provided transcripts in the case of Fzd4 (Shi and Boucaut, 2000) and for Fzd4s an additional RA-independent expression is conceivable.

The described loss-of-function studies through morpholino-oligonucleotide and CRISPR/Cas-system should affect both Fzd4 variants. Therefore, Fzd4 and Fzd4s were designated as Fzd4/Fzd4s in this context. We demonstrated that both, the knockdown of Fzd4/Fzd4s by morpholino-oligonucleotide and the knockout by RNAguided Cas9 in pancreatic organoids showed a similar decrease of pancreatic marker gene expression. For the knockdown approach, several strategies failed to rescue the observed phenotype in order to demonstrate the specifity of the used morpholino-oligonucleotide. As mentioned in the results section, this might have several reasons. On the one hand, we were not able to provide active Fzd4 or Fzd4s specifically during gastrulation. On the other hand, we are not aware of the variant that is required and the appropriate level of active protein necessary for a proper pancreas development.

Both variants were not described to be involved in pancreas development so far. The transmembrane Fzd4 is described as mediator of both, canonical and noncanonical Wnt-signaling downstream of Wnt5a (Umbhauer et al., 2000; Chen et al., 2003; Mikels and Nusse, 2006; Xu et al., 2004). The activation of noncanonical/PCP pathway by Wnt5a/Fzd4-interaction was shown to be required for arterial network formation in mouse (Descamps et al., 2012). Furthermore, Fzd4 is the only known Wnt-receptor that binds Norrin and thereby activates canonical Wntsignaling promoting retinal vascularization in mouse (Xu et al., 2004; reviewed in Ye et al., 2010). Fzd4s shows structural similarities to secreted frizzled-related proteins (sfrps) and is therefore considered to be secreted (Rattner et al., 1997). Fzd4s still contains the Wnt-ligand binding domain and was described in Xenopus as an activator or inhibitor of canonical Wnt-signaling dependent on its ligand. Steinbeisser and colleagues showed that dependent on the corresponding Wnt-ligand, the Wnt/Fzd4s complex is recognized by the LRP5/6-co-receptor and mediates Wntsignaling or it is not recognized (Swain et al., 2005). Following studies revealed that the activatory or inhibitory function of Fzd4s is further dependent on its concentration. Low Fzd4s concentrations enhance and high concentrations repress Wnt-signaling in the presence of low Wnt-ligand levels (Gorny et al., 2013). This supports the assumption of the importance of appropriate Fzd4/Fzd4s levels 
required to rescue the morpholino-mediated knockdown phenotype. Findings by the Fzd4/Fzd4s-knockout confirm the results of Fzd4/Fzd4s-knockdown in pancreatic organoids. We could demonstrate that the observed decrease of pancreatic marker gene expression is due to specific mutations within the Fzd4 gene locus as tested potential off-targets showed no mutations.

The next important question that needs to be answered is whether the function of Fzd4/Fzd4s in Wnt-signaling is required for pancreas development. For Fzd4 also a signaling function outside of the classical Wnt-signaling pathway was described. During Wnt-signaling, Fzd4 usually binds intracellular Disheveled via a PDZ-binding motif that mediates Wnt-signaling (Wong et al., 2003; reviewed in Niehrs, 2012). In a mouse neuronal cell culture, the intracellular PDZ-binding motif of Fzd4 was shown to interact with other PDZ-domain containing proteins than Dvl and thereby promoting dendrite outgrowth (Bian et al., 2015). Therefore, a function of Fzd4 in pancreas development independent from the Wnt-signaling pathway is possible. However, results from luciferase reporter assays showed an inhibitory effect of RAtreatment on canonical as well as non-canonical reporter activity in Vegt/Nogginprogramed explants. The activatory effect of the Fzd4-knockdown on non-canonical reporter activity in these explants suggests the involvement of Wnt-signaling in early pancreas development. This issue will be discussed in the next section. In summary, we identified Fzd4 and its splice variant Fzd4s as direct RA-target genes. Our results strongly suggest the requirement of $\mathrm{Fzd} 4$ and/or Fzd4s in pancreas development.

\subsection{Wnt- and RA-signaling in pancreas development}

We identified the Wnt-receptor Fzd4 and its secreted variant Fzd4s as direct RAtargets with a possible role in pancreas development. Therefore, we asked about the role of Wnt-signaling in pancreas development. Previous studies proposed that Wnt-signaling needs to be repressed for foregut maintenance and therefore to allow a proper pancreas development. In Xenopus, McLin and colleagues found several foregut markers repressed upon Wnt8 over-expression (McLin etal., 2007). Furthermore, Li and colleagues identified the secreted Wnt-inhibitor sfrp5 to be expressed in the early foregut epithelium of Xenopus embryos. A downregulation of functional sfrp5 leads to smaller foregut domains and in contrast the ectopic sfrp5 expression results in an expanded foregut domain at the expense of the hindgut ( $\mathrm{Li}$ et al., 2008). However, a more recent study found evidences for the requirement of 
low Wnt-signaling activity for foregut maintenance. It was shown that the depletion of Wnt-receptor Fzd7 in the foregut results in pancreas agenesis in Xenopus and that a low expression level of this Wnt-receptor is essential for foregut maintenance (Zhang et al., 2013a). Moreover, sfrps that were initially thought to be exclusively negative Wnt-signaling modulators emerged as biphasic regulators in a concentration dependent manner (Mii and Taira, 2009). These findings suggest a regulatory mechanism that ensures an appropriate Wnt-signaling activity in the foregut.

Our finding of Wnt-receptors Fzd4 and Fzd4s involved in pancreas development is further supported by transcriptome analysis of hepatic and pancreatic progenitors in mouse. The transcriptome of bi-potential hepato-pancreatic progenitors was compared to the transcriptome of developed dorsal and ventral pancreatic buds and the liver bud (Rodríguez-Seguel et al., 2013). They found intracellular Wnt-signaling transducers like Disheveled to be expressed equally in all samples whereas Wntligands, receptors and co-receptors were strongly downregulated in liver progenitors. Among these differentially expressed Wnt-components, Fzd4 and its ligand Wnt5a were found. It was demonstrated that endodermal explants from Xenopus embryos treated with soluble Wnt5a exhibit an enhanced expression of $\mathrm{Pdx} 1$ and Ptf1a. Furthermore, liver cells treated with Wnt5a strongly induce Pdx1 expression (Rodríguez-Seguel et al., 2013). They suggest that non-canonical Wntsignaling is a potential promotor of pancreatic fate. We found only Fzd4/Fzd4s expression regulated by RA and not Wnt5a expression. However, we observed an effect of RA-treatment on Wnt-signaling in our explant system. Two hours after RAaddition the activity of both, canonical and non-canonical Wnt-signaling reporter was decreased. Thereby, the non-canonical Wnt-reporter was slightly stronger affected. The described effect of RA-treatment on canonical Wnt-signaling is consistent with several other studies. Zhang and colleagues identified Ndrg1a as RA-target and demonstrated that the inhibitory function of Ndrg1 on canonical Wnt-signaling is required for foregut development (Zhang et al., 2013b). However, Ndrg1 was not differentially expressed upon RA-treatment in our system. One explanation for this could be the late time point of Ndrg1 induction by RA that was observed in stage 16 embryos earliest, but we searched for RA-targets that were induced within two hours after RA-addition. Another study using mouse ESCs also found the negative regulatory effect of RA on canonical Wnt-signaling (Osei-sarfo and Gudas, 2014). In addition, they found non-canonical Wnt-signaling activated by RA. This activatory effect of RA on non-canonical Wnt-signaling was also described by Harada and colleagues (Harada et al., 2007). There, RA-inducible G-protein-coupled receptors 
were found to bind Wnt-receptors and thereby activating non-canonical Wntsignaling. These findings seem to be contradictory to our observed negative regulation of non-canonical Wnt-reporter activity by RA-treatment. However, the term "non-canonical Wnt-signaling" comprises two different pathways. The planar cell polarity (PCP) pathway that involves Rho GTPase and JNK and on the other hand the calcium pathway that involves calcium-sensitive kinases and PKC (reviewed in Nusse, 2012). Our non-canonical Wnt-reporter system is based on an Atf2-response element that is activated by the PCP-pathway (Ohkawara and Niehrs, 2010). In contrast, both studies used a reporter that contains a binding site for the transcription factor NFAT. This transcription factor is activated by the $\mathrm{Wnt} /$ calcium pathway (Dejmek et al., 2006). Thus, the effect of RA on the NFAT-reporter needs to be tested in our system. It is possible that RA-signaling has a biphasic activity on different non-canonical Wnt-signaling pathways.

We further examined the effect of Fzd4-knockdown on Wnt-signaling reporter activity in the explant system. We found canonical Wnt-reporter activity only slightly and not significantly increased upon Fzd4-downregulation. In contrast, noncanonical Atf2-reporter activity was significantly increased. This finding complies with the observed decrease in non-canonical Wnt-reporter activity upon RA addition. Hence, these data suggest that the negative regulatory effect of RA on noncanonical Wnt-signaling is mediated by Fzd4 and/or Fzd4s. However, Fzd4 as well as Fzd4s were shown to positively regulate non-canonical Wnt/PCP-signaling (Descamps et al., 2012; Gorny et al., 2013). Therefore, the effect of Fzd4downregulation on non-canonical Wnt-signaling needs to be further investigated. Moreover, it remains to be tested whether the downregulation of Wnt-signaling by $\mathrm{RA}$ is mediated by Fzd4 and if this Fzd4-function is required for pancreas specification.

A connection of Hnf1b and Wnt-signaling was shown in zebrafish. Lancman and colleagues demonstrated that Hnf1b and Wnt2b synergistically function in the specification of hepato-pancreatic progenitors (Lancman et al., 2013). Thus, it is necessary to examine if a combined activity of Hnf1b and Fzd4 and/or Fzd4s is sufficient to substitute for RA in pancreas specification. 


\subsection{Conclusions}

In this study, a system of in vitro generated pancreatic organoids was used for the identification of RA-target genes involved in the early pancreas development. We could identify 22 RA-responsive genes, some of which have previously been described as RA-targets. For the transcription factor Hnf1b and the Wnt-receptor Fzd4, we found RA-responsive expression domains within the dorsal endoderm, the origin of pancreatic precursor cells, during gastrulation. Functional analysis for both factors revealed their requirement for pancreas development. We provide the evidence for an early function of Hnf1b in pancreatic progenitor formation. Moreover, we found non-canonical Wnt-signaling activity decreased upon RA-treatment, which is probably mediated by Fzd4. Based on our data we suggest the following model. During gastrulation, RA is secreted from the dorsal mesoderm and directly induces the expression of Hnf1b and Fzd4 in the dorsal endoderm. The overlapping activity of these two RA-target genes establishes a pre-pancreatic domain in the dorsal endoderm. Fzd4 activity down-regulates non-canonical Wnt-signaling which allows the specification of the pancreatic epithelium in the foregut endoderm characterized by the co-expression of Ptf1a and Pdx1. Hnf1b induces a transcriptional cascade via its direct target Hnf6 that leads to the expression of Pdx1. Finally, pancreatic progenitors proliferate and differentiate to endocrine and exocrine tissue constituting the pancreas (Fig. 4.1).

In order to extend and further confirm the suggested model, a few aspects remain to be investigated. It would be interesting to uncover which of the two Fzd4 splice variants is required for pancreas specification. Moreover, a further evidence is needed that the regulatory function of Fzd4 in respect to Wnt-signaling is involved in pancreas development. For the function of Hnf1b in pancreas development it would be interesting to search for additional direct targets in this context. Most importantly, it remains to be proven whether the combined activity of Hnf1b and Fzd4 is sufficient for pancreas development or if additional RA-regulated factors are required. 
endoderm

formation and

pre-patterning

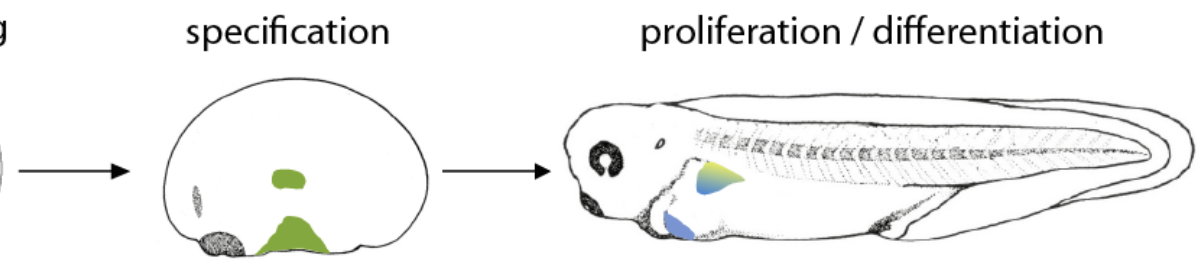

non-canonical

Wnt-signaling

T

$\longrightarrow$

Ptf1a

Insulin

Fzd4

$\mathrm{Hnf1b} \longrightarrow \mathrm{Hnf6}$

Pdia2

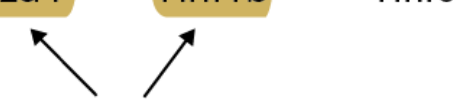

RA

endoderm pre-pancreatic endoderm pancreatic progenitors

endocrine tissue

exocrine tissue

Fig. 4.1 Model of pancreas specification involving direct RA-targets, Fzd4 and Hnf1b, in Xenopus embryos.

During gastrulation, the expression of $\mathrm{Fzd} 4$ and $\mathrm{Hnf} 1 \mathrm{~b}$ is directly induced by RA. The overlapping activity of $\mathrm{Fzd} 4$ and Hnf1b establishes a pre-pancreatic domain within the dorsal endoderm. Fzd4 down-regulates non-canonical Wnt-signaling activity, which allows the specification of pancreatic progenitors characterized by the co-expression of Ptf1a and Pdx1. In addition, Hnf1b directs endodermal cells into pancreatic progenitors through a sequential transcriptional cascade including $\mathrm{Hnf} 6$ and $\mathrm{Pd} \times 1$. Pancreatic progenitors subsequently proliferate and differentiate into endocrine and exocrine tissue. 


\section{References}

Abu-Abed, S.S., Beckett, B.R., Chiba, H., Chithalen, J.V., Jones, G., Metzger, D., Chambon, P. and Petkovich, M. (1998) Mouse P450RAI (CYP26) expression and retinoic acid-inducible retinoic acid metabolism in F9 cells are regulated by retinoic acid receptor gamma and retinoid $X$ receptor alpha. J Biol Chem, 273, 2409-2415.

Afelik, S., Chen, Y. and Pieler, T. (2004) Pancreatic protein disulfide isomerase (XPDIp) is an early marker for the exocrine lineage of the developing pancreas in Xenopus laevis embryos. Gene Expr Patterns, 4, 71-76.

Afelik, S., Chen, Y. and Pieler, T. (2006) Combined ectopic expression of Pdx1 and Ptf1a/p48 results in the stable conversion of posterior endoderm into endocrine and exocrine pancreatic tissue. Genes Dev, 20, 1441-1446.

Agius, E., Oelgeschlager, M., Wessely, O., Kemp, C. and De Robertis, E.M. (2000) Endodermal Nodal-related signals and mesoderm induction in Xenopus. Development, 127, 1173-1183.

Apelqvist, A., Li, H., Sommer, L., Beatus, P., Anderson, D.J., Honjo, T., Hrabe de Angelis, M., Lendahl, U. and Edlund, H. (1999) Notch signalling controls pancreatic cell differentiation. Nature, 400, 877-881.

Ariizumi, T. and Asashima, M. (1995) Control of the embryonic body plan by activin during amphibian development. Zoolog Sci, 12, 509-521.

Ariizumi, T., Kinoshita, M., Yokota, C., Takano, K., Fukuda, K., Moriyama, N., Malacinski, G.M. and Asashima, M. (2003) Amphibian in vitro heart induction: a simple and reliable model for the study of vertebrate cardiac development. Int J Dev Biol, 47, 405-410.

Ariizumi, T., Sawamura, K., Uchiyama, H. and Asashima, M. (1991) Dose and timedependent mesoderm induction and outgrowth formation by activin $A$ in Xenopus laevis. Int J Dev Biol, 35, 407-414.

Arnes, L. and Sussel, L. (2015) Epigenetic modifications and long noncoding RNAs influence pancreas development and function. Trends Genet, 31, 290-299.

Asashima, M., Ito, Y., Chan, T., Michiue, T., Nakanishi, M., Suzuki, K., Hitachi, K., Okabayashi, K., Kondow, A. and Ariizumi, T. (2009) In vitro organogenesis from undifferentiated cells in Xenopus. Dev Dyn, 238, 1309-1320.

Bae, S., Reid, C.D. and Kessler, D.S. (2011) Siamois and Twin are redundant and essential in formation of the Spemann organizer. Dev Biol, 352, 367-381.

Balmer, J.E. and Blomhoff, R. (2002) Gene expression regulation by retinoic acid. J Lipid Res, 43, 1773-1808.

Barbacci, E., Reber, M., Ott, M.O., Breillat, C., Huetz, F. and Cereghini, S. (1999) Variant hepatocyte nuclear factor 1 is required for visceral endoderm specification. Development, 126, 4795-4805.

Barker, N., Huch, M., Kujala, P., van de Wetering, M., Snippert, H.J., van Es, J.H., Sato, T., Stange, D.E., Begthel, H., van den Born, M., Danenberg, E., van den Brink, S., Korving, J., Abo, A., Peters, P.J., Wright, N., Poulsom, R. and Clevers, H. (2010) Lgr5(+ve) stem cells drive self-renewal in the stomach and build long-lived gastric units in vitro. Cell Stem Cell, 6, 25-36.

Basford, C.L., Prentice, K.J., Hardy, A.B., Sarangi, F., Micallef, S.J., Li, X., Guo, Q., Elefanty, A.G., Stanley, E.G., Keller, G., Allister, E.M., Nostro, M.C. and Wheeler, M.B. (2012) The functional and molecular characterisation of human embryonic stem cell-derived insulin-positive cells compared with adult pancreatic beta cells. Diabetologia, 55, 358-371.

Beres, T.M., Masui, T., Swift, G.H., Shi, L., Henke, R.M. and MacDonald, R.J. (2006) PTF1 is an organ-specific and Notch-independent basic helix-loop-helix complex containing the mammalian Suppressor of Hairless (RBP-J) or its paralogue, RBP-L. Mol Cell Biol, 26, 117-130.

Beucher, A., Gjernes, E., Collin, C., Courtney, M., Meunier, A., Collombat, P. and Gradwohl, G. (2012) The homeodomain-containing transcription factors Arx and Pax4 control enteroendocrine subtype specification in mice. PLoS One, 7, e36449.

Bian, W.J., Miao, W.Y., He, S.J., Wan, Z.F., Luo, Z.G. and Yu, X. (2015) A novel Wnt5aFrizzled4 signaling pathway mediates activity-independent dendrite morphogenesis via the distal PDZ motif of Frizzled 4. Dev Neurobiol, 75, 805-822.

Blitz, I.L., Biesinger, J., Xie, X. and Cho, K.W. (2013) Biallelic genome modification in F(0) 
Xenopus tropicalis embryos using the CRISPR/Cas system. Genesis, $\mathbf{5 1}$, 827-834.

Boj, S.F., Hwang, C.I., Baker, L.A., Chio, II, Engle, D.D., Corbo, V., Jager, M., PonzSarvise, M., Tiriac, H., Spector, M.S., Gracanin, A., Oni, T., Yu, K.H., van Boxtel, R., Huch, M., Rivera, K.D., Wilson, J.P., Feigin, M.E., Ohlund, D., HandlySantana, A., Ardito-Abraham, C.M., Ludwig, M., Elyada, E., Alagesan, B., Biffi, G., Yordanov, G.N., Delcuze, B., Creighton, B., Wright, K., Park, Y., Morsink, F.H., Molenaar, I.Q., Borel Rinkes, I.H., Cuppen, E., Hao, Y., Jin, Y., Nijman, I.J., lacobuzio-Donahue, C., Leach, S.D., Pappin, D.J., Hammell, M., Klimstra, D.S., Basturk, O., Hruban, R.H., Offerhaus, G.J., Vries, R.G., Clevers, H. and Tuveson, D.A. (2015) Organoid models of human and mouse ductal pancreatic cancer. Cell, 160, 324-338.

Borchers, A. and Pieler, T. (2010) Programing pluripotent precursor cells derived from Xenopus embryos to generate specific tissues and organs. Genes (Basel), 1, 413426.

Bort, R., Signore, M., Tremblay, K., Martinez Barbera, J.P. and Zaret, K.S. (2006) Hex homeobox gene controls the transition of the endoderm to a pseudostratified, cell emergent epithelium for liver bud development. Dev Biol, 290, 44-56.

Bouillet, P., Chazaud, C., Oulad-Abdelghani, M., Dolle, P. and Chambon, P. (1995) Sequence and expression pattern of the Stra7 (Gbx-2) homeobox-containing gene induced by retinoic acid in P19 embryonal carcinoma cells. Dev Dyn, 204, 372-382.

Bourguignon, C., Li, J. and Papalopulu, N. (1998) XBF-1, a winged helix transcription factor with dual activity, has a role in positioning neurogenesis in Xenopus competent ectoderm. Development, 125, 4889-4900.

Boylan, J.F., Lohnes, D., Taneja, R., Chambon, P. and Gudas, L.J. (1993) Loss of retinoic acid receptor gamma function in F9 cells by gene disruption results in aberrant Hoxa-1 expression and differentiation upon retinoic acid treatment. Proc Natl Acad Sci U S A, 90, 9601-9605.

Brannon, M., Gomperts, M., Sumoy, L., Moon, R.T. and Kimelman, D. (1997) A betacatenin/XTcf-3 complex binds to the siamois promoter to regulate dorsal axis specification in Xenopus. Genes Dev, 11, 2359-2370.

Brannon, M. and Kimelman, D. (1996) Activation of Siamois by the Wnt pathway. Dev Biol, 180, 344-347.

Brereton, M.F., Vergari, E., Zhang, Q. and Clark, A. (2015) Alpha-, Delta- and PP-cells: Are They the Architectural Cornerstones of Islet Structure and Co-ordination? J Histochem Cytochem, 63, 575-591.

Burlison, J.S., Long, Q., Fujitani, Y., Wright, C.V. and Magnuson, M.A. (2008) Pdx-1 and Ptf1a concurrently determine fate specification of pancreatic multipotent progenitor cells. Dev Biol, 316, 74-86.

Carrasco, M., Delgado, I., Soria, B., Martin, F. and Rojas, A. (2012) GATA4 and GATA6 control mouse pancreas organogenesis. J Clin Invest, 122, 3504-3515.

Chalmers, A.D. and Slack, J.M. (2000) The Xenopus tadpole gut: fate maps and morphogenetic movements. Development, 127, 381-392.

Chan, T.C., Ariizumi, T. and Asashima, M. (1999) A model system for organ engineering: transplantation of in vitro induced embryonic kidney. Naturwissenschaften, 86, 224227.

Chang, Y.F., Imam, J.S. and Wilkinson, M.F. (2007) The nonsense-mediated decay RNA surveillance pathway. Annual review of biochemistry, 76, 51-74.

Chen, J.Y., Penco, S., Ostrowski, J., Balaguer, P., Pons, M., Starrett, J.E., Reczek, P., Chambon, P. and Gronemeyer, H. (1995) RAR-specific agonist/antagonists which dissociate transactivation and AP1 transrepression inhibit anchorage-independent cell proliferation. Embo j, 14, 1187-1197.

Chen, W., ten Berge, D., Brown, J., Ahn, S., Hu, L.A., Miller, W.E., Caron, M.G., Barak, L.S., Nusse, R. and Lefkowitz, R.J. (2003) Dishevelled 2 recruits beta-arrestin 2 to mediate Wnt5A-stimulated endocytosis of Frizzled 4. Science, 301, 1391-1394.

Chen, Y., Pan, F.C., Brandes, N., Afelik, S., Solter, M. and Pieler, T. (2004) Retinoic acid signaling is essential for pancreas development and promotes endocrine at the expense of exocrine cell differentiation in Xenopus. Dev Biol, 271, 144-160.

Chen, Y., Pollet, N., Niehrs, C. and Pieler, T. (2001) Increased XRALDH2 activity has a posteriorizing effect on the central nervous system of Xenopus embryos. Mech Dev, 101, 91-103.

Chiang, M.K. and Melton, D.A. (2003) Single-cell transcript analysis of pancreas development. Dev Cell, 4, 383-393. 
Chitnis, A., Henrique, D., Lewis, J., Ish-Horowicz, D. and Kintner, C. (1995) Primary neurogenesis in Xenopus embryos regulated by a homologue of the Drosophila neurogenic gene Delta. Nature, 375, 761-766.

Chung, W.S., Shin, C.H. and Stainier, D.Y. (2008) Bmp2 signaling regulates the hepatic versus pancreatic fate decision. Dev Cell, 15, 738-748.

Clagett-Dame, M. and DeLuca, H.F. (2002) The role of vitamin A in mammalian reproduction and embryonic development. Annu Rev Nutr, 22, 347-381.

Clements, D., Friday, R.V. and Woodland, H.R. (1999) Mode of action of VegT in mesoderm and endoderm formation. Development, 126, 4903-4911.

Cockell, M., Stevenson, B.J., Strubin, M., Hagenbuchle, O. and Wellauer, P.K. (1989) Identification of a cell-specific DNA-binding activity that interacts with a transcriptional activator of genes expressed in the acinar pancreas. Mol Cell Biol, $\mathbf{9}$, 2464-2476.

Conlon, F.L., Lyons, K.M., Takaesu, N., Barth, K.S., Kispert, A., Herrmann, B. and Robertson, E.J. (1994) A primary requirement for nodal in the formation and maintenance of the primitive streak in the mouse. Development, 120, 1919-1928.

Damianitsch, K., Melchert, J. and Pieler, T. (2009) XsFRP5 modulates endodermal organogenesis in Xenopus laevis. Dev Biol, 329, 327-337.

D'Amour, K.A., Bang, A.G., Eliazer, S., Kelly, O.G., Agulnick, A.D., Smart, N.G., Moorman, M.A., Kroon, E., Carpenter, M.K. and Baetge, E.E. (2006) Production of pancreatic hormone-expressing endocrine cells from human embryonic stem cells. Nat Biotechnol, 24, 1392-1401.

De Genaro, P., Simon, M.V., Rotstein, N.P. and Politi, L.E. (2013) Retinoic acid promotes apoptosis and differentiation in photoreceptors by activating the P38 MAP kinase pathway. Invest Ophthalmol Vis Sci, 54, 3143-3156.

De Simone, V., De Magistris, L., Lazzaro, D., Gerstner, J., Monaci, P., Nicosia, A. and Cortese, R. (1991) LFB3, a heterodimer-forming homeoprotein of the LFB1 family, is expressed in specialized epithelia. Embo j, 10, 1435-1443.

De Vas, M.G., Kopp, J.L., Heliot, C., Sander, M., Cereghini, S. and Haumaitre, C. (2015) Hnf1b controls pancreas morphogenesis and the generation of Ngn3+ endocrine progenitors. Development, 142, 871-882.

Decker, K., Goldman, D.C., Grasch, C.L. and Sussel, L. (2006) Gata6 is an important regulator of mouse pancreas development. Dev Biol, 298, 415-429.

Dejmek, J., Safholm, A., Kamp Nielsen, C., Andersson, T. and Leandersson, K. (2006) Wnt-5a/Ca2+-induced NFAT activity is counteracted by Wnt-5a/Yes-Cdc42-casein kinase 1alpha signaling in human mammary epithelial cells. Molecular and cellular biology, 26, 6024-6036.

Dersch, H. and Zile, M.H. (1993) Induction of normal cardiovascular development in the vitamin A-deprived quail embryo by natural retinoids. Dev Biol, 160, 424-433.

Descamps, B., Sewduth, R., Ferreira Tojais, N., Jaspard, B., Reynaud, A., Sohet, F., Lacolley, P., Allieres, C., Lamaziere, J.M., Moreau, C., Dufourcq, P., Couffinhal, T. and Duplaa, C. (2012) Frizzled 4 regulates arterial network organization through noncanonical Wnt/planar cell polarity signaling. Circ Res, 110, 47-58.

Dessimoz, J., Bonnard, C., Huelsken, J. and Grapin-Botton, A. (2005) Pancreas-specific deletion of beta-catenin reveals Wnt-dependent and Wnt-independent functions during development. Curr Biol, 15, 1677-1683.

Dessimoz, J., Opoka, R., Kordich, J.J., Grapin-Botton, A. and Wells, J.M. (2006) FGF signaling is necessary for establishing gut tube domains along the anterior-posterior axis in vivo. Mech Dev, 123, 42-55.

Deutsch, G., Jung, J., Zheng, M., Lora, J. and Zaret, K.S. (2001) A bipotential precursor population for pancreas and liver within the embryonic endoderm. Development, 128, 871-881.

Dichmann, D.S. and Harland, R.M. (2011) Nkx6 genes pattern the frog neural plate and Nkx6.1 is necessary for motoneuron axon projection. Dev Biol, 349, 378-386.

Dickman, E.D., Thaller, C. and Smith, S.M. (1997) Temporally-regulated retinoic acid depletion produces specific neural crest, ocular and nervous system defects. Development, 124, 3111-3121.

Djiane, A., Riou, J., Umbhauer, M., Boucaut, J. and Shi, D. (2000) Role of frizzled 7 in the regulation of convergent extension movements during gastrulation in Xenopus laevis. Development, 127, 3091-3100.

Duester, G. (2008) Retinoic acid synthesis and signaling during early organogenesis. Cell, 134, 921-931. 
Edlund, H. (2002) Pancreatic organogenesis--developmental mechanisms and implications for therapy. Nat Rev Genet, 3, 524-532.

Ellis, P., Fagan, B.M., Magness, S.T., Hutton, S., Taranova, O., Hayashi, S., McMahon, A., Rao, M. and Pevny, L. (2004) SOX2, a persistent marker for multipotential neural stem cells derived from embryonic stem cells, the embryo or the adult. Dev Neurosci, 26, 148-165.

Engleka, M.J., Craig, E.J. and Kessler, D.S. (2001) VegT activation of Sox17 at the midblastula transition alters the response to nodal signals in the vegetal endoderm domain. Dev Biol, 237, 159-172.

Fineran, P.C. and Dy, R.L. (2014) Gene regulation by engineered CRISPR-Cas systems. Curr Opin Microbiol, 18, 83-89.

Finkbeiner, S.R., Freeman, J.J., Wieck, M.M., El-Nachef, W., Altheim, C.H., Tsai, Y.H., Huang, S., Dyal, R., White, E.S., Grikscheit, T.C., Teitelbaum, D.H. and Spence, J.R. (2015) Generation of tissue-engineered small intestine using embryonic stem cell-derived human intestinal organoids. Biol Open, 4, 1462-1472.

Frasch, M., Chen, X. and Lufkin, T. (1995) Evolutionary-conserved enhancers direct region-specific expression of the murine Hoxa-1 and Hoxa-2 loci in both mice and Drosophila. Development, 121, 957-974.

Fukuda, K. and Kikuchi, Y. (2005) Endoderm development in vertebrates: fate mapping, induction and regional specification. Dev Growth Differ, 47, 343-355.

Fukui, Y., Furue, M., Myoishi, Y., Sato, J.D., Okamoto, T. and Asashima, M. (2003) Long-term culture of Xenopus presumptive ectoderm in a nutrient-supplemented culture medium. Dev Growth Differ, 45, 499-506.

Gammill, L.S. and Sive, H. (1997) Identification of otx2 target genes and restrictions in ectodermal competence during Xenopus cement gland formation. Development, 124, 471-481.

Gao, D., Vela, I., Sboner, A., laquinta, P.J., Karthaus, W.R., Gopalan, A., Dowling, C., Wanjala, J.N., Undvall, E.A., Arora, V.K., Wongvipat, J., Kossai, M., Ramazanoglu, S., Barboza, L.P., Di, W., Cao, Z., Zhang, Q.F., Sirota, I., Ran, L., MacDonald, T.Y., Beltran, H., Mosquera, J.M., Touijer, K.A., Scardino, P.T., Laudone, V.P., Curtis, K.R., Rathkopf, D.E., Morris, M.J., Danila, D.C., Slovin, S.F., Solomon, S.B., Eastham, J.A., Chi, P., Carver, B., Rubin, M.A., Scher, H.I., Clevers, H., Sawyers, C.L. and Chen, Y. (2014) Organoid cultures derived from patients with advanced prostate cancer. Cell, 159, 176-187.

Germain, P., Chambon, P., Eichele, G., Evans, R.M., Lazar, M.A., Leid, M., De Lera, A.R., Lotan, R., Mangelsdorf, D.J. and Gronemeyer, H. (2006) International Union of Pharmacology. LX. Retinoic acid receptors. Pharmacol Rev, 58, 712-725.

Germain, P., lyer, J., Zechel, C. and Gronemeyer, H. (2002) Co-regulator recruitment and the mechanism of retinoic acid receptor synergy. Nature, 415, 187-192.

Gilchrist, M.J., Zorn, A.M., Voigt, J., Smith, J.C., Papalopulu, N. and Amaya, E. (2004) Defining a large set of full-length clones from a Xenopus tropicalis EST project. Dev Biol, 271, 498-516.

Gittes, G.K. (2009) Developmental biology of the pancreas: a comprehensive review. Dev Biol, 326, 4-35.

Glover, J.C., Renaud, J.S. and Rijli, F.M. (2006) Retinoic acid and hindbrain patterning. J Neurobiol, 66, 705-725.

Gonzalez, E. and Joly, S. (2013) Impact of RNA-seq attributes on false positive rates in differential expression analysis of de novo assembled transcriptomes. BMC Res Notes, 6, 503.

Gorny, A.K., Kaufmann, L.T., Swain, R.K. and Steinbeisser, H. (2013) A secreted splice variant of the Xenopus frizzled-4 receptor is a biphasic modulator of Wnt signalling. Cell Commun Signal, 11, 89.

Gouzi, M., Kim, Y.H., Katsumoto, K., Johansson, K. and Grapin-Botton, A. (2011) Neurogenin3 initiates stepwise delamination of differentiating endocrine cells during pancreas development. Dev Dyn, 240, 589-604.

Gradwohl, G., Dierich, A., LeMeur, M. and Guillemot, F. (2000) neurogenin3 is required for the development of the four endocrine cell lineages of the pancreas. Proc Natl Acad Sci U S A, 97, 1607-1611.

Grapin-Botton, A. and Constam, D. (2007) Evolution of the mechanisms and molecular control of endoderm formation. Mech Dev, 124, 253-278.

Gu, G., Dubauskaite, J. and Melton, D.A. (2002) Direct evidence for the pancreatic lineage: NGN3+ cells are islet progenitors and are distinct from duct progenitors. 
Development, 129, 2447-2457.

Guenette, S.A., Giroux, M.C. and Vachon, P. (2013) Pain perception and anaesthesia in research frogs. Exp Anim, 62, 87-92.

Guo, X., Zhang, T., Hu, Z., Zhang, Y., Shi, Z., Wang, Q., Cui, Y., Wang, F., Zhao, H. and Chen, Y. (2014) Efficient RNA/Cas9-mediated genome editing in Xenopus tropicalis. Development, 141, 707-714.

Haeseleer, F., Huang, J., Lebioda, L., Saari, J.C. and Palczewski, K. (1998) Molecular characterization of a novel short-chain dehydrogenase/reductase that reduces alltrans-retinal. J Biol Chem, 273, 21790-21799.

Harada, Y., Yokota, C., Habas, R., Slusarski, D.C. and He, X. (2007) Retinoic acidinducible $G$ protein-coupled receptors bind to frizzled receptors and may activate non-canonical Wnt signaling. Biochemical and biophysical research communications, 358, 968-975.

Harland, R.M. (1991) In situ hybridization: an improved whole-mount method for Xenopus embryos. Methods Cell Biol, 36, 685-695.

Hashimoto, M., Nakamura, T., Inoue, S., Kondo, T., Yamada, R., Eto, Y., Sugino, H. and Muramatsu, M. (1992) Follistatin is a developmentally regulated cytokine in neural differentiation. J Biol Chem, 267, 7203-7206.

Hattersley, A.T. (1998) Maturity-onset diabetes of the young: clinical heterogeneity explained by genetic heterogeneity. Diabet Med, 15, 15-24.

Haumaitre, C., Barbacci, E., Jenny, M., Ott, M.O., Gradwohl, G. and Cereghini, S. (2005) Lack of TCF2/VHNF1 in mice leads to pancreas agenesis. Proc Natl Acad Sci U S A, 102, 1490-1495.

Haumaitre, C., Fabre, M., Cormier, S., Baumann, C., Delezoide, A.L. and Cereghini, S. (2006) Severe pancreas hypoplasia and multicystic renal dysplasia in two human fetuses carrying novel HNF1beta/MODY5 mutations. Hum Mol Genet, 15, 23632375.

Haumaitre, C., Reber, M. and Cereghini, S. (2003) Functions of HNF1 family members in differentiation of the visceral endoderm cell lineage. J Biol Chem, 278, 40933-40942.

Heasman, J. (2006) Maternal determinants of embryonic cell fate. Semin Cell Dev Biol, 17, 93-98.

Hebrok, M., Kim, S.K. and Melton, D.A. (1998) Notochord repression of endodermal Sonic hedgehog permits pancreas development. Genes Dev, 12, 1705-1713.

Hebrok, M., Kim, S.K., St Jacques, B., McMahon, A.P. and Melton, D.A. (2000) Regulation of pancreas development by hedgehog signaling. Development, 127, 4905-4913.

Henry, G.L., Brivanlou, I.H., Kessler, D.S., Hemmati-Brivanlou, A. and Melton, D.A. (1996) TGF-beta signals and a pattern in Xenopus laevis endodermal development. Development, 122, 1007-1015.

Henseleit, K.D., Nelson, S.B., Kuhlbrodt, K., Hennings, J.C., Ericson, J. and Sander, M. (2005) NKX6 transcription factor activity is required for alpha- and beta-cell development in the pancreas. Development, 132, 3139-3149.

Hernandez, R.E., Rikhof, H.A., Bachmann, R. and Moens, C.B. (2004) vhnf1 integrates global RA patterning and local FGF signals to direct posterior hindbrain development in zebrafish. Development, 131, 4511-4520.

Hesselson, D., Anderson, R.M. and Stainier, D.Y. (2011) Suppression of Ptf1a activity induces acinar-to-endocrine conversion. Curr Biol, 21, 712-717.

Hollemann, T., Chen, Y., Grunz, H. and Pieler, T. (1998) Regionalized metabolic activity establishes boundaries of retinoic acid signalling. Embo j, 17, 7361-7372.

Hollemann, T. and Pieler, T. (1999) Xpitx-1: a homeobox gene expressed during pituitary and cement gland formation of Xenopus embryos. Mech Dev, 88, 249-252.

Holtzinger, A. and Evans, T. (2005) Gata4 regulates the formation of multiple organs. Development, 132, 4005-4014.

Horb, M.E., Shen, C.N., Tosh, D. and Slack, J.M. (2003) Experimental conversion of liver to pancreas. Curr Biol, 13, 105-115.

Horb, M.E. and Slack, J.M. (2001) Endoderm specification and differentiation in Xenopus embryos. Dev Biol, 236, 330-343.

Horb, M.E. and Slack, J.M. (2002) Expression of amylase and other pancreatic genes in Xenopus. Mech Dev, 113, 153-157.

Hsu, P.D., Lander, E.S. and Zhang, F. (2014) Development and applications of CRISPRCas9 for genome engineering. Cell, 157, 1262-1278.

Hsu, P.D., Scott, D.A., Weinstein, J.A., Ran, F.A., Konermann, S., Agarwala, V., Li, Y., 
Fine, E.J., Wu, X., Shalem, O., Cradick, T.J., Marraffini, L.A., Bao, G. and Zhang, F. (2013) DNA targeting specificity of RNA-guided Cas9 nucleases. Nat Biotechnol, 31, 827-832.

Huang, D., Chen, S.W. and Gudas, L.J. (2002) Analysis of two distinct retinoic acid response elements in the homeobox gene Hoxb1 in transgenic mice. Dev Dyn, 223, 353-370.

Huang, H.P., Liu, M., El-Hodiri, H.M., Chu, K., Jamrich, M. and Tsai, M.J. (2000) Regulation of the pancreatic islet-specific gene BETA2 (neuroD) by neurogenin 3. Mol Cell Biol, 20, 3292-3307.

Huang, P., Chandra, V. and Rastinejad, F. (2014b) Retinoic acid actions through mammalian nuclear receptors. Chem Rev, 114, 233-254.

Huang, W., Wang, G., Delaspre, F., Vitery Mdel, C., Beer, R.L. and Parsons, M.J. (2014a) Retinoic acid plays an evolutionarily conserved and biphasic role in pancreas development. Dev Biol, 394, 83-93.

Huch, M., Boj, S.F. and Clevers, H. (2013b) Lgr5(+) liver stem cells, hepatic organoids and regenerative medicine. Regen Med, 8, 385-387.

Huch, M., Bonfanti, P., Boj, S.F., Sato, T., Loomans, C.J., van de Wetering, M., Sojoodi, M., Li, V.S., Schuijers, J., Gracanin, A., Ringnalda, F., Begthel, H., Hamer, K., Mulder, J., van Es, J.H., de Koning, E., Vries, R.G., Heimberg, H. and Clevers, H. (2013a) Unlimited in vitro expansion of adult bi-potent pancreas progenitors through the Lgr5/R-spondin axis. Embo j, 32, 2708-2721.

Hufton, A.L., Vinayagam, A., Suhai, S. and Baker, J.C. (2006) Genomic analysis of Xenopus organizer function. BMC Dev Biol, 6, 27.

Hutchison, K.A., Scherrer, L.C., Czar, M.J., Stancato, L.F., Chow, Y.H., Jove, R. and Pratt, W.B. (1993) Regulation of glucocorticoid receptor function through assembly of a receptor-heat shock protein complex. Ann N Y Acad Sci, 684, 35-48.

Hwang, W.Y., Fu, Y., Reyon, D., Maeder, M.L., Tsai, S.Q., Sander, J.D., Peterson, R.T., Yeh, J.R. and Joung, J.K. (2013) Efficient genome editing in zebrafish using a CRISPR-Cas system. Nat Biotechnol, 31, 227-229.

Hyde, C.E. and Old, R.W. (2000) Regulation of the early expression of the Xenopus nodalrelated 1 gene, Xnr1. Development, 127, 1221-1229.

Ikuzawa, M., Kobayashi, K., Yasumasu, S. and luchi, I. (2005) Expression of CCAAT/enhancer binding protein delta is closely associated with degeneration of surface mucous cells of larval stomach during the metamorphosis of Xenopus laevis. Comp Biochem Physiol B Biochem Mol Biol, 140, 505-511.

Ishioka, A., Jindo, T., Kawanabe, T., Hatta, K., Parvin, M.S., Nikaido, M., Kuroyanagi, Y., Takeda, H. and Yamasu, K. (2011) Retinoic acid-dependent establishment of positional information in the hindbrain was conserved during vertebrate evolution. Dev Biol, 350, 154-168.

Ismadi, S.D. and Olson, J.A. (1982) Dynamics of the fetal distribution and transfer of Vitamin A between rat fetuses and their mother. Int $J$ Vitam Nutr Res, 52, 112-119.

Jacquemin, P., Lemaigre, F.P. and Rousseau, G.G. (2003) The Onecut transcription factor HNF-6 (OC-1) is required for timely specification of the pancreas and acts upstream of Pdx-1 in the specification cascade. Dev Biol, 258, 105-116.

Jacquemin, P., Yoshitomi, H., Kashima, Y., Rousseau, G.G., Lemaigre, F.P. and Zaret, K.S. (2006) An endothelial-mesenchymal relay pathway regulates early phases of pancreas development. Dev Biol, 290, 189-199.

Jarikji, Z., Horb, L.D., Shariff, F., Mandato, C.A., Cho, K.W. and Horb, M.E. (2009) The tetraspanin Tm4sf3 is localized to the ventral pancreas and regulates fusion of the dorsal and ventral pancreatic buds. Development, 136, 1791-1800.

Jarikji, Z.H., Vanamala, S., Beck, C.W., Wright, C.V., Leach, S.D. and Horb, M.E. (2007) Differential ability of Ptf1a and Ptf1a-VP16 to convert stomach, duodenum and liver to pancreas. Dev Biol, 304, 786-799.

Jensen, J., Heller, R.S., Funder-Nielsen, T., Pedersen, E.E., Lindsell, C., Weinmaster, G., Madsen, O.D. and Serup, P. (2000) Independent development of pancreatic alpha- and beta-cells from neurogenin3-expressing precursors: a role for the notch pathway in repression of premature differentiation. Diabetes, 49, 163-176.

Johansson, K.A., Dursun, U., Jordan, N., Gu, G., Beermann, F., Gradwohl, G. and Grapin-Botton, A. (2007) Temporal control of neurogenin3 activity in pancreas progenitors reveals competence windows for the generation of different endocrine cell types. Dev Cell, 12, 457-465.

Jones, E.A. (1985) Epidermal development in Xenopus laevis: the definition of a monoclonal 
antibody to an epidermal marker. J Embryol Exp Morphol, 89 Suppl, 155-166.

Jorgensen, M.C., Ahnfelt-Ronne, J., Hald, J., Madsen, O.D., Serup, P. and HecksherSorensen, J. (2007) An illustrated review of early pancreas development in the mouse. Endocr Rev, 28, 685-705.

Jung, J., Zheng, M., Goldfarb, M. and Zaret, K.S. (1999) Initiation of mammalian liver development from endoderm by fibroblast growth factors. Science, 284, 1998-2003.

Kam, R.K., Chen, Y., Chan, S.O., Chan, W.Y., Dawid, I.B. and Zhao, H. (2010) Developmental expression of Xenopus short-chain dehydrogenase/reductase 3. Int J Dev Biol, 54, 1355-1360.

Kam, R.K., Shi, W., Chan, S.O., Chen, Y., Xu, G., Lau, C.B., Fung, K.P., Chan, W.Y. and Zhao, H. (2013) Dhrs3 protein attenuates retinoic acid signaling and is required for early embryonic patterning. J Biol Chem, 288, 31477-31487.

Karthaus, W.R., laquinta, P.J., Drost, J., Gracanin, A., van Boxtel, R., Wongvipat, J., Dowling, C.M., Gao, D., Begthel, H., Sachs, N., Vries, R.G., Cuppen, E., Chen, Y., Sawyers, C.L. and Clevers, H.C. (2014) Identification of multipotent luminal progenitor cells in human prostate organoid cultures. Cell, 159, 163-175.

Katoh, M. (2002) Regulation of WNT signaling molecules by retinoic acid during neuronal differentiation in NT2 cells: threshold model of WNT action (review). Int $\mathrm{J} \mathrm{Mol} \mathrm{Med,}$ 10, 683-687.

Kawaguchi, R., Yu, J., Honda, J., Hu, J., Whitelegge, J., Ping, P., Wiita, P., Bok, D. and Sun, H. (2007) A membrane receptor for retinol binding protein mediates cellular uptake of vitamin A. Science, 315, 820-825.

Kawaguchi, Y., Cooper, B., Gannon, M., Ray, M., MacDonald, R.J. and Wright, C.V. (2002) The role of the transcriptional regulator Ptfla in converting intestinal to pancreatic progenitors. Nat Genet, 32, 128-134.

Keller, R. (1991) Early embryonic development of Xenopus laevis. Methods Cell Biol, 61113.

Kelly, O.G. and Melton, D.A. (2000) Development of the pancreas in Xenopus laevis. Dev Dyn, 218, 615-627.

Kim, S.K., Hebrok, M. and Melton, D.A. (1997) Notochord to endoderm signaling is required for pancreas development. Development, 124, 4243-4252.

Kirchgessner, A.L. and Gershon, M.D. (1990) Innervation of the pancreas by neurons in the gut. J Neurosci, 10, 1626-1642.

Klein, S.L., Strausberg, R.L., Wagner, L., Pontius, J., Clifton, S.W. and Richardson, P. (2002) Genetic and genomic tools for Xenopus research: The NIH Xenopus initiative. Dev Dyn, 225, 384-391.

Klisch, T. (2006) Transcriptional control in the context of primary neurogenesis $P h D$ thesis.

Kofron, M., Puck, H., Standley, H., Wylie, C., Old, R., Whitman, M. and Heasman, J. (2004) New roles for FoxH1 in patterning the early embryo. Development, 131, 5065-5078.

Kogawa, K., Nakamura, T., Sugino, K., Takio, K., Titani, K. and Sugino, H. (1991) Activin-binding protein is present in pituitary. Endocrinology, 128, 1434-1440.

Kolm, P.J. and Sive, H.L. (1994) Complex regulation of Xenopus HoxAl and HoxDI. Biochem Soc Trans, 22, 579-584.

Kopp, J.L., Dubois, C.L., Hao, E., Thorel, F., Herrera, P.L. and Sander, M. (2011a) Progenitor cell domains in the developing and adult pancreas. Cell Cycle, 10, 19211927.

Kopp, J.L., Dubois, C.L., Schaffer, A.E., Hao, E., Shih, H.P., Seymour, P.A., Ma, J. and Sander, M. (2011b) Sox9+ ductal cells are multipotent progenitors throughout development but do not produce new endocrine cells in the normal or injured adult pancreas. Development, 138, 653-665.

Krapp, A., Knofler, M., Ledermann, B., Burki, K., Berney, C., Zoerkler, N., Hagenbuchle, O. and Wellauer, P.K. (1998) The bHLH protein PTF1-p48 is essential for the formation of the exocrine and the correct spatial organization of the endocrine pancreas. Genes Dev, 12, 3752-3763.

Kroon, E., Martinson, L.A., Kadoya, K., Bang, A.G., Kelly, O.G., Eliazer, S., Young, H., Richardson, M., Smart, N.G., Cunningham, J., Agulnick, A.D., D'Amour, K.A., Carpenter, M.K. and Baetge, E.E. (2008) Pancreatic endoderm derived from human embryonic stem cells generates glucose-responsive insulin-secreting cells in vivo. Nat Biotechnol, 26, 443-452.

Kubo, A., Shinozaki, K., Shannon, J.M., Kouskoff, V., Kennedy, M., Woo, S., Fehling, H.J. and Keller, G. (2004) Development of definitive endoderm from embryonic 
stem cells in culture. Development, 131, 1651-1662.

Kurisaki, A., Ito, Y., Onuma, Y., Intoh, A. and Asashima, M. (2010) In vitro organogenesis using multipotent cells. Hum Cell, 23, 1-14.

Lalevee, S., Anno, Y.N., Chatagnon, A., Samarut, E., Poch, O., Laudet, V., Benoit, G., Lecompte, O. and Rochette-Egly, C. (2011) Genome-wide in silico identification of new conserved and functional retinoic acid receptor response elements (direct repeats separated by 5 bp). J Biol Chem, 286, 33322-33334.

Lammert, E., Cleaver, O. and Melton, D. (2001) Induction of pancreatic differentiation by signals from blood vessels. Science, 294, 564-567.

Lancaster, M.A. and Knoblich, J.A. (2014) Organogenesis in a dish: modeling development and disease using organoid technologies. Science, 345, 1247125.

Lancaster, M.A., Renner, M., Martin, C.A., Wenzel, D., Bicknell, L.S., Hurles, M.E., Homfray, T., Penninger, J.M., Jackson, A.P. and Knoblich, J.A. (2013) Cerebral organoids model human brain development and microcephaly. Nature, 501, 373379.

Lancman, J.J., Zvenigorodsky, N., Gates, K.P., Zhang, D., Solomon, K., Humphrey, R.K., Kuo, T., Setiawan, L., Verkade, H., Chi, Y.I., Jhala, U.S., Wright, C.V., Stainier, D.Y. and Dong, P.D. (2013) Specification of hepatopancreas progenitors in zebrafish by hnf1ba and wnt2bb. Development, 140, 2669-2679.

Landry, C., Clotman, F., Hioki, T., Oda, H., Picard, J.J., Lemaigre, F.P. and Rousseau, G.G. (1997) HNF-6 is expressed in endoderm derivatives and nervous system of the mouse embryo and participates to the cross-regulatory network of liver-enriched transcription factors. Dev Biol, 192, 247-257.

Langmead, B. and Salzberg, S.L. (2012) Fast gapped-read alignment with Bowtie 2. Nat Methods, 9, 357-359.

Langston, A.W., Thompson, J.R. and Gudas, L.J. (1997) Retinoic acid-responsive enhancers located $3^{\prime}$ of the Hox A and Hox B homeobox gene clusters. Functional analysis. J Biol Chem, 272, 2167-2175.

Lausen, J., Thomas, H., Lemm, l., Bulman, M., Borgschulze, M., Lingott, A., Hattersley, A.T. and Ryffel, G.U. (2000) Naturally occurring mutations in the human HNF4alpha gene impair the function of the transcription factor to a varying degree. Nucleic Acids Res, 28, 430-437.

Lee, J.C., Smith, S.B., Watada, H., Lin, J., Scheel, D., Wang, J., Mirmira, R.G. and German, M.S. (2001) Regulation of the pancreatic pro-endocrine gene neurogenin3. Diabetes, 50, 928-936.

Lee, Y.H. and Saint-Jeannet, J.P. (2003) Sox9, a novel pancreatic marker in Xenopus. Int J Dev Biol, 47, 459-462.

Li, H., Handsaker, B., Wysoker, A., Fennell, T., Ruan, J., Homer, N., Marth, G., Abecasis, G. and Durbin, R. (2009) The Sequence Alignment/Map format and SAMtools. Bioinformatics, 25, 2078-2079.

Li, M.L., Aggeler, J., Farson, D.A., Hatier, C., Hassell, J. and Bissell, M.J. (1987) Influence of a reconstituted basement membrane and its components on casein gene expression and secretion in mouse mammary epithelial cells. Proc Natl Acad Sci U S A, 84, 136-140.

Li, Y., Rankin, S.A., Sinner, D., Kenny, A.P., Krieg, P.A. and Zorn, A.M. (2008) Sfrp5 coordinates foregut specification and morphogenesis by antagonizing both canonical and noncanonical Wnt11 signaling. Genes Dev, 22, 3050-3063.

Lioubinski, O., Muller, M., Wegner, M. and Sander, M. (2003) Expression of Sox transcription factors in the developing mouse pancreas. Dev Dyn, 227, 402-408.

Logan, C.Y. and Nusse, R. (2004) The Wnt signaling pathway in development and disease. Annu Rev Cell Dev Biol, 20, 781-810.

Lohnes, D., Mark, M., Mendelsohn, C., Dolle, P., Dierich, A., Gorry, P., Gansmuller, A. and Chambon, P. (1994) Function of the retinoic acid receptors (RARs) during development (I). Craniofacial and skeletal abnormalities in RAR double mutants. Development, 120, 2723-2748.

Loudig, O., Babichuk, C., White, J., Abu-Abed, S., Mueller, C. and Petkovich, M. (2000) Cytochrome P450RAI(CYP26) promoter: a distinct composite retinoic acid response element underlies the complex regulation of retinoic acid metabolism. Molecular endocrinology (Baltimore, Md.), 14, 1483-1497.

Loudig, O., Maclean, G.A., Dore, N.L., Luu, L. and Petkovich, M. (2005) Transcriptional co-operativity between distant retinoic acid response elements in regulation of Cyp26A1 inducibility. Biochem J, 392, 241-248. 
Lynn, F.C., Smith, S.B., Wilson, M.E., Yang, K.Y., Nekrep, N. and German, M.S. (2007) Sox9 coordinates a transcriptional network in pancreatic progenitor cells. Proc Natl Acad Sci U S A, 104, 10500-10505.

Maake, C., Hanke, W. and Reinecke, M. (1998) An immunohistochemical and morphometric analysis of insulin, insulin-like growth factor I, glucagon, somatostatin, and PP in the development of the gastro-entero-pancreatic system of Xenopus laevis. Gen Comp Endocrinol, 110, 182-195.

Maczkowiak, F., Mateos, S., Wang, E., Roche, D., Harland, R. and Monsoro-Burq, A.H. (2010) The Pax3 and Pax7 paralogs cooperate in neural and neural crest patterning using distinct molecular mechanisms, in Xenopus laevis embryos. Dev Biol, 340, 381-396.

Maestro, M.A., Boj, S.F., Luco, R.F., Pierreux, C.E., Cabedo, J., Servitja, J.M., German, M.S., Rousseau, G.G., Lemaigre, F.P. and Ferrer, J. (2003) Hnf6 and Tcf2 (MODY5) are linked in a gene network operating in a precursor cell domain of the embryonic pancreas. Hum Mol Genet, 12, 3307-3314.

Magenheim, J., Klein, A.M., Stanger, B.Z., Ashery-Padan, R., Sosa-Pineda, B., Gu, G. and Dor, Y. (2011) Ngn3(+) endocrine progenitor cells control the fate and morphogenesis of pancreatic ductal epithelium. Dev Biol, 359, 26-36.

Mark, M., Ghyselinck, N.B. and Chambon, P. (2006) Function of retinoid nuclear receptors: lessons from genetic and pharmacological dissections of the retinoic acid signaling pathway during mouse embryogenesis. Annu Rev Pharmacol Toxicol, 46, 451-480.

Martin, M., Gallego-Llamas, J., Ribes, V., Kedinger, M., Niederreither, K., Chambon, P., Dolle, P. and Gradwohl, G. (2005) Dorsal pancreas agenesis in retinoic aciddeficient Raldh2 mutant mice. Dev Biol, 284, 399-411.

Masui, T., Long, Q., Beres, T.M., Magnuson, M.A. and MacDonald, R.J. (2007) Early pancreatic development requires the vertebrate Suppressor of Hairless (RBPJ) in the PTF1 bHLH complex. Genes Dev, 21, 2629-2643.

Masui, T., Swift, G.H., Deering, T., Shen, C., Coats, W.S., Long, Q., Elsasser, H.P., Magnuson, M.A. and MacDonald, R.J. (2010) Replacement of Rbpj with Rbpjl in the PTF1 complex controls the final maturation of pancreatic acinar cells. Gastroenterology, 139, 270-280.

Masui, T., Swift, G.H., Hale, M.A., Meredith, D.M., Johnson, J.E. and Macdonald, R.J. (2008) Transcriptional autoregulation controls pancreatic Ptf1a expression during development and adulthood. Mol Cell Biol, 28, 5458-5468.

McCracken, K.W., Cata, E.M., Crawford, C.M., Sinagoga, K.L., Schumacher, M., Rockich, B.E., Tsai, Y.H., Mayhew, C.N., Spence, J.R., Zavros, Y. and Wells, J.M. (2014) Modelling human development and disease in pluripotent stem-cellderived gastric organoids. Nature, 516, 400-404.

McCracken, K.W. and Wells, J.M. (2012) Molecular pathways controlling pancreas induction. Semin Cell Dev Biol, 23, 656-662.

McLin, V.A., Rankin, S.A. and Zorn, A.M. (2007) Repression of Wnt/beta-catenin signaling in the anterior endoderm is essential for liver and pancreas development. Development, 134, 2207-2217.

Medina, A., Reintsch, W. and Steinbeisser, H. (2000) Xenopus frizzled 7 can act in canonical and non-canonical Wht signaling pathways: implications on early patterning and morphogenesis. Mech Dev, 92, 227-237.

Mellitzer, G., Bonne, S., Luco, R.F., Van De Casteele, M., Lenne-Samuel, N., Collombat, P., Mansouri, A., Lee, J., Lan, M., Pipeleers, D., Nielsen, F.C., Ferrer, J., Gradwohl, G. and Heimberg, H. (2006) IA1 is NGN3-dependent and essential for differentiation of the endocrine pancreas. Embo j, 25, 1344-1352.

Mendelsohn, C., Lohnes, D., Decimo, D., Lufkin, T., LeMeur, M., Chambon, P. and Mark, M. (1994) Function of the retinoic acid receptors (RARs) during development (II). Multiple abnormalities at various stages of organogenesis in RAR double mutants. Development, 120, 2749-2771.

Mii, Y. and Taira, M. (2009) Secreted Frizzled-related proteins enhance the diffusion of Wnt ligands and expand their signalling range. Development, 136, 4083-4088.

Mikels, A.J. and Nusse, R. (2006) Purified Wnt5a protein activates or inhibits beta-cateninTCF signaling depending on receptor context. PLoS Biol, 4, e115.

Mitchell, D.M., Stevens, C.B., Frey, R.A., Hunter, S.S., Ashino, R., Kawamura, S. and Stenkamp, D.L. (2015) Retinoic Acid Signaling Regulates Differential Expression of 
the Tandemly-Duplicated Long Wavelength-Sensitive Cone Opsin Genes in Zebrafish. PLoS Genet, 11, e1005483.

Miyatsuka, T., Matsuoka, T.A., Shiraiwa, T., Yamamoto, T., Kojima, I. and Kaneto, H. (2007) Ptf1a and RBP-J cooperate in activating Pdx1 gene expression through binding to Area III. Biochem Biophys Res Commun, 362, 905-909.

Molotkov, A., Molotkova, N. and Duester, G. (2005) Retinoic acid generated by Raldh2 in mesoderm is required for mouse dorsal endodermal pancreas development. Dev Dyn, 232, 950-957.

Moon, R.T. and Kimelman, D. (1998) From cortical rotation to organizer gene expression: toward a molecular explanation of axis specification in Xenopus. Bioessays, 20, 536545.

Moore, F., Santin, I., Nogueira, T.C., Gurzov, E.N., Marselli, L., Marchetti, P. and Eizirik, D.L. (2012) The transcription factor C/EBP delta has anti-apoptotic and antiinflammatory roles in pancreatic beta cells. PLoS One, 7, e31062.

Moriya, N., Komazaki, S. and Asashima, M. (2000a) In vitro organogenesis of pancreas in Xenopus laevis dorsal lips treated with retinoic acid. Dev Growth Differ, 42, 175-185.

Moriya, N., Komazaki, S., Takahashi, S., Yokota, C. and Asashima, M. (2000b) In vitro pancreas formation from Xenopus ectoderm treated with activin and retinoic acid. Dev Growth Differ, 42, 593-602.

Mukhi, S., Mao, J. and Brown, D.D. (2008) Remodeling the exocrine pancreas at metamorphosis in Xenopus laevis. Proc Natl Acad Sci U S A, 105, 8962-8967.

Mullis, K., Faloona, F., Scharf, S., Saiki, R., Horn, G. and Erlich, H. (1986) Specific enzymatic amplification of DNA in vitro: the polymerase chain reaction. Cold Spring Harb Symp Quant Biol, 51 Pt 1, 263-273.

Murtaugh, L.C., Law, A.C., Dor, Y. and Melton, D.A. (2005) Beta-catenin is essential for pancreatic acinar but not islet development. Development, 132, 4663-4674.

Nakayama, T., Fish, M.B., Fisher, M., Oomen-Hajagos, J., Thomsen, G.H. and Grainger, R.M. (2013) Simple and efficient CRISPR/Cas9-mediated targeted mutagenesis in Xenopus tropicalis. Genesis, 51, 835-843.

Napolitano, T., Avolio, F., Courtney, M., Vieira, A., Druelle, N., Ben-Othman, N., Hadzic, B., Navarro, S. and Collombat, P. (2015) Pax4 acts as a key player in pancreas development and plasticity. Semin Cell Dev Biol, 44, 107-114.

Nieber, F., Pieler, T. and Henningfeld, K.A. (2009) Comparative expression analysis of the neurogenins in Xenopus tropicalis and Xenopus laevis. Dev Dyn, 238, 451-458.

Niederreither, K., McCaffery, P., Drager, U.C., Chambon, P. and Dolle, P. (1997) Restricted expression and retinoic acid-induced downregulation of the retinaldehyde dehydrogenase type 2 (RALDH-2) gene during mouse development. Mech Dev, 62, 67-78.

Niehrs, C. (2012) The complex world of WNT receptor signalling. Nat Rev Mol Cell Biol, 13, 767-779.

Nieto, M.A., Bradley, L.C., Hunt, P., Das Gupta, R., Krumlauf, R. and Wilkinson, D.G. (1992) Molecular mechanisms of pattern formation in the vertebrate hindbrain. Ciba Found Symp, 165, 92-102; discussion 102-107.

Nieuwkoop, P.D. (1963) Pattern formation in artificially activated ectoderm (Rana pipiens and Ambystoma punctatum). Dev Biol, 6, 255-279.

Nieuwkoop, P.D. and Faber, J. (1967) Normal table of Xenopus laevis. (Daudin) North Holland Publishing Co. Amsterdam, 2nd ed.

Nolte, C., Amores, A., Nagy Kovacs, E., Postlethwait, J. and Featherstone, M. (2003) The role of a retinoic acid response element in establishing the anterior neural expression border of Hoxd4 transgenes. Mech Dev, 120, 325-335.

Nusse, R. (2012) Wnt signaling. Cold Spring Harbor perspectives in biology, 4.

Obata, J., Yano, M., Mimura, H., Goto, T., Nakayama, R., Mibu, Y., Oka, C. and Kawaichi, M. (2001) p48 subunit of mouse PTF1 binds to RBP-Jkappa/CBF-1, the intracellular mediator of Notch signalling, and is expressed in the neural tube of early stage embryos. Genes Cells, 6, 345-360.

Ohkawara, B. and Niehrs, C. (2011) An ATF2-based luciferase reporter to monitor noncanonical Wnt signaling in Xenopus embryos. Dev Dyn, 240, 188-194.

Okabayashi, K. and Asashima, M. (2006) In Vitro organogenesis using amphibian pluripotent cells. Proc Jpn Acad Ser B Phys Biol Sci, 82, 197-207.

Osei-Sarfo, K. and Gudas, L.J. (2014) Retinoic acid suppresses the canonical Wnt signaling pathway in embryonic stem cells and activates the noncanonical Wnt signaling pathway. Stem cells (Dayton, Ohio), 32, 2061-2071. 
Oster, A., Jensen, J., Serup, P., Galante, P., Madsen, O.D. and Larsson, L.I. (1998) Rat endocrine pancreatic development in relation to two homeobox gene products (Pdx1 and Nkx 6.1). J Histochem Cytochem, 46, 707-715.

Pagliuca, F.W., Millman, J.R., Gurtler, M., Segel, M., Van Dervort, A., Ryu, J.H., Peterson, Q.P., Greiner, D. and Melton, D.A. (2014) Generation of functional human pancreatic beta cells in vitro. Cell, 159, 428-439.

Pan, F.C., Bankaitis, E.D., Boyer, D., Xu, X., Van de Casteele, M., Magnuson, M.A., Heimberg, H. and Wright, C.V. (2013) Spatiotemporal patterns of multipotentiality in Ptf1a-expressing cells during pancreas organogenesis and injury-induced facultative restoration. Development, 140, 751-764.

Pan, F.C., Chen, Y., Bayha, E. and Pieler, T. (2007) Retinoic acid-mediated patterning of the pre-pancreatic endoderm in Xenopus operates via direct and indirect mechanisms. Mech Dev, 124, 518-531.

Pan, F.C. and Wright, C. (2011) Pancreas organogenesis: from bud to plexus to gland. Dev Dyn, 240, 530-565.

Pearl, E.J., Bilogan, C.K., Mukhi, S., Brown, D.D. and Horb, M.E. (2009) Xenopus pancreas development. Dev Dyn, 238, 1271-1286.

Pera, E.M., Martinez, S.L., Flanagan, J.J., Brechner, M., Wessely, O. and De Robertis, E.M. (2003) Darmin is a novel secreted protein expressed during endoderm development in Xenopus. Gene Expr Patterns, 3, 147-152.

Perron, M., Opdecamp, K., Butler, K., Harris, W.A. and Bellefroid, E.J. (1999) X-ngnr-1 and Xath3 promote ectopic expression of sensory neuron markers in the neurula ectoderm and have distinct inducing properties in the retina. Proc Natl Acad Sci U S A, 96, 14996-15001.

Piccolo, S., Sasai, Y., Lu, B. and De Robertis, E.M. (1996) Dorsoventral patterning in Xenopus: inhibition of ventral signals by direct binding of chordin to BMP-4. Cell, 86, 589-598.

Pictet, R.L., Clark, W.R., Williams, R.H. and Rutter, W.J. (1972) An ultrastructural analysis of the developing embryonic pancreas. Dev Biol, 29, 436-467.

Pieler, T. and Chen, Y. (2006) Forgotten and novel aspects in pancreas development. Biol Cell, 98, 79-88.

Poll, A.V., Pierreux, C.E., Lokmane, L., Haumaitre, C., Achouri, Y., Jacquemin, P., Rousseau, G.G., Cereghini, S. and Lemaigre, F.P. (2006) A vHNF1/TCF2-HNF6 cascade regulates the transcription factor network that controls generation of pancreatic precursor cells. Diabetes, 55, 61-69.

Pouilhe, M., Gilardi-Hebenstreit, P., Desmarquet-Trin Dinh, C. and Charnay, P. (2007) Direct regulation of vHnf1 by retinoic acid signaling and MAF-related factors in the neural tube. Dev Biol, 309, 344-357.

Power, S.C. and Cereghini, S. (1996) Positive regulation of the vHNF1 promoter by the orphan receptors COUP-TF1/Ear3 and COUP-TFII/Arp1. Mol Cell Biol, 16, 778-791.

Quadro, L., Blaner, W.S., Salchow, D.J., Vogel, S., Piantedosi, R., Gouras, P., Freeman, S., Cosma, M.P., Colantuoni, V. and Gottesman, M.E. (1999) Impaired retinal function and vitamin A availability in mice lacking retinol-binding protein. Embo j, 18, 4633-4644.

Rankin, S.A., Kormish, J., Kofron, M., Jegga, A. and Zorn, A.M. (2011) A gene regulatory network controlling hhex transcription in the anterior endoderm of the organizer. Dev Biol, 351, 297-310.

Rattner, A., Hsieh, J.C., Smallwood, P.M., Gilbert, D.J., Copeland, N.G., Jenkins, N.A. and Nathans, J. (1997) A family of secreted proteins contains homology to the cysteine-rich ligand-binding domain of frizzled receptors. Proc Natl Acad Sci U S A, 94, 2859-2863.

Rausa, F., Samadani, U., Ye, H., Lim, L., Fletcher, C.F., Jenkins, N.A., Copeland, N.G. and Costa, R.H. (1997) The cut-homeodomain transcriptional activator HNF-6 is coexpressed with its target gene HNF-3 beta in the developing murine liver and pancreas. Dev Biol, 192, 228-246.

Ray, W.J., Bain, G., Yao, M. and Gottlieb, D.I. (1997) CYP26, a novel mammalian cytochrome P450, is induced by retinoic acid and defines a new family. J Biol Chem, 272, 18702-18708.

Rehorn, K.P., Thelen, H., Michelson, A.M. and Reuter, R. (1996) A molecular aspect of hematopoiesis and endoderm development common to vertebrates and Drosophila. Development, 122, 4023-4031.

Rezania, A., Bruin, J.E., Riedel, M.J., Mojibian, M., Asadi, A., Xu, J., Gauvin, R., 
Narayan, K., Karanu, F., O'Neil, J.J., Ao, Z., Warnock, G.L. and Kieffer, T.J. (2012) Maturation of human embryonic stem cell-derived pancreatic progenitors into functional islets capable of treating pre-existing diabetes in mice. Diabetes, 61, 2016-2029.

Rhinn, M. and Dolle, P. (2012) Retinoic acid signalling during development. Development 139, 843-858.

Richard-Parpaillon, L., Heligon, C., Chesnel, F., Boujard, D. and Philpott, A. (2002) The IGF pathway regulates head formation by inhibiting Wnt signaling in Xenopus. Dev Biol, 244, 407-417.

Rindi, G., Torsello, A., Locatelli, V. and Solcia, E. (2004) Ghrelin expression and actions: a novel peptide for an old cell type of the diffuse endocrine system. Exp Biol Med (Maywood), 229, 1007-1016.

Robinson, M.D., McCarthy, D.J. and Smyth, G.K. (2010) edgeR: a Bioconductor package for differential expression analysis of digital gene expression data. Bioinformatics, 26, 139-140.

Rochette-Egly, C. and Germain, P. (2009) Dynamic and combinatorial control of gene expression by nuclear retinoic acid receptors (RARs). Nucl Recept Signal, 7, e005.

Rock, J.R., Onaitis, M.W., Rawlins, E.L., Lu, Y., Clark, C.P., Xue, Y., Randell, S.H. and Hogan, B.L. (2009) Basal cells as stem cells of the mouse trachea and human airway epithelium. Proc Natl Acad Sci U S A, 106, 12771-12775.

Rodriguez-Seguel, E., Mah, N., Naumann, H., Pongrac, I.M., Cerda-Esteban, N., Fontaine, J.F., Wang, Y., Chen, W., Andrade-Navarro, M.A. and Spagnoli, F.M. (2013) Mutually exclusive signaling signatures define the hepatic and pancreatic progenitor cell lineage divergence. Genes Dev, 27, 1932-1946.

Rodriguez-Segui, S., Akerman, I. and Ferrer, J. (2012) GATA believe it: new essential regulators of pancreas development. J Clin Invest, 122, 3469-3471.

Rookmaaker, M.B., Schutgens, F., Verhaar, M.C. and Clevers, H. (2015) Development and application of human adult stem or progenitor cell organoids. Nat Rev Nephrol, 11, 546-554.

Rose, S.D., Swift, G.H., Peyton, M.J., Hammer, R.E. and MacDonald, R.J. (2001) The role of PTF1-P48 in pancreatic acinar gene expression. $\mathrm{J}$ Biol Chem, 276, 4401844026.

Rossi, J.M., Dunn, N.R., Hogan, B.L. and Zaret, K.S. (2001) Distinct mesodermal signals, including BMPs from the septum transversum mesenchyme, are required in combination for hepatogenesis from the endoderm. Genes Dev, 15, 1998-2009.

Rubenstein, A., Merriam, J. and Klymkowsky, M.W. (1997) Localizing the adhesive and signaling functions of plakoglobin. Dev Genet, 20, 91-102.

Rukstalis, J.M. and Habener, J.F. (2007) Snail2, a mediator of epithelial-mesenchymal transitions, expressed in progenitor cells of the developing endocrine pancreas. Gene Expr Patterns, 7, 471-479.

Rupp, R.A., Snider, L. and Weintraub, H. (1994) Xenopus embryos regulate the nuclear localization of XMyoD. Genes Dev, 8, 1311-1323.

Ryffel, G.U. (2001) Mutations in the human genes encoding the transcription factors of the hepatocyte nuclear factor (HNF)1 and HNF4 families: functional and pathological consequences. J Mol Endocrinol, 27, 11-29.

Sagara, N., Kirikoshi, H., Terasaki, H., Yasuhiko, Y., Toda, G., Shiokawa, K. and Katoh, M. (2001) FZD4S, a splicing variant of frizzled-4, encodes a soluble-type positive regulator of the WNT signaling pathway. Biochem Biophys Res Commun, 282, 750756.

Saiki, R.K., Scharf, S., Faloona, F., Mullis, K.B., Horn, G.T., Erlich, H.A. and Arnheim, N. (1985) Enzymatic amplification of beta-globin genomic sequences and restriction site analysis for diagnosis of sickle cell anemia. Science, 230, 1350-1354.

Salzberg, A., Elias, S., Nachaliel, N., Bonstein, L., Henig, C. and Frank, D. (1999) A Meis family protein caudalizes neural cell fates in Xenopus. Mech Dev, 80, 3-13.

Sambrook, J. and Russel, D.W. (2001) Molecular Cloning: a laboratory manual. Cold Spring Harbour Laboratory Press, Cold Spring Harbour, New York., 3rd edition.

Sander, J.D. and Joung, J.K. (2014) CRISPR-Cas systems for editing, regulating and targeting genomes. Nat Biotechnol, 32, 347-355.

Sander, J.D., Maeder, M.L., Reyon, D., Voytas, D.F., Joung, J.K. and Dobbs, D. (2010) ZiFiT (Zinc Finger Targeter): an updated zinc finger engineering tool. Nucleic Acids Res, 38, W462-468.

Sander, J.D., Zaback, P., Joung, J.K., Voytas, D.F. and Dobbs, D. (2007) Zinc Finger 
Targeter (ZiFiT): an engineered zinc finger/target site design tool. Nucleic Acids Res, 35, W599-605.

Sanger, F., Nicklen, S. and Coulson, A.R. (1977) DNA sequencing with chain-terminating inhibitors. Proc Natl Acad Sci U S A, 74, 5463-5467.

Sato, T., van Es, J.H., Snippert, H.J., Stange, D.E., Vries, R.G., van den Born, M., Barker, N., Shroyer, N.F., van de Wetering, M. and Clevers, H. (2011) Paneth cells constitute the niche for Lgr5 stem cells in intestinal crypts. Nature, 469, 415418.

Sato, T., Vries, R.G., Snippert, H.J., van de Wetering, M., Barker, N., Stange, D.E., van Es, J.H., Abo, A., Kujala, P., Peters, P.J. and Clevers, H. (2009) Single Lgr5 stem cells build crypt-villus structures in vitro without a mesenchymal niche. Nature, 459, 262-265.

Schaffer, A.E., Freude, K.K., Nelson, S.B. and Sander, M. (2010) Nkx6 transcription factors and Ptf1a function as antagonistic lineage determinants in multipotent pancreatic progenitors. Dev Cell, 18, 1022-1029.

Schier, A.F. and Talbot, W.S. (2005) Molecular genetics of axis formation in zebrafish. Annu Rev Genet, 39, 561-613.

Schiesser, J.V., Micallef, S.J., Hawes, S., Elefanty, A.G. and Stanley, E.G. (2014) Derivation of insulin-producing beta-cells from human pluripotent stem cells. Rev Diabet Stud, 11, 6-18.

Schneider-Poetsch, T., Ju, J., Eyler, D.E., Dang, Y., Bhat, S., Merrick, W.C., Green, R., Shen, B. and Liu, J.O. (2010) Inhibition of eukaryotic translation elongation by cycloheximide and lactimidomycin. Nature chemical biology, 6, 209-217.

Schulz, T.C., Young, H.Y., Agulnick, A.D., Babin, M.J., Baetge, E.E., Bang, A.G., Bhoumik, A., Cepa, I., Cesario, R.M., Haakmeester, C., Kadoya, K., Kelly, J.R., Kerr, J., Martinson, L.A., McLean, A.B., Moorman, M.A., Payne, J.K., Richardson, M., Ross, K.G., Sherrer, E.S., Song, X., Wilson, A.Z., Brandon, E.P., Green, C.E., Kroon, E.J., Kelly, O.G., D'Amour, K.A. and Robins, A.J. (2012) A scalable system for production of functional pancreatic progenitors from human embryonic stem cells. PLoS One, 7, e37004.

Schwank, G., Koo, B.K., Sasselli, V., Dekkers, J.F., Heo, I., Demircan, T., Sasaki, N., Boymans, S., Cuppen, E., van der Ent, C.K., Nieuwenhuis, E.E., Beekman, J.M. and Clevers, H. (2013) Functional repair of CFTR by CRISPR/Cas9 in intestinal stem cell organoids of cystic fibrosis patients. Cell Stem Cell, 13, 653-658.

Schwitzgebel, V.M., Scheel, D.W., Conners, J.R., Kalamaras, J., Lee, J.E., Anderson, D.J., Sussel, L., Johnson, J.D. and German, M.S. (2000) Expression of neurogenin3 reveals an islet cell precursor population in the pancreas. Development, 127, 3533-3542.

Seymour, P.A. (2014) Sox9: a master regulator of the pancreatic program. Rev Diabet Stud, 11, 51-83.

Seymour, P.A., Freude, K.K., Tran, M.N., Mayes, E.E., Jensen, J., Kist, R., Scherer, G. and Sander, M. (2007) SOX9 is required for maintenance of the pancreatic progenitor cell pool. Proc Natl Acad Sci U S A, 104, 1865-1870.

Shaer, A., Azarpira, N., Vahdati, A., Karimi, M.H. and Shariati, M. (2015) Differentiation of human-induced pluripotent stem cells into insulin-producing clusters. Exp Clin Transplant, 13, 68-75.

Sharp, P.A., Sugden, B. and Sambrook, J. (1973) Detection of two restriction endonuclease activities in Haemophilus parainfluenzae using analytical agarose-ethidium bromide electrophoresis. Biochemistry, 12, 3055-3063.

Sharpe, C.R., Pluck, A. and Gurdon, J.B. (1989) XIF3, a Xenopus peripherin gene, requires an inductive signal for enhanced expression in anterior neural tissue. Development, 107, 701-714.

Shen, M.M. (2007) Nodal signaling: developmental roles and regulation. Development, 134, 1023-1034.

Sherwood, R.I., Maehr, R., Mazzoni, E.O. and Melton, D.A. (2011) Wnt signaling specifies and patterns intestinal endoderm. Mech Dev, 128, 387-400.

Shi, D.L. and Boucaut, J.C. (2000) Xenopus frizzled 4 is a maternal mRNA and its zygotic expression is localized to the neuroectoderm and trunk lateral plate mesoderm. Mech Dev, 94, 243-245.

Shih, H.P., Kopp, J.L., Sandhu, M., Dubois, C.L., Seymour, P.A., Grapin-Botton, A. and Sander, M. (2012) A Notch-dependent molecular circuitry initiates pancreatic endocrine and ductal cell differentiation. Development, 139, 2488-2499. 
Shih, H.P., Seymour, P.A., Patel, N.A., Xie, R., Wang, A., Liu, P.P., Yeo, G.W., Magnuson, M.A. and Sander, M. (2015) A Gene Regulatory Network Cooperatively Controlled by Pdx1 and Sox9 Governs Lineage Allocation of Foregut Progenitor Cells. Cell Rep, 13, 326-336.

Shih, H.P., Wang, A. and Sander, M. (2013) Pancreas organogenesis: from lineage determination to morphogenesis. Annu Rev Cell Dev Biol, 29, 81-105.

Shuldiner, A.R., Phillips, S., Roberts, C.T., Jr., LeRoith, D. and Roth, J. (1989) Xenopus laevis contains two nonallelic preproinsulin genes. cDNA cloning and evolutionary perspective. J Biol Chem, 264, 9428-9432.

Singer, G.a. (2006) Developmental Biology. Sinauer Associates Inc., 8th edition.

Sive, H.L. and Cheng, P.F. (1991) Retinoic acid perturbs the expression of Xhox.lab genes and alters mesodermal determination in Xenopus laevis. Genes Dev, 5, 1321-1332.

Skirkanich, J., Luxardi, G., Yang, J., Kodjabachian, L. and Klein, P.S. (2011) An essential role for transcription before the MBT in Xenopus laevis. Dev Biol, 357, 478491.

Slack, J.M. (1995) Developmental biology of the pancreas. Development, 121, 1569-1580.

Smith, W.C. and Harland, R.M. (1991) Injected Xwnt-8 RNA acts early in Xenopus embryos to promote formation of a vegetal dorsalizing center. Cell, 67, 753-765.

Smith, W.C., Knecht, A.K., Wu, M. and Harland, R.M. (1993) Secreted noggin protein mimics the Spemann organizer in dorsalizing Xenopus mesoderm. Nature, 361, 547549.

Sogame, A., Hayata, T. and Asashima, M. (2003) Screening for novel pancreatic genes from in vitro-induced pancreas in Xenopus. Dev Growth Differ, 45, 143-152.

Solar, M., Cardalda, C., Houbracken, I., Martin, M., Maestro, M.A., De Medts, N., Xu, X., Grau, V., Heimberg, H., Bouwens, L. and Ferrer, J. (2009) Pancreatic exocrine duct cells give rise to insulin-producing beta cells during embryogenesis but not after birth. Dev Cell, 17, 849-860.

Soyer, J., Flasse, L., Raffelsberger, W., Beucher, A., Orvain, C., Peers, B., Ravassard, P., Vermot, J., Voz, M.L., Mellitzer, G. and Gradwohl, G. (2010) Rfx6 is an Ngn3dependent winged helix transcription factor required for pancreatic islet cell development. Development, 137, 203-212.

Spagnoli, F.M. and Brivanlou, A.H. (2008) The Gata5 target, TGIF2, defines the pancreatic region by modulating BMP signals within the endoderm. Development, 135, 451461.

Stafford, D., Hornbruch, A., Mueller, P.R. and Prince, V.E. (2004) A conserved role for retinoid signaling in vertebrate pancreas development. Dev Genes Evol, 214, 432441.

Stafford, D. and Prince, V.E. (2002) Retinoic acid signaling is required for a critical early step in zebrafish pancreatic development. Curr Biol, 12, 1215-1220.

Stemmer, M., Thumberger, T., Del Sol Keyer, M., Wittbrodt, J. and Mateo, J.L. (2015) CCTop: An Intuitive, Flexible and Reliable CRISPR/Cas9 Target Prediction Tool. PLoS One, 10, e0124633.

Strate, I., Min, T.H., Iliev, D. and Pera, E.M. (2009) Retinol dehydrogenase 10 is a feedback regulator of retinoic acid signalling during axis formation and patterning of the central nervous system. Development, 136, 461-472.

Swain, R.K., Katoh, M., Medina, A. and Steinbeisser, H. (2005) Xenopus frizzled-4S, a splicing variant of $\mathrm{Xfz} 4$ is a context-dependent activator and inhibitor of Wnt/betacatenin signaling. Cell Commun Signal, 3, 12.

Taira, M., Otani, H., Jamrich, M. and Dawid, I.B. (1994) Expression of the LIM class homeobox gene Xlim-1 in pronephros and CNS cell lineages of Xenopus embryos is affected by retinoic acid and exogastrulation. Development, 120, 1525-1536.

Takahashi, S., Yokota, C., Takano, K., Tanegashima, K., Onuma, Y., Goto, J. and Asashima, M. (2000) Two novel nodal-related genes initiate early inductive events in Xenopus Nieuwkoop center. Development, 127, 5319-5329.

Takasato, M., Er, P.X., Chiu, H.S., Maier, B., Baillie, G.J., Ferguson, C., Parton, R.G., Wolvetang, E.J., Roost, M.S., Chuva de Sousa Lopes, S.M. and Little, M.H. (2015) Kidney organoids from human iPS cells contain multiple lineages and model human nephrogenesis. Nature, 526, 564-568.

Tam, P.P. and Loebel, D.A. (2007) Gene function in mouse embryogenesis: get set for gastrulation. Nat Rev Genet, 8, 368-381.

Tamai, K., Yokota, C., Ariizumi, T. and Asashima, M. (1999) Cytochalasin B inhibits morphogenetic movement and muscle differentiation of activin-treated ectoderm in 
Xenopus. Dev Growth Differ, 41, 41-49.

Tashiro, K., Yamada, R., Asano, M., Hashimoto, M., Muramatsu, M. and Shiokawa, K. (1991) Expression of mRNA for activin-binding protein (follistatin) during early embryonic development of Xenopus laevis. Biochem Biophys Res Commun, 174, 1022-1027.

Terns, R.M. and Terns, M.P. (2014) CRISPR-based technologies: prokaryotic defense weapons repurposed. Trends Genet, 30, 111-118.

Thomas, H., Jaschkowitz, K., Bulman, M., Frayling, T.M., Mitchell, S.M., Roosen, S., Lingott-Frieg, A., Tack, C.J., Ellard, S., Ryffel, G.U. and Hattersley, A.T. (2001) A distant upstream promoter of the HNF-4alpha gene connects the transcription factors involved in maturity-onset diabetes of the young. Hum Mol Genet, 10, 20892097.

Tsai, M.J. and O'Malley, B.W. (1994) Molecular mechanisms of action of steroid/thyroid receptor superfamily members. Annual review of biochemistry, 63, 451-486.

Turner, D.L. and Weintraub, H. (1994) Expression of achaete-scute homolog 3 in Xenopus embryos converts ectodermal cells to a neural fate. Genes Dev, 8, 1434-1447.

Umbhauer, M., Djiane, A., Goisset, C., Penzo-Mendez, A., Riou, J.F., Boucaut, J.C. and Shi, D.L. (2000) The C-terminal cytoplasmic Lys-thr-X-X-X-Trp motif in frizzled receptors mediates Wnt/beta-catenin signalling. Embo j, 19, 4944-4954.

van der Sanden, M.H., Meems, H., Houweling, M., Helms, J.B. and Vaandrager, A.B. (2004) Induction of CCAAT/enhancer-binding protein (C/EBP)-homologous protein/growth arrest and DNA damage-inducible protein 153 expression during inhibition of phosphatidylcholine synthesis is mediated via activation of a C/EBP. activating transcription factor-responsive element. J Biol Chem, 279, 52007-52015.

Vignali, R., Poggi, L., Madeddu, F. and Barsacchi, G. (2000) HNF1(beta) is required for mesoderm induction in the Xenopus embryo. Development, 127, 1455-1465.

Villasenor, A., Chong, D.C., Henkemeyer, M. and Cleaver, O. (2010) Epithelial dynamics of pancreatic branching morphogenesis. Development, 137, 4295-4305.

Wang, F., Shi, Z., Cui, Y., Guo, X., Shi, Y.B. and Chen, Y. (2015) Targeted gene disruption in Xenopus laevis using CRISPR/Cas9. Cell Biosci, 5, 15.

Wang, L., Coffinier, C., Thomas, M.K., Gresh, L., Eddu, G., Manor, T., Levitsky, L.L., Yaniv, M. and Rhoads, D.B. (2004) Selective deletion of the Hnf1beta (MODY5) gene in beta-cells leads to altered gene expression and defective insulin release. Endocrinology, 145, 3941-3949.

Waters, C.A., Strande, N.T., Wyatt, D.W., Pryor, J.M. and Ramsden, D.A. (2014) Nonhomologous end joining: a good solution for bad ends. DNA Repair (Amst), 17, 39-51.

Watt, A.J., Zhao, R., Li, J. and Duncan, S.A. (2007) Development of the mammalian liver and ventral pancreas is dependent on GATA4. BMC Dev Biol, 7, 37.

Wells, J.M. and Melton, D.A. (2000) Early mouse endoderm is patterned by soluble factors from adjacent germ layers. Development, 127, 1563-1572.

Wheeler, G.N. and Hoppler, S. (1999) Two novel Xenopus frizzled genes expressed in developing heart and brain. Mech Dev, 86, 203-207.

White, J.A., Guo, Y.D., Baetz, K., Beckett-Jones, B., Bonasoro, J., Hsu, K.E., Dilworth, F.J., Jones, G. and Petkovich, M. (1996) Identification of the retinoic acid-inducible all-trans-retinoic acid 4-hydroxylase. J Biol Chem, 271, 29922-29927.

Wierup, N., Sundler, F. and Heller, R.S. (2014) The islet ghrelin cell. J Mol Endocrinol, 52 , R35-49.

Willyard, C. (2015) The boom in mini stomachs, brains, breasts, kidneys and more. Nature, 523, 520-522.

Wong, H.C., Bourdelas, A., Krauss, A., Lee, H.J., Shao, Y., Wu, D., Mlodzik, M., Shi, D.L. and Zheng, J. (2003) Direct binding of the PDZ domain of Dishevelled to a conserved internal sequence in the C-terminal region of Frizzled. Mol Cell, 12, 12511260.

Wright, C.V., Schnegelsberg, P. and De Robertis, E.M. (1989) XIHbox 8: a novel Xenopus homeo protein restricted to a narrow band of endoderm. Development, 105, 787794.

Xanthos, J.B., Kofron, M., Wylie, C. and Heasman, J. (2001) Maternal VegT is the initiator of a molecular network specifying endoderm in Xenopus laevis. Development, 128, 167-180.

Xu, C.R., Cole, P.A., Meyers, D.J., Kormish, J., Dent, S. and Zaret, K.S. (2011) Chromatin "prepattern" and histone modifiers in a fate choice for liver and pancreas. Science, 
332, 963-966.

Xu, Q., Wang, Y., Dabdoub, A., Smallwood, P.M., Williams, J., Woods, C., Kelley, M.W., Jiang, L., Tasman, W., Zhang, K. and Nathans, J. (2004) Vascular development in the retina and inner ear: control by Norrin and Frizzled-4, a high-affinity ligandreceptor pair. Cell, 116, 883-895.

Xuan, S., Borok, M.J., Decker, K.J., Battle, M.A., Duncan, S.A., Hale, M.A., Macdonald, R.J. and Sussel, L. (2012) Pancreas-specific deletion of mouse Gata4 and Gata6 causes pancreatic agenesis. J Clin Invest, 122, 3516-3528.

Yam, J.W., Chan, K.W., Ngan, E.S. and Hsiao, W.L. (2005) Genomic structure, alternative splicing and tissue expression of $\mathrm{rFrp} / \mathrm{sFRP}-4$, the rat frizzled related protein gene. Gene, 357, 55-62.

Yamagata, K., Furuta, H., Oda, N., Kaisaki, P.J., Menzel, S., Cox, N.J., Fajans, S.S., Signorini, S., Stoffel, M. and Bell, G.I. (1996) Mutations in the hepatocyte nuclear factor-4alpha gene in maturity-onset diabetes of the young (MODY1). Nature, 384, 458-460.

Yasuhara, R., Yuasa, T., Williams, J.A., Byers, S.W., Shah, S., Pacifici, M., Iwamoto, M. and Enomoto-Iwamoto, M. (2010) Wnt/beta-catenin and retinoic acid receptor signaling pathways interact to regulate chondrocyte function and matrix turnover. $\mathrm{J}$ Biol Chem, 285, 317-327.

Yasuo, H. and Lemaire, P. (1999) A two-step model for the fate determination of presumptive endodermal blastomeres in Xenopus embryos. Curr Biol, 9, 869-879.

Ye, X., Wang, Y. and Nathans, J. (2010) The Norrin/Frizzled4 signaling pathway in retinal vascular development and disease. Trends Mol Med, 16, 417-425.

Yoshitomi, H. and Zaret, K.S. (2004) Endothelial cell interactions initiate dorsal pancreas development by selectively inducing the transcription factor Ptf1a. Development, 131, 807-817.

Zaret, K.S. and Grompe, M. (2008) Generation and regeneration of cells of the liver and pancreas. Science, 322, 1490-1494.

Zeynali, B., Kalionis, B. and Dixon, K.E. (2000) Determination of anterior endoderm in Xenopus embryos. Dev Dyn, 218, 531-536.

Zhang, C., Basta, T., Fawcett, S.R. and Klymkowsky, M.W. (2005) SOX7 is an immediateearly target of VegT and regulates Nodal-related gene expression in Xenopus. Dev Biol, 278, 526-541.

Zhang, D., Jiang, W., Liu, M., Sui, X., Yin, X., Chen, S., Shi, Y. and Deng, H. (2009) Highly efficient differentiation of human ES cells and iPS cells into mature pancreatic insulin-producing cells. Cell Res, 19, 429-438.

Zhang, J., Houston, D.W., King, M.L., Payne, C., Wylie, C. and Heasman, J. (1998) The role of maternal VegT in establishing the primary germ layers in Xenopus embryos. Cell, 94, 515-524.

Zhang, J. and King, M.L. (1996) Xenopus VegT RNA is localized to the vegetal cortex during oogenesis and encodes a novel T-box transcription factor involved in mesodermal patterning. Development, 122, 4119-4129.

Zhang, K., Harada, Y., Wei, X., Shukla, D., Rajendran, A., Tawansy, K., Bedell, M., Lim, S., Shaw, P.X., He, X. and Yang, Z. (2011) An essential role of the cysteine-rich domain of FZD4 in Norrin/Wnt signaling and familial exudative vitreoretinopathy. $J$ Biol Chem, 286, 10210-10215.

Zhang, T., Guo, X. and Chen, Y. (2013b) Retinoic acid-activated Ndrg1a represses Wnt/beta-catenin signaling to allow Xenopus pancreas, oesophagus, stomach, and duodenum specification. PLoS One, 8, e65058.

Zhang, Y.Q., Cleary, M.M., Si, Y., Liu, G., Eto, Y., Kritzik, M., Dabernat, S., Kayali, A.G. and Sarvetnick, N. (2004) Inhibition of activin signaling induces pancreatic epithelial cell expansion and diminishes terminal differentiation of pancreatic beta-cells. Diabetes, 53, 2024-2033.

Zhang, Z., Rankin, S.A. and Zorn, A.M. (2013a) Different thresholds of Wnt-Frizzled 7 signaling coordinate proliferation, morphogenesis and fate of endoderm progenitor cells. Dev Biol, 378, 1-12.

Zhou, Q., Law, A.C., Rajagopal, J., Anderson, W.J., Gray, P.A. and Melton, D.A. (2007) A multipotent progenitor domain guides pancreatic organogenesis. Dev Cell, 13, 103-114.

Zimmerman, L.B., De Jesus-Escobar, J.M. and Harland, R.M. (1996) The Spemann organizer signal noggin binds and inactivates bone morphogenetic protein 4. Cell, 86, 599-606. 
Zorn, A.M., Butler, K. and Gurdon, J.B. (1999) Anterior endomesoderm specification in Xenopus by Wnt/beta-catenin and TGF-beta signalling pathways. Dev Biol, 209, 282-297.

Zorn, A.M. and Wells, J.M. (2009) Vertebrate endoderm development and organ formation. Annu Rev Cell Dev Biol, 25, 221-251. 


\section{Appendix}

\subsection{Formation of pancreatic organoids that recapitulate the in vivo program of pancreas development}

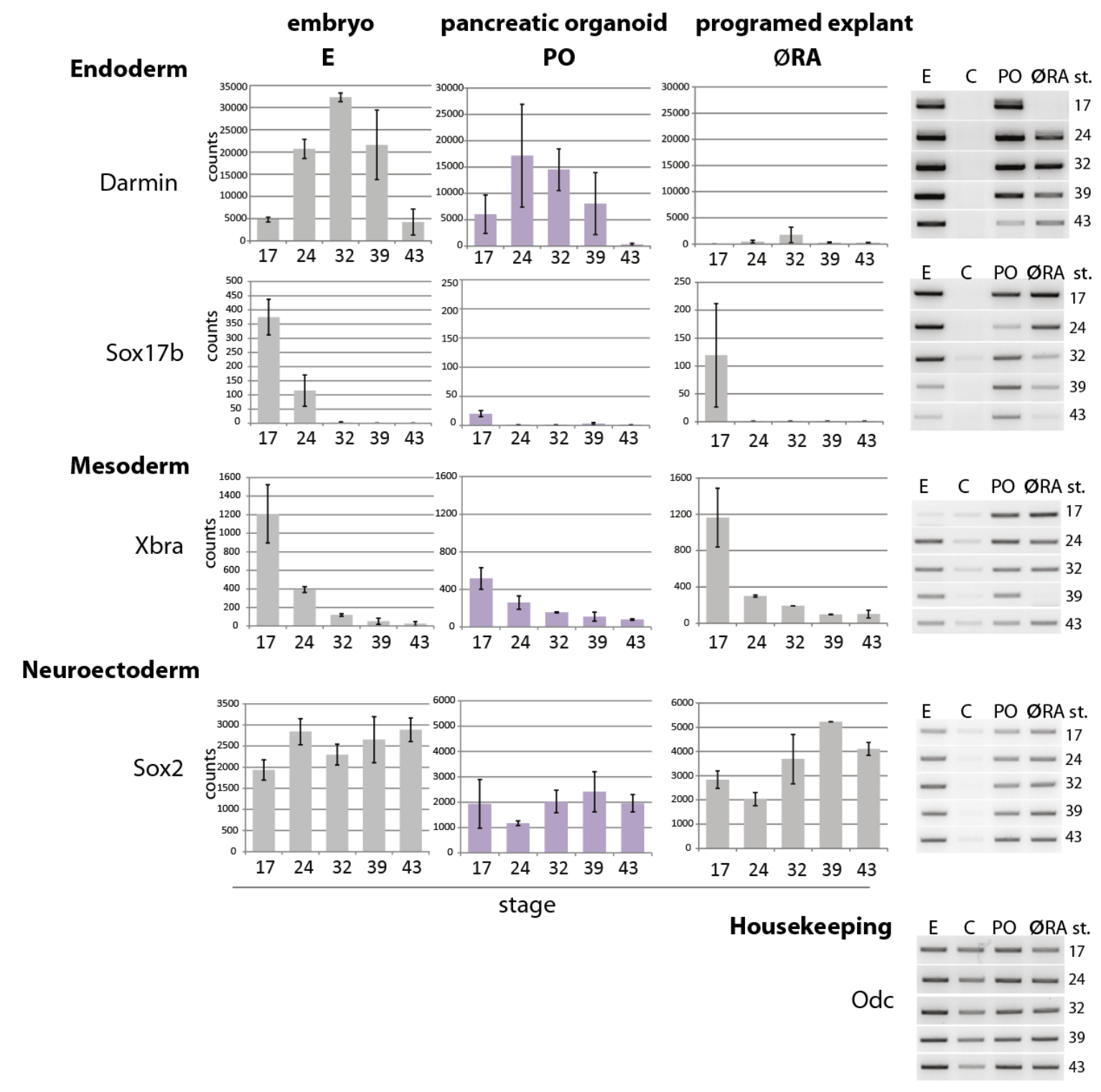

Fig. 6.1 Detection of endodermal, mesodermal and neuro-ectodermal marker genes

Nanostring analysis and RT-PCR results for whole embryos (embryo, E), Vegt/Noggin/RAprogramed explants (pancreatic organoids, PO), Vegt/Noggin/Cyp26a1-programed explants (ØRA) and un-programed explants (C) are shown. Diagrams indicate the mean of normalized Nanostring counts from two independent experiments. Figures on the right show RT-PCR results for indicated marker genes. 


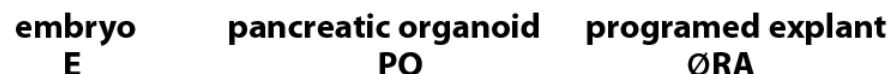

pancreatic progenitors
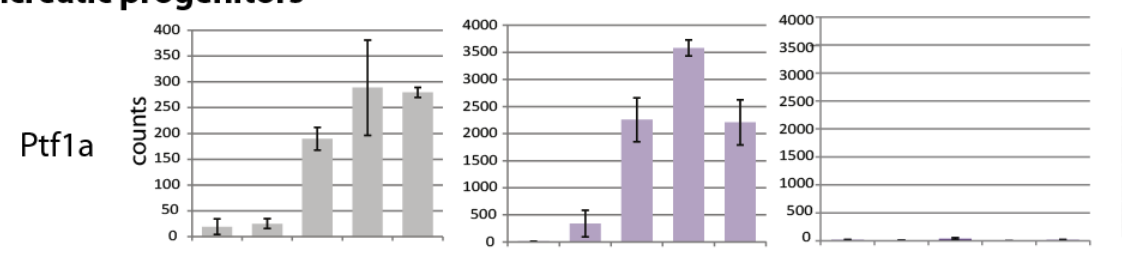

E C PO ØRA st.

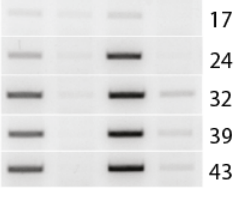

Gata4
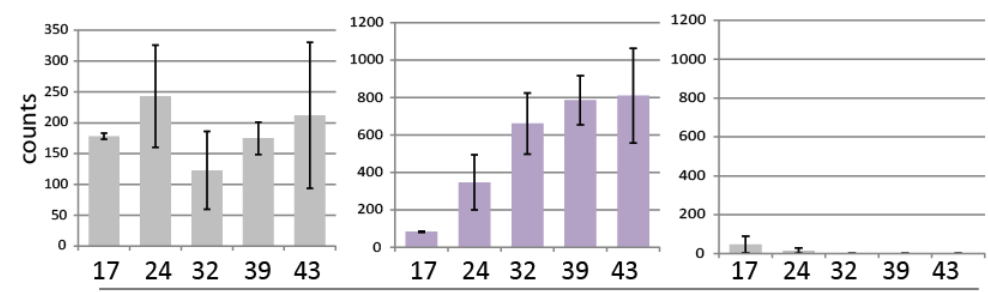

stage

exocrine

Amy2a
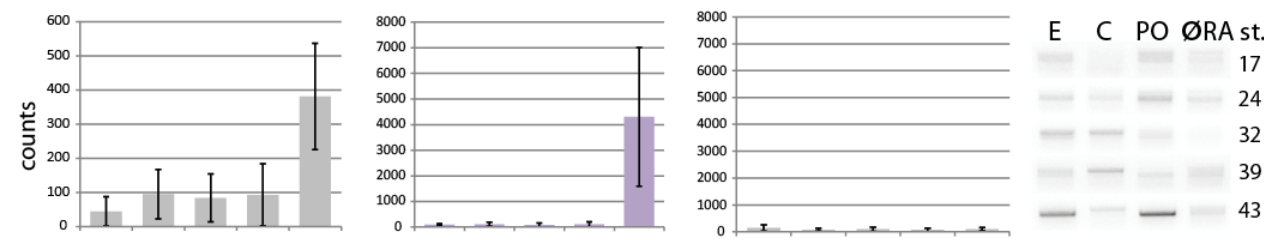

pancreatic fusion

Tm4sf3
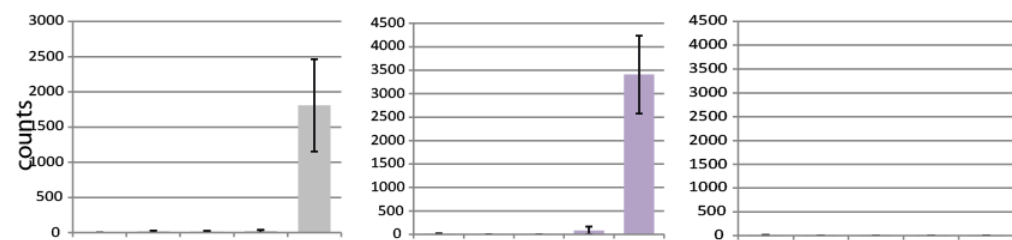

endocrine

Ngn3
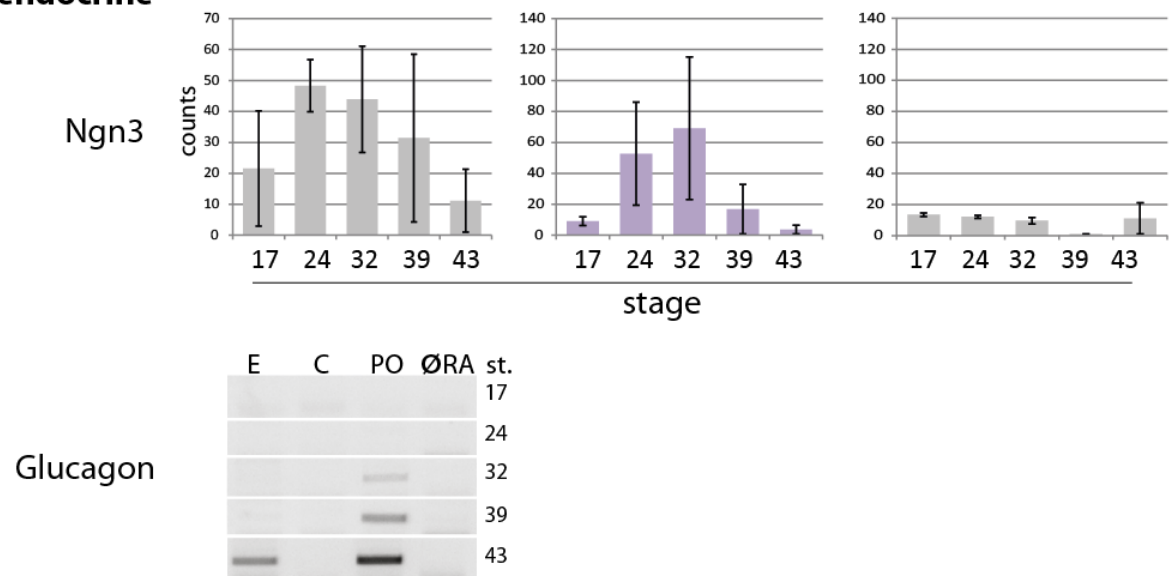

Fig. 6.2 Detection of marker genes for pancreatic structures

Nanostring analysis and RT-PCR results for whole embryos (embryo, E), Vegt/Noggin/RAprogramed explants (pancreatic organoids, PO), Vegt/Noggin/Cyp26a1-programed explants (ØRA) and un-programed explants (C) are shown. Diagrams indicate the mean of normalized Nanostring counts from two independent experiments. Figures on the right and at the bottom show RT-PCR results for indicated marker genes. 


\subsection{Identification, verification and expression characteristics of early RA-responsive genes}
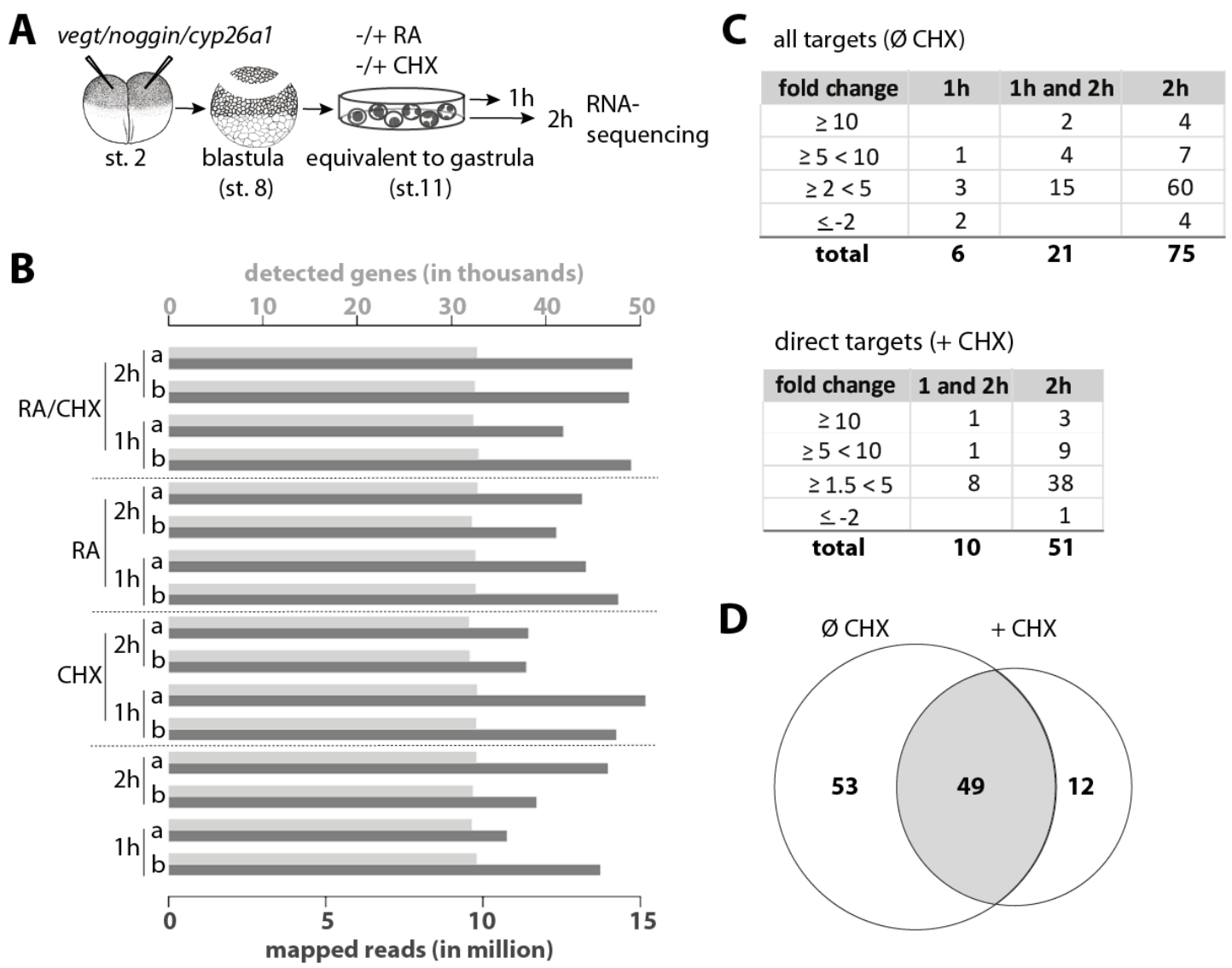

direct targets (+ $\mathrm{CHX}$ )

\begin{tabular}{|c|c|c|}
\hline fold change & $\mathbf{1}$ and $\mathbf{2 h}$ & $\mathbf{2 h}$ \\
\hline$\geq 10$ & 1 & 3 \\
$\geq 5<10$ & 1 & 9 \\
\hline $1.5<5$ & 8 & 38 \\
\hline$\leq-2$ & & 1 \\
\hline total & $\mathbf{1 0}$ & $\mathbf{5 1}$ \\
\hline
\end{tabular}

D

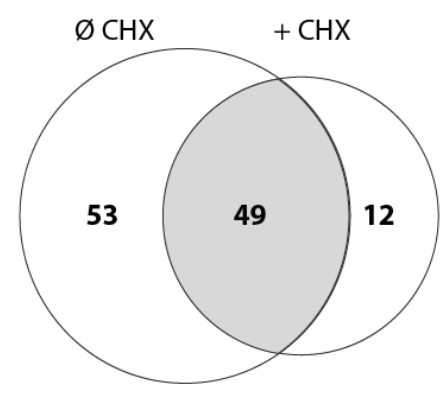

Fig. 6.3 Detection of early direct and indirect RA-target genes by RNAsequencing

(A) Vegt (500pg), noggin (500pg) and cyp26a1 (2000pg) RNAs were co-injected into the animal pole of a two-cell stage embryo. Blastocoel roof tissue was explanted at blastula stage. Explants were treated with $5 \mu \mathrm{M}$ RA and cycloheximide $(\mathrm{CHX})$ at the equivalent of gastrula stage. One and two hours after RA addition, total RNA was isolated and analyzed by RNA-sequencing. (B) Number of detected genes and mapped reads for each sample (a and $b$ indicate two independent experiments) are shown. (C) Fold changes are shown for differentially expressed genes in RA-treated versus untreated pancreatic organoids. Tables are shown indicating all targets (absence of $\mathrm{CHX}$ ) and putative direct targets (presence of $\mathrm{CHX).} \mathrm{(D)} \mathrm{Comparison} \mathrm{of} \mathrm{differentially} \mathrm{expressed} \mathrm{genes} \mathrm{in} \mathrm{the} \mathrm{presence} \mathrm{versus} \mathrm{absence} \mathrm{of}$ $\mathrm{CHX}$. The overlap indicates the number of putative direct RA-targets. 


\section{A RA-inducibility}
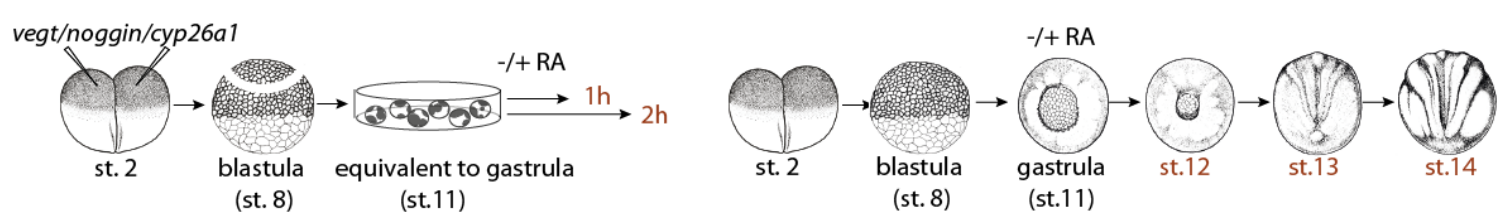

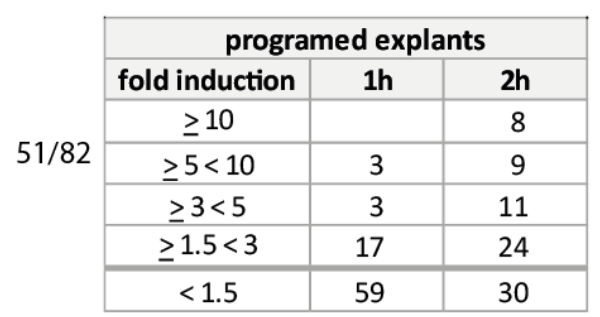

\begin{tabular}{|c|c|c|c|c|}
\hline \multirow{7}{*}{$48 / 82$} & \multicolumn{4}{|c|}{ whole embryos } \\
\hline & fold induction & st.12 & st.13 & st.14 \\
\hline & $\geq 10$ & 2 & 1 & 2 \\
\hline & $\geq 5<10$ & 2 & 5 & 3 \\
\hline & $\geq 3<5$ & 9 & 8 & 11 \\
\hline & $\geq 1.5<3$ & 26 & 31 & 26 \\
\hline & $<1.5$ & 43 & 37 & 40 \\
\hline
\end{tabular}

\section{B RA-dependency}
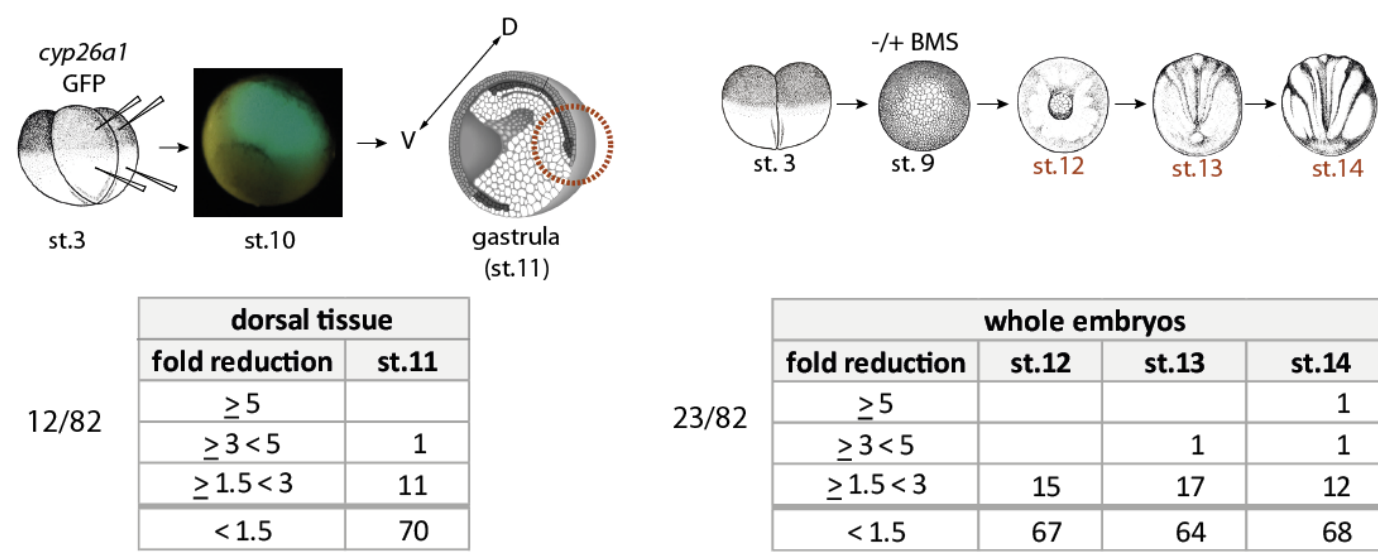

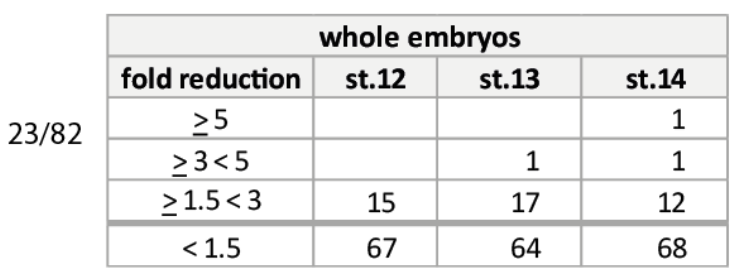

\section{Fig. 6.4 Confirmation of RA-responsiveness}

(A) RA-inducibility was analyzed using two different experimental approaches. Ectodermal explants from vegt/nog/cyp26a1-injected embryos or whole embryos were treated with RA for one hour or left untreated. Candidate gene expression was analyzed by the use of quantitative Nanostring analysis for the time points indicated in red.

(B) RA-dependency was determined by the use of two different concepts. Embryos were injected with RNAs encoding Cyp26a1 (2000 pg) and GFP (200 pg) into the two dorsal blastomers of a four-cell stage embryo. At stage 10, dorsal GFP-positive embryos were selected. At stage 11 the tissue sourrounding the dorsal blastopore lip (red dotted line = dorsal part) was resected and again verified for a GFP signal. In total, 10 dorsal tissues from injected and un-injected embryos were analyzed. Additionally, embryos were treated with the RA-antagonist BMS453 at stage 9. Five embryos of stages 12 to 14 were analyzed. Numbers in front of the tables indicate confirmed candidates. The tables show number of genes and their level of induction or reduction for the individual approaches analyzed by Nanostring. 


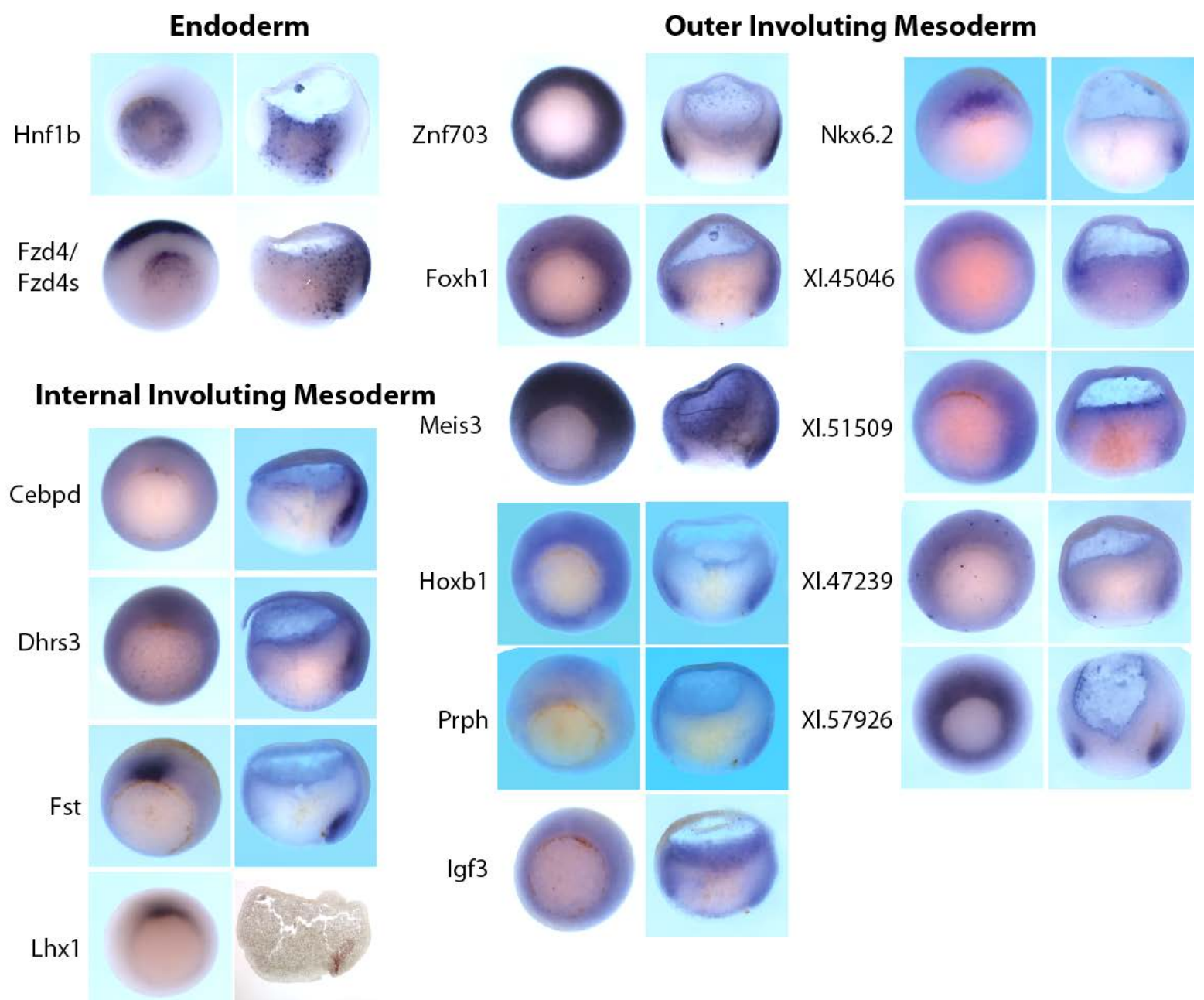

Neuroectoderm and Outer Involuting Mesoderm
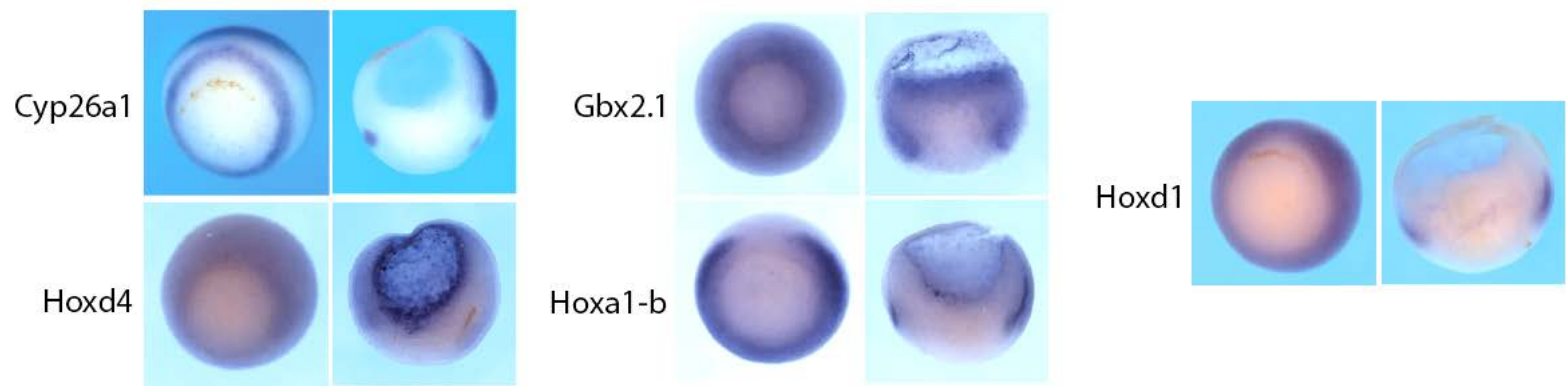

Fig. 6.5 Expression of RA-responsive genes at gastrula stage by WMISH

Expression pattern of the 22 confirmed RA-responsive genes at gastrula stage 11 by WMISH. On the left side, whole embryos (dorsal side up) and on right side bisected embryos (dorsal side right) are shown. Candidates were grouped according to their expression domains in four groups. Hnf $1 \mathrm{~b}$ and Fzd4 exhibit an endodermal expression. Cebpd, Dhrs3, Fst and Lhx1 are expressed in the dorsal internal involuting mesoderm. Znf703, Foxh1, Meis3, Hoxb1, Prph, Igf3, Nkx6.2, XI.45046, XI.51509, XI.47239 and XI.57926 are expressed in the outer involuting mesoderm. Cyp26a1, Hoxd4, Gbx2.1, Hoxa1-b and Hoxd1 are expressed in both, prospective neuro-ectoderm and outer involuting mesoderm. 
A

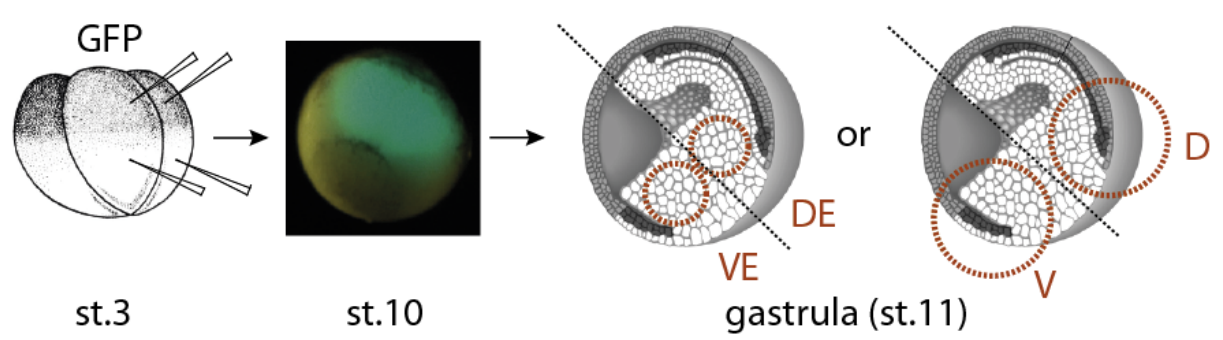

B endoderm

mesoderm

ectoderm
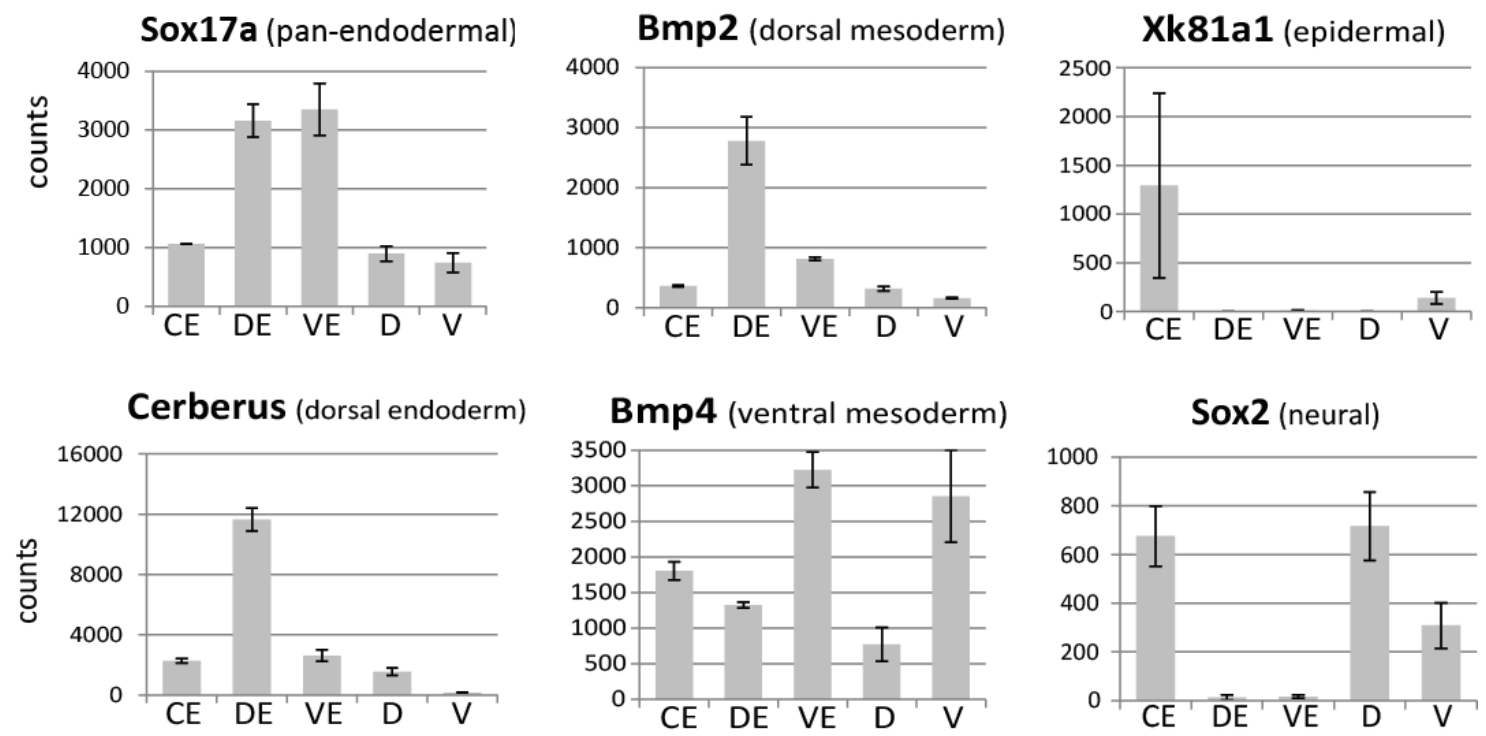

Fig. 6.6 Expression characteristics of endodermal, mesodermal and ectodermal markers at gastrula stage by Nanostring analysis

(A) Four-cell stage embryos were injected with RNA coding for GFP into the two dorsal blastomeres. At stage 10, embryos with a GFP signal at the dorsal site were selected for further cultivation. At stage 11, either dorsal and ventral endoderm was isolated or the whole tissue surrounding the dorsal blastoporus lip and the corresponding ventral tissue were isolated. (B) Graphs show Nanostring counts for endodermal, mesodermal and ectodermal marker genes for the different preparations of two independent experiments. $\mathrm{CE}=$ control embryo, $\mathrm{DE}=$ dorsal endoderm, $\mathrm{VE}=$ ventral endoderm, $\mathrm{D}=$ dorsal part, $\mathrm{V}=$ ventral part 


\section{A dorsally enriched}
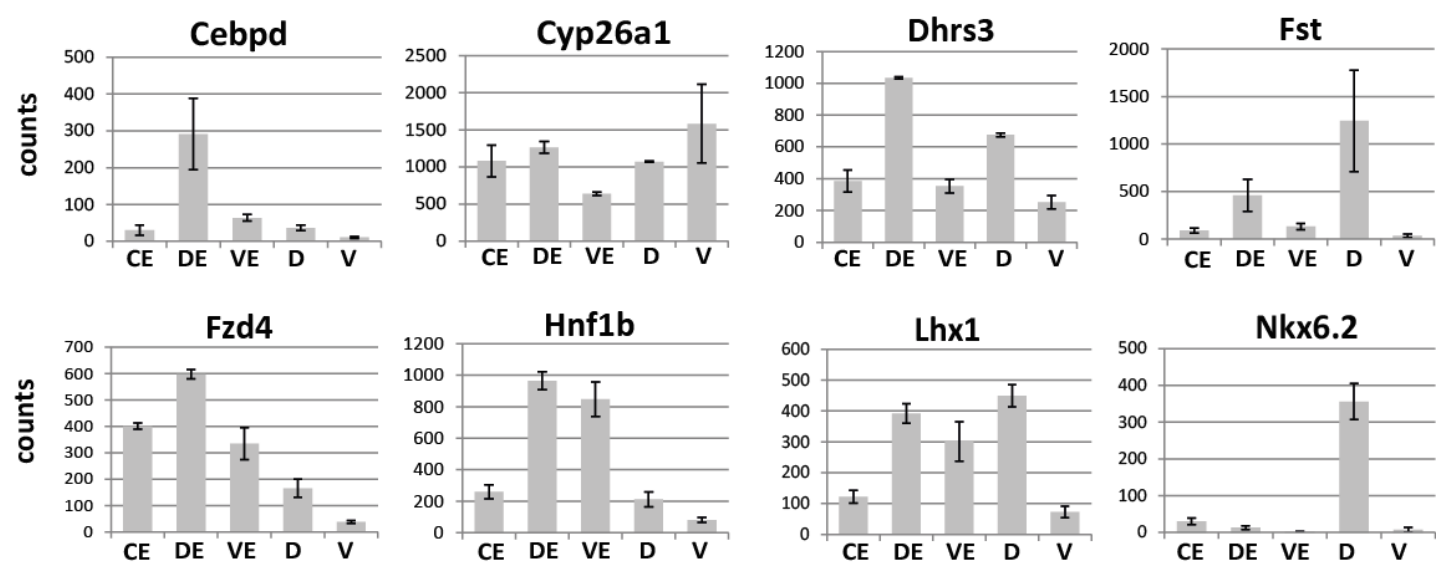

B ventrally enriched
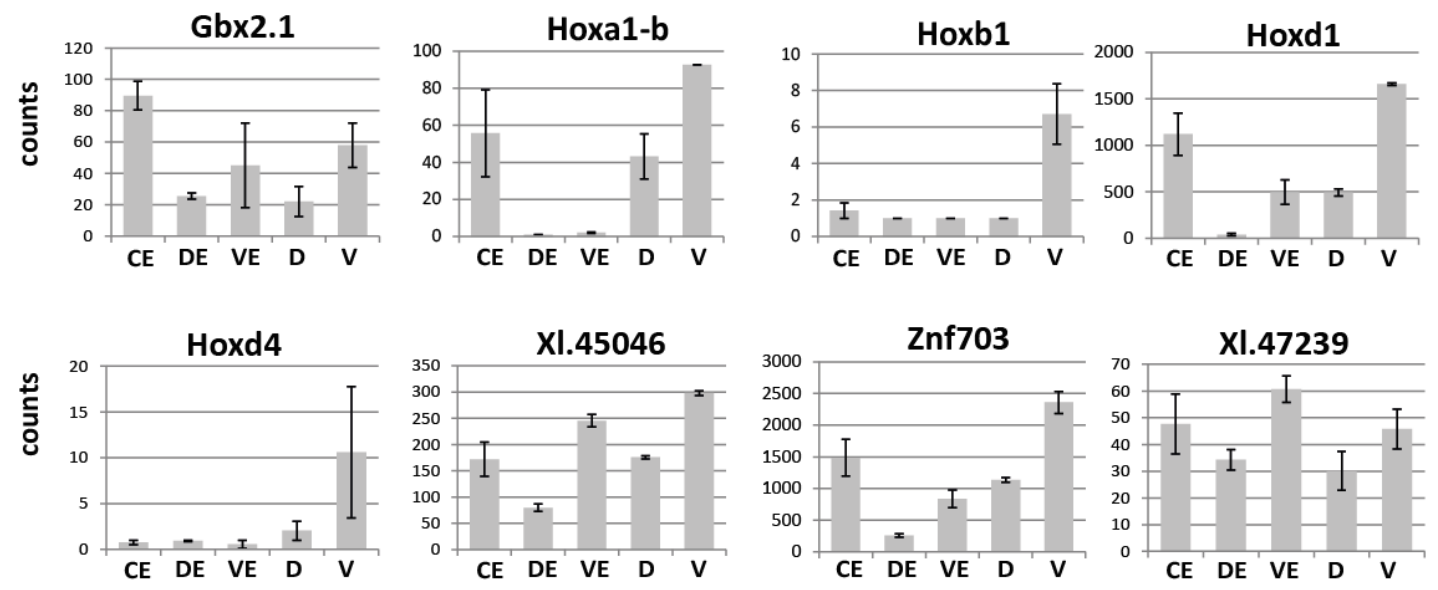

\section{C equally distributed}
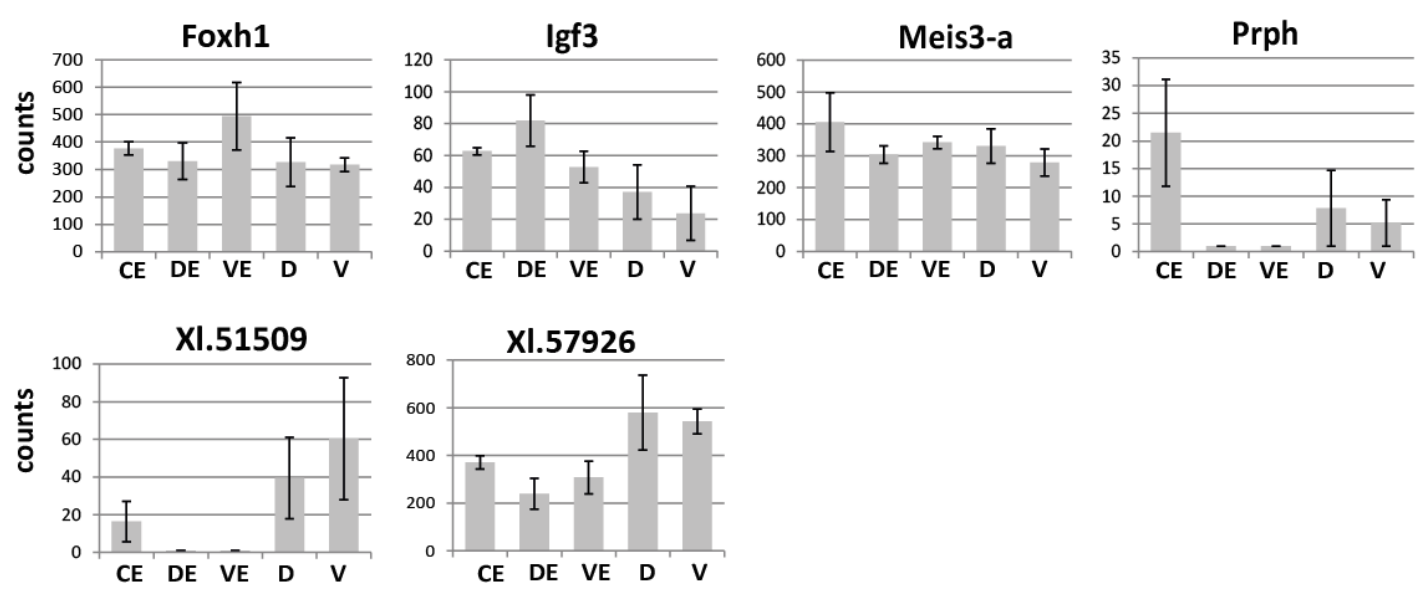

Fig. 6.7 Expression characteristics of RA-responsive genes at gastrula stage by Nanostring analysis

Samples were prepared like described in Fig. 6.6. The 22 RA-responsive genes were grouped according to a dorsal (A) or ventral enrichment (B) in the different preparations or for their equal distribution (C). Graphs indicate Nanostring counts for two independent experiments. Error bars indicate the SEM. DE=dorsal endoderm; VE= ventral endoderm; $\mathrm{D}=$ dorsal blastopore; $\mathrm{V}=$ ventral blastopore 


\subsection{Expression and functional analysis of the RA-target Hnf1b}

A

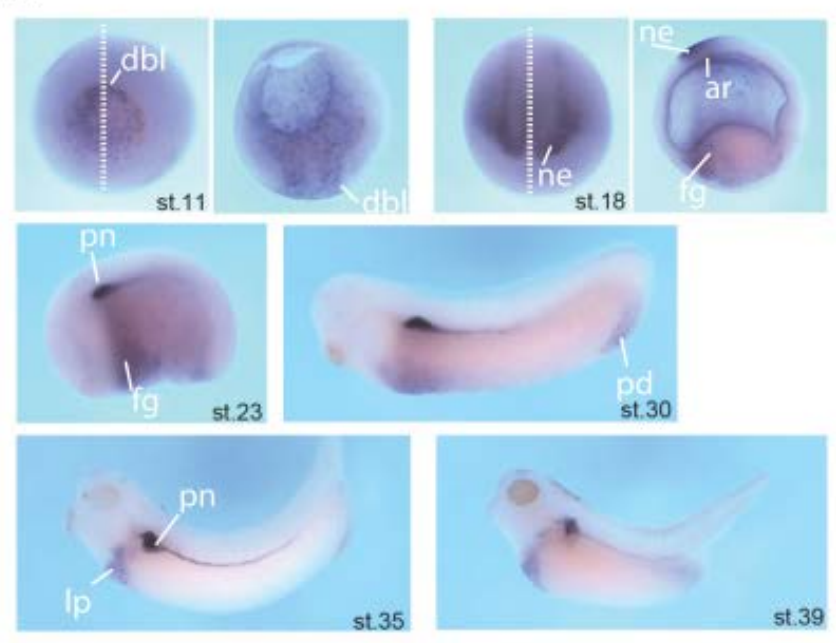

C

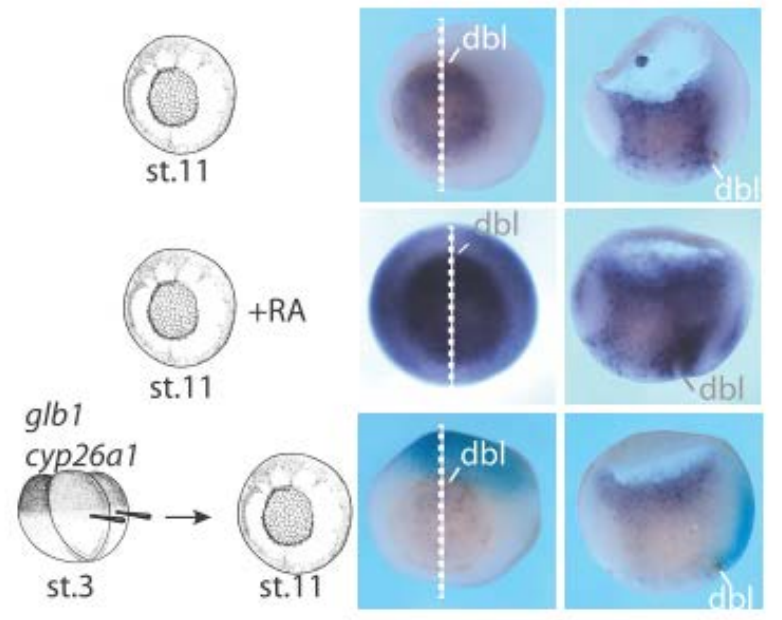

B

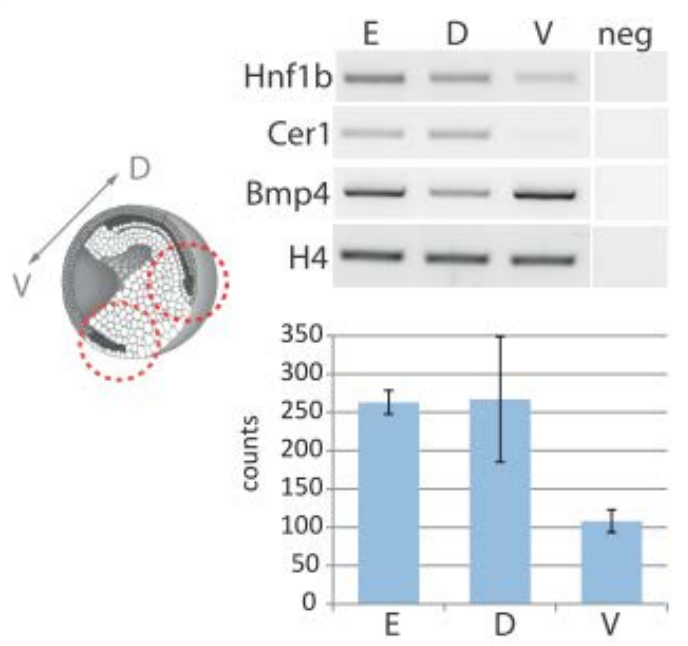

D
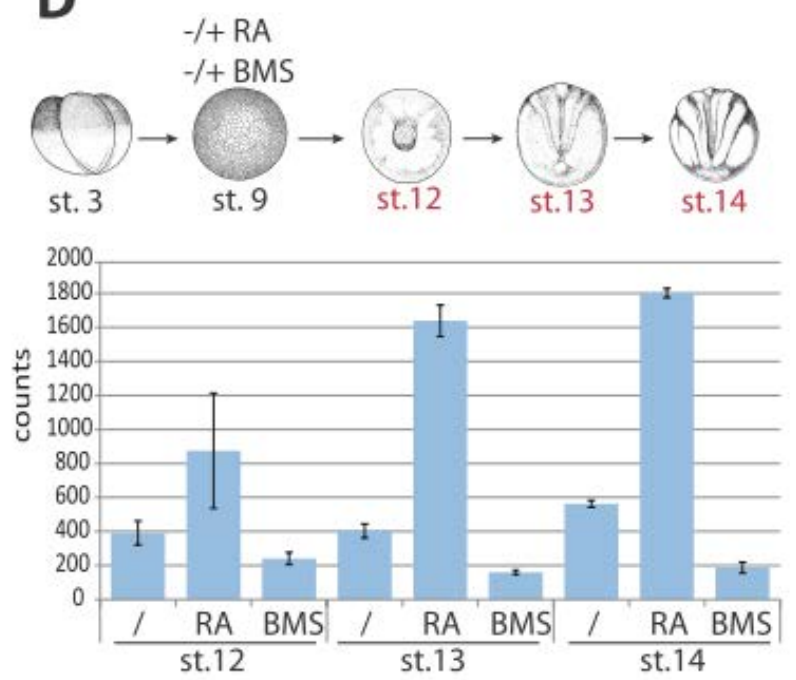

Fig. 6.8 RA-responsive expression of Hnf1b in the dorsal endoderm

(A) Expression pattern of Hnf1b during embryogenesis. WMISH against Hnf1b in embryos at gastrula stage (stage 11), neurula stage (stage 18) and at stages 23 to 39 are shown. (B) Enrichment of Hnf1b in the dorsal part. The tissue surrounding the dorsal blastopore lip and the corresponding ventral half were isolated from 10 gastrula stage embryos. Hnf1b transcripts were detected by RTPCR and Nanostring analysis and were compared to the levels in whole embryos. Cerberus (Cer1) was used as dorsal anterior marker and bmp4 as ventral marker. $E=$ whole embryo, $D=$ dorsal tissue, $\mathrm{V}=$ ventral tissue (C) The expression domain of Hnf1b in the dorsal endoderm during gastrulation is RA-responsive. WMISH of untreated, RA-treated and cyp26a1(2000pg)/g/b1(200pg)injected gastrula stage embryos stained with X-Gal. (D) Quantitative analysis of Hnf1b RAresponsiveness in whole embryos at stages 11,12 and 13 (five embryos each) treated with RA or BMS453. dorsal blastopore lip $=\mathrm{dbl}$, neuroectoderm $=$ ne, archenteron roof $=$ ar, prospective foregut $=\mathrm{fg}$, pronephros $=\mathrm{pn}$, liver primordium $=\mathrm{lp}$, proctodeum $=\mathrm{pd}$ 
A

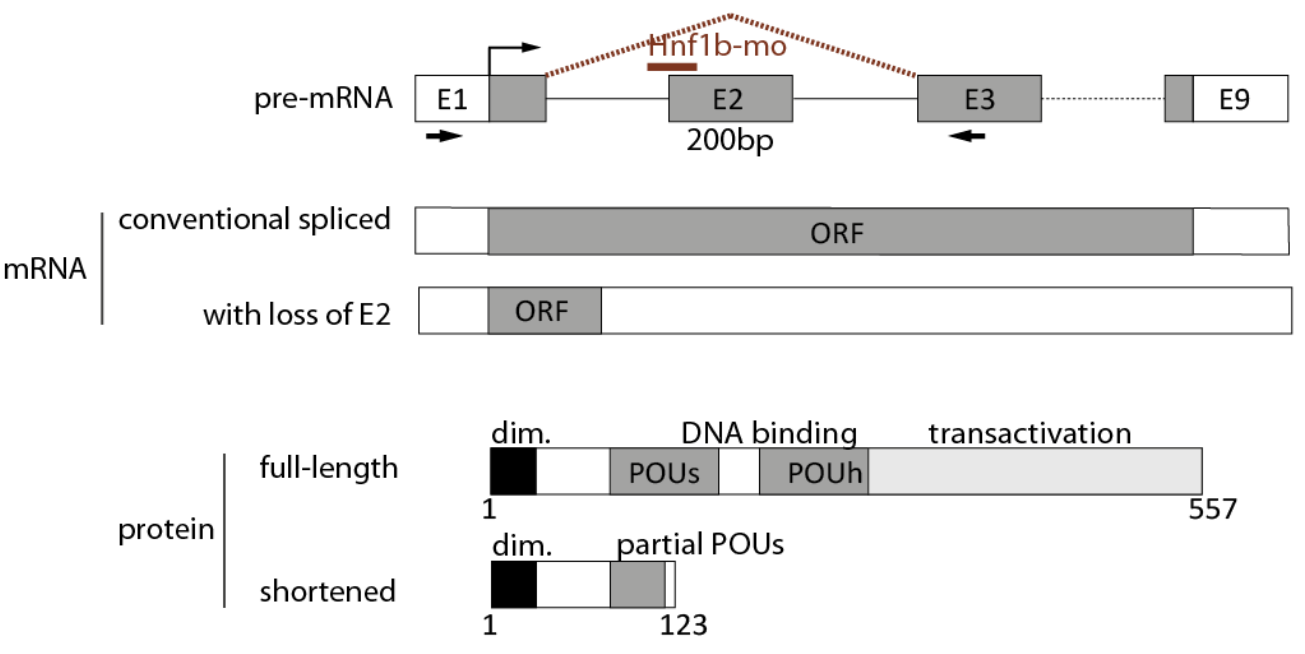

B

C
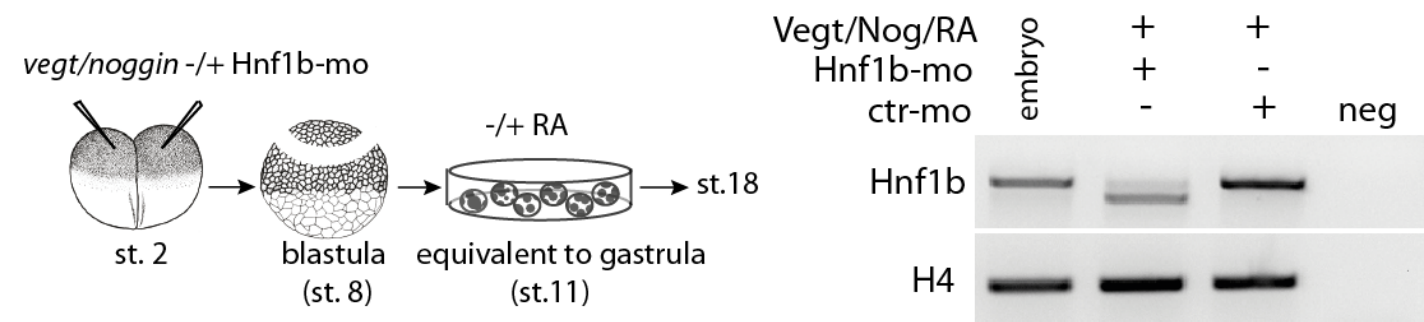

Fig. 6.9 Knockdown of Hnf1b through loss of exon 2

(A) Hnf1b-morpholino (Hnf1b-mo) targets the intron1/exon2 boundary (E1/E2) in Hnf1b premRNA. Conventional splicing is prevented and exon 2 is retained. This leads to a shortened open reading frame (ORF) in the Hnf1b mRNA. A shortened Hnf1b protein is translated that lacks functional DNA-binding domains (POUs and POUh) and the transactivation domain. (B) Functionality of the Hnf1b-morpholino was tested. The Hnf1b-morpholino (55ng) or the control-morpholino (55ng) (ctr-mo) were co-injected with vegt and noggin RNA into two-cell stage embryos. At blastula stage, blastocoel roof tissue was explanted, cultivated and treated with RA at the equivalent of gastrula stage. At the equivalent of stage 18, total RNA was isolated and subjected to RT-PCR. (C) RT-PCR result with amplified Hnf1b cDNA using the oligonucleotides that bind to exon 1 and exon3 (indicated as arrows in (A)). Hnf1b amplicon is shifted to smaller size in the presence of the Hnf1b-morpholino, indicating the loss of exon2. 


\subsection{Expression and functional analysis of RA-target Fzd4/Fzd4s}
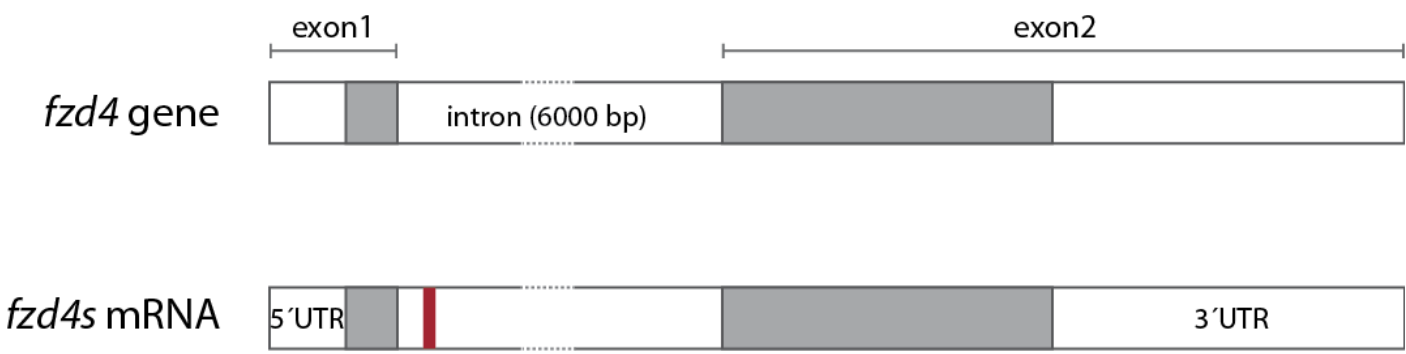

protein $\quad$ CRD

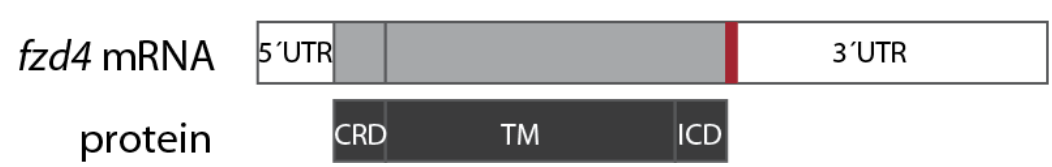

\section{Fig. 6.10 Fzd4 has an alternative splice variant Fzd4s}

The Fzd4 gene consists of two exons and one large intronic region. Conventional splicing results in the loss of the intron and a protein is generated containing a small extracellular cysteine-rich domain (CRD), a seven transmembrane domain (TM) and an intracellular domain (ICD) (Shi and Boucaut, 2000). Through alternative splicing, the intron sequence is retained. Thereby, a premature stop codon in the intron is used resulting in a shortened open reading frame. The alternative splice variant Fzd4s gives rise to a secreted protein containing the Wnt-ligand binding cysteine-rich domain (Swain et al., 2005). 

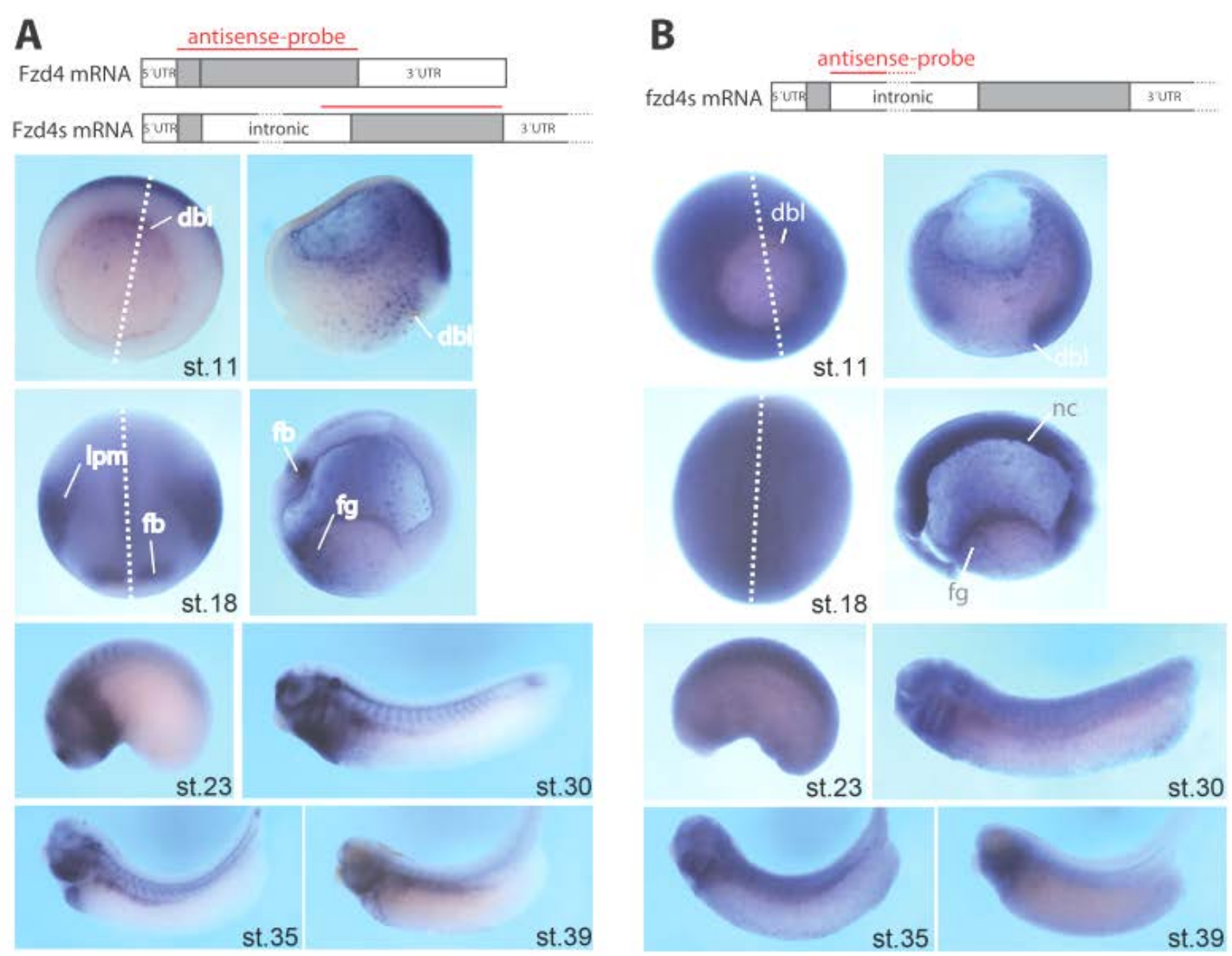

st.11
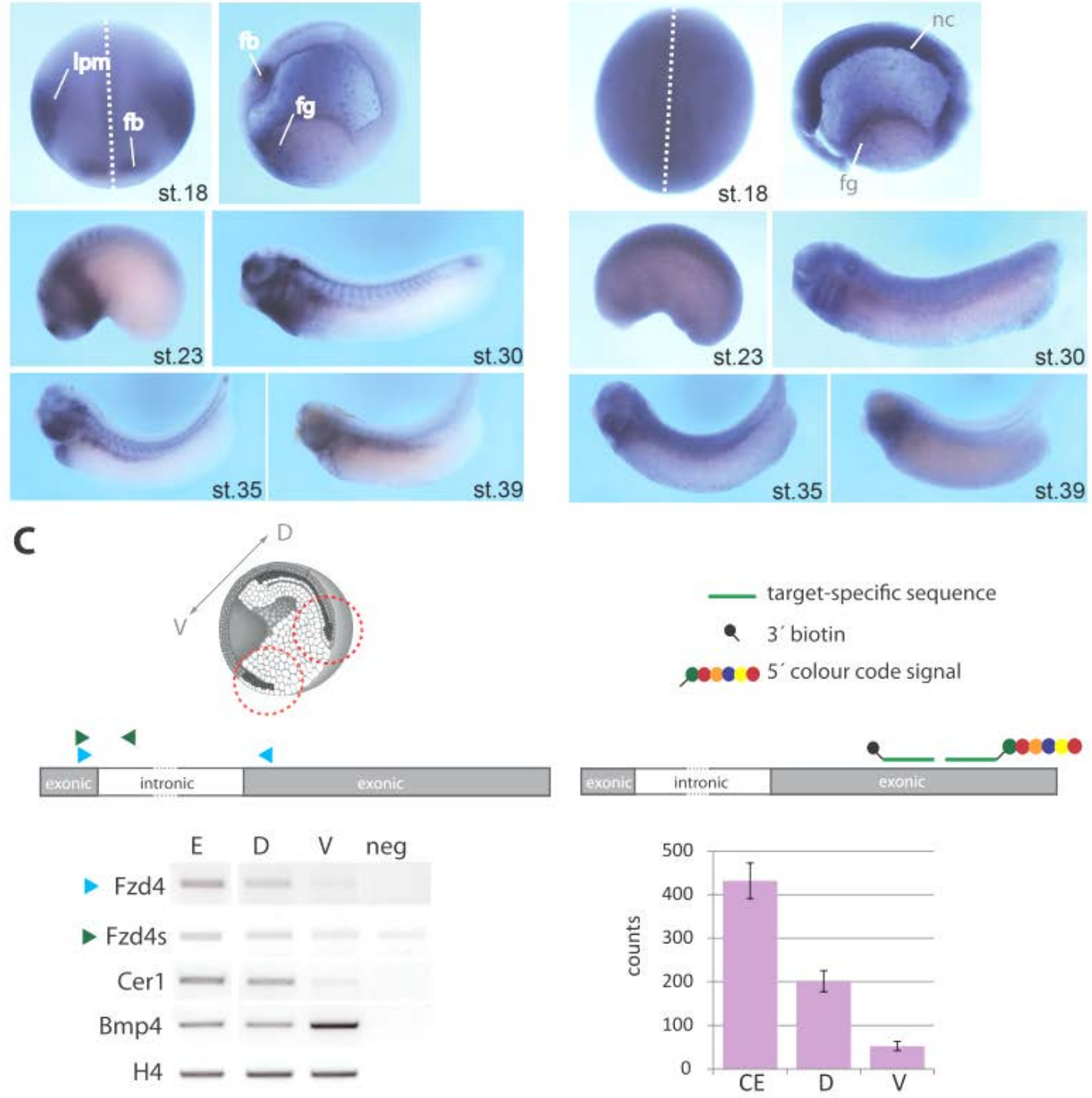

Fig. 6.11 Fzd4/Fzd4s expression analysis

(A) WMISH using an antisense probe that targets transcripts of both splice variants, Fzd4 and Fzd4s. (B) WMISH with an antisense probe that specifically targets the alternative splice variant Fzd4s. Embryos at indicated developmental stages were used. (C) Gastrula stage embryos were used for preparations of the tissue surrounding the dorsal blastopore lip and the corresponding ventral part. RT-PCR for Fzd4 and Fzd4s using the indicated oligonucleotides is shown. Cerberus (Cer1) was used as dorsal anterior marker and bmp4 as ventral marker. Nanostring analysis was done to quantify the dorsal enrichment of Fzd4/Fzd4s.

$\mathrm{E}=$ whole embryo, $\mathrm{D}=$ dorsal tissue, $\mathrm{V}=$ ventral tissue, dorsal blastopore lip $=\mathrm{dbl}$, lateral plate mesoderm $=\mathrm{lpm}$, forebrain $=\mathrm{fb}$, foregut $=\mathrm{fg}$, notochord $=\mathrm{nc}$ 
A

\begin{tabular}{l|l|l|}
\multicolumn{3}{c}{ fzd4 mRNA 5 antisense-probe } \\
\cline { 2 - 3 } 5UTR & 3'UTR \\
\cline { 1 - 3 }
\end{tabular}

fzd4s mRNA
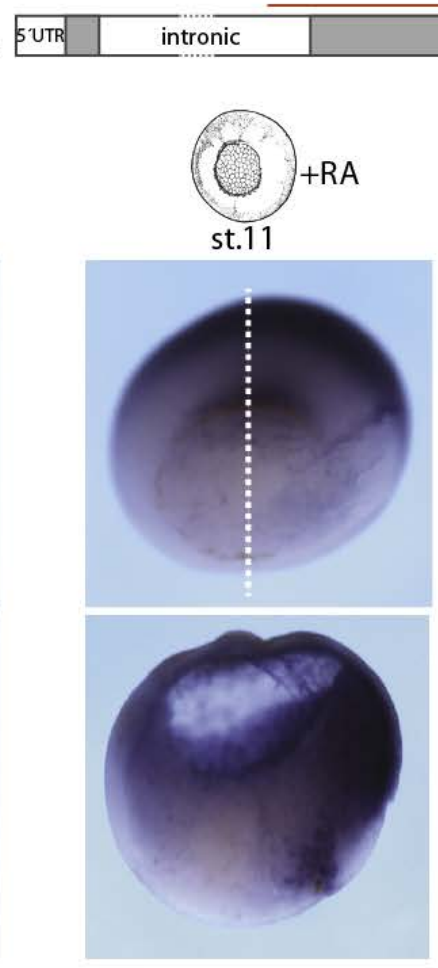
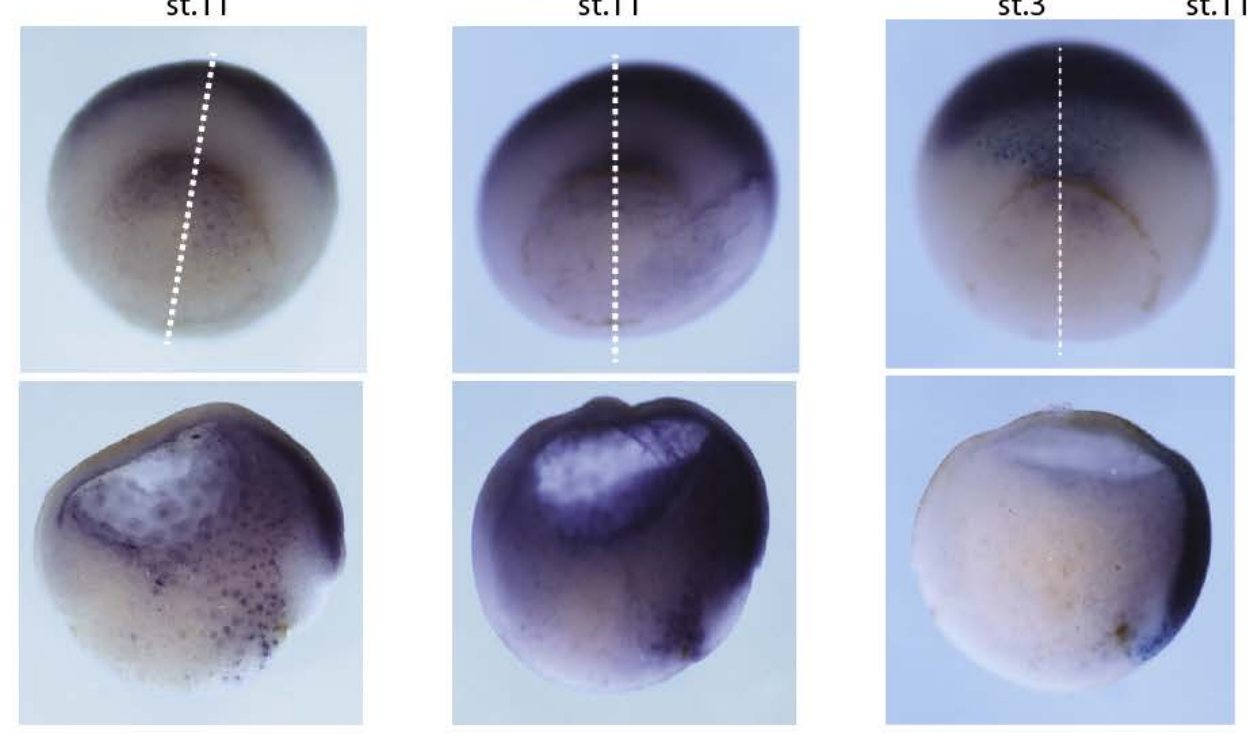

B
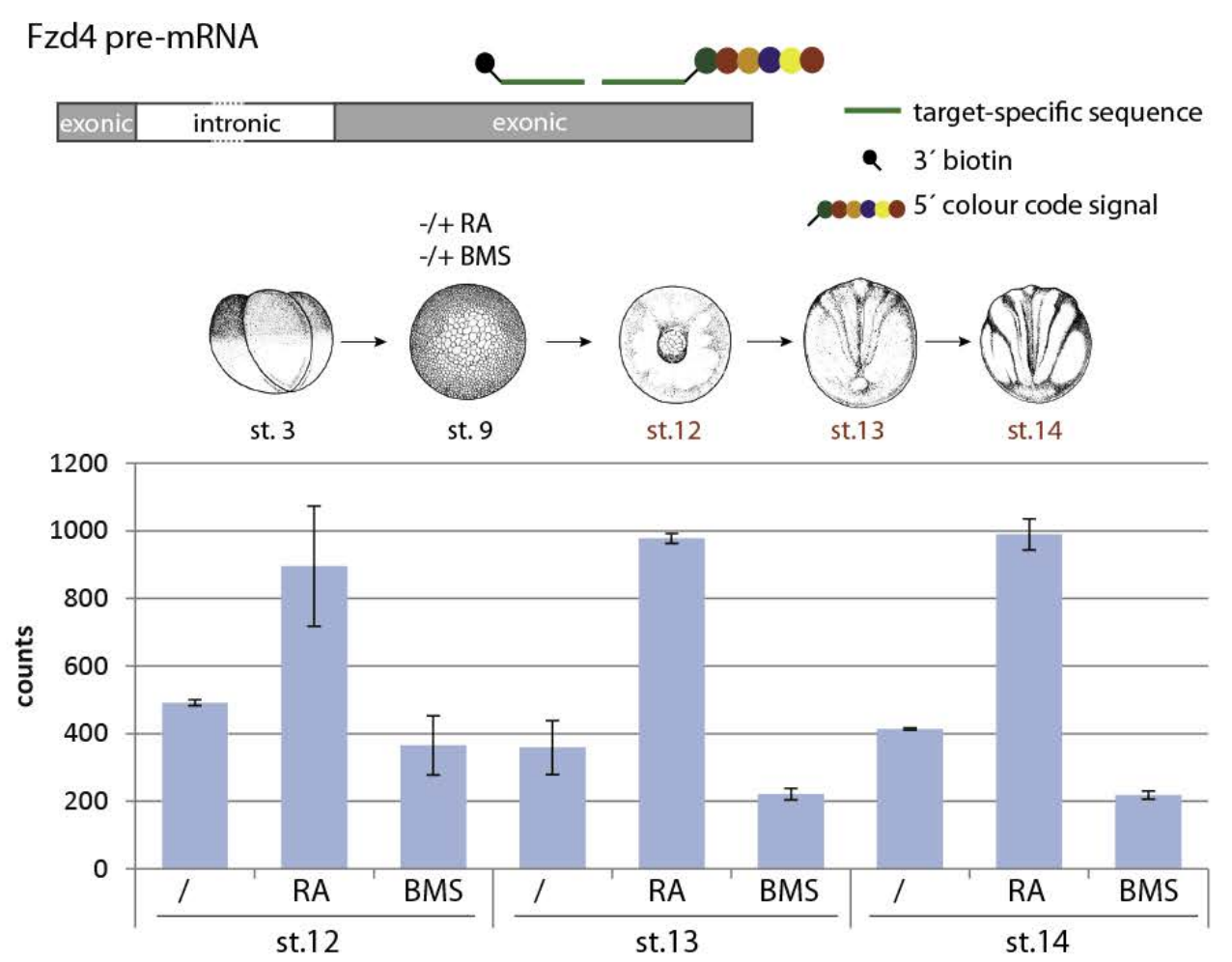

Fig. 6.12 RA-responsive expression of Fzd4/Fzd4s

(A) WMISH against Fzd4/Fzd4s during gastrulation in untreated, RA-treated and cyp26a1injected embryos. Upper images show whole embryos (dorsal side up) and lower images show bisected embryos (dorsal side right). (B) Nanostring analysis of untreated, RA- and BMS-treated embryos. Indicated is the number of counts for Fzd4/Fzd4s transcripts in embryos at stages 12, 13 and 14 . 
A

Cas9

\begin{tabular}{|c|c|c|c|c|c|c|}
\hline VT & AGCGGCGCTG & & & & & \\
\hline 1 & AGCGGCGCTG & CGACCCCATC & AGGATCACCA & TGTGCCAGAA & CCTCGGCTAC & \\
\hline 2 & AGCGGCGCTG & CGACCCCATC & AGGATCACCA & TGTGCCAGAA & CCTCGGCTAC & $\| G I C A C$ \\
\hline 3 & AGCGGCGCTG & CGACCCCATC & AGGATCACCA & TGTGCCAGAA & CCTCGGCTAC & \\
\hline 4 & AGCGGCGCTG & CGACCCCATC & AGGATCACCA & TGTGCCAGAA & CCTCGGCTAC & \\
\hline 5 & AGCGGCGCTG & CGACCCCATC & AGGATCACCA & TGTGCCAGAA & CCTCGGCTAC & \\
\hline 6 & AGCGGCGCTG & CGACCCCATC & AGGATCACCA & TGTGCCAGAA & CCTCGGCTAC & I t \\
\hline 7 & AGCGGCGCTG & CGACCCCATC & AGGATCACCA & TGTGCCAGAA & CCTCGGCTAC & CAC \\
\hline 8 & AGCGGCGCTG & CGACCCCATC & AGGATCACCA & TGTGCCAGAA & CCTCGGCTAC & \\
\hline 9 & AGCGGCGCTG & CGACCCCATC & AGGATCACCA & TGTGCCAGAA & CCTCGGCTAC & \\
\hline & AGCGGGGCTG & CGAGCCCATC & AGGATCACCA & TGTG & $\mathrm{GC}$ & \\
\hline & AGCGGCGCTG & CGACCCCATC & AGGATCACCA & TGTGCCAGA & CCTCG & \\
\hline
\end{tabular}

Cas9 + Fzd4-gRNA

\begin{tabular}{|c|c|c|c|c|c|c|c|}
\hline $\begin{array}{l}\text { WT } \\
1 \\
2\end{array}$ & $\begin{array}{l}\text { AGCGGCGCTG } \\
\text { AGCGGCGCTG } \\
\text { AGCGGCGCTG }\end{array}$ & $\begin{array}{l}\text { CGACCCCATC } \\
\text { CGACCCCA- } \\
\text { CGACCCCA- }\end{array}$ & $\begin{array}{l}\text { AGGATCACCA } \\
\text { - GGATCACCA } \\
\text { - GGATCACCA }\end{array}$ & $\begin{array}{l}\text { TGTGCCAGAA } \\
\text { TGTGCCAGAA } \\
\text { TGTGCCAGAA }\end{array}$ & $\begin{array}{l}\text { CCTCGGCTAC } \\
\text { CCTCGGCTAC } \\
\text { CCTCGGCTAC }\end{array}$ & $\begin{array}{l}\text { AATGTCACCA } \\
\text { AATGTCACCA } \\
\text { AATGTCACCA }\end{array}$ & $\begin{array}{l}-3 \\
-3\end{array}$ \\
\hline 3 & AGCGGCGCTG & CGACCCCAT. & - GGATCACCA & TGTGCCAGAA & CCTCGGCTAC & AATGTCACCA & \\
\hline 4 & AGNGGCGCTG & NGNCCCCA.- & $\ldots \ldots$ & TGTGCCAGAA & $T A C$ & AATGTCACCA & \\
\hline 5 & AGCGGCGC & A - : & & TGTGCCAGAA & TAC & CA & -1 \\
\hline 6 & AGCGGCGC & $C \ldots$ & $\ldots \ldots$ & $\cdots G A A$ & ССTCGGCTAC & AATGTCACCA & -20 \\
\hline 7 & AGC & & $\cdots$ & $\ldots \ldots \ldots$ & $\ldots \ldots \ldots$ & $A T G T C A C C A$ & -34 \\
\hline 8 & AGCGGCGCTG & & & & $\ldots .$. & $\cdots$ & 140 \\
\hline 9 & AGCGGCGCTG & $c \cdots$ & $\cdots \cdots$ & $\cdots \cdots$ & $\cdots \cdots$ & $\cdots \ldots$ & -140 \\
\hline 10 & AGCGGCGCTG & CGACYCM - . & $\cdots \cdots$ & $\cdots \cdots$ & $\cdots \cdots$ & $\cdots \cdots$ & -142 \\
\hline 11 & AGCGGCGCTG & CGACMCN $\cdots$ & $\cdots \cdots$ & $\cdots \cdots$ & $\cdots \cdots$ & $\cdots \cdots$ & -140 \\
\hline 12 & AGCGGCGCTG & CGACCCC . & $\ldots \ldots$ & $\ldots \ldots$ & $\ldots \ldots$ & $\ldots \ldots \ldots$ & -140 \\
\hline
\end{tabular}

B $\underline{\text { Cas } 9}$

\begin{tabular}{|c|c|c|c|c|c|c|}
\hline & & & & & & \\
\hline & & & & & & \\
\hline 2 & GC & AA & CT & T G & GG & C \\
\hline $\begin{array}{l}3 \\
4\end{array}$ & $\begin{array}{l}\text { ACACAGC } \\
\text { ACACAGC }\end{array}$ & & $\begin{array}{l}\text { TG } \\
\text { TG }\end{array}$ & & & \\
\hline & & & & & & $A C$ \\
\hline & $\begin{array}{l}\text { ACCACACAGC } \\
\text { ACCACACAGC }\end{array}$ & $\begin{array}{l}\text { ACT TGTACAA } \\
\text { ACTTGTACAA }\end{array}$ & $\begin{array}{l}\text { TGCTCAC } \\
\text { TGCTCAC }\end{array}$ & $\begin{array}{l}\text { TTG } \\
T \text { T }\end{array}$ & $\begin{array}{l}\text { GG } \\
\text { GG }\end{array}$ & \\
\hline & $A C C A C A C A G C$ & ACT TGTACAA & TGCTCAGTCT & GATCCTGATG & GGGAGCTAGG & GCTGGGCAAC \\
\hline
\end{tabular}

\section{Cas9+Fzd4-gRNA}

\begin{tabular}{|c|c|c|c|c|c|c|}
\hline & $\begin{array}{l}\text { ACCACACAGC } \\
A C C A C A C A G C \\
A C C A C A C A G C\end{array}$ & $\begin{array}{l}\text { CT TGTACAA } \\
\text { CT TGTACAA } \\
\text { CT TGTACAA }\end{array}$ & $\begin{array}{l}\text { TGCTCAGTCT } \\
\text { TGCTCAGTCT } \\
\text { TGCTCAGTCT }\end{array}$ & $\begin{array}{l}\text { GATCCTGATG } \\
\text { GATCCTGATG } \\
\text { GATCCTGATG }\end{array}$ & $\begin{array}{l}\text { GGGAGCTAGG } \\
\text { GGGAGCTAGG } \\
\text { GGGAGCTAGG }\end{array}$ & $A C$ \\
\hline & & & TCT & & & \\
\hline & & & & & & \\
\hline & & & & & & \\
\hline 6 & & & & & & \\
\hline 7 & & & & & & \\
\hline $\begin{array}{l}8 \\
9\end{array}$ & $\begin{array}{l}\text { CCACACAGC } \\
\text { CCACACAGC }\end{array}$ & $\begin{array}{l}\text { АСT T } \\
\text { АСT T }\end{array}$ & & & & \\
\hline 10 & CCACACAGC & ACT TGTACAA & TGCTCAGTCT & GATCCTGATG & GGGAGCTAGG & GCTGGGCAAC \\
\hline
\end{tabular}

Fig. 6.13 Mutation analysis of CRISPR/Cas-treated pancreatic organoids by DNA sequencing

The Fzd4-gRNA target sequence is yellow highlighted and number of deleted nucleotides is indicated.

(A) Cloned Fzd4 amplicons from Cas9 only or Cas9 with Fzd4-gRNA treated pancreatic organoids aligned to genomic Fzd4 X.leavis sequence. (B) Cloned Kremen2 amplicons from treated pancreatic organoids aligned to genomic Kremen2 X.leavis sequence. 


\subsection{Nanostring analysis data for in vitro generation of pancreatic organoids}

\section{Tab. 6.1 Normalized data of two independent experiments}

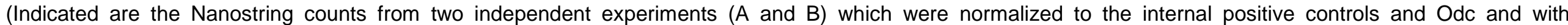
substracted mean of the negative controls as described in the "Materials and Methods" section).

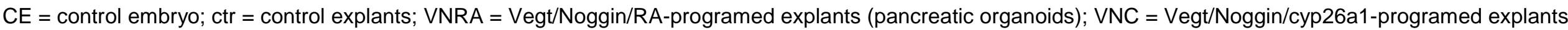

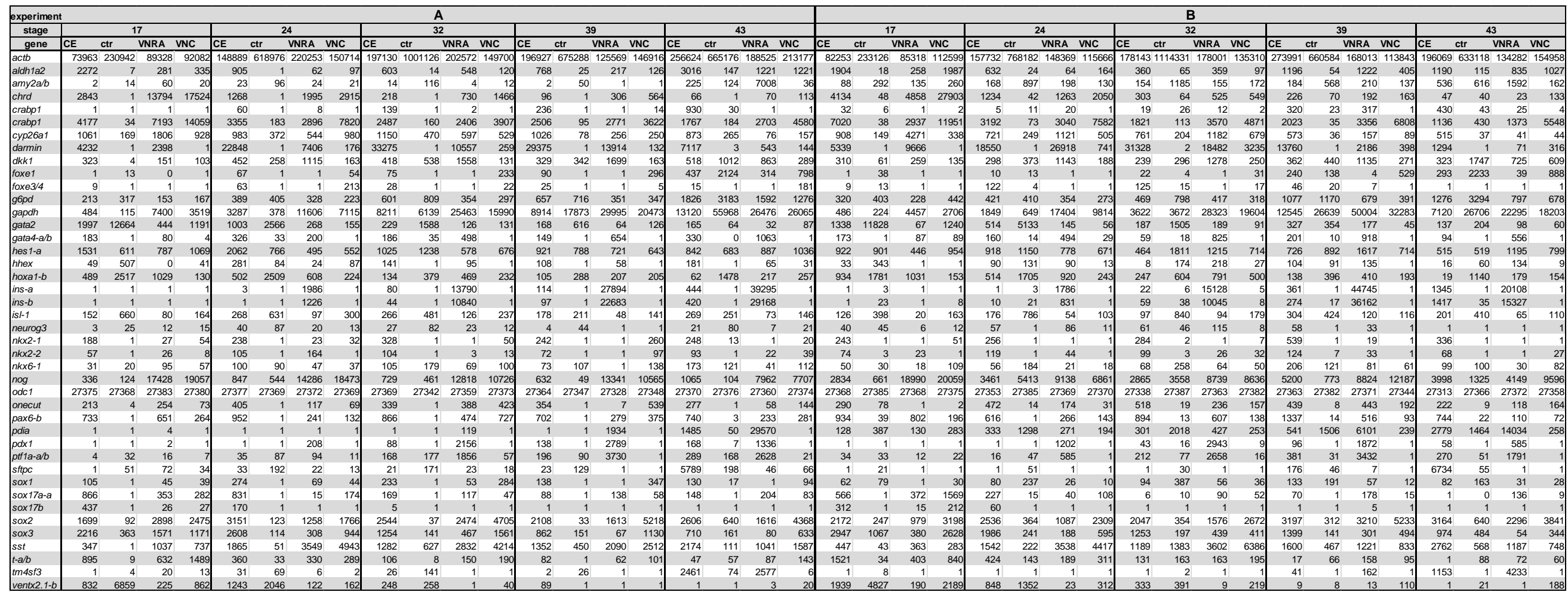


Tab. 6.2 Calculated mean and standard error of mean (SEM)

(Mean and SEM were calculated from the normalized Nanostring counts of two independent experiments)

$\mathrm{CE}=$ control embryo; $\mathrm{ctr}=$ control explants; VNRA = Vegt/Noggin/RA-programed explants (pancreatic organoids); VNC = Vegt/Noggin/cyp26a1-programed explants

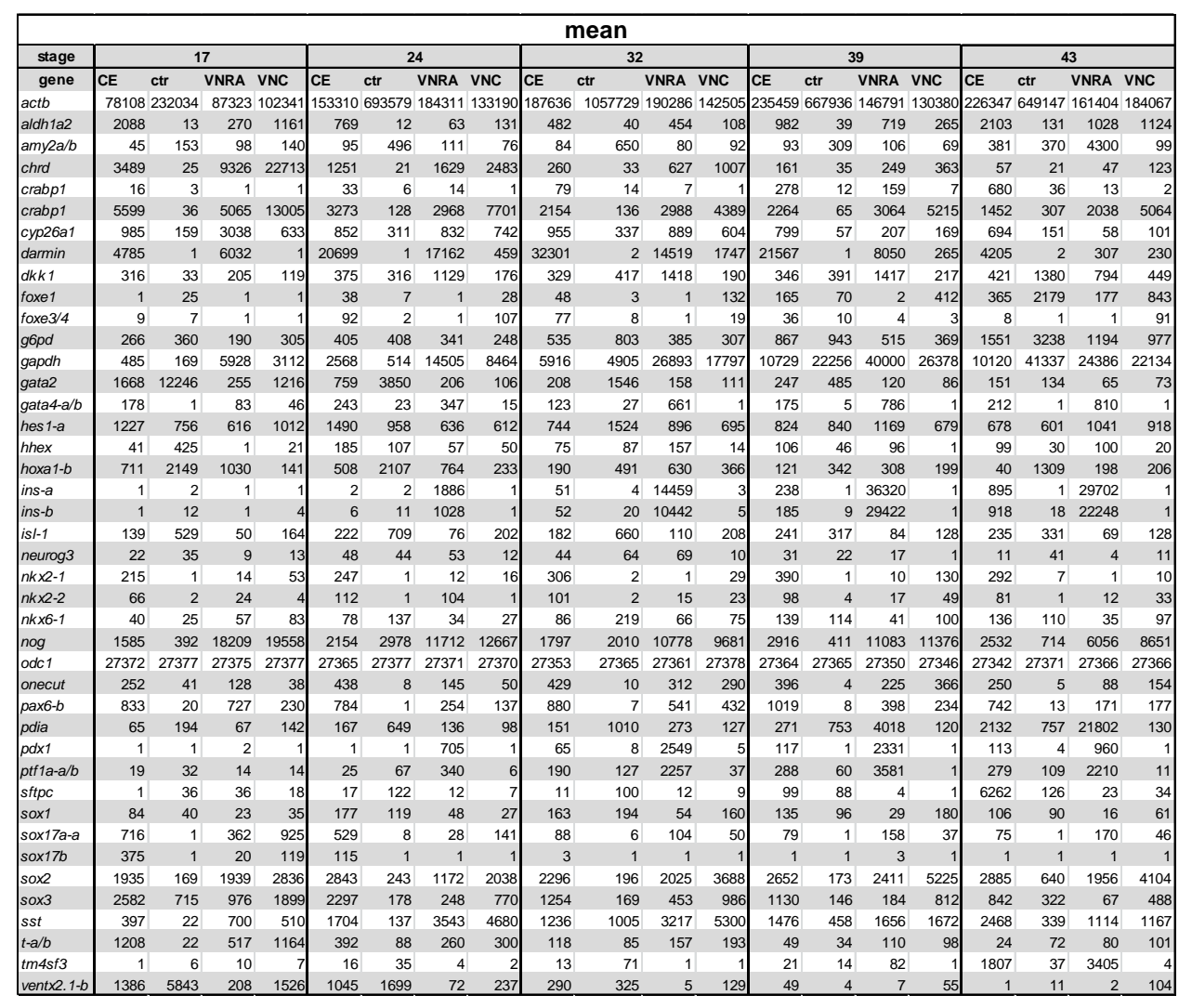

\begin{tabular}{|c|c|c|c|c|c|c|c|c|c|c|c|c|c|c|c|c|c|c|c|}
\hline \multicolumn{20}{|c|}{ SEM } \\
\hline stage & \multicolumn{4}{|c|}{17} & \multicolumn{4}{|c|}{24} & \multicolumn{4}{|c|}{32} & \multicolumn{3}{|c|}{39} & \multicolumn{4}{|c|}{43} \\
\hline gene & CE & & VNRA & VNC & $\begin{array}{lll}C E & \mathrm{ct}\end{array}$ & & VNRA V & & CE $\quad c$ & & VNRA VI & & & & VNRA VNC & & & VNRA & \\
\hline acto & 4145 & 1092 & 2005 & & 44217 & & & & \begin{tabular}{|l|l|}
9494 \\
\end{tabular} & 56603 & 12285 & 7195 & 38532 & & & & & & \\
\hline $0,2 a d b$ & $\begin{array}{r}184 \\
43\end{array}$ & & ${ }_{37}^{11}$ & & $\begin{array}{l}136 \\
72\end{array}$ & ${ }^{11}$ & $\begin{array}{r}1 \\
87\end{array}$ & $\begin{array}{r}33 \\
54\end{array}$ & 121 & $\begin{array}{r}26 \\
534\end{array}$ & 94 & & ${ }_{914}^{214}$ & $\begin{array}{r}15 \\
259\end{array}$ & $\begin{array}{l}503 \\
105\end{array}$ & 913 & 16 & 193 & \\
\hline & 645 & & 4468 & & & 20 & 366 & & & 32 & & 450 & & 34 & 105 & & 20 & & \\
\hline$a b p 1$ & 15 & & & & 27 & 5 & 6 & & 60 & 13 & 5 & & 42 & 11 & 158 & 250 & 6 & 12 & \\
\hline & 1421 & 2 & 2128 & 1054 & 81 & 55 & 72 & 119 & 333 & 24 & 582 & 482 & 241 & 30 & 292 & 315 & 123 & 665 & \\
\hline & & 10 & 1233 & & 131 & 62 & 289 & & 194 & 133 & 293 & & 226 & 21 & 50 & 179 & 114 & & \\
\hline & 553 & & & & 2149 & & & & & & & & & & 5864 & 2911 & & & \\
\hline & & 29 & 54 & 16 & 77 & 58 & 14 & & & 121 & 40 & & 16 & 49 & & 97 & 368 & & \\
\hline & & 13 & & & 29 & 6 & 0 & & & 2 & 0 & & & 69 & 117 & & 54 & & \\
\hline $\mathrm{x} 33 / 4$ & 0 & 6 & 0 & & 29 & 1 & & 106 & & 7 & 0 & & & & 3 & & 0 & & \\
\hline & 54 & & 37 & 138 & 16 & 2 & 13 & & & 6 & 31 & 11 & 210 & & 164 & 275 & & & \\
\hline odh & 1 & 54 & 1472 & & 719 & 135 & 2899 & 1350 & 2295 & 1234 & 1430 & 1807 & 1816 & 43831 & 10004 & 3000 & 14631 & 2001 & \\
\hline & 329 & & 189 & & 245 & 1284 & 62 & & 21 & 41 & 31 & & 79 & 131 & 57 & 14 & 70 & 33 & \\
\hline & 5 & 0 & 3 & & 83 & 9 & 147 & & 63 & 8 & 164 & & 26 & & 132 & 118 & 0 & 253 & \\
\hline & & & & & & & & & & & & & & & & 163 & & & \\
\hline & 8 & 82 & 0 & & 96 & 24 & 3 & & & 86 & & & & & & & 29 & & \\
\hline & 200 & & 1 & & 1 & 402 & . & & & ts & 1010 & & 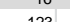 & & & & 109 & & \\
\hline & 0 & 11 & 0 & & 5 & 10 & 197 & & & & 397 & & 89 & & & 年 & 17 & 9593 & \\
\hline & & & 30 & & 46 & 78 & 21 & & & 180 & 16 & & 6 & 107 & & 34 & 79 & & \\
\hline & & & 3 & & 8 & 43 & 33 & & & 18 & 46 & & & 21 & & 16 & & & \\
\hline & 28 & 0 & 13 & & 9 & 0 & 11 & & & & 0 & & & 0 & 129 & & & & \\
\hline & 9 & 1 & 1 & & 7 & 8 & 60 & & & 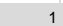 & 11 & & 2 & 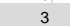 & 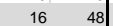 & & 0 & & \\
\hline & 10 & 5 & 39 & & 22 & 47 & 13 & & & 39 & 3 & & & 7 & & 3 & 11 & & \\
\hline & 1249 & 268 & 781 & & 1307 & 2435 & 2574 & & 1068 & 1548 & 2040 & & 2284 & 362 & 2258 & 1467 & 610 & & \\
\hline & 3 & 9 & 7 & & 12 & 8 & 1 & & & 22 & 2 & & & 17 & & 29 & 5 & & \\
\hline & & 37 & 127 & & & 7 & $2 \mathrm{~s}$ & & & & 76 & & & 3 & & & 4 & & \\
\hline & 100 & 19 & 75 & & 168 & 0 & 13 & & & 6 & 66 & & 31 & & 141 & 2 & 9 & & \\
\hline & 64 & 193 & 63 & & 166 & 648 & 13. & & & 1009 & 154 & & 27 & 752 & & 647 & 707 & & \\
\hline & 0 & 0 & 1 & & 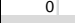 & 0 & 49 & & & 1 & & & & 0 & & & 3 & & \\
\hline & 15 & & 25 & & & 78 & & & & & & & & & & & & & \\
\hline & & 10 & 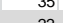 & & 10 & 10 & 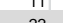 & & & 11 & 11 & & & . & & $4 / 2$ & 71 & & \\
\hline & 150 & 0 & 100 & & 302 & 7 & 12 & & & 5 & 13 & & & & 20 & & & & \\
\hline & & 0 & 5 & & 55 & 0 & 0 & & & 0 & $\begin{array}{l}13 \\
0\end{array}$ & & & 0 & 2 & $r$ & 0 & & \\
\hline & 237 & & 960 & $361 \mid$ & 308 & 121 & 85 & & 248 & 158 & 449 & 1016 & 54 & 140 & 799 & 279 & 0 & 34 & \\
\hline & 366 & & & & & & 60 & & & & & & & & 117 & 132 & & & \\
\hline & & & & 227 & 162 & 86 & 5 & & 4 & 378 & 385 & 1086 & 124 & 8 & 435 & 294 & 229 & & \\
\hline & 313 & 12 & 114 & 325 & & 55 & 71 & & & & & & 33 & 33 & 48 & & & & \\
\hline & & & & & & & & & & & & & & 13 & 81 & 654 & 36 & 828 & \\
\hline & 554 & 1016 & 17 & & 198 & & & & & & & & & & & & & & \\
\hline
\end{tabular}




\subsection{RNA-sequencing data for the identification of RA-target genes}

Tab. 6.3 Summary of 102 differentially expressed genes in the absence of CHX.

Fold changes of indicated genes in explants $1 \mathrm{~h}$ and $2 \mathrm{~h}$ after RA addition (-RA versus $+\mathrm{RA} ; \varnothing$ $\mathrm{CHX})$

* no sequence known for $X$.laevis

$\dagger$ no nanostring probe designable

\begin{tabular}{|c|c|c|c|c|c|c|c|}
\hline \multicolumn{2}{|c|}{ 1h (6) } & \multicolumn{2}{|c|}{1 and $2 h(21)$} & \multicolumn{4}{|c|}{$2 \mathrm{~h}(75)$} \\
\hline gene & FC & gene & FC & gene & FC & gene & FC \\
\hline hoxb1 & 6.79 & hoxa1 & $16.62 / 26.39$ & mesp2* & 27.90 & $X I .80298^{*}$ & 2.60 \\
\hline$X 1.32109$ & 2.32 & hoxa2 & $15.43 / 21.88$ & XI.8753 & 16.22 & Xetrov72036339* & 2.57 \\
\hline olig4* & 2.20 & dusp6 & $5.75 / 8.51$ & mespa & 15.57 & Xetrov72039973* & 2.55 \\
\hline$X I .59256$ & 2.19 & $X I .9874$ & $5.04 / 7.31$ & hoxa3 & 13.07 & gadd45at & 2.52 \\
\hline$X 1.9822$ & -2.16 & $X 1.67202$ & $4.85 / 5.63$ & cebpd & 7.04 & stox1 & 2.50 \\
\hline sp9* & -3.14 & dhrs3 & $4.36 / 7.23$ & s1pr5* & 6.74 & $f z d 4$ & 2.50 \\
\hline & & znf703 & $3.94 / 7.73$ & hoxb4 & 6.51 & hoxa4* & 2.50 \\
\hline & & hoxa5 & $3.85 / 7.26$ & cyp26a1 & 6.27 & $X I .68408$ & 2.48 \\
\hline & & bhlhe40 & 3.77 / 4.97 & nodal1 & 5.84 & dusp5 & 2.40 \\
\hline & & hoxd1 & $3.75 / 5.36$ & sox9 & 5.12 & $X I .80297$ & 2.40 \\
\hline & & neurog2 & $3.46 / 4.13$ & $X I .82687^{*}$ & 5.11 & tril* & 2.39 \\
\hline & & cxcr7 & $2.81 / 3.57$ & prph & 4.70 & XI.57926 & 2.36 \\
\hline & & rgs2 & $2.81 / 4.33$ & $n k \times 6-2$ & 4.39 & erf & 2.32 \\
\hline & & $c d \times 4$ & $2.72 / 2.62$ & $X I .45046$ & 4.38 & $X I .70850$ & 2.31 \\
\hline & & $X I .15091$ & $2.68 / 12.58$ & mespb & 4.23 & $\operatorname{lh} \times 1$ & 2.27 \\
\hline & & $X I .71159$ & $2.42 / 2.72$ & $n r 2 f 5$ & 4.20 & hoxd4 & 2.25 \\
\hline & & $h n f 1 b$ & $2.37 / 5.55$ & trib1 & 4.08 & fhdc1* & 2.25 \\
\hline & & $X I .58101$ & $2.35 / 3.69$ & $X I .84363^{*}$ & 3.84 & rgs14 & 2.22 \\
\hline & & fgf16 + & $2.32 / 2.68$ & fst & 3.83 & kirrel2 & 2.16 \\
\hline & & txnip & $2.22 / 2.83$ & c10orf140 & 3.59 & pim1 & 2.16 \\
\hline & & $X I .4906$ & $2.21 / 3.09$ & Xetrov72011149t & 3.55 & dact1 & 2.15 \\
\hline & & & & $X 1.51509$ & 3.53 & $X I .47239$ & 2.14 \\
\hline & & & & $X I .6091$ & 3.47 & rara & 2.12 \\
\hline & & & & hunk & 3.06 & pkdcc. 1 & 2.10 \\
\hline & & & & tmem 72 & 3.00 & $m x i 1$ & 2.07 \\
\hline & & & & $X I .16263$ & 2.85 & cass4 & 2.06 \\
\hline & & & & nodal2 & 2.81 & $X I .79790$ & 2.06 \\
\hline & & & & foxh1 & 2.78 & $X I .82247^{*}$ & 2.04 \\
\hline & & & & kiaa0182 & 2.76 & fgfr4 & 2.03 \\
\hline & & & & meis3 & 2.74 & slc38a8* & 2.03 \\
\hline & & & & znf503* & 2.72 & hey1 & 2.02 \\
\hline & & & & $m y c$ & 2.70 & hoxb3 & 2.02 \\
\hline & & & & igf3 & 2.70 & $m n 1^{*}$ & 2.02 \\
\hline & & & & $X I .74263$ & 2.68 & spry2 & 2.02 \\
\hline & & & & $t d g f 1 p 2$ & 2.66 & XI.85251 & -2.08 \\
\hline & & & & $g b \times 2.1$ & 2.65 & XI.13572* & -2.09 \\
\hline & & & & $X I .57027$ & 2.60 & twist1 & -2.26 \\
\hline & & & & & & $p d g f b$ & -3.02 \\
\hline
\end{tabular}


Tab. 6.4 Summary of differentially expressed genes in the presence of CHX

Fold changes of indicated genes in explants $1 \mathrm{~h}$ and $2 \mathrm{~h}$ after RA addition (-RA versus + RA; $+\mathrm{CHX}$ )

\begin{tabular}{|c|c|c|c|c|c|}
\hline \multicolumn{2}{|c|}{1 and $2 \mathrm{~h}(10)$} & \multicolumn{4}{|c|}{$2 \mathrm{~h}(51)$} \\
\hline gene & FC & symbol & FC & symbol & FC \\
\hline hoxa1 & 11.94 / 28.34 & hoxa3 & 24.89 & XI.29118 & 3.26 \\
\hline hoxa2 & $6.47 / 14.11$ & hoxb4 & 12.79 & foxh1 & 3.07 \\
\hline$X I .9874$ & 4.62 / 4.61 & foxi4. 2 & 10.73 & XI.58101 & 3.02 \\
\hline$X I .15091$ & $3.98 / 3.93$ & hoxb1 & 9.61 & $X I .6091$ & 2.93 \\
\hline dusp6 & 3.82 / 4.92 & $X I .78389$ & 9.49 & $k l b$ & 2.86 \\
\hline$n k \times 6-2$ & $3.20 / 3.26$ & $X 1.67202$ & 6.59 & hoxd4 & 2.81 \\
\hline dhrs3 & $2.91 / 4.74$ & cyp26c1 & 6.58 & olig4 & 2.75 \\
\hline hoxa5 & $2.89 / 8.13$ & znf703 & 6.53 & hoxb8 & 2.68 \\
\hline rgs2 & $2.18 / 3.23$ & сур 26a1 & 5.64 & $X I .84363$ & 2.58 \\
\hline \multirow[t]{17}{*}{ bhlhe40 } & $2.05 / 4.24$ & s1pr5 & 5.59 & hoxb3 & 2.55 \\
\hline & & $X I .82687$ & 5.54 & hoxa4 & 2.45 \\
\hline & & sox9 & 5.06 & $X I .13431$ & 2.43 \\
\hline & & $X I .45046$ & 4.76 & rara & 2.31 \\
\hline & & trib1 & 4.69 & $m y c$ & 2.30 \\
\hline & & hoxd1 & 4.66 & igf3 & 2.30 \\
\hline & & cebpd & 4.48 & $n r 2 f 5$ & 2.28 \\
\hline & & $h n f 1 b$ & 4.21 & edn1 & 2.24 \\
\hline & & $g b \times 2.2$ & 4.06 & hunk & 2.22 \\
\hline & & hoxc4 & 3.93 & $X I .79790$ & 2.20 \\
\hline & & $X 1.51509$ & 3.93 & $X I .41047$ & 2.15 \\
\hline & & tmem 72 & 3.90 & meis3 & 2.14 \\
\hline & & gata3 & 3.87 & kiaa0182 & 2.12 \\
\hline & & neurog2 & 3.56 & tril & 2.07 \\
\hline & & c10orf140 & 3.52 & $X I .74263$ & 2.05 \\
\hline & & $g b \times 2.1$ & 3.46 & $f z d 4$ & 1.90 \\
\hline & & & & twist1 & -2.14 \\
\hline
\end{tabular}


Tab. 6.5 Comparison of differentially expressed genes absence versus presence of $\mathrm{CHX}$ (putative direct RA-targets).

\begin{tabular}{|c|c|c|}
\hline $\begin{array}{c}-\mathrm{CHX} \text { only } \\
\text { (53) }\end{array}$ & $\begin{array}{c}+\mathrm{CHX} \text { only } \\
\text { (12) }\end{array}$ & $\begin{array}{c}-\mathrm{CHX} \text { and }+\mathrm{CHX} \\
\text { (putative direct targets) } \\
\text { (49) }\end{array}$ \\
\hline cass4 & сур $26 c 1$ & bhlhe40 \\
\hline$c d \times 4$ & edn1 & c10orf140 \\
\hline cxcr7 & foxi4.2 & cebpd \\
\hline dact1 & gata3 & сур26a1 \\
\hline dusp5 & $g b \times 2.2$ & dhrs3 \\
\hline erf & hoxb8 & dusp6 \\
\hline$f g f 16$ & hoxc4 & $f z d 4$ \\
\hline fgfr4 & $k l b$ & foxh1 \\
\hline fhdc1 & $X I .13431$ & $g b \times 2.1$ \\
\hline fst & $X I .29118$ & $h n f 1 b$ \\
\hline gadd $45 a$ & $X I .41047$ & hoxa1 \\
\hline hey1 & $X I .78389$ & hoxa2 \\
\hline kirrel2 & & hохаз \\
\hline $\operatorname{lh} \times 1$ & & hoxa4 \\
\hline mesp2 & & hoxa5 \\
\hline mespa & & hoxb1 \\
\hline mespb & & hoxb3 \\
\hline$m n 1$ & & hoxb4 \\
\hline$m x i 1$ & & hoxd1 \\
\hline nodal1 & & hoxd4 \\
\hline nodal2 & & hunk \\
\hline$p d g f b$ & & igf3 \\
\hline pim1 & & kiaa0182 \\
\hline$p k d c c .1$ & & meis3 \\
\hline prph & & $m y c$ \\
\hline rgs14 & & neurog2 \\
\hline$s / c 38 a 8$ & & $n k x 6-2$ \\
\hline sp9 & & $n r 2 f 5$ \\
\hline spry2 & & olig4 \\
\hline stox1 & & rara \\
\hline $\operatorname{tdgf1p2}$ & & rgs2 \\
\hline txnip & & s1pr5 \\
\hline \multicolumn{2}{|c|}{ Xetrov72011149 } & sox9 \\
\hline \multicolumn{2}{|c|}{ Xetrov72036339 } & tmem 72 \\
\hline \multicolumn{2}{|c|}{ Xetrov72039973 } & trib1 \\
\hline$X I .13572$ & & tril \\
\hline$X I .16263$ & & twist1 \\
\hline$X I .32109$ & & XI.15091 \\
\hline$X I .47239$ & & $X I .45046$ \\
\hline$X I .4906$ & & $X I .51509$ \\
\hline$X I .57027$ & & $X I .58101$ \\
\hline$X I .57926$ & & $X I .6091$ \\
\hline$X I .59256$ & & XI.67202 \\
\hline$X I .68408$ & & $X I .74263$ \\
\hline$X I .70850$ & & XI.79790 \\
\hline$X I .80297$ & & $X I .82687$ \\
\hline$X I .80298$ & & $X I .84363$ \\
\hline$X I .82120$ & & XI.9874 \\
\hline$X I .82247$ & & znf703 \\
\hline \multicolumn{3}{|l|}{$X I .85251$} \\
\hline \multicolumn{3}{|l|}{$X I .8753$} \\
\hline \multicolumn{3}{|l|}{$X I .9822$} \\
\hline znf503 & & \\
\hline
\end{tabular}


Tab. 6.6 RNA-sequencing of explants $1 \mathrm{~h}$ after RA addition in the absence of $\mathrm{CHX}$. Normalized data (1h Ø CHX).

Genes with > 60 mapped reads in samples with RA.

\begin{tabular}{|c|c|c|c|c|c|c|c|c|c|c|}
\hline \multirow{2}{*}{ no. } & \multirow{2}{*}{ symbol } & \multirow{2}{*}{ accession number } & \multirow{2}{*}{ gene function } & \multirow{2}{*}{ UnigeneID_XL } & \multirow{2}{*}{$\begin{array}{l}\log 2 \mathrm{FC} \\
+\mathrm{RA} /-\mathrm{RA} \\
\end{array}$} & \multirow{2}{*}{ FDR } & \multicolumn{2}{|c|}{- RA } & \multicolumn{2}{|c|}{$+\mathrm{RA}$} \\
\hline & & & & & & & 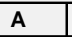 & B & A & B \\
\hline 1 & hoxa1 & \begin{tabular}{|l|} 
BC080895,NM_001 \\
O08016
\end{tabular} & homeodomain transcription factor & $\mathrm{XI} .23512, \mathrm{XI} .283$ & 4.05 & $0.00 \%$ & 38 & 31 & 699 & 499 \\
\hline 2 & hoxa2 & CU025162 & homeodomain transcription factor & XI.12098,XI.751 & 3.95 & $0.00 \%$ & 8 & 7 & 157 & 103 \\
\hline 3 & hoxb1 & XM_002938017 & homeodomain transcription factor & XI.85422 & 2.76 & $0.40 \%$ & 10 & 11 & 105 & 48 \\
\hline 4 & dusp 6 & \begin{tabular}{|l|} 
NM_001045578,CR \\
$848381, B C 118778$, \\
\end{tabular} & MAP kinase phosphatase & \begin{tabular}{|l|}
$\mathrm{XI} .31935, \mathrm{XI} .49155$ \\
$, \mathrm{XI} .52935, \mathrm{XI} .8002$ \\
\end{tabular} & 2.52 & $0.00 \%$ & 669 & 562 & 4471 & 2737 \\
\hline 5 & XI.9874 & & & XI.9874 & 2.33 & $0.00 \%$ & 10 & 28 & 69 & 123 \\
\hline 6 & XI.67202 & & & XI.67202 & 2.28 & $0.81 \%$ & 29 & 34 & 210 & 87 \\
\hline 7 & dhrs3 & \begin{tabular}{|l|} 
NM_001008431,CR \\
$848157, B C 080136$, \\
\end{tabular} & & \begin{tabular}{|l|}
$\mathrm{XI} .77868, \mathrm{XI} .59231$ \\
$\mathrm{XI} .83090$
\end{tabular} & 2.13 & $0.00 \%$ & 992 & 1367 & 4475 & 5786 \\
\hline 8 & $z n f 703$ & \begin{tabular}{|l|} 
NM_001030507,BC \\
093467
\end{tabular} & Zn-finger & \begin{tabular}{|l|}
$X I .1529, X I .23634$, \\
XI.69973
\end{tabular} & 1.98 & $4.87 \%$ & 950 & 500 & 4766 & 1416 \\
\hline 9 & hoxa5 & \begin{tabular}{|l|} 
BC088772,NM_001 \\
011405
\end{tabular} & homeodomain transcription factor & XI.58357 & 1.94 & $0.00 \%$ & 32 & 28 & 131 & 103 \\
\hline 10 & bhlhe40 & \begin{tabular}{|l|} 
BC168818,BC16913 \\
9,XM_002938312, B \\
\end{tabular} & $\begin{array}{l}\text { basic helix-loop-helix (bHLH) } \\
\text { transcription factor, circadian regulator }\end{array}$ & XI.19778,XI.26256 & 1.92 & $0.00 \%$ & 75 & 48 & 270 & 200 \\
\hline 11 & hoxd1 & $\begin{array}{l}\text { CR760220,NM_001 } \\
\text { 016678,BC170965, }\end{array}$ & homeodomain transcription factor & \begin{tabular}{|l|}
$\mathrm{XI.53491,XI.11612}$ \\
, $\mathrm{XI} .76294$
\end{tabular} & 1.91 & $0.40 \%$ & 813 & 398 & 2970 & 1553 \\
\hline 12 & neurog2 & XM_002934243 & $\begin{array}{l}\text { basic helix-loop-helix transcription } \\
\text { factor }\end{array}$ & \begin{tabular}{|l|}
$\mathrm{XI} .369, \mathrm{XI} .370, \mathrm{XI} .7$ \\
9613
\end{tabular} & 1.79 & $1.80 \%$ & 30 & 15 & 137 & 34 \\
\hline 13 & $c x c r 7$ & $\begin{array}{l}\text { NM_001030434,BC } \\
091057\end{array}$ & glycoprotein hormone receptor & XI.15037 & 1.49 & $2.16 \%$ & 247 & 379 & 745 & 1004 \\
\hline 14 & rgs 2 & \begin{tabular}{|l|} 
BC091089,NM_001 \\
030451
\end{tabular} & & \begin{tabular}{|l|}
$\mathrm{XI} .49812, \mathrm{XI} .76046$ \\
, $\mathrm{XI} .85582$
\end{tabular} & 1.49 & $0.00 \%$ & 97 & 42 & 281 & 117 \\
\hline 15 & $c d \times 4$ & $\begin{array}{l}\text { AF417199,CT03048 } \\
\text { 3,BC167866,NM_2 } \\
\end{array}$ & homeodomain transcription factor & XI.26888 & 1.44 & $0.05 \%$ & 1728 & 866 & 5059 & 2168 \\
\hline 16 & $X I .15091$ & & & XI.15091 & 1.42 & $0.53 \%$ & 26 & 29 & 80 & 69 \\
\hline 17 & $X I .71159$ & & & $X I .71159$ & 1.28 & $0.00 \%$ & 175 & 147 & 454 & 330 \\
\hline 18 & $h n f 1 b$ & \begin{tabular}{|l|} 
CR760425,XM_002 \\
939634,XM__002939
\end{tabular} & homeodomain transcription factor & $|\mathrm{XI} .65135, \mathrm{XI} .12667|$ & 1.25 & $0.01 \%$ & 454 & 490 & 1010 & 1230 \\
\hline 19 & XI.58101 & & & XI.58101 & 1.23 & $0.13 \%$ & 117 & 127 & 210 & 381 \\
\hline 20 & $X I .32109$ & & & Xl.32109 & 1.21 & $0.11 \%$ & 86 & 74 & 200 & 174 \\
\hline 21 & fgf16 & XM_002931813 & heparin-binding growth factor & & 1.21 & $0.00 \%$ & 245 & 219 & 547 & 532 \\
\hline 22 & txnip & \begin{tabular}{|l|} 
XM_002938464,BC \\
121658 \\
\end{tabular} & & \begin{tabular}{|l|}
$\mathrm{XI} .77253, \mathrm{XI} .13359$ \\
$, \mathrm{XI} .57371, \mathrm{XI} .7636$ \\
\end{tabular} & 1.15 & $2.96 \%$ & 155 & 102 & 388 & 194 \\
\hline 23 & $X I .4906$ & & & XI.4906 & 1.15 & $2.27 \%$ & 351 & 460 & 933 & 803 \\
\hline 24 & olig4 & \begin{tabular}{|l|} 
BC161514,NM_001 \\
045715,CR848409
\end{tabular} & transcription factor & XI.72230 & 1.14 & $0.84 \%$ & 33 & 42 & 73 & 95 \\
\hline 25 & $X I .59256$ & & & XI.59256 & 1.13 & $1.45 \%$ & 51 & 46 & 107 & 108 \\
\hline 26 & $X 1.9822$ & & & XI.9822 & -1.11 & $1.45 \%$ & 130 & 133 & 74 & 47 \\
\hline 27 & $s p 9$ & \begin{tabular}{|l|} 
BC121268,NM_001 \\
078801
\end{tabular} & & & -1.65 & $0.14 \%$ & 64 & 58 & 28 & 10 \\
\hline
\end{tabular}


Tab. 6.7 RNA-sequencing of explants $2 \mathrm{~h}$ after RA addition in the absence of $\mathrm{CHX}$. Normalized data (2h Ø $\mathrm{CHX}$ ).

Genes with > 60 mapped reads in samples with RA.

\begin{tabular}{|c|c|c|c|c|c|c|c|c|c|c|}
\hline \multirow{2}{*}{ no. } & \multirow{2}{*}{ symbol } & \multirow{2}{*}{ accession } & \multirow{2}{*}{ gene function } & \multirow{2}{*}{ UnigeneID_XL } & \multirow{2}{*}{$\begin{array}{l}\log 2 \mathrm{FC} \\
+\mathrm{RA} /-\mathrm{RA}\end{array}$} & \multirow{2}{*}{ FDR } & \multicolumn{2}{|c|}{ - RA } & \multicolumn{2}{|c|}{$+\mathrm{RA}$} \\
\hline & & & & & & & A & B & A & B \\
\hline 1 & mesp2 & & & & 4.80 & $0.11 \%$ & 1 & 1 & 138 & 10 \\
\hline 2 & hoxa1 & \begin{tabular}{|l|} 
BC080895,NM_00 \\
1008016
\end{tabular} & $\begin{array}{l}\text { homeodomain } \\
\text { transcription factor }\end{array}$ & XI.23512,XI.283 & 4.72 & $0.00 \%$ & 70 & 24 & 1295 & 1129 \\
\hline 3 & hoxa2 & CU025162 & $\begin{array}{l}\text { homeodomain } \\
\text { transcription factor }\end{array}$ & XI.12098,XI.751 & 4.45 & $0.00 \%$ & 18 & 9 & 334 & 276 \\
\hline 4 & $X I .8753$ & & & XI.8753 & 4.02 & $0.00 \%$ & 2 & 4 & 64 & 65 \\
\hline 5 & mespa & \begin{tabular}{|l|} 
CR848619,NM_00 \\
1045719,BC15731
\end{tabular} & transcription factor & XI.55,XI.54 & 3.96 & $0.17 \%$ & 89 & 96 & 3066 & 248 \\
\hline 6 & hoxa3 & \begin{tabular}{|l|} 
NM_001127429,B \\
C166398
\end{tabular} & $\begin{array}{l}\text { homeodomain } \\
\text { transcription factor }\end{array}$ & XI.9439 & 3.71 & $0.00 \%$ & 18 & 6 & 153 & 173 \\
\hline 7 & XI.15091 & & & XI.15091 & 3.65 & $0.00 \%$ & 11 & 16 & 224 & 157 \\
\hline 8 & dusp6 & $\mid \begin{array}{l}\text { NM_001045578,C } \\
\text { R848381,BC11877 }\end{array}$ & $\begin{array}{l}\text { MAP kinase } \\
\text { phosphatase }\end{array}$ & $\begin{array}{l}\text { XI.31935,XI.49155, } \\
\text { XI.52935,XI.80026 }\end{array}$ & 3.09 & $0.00 \%$ & 619 & 512 & 6084 & 3726 \\
\hline 9 & znf703 & \begin{tabular}{|l|} 
NM_001030507,B \\
C093467
\end{tabular} & Zn-finger & \begin{tabular}{|l|}
$\mathrm{XI} .1529, \mathrm{XI} .23634, \mathrm{XI}$ \\
.69973
\end{tabular} & 2.95 & $0.00 \%$ & 1013 & 263 & 4168 & 4062 \\
\hline 10 & $X I .9874$ & & & XI.9874 & 2.87 & $0.00 \%$ & 18 & 35 & 160 & 240 \\
\hline 11 & hoxa5 & \begin{tabular}{|l|} 
BC088772,NM_00 \\
1011405
\end{tabular} & $\begin{array}{l}\text { homeodomain } \\
\text { transcription factor }\end{array}$ & XI.58357 & 2.86 & $0.00 \%$ & 29 & 35 & 226 & 252 \\
\hline 12 & $d h r s 3$ & $\begin{array}{l}\text { NM_001008431,C } \\
\text { R848157,BC08013 }\end{array}$ & & $\begin{array}{l}\text { XI.77868,XI.59231, } \\
\text { XI.83090 }\end{array}$ & 2.85 & $0.00 \%$ & 1015 & 1453 & 8872 & 8548 \\
\hline 13 & cebpd & \begin{tabular}{l|} 
NM_001030414,C \\
R942782,BC09102
\end{tabular} & $\begin{array}{l}\text { basic-leucine zipper } \\
\text { (bZIP) transcription }\end{array}$ & $\begin{array}{l}\text { XI.29876,XI.25857, } \\
\text { XI.81500 }\end{array}$ & 2.82 & $0.00 \%$ & 38 & 24 & 158 & 238 \\
\hline 14 & s1pr5 & \begin{tabular}{|l|} 
BC161459,NM_00 \\
1127068
\end{tabular} & & XI.75787,XI.9971 & 2.75 & $0.03 \%$ & 82 & 43 & 390 & 481 \\
\hline 15 & hoxb4 & \begin{tabular}{|l|} 
BC090114,BC1615 \\
50,NM_00112301
\end{tabular} & $\begin{array}{l}\text { Transcription factor } \\
\text { zerknullt and related }\end{array}$ & $\begin{array}{l}\text { XI.54392,XI.49715, } \\
\text { XI.72333 }\end{array}$ & 2.70 & $0.00 \%$ & 51 & 14 & 192 & 200 \\
\hline 16 & cyp26a1 & \begin{tabular}{|l|} 
NM_001016147,C \\
R761993,BC17108
\end{tabular} & $\begin{array}{l}\text { retinoic acid } \\
\text { hydroxylase }\end{array}$ & XI.50113,XI.456 & 2.65 & $0.00 \%$ & 3148 & 3665 & 18022 & 26048 \\
\hline 17 & nodal1 & \begin{tabular}{|l|} 
BC171037,CR7614 \\
56,NM_00101632
\end{tabular} & $\begin{array}{l}\text { transforming growth } \\
\text { factor beta and bone }\end{array}$ & XI.1037,XI.85704 & 2.55 & $0.04 \%$ & 21 & 35 & 240 & 75 \\
\hline 18 & $X 1.67202$ & & & XI.67202 & 2.49 & $0.04 \%$ & 37 & 22 & 154 & 193 \\
\hline 19 & $h n f 1 b$ & \begin{tabular}{|l|} 
CR760425,XM_00 \\
2939634,XM_0029
\end{tabular} & $\begin{array}{l}\text { homeodomain } \\
\text { transcription factor }\end{array}$ & XI.65135,XI.12667 & 2.47 & $0.00 \%$ & 518 & 518 & 2808 & 2942 \\
\hline 20 & hoxd1 & \begin{tabular}{|l|} 
CR760220,NM_00 \\
1016678,BC17096
\end{tabular} & $\begin{array}{l}\text { homeodomain } \\
\text { transcription factor }\end{array}$ & $\begin{array}{l}\text { XI.53491,XI.11612, } \\
\text { XI.76294 }\end{array}$ & 2.42 & $0.00 \%$ & 1020 & 306 & 3243 & 3015 \\
\hline 21 & $\operatorname{sox} 9$ & \begin{tabular}{|l|} 
NM_001016853,C \\
R855424
\end{tabular} & $\begin{array}{l}\text { HMG-box transcription } \\
\text { factor }\end{array}$ & \begin{tabular}{|l|}
$\mathrm{XI} .28992, \mathrm{XI} .1690, \mathrm{XI}$ \\
.82068
\end{tabular} & 2.36 & $0.00 \%$ & 51 & 29 & 157 & 270 \\
\hline 22 & $X I .82687$ & & & XI.82687 & 2.35 & $0.00 \%$ & 12 & 13 & 39 & 103 \\
\hline 23 & bhlhe40 & \begin{tabular}{|l|} 
BC168818,BC1691 \\
39,XM_002938312
\end{tabular} & $\begin{array}{l}\text { basic helix-loop-helix } \\
(\mathrm{bHLH}) \text { transcription }\end{array}$ & XI.19778,XI.26256 & 2.31 & $0.00 \%$ & 79 & 76 & 399 & 375 \\
\hline 24 & prph & \begin{tabular}{|l|} 
NM_001001235,B \\
C067967
\end{tabular} & intermediate filament & XI.27,XI.16232 & 2.23 & $4.01 \%$ & 9 & 7 & 11 & 93 \\
\hline 25 & $n k x 6-2$ & $\begin{array}{l}\text { СТ010540,XM_00 } \\
2937790\end{array}$ & $\begin{array}{l}\text { homeodomain } \\
\text { transcription factor }\end{array}$ & XI.55860,XI.49827 & 2.13 & $0.00 \%$ & 111 & 203 & 419 & 1028 \\
\hline 26 & $X I .45046$ & & & XI.45046 & 2.13 & $0.00 \%$ & 82 & 42 & 268 & 267 \\
\hline 27 & rgs2 & \begin{tabular}{|l|} 
BC091089,NM_00 \\
1030451
\end{tabular} & & $\begin{array}{l}\text { XI.49812,XI.76046, } \\
\text { XI.85582 }\end{array}$ & 2.11 & $0.00 \%$ & 52 & 26 & 234 & 111 \\
\hline 28 & mespb & \begin{tabular}{|l|} 
NM_001016653,C \\
R760340
\end{tabular} & transcription factor & XI.51050,XI.81675 & 2.08 & $4.27 \%$ & 583 & 240 & 4138 & 453 \\
\hline 29 & $n r 2 f 5$ & XM_002938463 & hormone receptor & \begin{tabular}{|l|}
$\mathrm{XI} .1157, \mathrm{XI} .13423, \mathrm{XI}$ \\
$.81917, \mathrm{XI} .83500$
\end{tabular} & 2.07 & $0.00 \%$ & 28 & 28 & 149 & 97 \\
\hline 30 & neurog2 & XM_002934243 & $\begin{array}{l}\text { basic helix-loop-helix } \\
\text { transcription factor }\end{array}$ & $\begin{array}{l}\text { XI.369,XI.370,XI.79 } \\
613\end{array}$ & 2.05 & $0.04 \%$ & 35 & 8 & 102 & 62 \\
\hline 31 & trib1 & XM_002938661 & & XI.75411,XI.81850 & 2.03 & $0.88 \%$ & 13 & 16 & 46 & 83 \\
\hline
\end{tabular}




\section{Appendix}

\begin{tabular}{|c|c|c|c|c|c|c|c|c|c|c|}
\hline \multirow{2}{*}{ no. } & \multirow{2}{*}{ symbol } & \multirow{2}{*}{ accession } & \multirow{2}{*}{ gene function } & \multirow{2}{*}{ UnigeneID_XL } & \multirow{2}{*}{$\begin{array}{l}\log 2 \mathrm{FC} \\
+\mathrm{RA} / \mathrm{RA}\end{array}$} & \multirow{2}{*}{ FDR } & \multicolumn{2}{|c|}{ - RA } & \multicolumn{2}{|c|}{$+\mathrm{RA}$} \\
\hline & & & & & & & A & B & A & B \\
\hline 32 & $X I .84363$ & & & XI.84363 & 1.94 & $0.00 \%$ & 37 & 38 & 158 & 137 \\
\hline 33 & fst & $\begin{array}{l}\text { NM_001008056,B } \\
\text { C080943 }\end{array}$ & $\begin{array}{l}\text { activin and bmp7 } \\
\text { antagonist, also }\end{array}$ & $\begin{array}{l}\text { XI.1094,XI.75880,XI } \\
.79342, X I .83742 \\
\end{array}$ & 1.94 & $3.02 \%$ & 131 & 974 & 1323 & 1718 \\
\hline 34 & XI.58101 & & & XI.58101 & 1.88 & $0.00 \%$ & 98 & 170 & 508 & 462 \\
\hline 35 & c10orf140 & $\begin{array}{l}\text { NM_001126649,B } \\
\text { C159015, BC15904 }\end{array}$ & & XI.52870,XI.80054 & 1.84 & $0.00 \%$ & 119 & 59 & 294 & 340 \\
\hline 36 & cxcr7 & $\begin{array}{l}\text { NM_001030434,B } \\
\text { C091057 }\end{array}$ & $\begin{array}{l}\text { glycoprotein hormone } \\
\text { receptor }\end{array}$ & XI.15037 & 1.84 & $0.01 \%$ & 216 & 466 & 1409 & 817 \\
\hline 37 & \begin{tabular}{|l} 
Xetrov72 \\
011149
\end{tabular} & & & & 1.83 & $0.00 \%$ & 28 & 29 & 94 & 111 \\
\hline 38 & $X I .51509$ & & & XI.51509 & 1.82 & $0.11 \%$ & 35 & 17 & 90 & 87 \\
\hline 39 & $X I .6091$ & & & XI.6091 & 1.80 & $0.00 \%$ & 32 & 21 & 109 & 81 \\
\hline 40 & $X I .4906$ & & & XI.4906 & 1.63 & $0.00 \%$ & 289 & 329 & 891 & 1022 \\
\hline 41 & hunk & $\begin{array}{l}\text { NM_001127077,B } \\
\text { C161479 }\end{array}$ & & $\begin{array}{l}\text { XI.24322,XI.12483, } \\
\text { XI.83064 }\end{array}$ & 1.61 & $0.00 \%$ & 449 & 355 & 1193 & 1254 \\
\hline 42 & tmem 72 & $\begin{array}{l}\text { BC158380,XM_00 } \\
2939555\end{array}$ & & XI.17444 & 1.59 & $0.10 \%$ & 38 & 24 & 97 & 87 \\
\hline 43 & XI.16263 & & & XI.16263 & 1.51 & $0.00 \%$ & 146 & 92 & 403 & 274 \\
\hline 44 & txnip & $\begin{array}{l}\text { XM_002938464,B } \\
\text { C121658 }\end{array}$ & & $\begin{array}{l}\text { XI.77253,XI.13359, } \\
\text { XI.57371,XI.76363, }\end{array}$ & 1.50 & $0.00 \%$ & 123 & 111 & 470 & 224 \\
\hline 45 & nodal2 & XM_002932721 & $\begin{array}{l}\text { transforming growth } \\
\text { factor beta and bone }\end{array}$ & XI.1038 & 1.49 & $0.33 \%$ & 88 & 279 & 506 & 412 \\
\hline 46 & foxh1 & $\begin{array}{l}\text { CR761447,NM_00 } \\
1017084\end{array}$ & $\begin{array}{l}\text { winged helix } \\
\text { transcription factor }\end{array}$ & XI.381 & 1.47 & $0.68 \%$ & 70 & 66 & 183 & 200 \\
\hline 47 & kiaa0182 & $\begin{array}{l}\text { XM_002936097,B } \\
\text { C125792 }\end{array}$ & & $\begin{array}{l}\text { XI.78028,XI.1433,XI } \\
.78276\end{array}$ & 1.47 & $0.00 \%$ & 290 & 169 & 553 & 706 \\
\hline 48 & meis3 & $\begin{array}{l}\text { CR760178,BC0755 } \\
\text { 89,NM_00100678 }\end{array}$ & $\begin{array}{l}\text { homeodomain } \\
\text { transcription factor }\end{array}$ & $\begin{array}{l}\text { XI.23066,XI.452,XI. } \\
80020, X I .82977\end{array}$ & 1.46 & $0.00 \%$ & 2458 & 1457 & 5909 & 4590 \\
\hline 49 & $X I .71159$ & & & XI.71159 & 1.45 & $0.00 \%$ & 160 & 153 & 556 & 316 \\
\hline 50 & znf503 & \begin{tabular}{|l} 
BC124040,NM_00 \\
1079230
\end{tabular} & zinc finger, $\mathrm{C} 2 \mathrm{H} 2$ type & XI.60819,XI.80579 & 1.44 & $0.00 \%$ & 437 & 380 & 926 & 1324 \\
\hline 51 & myc & \begin{tabular}{|l} 
BC064880,NM_20 \\
4059,CR761143
\end{tabular} & & XI.826,XI.1155 & 1.43 & $0.00 \%$ & 1447 & 527 & 2915 & 1943 \\
\hline 52 & igf3 & $\begin{array}{l}\text { BC161167,CR8483 } \\
\text { 77,NM_00112694 }\end{array}$ & & XI.12078,XI.78527 & 1.43 & $0.00 \%$ & 49 & 35 & 122 & 107 \\
\hline 53 & fgf16 & XM_002931813 & $\begin{array}{l}\text { heparin-binding } \\
\text { growth factor }\end{array}$ & & 1.42 & $0.00 \%$ & 289 & 322 & 893 & 746 \\
\hline 54 & $X I .74263$ & & & XI.74263 & 1.42 & $0.01 \%$ & 153 & 82 & 262 & 339 \\
\hline 55 & $t d g f 1 p 2$ & XM_002940604 & EGF-like growth factor & XI.15503,XI.51406 & 1.41 & $0.10 \%$ & 32 & 42 & 112 & 88 \\
\hline 56 & $g b \times 2.1$ & $\begin{array}{l}\text { XM_002932000,X } \\
\text { M_002932001 }\end{array}$ & $\begin{array}{l}\text { homeodomain } \\
\text { transcription factor }\end{array}$ & XI.77345 & 1.41 & $0.08 \%$ & 74 & 53 & 169 & 166 \\
\hline 57 & $c d \times 4$ & $\begin{array}{l}\text { AF417199,CT0304 } \\
\text { 83,BC167866,NM }\end{array}$ & $\begin{array}{l}\text { homeodomain } \\
\text { transcription factor }\end{array}$ & XI.26888 & 1.39 & $0.03 \%$ & 2515 & 1094 & 6302 & 3014 \\
\hline 58 & $X I .57027$ & & & $X I .57027$ & 1.38 & $0.10 \%$ & 29 & 34 & 78 & 88 \\
\hline 59 & $X 1.80298$ & & & XI.80298 & 1.38 & $0.21 \%$ & 47 & 32 & 106 & 99 \\
\hline 60 & \begin{tabular}{|l} 
Xetrov72 \\
036339
\end{tabular} & & & & 1.36 & $0.00 \%$ & 108 & 135 & 213 & 440 \\
\hline 61 & $\begin{array}{l}\text { Xetrov72 } \\
039973\end{array}$ & & & & 1.35 & $3.33 \%$ & 27 & 31 & 63 & 88 \\
\hline 62 & gadd $45 a$ & $\begin{array}{l}\text { NM_001016151,B } \\
\text { C167898,BC12153 }\end{array}$ & $\begin{array}{l}\text { 40S ribosomal protein } \\
\text { S12 }\end{array}$ & $\begin{array}{l}\text { XI.79095,XI.71159, } \\
\text { XI.81981 }\end{array}$ & 1.33 & $0.22 \%$ & 1216 & 1180 & 4394 & 1907 \\
\hline
\end{tabular}




\begin{tabular}{|c|c|c|c|c|c|c|c|c|c|c|}
\hline \multirow{2}{*}{ no. } & \multirow{2}{*}{ symbol } & \multirow{2}{*}{ accession } & \multirow{2}{*}{ gene function } & \multirow{2}{*}{ UnigeneID_XL } & \multirow{2}{*}{$\begin{array}{r}\log 2 \mathrm{FC} \\
+\mathrm{RA} /-\mathrm{RA} \\
\end{array}$} & \multirow{2}{*}{ FDR } & \multicolumn{2}{|c|}{ - RA } & \multicolumn{2}{|c|}{ + RA } \\
\hline & & & & & & & A & B & A & B \\
\hline 63 & stox 1 & XM_002936869 & $\begin{array}{l}\text { Conserved protein } \\
\text { Knockout }\end{array}$ & ,XI.15086 & 1.32 & $0.02 \%$ & 1164 & 668 & 1955 & 2459 \\
\hline 64 & $f z d 4$ & XM_002936543 & $\begin{array}{l}\text { transmembrane } \\
\text { receptor in the wnt }\end{array}$ & XI.460,XI.53888 & 1.32 & $0.00 \%$ & 681 & 503 & 1365 & 1581 \\
\hline 65 & hoxa4 & & $\begin{array}{l}\text { homeodomain } \\
\text { transcription factor }\end{array}$ & & 1.32 & $0.72 \%$ & 163 & 85 & 318 & 286 \\
\hline 66 & $X I .68408$ & & & XI.68408 & 1.31 & $1.33 \%$ & 35 & 45 & 59 & 151 \\
\hline 67 & dusp5 & $\begin{array}{l}\text { BC090366,NM_00 } \\
1015856\end{array}$ & $\begin{array}{l}\text { MAP kinase } \\
\text { phosphatase }\end{array}$ & XI.79939,XI.15374 & 1.26 & $0.11 \%$ & 799 & 620 & 1677 & 1690 \\
\hline 68 & $X I .80297$ & & & XI.80297 & 1.26 & $0.45 \%$ & 213 & 316 & 813 & 440 \\
\hline 69 & tril & XM_002933390 & & XI.73400,XI.78580 & 1.26 & $0.00 \%$ & 821 & 401 & 1596 & 1160 \\
\hline 70 & $X I .57926$ & & & XI.57926 & 1.24 & $0.03 \%$ & 867 & 751 & 2007 & 1811 \\
\hline 71 & erf & $\begin{array}{l}\text { NM_001015821,B } \\
\text { C090103 }\end{array}$ & transcription factor & $\begin{array}{l}\text { XI.41820,XI.47734, } \\
\text { XI.78801 }\end{array}$ & 1.22 & $2.07 \%$ & 691 & 307 & 961 & 1174 \\
\hline 72 & $X I .70850$ & & & XI.70850 & 1.21 & $0.00 \%$ & 81 & 121 & 176 & 298 \\
\hline 73 & $\operatorname{lh} \times 1$ & $\begin{array}{l}\text { NM_001100228,B } \\
\text { C135731 }\end{array}$ & $\begin{array}{l}\text { LIM and } \\
\text { homeodomain }\end{array}$ & $\begin{array}{l}\text { XI.32655,XI.79655, } \\
\text { XI.81258,XI.82262 }\end{array}$ & 1.19 & $0.18 \%$ & 2515 & 4358 & 8329 & 6707 \\
\hline 74 & hoxd4 & $\begin{array}{l}\text { XM_002935672,X } \\
\text { M_002935673 }\end{array}$ & $\begin{array}{l}\text { homeodomain } \\
\text { transcription factor }\end{array}$ & XI.34346,XI.85858 & 1.17 & $4.14 \%$ & 60 & 65 & 91 & 207 \\
\hline 75 & fhdc1 & $\begin{array}{l}\text { CR942785,XM_00 } \\
2933493\end{array}$ & & & 1.17 & $0.07 \%$ & 897 & 632 & 1490 & 1885 \\
\hline 76 & rgs14 & XM_002938062 & & XI.14836 & 1.15 & $0.64 \%$ & 48 & 54 & 88 & 142 \\
\hline 77 & kirrel2 & $\begin{array}{l}\text { NM_001142126,B } \\
\text { C168130 }\end{array}$ & $\begin{array}{l}\text { Immunoglobulin C-2 } \\
\text { Type/fibronectin type }\end{array}$ & $\begin{array}{l}\text { XI.76492,XI.60720, } \\
\text { XI.76486,XI.81573 }\end{array}$ & 1.11 & $1.50 \%$ & 541 & 706 & 1333 & 1350 \\
\hline 78 & pim1 & $\begin{array}{l}\text { NM_001008131,B } \\
\text { C081340,CR76042 }\end{array}$ & & $\begin{array}{l}\text { XI.48564,XI.68517, } \\
\text { XI.78542 }\end{array}$ & 1.11 & $0.30 \%$ & 1575 & 1405 & 4311 & 2312 \\
\hline 79 & dact1 & $\begin{array}{l}\text { BC080457,CR7619 } \\
\text { 83,NM_00100794 }\end{array}$ & $\begin{array}{l}\text { antagonist of beta- } \\
\text { catenin and c-jun N- }\end{array}$ & XI.7602,XI.19185 & 1.11 & $0.05 \%$ & 3586 & 2838 & 8553 & 5424 \\
\hline 80 & $X I .47239$ & & & XI.47239 & 1.10 & $0.07 \%$ & 126 & 96 & 264 & 211 \\
\hline 81 & rara & $\begin{array}{l}\text { NM_001171194,C } \\
\text { T025407 }\end{array}$ & $\begin{array}{l}\text { transcription factor, } \\
\text { steroid receptor class }\end{array}$ & XI.158,XI.57139 & 1.09 & $1.03 \%$ & 250 & 162 & 387 & 472 \\
\hline 82 & pkdcc.1 & \begin{tabular}{|l} 
BC168090,NM_00 \\
1142110
\end{tabular} & & $\begin{array}{l}\text { XI.80884,XI.55752, } \\
\text { XI.59589 }\end{array}$ & 1.07 & $0.98 \%$ & 774 & 1264 & 2368 & 1864 \\
\hline 83 & $m x i 1$ & $\begin{array}{l}\text { NM_001008128,C } \\
\text { R942348, BC08133 }\end{array}$ & transcription factor & XI.50498 & 1.05 & $1.27 \%$ & 286 & 218 & 398 & 635 \\
\hline 84 & cass4 & XM_002942273 & $\begin{array}{l}\text { p53-interacting } \\
\text { protein 53BP/ASPP, }\end{array}$ & XI.22909 & 1.04 & $0.51 \%$ & 258 & 324 & 507 & 700 \\
\hline 85 & $X I .79790$ & & & XI.79790 & 1.04 & $2.54 \%$ & 29 & 38 & 41 & 103 \\
\hline 86 & $X I .82247$ & & & XI.82247 & 1.03 & $0.62 \%$ & 213 & 190 & 459 & 371 \\
\hline 87 & fgfr4 & $\begin{array}{l}\text { CR761450,NM_00 } \\
\text { 1016323,BC17095 } \\
\end{array}$ & $\begin{array}{l}\text { receptor tyrosine } \\
\text { kinase }\end{array}$ & XI.1008,XI.1016 & 1.02 & $0.00 \%$ & 2621 & 2113 & 5280 & 4345 \\
\hline 88 & slc38a8 & \begin{tabular}{|l|} 
CR760593,NM_00 \\
1044435
\end{tabular} & & XI.70342,XI.78242 & 1.02 & $1.11 \%$ & 550 & 803 & 1420 & 1269 \\
\hline 89 & hey1 & \begin{tabular}{|l} 
CR760684,NM_00 \\
1007910,BC08034
\end{tabular} & $\begin{array}{l}\text { helix-loop-helix } \\
\text { transcription }\end{array}$ & $\begin{array}{l}\text { XI.469,XI.7544,XI.7 } \\
6323\end{array}$ & 1.02 & $0.02 \%$ & 185 & 162 & 396 & 311 \\
\hline 90 & hoxb3 & $\begin{array}{l}\text { CR848404,BC1354 } \\
\text { 06,NM_00101597 }\end{array}$ & $\begin{array}{l}\text { homeodomain } \\
\text { transcription factor }\end{array}$ & XI.79878,XI.80826 & 1.02 & $0.09 \%$ & 117 & 92 & 193 & 230 \\
\hline 91 & $m n 1$ & $\begin{array}{l}\text { NM_001100202,B } \\
\text { C135419 }\end{array}$ & Chitinase & $\begin{array}{l}\text { XI.57334,XI.69093, } \\
\text { XI.82818 } \\
\end{array}$ & 1.01 & $0.98 \%$ & 598 & 499 & 1198 & 1017 \\
\hline 92 & spry2 & $\begin{array}{l}\text { AY714335,BC0642 } \\
\text { O4,NM_00100693 }\end{array}$ & $\begin{array}{l}\text { regulation of signal } \\
\text { transduction }\end{array}$ & XI.11964,XI.11965 & 1.01 & $0.99 \%$ & 1781 & 1675 & 3743 & 3226 \\
\hline 93 & $X I .85251$ & & & XI.85251 & -1.06 & $0.75 \%$ & 85 & 80 & 43 & 35 \\
\hline 94 & XI.13572 & & & XI.13572 & -1.06 & $0.47 \%$ & 130 & 112 & 56 & 59 \\
\hline 95 & twist1 & $\begin{array}{l}\text { NM_204084,BK00 } \\
6266, B C 074558, C\end{array}$ & transcription factor & \begin{tabular}{|l|} 
XI.879,XI.23366,XI. \\
$68451, X I .82751, X I .8$
\end{tabular} & -1.17 & $1.23 \%$ & 217 & 260 & 101 & 108 \\
\hline 96 & $p d g f b$ & XM_002933750 & growth factor & XI.14185,XI.13630 & -1.59 & $0.00 \%$ & 218 & 252 & 102 & 61 \\
\hline
\end{tabular}


Tab. 6.8 RNA-sequencing of explants $1 \mathrm{~h}$ after RA addition in the presence of $\mathrm{CHX}$. Normalized data (1h + CHX).

Genes with > 60 mapped reads in samples with RA.

\begin{tabular}{|c|c|c|c|c|c|c|c|c|c|c|}
\hline \multirow{2}{*}{ no. } & \multirow{2}{*}{ symbol } & \multirow{2}{*}{ accession } & \multirow{2}{*}{ gene function } & \multirow{2}{*}{ UnigeneID_XL } & \multirow{2}{*}{\begin{tabular}{|c|}
$\log 2 \mathrm{FC}$ \\
$+\mathrm{RA} /-\mathrm{RA}$
\end{tabular}} & \multirow{2}{*}{ FDR } & \multicolumn{2}{|c|}{$-\mathrm{RA}+\mathrm{CHX}$} & \multicolumn{2}{|c|}{$+\mathrm{RA}+\mathrm{CHX}$} \\
\hline & & & & & & & \begin{tabular}{l|l}
$A$ &
\end{tabular} & B & \begin{tabular}{l|l}
$\mathbf{A}$ & \\
\end{tabular} & B \\
\hline 1 & hoxa1 & $\begin{array}{l}\text { BC080895,NM_0010 } \\
08016\end{array}$ & $\begin{array}{l}\text { homeodomain } \\
\text { transcription factor }\end{array}$ & XI.23512,XI.283 & 3.58 & $0.00 \%$ & 65 & 17 & 307 & 602 \\
\hline 2 & hoxa2 & CU025162 & $\begin{array}{l}\text { homeodomain } \\
\text { transcription factor }\end{array}$ & XI.12098,XI.751 & 2.69 & $0.00 \%$ & 21 & 6 & 70 & 108 \\
\hline 3 & $X I .9874$ & & & $\mathrm{XI} .9874$ & 2.21 & $0.00 \%$ & 24 & 29 & 98 & 159 \\
\hline 4 & XI.15091 & & & XI.15091 & 1.99 & $0.00 \%$ & 17 & 12 & 81 & 39 \\
\hline 5 & dusp6 & $\begin{array}{l}\text { NM_001045578,CR8 } \\
48381, B C 118778, B C\end{array}$ & $\begin{array}{l}\text { MAP kinase } \\
\text { phosphatase }\end{array}$ & $\begin{array}{l}\text { XI.31935,XI.49155, } \\
\text { XI.52935,XI.80026 }\end{array}$ & 1.94 & $0.00 \%$ & 1009 & 773 & 3103 & 3568 \\
\hline 6 & $n k \times 6-2$ & $\begin{array}{l}\text { CT010540,XM_0029 } \\
37790\end{array}$ & $\begin{array}{l}\text { homeodomain } \\
\text { transcription factor }\end{array}$ & XI.55860,XI.49827 & 1.68 & $0.00 \%$ & 116 & 146 & 282 & 622 \\
\hline 7 & dhrs3 & \begin{tabular}{|l|} 
NM_001008431,CR8 \\
48157,BC080136,CR
\end{tabular} & & $\begin{array}{l}\text { XI.77868,XI.59231, } \\
\text { XI.83090 }\end{array}$ & 1.54 & $0.00 \%$ & 1238 & 1702 & 3530 & 5058 \\
\hline 8 & hoxa5 & \begin{tabular}{|l|} 
BC088772,NM_0010 \\
11405
\end{tabular} & $\begin{array}{l}\text { homeodomain } \\
\text { transcription factor }\end{array}$ & $\mathrm{Xl} .58357$ & 1.53 & $2.29 \%$ & 32 & 27 & 62 & 117 \\
\hline 9 & rgs2 & $\begin{array}{l}\text { BC091089,NM_0010 } \\
30451\end{array}$ & & $\begin{array}{l}\text { XI.49812,XI.76046, } \\
\text { XI.85582 }\end{array}$ & 1.13 & $0.01 \%$ & 89 & 43 & 218 & 79 \\
\hline 10 & bhlhe 40 & $\begin{array}{l}\text { BC168818,BC169139 } \\
\text {,XM_002938312,BC1 }\end{array}$ & $\begin{array}{l}\text { basic helix-loop- } \\
\text { helix (bHLH) }\end{array}$ & XI.19778,XI.26256 & 1.04 & $4.91 \%$ & 114 & 47 & 191 & 131 \\
\hline
\end{tabular}


Tab. 6.9 RNA-sequencing of explants $2 \mathrm{~h}$ after RA addition in the presence of $\mathrm{CHX}$. Normalized data $(2 \mathrm{~h}+\mathrm{CHX})$

genes with > 60 mapped reads in samples with RA

\begin{tabular}{|c|c|c|c|c|c|c|c|c|c|c|}
\hline \multirow{2}{*}{ no. } & \multirow{2}{*}{ symbol } & \multirow{2}{*}{ accession } & \multirow{2}{*}{ gene function } & \multirow{2}{*}{ UnigeneID_XL } & \multirow{2}{*}{$\begin{array}{c}\log 2 \mathrm{FC} \\
+\mathrm{RA} /-\mathrm{RA}\end{array}$} & \multirow{2}{*}{ FDR } & \multicolumn{2}{|c|}{$-\mathrm{RA}+\mathrm{CHX}$} & \multicolumn{2}{|c|}{$+\mathrm{RA}+\mathrm{CHX}$} \\
\hline & & & & & & & \begin{tabular}{l|l}
$A$ &
\end{tabular} & B & \begin{tabular}{l|l} 
A & \\
\end{tabular} & B \\
\hline 1 & hoxa1 & $\begin{array}{l}\text { BC080895,NM_0 } \\
01008016\end{array}$ & $\begin{array}{l}\text { homeodomain } \\
\text { transcription factor }\end{array}$ & XI.23512,XI.283 & 4.82 & $0.00 \%$ & 53 & 52 & 1352 & 1639 \\
\hline 2 & hoxa3 & $\begin{array}{l}\text { NM_001127429, } \\
\text { BC166398 }\end{array}$ & $\begin{array}{l}\text { homeodomain } \\
\text { transcription factor }\end{array}$ & XI.9439 & 4.64 & $0.00 \%$ & 9 & 7 & 167 & 286 \\
\hline 3 & hoxa2 & CU025162 & $\begin{array}{l}\text { homeodomain } \\
\text { transcription factor }\end{array}$ & XI.12098,XI.751 & 3.82 & $0.00 \%$ & 21 & 21 & 314 & 304 \\
\hline 4 & hoxb4 & $\begin{array}{l}\text { BC090114,BC161 } \\
550, N M \_001123 \\
\end{array}$ & $\begin{array}{l}\text { Transcription factor } \\
\text { zerknullt and related }\end{array}$ & \begin{tabular}{|l|} 
XI.54392,XI.4971 \\
$5, X I .72333$
\end{tabular} & 3.68 & $0.00 \%$ & 27 & 14 & 216 & 286 \\
\hline 5 & foxi4.2 & \begin{tabular}{|l|} 
BC158378,NM_2 \\
$03934, B C 064241$ \\
\end{tabular} & $\begin{array}{l}\text { forkhead domain } \\
\text { transcription factor }\end{array}$ & XI.5369,XI.34912 & 3.42 & $4.51 \%$ & 140 & 14 & 658 & 512 \\
\hline 6 & hoxb1 & XM_002938017 & $\begin{array}{l}\text { homeodomain } \\
\text { transcription factor }\end{array}$ & XI.85422 & 3.27 & $0.00 \%$ & 11 & 12 & 130 & 102 \\
\hline 7 & XI. 78389 & & & XI.78389 & 3.25 & $4.90 \%$ & 61 & 10 & 271 & 245 \\
\hline 8 & XI.15091 & & & XI.15091 & 3.07 & $0.00 \%$ & 13 & 5 & 84 & 76 \\
\hline 9 & hoxa5 & \begin{tabular}{|l|} 
BC088772,NM_0 \\
01011405
\end{tabular} & $\begin{array}{l}\text { homeodomain } \\
\text { transcription factor }\end{array}$ & XI.58357 & 3.02 & $0.00 \%$ & 29 & 43 & 209 & 403 \\
\hline 10 & XI.67202 & & & XI.67202 & 2.72 & $0.01 \%$ & 26 & 58 & 220 & 338 \\
\hline 11 & cyp $26 c 1$ & XM_002939091 & electron transport & $\mid \begin{array}{l}X I .82105, X I .1946 \\
, X I .41946\end{array}$ & 2.72 & $0.00 \%$ & 6 & 9 & 51 & 58 \\
\hline 12 & znf703 & \begin{tabular}{|l|} 
NM_001030507, \\
BC093467
\end{tabular} & Zn-finger & $\begin{array}{l}\text { XI.1529,XI.23634 } \\
\text { XI.69973 }\end{array}$ & 2.71 & $0.01 \%$ & 1404 & 778 & 5731 & 6984 \\
\hline 13 & cyp26a1 & $\begin{array}{l}\text { NM_001016147, } \\
\text { CR761993,BC171 }\end{array}$ & $\begin{array}{l}\text { retinoic acid } \\
\text { hydroxylase }\end{array}$ & XI.50113,XI.456 & 2.50 & $0.00 \%$ & 2167 & 5301 & 16230 & 22874 \\
\hline 14 & s1pr5 & \begin{tabular}{|l|} 
BC161459,NM_0 \\
01127068
\end{tabular} & & XI.75787,XI.9971 & 2.48 & $0.25 \%$ & 83 & 129 & 532 & 657 \\
\hline 15 & $X I .82687$ & & & XI.82687 & 2.47 & $0.00 \%$ & 9 & 9 & 28 & 91 \\
\hline 16 & $\operatorname{sox} 9$ & $\begin{array}{l}\text { NM_001016853, } \\
\text { CR855424 }\end{array}$ & $\begin{array}{l}\text { HMG-box transcription } \\
\text { factor }\end{array}$ & \begin{tabular}{|l|}
$\mid X I .28992, X I .1690$ \\
,XI.82068
\end{tabular} & 2.34 & $0.00 \%$ & 39 & 70 & 179 & 382 \\
\hline 17 & dusp6 & \begin{tabular}{|l|} 
NM_001045578, \\
CR848381,BC118 \\
\end{tabular} & $\begin{array}{l}\text { MAP kinase } \\
\text { phosphatase }\end{array}$ & \begin{tabular}{|l|}
$\mathrm{XI} .31935, \mathrm{XI} .4915$ \\
$5, \mathrm{XI} .52935, \mathrm{XI} .80$ \\
\end{tabular} & 2.30 & $0.00 \%$ & 1363 & 1003 & 5859 & 5629 \\
\hline 18 & $X I .45046$ & & & XI.45046 & 2.25 & $0.00 \%$ & 80 & 51 & 279 & 334 \\
\hline 19 & dhrs3 & \begin{tabular}{|l|} 
NM_001008431, \\
CR848157,BC080 \\
\end{tabular} & & \begin{tabular}{|l|} 
XI.77868,XI.5923 \\
$1, \mathrm{XI} .83090$
\end{tabular} & 2.24 & $0.00 \%$ & 1510 & 1943 & 7370 & 8933 \\
\hline 20 & trib1 & XM_002938661 & & \begin{tabular}{|l|}
$\mathrm{XI} .75411, \mathrm{XI} .8185$ \\
0
\end{tabular} & 2.23 & $0.15 \%$ & 32 & 14 & 77 & 154 \\
\hline 21 & hoxd1 & \begin{tabular}{|l|} 
CR760220,NM_0 \\
$01016678, B C 170$ \\
\end{tabular} & $\begin{array}{l}\text { homeodomain } \\
\text { transcription factor }\end{array}$ & \begin{tabular}{|l|} 
XI.53491,XI.1161 \\
$2, X I .76294$
\end{tabular} & 2.22 & $0.01 \%$ & 744 & 614 & 3612 & 2775 \\
\hline 22 & $X I .9874$ & & & XI.9874 & 2.20 & $0.00 \%$ & 31 & 55 & 154 & 243 \\
\hline 23 & cebpd & $\begin{array}{l}\text { NM_001030414, } \\
\text { CR942782,BC091 }\end{array}$ & $\begin{array}{l}\text { basic-leucine zipper } \\
\text { (bZIP) transcription }\end{array}$ & \begin{tabular}{|l|}
$\mathrm{XI} .29876, \mathrm{XI} .2585$ \\
$7, \mathrm{XI} .81500$
\end{tabular} & 2.16 & $0.63 \%$ & 122 & 27 & 332 & 252 \\
\hline 24 & bhlhe40 & $\begin{array}{l}\text { BC168818,BC169 } \\
139, X M \_002938\end{array}$ & $\begin{array}{l}\text { basic helix-loop-helix } \\
(\mathrm{bHLH}) \text { transcription }\end{array}$ & $\begin{array}{l}\mathrm{XI} .19778, \mathrm{XI} .2625 \\
6\end{array}$ & 2.08 & $0.00 \%$ & 129 & 71 & 508 & 337 \\
\hline 25 & $h n f 1 b$ & $\begin{array}{l}\text { CR760425,XM_0 } \\
\text { 02939634,XM_0 }\end{array}$ & $\begin{array}{l}\text { homeodomain } \\
\text { transcription factor }\end{array}$ & \begin{tabular}{|l|}
$\mathrm{XI} .65135, \mathrm{XI} .1266$ \\
7
\end{tabular} & 2.08 & $0.00 \%$ & 636 & 480 & 2760 & 1958 \\
\hline 26 & $g b \times 2.2$ & $\begin{array}{l}\text { BC088605,NM_0 } \\
01011472\end{array}$ & $\begin{array}{l}\text { homeodomain } \\
\text { transcription factor }\end{array}$ & $X 1.973$ & 2.02 & $0.96 \%$ & 12 & 17 & 60 & 65 \\
\hline 27 & hoxc4 & XM_002936638 & $\begin{array}{l}\text { homeodomain } \\
\text { transcription factor }\end{array}$ & XI.69442 & 1.97 & $0.25 \%$ & 29 & 21 & 89 & 110 \\
\hline 28 & XI.51509 & & & XI.51509 & 1.97 & $0.07 \%$ & 31 & 13 & 94 & 74 \\
\hline 29 & tmem 72 & \begin{tabular}{|l|} 
BC158380,XM_0 \\
02939555
\end{tabular} & & XI.17444 & 1.96 & $0.01 \%$ & 25 & 9 & 102 & 39 \\
\hline 30 & gata3 & $\begin{array}{l}\text { NM_001004967, } \\
\text { BC075479 }\end{array}$ & $\begin{array}{l}\text { zinc finger transcription } \\
\text { factor }\end{array}$ & XI.28002 & 1.95 & $4.90 \%$ & 186 & 73 & 389 & 471 \\
\hline
\end{tabular}




\section{Appendix}

\begin{tabular}{|c|c|c|c|c|c|c|c|c|c|c|}
\hline \multirow{2}{*}{ no. } & \multirow{2}{*}{ symbol } & \multirow{2}{*}{ accession } & \multirow{2}{*}{ gene function } & \multirow{2}{*}{ UnigeneID_XL } & \multirow{2}{*}{$\begin{array}{l}\log 2 \mathrm{FC} \\
+\mathrm{RA} /-\mathrm{RA}\end{array}$} & \multirow{2}{*}{ FDR } & \multicolumn{2}{|c|}{$-\mathrm{RA}+\mathrm{CHX}$} & \multicolumn{2}{|c|}{$+\mathrm{RA}+\mathrm{CHX}$} \\
\hline & & & & & & & \begin{tabular}{|l|}
$\mathbf{A}$ \\
\end{tabular} & B & \begin{tabular}{l|l}
$\mathbf{A}$ & \\
\end{tabular} & $B$ \\
\hline 31 & neurog2 & XM_002934243 & $\begin{array}{l}\text { basic helix-loop-helix } \\
\text { transcription factor }\end{array}$ & \begin{tabular}{|l|} 
XI.369,XI.370,XI. \\
79613
\end{tabular} & 1.83 & $0.18 \%$ & 60 & 23 & 156 & 119 \\
\hline 32 & c10orf140 & $\begin{array}{l}\text { NM_001126649, } \\
\text { BC159015,BC159 }\end{array}$ & & \begin{tabular}{|l|}
$X I .52870, X I .8005$ \\
4
\end{tabular} & 1.81 & $0.00 \%$ & 126 & 128 & 355 & 532 \\
\hline 33 & $g b \times 2.1$ & $\begin{array}{l}\text { XM_002932000, } \\
\text { XM_002932001 }\end{array}$ & $\begin{array}{l}\text { homeodomain } \\
\text { transcription factor }\end{array}$ & $\mathrm{XI.77345}$ & 1.79 & $0.00 \%$ & 75 & 75 & 253 & 272 \\
\hline 34 & $n k x 6-2$ & \begin{tabular}{|l|} 
CT010540,XM_0 \\
02937790
\end{tabular} & $\begin{array}{l}\text { homeodomain } \\
\text { transcription factor }\end{array}$ & \begin{tabular}{|l|}
$\mathrm{XI} .55860, \mathrm{XI} .4982$ \\
7
\end{tabular} & 1.71 & $0.00 \%$ & 249 & 281 & 580 & 1333 \\
\hline 35 & $X I .29118$ & & & XI.29118 & 1.70 & $1.64 \%$ & 11 & 36 & 63 & 83 \\
\hline 36 & rgs2 & \begin{tabular}{|l} 
BC091089,NM_0 \\
01030451
\end{tabular} & & \begin{tabular}{|l|} 
XI.49812,XI.7604 \\
$6, X I .85582$
\end{tabular} & 1.69 & $0.00 \%$ & 88 & 28 & 249 & 119 \\
\hline 37 & foxh1 & \begin{tabular}{|l|} 
CR761447,NM_0 \\
01017084
\end{tabular} & $\begin{array}{l}\text { winged helix } \\
\text { transcription factor }\end{array}$ & XI.381 & 1.62 & $0.17 \%$ & 85 & 80 & 201 & 316 \\
\hline 38 & $X I .58101$ & & & XI.58101 & 1.59 & $0.00 \%$ & 101 & 109 & 283 & 358 \\
\hline 39 & $X 1.6091$ & & & XI.6091 & 1.55 & $0.01 \%$ & 35 & 26 & 90 & 91 \\
\hline 40 & $k l b$ & XM_002933470 & beta-glucosidase & & 1.52 & $0.00 \%$ & 31 & 24 & 79 & 81 \\
\hline 41 & hoxd4 & $\begin{array}{l}\text { XM_002935672, } \\
\text { XM_002935673 }\end{array}$ & $\begin{array}{l}\text { homeodomain } \\
\text { transcription factor }\end{array}$ & \begin{tabular}{|l|}
$X \mathrm{XI.34346,XI.8585}$ \\
8
\end{tabular} & 1.49 & $0.19 \%$ & 47 & 51 & 128 & 150 \\
\hline 42 & olig4 & $\begin{array}{l}\text { BC161514,NM_0 } \\
\text { 01045715,CR848 }\end{array}$ & transcription factor & XI.72230 & 1.46 & $0.00 \%$ & 45 & 35 & 113 & 109 \\
\hline 43 & hoxb8 & XM_002938021 & $\begin{array}{l}\text { homeodomain } \\
\text { transcription factor }\end{array}$ & XI.79158,XI.9701 & 1.42 & $0.13 \%$ & 39 & 17 & 105 & 49 \\
\hline 44 & XI.84363 & & & XI.84363 & 1.37 & $0.45 \%$ & 67 & 58 & 146 & 178 \\
\hline 45 & hoxb3 & $\begin{array}{l}\text { CR848404,BC135 } \\
\text { 406,NM_001015 } \\
\end{array}$ & $\begin{array}{l}\text { homeodomain } \\
\text { transcription factor }\end{array}$ & \begin{tabular}{|l|}
$\mathrm{XI} .79878, \mathrm{XI} .8082$ \\
6
\end{tabular} & 1.35 & $0.00 \%$ & 69 & 75 & 202 & 171 \\
\hline 46 & hoxa4 & & $\begin{array}{l}\text { homeodomain } \\
\text { transcription factor }\end{array}$ & & 1.29 & $1.70 \%$ & 156 & 97 & 232 & 360 \\
\hline 47 & XI.13431 & & & XI.13431 & 1.28 & $4.51 \%$ & 23 & 17 & 61 & 41 \\
\hline 48 & rara & $\begin{array}{l}\text { NM_001171194, } \\
\text { CT025407 }\end{array}$ & $\begin{array}{l}\text { transcription factor, } \\
\text { steroid receptor class }\end{array}$ & XI.158,XI.57139 & 1.21 & $0.24 \%$ & 258 & 174 & 429 & 555 \\
\hline 49 & $m y c$ & $\begin{array}{l}\text { BC064880,NM_2 } \\
\text { 04059,CR761143 } \\
\end{array}$ & & XI.826,XI.1155 & 1.20 & $0.25 \%$ & 1560 & 767 & 3167 & 1987 \\
\hline 50 & igf3 & $\begin{array}{l}\text { BC161167,CR848 } \\
\text { 377,NM_001126 }\end{array}$ & & \begin{tabular}{|l|} 
XI.12078,XI.7852 \\
7
\end{tabular} & 1.20 & $0.18 \%$ & 54 & 32 & 108 & 91 \\
\hline 51 & $n r 2 f 5$ & XM_002938463 & hormone receptor & $\begin{array}{l}\mathrm{XI} .1157, \mathrm{XI} .13423 \\
\mathrm{XI} .81917, \mathrm{XI} .835 \\
\end{array}$ & 1.19 & $1.22 \%$ & 46 & 43 & 136 & 74 \\
\hline 52 & edn1 & $\mid \begin{array}{l}\text { XM_002932664, } \\
\text { BC161190 }\end{array}$ & $\begin{array}{l}\text { secreted peptide } \\
\text { precursor }\end{array}$ & $\left|\begin{array}{l}\mathrm{XI} .68930, \mathrm{XI} .6379 \\
6\end{array}\right|$ & 1.16 & $0.57 \%$ & 54 & 52 & 115 & 125 \\
\hline 53 & hunk & \begin{tabular}{|l|} 
NM_001127077, \\
BC161479
\end{tabular} & & \begin{tabular}{|l|}
$\mathrm{XI} .24322, \mathrm{XI} .1248$ \\
$3, \mathrm{XI} .83064$ \\
\end{tabular} & 1.15 & $0.03 \%$ & 649 & 566 & 1215 & 1468 \\
\hline 54 & XI.79790 & & & XI.79790 & 1.14 & $0.80 \%$ & 22 & 49 & 55 & 102 \\
\hline 55 & $X I .41047$ & & & XI.41047 & 1.11 & $0.56 \%$ & 68 & 52 & 188 & 86 \\
\hline 56 & meis3 & \begin{tabular}{|l|} 
CR760178,BC075 \\
589,NM_001006 \\
\end{tabular} & $\begin{array}{l}\text { homeodomain } \\
\text { transcription factor }\end{array}$ & \begin{tabular}{|l|}
$X I .23066, X I .452$, \\
$X I .80020, X I .8297$ \\
\end{tabular} & 1.09 & $0.07 \%$ & 3434 & 2009 & 6277 & 5044 \\
\hline 57 & kiaa0182 & $\mid \begin{array}{l}\text { XM_002936097, } \\
\text { BC125792 }\end{array}$ & & $\mid \begin{array}{l}\text { XI.78028,XI.1433 } \\
\text {,XI.78276 }\end{array}$ & 1.08 & $0.46 \%$ & 329 & 297 & 615 & 709 \\
\hline 58 & tril & XM_002933390 & & $\left|\begin{array}{l}X I .73400, X I .7858 \\
0\end{array}\right|$ & 1.05 & $0.16 \%$ & 895 & 413 & 1695 & 941 \\
\hline 59 & $X I .74263$ & & & $\mathrm{XI} .74263$ & 1.04 & $4.90 \%$ & 136 & 89 & 242 & 216 \\
\hline 61 & $f z d 4$ & XM_002936543 & $\begin{array}{l}\text { transmembrane } \\
\text { receptor in the wnt }\end{array}$ & XI.460,XI.53888 & 0.93 & $0.69 \%$ & 1032 & 741 & 1679 & 1690 \\
\hline 60 & twist1 & $\begin{array}{l}\text { NM_204084,BKO } \\
\text { 06266,BC074558 }\end{array}$ & transcription factor & $\mid$\begin{tabular}{|}
$\mathrm{XI} .879, \mathrm{XI} .23366$, \\
$\mathrm{XI} .68451, \mathrm{XI} .8275$
\end{tabular} & -1.10 & $4.50 \%$ & 261 & 222 & 82 & 155 \\
\hline
\end{tabular}




\subsection{Nanostring analysis data for the verification of RA-responsiveness of putative RA-target genes}

Tab. 6.10 Overview of Nanostring analysis results.

Indicated is the fold induction upon activation of RA-signaling and fold reduction upon inhibition of RA-signaling. Candidates highlighted in grey are confirmed RA-responsive genes.

\begin{tabular}{|c|c|c|c|c|c|c|c|c|c|c|c|}
\hline \multirow{3}{*}{ no. } & \multirow{3}{*}{ direct } & \multirow{3}{*}{ gene } & \multicolumn{5}{|c|}{$\begin{array}{c}\text { RA-inducibility } \\
\text { fold induction +RA/-RA }\end{array}$} & \multicolumn{4}{|c|}{$\begin{array}{c}\text { RA-dependency } \\
\text { fold reduction +RA/-RA }\end{array}$} \\
\hline & & & \multicolumn{2}{|c|}{ pancreatic organoids } & \multicolumn{3}{|c|}{ whole embryo } & \multirow{2}{*}{\begin{tabular}{|c|} 
dorsal tissue \\
st.11 \\
\end{tabular}} & \multicolumn{3}{|c|}{ whole embryo } \\
\hline & & & $1 \mathrm{~h}$ & $2 \mathrm{~h}$ & st.12 & st.13 & st.14 & & st.12 & st.13 & st.14 \\
\hline 1 & $\bullet$ & bhlhe40 & 2.05 & 4.01 & 4.72 & 3.24 & 3.78 & 1 & 1 & 1 & 1 \\
\hline 2 & $\bullet$ & c10orf140 & 1 & 1 & 1 & 1 & 1 & 1.00 & 1 & 1 & 1 \\
\hline 3 & & cass4 & 0.64 & 1.49 & 1.17 & 1.54 & 1.61 & 0.91 & 1.19 & 1.09 & 1.09 \\
\hline 4 & & $c d x 4$ & 1.45 & 2.09 & 1.26 & 1.24 & 1.00 & 1.15 & 1.14 & 1.25 & 1.19 \\
\hline 5 & $\bullet$ & cebpd & 1.44 & 18.71 & 3.35 & 7.92 & 7.14 & 2.05 & 0.96 & 0.82 & 1.33 \\
\hline 6 & & cxcr7 & 1.54 & 1.69 & 1 & 1 & 1 & 1.23 & 0.96 & 1.04 & 0.95 \\
\hline 7 & $\bullet$ & cyp26a1 & 4.39 & 14.23 & 6.70 & 13.96 & 14.19 & 2.29 & 1.81 & 1.44 & 1.53 \\
\hline 8 & & dact1- $a / b$ & 0.89 & 1.51 & 1.82 & 1.89 & 1.74 & 1.07 & 1.08 & 1.01 & 1.28 \\
\hline 9 & $\bullet$ & dhrs3 & 5.87 & 8.86 & 4.13 & 7.43 & 7.80 & 2.76 & 1.78 & 1.56 & 2.05 \\
\hline 10 & & $d u s p 5$ & 0.91 & 2.28 & 1.96 & 2.42 & 2.29 & 1.20 & 1.49 & 1.31 & 1.31 \\
\hline 11 & $\bullet$ & dusp6 & 2.86 & 5.39 & 3.55 & 4.47 & 4.43 & 1.31 & 1.05 & 1.27 & 0.99 \\
\hline 12 & & erf & 0.62 & 1.50 & 1.23 & 1.90 & 1.71 & 0.97 & 1.09 & 1.06 & 1.30 \\
\hline 13 & & $f g f r 4-a / b$ & 1.03 & 1.40 & 1.09 & 1.00 & 0.89 & 0.99 & 0.94 & 0.99 & 1.05 \\
\hline 14 & $\bullet$ & foxh1 & 0.65 & 2.49 & 1.42 & 1.56 & 1.49 & 1.02 & 1.32 & 1.72 & 1.41 \\
\hline 15 & & fst & 1.59 & 1.73 & 1.75 & 2.57 & 2.91 & 2.44 & 1.91 & 1.13 & 1.52 \\
\hline 16 & $\bullet$ & $f z d 4$ & 1.27 & 2.26 & 1.92 & 2.87 & 2.51 & 0.90 & 1.43 & 1.61 & 1.90 \\
\hline 17 & $\bullet$ & $g b \times 2.1$ & 2.21 & 4.56 & 2.07 & 2.92 & 2.99 & 1.45 & 1.76 & 1.74 & 1.64 \\
\hline 18 & & hey1 & 1.06 & 1.57 & 0.92 & 1.30 & 1.41 & 1.48 & 1.11 & 1.20 & 1.25 \\
\hline 19 & $\bullet$ & $h n f 1 b$ & 1.95 & 3.00 & 2.47 & 4.09 & 3.21 & 1.08 & 1.71 & 2.51 & 3.11 \\
\hline 20 & $\bullet$ & hoxa1-b & 9.53 & 44.33 & 3.27 & 4.21 & 4.33 & 4.24 & 2.66 & 3.42 & 7.32 \\
\hline 21 & $\bullet$ & hoxa2 & 1 & 1 & 0.98 & 1.82 & 1.26 & 1 & 1 & 1 & 1 \\
\hline 22 & $\bullet$ & hoxa3 & 1 & 1 & 108.71 & 9.31 & 10.49 & 1 & 1 & 1 & 1 \\
\hline 23 & $\bullet$ & hoxa5 & 1 & 1 & 1 & 1 & 1 & 1 & 1 & 1 & 7 \\
\hline 24 & $\bullet$ & hoxb1 & 9.81 & 21.42 & 2.56 & 2.69 & 2.31 & 1 & 1.81 & 2.31 & 1.62 \\
\hline 25 & $\bullet$ & hoxb3 & 1 & 1 & 25.56 & 5.85 & 5.01 & 1 & 1 & 1 & 1 \\
\hline 26 & $\bullet$ & hoxb4 & 0.85 & 2.23 & 2.46 & 2.64 & 3.43 & 0.83 & 0.93 & 1.37 & 1.37 \\
\hline 27 & $\bullet$ & hoxd1 & 2.54 & 6.94 & 1.75 & 1.97 & 2.09 & 1.17 & 1.39 & 1.54 & 1.70 \\
\hline 28 & $\bullet$ & hoxd4 & 2.33 & 41.83 & 6.07 & 4.26 & 4.46 & 1.43 & 1.22 & 1.66 & 0.81 \\
\hline 29 & $\bullet$ & hunk & 0.77 & 2.64 & 2.02 & 2.25 & 2.11 & 1.00 & 1.26 & 1.31 & 1.31 \\
\hline 30 & $\bullet$ & igf3 & 3.45 & 3.38 & 1.92 & 2.29 & 2.61 & 1.69 & 1.53 & 1.41 & 0.88 \\
\hline 31 & $\bullet$ & kiaa0182 & 0.47 & 2.04 & 1.07 & 1.56 & 1.85 & 1.01 & 1.30 & 1.31 & 1.12 \\
\hline 32 & & kirrel2 & 0.66 & 1.87 & 1.41 & 1.22 & 1.21 & 1.39 & 1.09 & 1.17 & 1.23 \\
\hline 33 & & $\operatorname{lh} \times 1$ & 0.98 & 2.42 & 4.00 & 3.96 & 4.43 & 1.34 & 1.16 & 1.51 & 1.53 \\
\hline 34 & $\bullet$ & meis- $a$ & 1.35 & 2.59 & 1.96 & 2.58 & 2.72 & 1.39 & 1.74 & 1.56 & 1.41 \\
\hline 35 & & mespa & 2.86 & 7.19 & 1.73 & 2.19 & 1.35 & 1.39 & 1 & 1 & 1 \\
\hline 36 & & mespb & 3.53 & 2.51 & 1.02 & 1.26 & 1.05 & 1.04 & 0.96 & 0.81 & 1.09 \\
\hline 37 & & $m x i 1$ & 0.91 & 3.05 & 2.08 & 2.05 & 1.97 & 1.18 & 1.54 & 1.19 & 1.45 \\
\hline 38 & $\bullet$ & $m y c-a / b$ & 0.60 & 1.99 & 1.19 & 1.30 & 1.26 & 1 & 1.14 & 1.56 & 1.22 \\
\hline 39 & $\bullet$ & neurog2 & 1 & 1 & 1.02 & 0.88 & 0.99 & 1.65 & 1.21 & 0.93 & 1.11 \\
\hline 40 & $\bullet$ & $n k \times 6-2$ & 1.52 & 4.32 & 2.49 & 2.38 & 3.48 & 2.09 & 1.84 & 1.37 & 1.07 \\
\hline 41 & & nodal1 & 1.21 & 1.75 & / & / & / & 1.39 & / & / & 1 \\
\hline
\end{tabular}




\begin{tabular}{|c|c|c|c|c|c|c|c|c|c|c|c|}
\hline \multirow{3}{*}{ no. } & \multirow{3}{*}{ direct } & \multirow{3}{*}{ gene } & \multicolumn{5}{|c|}{$\begin{array}{c}\text { RA-inducibility } \\
\text { fold induction +RA/-RA }\end{array}$} & \multicolumn{4}{|c|}{$\begin{array}{c}\text { RA-dependency } \\
\text { fold reduction +RA/-RA } \\
\end{array}$} \\
\hline & & & \multicolumn{2}{|c|}{ pancreatic organoids } & \multicolumn{3}{|c|}{ whole embryo } & \multirow{2}{*}{\begin{tabular}{|c|} 
dorsal tissue \\
st.11 \\
\end{tabular}} & \multicolumn{3}{|c|}{ whole embryo } \\
\hline & & & $1 \mathrm{~h}$ & $2 \mathrm{~h}$ & st.12 & st.13 & st.14 & & st.12 & st.13 & st.14 \\
\hline 42 & & nodal2 & 1.07 & 1.36 & 1 & 1 & 1 & 0.86 & 1 & $/$ & 1 \\
\hline 43 & $\bullet$ & $n r 2 f 5$ & 1 & I & 1 & 4.38 & 3.97 & 0.93 & 1 & 1 & 1 \\
\hline 44 & & $p d g f b$ & 0.71 & 1.24 & 3.87 & 1.23 & 1.76 & 1.22 & 1 & 1 & 1 \\
\hline 45 & & $\operatorname{pim1}$ & 1.23 & 1.76 & 1.33 & 1.96 & 1.59 & 1.33 & 1.14 & 1.31 & 1.20 \\
\hline 46 & & $p k d c c .1$ & 1.02 & 1.05 & 1.32 & 1.16 & 1.23 & 1.07 & 0.97 & 1.05 & 1.44 \\
\hline 47 & & prph & 1 & 31.75 & 4.68 & 5.07 & 3.60 & 1 & 2.85 & 2.39 & 1.63 \\
\hline 48 & $\bullet$ & rara & 1 & 1 & 1.89 & 2.05 & 2.02 & 1.06 & 1.60 & 1.18 & 1.40 \\
\hline 49 & & rgs14 & 2.40 & 4.24 & 1 & 1 & 1 & 1 & 1 & 1 & 1 \\
\hline 50 & $\bullet$ & rgs2 & 1.93 & 4.71 & 2.43 & 1.73 & 1.68 & 1.40 & 1.07 & 1.11 & 1.08 \\
\hline 51 & $\bullet$ & $\operatorname{sox} 9$ & 1.60 & 6.45 & 2.96 & 2.21 & 2.40 & 0.84 & 1.15 & 1.44 & 0.87 \\
\hline 52 & & spry2 & 1.31 & 1.61 & 1.34 & 1.48 & 1.55 & 0.90 & 0.96 & 0.95 & 1.03 \\
\hline 53 & & stox 1 & 0.81 & 1.04 & 0.92 & 1.13 & 0.95 & 0.87 & 1.18 & 0.93 & 1.15 \\
\hline 54 & & $t d g f 1 p 2-a / b$ & 1 & 1 & 1 & 1 & 1 & 1 & 1 & 1 & 1 \\
\hline 55 & $\bullet$ & tmem72 & 2.74 & 5.25 & 1.77 & 2.50 & 1.69 & 1.46 & 1.15 & 1.33 & 1.49 \\
\hline 56 & $\bullet$ & trib1 & 0.52 & 4.58 & 0.73 & 1.27 & 1.00 & 1 & 1.10 & 0.90 & 1.04 \\
\hline 57 & $\bullet$ & twist1 & 0.55 & 0.71 & 0.77 & 0.30 & 0.34 & 1.52 & 0.99 & 0.91 & 0.75 \\
\hline 58 & & txnip & 2.72 & 7.40 & 1.37 & 2.10 & 1.36 & 1.14 & 0.91 & 1.06 & 1.25 \\
\hline 59 & $\bullet$ & XI.15091 & / & 1 & / & 1 & I & 1 & / & 1 & 1 \\
\hline 60 & & XI.16263 & 1 & 1 & 1 & 1 & 1 & 1 & 1 & 1 & 1 \\
\hline 61 & & XI.32109 & 1 & 1 & 1 & 1 & 1 & 1 & 1 & 1 & 1 \\
\hline 62 & $\bullet$ & $X I .45046$ & 1.27 & 7.03 & 1.84 & 2.27 & 2.50 & 1.53 & 2.02 & 1.97 & 1.39 \\
\hline 63 & & \begin{tabular}{|l}
$X 1.47239$ \\
\end{tabular} & 0.91 & 3.34 & 1.70 & 1.71 & 1.62 & 1.00 & 1.41 & 1.77 & 0.96 \\
\hline 64 & & $X 1.4906$ & 1.11 & 3.14 & 1.15 & 0.99 & 0.78 & 0.84 & 0.97 & 1.04 & 1.18 \\
\hline 65 & $\bullet$ & $X 1.51509$ & 1.63 & 30.14 & 3.88 & 4.96 & 4.33 & 2.23 & 1.74 & 1.78 & 1.90 \\
\hline 66 & & $X 1.57027$ & 0.64 & 14.12 & 2.72 & 1.49 & 1.44 & 0.76 & 1 & 1 & 1 \\
\hline 67 & & $X 1.57926$ & 0.84 & 1.99 & 2.37 & 2.99 & 2.91 & 1.27 & 1.43 & 1.53 & 1.56 \\
\hline 68 & $\bullet$ & $X 1.58101$ & 1 & 1 & 1 & 1 & 1 & 1 & 1 & 1 & 1 \\
\hline 69 & & $X 1.59256$ & 1 & 1 & 1 & 1 & 1 & 1 & 1 & 1 & 1 \\
\hline 70 & $\bullet$ & $X I .6091$ & 1 & 1 & 1 & 1 & 1 & 0.75 & 1 & 1 & 1 \\
\hline 71 & $\bullet$ & $X 1.67202$ & 1 & 1 & 1 & 1 & 1 & 1 & 1 & 1 & 1 \\
\hline 72 & & $X I .68408$ & 1 & 1 & 1 & 1 & 1 & 1 & 1 & 1 & 1 \\
\hline 73 & & $X I .70850$ & 1 & 1 & 1 & 1 & 1 & 1 & 1 & 1 & 1 \\
\hline 74 & & \begin{tabular}{|l}
$\mathrm{XI} .71159$ \\
\end{tabular} & 0.70 & 0.66 & 0.68 & 0.51 & 0.48 & 0.95 & 0.79 & 0.86 & 0.88 \\
\hline 75 & $\bullet$ & $X I .74263$ & 1 & 1 & 1 & 1 & 1 & 1 & 1 & 1 & 1 \\
\hline 76 & $\bullet$ & $X I .79790$ & 0.97 & 2.24 & 2.18 & 2.07 & 1.40 & 1 & 1 & 1 & 1 \\
\hline 77 & & $X I .80297$ & 1.64 & 1.90 & 2.08 & 1.94 & 1.99 & 1.23 & 1.19 & 1.01 & 1.00 \\
\hline 78 & & $X I .8753$ & I & 1 & 1 & 1 & 1 & 1.00 & 1 & 1 & 1 \\
\hline 79 & & $X I .85251$ & 1 & 1 & 1 & 1 & 1 & 1.00 & 1 & 1 & 1 \\
\hline 80 & & $X 1.9822$ & 0.54 & 2.23 & 1 & 1 & 1 & 2.29 & 1 & 1 & 1 \\
\hline 81 & $\bullet$ & $X 1.9874$ & 1 & 1 & 1 & 1 & 1 & 1 & 1 & 1 & 1 \\
\hline 82 & $\bullet$ & znf703 & 1.41 & 8.98 & 1.94 & 2.52 & 2.54 & 1.37 & 1.64 & 1.76 & 1.55 \\
\hline
\end{tabular}


Tab. 6.11 Raw data of RA-inducibility in explants

\begin{tabular}{|c|c|c|c|c|c|c|c|c|}
\hline \multirow{2}{*}{ gene } & \multicolumn{4}{|c|}{ 1h } & \multicolumn{4}{|c|}{$2 h$} \\
\hline & 1 & RA & CHX & $\mathrm{RA} / \mathrm{CHX}$ & 1 & RA & CHX & $\mathrm{RA} / \mathrm{CHX}$ \\
\hline bhlhe 40 & 23 & 72 & 2 & 81 & 53 & 94 & 48 & 123 \\
\hline c10orf140 & 2 & 7 & 1 & 6 & 6 & 6 & 5 & 9 \\
\hline cass4 & 251 & 266 & 5 & 345 & 310 & 261 & 386 & 492 \\
\hline $\mathrm{cdx} 4$ & 429 & 988 & 12 & 1212 & 780 & 808 & 1150 & 1422 \\
\hline cebpd & 40 & 91 & 1 & 51 & 24 & 122 & 28 & 154 \\
\hline cxcr7 & 197 & 487 & 4 & 637 & 339 & 309 & 539 & 677 \\
\hline сур26a1 & 460 & 3225 & 6 & 2458 & 572 & 4387 & 599 & 5018 \\
\hline dact1-a & 334 & 349 & 5 & 321 & 327 & 247 & 378 & 516 \\
\hline dact1-b & 1509 & 2175 & 41 & 2537 & 1822 & 1511 & 2206 & 3092 \\
\hline dhrs3 & 184 & 1779 & 5 & 1419 & 317 & 1496 & 413 & 2206 \\
\hline dusp5 & 796 & 1165 & 31 & 1166 & 876 & 1089 & 1091 & 1753 \\
\hline dusp6 & 342 & 1411 & 10 & 1632 & 463 & 1342 & 679 & 2460 \\
\hline e_Cerberus & 8391 & 14195 & 241 & 13089 & 14634 & 6226 & 11690 & 11101 \\
\hline e_darmin & 4 & 12 & 1 & 5 & 10 & 10 & 4 & 6 \\
\hline e_Sox17alph & 2721 & 3750 & 91 & 4838 & 3950 & 2018 & 5675 & 5995 \\
\hline e_Sox17beta & 1512 & 2351 & 72 & 2890 & 1840 & 974 & 3285 & 3269 \\
\hline e_TGIF2 & 1048 & 1219 & 8 & 1276 & 1388 & 607 & 1479 & 1514 \\
\hline e_vpp1 & 40 & 46 & 1 & 36 & 55 & 32 & 38 & 36 \\
\hline ec_Sox 2 & 284 & 463 & 3 & 509 & 195 & 538 & 564 & 882 \\
\hline ec_tubb2b & 18 & 27 & 1 & 33 & 41 & 23 & 28 & 25 \\
\hline ec_xk81a1 & 468 & 308 & 8 & 110 & 105 & 290 & 104 & 351 \\
\hline em_BMP2-a & 426 & 631 & 6 & 519 & 689 & 337 & 471 & 541 \\
\hline em_BMP4-a & 1432 & 2078 & 37 & 2404 & 2363 & 1291 & 2235 & 3053 \\
\hline \begin{tabular}{|l|} 
erf \\
\end{tabular} & 369 & 376 & 14 & 394 & 394 & 319 & 396 & 494 \\
\hline fgfr4-a & 735 & 1226 & 26 & 1043 & 917 & 700 & 898 & 1050 \\
\hline fgfr4-b & 471 & 833 & 18 & 810 & 614 & 487 & 652 & 964 \\
\hline foxh1 & 291 & 313 & 5 & 350 & 234 & 308 & 285 & 447 \\
\hline fst & 240 & 614 & 8 & 958 & 525 & 493 & 482 & 1494 \\
\hline $\mathrm{fzd} 4$ & 362 & 741 & 13 & 783 & 444 & 542 & 579 & 1102 \\
\hline gbx2.1 & 28 & 91 & 1 & 108 & 39 & 71 & 43 & 190 \\
\hline hey1 & 92 & 176 & 3 & 146 & 117 & 87 & 122 & 174 \\
\hline hk_g6pd & 261 & 392 & 10 & 385 & 404 & 226 & 337 & 298 \\
\hline hk_gapdh & 177 & 328 & 5 & 243 & 307 & 188 & 263 & 244 \\
\hline hk_H4 & 81031 & 121843 & 2423 & 146020 & 130802 & 67733 & 157329 & 153353 \\
\hline hk_ODC & 6783 & 10937 & 313 & 10021 & 12306 & 6377 & 9821 & 9213 \\
\hline hnf1b & 194 & 603 & 8 & 445 & 413 & 665 & 300 & 905 \\
\hline hoxa1-a & 1 & 25 & 1 & 35 & 1 & 40 & 6 & 66 \\
\hline hoxa1-b & 14 & 127 & 3 & 164 & 14 & 193 & 22 & 407 \\
\hline hoxa2-a & 14 & 33 & 1 & 31 & 22 & 15 & 21 & 29 \\
\hline hoxa3 & 5 & 30 & 1 & 30 & 8 & 52 & 7 & 130 \\
\hline hoxa5 & 2 & 21 & 1 & 20 & 1 & 22 & 3 & 59 \\
\hline hoxb1 & 7 & 32 & 1 & 26 & 12 & 21 & 12 & 44 \\
\hline hoxb3 & 6 & 15 & 1 & 21 & 11 & 26 & 15 & 27 \\
\hline hoxb4 & 35 & 52 & 2 & 65 & 47 & 47 & 49 & 101 \\
\hline hoxd1 & 265 & 1114 & 12 & 1386 & 393 & 1459 & 704 & 2098 \\
\hline hoxd4 & 2 & 15 & 1 & 18 & 4 & 38 & 7 & 66 \\
\hline hunk & 351 & 438 & 6 & 562 & 367 & 520 & 451 & 972 \\
\hline igf3 & 17 & 75 & 1 & 86 & 42 & 62 & 35 & 119 \\
\hline kiaa0182 & 311 & 244 & 1 & 212 & 236 & 256 & 247 & 435 \\
\hline kirrel2 & 98 & 119 & 2 & 158 & 109 & 104 & 128 & 221 \\
\hline Ihx1 & 223 & 356 & 6 & 550 & 241 & 309 & 355 & 879 \\
\hline m_Chordin & 14236 & 20467 & 597 & 20254 & 21964 & 11183 & 18640 & 20494 \\
\hline m_Xbra-a/b & 1938 & 2480 & 56 & 3743 & 3593 & 1911 & 4577 & 3849 \\
\hline m_Xwnt8 & 1109 & 1508 & 33 & 1488 & 1577 & 739 & 1655 & 1494 \\
\hline me_ventx2. & 612 & 1298 & 40 & 2482 & 752 & 428 & 3005 & 4569 \\
\hline meis3-a & 333 & 725 & 3 & 729 & 457 & 658 & 489 & 1127 \\
\hline mespa-a & 14 & 50 & 1 & 187 & 21 & 38 & 87 & 280 \\
\hline
\end{tabular}




\begin{tabular}{|c|c|c|c|c|c|c|c|c|}
\hline \multirow{2}{*}{ gene } & \multicolumn{4}{|c|}{ 1h } & \multicolumn{4}{|c|}{$2 \mathrm{~h}$} \\
\hline & I & RA & $\mathrm{CHX}$ & $\mathrm{RA} / \mathrm{CH} X$ & 1 & RA & $\mathrm{CHX}$ & RA / CHX \\
\hline mespb & 76 & 412 & 11 & 1044 & 236 & 313 & 475 & 1368 \\
\hline mxi1 & 164 & 244 & 1 & 221 & 174 & 276 & 169 & 373 \\
\hline myc-a & 46 & 51 & 1 & 57 & 54 & 50 & 45 & 105 \\
\hline myc-b & 217 & 458 & 6 & 435 & 315 & 537 & 306 & 587 \\
\hline ndrg1-a & 1 & 2 & 1 & 3 & 1 & 1 & 2 & 4 \\
\hline ndrg1-b & 2 & 3 & 1 & 2 & 1 & 1 & 3 & 1 \\
\hline neurog2 & 18 & 29 & 1 & 30 & 18 & 45 & 26 & 50 \\
\hline nkx6-2 & 251 & 613 & 1 & 522 & 254 & 579 & 224 & 945 \\
\hline nodal1 & 89 & 160 & 6 & 311 & 107 & 88 & 219 & 500 \\
\hline nodal2 & 454 & 788 & 20 & 887 & 510 & 376 & 944 & 968 \\
\hline nr2f5 & 15 & 28 & 1 & 29 & 18 & 21 & 13 & 32 \\
\hline pdgfb & 32 & 43 & 1 & 41 & 32 & 18 & 44 & 35 \\
\hline pim1 & 140 & 277 & 6 & 408 & 237 & 222 & 330 & 614 \\
\hline pkdcc. 1 & 648 & 1072 & 42 & 1181 & 930 & 534 & 1009 & 1140 \\
\hline prph & 12 & 14 & 1 & 9 & 7 & 30 & 7 & 18 \\
\hline rara & 26 & 23 & 1 & 20 & 31 & 36 & 24 & 39 \\
\hline rgs14 & 29 & 102 & 1 & 47 & 41 & 71 & 32 & 45 \\
\hline rgs2 & 42 & 125 & 1 & 69 & 37 & 68 & 41 & 135 \\
\hline sox9-a/b & 26 & 64 & 1 & 78 & 30 & 67 & 34 & 118 \\
\hline spry2 & 769 & 1627 & 27 & 1854 & 1316 & 1172 & 1807 & 2027 \\
\hline stox 2 & 318 & 420 & 6 & 398 & 485 & 274 & 366 & 468 \\
\hline tdgf1p2-a & 9 & 33 & 1 & 40 & 10 & 18 & 18 & 63 \\
\hline tdgf1p2-b & 6 & 10 & 1 & 6 & 7 & 7 & 12 & 23 \\
\hline tmem72 & 29 & 115 & 1 & 58 & 39 & 81 & 26 & 75 \\
\hline trib1 & 30 & 32 & 1 & 40 & 25 & 36 & 32 & 93 \\
\hline twist1-a & 185 & 163 & 5 & 199 & 241 & 94 & 211 & 146 \\
\hline txnip & 27 & 105 & 2 & 133 & 36 & 92 & 116 & 297 \\
\hline XI.15091 & 1 & 1 & 1 & 2 & 1 & 1 & 1 & 2 \\
\hline XI.16263 & 3 & 2 & 1 & 1 & 2 & 1 & 2 & 2 \\
\hline XI.32109 & 4 & 12 & 1 & 10 & 9 & 3 & 12 & 19 \\
\hline XI.45046 & 88 & 180 & 4 & 206 & 77 & 246 & 96 & 460 \\
\hline XI.47239 & 52 & 80 & 1 & 67 & 48 & 69 & 50 & 93 \\
\hline XI.4906 & 306 & 547 & 9 & 700 & 310 & 544 & 690 & 1161 \\
\hline XI.51509 & 18 & 44 & 1 & 50 & 18 & 94 & 23 & 111 \\
\hline XI.57027 & 29 & 36 & 1 & 31 & 16 & 31 & 34 & 61 \\
\hline XI.57926 & 331 & 451 & 2 & 428 & 372 & 398 & 426 & 783 \\
\hline XI.58101 & 4 & 1 & 1 & 1 & 2 & 1 & 1 & 5 \\
\hline XI.59256 & 1 & 2 & 1 & 2 & 3 & 1 & 1 & 2 \\
\hline XI.6091 & 14 & 21 & 1 & 31 & 22 & 7 & 29 & 22 \\
\hline XI.67202 & 2 & 7 & 1 & 3 & 5 & 5 & 1 & 3 \\
\hline XI.68408 & 1 & 1 & 1 & 2 & 1 & 1 & 1 & 3 \\
\hline XI.70850 & 3 & 5 & 1 & 2 & 4 & 6 & 5 & 12 \\
\hline XI.71159 & 567 & 644 & 20 & 1114 & 829 & 303 & 1403 & 1191 \\
\hline XI.74263 & 5 & 6 & 1 & 6 & 3 & 1 & 5 & 6 \\
\hline XI.79790 & 20 & 34 & 1 & 30 & 25 & 20 & 23 & 48 \\
\hline XI.80297 & 233 & 610 & 11 & 816 & 488 & 501 & 695 & 1084 \\
\hline XI.85251 & 1 & 3 & 1 & 2 & 3 & 1 & 6 & 3 \\
\hline XI.8753 & 2 & 8 & 1 & 3 & 5 & 5 & 3 & 5 \\
\hline XI.9822 & 36 & 38 & 1 & 24 & 33 & 29 & 27 & 41 \\
\hline XI.9874 & 5 & 13 & 1 & 10 & 5 & 13 & 8 & 13 \\
\hline znf703 & 538 & 1221 & 8 & 1628 & 430 & 2069 & 817 & 3756 \\
\hline NEG_A & 3 & 1 & 1 & 4 & 3 & 3 & 1 & 1 \\
\hline NEG_B & 3 & 1 & 1 & 4 & 5 & 3 & 6 & 3 \\
\hline NEG_C & 3 & 1 & 1 & 1 & 1 & 1 & 1 & 1 \\
\hline NEG_D & 4 & 4 & 1 & 2 & 4 & 3 & 1 & 1 \\
\hline NEG_E & 4 & 4 & 1 & 3 & 3 & 3 & 3 & 3 \\
\hline NEG_F & 7 & 9 & 1 & 16 & 9 & 6 & 11 & 11 \\
\hline NEG_G & 2 & 1 & 1 & 1 & 1 & 1 & 1 & 1 \\
\hline NEG_H & 3 & 2 & 1 & 1 & 3 & 1 & 4 & 3 \\
\hline POS_A & 5323 & 5854 & 114 & 5160 & 6512 & 4601 & 5552 & 6190 \\
\hline POS_B & 1789 & 2124 & 32 & 1839 & 2227 & 1663 & 1784 & 2095 \\
\hline POS_C & 519 & 637 & 12 & 580 & 713 & 507 & 577 & 700 \\
\hline POS_D & 127 & 151 & 3 & 106 & 126 & 94 & 134 & 162 \\
\hline POS_E & 18 & 21 & 1 & 24 & 27 & 17 & 31 & 26 \\
\hline POS_F & 10 & 14 & 1 & 13 & 14 & 7 & 14 & 17 \\
\hline
\end{tabular}


Tab. 6.12 Normalized data of RA-inducibility in explants

Indicated are the Nanostring counts which were normalized to the internal positive controls and Odc and with substracted mean of the negative controls as described in the "Materials and Methods" section.

\begin{tabular}{|c|c|c|c|c|c|c|c|c|}
\hline \multirow{2}{*}{ gene } & \multicolumn{4}{|c|}{ 1h } & \multicolumn{4}{|c|}{$2 \mathrm{~h}$} \\
\hline & 1 & RA & $\mathrm{CHX}$ & RA / CHX & 1 & RA & CHX & RA / CHX \\
\hline bhlhe40 & 20 & 43 & 65 & \begin{tabular}{r|}
48 \\
\end{tabular} & 28 & 112 & 26 & 91 \\
\hline c10orf140 & 1 & 1 & 32 & 1 & 1 & 2 & 1 & 1 \\
\hline cass4 & 285 & 183 & 165 & 249 & 206 & 322 & 283 & 393 \\
\hline $\mathrm{cdx} 4$ & 491 & 703 & 397 & 909 & 532 & 1011 & 865 & 1153 \\
\hline cebpd & 40 & 57 & 32 & 25 & 8 & 148 & 11 & 116 \\
\hline cxcr7 & 222 & 342 & 132 & 471 & 226 & 383 & 400 & 544 \\
\hline сур26a1 & 527 & 2313 & 198 & 1857 & 388 & 5514 & 445 & 4095 \\
\hline dact1-a & 381 & 243 & 165 & 230 & 218 & 305 & 277 & 412 \\
\hline dact1-b & 1744 & 1557 & 1360 & 1918 & 1254 & 1895 & 1669 & 2520 \\
\hline dhrs3 & 207 & 1272 & 165 & 1066 & 211 & 1876 & 304 & 1795 \\
\hline dusp5 & 917 & 830 & 1028 & 874 & 598 & 1364 & 820 & 1424 \\
\hline dusp6 & 390 & 1007 & 331 & 1229 & 312 & 1683 & 506 & 2003 \\
\hline e_Cerberus & 9728 & 10209 & 7997 & 9952 & 10130 & 7828 & 8889 & 9071 \\
\hline e_darmin & 1 & 0 & 32 & 1 & 1 & 7 & 1 & 1 \\
\hline e_Sox17alpha & 3150 & 2691 & 3019 & 3669 & 2728 & 2533 & 4310 & 4894 \\
\hline e_Sox17beta & 1748 & 1684 & 2389 & 2186 & 1266 & 1220 & 2490 & 2664 \\
\hline e_TGIF2 & 1209 & 869 & 265 & 957 & 953 & 758 & 1115 & 1229 \\
\hline e_vpp1 & 40 & 25 & 32 & 13 & 29 & 34 & 18 & 20 \\
\hline ec_Sox2 & 323 & 325 & 99 & 374 & 126 & 671 & 419 & 712 \\
\hline ec_tubb2b & 14 & 11 & 32 & 11 & 20 & 23 & 11 & 11 \\
\hline ec_xk81a1 & 536 & 213 & 265 & 70 & 64 & 359 & 69 & 277 \\
\hline em_BMP2-a/b & 488 & 446 & 198 & 381 & 469 & 418 & 348 & 433 \\
\hline em_BMP4-a/b & 1655 & 1487 & 1227 & 1816 & 1628 & 1618 & 1691 & 2488 \\
\hline erf & 421 & 262 & 464 & 286 & 264 & 395 & 291 & 394 \\
\hline fgfr4-a & 846 & 874 & 862 & 780 & 627 & 875 & 673 & 849 \\
\hline fgfr4-b & 540 & 591 & 596 & 603 & 417 & 607 & 486 & 779 \\
\hline foxh1 & 331 & 217 & 165 & 252 & 153 & 382 & 206 & 356 \\
\hline fst & 272 & 433 & 265 & 715 & 355 & 614 & 356 & 1212 \\
\hline $\mathrm{fzd} 4$ & 413 & 525 & 430 & 582 & 299 & 676 & 430 & 892 \\
\hline $\mathrm{gb} \times 2.1$ & 26 & 57 & 32 & 68 & 18 & 83 & 22 & 146 \\
\hline hey1 & 100 & 118 & 99 & 97 & 72 & 103 & 82 & 133 \\
\hline hk_g6pd & 296 & 274 & 331 & 279 & 271 & 278 & 246 & 234 \\
\hline hk_gapdh & 199 & 228 & 165 & 171 & 204 & 231 & 190 & 190 \\
\hline hk_H4 & 94003 & 87697 & 80412 & 111161 & 90615 & 85220 & 119763 & 125437 \\
\hline hk_ODC & 7863 & 7864 & 10387 & 7616 & 8517 & 8018 & 7466 & 7527 \\
\hline hnf1b & 218 & 426 & 265 & 325 & 277 & 831 & 218 & 731 \\
\hline hoxa1-a & 1 & 10 & 32 & 13 & 1 & 44 & 1 & 44 \\
\hline hoxa1-b & 10 & 83 & 99 & 111 & 1 & 237 & 6 & 323 \\
\hline hoxa2-a & 10 & 15 & 32 & 10 & 6 & 13 & 5 & 14 \\
\hline hoxa3 & 1 & 13 & 32 & 9 & 1 & 59 & 1 & 97 \\
\hline hoxa5 & 1 & 7 & 32 & 1 & 1 & 22 & 1 & 38 \\
\hline hoxb1 & 1 & 15 & 32 & 6 & 1 & 20 & 1 & 26 \\
\hline hoxb3 & 0 & 2 & 32 & 2 & 1 & 27 & 1 & 12 \\
\hline hoxb4 & 34 & 29 & 65 & 35 & 24 & 53 & 27 & 73 \\
\hline hoxd1 & 301 & 793 & 397 & 1041 & 264 & 1830 & 525 & 1706 \\
\hline hoxd4 & 1 & 2 & 32 & 1 & 1 & 42 & 1 & 44 \\
\hline hunk & 401 & 307 & 198 & 414 & 246 & 648 & 333 & 785 \\
\hline igf3 & 13 & 46 & 32 & 51 & 20 & 72 & 16 & 88 \\
\hline kiaa0182 & 354 & 167 & 32 & 147 & 155 & 316 & 177 & 346 \\
\hline kirrel2 & 107 & $77 \mid$ & 65 & 106 & 67 & 125 & 87 & 171 \\
\hline Ihx1 & 252 & 248 & 198 & 405 & 158 & 383 & 260 & 709 \\
\hline
\end{tabular}




\begin{tabular}{|c|c|c|c|c|c|c|c|c|}
\hline \multirow{2}{*}{ gene } & \multicolumn{4}{|c|}{ 1h } & \multicolumn{4}{|c|}{$2 \mathrm{~h}$} \\
\hline & 1 & RA & $\mathrm{CHX}$ & RA / CHX & 1 & RA & $\mathrm{CHX}$ & RA / CHX \\
\hline m_Chordin & 16509 & 14724 & 19812 & 15407 & 15209 & 14065 & 14180 & 16755 \\
\hline m_Xbra-a/b & 2242 & 1777 & 1858 & 2836 & 2481 & 2399 & 3474 & 3139 \\
\hline m_Xwnt8 & 1280 & 1077 & 1094 & 1119 & 1084 & 924 & 1249 & 1212 \\
\hline me_ventx2.1-b & 703 & 926 & 1327 & 1876 & 512 & 533 & 2277 & 3728 \\
\hline \begin{tabular}{|l|} 
meis3-a \\
\end{tabular} & 380 & 513 & 99 & 541 & 308 & 822 & 362 & 912 \\
\hline mespa-a & 10 & 28 & 32 & 128 & 6 & 42 & 56 & 219 \\
\hline mespb & 82 & 288 & 364 & 781 & 155 & 388 & 351 & 1109 \\
\hline mxi1 & 184 & 167 & 32 & 154 & 112 & 341 & 118 & 295 \\
\hline myc-a & 47 & 28 & 32 & 29 & 29 & 57 & 24 & 76 \\
\hline myc-b & 245 & 321 & 198 & 317 & 209 & 670 & 222 & 470 \\
\hline ndrg1-a & 1 & 1 & 32 & 1 & 1 & 1 & 1 & 1 \\
\hline ndrg1-b & 1 & 1 & 32 & 1 & 1 & 1 & 1 & 1 \\
\hline neurog2 & 14 & 12 & 32 & 9 & 4 & 51 & 9 & 31 \\
\hline$n k x 6-2$ & 285 & 433 & 32 & 383 & 167 & 723 & 160 & 763 \\
\hline nodal1 & 97 & 107 & 198 & 223 & 65 & 105 & 156 & 399 \\
\hline nodal2 & 520 & 559 & 663 & 661 & 345 & 467 & 708 & 782 \\
\hline$n r 2 f 5$ & 11 & 12 & 32 & 8 & 4 & 20 & 1 & 16 \\
\hline pdgfb & 30 & 22 & 32 & 17 & 13 & 17 & 23 & 19 \\
\hline pim1 & 156 & 191 & 198 & 297 & 155 & 273 & 241 & 493 \\
\hline pkdcc.1 & 745 & 763 & 1393 & 885 & 636 & 666 & 758 & 923 \\
\hline prph & 7 & 2 & 32 & 1 & 1 & 32 & 1 & 5 \\
\hline rara & 24 & 8 & 32 & 1 & 13 & 39 & 8 & 22 \\
\hline rgs14 & 27 & 65 & 32 & 22 & 20 & 83 & 14 & 27 \\
\hline rgs2 & 42 & 82 & 32 & 39 & 17 & 80 & 21 & 101 \\
\hline sox9-a/b & 24 & 38 & 32 & 45 & 12 & 78 & 15 & 87 \\
\hline spry2 & 886 & 1163 & 895 & 1398 & 903 & 1469 & 1365 & 1648 \\
\hline stox2 & 362 & 294 & 198 & 289 & 327 & 339 & 268 & 373 \\
\hline tdgf1p2-a & 4 & 15 & 32 & 16 & 1 & 17 & 3 & 42 \\
\hline tdgf1p2-b & 0 & 1 & 32 & 1 & 1 & 3 & 1 & 9 \\
\hline tmem72 & 27 & 74 & 32 & 30 & 18 & 96 & 9 & 52 \\
\hline trib1 & 28 & 15 & 32 & 16 & 9 & 39 & 14 & 66 \\
\hline twist1-a & 208 & 109 & 165 & 137 & 158 & 112 & 150 & 110 \\
\hline txnip & 25 & 67 & 65 & 87 & 16 & 110 & 78 & 233 \\
\hline XI.15091 & 1 & 1 & 32.19 & 1 & 1 & 1 & 1 & 1 \\
\hline XI.16263 & 1 & 1 & 32 & 1 & 1 & 1 & 1 & 1 \\
\hline XI.32109 & 1 & 0 & 32 & 1 & 1 & 1 & 1 & 6 \\
\hline XI.45046 & 95 & 121 & 132 & 143 & 45 & 304 & 62 & 367 \\
\hline XI.47239 & 54 & 49 & 32 & 37 & 25 & 81 & 27 & 66 \\
\hline XI.4906 & 348 & 385 & 298 & 519 & 206 & 678 & 515 & 940 \\
\hline XI.51509 & 14 & 23 & 32 & 24 & 4 & 112 & 7 & 81 \\
\hline XI.57027 & 27 & 17 & 32 & 10 & 2 & 33 & 15 & 40 \\
\hline XI.57926 & 377 & 316 & 65 & 312 & 249 & 495 & 314 & 631 \\
\hline XI.58101 & 1 & 1 & 32 & 1 & 1 & 1 & 1 & 1 \\
\hline XI.59256 & 1 & 1 & 32 & 1 & 1 & 1 & 1 & 1 \\
\hline XI.6091 & 10 & 7 & 32 & 10 & 6 & 3 & 11 & 8 \\
\hline XI.67202 & 1 & 1 & 32 & 1 & 1 & 0 & 1 & 1 \\
\hline XI.68408 & 1 & 1 & 32 & 1 & 1 & 1 & 1 & 1 \\
\hline XI.70850 & 1 & 1 & 32 & 1 & 1 & 2 & 1 & 0 \\
\hline XI.71159 & 651 & 455 & 663 & 834 & 566 & 375 & 1058 & 965 \\
\hline XI.74263 & 1 & 1 & 32 & 1 & 1 & 1 & 1 & 1 \\
\hline XI.79790 & 17 & 16 & 32 & 9 & 9 & 19 & 7 & 30 \\
\hline XI.80297 & 264 & 431 & 364 & 607 & 329 & 624 & 519 & 877 \\
\hline XI.85251 & 1 & 1 & 32 & 1 & 1 & 1 & 1 & 1 \\
\hline XI.8753 & 1 & 1 & 32 & 1 & 1 & 0 & 1 & 1 \\
\hline $\mathrm{XI} .9822$ & 35 & 19 & 32 & 4 & 14 & 30 & 10 & 24 \\
\hline XI.9874 & 1 & 1 & 32 & 1 & 1 & 10 & 1 & 1 \\
\hline znf703 & 618 & 870 & 265 & 1225 & 289 & 2597 & 611 & 3063 \\
\hline
\end{tabular}


Tab. 6.13 Raw data of RA-inducibility in whole embryos

$\mathrm{CE}=$ control embryo; $\mathrm{RA}=\mathrm{CE}+\mathrm{RA}$

\begin{tabular}{|c|c|c|c|c|c|c|c|c|c|c|c|c|}
\hline \multirow{3}{*}{ gene } & \multicolumn{6}{|c|}{$s 22(A)$} & \multicolumn{6}{|c|}{ s23 (B) } \\
\hline & \multicolumn{2}{|c|}{ st.12 } & \multicolumn{2}{|c|}{ st.13 } & \multicolumn{2}{|c|}{ st.14 } & \multicolumn{2}{|c|}{ st.12 } & \multicolumn{2}{|c|}{ st.13 } & \multicolumn{2}{|c|}{ st.14 } \\
\hline & CE & RA & CE & RA & CE & RA & CE & RA & CE & RA & CE & RA \\
\hline bhlhe40 & 21 & 30 & 29 & 77 & 22 & 119 & 19 & 20 & 32 & 68 & 49 & 84 \\
\hline c10orf140 & 5 & 2 & 4 & 6 & 5 & 8 & 6 & 3 & 3 & 3 & 1 & 3 \\
\hline cass4 & 189 & 147 & 241 & 303 & 151 & 356 & 207 & 173 & 172 & 328 & 273 & 362 \\
\hline $\mathrm{cdx} 4$ & 1484 & 1521 & 1873 & 2325 & 1313 & 2015 & 1863 & 1425 & 1615 & 2082 & 2163 & 1852 \\
\hline cebpd & 42 & 74 & 28 & 169 & 63 & 215 & 82 & 192 & 47 & 229 & 38 & 259 \\
\hline cxcr7 & 148 & 111 & 114 & 162 & 96 & 161 & 201 & 166 & 128 & 148 & 131 & 161 \\
\hline cyp26a1 & 814 & 2337 & 884 & 11847 & 612 & 12983 & 1491 & 8338 & 740 & 11128 & 951 & 11009 \\
\hline dact1-a & 99 & 82 & 75 & 104 & 67 & 99 & 121 & 139 & 91 & 129 & 147 & 99 \\
\hline dact1-b & 655 & 759 & 630 & 1099 & 493 & 1217 & 974 & 1272 & 613 & 1204 & 794 & 1227 \\
\hline dhrs3 & 378 & 730 & 432 & 2631 & 308 & 3191 & 630 & 2268 & 359 & 3272 & 429 & 2960 \\
\hline dusp5 & 308 & 319 & 308 & 694 & 245 & 841 & 390 & 631 & 339 & 884 & 433 & 839 \\
\hline dusp6 & 349 & 506 & 491 & 1709 & 322 & 2063 & 314 & 1002 & 304 & 1732 & 481 & 1842 \\
\hline e_Cerberus & 254 & 457 & 89 & 118 & 80 & 66 & 1461 & 943 & 491 & 695 & 556 & 313 \\
\hline e_darmin & 693 & 873 & 480 & 1189 & 911 & 1490 & 959 & 519 & 530 & 1094 & 1209 & 1108 \\
\hline e_Sox17alph & 525 & 531 & 623 & 478 & 516 & 455 & 807 & 420 & 565 & 576 & 725 & 459 \\
\hline e_Sox17beta & 563 & 509 & 570 & 521 & 426 & 444 & 736 & 409 & 577 & 487 & 684 & 438 \\
\hline e_TGIF2 & 192 & 190 & 310 & 234 & 186 & 243 & 460 & 293 & 299 & 281 & 243 & 141 \\
\hline e_vpp1 & 70 & 78 & 35 & 52 & 71 & 35 & 162 & 81 & 93 & 95 & 123 & 52 \\
\hline ec_Sox2 & 890 & 659 & 1139 & 841 & 812 & 1039 & 1430 & 1068 & 1001 & 1412 & 1067 & 1340 \\
\hline ec_tubb2b & 43 & 37 & 70 & 77 & 52 & 119 & 39 & 21 & 43 & 43 & 55 & 60 \\
\hline ec_xk81a1 & 3456 & 2400 & 6564 & 6466 & 5765 & 8264 & 4112 & 2638 & 6402 & 8453 & 10511 & 8033 \\
\hline em_BMP2-a & 457 & 458 & 324 & 399 & 368 & 494 & 661 & 238 & 443 & 308 & 668 & 416 \\
\hline em_BMP4-a & 1624 & 1501 & 1377 & 1240 & 1026 & 1058 & 2915 & 1512 & 1660 & 1298 & 1863 & 1000 \\
\hline erf & 221 & 151 & 193 & 299 & 174 & 422 & 304 & 302 & 219 & 508 & 308 & 454 \\
\hline fgfr4-a & 1100 & 927 & 1113 & 971 & 894 & 905 & 1742 & 972 & 1357 & 1228 & 1511 & 1133 \\
\hline fgfr4-b & 469 & 429 & 526 & 498 & 396 & 510 & 781 & 521 & 468 & 520 & 608 & 485 \\
\hline foxh1 & 236 & 197 & 282 & 445 & 165 & 402 & 290 & 321 & 257 & 406 & 338 & 403 \\
\hline fst & 169 & 183 & 241 & 373 & 183 & 606 & 407 & 559 & 215 & 814 & 276 & 828 \\
\hline $\mathrm{fzd} 4$ & 450 & 537 & 311 & 1006 & 328 & 999 & 641 & 871 & 383 & 938 & 448 & 1037 \\
\hline $\mathrm{gb} \times 2.1$ & 384 & 330 & 351 & 910 & 253 & 1058 & 379 & 710 & 330 & 1003 & 466 & 1220 \\
\hline hey1 & 118 & 117 & 156 & 213 & 101 & 192 & 165 & 68 & 165 & 190 & 163 & 207 \\
\hline hk_g6pd & 186 & 147 & 192 & 161 & 124 & 147 & 296 & 147 & 137 & 165 & 207 & 152 \\
\hline hk_gapdh & 125 & 101 & 165 & 156 & 125 & 245 & 123 & 102 & 120 & 158 & 120 & 186 \\
\hline hk_H4 & 66093 & 54788 & 86152 & 78237 & 62183 & 66486 & 146073 & 84833 & 93587 & 84235 & 112190 & 93322 \\
\hline hk_ODC & 7511 & 6970 & 8871 & 9650 & 6875 & 9427 & 9779 & 6085 & 6549 & 7328 & 8264 & 6815 \\
\hline hnf1b & 416 & 402 & 398 & 1562 & 425 & 1928 & 432 & 983 & 388 & 1678 & 630 & 1769 \\
\hline hoxa1-a & 18 & 31 & 27 & 140 & 35 & 149 & 40 & 150 & 36 & 208 & 51 & 225 \\
\hline hoxa1-b & 153 & 247 & 251 & 897 & 196 & 994 & 264 & 711 & 236 & 1060 & 280 & 1209 \\
\hline hoxa2-a & 17 & 15 & 31 & 38 & 19 & 32 & 30 & 16 & 17 & 32 & 35 & 34 \\
\hline hoxa3 & 61 & 48 & 79 & 345 & 47 & 498 & 12 & 183 & 33 & 401 & 57 & 491 \\
\hline hoxa5 & 4 & 8 & 11 & 61 & 12 & 70 & 5 & 114 & 12 & 200 & 12 & 143 \\
\hline hoxb1 & 132 & 129 & 199 & 507 & 158 & 519 & 98 & 208 & 151 & 422 & 203 & 390 \\
\hline hoxb3 & 18 & 19 & 24 & 73 & 24 & 99 & 16 & 49 & 20 & 102 & 34 & 123 \\
\hline hoxb4 & 39 & 35 & 60 & 129 & 40 & 160 & 57 & 96 & 52 & 141 & 62 & 169 \\
\hline hoxd1 & 1309 & 1648 & 1378 & 2785 & 931 & 2703 & 2262 & 2791 & 1570 & 3129 & 1451 & 2769 \\
\hline hoxd4 & 100 & 106 & 193 & 400 & 106 & 650 & 49 & 230 & 107 & 708 & 228 & 892 \\
\hline hunk & 623 & 582 & 684 & 1197 & 468 & 1414 & 846 & 1517 & 531 & 1550 & 801 & 1468 \\
\hline igf3 & 73 & 74 & 116 & 236 & 93 & 291 & 94 & 134 & 127 & 315 & 123 & 327 \\
\hline kiaa0182 & 274 & 215 & 361 & 519 & 215 & 631 & 578 & 435 & 376 & 645 & 498 & 700 \\
\hline kirrel2 & 180 & 174 & 212 & 287 & 195 & 359 & 361 & 363 & 360 & 388 & 403 & 398 \\
\hline Ihx1 & 83 & 126 & 120 & 327 & 81 & 347 & 90 & 287 & 87 & 468 & 99 & 447 \\
\hline
\end{tabular}




\begin{tabular}{|c|c|c|c|c|c|c|c|c|c|c|c|c|}
\hline \multirow{3}{*}{ gene } & \multicolumn{6}{|c|}{$s 22(A)$} & \multicolumn{6}{|c|}{ s23 (B) } \\
\hline & \multicolumn{2}{|c|}{ st.12 } & \multicolumn{2}{|c|}{ st.13 } & \multicolumn{2}{|c|}{ st.14 } & st. & .12 & st.1. & .13 & st.1 & 14 \\
\hline & CE & RA & CE & RA & CE & RA & CE & RA & CE & RA & CE & RA \\
\hline m_Chordin & 1302 & 1330 & 1451 & 849 & 1085 & 1034 & 2672 & 1872 & 1554 & 1423 & 1495 & 1220 \\
\hline m_Xbra-a/b & 955 & 912 & 961 & 439 & 533 & 233 & 2013 & 1001 & 829 & 440 & 888 & 310 \\
\hline $\mathrm{m} \_\mathrm{Xwnt8}$ & 396 & 297 & 491 & 240 & 233 & 191 & 859 & 585 & 383 & 514 & 337 & 366 \\
\hline me_ventx 2 . & 1328 & 748 & 992 & 1118 & 179 & 921 & 2356 & 1431 & 1200 & 2000 & 1507 & 848 \\
\hline meis3-a & 703 & 649 & 706 & 1715 & 505 & 2034 & 794 & 1393 & 633 & 1803 & 934 & 2026 \\
\hline mespa-a & 28 & 45 & 27 & 45 & 32 & 61 & 43 & 30 & 37 & 68 & 46 & 47 \\
\hline mespb & 196 & 146 & 180 & 199 & 94 & 151 & 348 & 246 & 164 & 243 & 193 & 164 \\
\hline mxi1 & 359 & 397 & 330 & 514 & 278 & 657 & 434 & 735 & 309 & 816 & 384 & 769 \\
\hline myc-a & 88 & 101 & 130 & 156 & 101 & 181 & 103 & 60 & 91 & 130 & 144 & 156 \\
\hline myc-b & 1067 & 1042 & 1490 & 1579 & 907 & 1505 & 1378 & 841 & 1299 & 1057 & 1730 & 1082 \\
\hline ndrg1-a & 1 & 1 & 2 & 1 & 3 & 1 & 1 & 2 & 1 & 1 & 1 & 2 \\
\hline ndrg1-b & 1 & 2 & 2 & 1 & 2 & 6 & 1 & 2 & 2 & 3 & 6 & 2 \\
\hline neurog2 & 93 & 73 & 83 & 68 & 49 & 74 & 174 & 115 & 119 & 110 & 86 & 69 \\
\hline$n k x 6-2$ & 94 & 126 & 112 & 224 & 73 & 352 & 159 & 305 & 124 & 334 & 138 & 378 \\
\hline nodal1 & 12 & 6 & 18 & 11 & 10 & 11 & 10 & 13 & 3 & 8 & 3 & 9 \\
\hline nodal2 & 13 & 6 & 13 & 4 & 1 & 4 & 14 & 6 & 6 & 5 & 8 & 7 \\
\hline $\mathrm{nr} 2 \mathrm{f5}$ & 19 & 16 & 90 & 347 & 103 & 590 & 18 & 41 & 72 & 311 & 144 & 437 \\
\hline pdgfb & 15 & 12 & 21 & 22 & 19 & 28 & 17 & 16 & 16 & 19 & 23 & 29 \\
\hline pim1 & 174 & 179 & 166 & 354 & 133 & 371 & 328 & 284 & 210 & 357 & 317 & 319 \\
\hline pkdcc.1 & 213 & 233 & 265 & 266 & 229 & 343 & 512 & 399 & 338 & 470 & 387 & 484 \\
\hline prph & 50 & 39 & 82 & 312 & 56 & 237 & 49 & 179 & 89 & 493 & 114 & 368 \\
\hline rara & 54 & 41 & 88 & 97 & 54 & 146 & 52 & 72 & 58 & 175 & 115 & 190 \\
\hline rgs14 & 1 & 1 & 1 & 1 & 1 & 1 & 1 & 3 & 1 & 3 & 1 & 4 \\
\hline rgs2 & 66 & 144 & 180 & 273 & 119 & 346 & 112 & 131 & 93 & 202 & 124 & 142 \\
\hline sox9-a/b & 107 & 176 & 222 & 483 & 164 & 474 & 147 & 358 & 184 & 431 & 271 & 667 \\
\hline spry2 & 290 & 339 & 347 & 465 & 239 & 539 & 509 & 395 & 344 & 579 & 389 & $52 C$ \\
\hline stox2 & 211 & 147 & 169 & 206 & 153 & 204 & 274 & 169 & 171 & 178 & 254 & 210 \\
\hline tdgf1p2-a & 25 & 33 & 42 & 72 & 38 & 92 & 37 & 64 & 28 & 97 & 46 & 110 \\
\hline tdgf1p2-b & 9 & 6 & 3 & 9 & 9 & 18 & 3 & 10 & 4 & 11 & 11 & 14 \\
\hline tmem72 & 104 & 98 & 142 & 314 & 169 & 342 & 154 & 233 & 202 & 541 & 293 & 499 \\
\hline trib1 & 52 & 40 & 51 & 75 & 37 & 60 & 53 & 21 & 51 & 45 & 63 & 48 \\
\hline twist1-a & 215 & 188 & 287 & 92 & 244 & 129 & 261 & 83 & 267 & 96 & 374 & 110 \\
\hline txnip & 231 & 280 & 207 & 465 & 233 & 488 & 110 & 83 & 115 & 207 & 209 & 212 \\
\hline XI.15091 & 1 & 2 & 6 & 2 & 2 & 2 & 2 & 1 & 1 & 3 & 4 & 34 \\
\hline XI.16263 & 2 & 2 & 1 & 1 & 1 & 3 & 3 & 1 & 2 & 1 & 5 & 2 \\
\hline XI.32109 & 11 & 5 & 8 & 14 & 3 & 8 & 11 & 7 & 10 & 7 & 5 & 19 \\
\hline $\mathrm{XI} .45046$ & 324 & 316 & 497 & 919 & 311 & 1116 & 508 & 773 & 478 & 1342 & 694 & 1333 \\
\hline XI.47239 & 65 & 54 & 82 & 130 & 72 & 160 & 50 & 58 & 76 & 131 & 101 & 137 \\
\hline $\mathrm{XI} .4906$ & 603 & 455 & 578 & 568 & 489 & 626 & 1131 & 880 & 744 & 773 & 1062 & 614 \\
\hline XI.51509 & 66 & 79 & 94 & 339 & 74 & 447 & 58 & 166 & 72 & 420 & 133 & 446 \\
\hline XI.57027 & 35 & 33 & 44 & 60 & 35 & 75 & 42 & 76 & 38 & 52 & 70 & 74 \\
\hline XI.57926 & 232 & 350 & 276 & 693 & 160 & 862 & 430 & 757 & 309 & 1003 & 511 & 823 \\
\hline XI.58101 & 2 & 1 & 2 & 1 & 1 & 5 & 3 & 3 & 2 & 3 & 2 & 1 \\
\hline XI.59256 & 1 & 1 & 1 & 2 & 1 & 2 & 1 & 1 & 1 & 1 & 2 & 5 \\
\hline XI.6091 & 10 & 9 & 16 & 11 & 7 & 14 & 17 & 8 & 14 & 19 & 17 & $1 \varepsilon$ \\
\hline $\mathrm{XI} .67202$ & 2 & 2 & 4 & 2 & 3 & 1 & 7 & 4 & 3 & 7 & 5 & 1 \\
\hline XI.68408 & 1 & 2 & 3 & 3 & 1 & 3 & 1 & 1 & 1 & 3 & 1 & 1 \\
\hline XI.70850 & 3 & 6 & 5 & 6 & 4 & 11 & 17 & 8 & 7 & 12 & 10 & 13 \\
\hline XI.71159 & 608 & 431 & 775 & 413 & 494 & 382 & 929 & 306 & 629 & 316 & \begin{tabular}{l|l}
652 \\
\end{tabular} & 258 \\
\hline XI.74263 & 6 & 1 & 1 & 5 & 2 & 3 & 2 & 3 & 1 & 7 & 2 & 4 \\
\hline XI.79790 & 29 & 23 & 60 & 82 & 50 & 75 & 49 & 77 & 47 & 113 & 86 & 114 \\
\hline XI.80297 & 63 & 77 & 72 & 134 & 61 & 135 & 110 & 161 & 77 & 139 & 69 & 127 \\
\hline XI.85251 & 1 & 1 & 5 & 1 & 2 & 4 & 6 & 3 & 3 & 2 & 4 & 2 \\
\hline XI.8753 & 1 & 1 & 1 & 2 & 1 & 5 & 2 & 3 & 2 & 5 & 5 & 2 \\
\hline XI.9822 & 13 & 12 & 23 & 8 & 11 & 10 & 23 & 18 & 16 & 23 & 32 & 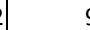 \\
\hline XI.9874 & 4 & 4 & 8 & 3 & 5 & 7 & 5 & 3 & 2 & 3 & 2 & 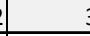 \\
\hline znf703 & 2494 & 2707 & 2278 & 5979 & 1642 & 6561 & 2789 & 4455 & 2505 & 6285 & 3439 & 686 \\
\hline NEG_A & 2 & 2 & 6 & 4 & 2 & 4 & 5 & 3 & 2 & 4 & 5 & 5 \\
\hline NEG_B & 2 & 4 & 3 & 3 & 1 & 2 & 5 & 3 & 3 & 7 & 5 & 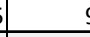 \\
\hline NEG_C & 1 & 1 & 1 & 1 & 1 & 1 & 1 & 1 & 1 & 1 & 1 & L \\
\hline NEG_D & 1 & 1 & 2 & 1 & 2 & 1 & 1 & 3 & 2 & 5 & 4 & t. \\
\hline NEG_E & 2 & 1 & 6 & 2 & 2 & 7 & 4 & 4 & 3 & 2 & 4 & t. \\
\hline NEG_F & 8 & 5 & 12 & 10 & 9 & 8 & 14 & 13 & 9 & 9 & 16 & 5 \\
\hline NEG_G & 1 & 1 & 1 & 1 & 1 & 2 & 1 & 1 & 1 & 1 & 1 & 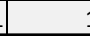 \\
\hline NEG_H & 1 & 3 & 1 & 1 & 1 & 5 & 1 & 3 & 1 & 1 & 2 & 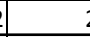 \\
\hline POS_A & 4427 & 5014 & 6295 & 5330 & 5094 & 4745 & 6726 & 4348 & 5441 & 6022 & 7964 & 760 \\
\hline POS_B & 1739 & 1975 & 2557 & 2088 & 2053 & 1910 & 2829 & 1780 & 2058 & 2343 & 2986 & 284 \\
\hline POS_C & 511 & 607 & 814 & 686 & 616 & 580 & 787 & 555 & 592 & 718 & 938 & 78 \\
\hline POS_D & 124 & 135 & 160 & 131 & 144 & 121 & 158 & 105 & 133 & 135 & 176 & 18 \\
\hline POS_E & 15 & 21 & 31 & 30 & 18 & 18 & 22 & 35 & 16 & 23 & 38 & 36 \\
\hline POS_F & 4 & 13 & 13 & 6 & 13 & 7 & 13 & 10 & 17 & 10 & 20 & 2. \\
\hline
\end{tabular}


Tab. 6.14 Normalized data of RA-inducibility in whole embryos

Indicated are the Nanostring counts which were normalized to the internal positive controls and Odc and with substracted mean of the negative controls as described in the "Materials and Methods" section.

$\mathrm{CE}=$ control embryo; $\mathrm{RA}=\mathrm{CE}+\mathrm{RA}$

\begin{tabular}{|c|c|c|c|c|c|c|c|c|c|c|c|c|}
\hline \multirow{3}{*}{ gene } & \multicolumn{6}{|c|}{ A } & \multicolumn{6}{|c|}{ B } \\
\hline & \multicolumn{2}{|c|}{ st. 12} & \multicolumn{2}{|c|}{ st. 13} & \multicolumn{2}{|c|}{ st. 14} & \multicolumn{2}{|c|}{ st. 12} & \multicolumn{2}{|c|}{ st. 13} & \multicolumn{2}{|c|}{ st. 14} \\
\hline & \begin{tabular}{l|l|} 
CE & \\
\end{tabular} & RA & \begin{tabular}{l|l|}
$\mathrm{CE}$ & \\
\end{tabular} & RA & \begin{tabular}{l|l|} 
CE & \\
\end{tabular} & RA & \begin{tabular}{l|l|}
$\mathrm{CE}$ & \\
\end{tabular} & RA & \begin{tabular}{l|l|}
$\mathrm{CE}$ & \\
\end{tabular} & RA & \begin{tabular}{l|l|}
$\mathrm{CE}$ & \\
\end{tabular} & RA \\
\hline bhlhe40 & 17 & 35 & 15 & 68 & 22 & 114 & 2 & 13 & 29 & 62 & 32 & 74 \\
\hline c11orf140 & 1 & 1 & 1 & 1 & 1 & 1 & 1 & 1 & 1 & 1 & 1 & \\
\hline cass4 & 216 & 193 & 214 & 293 & 188 & 331 & 147 & 214 & 193 & 331 & 245 & 354 \\
\hline $\mathrm{cdx} 4$ & 1666 & 2143 & 1743 & 2316 & 1693 & 1912 & 1429 & 1764 & 1876 & 2151 & 2138 & 1858 \\
\hline cebpd & 41 & 94 & 15 & 159 & 74 & 196 & 52 & 228 & 47 & 228 & 22 & 251 \\
\hline cxcr7 & 160 & 144 & 95 & 152 & 117 & 144 & 143 & 195 & 141 & 144 & 120 & 152 \\
\hline cyp26a1 & 912 & 3142 & 816 & 11789 & 785 & 12369 & 1142 & 11377 & 855 & 11536 & 888 & 11199 \\
\hline dact1-a & 115 & 115 & 59 & 95 & 79 & 85 & 82 & 162 & 98 & 124 & 125 & 89 \\
\hline dact1-b & 731 & 1117 & 579 & 1185 & 632 & 1151 & 742 & 1573 & 717 & 1239 & 739 & 1227 \\
\hline dhrs3 & 419 & 978 & 393 & 2611 & 391 & 3133 & 475 & 2814 & 412 & 3385 & 393 & 2976 \\
\hline dusp5 & 341 & 424 & 277 & 682 & 320 & 793 & 289 & 775 & 387 & 917 & 396 & 836 \\
\hline dusp6 & 386 & 676 & 448 & 1693 & 419 & 1958 & 231 & 1237 & 347 & 1787 & 442 & 1848 \\
\hline e_Cerberus & 279 & 611 & 72 & 118 & 96 & 54 & 1118 & 1163 & 565 & 711 & 513 & 315 \\
\hline e_darmin & 774 & 1171 & 438 & 1175 & 1172 & 1411 & 729 & 635 & 611 & 1125 & 1133 & 1117 \\
\hline e_Sox17alpha & 585 & 720 & 572 & 467 & 662 & 425 & 612 & 512 & 652 & 588 & 673 & 452 \\
\hline e_Sox17beta & 628 & 681 & 522 & 520 & 544 & 414 & 557 & 498 & 665 & 495 & 634 & 431 \\
\hline e_TGIF2 & 219 & 251 & 279 & 224 & 233 & 223 & 343 & 354 & 342 & 282 & 216 & 131 \\
\hline e_vpp1 & 72 & 100 & 21 & 43 & 84 & 24 & 112 & 89 & 111 & 89 & 112 & 42 \\
\hline ec_Sox2 & 996 & 882 & 1155 & 828 & 1144 & 981 & 1194 & 1319 & 1160 & 1455 & 998 & 1341 \\
\hline ec_tubb2b & 41 & 44 & 54 & 68 & 60 & 114 & 17 & 15 & 42 & 35 & 38 & 50 \\
\hline ec_xk81a1 & 3890 & 3227 & 6138 & 6431 & 7459 & 7870 & 3169 & 3275 & 7459 & 8762 & 9957 & 8196 \\
\hline em_BMP2-a/b & 518 & 611 & 292 & 388 & 469 & 462 & 499 & 285 & 519 & 320 & 619 & 419 \\
\hline em_BMP4-a/b & 1824 & 2116 & 1278 & 1226 & 1321 & 1000 & 2243 & 1872 & 1928 & 1337 & 1753 & 998 \\
\hline erf & 242 & 198 & 169 & 289 & 218 & 393 & 222 & 365 & 247 & 517 & 278 & 447 \\
\hline fgfr4-a & 1233 & 1243 & 1131 & 958 & 1151 & 854 & 1335 & 1200 & 1575 & 1264 & 1419 & 1133 \\
\hline fgfr4-b & 522 & 572 & 481 & 487 & 515 & 477 & 592 & 638 & 538 & 530 & 562 & 479 \\
\hline foxh1 & 259 & 260 & 252 & 434 & 216 & 374 & 212 & 388 & 292 & 411 & 316 & 396 \\
\hline fst & 184 & 241 & 214 & 362 & 229 & 569 & 312 & 685 & 243 & 835 & 247 & 825 \\
\hline $\mathrm{fzd} 4$ & 511 & 718 & 280 & 993 & 417 & 943 & \begin{tabular}{ll|}
483 \\
\end{tabular} & 1174 & 439 & 963 & 412 & 1136 \\
\hline $\mathrm{gb} \times 2.1$ & 426 & 439 & 317 & 897 & 320 & 1000 & 281 & 873 & 377 & 1132 & 428 & 1221 \\
\hline hey1 & 126 & 152 & 134 & 213 & 123 & 174 & 115 & 73 & 184 & 187 & 141 & 198 \\
\hline hnf1b & 462 & 536 & 361 & 1547 & 543 & 1829 & 321 & 1213 & 445 & 1731 & 583 & 1774 \\
\hline hoxa1-a & 13 & 36 & 14 & 131 & 38 & 133 & 18 & 175 & 34 & 216 & 34 & 216 \\
\hline hoxa1-b & 166 & 327 & 223 & 884 & 246 & 939 & 191 & 874 & 267 & 1190 & 251 & 1219 \\
\hline hoxa2-a & 12 & 15 & 17 & 29 & 17 & 21 & 11 & 8 & 12 & 23 & 19 & 23 \\
\hline hoxa3 & \begin{tabular}{l|l}
62 \\
\end{tabular} & 59 & \begin{tabular}{l|l}
62 \\
\end{tabular} & 335 & \begin{tabular}{l|l}
53 \\
\end{tabular} & 466 & 1 & 216 & 31 & 416 & 40 & 485 \\
\hline hoxa5 & 1 & 5 & 1 & 52 & 8 & 58 & 1 & 131 & 6 & 198 & 1 & 133 \\
\hline hoxb1 & 142 & 168 & 175 & 496 & 197 & 486 & 63 & 248 & 168 & 428 & 178 & 383 \\
\hline hoxb3 & 13 & 21 & 12 & 64 & 23 & 85 & 1 & 50 & 15 & 96 & 18 & 113 \\
\hline hoxb4 & 37 & 42 & 45 & 119 & 44 & 143 & 31 & 118 & 53 & 136 & 44 & 160 \\
\hline hoxd1 & 1469 & 2214 & 1279 & 2764 & 1198 & 2568 & 1738 & 3466 & 1823 & 3237 & 1362 & 2784 \\
\hline hoxd4 & 116 & 137 & 169 & 389 & 129 & 612 & 25 & 275 & 117 & 725 & 212 & 889 \\
\hline hunk & 695 & 778 & 629 & 1183 & 598 & 1339 & 642 & 1879 & 611 & 1598 & 745 & 1472 \\
\hline igf3 & 75 & 94 & 97 & 226 & 113 & 268 & 60 & 155 & 141 & 317 & 112 & 319 \\
\hline kiaa1182 & 312 & 284 & 327 & 518 & 272 & 592 & 434 & 531 & 432 & 659 & 458 & 696 \\
\hline kirrel2 & 196 & 229 & 187 & 277 & 245 & 333 & 266 & 442 & 412 & 393 & 368 & 392 \\
\hline Ihx1 & 87 & 164 & 112 & 317 & 97 & 322 & 57 & 346 & 93 & 476 & 80 & 441 \\
\hline
\end{tabular}




\begin{tabular}{|c|c|c|c|c|c|c|c|c|c|c|c|c|}
\hline \multirow{3}{*}{ gene } & \multicolumn{6}{|c|}{ A } & \multicolumn{6}{|c|}{ B } \\
\hline & \multicolumn{2}{|c|}{ st. 12} & \multicolumn{2}{|c|}{ st. 13} & \multicolumn{2}{|c|}{ st. 14} & \multicolumn{2}{|c|}{ st. 12} & \multicolumn{2}{|c|}{ st. 13} & \multicolumn{2}{|c|}{ st. 14} \\
\hline & CE & RA & \begin{tabular}{l|l} 
CE & \\
\end{tabular} & RA & \begin{tabular}{l|l} 
CE & \\
\end{tabular} & $\mathrm{RA}$ & \begin{tabular}{l|l} 
CE & \\
\end{tabular} & RA & \begin{tabular}{l|l}
$\mathrm{CE}$ & \\
\end{tabular} & RA & CE & RA \\
\hline m_Chordin & 1462 & 1786 & 1348 & 836 & 1398 & 977 & 2155 & 2322 & 1815 & 1467 & 1414 & 1221 \\
\hline m_Xbra-a/b & 1170 & 1223 & 889 & 428 & 683 & 213 & 1545 & 1236 & 959 & 447 & 828 & 312 \\
\hline m_Xwnt8 & 439 & 395 & 448 & 230 & 294 & 173 & 652 & 717 & 439 & 523 & 315 & 358 \\
\hline me_ventx2.1-b & 1491 & 1112 & 918 & 1114 & 224 & 869 & 1811 & 1771 & 1392 & 2165 & 1415 & 845 \\
\hline meis3-a & 786 & 869 & 650 & 1699 & 646 & 1931 & 612 & 1724 & 731 & 1862 & 872 & 2134 \\
\hline mespa-a & 25 & 55 & 14 & 36 & 34 & 49 & 21 & 26 & 35 & 62 & 29 & 37 \\
\hline mespb & 214 & 191 & 157 & 189 & 114 & 135 & 256 & 295 & 183 & 242 & 169 & 155 \\
\hline mxi1 & 398 & 529 & 297 & 513 & 352 & 617 & 323 & 914 & 352 & 837 & 350 & 765 \\
\hline myc-a & 92 & 132 & 111 & 146 & 123 & 163 & 67 & 63 & 98 & 125 & 122 & 147 \\
\hline myc-b & 1196 & 1398 & 1384 & 1563 & 1167 & 1426 & 1154 & 1136 & 1517 & 1187 & 1627 & 1181 \\
\hline ndrg1-a & 1 & 1 & 1 & 1 & 1 & 1 & 1 & 1 & 1 & 1 & 1 & 1 \\
\hline ndrg1-b & 1 & 1 & 1 & 1 & 1 & 1 & 1 & 1 & 1 & 1 & 1 & 1 \\
\hline neurog2 & 98 & 93 & 66 & 59 & 56 & 61 & 122 & 132 & 132 & 114 & 67 & 59 \\
\hline nkx6-2 & 99 & 164 & 93 & 214 & 87 & 326 & 111 & 368 & 137 & 337 & 116 & 372 \\
\hline nodal1 & 7 & 3 & 5 & 2 & 5 & 1 & 1 & 5 & 1 & 1 & 1 & 1 \\
\hline nodal2 & 8 & 3 & 1 & 1 & 1 & 1 & 1 & 1 & 1 & 1 & 1 & 1 \\
\hline $\mathrm{nr} 2 \mathrm{f5}$ & 14 & 16 & 73 & 337 & 126 & 553 & 1 & 40 & 76 & 313 & 122 & 431 \\
\hline pdgfb & 10 & 12 & 8 & 13 & 17 & 18 & 1 & 8 & 12 & 10 & 7 & 18 \\
\hline pim1 & 189 & 236 & 144 & 343 & 164 & 345 & 242 & 342 & 237 & 362 & 286 & 311 \\
\hline pkdcc. 1 & 233 & 318 & 237 & 256 & 289 & 318 & 383 & 486 & 386 & 478 & 353 & 478 \\
\hline prph & 49 & 47 & 65 & 312 & 65 & 217 & 25 & 211 & 96 & 512 & 94 & 361 \\
\hline rara & 54 & 50 & 72 & 88 & 62 & 131 & 27 & 78 & 60 & 172 & 95 & 182 \\
\hline rgs14 & 1 & 1 & 1 & 1 & 1 & 1 & 1 & 1 & 1 & 1 & 1 & 1 \\
\hline rgs2 & 67 & 189 & 157 & 263 & 146 & 322 & 74 & 152 & 111 & 200 & 113 & 132 \\
\hline sox9-a/b & 114 & 232 & 196 & 472 & 215 & 443 & 112 & 434 & 217 & 437 & 243 & 662 \\
\hline spry2 & 320 & 451 & 313 & 454 & 312 & 515 & 381 & 482 & 393 & 592 & 355 & 514 \\
\hline stox2 & 232 & 193 & 147 & 196 & 191 & 185 & 199 & 199 & 191 & 175 & 227 & 211 \\
\hline tdgf1p2-a & 21 & 39 & 28 & 63 & 41 & 79 & 16 & 68 & 25 & 92 & 29 & 111 \\
\hline tdgf1p2-b & 3 & 3 & 1 & 1 & 4 & 8 & 1 & 2 & 1 & 2 & 1 & 3 \\
\hline tmem72 & 111 & 127 & 121 & 314 & 211 & 317 & 116 & 279 & 228 & 551 & 264 & 493 \\
\hline trib1 & 52 & 48 & 36 & 66 & 41 & 48 & 28 & 15 & 51 & 37 & 45 & 38 \\
\hline twist1-a & 235 & 248 & 257 & 83 & 318 & 114 & 189 & 92 & 313 & 90 & 341 & 111 \\
\hline txnip & 253 & 372 & 182 & 454 & 294 & 456 & 72 & 92 & 126 & 215 & 184 & 213 \\
\hline XI.15091 & 1 & 1 & 1 & 1 & 1 & 1 & 1 & 1 & 1 & 1 & 1 & 1 \\
\hline XI.16263 & 1 & 1 & 1 & 1 & 1 & 1 & 1 & 1 & 1 & 1 & 1 & 1 \\
\hline $\mathrm{XI} .32109$ & 5 & 1 & 1 & 5 & 1 & 1 & 1 & 1 & 4 & 1 & 1 & 8 \\
\hline XI.45046 & 358 & 421 & 454 & 916 & 395 & 1155 & 381 & 952 & 549 & 1383 & 644 & 1334 \\
\hline XI.47239 & 66 & 67 & 65 & 121 & 85 & 143 & 26 & 62 & 82 & 126 & 81 & 127 \\
\hline XI.4906 & 673 & 617 & 530 & 557 & 626 & 588 & 862 & 1185 & 860 & 792 & 993 & 619 \\
\hline XI.51509 & 67 & 112 & 76 & 329 & 88 & 417 & 32 & 195 & 76 & 426 & 112 & 439 \\
\hline XI.57027 & 32 & 39 & 30 & 52 & 38 & 62 & 20 & 83 & 36 & 44 & 52 & 64 \\
\hline XI.57926 & 255 & 466 & 247 & 681 & 199 & 813 & 320 & 932 & 352 & 1132 & 471 & 820 \\
\hline XI.58101 & 1 & 1 & 1 & 1 & 1 & 1 & 1 & 1 & 1 & 1 & 1 & 1 \\
\hline $\mathrm{XI} .59256$ & 1 & 1 & 1 & 1 & 1 & 1 & 1 & 1 & 1 & 1 & 1 & 1 \\
\hline XI.6091 & 4 & 7 & 3 & 2 & 1 & 4 & 1 & 1 & 8 & 10 & 2 & 7 \\
\hline XI.67202 & 1 & 1 & 1 & 1 & 1 & 1 & 1 & 1 & 1 & 1 & 1 & 1 \\
\hline $\mathrm{XI} .68408$ & 1 & 1 & 1 & 1 & 1 & 1 & 1 & 1 & 1 & 1 & 1 & 1 \\
\hline XI.71159 & 679 & 575 & 714 & 412 & 632 & 355 & 716 & 370 & 726 & 318 & 614 & 249 \\
\hline XI.70850 & 1 & 3 & 1 & 1 & 1 & 1 & 1 & 1 & 1 & 3 & 1 & 2 \\
\hline XI.74263 & 1 & 1 & 1 & 1 & 1 & 1 & 1 & 1 & 1 & 1 & 1 & 1 \\
\hline XI.79790 & 26 & 26 & 45 & 73 & 57 & 62 & 25 & 84 & 47 & 117 & 67 & 114 \\
\hline XI.81297 & 64 & 98 & 56 & 124 & 71 & 120 & 72 & 189 & 82 & 134 & 51 & 117 \\
\hline XI.85251 & 1 & 1 & 1 & 1 & 1 & 1 & 1 & 1 & 1 & 1 & 1 & 1 \\
\hline XI.8753 & 1 & 1 & 1 & 1 & 1 & 1 & 1 & 1 & 1 & 1 & 1 & 1 \\
\hline $\mathrm{XI.9822}$ & 8 & 12 & 10 & 1 & 6 & 1 & 5 & 12 & 12 & 14 & 16 & 1 \\
\hline XI.9874 & 1 & 1 & 1 & 1 & 1 & 1 & 1 & 1 & 1 & 1 & 1 & 1 \\
\hline znf703 & 2815 & 3641 & 2122 & 5945 & 2119 & 6246 & 2145 & 5539 & 2914 & 6511 & 3248 & 6915 \\
\hline
\end{tabular}


Tab. 6.15 Raw data of RA-dependency in dorsal tissue

\begin{tabular}{|c|c|c|c|c|}
\hline \multirow{2}{*}{ gene } & \multicolumn{2}{|c|}{ A } & \multicolumn{2}{|c|}{ B } \\
\hline & D & $D+C Y P$ & D & $D+C Y P$ \\
\hline bhlhe 40 & 25 & 20 & 40 & 18 \\
\hline c10orf140 & 3 & 2 & 3 & 3 \\
\hline cass4 & 107 & 154 & 274 & 254 \\
\hline $\mathrm{cdx} 4$ & 381 & 345 & 517 & 485 \\
\hline cebpd & 66 & 47 & 77 & 34 \\
\hline cxcr7 & 146 & 136 & 206 & 169 \\
\hline сур26a1 & 813 & 527 & 1572 & 546 \\
\hline dact1-a & 227 & 263 & 420 & 348 \\
\hline dact1-b & 1191 & 1406 & 1947 & 1700 \\
\hline dhrs3 & 757 & 449 & 972 & 286 \\
\hline dusp5 & 950 & 994 & 1417 & 1051 \\
\hline dusp6 & 512 & 477 & 644 & 447 \\
\hline e_Cerberus & 1191 & 2085 & 2607 & 2209 \\
\hline e_darmin & 179 & 196 & 225 & 120 \\
\hline e_Sox17alpha & 601 & 777 & 1121 & 770 \\
\hline e_Sox17beta & 503 & 778 & 735 & 702 \\
\hline e_TGIF2 & 420 & 486 & 889 & 792 \\
\hline e_vpp1 & 135 & 198 & 85 & 56 \\
\hline ec_Sox2 & 1054 & 1003 & 1252 & 1169 \\
\hline ec_tubb2b & 24 & 27 & 20 & 30 \\
\hline ec_xk81a1 & 4 & 12 & 17 & 24 \\
\hline em_BMP2-a/b & 305 & 324 & 410 & 298 \\
\hline em_BMP4-a/b & 683 & 675 & 783 & 752 \\
\hline erf & 246 & 316 & 416 & 377 \\
\hline fgfr4-a & 421 & 524 & 557 & 507 \\
\hline fgfr4-b & 221 & 345 & 364 & 342 \\
\hline foxh1 & 314 & 353 & 613 & 567 \\
\hline fst & 1177 & 705 & 1039 & 373 \\
\hline $\mathrm{fzd} 4$ & 197 & 233 & 302 & 342 \\
\hline gbx2.1 & 36 & 26 & 59 & 48 \\
\hline hey1 & 87 & 66 & 125 & 86 \\
\hline hk_g6pd & 150 & 211 & 286 & 268 \\
\hline hk_gapdh & 128 & 151 & 297 & 280 \\
\hline hk_H4 & 69695 & 88424 & 135977 & 123588 \\
\hline hk_ODC & 5149 & 5869 & 7514 & 7283 \\
\hline hnf1b & 174 & 205 & 250 & 193 \\
\hline hoxa1-a & 8 & 6 & 14 & 6 \\
\hline hoxa1-b & 36 & 13 & 58 & 22 \\
\hline hoxa2-a & 12 & 11 & 16 & 10 \\
\hline hoxa3 & 5 & 1 & 2 & 5 \\
\hline hoxa5 & 4 & 1 & 2 & 1 \\
\hline hoxb1 & 14 & 3 & 9 & 6 \\
\hline hoxb3 & 7 & 8 & 21 & 8 \\
\hline hoxb4 & 25 & 43 & 45 & 39 \\
\hline hoxd1 & 356 & 363 & 669 & 526 \\
\hline hoxd4 & 8 & 9 & 11 & 5 \\
\hline hunk & 381 & 534 & 1017 & 889 \\
\hline igf3 & 51 & 35 & 42 & 26 \\
\hline kiaa0182 & 283 & 338 & 519 & 469 \\
\hline kirrel2 & 328 & 331 & 325 & 192 \\
\hline Ihx1 & 469 & 450 & 611 & 404 \\
\hline m_Chordin & 10753 & 11169 & 11641 & 8870 \\
\hline$m$ Xbra-a/b & 3423 & 5124 & 4320 & 4792 \\
\hline
\end{tabular}

\begin{tabular}{|c|c|c|c|c|}
\hline \multirow{2}{*}{ gene } & \multicolumn{2}{|c|}{ A } & \multicolumn{2}{|c|}{ B } \\
\hline & D & D + CYP & D & D + CYP \\
\hline m Xwnt8 & 210 & 299 & 395 & 333 \\
\hline me_ventx2.1-b & 535 & 880 & 754 & 863 \\
\hline meis3-a & 273 & 254 & 568 & 360 \\
\hline mespa-a & 12 & 15 & 34 & 18 \\
\hline mespb & 221 & 253 & 316 & 278 \\
\hline mxi1 & 277 & 296 & 371 & 278 \\
\hline myc-a & 46 & 33 & 64 & 41 \\
\hline myc-b & 454 & 523 & 490 & 415 \\
\hline ndrg1-a & 1 & 1 & 1 & 1 \\
\hline ndrg1-b & 1 & 2 & 2 & 1 \\
\hline \begin{tabular}{|l|} 
neurog2 \\
\end{tabular} & 33 & 21 & 59 & 47 \\
\hline nkx6-2 & 539 & 316 & 457 & 203 \\
\hline nodal1 & 88 & 76 & 115 & 78 \\
\hline nodal2 & 56 & 127 & 110 & 91 \\
\hline nr2f5 & 3 & 7 & 2 & 2 \\
\hline pdgfb & 15 & 12 & 21 & 24 \\
\hline pim1 & 164 & 151 & 341 & 234 \\
\hline pkdcc.1 & 511 & 626 & 709 & 563 \\
\hline prph & 15 & 35 & 10 & 15 \\
\hline rara & 25 & 26 & 34 & 30 \\
\hline rgs14 & 1 & 3 & 2 & 3 \\
\hline rgs2 & 262 & 233 & 241 & 152 \\
\hline sox9-a/b & 61 & 75 & 61 & 71 \\
\hline spry2 & 1018 & 1418 & 1544 & 1522 \\
\hline stox 2 & 171 & 237 & 259 & 262 \\
\hline tdgf1p2-a & 18 & 5 & 23 & 7 \\
\hline tdgf1p2-b & 7 & 16 & 17 & 11 \\
\hline tmem72 & 37 & 34 & 66 & 40 \\
\hline \begin{tabular}{|l|} 
trib1 \\
\end{tabular} & 48 & 36 & 47 & 32 \\
\hline twist1-a & 195 & 114 & 52 & 47 \\
\hline txnip & 97 & 87 & 92 & 79 \\
\hline XI.15091 & 1 & 1 & 1 & 1 \\
\hline XI.16263 & 1 & 2 & 1 & 3 \\
\hline XI.32109 & 11 & 5 & 5 & 10 \\
\hline XI.45046 & 175 & 146 & 271 & 157 \\
\hline XI.47239 & 35 & 42 & 67 & 59 \\
\hline \begin{tabular}{|l}
$\mathrm{XI} .4906$ \\
\end{tabular} & 316 & 442 & 470 & 520 \\
\hline XI.51509 & 40 & 18 & 39 & 34 \\
\hline XI.57027 & 36 & 71 & 106 & 109 \\
\hline XI.57926 & 598 & 701 & 624 & 379 \\
\hline XI.58101 & 1 & 2 & 2 & 4 \\
\hline XI.59256 & 1 & 3 & 1 & 2 \\
\hline XI.6091 & 13 & 16 & 21 & 22 \\
\hline XI.67202 & 2 & 1 & 1 & 1 \\
\hline XI.68408 & 2 & 1 & 2 & 1 \\
\hline XI.70850 & 9 & 15 & 13 & 9 \\
\hline XI.71159 & 365 & 462 & 462 & 440 \\
\hline XI.74263 & 4 & 1 & 3 & 3 \\
\hline XI.79790 & 12 & 13 & 24 & 12 \\
\hline \begin{tabular}{|l}
$\mathrm{XI} .80297$ \\
\end{tabular} & 210 & 201 & 424 & 171 \\
\hline XI.85251 & 1 & 1 & 1 & 1 \\
\hline XI.8753 & 3 & 1 & 3 & 5 \\
\hline XI.9822 & 27 & 13 & 30 & 33 \\
\hline XI.9874 & 1 & 5 & 4 & 1 \\
\hline znf703 & 1109 & 921 & 1600 & 1143 \\
\hline NEG_A & 1 & 5 & 2 & 1 \\
\hline NEG_B & 5 & 2 & 3 & 5 \\
\hline NEG_C & 1 & 1 & 1 & 1 \\
\hline NEG_D & 2 & 2 & 1 & 1 \\
\hline NEG_E & 4 & 3 & 9 & 3 \\
\hline NEG_F & 12 & 10 & 6 & 7 \\
\hline NEG_G & 1 & 1 & 2 & 1 \\
\hline NEG_H & 3 & 1 & 4 & \\
\hline POS_A & 4833 & 6975 & 6828 & 5936 \\
\hline POS_B & 1783 & 2551 & 2451 & 2069 \\
\hline POS_C & 567 & 745 & 711 & 596 \\
\hline POS_D & 118 & 150 & 175 & 164 \\
\hline POS_E & 15 & 27 & 21 & 20 \\
\hline POS F & 7 & 12 & 14 & \\
\hline
\end{tabular}


Tab. 6.16 Normalized data of RA-dependency in dorsal tissue

Indicated are the Nanostring counts which were normalized to the internal positive controls and Odc and with substracted mean of the negative controls as described in the "Materials and Methods" section.

\begin{tabular}{|c|c|c|c|c|}
\hline \multirow{2}{*}{ gene } & \multicolumn{2}{|c|}{ A } & \multicolumn{2}{|c|}{ B } \\
\hline & D & $D+C Y P$ & D & $D+C Y P$ \\
\hline bhlhe40 & 27 & 17 & 33 & 12 \\
\hline c10orf140 & 1 & 1 & 1 & \\
\hline cass4 & 152 & 197 & 277 & 267 \\
\hline $\mathrm{cdx} 4$ & 570 & 452 & 532 & 516 \\
\hline cebpd & 90 & 54 & 71 & 29 \\
\hline cxcr7 & 212 & 173 & 216 & 175 \\
\hline сур26a1 & 1228 & 695 & 1633 & 581 \\
\hline dact1-a & 335 & 342 & 430 & 368 \\
\hline dact1-b & 1814 & 1872 & 2125 & 1825 \\
\hline dhrs3 & 1143 & 591 & 1116 & 311 \\
\hline dusp5 & 1437 & 1320 & 1471 & 1125 \\
\hline dusp6 & 769 & 629 & 664 & 475 \\
\hline e_Cerberus & 1814 & 2779 & 2714 & 2373 \\
\hline e_darmin & 262 & 253 & 226 & 122 \\
\hline e_Sox17alpha & 915 & 1130 & 1162 & 823 \\
\hline e_Sox17beta & 756 & 1131 & 759 & 749 \\
\hline e_TGIF2 & 629 & 642 & 920 & 846 \\
\hline e_vpp1 & 195 & 255 & 80 & 53 \\
\hline ec_Sox2 & 1596 & 1332 & 1299 & 1253 \\
\hline ec_tubb2b & 26 & 27 & 12 & 25 \\
\hline ec_xk81a1 & 1 & 7 & 9 & 19 \\
\hline em_BMP2-a/b & 454 & 424 & 419 & 314 \\
\hline em_BMP4-a/b & 1131 & 893 & 819 & 813 \\
\hline erf & 364 & 413 & 425 & 399 \\
\hline fgfr4-a & 632 & 691 & 573 & 539 \\
\hline fgfr4-b & 326 & 452 & 371 & 361 \\
\hline foxh1 & 468 & 463 & 631 & 614 \\
\hline fst & 1783 & 934 & 1176 & 395 \\
\hline $\mathrm{fzd} 4$ & 289 & 312 & 316 & 361 \\
\hline$g b \times 2.1$ & 44 & 25 & 53 & 45 \\
\hline hey1 & 122 & 79 & 122 & 86 \\
\hline hk_g6pd & 218 & 273 & 290 & 282 \\
\hline hk_gapdh & 184 & 193 & 311 & 295 \\
\hline hk_H4 & 116227 & 118242 & 142126 & 133181 \\
\hline hk_ODC & 7838 & 7839 & 7840 & 7842 \\
\hline hnf1b & 254 & 265 & 252 & 212 \\
\hline hoxa1-a & 1 & 1 & 6 & 1 \\
\hline hoxa1-b & 44 & 8 & 52 & 17 \\
\hline hoxa2-a & 7 & 5 & 8 & 4 \\
\hline hoxa3 & 1 & 1 & 1 & 1 \\
\hline hoxa5 & 1 & 1 & 1 & 1 \\
\hline hoxb1 & 11 & 1 & 1 & 1 \\
\hline hoxb3 & 1 & 1 & 13 & 1 \\
\hline hoxb4 & 27 & 48 & 38 & 35 \\
\hline hoxd1 & 532 & 476 & 690 & 560 \\
\hline hoxd4 & 1 & 3 & 2 & 1 \\
\hline hunk & 570 & 715 & 1153 & 952 \\
\hline igf3 & 67 & 38 & 35 & 22 \\
\hline kiaa0182 & 421 & 443 & 533 & 498 \\
\hline kirrel2 & 489 & 433 & 331 & 200 \\
\hline Ihx1 & 714 & 592 & 629 & 428 \\
\hline m_Chordin & 16381 & 14927 & 12152 & 9552 \\
\hline m_Xbra-a/b & 5217 & 6843 & 4513 & 5157 \\
\hline
\end{tabular}

\begin{tabular}{|c|c|c|c|c|}
\hline \multirow{2}{*}{ gene } & \multicolumn{2}{|c|}{ A } & \multicolumn{2}{|c|}{ B } \\
\hline & D & $D+C Y P$ & D & $D+C Y P$ \\
\hline m Xwnt8 & 319 & 392 & 414 & 352 \\
\hline me_ventx2.1-b & 814 & 1168 & 779 & 923 \\
\hline meis3-a & 415 & 331 & 584 & 382 \\
\hline mespa-a & 7 & 12 & 26 & 12 \\
\hline mespb & 326 & 329 & 321 & 292 \\
\hline$m x i 1$ & 411 & 387 & 378 & 292 \\
\hline myc-a & 59 & 35 & 58 & 37 \\
\hline myc-b & 681 & 691 & 513 & 441 \\
\hline ndrg1-a & 1 & 1 & 1 & 1 \\
\hline ndrg1-b & 1 & 1 & 1 & 1 \\
\hline neurog2 & 39 & 19 & 53 & 43 \\
\hline$n k x 6-2$ & 812 & 413 & 468 & 212 \\
\hline nodal1 & 123 & 92 & 111 & 77 \\
\hline nodal2 & 74 & 162 & 116 & 92 \\
\hline $\mathrm{nr} 2 \mathrm{f5}$ & 1 & 1 & 1 & 1 \\
\hline pdgfb & 12 & 7 & 13 & 19 \\
\hline pim1 & 239 & 193 & 347 & 245 \\
\hline pkdcc.1 & 768 & 828 & 732 & 600 \\
\hline prph & 12 & 38 & 1 & 9 \\
\hline rara & 27 & 25 & 26 & 25 \\
\hline rgs14 & 1 & 1 & 1 & 1 \\
\hline rgs2 & 388 & 312 & 243 & 157 \\
\hline sox9-a/b & 82 & 92 & 55 & 69 \\
\hline spry2 & 1542 & 1887 & 1614 & 1633 \\
\hline stox2 & 250 & 318 & 261 & 275 \\
\hline tdgf1p2-a & 16 & 1 & 15 & 1 \\
\hline tdgf1p2-b & 1 & 12 & 9 & 5 \\
\hline tmem72 & 45 & 36 & 60 & 36 \\
\hline trib1 & 62 & 39 & 41 & 27 \\
\hline twist1-a & 286 & 143 & 45 & 43 \\
\hline txnip & 137 & 117 & 87 & 78 \\
\hline XI.15091 & 1 & 1 & 1 & 1 \\
\hline XI.16263 & 1 & 1 & 1 & 1 \\
\hline XI.32109 & 6 & 1 & 1 & 4 \\
\hline XI.45046 & 256 & 186 & 274 & 162 \\
\hline XI.47239 & 42 & 47 & 62 & 56 \\
\hline XI.4906 & 472 & 582 & 482 & 553 \\
\hline XI.51509 & 50 & 15 & 32 & 29 \\
\hline XI.57027 & 44 & 86 & 112 & 111 \\
\hline XI.57926 & 912 & 928 & 643 & 411 \\
\hline XI.58101 & 1 & 1 & 1 & 1 \\
\hline XI.59256 & 1 & 1 & 1 & 1 \\
\hline XI.6091 & 9 & 12 & 13 & 17 \\
\hline XI.67202 & 1 & 1 & 1 & 1 \\
\hline XI.68408 & 1 & 1 & 1 & 1 \\
\hline XI.70850 & 3 & 12 & 5 & 3 \\
\hline XI.71159 & 545 & 619 & 474 & 467 \\
\hline $\mathrm{XI} .74263$ & 1 & 1 & 1 & 1 \\
\hline XI.79790 & 7 & 8 & 16 & 6 \\
\hline XI.80297 & 319 & 259 & 434 & 177 \\
\hline XI.85251 & 1 & 1 & 1 & 1 \\
\hline XI.8753 & 1 & 1 & 1 & 1 \\
\hline XI.9822 & 31 & 8 & 22 & 28 \\
\hline XI.9874 & 1 & 1 & 1 & 1 \\
\hline znf703 & 1679 & 1222 & 1662 & 1225 \\
\hline
\end{tabular}


Tab. 6.17 Raw data of RA-dependency in whole embryos

\begin{tabular}{|c|c|c|c|c|c|c|c|c|c|c|c|c|}
\hline \multirow{3}{*}{ gene } & \multicolumn{6}{|c|}{ A } & \multicolumn{6}{|c|}{ B } \\
\hline & \multicolumn{2}{|c|}{ st.12 } & \multicolumn{2}{|c|}{ st.13 } & \multicolumn{2}{|c|}{ st.14 } & \multicolumn{2}{|c|}{ st.12 } & \multicolumn{2}{|c|}{ st.13 } & \multicolumn{2}{|c|}{ st.14 } \\
\hline & $\mathrm{CE}$ & BMS & CE & BMS & $\mathrm{CE}$ & BMS & $\mathrm{CE}$ & BMS & $\mathrm{CE}$ & BMS & CE & BMS \\
\hline bhlhe 40 & 21 & 16 & 29 & 28 & 22 & 34 & 19 & 12 & 32 & 29 & 49 & 42 \\
\hline c10orf140 & 5 & 6 & 4 & 4 & 5 & 6 & 6 & 7 & 3 & 1 & 1 & 9 \\
\hline cass4 & 189 & 162 & 241 & 164 & 151 & 218 & 207 & 137 & 172 & 161 & 273 & 266 \\
\hline $\mathrm{cdx} 4$ & 1484 & 1467 & 1873 & 959 & 1313 & 1637 & 1863 & 1156 & 1615 & 1457 & 2163 & 2023 \\
\hline cebpd & 42 & 64 & 28 & 40 & 63 & 64 & 82 & 48 & 47 & 46 & 38 & 39 \\
\hline cxcr7 & 148 & 147 & 114 & 77 & 96 & 136 & 201 & 173 & 128 & 126 & 131 & 167 \\
\hline сур26a1 & 814 & 407 & 884 & 364 & 612 & 575 & 1491 & 758 & 740 & 620 & 951 & 730 \\
\hline dact1-a & 99 & 84 & 75 & 58 & 67 & 94 & 121 & 111 & 91 & 87 & 147 & 96 \\
\hline dact1-b & 655 & 591 & 630 & 441 & 493 & 688 & 974 & 679 & 613 & 622 & 794 & 771 \\
\hline dhrs3 & 378 & 207 & 432 & 146 & 308 & 239 & 630 & 301 & 359 & 315 & 429 & 231 \\
\hline dusp5 & 308 & 171 & 308 & 128 & 245 & 288 & 390 & 270 & 339 & 339 & 433 & 364 \\
\hline dusp6 & 349 & 370 & 491 & 294 & 322 & 504 & 314 & 214 & 304 & 243 & 481 & 521 \\
\hline e_Cerberus & 254 & 408 & 89 & 161 & 80 & 105 & 1461 & 1437 & 491 & 788 & 556 & 417 \\
\hline e_darmin & 693 & 807 & 480 & 836 & 911 & 1262 & 959 & 638 & 530 & 629 & 1209 & 710 \\
\hline e_Sox17alpha & 525 & 642 & 623 & 432 & 516 & 642 & 807 & 623 & 565 & 598 & 725 & 629 \\
\hline e_Sox17beta & 563 & 626 & 570 & 340 & 426 & 561 & 736 & 644 & 577 & 551 & 684 & 641 \\
\hline e_TGIF2 & 192 & 251 & 310 & 240 & 186 & 339 & 460 & 441 & 299 & 252 & 243 & 285 \\
\hline e_vpp1 & 70 & 95 & 35 & 76 & 71 & 70 & 162 & 100 & 93 & 138 & 123 & 88 \\
\hline ec_Sox2 & 890 & 720 & 1139 & 674 & 812 & 895 & 1430 & 1140 & 1001 & 1096 & 1067 & 900 \\
\hline ec_tubb2b & 43 & 48 & 70 & 59 & 52 & 151 & 39 & 31 & 43 & 32 & 55 & 84 \\
\hline ec_xk81a1 & 3456 & 2773 & 6564 & 2941 & 5765 & 7215 & 4112 & 2439 & 6402 & 4839 & 10511 & 7752 \\
\hline em_BMP2-a/b & 457 & 499 & 324 & 355 & 368 & 591 & 661 & 476 & 443 & 511 & 668 & 598 \\
\hline em_BMP4-a/b & 1624 & 2012 & 1377 & 1005 & 1026 & 1612 & 2915 & 2402 & 1660 & 1893 & 1863 & 2073 \\
\hline erf & 221 & 196 & 193 & 120 & 174 & 213 & 304 & 233 & 219 & 224 & 308 & 252 \\
\hline fgfr4-a & 1100 & 1220 & 1113 & 825 & 894 & 1361 & 1742 & 1376 & 1357 & 1322 & 1511 & 1611 \\
\hline fgfr4-b & 469 & 519 & 526 & 350 & 396 & 538 & 781 & 649 & 468 & 546 & 608 & 634 \\
\hline foxh1 & 236 & 177 & 282 & 97 & 165 & 206 & 290 & 180 & 257 & 184 & 338 & 238 \\
\hline fst & 169 & 86 & 241 & 105 & 183 & 149 & 407 & 192 & 215 & 280 & 276 & 267 \\
\hline $\mathrm{fzd} 4$ & 450 & 267 & 311 & 130 & 328 & 247 & 641 & 453 & 383 & 261 & 448 & 285 \\
\hline$g b \times 2.1$ & 384 & 182 & 351 & 161 & 253 & 259 & 379 & 232 & 330 & 188 & 466 & 294 \\
\hline hey1 & 118 & 136 & 156 & 79 & 101 & 140 & 165 & 95 & 165 & 160 & 163 & 132 \\
\hline hk_g6pd & 186 & 188 & 192 & 105 & 124 & 186 & 296 & 222 & 137 & 212 & 207 & 209 \\
\hline hk_gapdh & 125 & 126 & 165 & 90 & 125 & 173 & 123 & 100 & 120 & 114 & 120 & 150 \\
\hline hk_H4 & 66093 & 64900 & 86152 & 51542 & 62183 & 91163 & 146073 & 113620 & 93587 & 121011 & 112190 & 126582 \\
\hline hk_ODC & 7511 & 9063 & 8871 & 5742 & 6875 & 11303 & 9779 & 6880 & 6549 & 7906 & 8264 & 8734 \\
\hline hnf1b & 416 & 199 & 398 & 94 & 425 & 263 & 432 & 280 & 388 & 194 & 630 & 198 \\
\hline hoxa1-a & 18 & 5 & 27 & 3 & 35 & 12 & 40 & 13 & 36 & 12 & 51 & 15 \\
\hline hoxa1-b & 153 & 60 & 251 & 38 & 196 & 37 & 264 & 89 & 236 & 107 & 280 & 80 \\
\hline hoxa2-a & 17 & 14 & 31 & 8 & 19 & 18 & 30 & 19 & 17 & 20 & 35 & 27 \\
\hline hoxa3 & 61 & 20 & 79 & 20 & 47 & 64 & 12 & 6 & 33 & 16 & 57 & 36 \\
\hline hoxa5 & 4 & 1 & 11 & 5 & 12 & 7 & 5 & 2 & 12 & 5 & 12 & 6 \\
\hline hoxb1 & 132 & 71 & 199 & 64 & 158 & 138 & 98 & 49 & 151 & 73 & 203 & 156 \\
\hline hoxb3 & 18 & 12 & 24 & 19 & 24 & 25 & 16 & 15 & 20 & 7 & 34 & 27 \\
\hline hoxb4 & 39 & 40 & 60 & 22 & 40 & 55 & 57 & 48 & 52 & 53 & 62 & 48 \\
\hline hoxd1 & 1309 & 890 & 1378 & 618 & 931 & 752 & 2262 & 1388 & 1570 & 1092 & 1451 & 1051 \\
\hline hoxd4 & 100 & 70 & 193 & 72 & 106 & 245 & 49 & 37 & 107 & 79 & 228 & 255 \\
\hline hunk & 623 & 463 & 684 & 352 & 468 & 523 & 846 & 578 & 531 & 443 & 801 & 698 \\
\hline igf3 & 73 & 50 & 116 & 57 & 93 & 170 & 94 & 51 & 127 & 97 & 123 & 143 \\
\hline kiaa0182 & 274 & 203 & 361 & 161 & 215 & 303 & 578 & 378 & 376 & 350 & 498 & 471 \\
\hline kirrel2 & 180 & 141 & 212 & 166 & 195 & 253 & 361 & 332 & 360 & 276 & 403 & 346 \\
\hline Ihx1 & 83 & 79 & 120 & 51 & 81 & 80 & 90 & 55 & 87 & 68 & 99 & 79 \\
\hline m_Chordin & 1302 & 1497 & 1451 & 1055 & 1085 & 1452 & 2672 & 1809 & 1554 & 1811 & 1495 & 1992 \\
\hline m_Xbra-a/b & 955 & 1354 & 961 & 647 & 533 & 699 & 2013 & 1624 & 829 & 1118 & 888 & 1049 \\
\hline m_Xwnt8 & 396 & 477 & 491 & 182 & 233 & 358 & 859 & 800 & 383 & 624 & 337 & 428 \\
\hline me_ventx2.1-b & 1328 & 2695 & 992 & 610 & 179 & 754 & 2356 & 1430 & 1200 & 889 & 1507 & 909 \\
\hline meis3-a & 703 & 344 & 706 & 322 & 505 & 655 & 794 & 463 & 633 & 426 & 934 & 630 \\
\hline
\end{tabular}




\begin{tabular}{|c|c|c|c|c|c|c|c|c|c|c|c|c|}
\hline \multirow{3}{*}{ gene } & \multicolumn{6}{|c|}{ A } & \multicolumn{6}{|c|}{ B } \\
\hline & \multicolumn{2}{|c|}{ st.12 } & \multicolumn{2}{|c|}{ st.13 } & \multicolumn{2}{|c|}{ st.14 } & \multicolumn{2}{|c|}{ st.12 } & \multicolumn{2}{|c|}{ st.13 } & st. & \\
\hline & CE & BMS & $\mathrm{CE}$ & BMS & CE & BMS & $\mathrm{CE}$ & BMS & $\mathrm{CE}$ & BMS & $\mathrm{CE}$ & BMS \\
\hline mespa-a & 28 & 21 & 27 & 18 & 32 & 30 & 43 & 14 & 37 & 28 & 46 & 30 \\
\hline mespb & 196 & 231 & 180 & 133 & 94 & 162 & 348 & 256 & 164 & 234 & 193 & 166 \\
\hline mxi1 & 359 & 253 & 330 & 185 & 278 & 287 & 434 & 211 & 309 & 284 & 384 & 300 \\
\hline myc-a & 88 & 83 & 130 & 60 & 101 & 144 & 103 & 62 & 91 & 61 & 144 & 104 \\
\hline myc-b & 1067 & 1046 & 1490 & 855 & 907 & 1730 & 1378 & 991 & 1299 & 1101 & 1730 & 1868 \\
\hline ndrg1-a & 1 & 1 & 2 & 3 & 3 & 4 & 1 & 1 & 1 & 3 & 1 & 2 \\
\hline ndrg1-b & 1 & 1 & 2 & 2 & 2 & 1 & 1 & 1 & 2 & 2 & 6 & 5 \\
\hline \begin{tabular}{|l|} 
neurog2 \\
\end{tabular} & 93 & 72 & 83 & 62 & 49 & 68 & 174 & 129 & 119 & 128 & 86 & 86 \\
\hline nkx6-2 & 94 & 45 & 112 & 53 & 73 & 108 & 159 & 102 & 124 & 101 & 138 & 137 \\
\hline nodal1 & 12 & 13 & 18 & 6 & 10 & 3 & 10 & 9 & 3 & 6 & 3 & 8 \\
\hline nodal2 & 13 & 11 & 13 & 4 & 1 & 7 & 14 & 11 & 6 & 7 & 8 & 8 \\
\hline$n r 2 f 5$ & 19 & 6 & 90 & 35 & 103 & 144 & 18 & 8 & 72 & 27 & 144 & 108 \\
\hline pdgfb & 15 & 13 & 21 & 9 & 19 & 19 & 17 & 11 & 16 & 12 & 23 & 20 \\
\hline pim1 & 174 & 174 & 166 & 112 & 133 & 216 & 328 & 204 & 210 & 149 & 317 & 243 \\
\hline pkdcc.1 & 213 & 250 & 265 & 187 & 229 & 226 & 512 & 366 & 338 & 322 & 387 & 322 \\
\hline prph & 50 & 19 & 82 & 27 & 56 & 60 & 49 & 27 & 89 & 43 & 114 & 77 \\
\hline rara & 54 & 30 & 88 & 40 & 54 & 60 & 52 & 39 & 58 & 64 & 115 & 95 \\
\hline rgs14 & 1 & 3 & 1 & 2 & 1 & 1 & 1 & 1 & 1 & 1 & 1 & 3 \\
\hline rgs2 & 66 & 72 & 180 & 82 & 119 & 220 & 112 & 65 & 93 & 98 & 124 & 91 \\
\hline sox9-a/b & 107 & 111 & 222 & 122 & 164 & 373 & 147 & 86 & 184 & 128 & 271 & 275 \\
\hline spry2 & 290 & 337 & 347 & 232 & 239 & 357 & 509 & 374 & 344 & 406 & 389 & 405 \\
\hline stox2 & 211 & 204 & 169 & 123 & 153 & 223 & 274 & 162 & 171 & 194 & 254 & 224 \\
\hline \begin{tabular}{|l|} 
tdgf1p2-a \\
\end{tabular} & 25 & 25 & 42 & 17 & 38 & 58 & 37 & 10 & 28 & 33 & 46 & 35 \\
\hline tdgf1p2-b & 9 & 6 & 3 & 6 & 9 & 8 & 3 & 5 & 4 & 1 & 11 & 4 \\
\hline tmem72 & 104 & 86 & 142 & 84 & 169 & 181 & 154 & 114 & 202 & 149 & 293 & 213 \\
\hline trib1 & 52 & 60 & 51 & 37 & 37 & 62 & 53 & 30 & 51 & 58 & 63 & 60 \\
\hline twist1-a & 215 & 238 & 287 & 260 & 244 & 522 & 261 & 190 & 267 & 279 & 374 & 512 \\
\hline txnip & 231 & 288 & 207 & 204 & 233 & 406 & 110 & 81 & 115 & 96 & 209 & 147 \\
\hline XI.15091 & 1 & 1 & 6 & 1 & 2 & 1 & 2 & 3 & 1 & 3 & 4 & 1 \\
\hline XI.16263 & 2 & 2 & 1 & 2 & 1 & 2 & 3 & 2 & 2 & 1 & 5 & 1 \\
\hline \begin{tabular}{|l|}
$X I .32109$ \\
\end{tabular} & 11 & 6 & 8 & 3 & 3 & 4 & 11 & 13 & 10 & 12 & 5 & 10 \\
\hline XI.45046 & 324 & 156 & 497 & 210 & 311 & 385 & 508 & 217 & 478 & 236 & 694 & 495 \\
\hline $\mathrm{XI.47239}$ & 65 & 42 & 82 & 29 & 72 & 101 & 50 & 34 & 76 & 53 & 101 & 127 \\
\hline $\mathrm{XI} .4906$ & 603 & 636 & 578 & 426 & 489 & 724 & 1131 & 902 & 744 & 704 & 1062 & 881 \\
\hline XI.51509 & 66 & 54 & 94 & 36 & 74 & 68 & 58 & 23 & 72 & 48 & 133 & 79 \\
\hline \begin{tabular}{|l|}
$X 1.57027$ \\
\end{tabular} & 35 & 26 & 44 & 23 & 35 & 35 & 42 & 26 & 38 & 24 & 70 & 63 \\
\hline XI.57926 & 232 & 148 & 276 & 102 & 160 & 178 & 430 & 277 & 309 & 256 & 511 & 329 \\
\hline XI.58101 & 2 & 4 & 2 & 2 & 1 & 1 & 3 & 1 & 2 & 3 & 2 & 1 \\
\hline XI.59256 & 1 & 1 & 1 & 1 & 1 & 2 & 1 & 1 & 1 & 1 & 2 & 3 \\
\hline XI.6091 & 10 & 6 & 16 & 7 & 7 & 17 & 17 & 15 & 14 & 19 & 17 & 24 \\
\hline \begin{tabular}{|l|}
$\mathrm{XI} .67202$ \\
\end{tabular} & 2 & 3 & 4 & 3 & 3 & 4 & 7 & 4 & 3 & 1 & 5 & 6 \\
\hline XI.68408 & 1 & 1 & 3 & 1 & 1 & 1 & 1 & 1 & 1 & 1 & 1 & 2 \\
\hline XI.70850 & 3 & 4 & 5 & 2 & 4 & 4 & 17 & 11 & 7 & 6 & 10 & 6 \\
\hline \begin{tabular}{|l}
$X I .71159$ \\
\end{tabular} & 608 & 916 & 775 & 544 & 494 & 756 & 929 & 796 & 629 & 870 & 652 & 921 \\
\hline XI.74263 & 6 & 2 & 1 & 1 & 2 & 2 & 2 & 2 & 1 & 1 & 2 & 1 \\
\hline \begin{tabular}{|l|}
$\mathrm{XI} .79790$ \\
\end{tabular} & 29 & 15 & 60 & 30 & 50 & 66 & 49 & 54 & 47 & 60 & 86 & 66 \\
\hline XI.80297 & 63 & 54 & 72 & 42 & 61 & 77 & 110 & 75 & 77 & 86 & 69 & 92 \\
\hline XI.85251 & 1 & 2 & 5 & 1 & 2 & 2 & 6 & 5 & 3 & 4 & 4 & 1 \\
\hline $\mathrm{XI} .8753$ & 1 & 2 & 1 & 1 & 1 & 3 & 2 & 1 & 2 & 3 & 5 & 2 \\
\hline $\mathrm{XI} .9822$ & 13 & 11 & 23 & 9 & 11 & 16 & 23 & 21 & 16 & 12 & 32 & 11 \\
\hline \begin{tabular}{|l|}
$X I .9874$ \\
\end{tabular} & 4 & 6 & 8 & 2 & 5 & 6 & 5 & 4 & 2 & 4 & 2 & 3 \\
\hline znf703 & 2494 & 1393 & 2278 & 965 & 1642 & 1713 & 2789 & 1514 & 2505 & 1443 & 3439 & 2292 \\
\hline NEG_A & 2 & 1 & 6 & 1 & 2 & 4 & 5 & 6 & 2 & 5 & 5 & 6 \\
\hline NEG_B & 2 & 1 & 3 & 3 & 1 & 3 & 5 & 1 & 3 & 4 & 5 & 7 \\
\hline NEG_C & 1 & 1 & 1 & 1 & 1 & 2 & 1 & 1 & 1 & 1 & 1 & 2 \\
\hline NEG_D & 1 & 2 & 2 & 2 & 2 & 3 & 1 & 1 & 2 & 2 & 4 & 4 \\
\hline NEG_E & 2 & 5 & 6 & 1 & 2 & 3 & 4 & 2 & 3 & 6 & 4 & 1 \\
\hline NEG_F & 8 & 10 & 12 & 4 & 9 & 8 & 14 & 8 & 9 & 10 & 16 & 15 \\
\hline NEG_G & 1 & 1 & 1 & 1 & 1 & 1 & 1 & 1 & 1 & 1 & 1 & 1 \\
\hline NEG_H & 1 & 1 & 1 & 1 & 1 & 2 & 1 & 2 & 1 & 1 & 2 & 2 \\
\hline POS_A & 4427 & 4213 & 6295 & 4057 & 5094 & 5428 & 6726 & 5523 & 5441 & 7361 & 7964 & 6236 \\
\hline POS_B & 1739 & 1768 & 2557 & 1674 & 2053 & 2292 & 2829 & 2204 & 2058 & 2961 & 2986 & 2478 \\
\hline POS_C & 511 & 536 & 814 & 473 & $\begin{array}{ll}616 \\
\end{array}$ & 662 & $\begin{array}{l}787 \\
\end{array}$ & 629 & 592 & 827 & 938 & 762 \\
\hline POS_D & 124 & 100 & 160 & 92 & 144 & 141 & 158 & 145 & 133 & 192 & 176 & 168 \\
\hline POS_E & 15 & 20 & 31 & 17 & 18 & 17 & 22 & 25 & 16 & 47 & 38 & 33 \\
\hline POS F F & 4 & 8 & 13 & 7 & 13 & 9 & 13 & 6 & 17 & 27 & 20 & 13 \\
\hline
\end{tabular}


Tab. 6.18 Normalized data of RA-dependency in whole embryos

Indicated are the Nanostring counts which were normalized to the internal positive controls and Odc and with substracted mean of the negative controls as described in the "Materials and Methods" section

\begin{tabular}{|c|c|c|c|c|c|c|c|c|c|c|c|c|}
\hline \multirow{3}{*}{ gene } & \multicolumn{6}{|c|}{ s22 } & \multicolumn{6}{|c|}{$s 23$} \\
\hline & \multicolumn{2}{|c|}{ st.12 } & \multicolumn{2}{|c|}{ st.13 } & \multicolumn{2}{|c|}{ st.14 } & \multicolumn{2}{|c|}{ st.12 } & \multicolumn{2}{|c|}{ st.13 } & \multicolumn{2}{|c|}{ st.14 } \\
\hline & CE & BMS & CE & BMS & CE & BMS & CE & BMS & CE & BMS & CE & BMS \\
\hline bhlhe 40 & 17 & 8 & 15 & \begin{tabular}{|r|}
41 \\
\end{tabular} & 21 & 22 & 2 & 4 & 29 & 17 & 32 & \begin{tabular}{|r|}
22 \\
\end{tabular} \\
\hline c10orf140 & 1 & 1 & 1 & 2 & 1 & 1 & 1 & 1 & 1 & 1 & 1 & 1 \\
\hline cass4 & 206 & 165 & 214 & 259 & 188 & 181 & 147 & 131 & 193 & 142 & 245 & 214 \\
\hline $\mathrm{cdx} 4$ & 1666 & 1569 & 1743 & 1537 & 1693 & 1410 & 1429 & 1169 & 1876 & 1371 & 2038 & 1719 \\
\hline cebpd & 40 & 60 & 15 & 60 & 74 & 48 & 51 & 41 & 47 & 33 & 22 & 19 \\
\hline cxcr7 & 160 & 149 & 95 & 120 & 117 & 110 & 143 & 168 & 141 & 109 & 110 & 129 \\
\hline сур26a1 & 911 & 429 & 816 & 581 & 785 & 490 & 1141 & 764 & 855 & 577 & 888 & 611 \\
\hline dact1-a & 105 & 81 & 59 & 89 & 79 & 74 & 81 & 105 & 98 & 72 & 125 & 68 \\
\hline dact1-b & 731 & 626 & 579 & 705 & 631 & 588 & 741 & 683 & 707 & 579 & 739 & 646 \\
\hline dhrs3 & 419 & 213 & 393 & 231 & 391 & 199 & 475 & 298 & 411 & 288 & 393 & 184 \\
\hline dusp5 & 340 & 175 & 277 & 202 & 310 & 242 & 289 & 267 & 387 & 311 & 396 & 298 \\
\hline dusp6 & 386 & 389 & 448 & 468 & 409 & 429 & 230 & 210 & 347 & 220 & 442 & 432 \\
\hline e_Cerberus & 279 & 430 & 72 & 255 & 96 & 83 & 1118 & 1455 & 565 & 737 & 513 & 343 \\
\hline e_darmin & 774 & 859 & 438 & 1339 & 1172 & 1085 & 729 & 641 & 610 & 586 & 1133 & 594 \\
\hline e_Sox17alpha & 585 & 681 & 572 & 690 & 661 & 549 & 612 & 626 & 651 & 557 & 673 & 525 \\
\hline e_Sox17beta & 628 & 664 & 522 & 542 & 544 & 478 & 557 & 648 & 665 & 512 & 634 & 535 \\
\hline e_TGIF2 & 209 & 261 & 279 & 382 & 233 & 286 & 343 & 441 & 341 & 229 & 216 & 230 \\
\hline e_vpp1 & 72 & 93 & 21 & 118 & 84 & 53 & 112 & 94 & 100 & 121 & 102 & 61 \\
\hline ec_Sox2 & 996 & 765 & 1055 & 1079 & 1044 & 768 & 1094 & 1153 & 1160 & 1029 & 998 & 757 \\
\hline ec_tubb2b & 41 & 42 & 54 & 91 & 60 & 123 & 17 & 23 & 42 & 20 & 38 & 58 \\
\hline ec_xk81a1 & 3890 & 2974 & 6138 & 4722 & 7459 & 6241 & 3169 & 2475 & 7459 & 4576 & 9957 & 6625 \\
\hline em_BMP2-a/b & 508 & 528 & 292 & 566 & 469 & 504 & 499 & 476 & 509 & 474 & 619 & 498 \\
\hline em_BMP4-a/b & 1824 & 2155 & 1278 & 1611 & 1321 & 1389 & 2243 & 2438 & 1928 & 1784 & 1753 & 1761 \\
\hline erf & 242 & 202 & 169 & 189 & 218 & 177 & 222 & 229 & 247 & 202 & 278 & 202 \\
\hline fgfr4-a & 1233 & 1303 & 1031 & 1322 & 1150 & 1171 & 1335 & 1393 & 1575 & 1243 & 1419 & 1366 \\
\hline fgfr4-b & 522 & 549 & 481 & 558 & 505 & 458 & 592 & 653 & 538 & 507 & 562 & 529 \\
\hline foxh1 & 259 & 181 & 252 & 152 & 206 & 171 & 212 & 175 & 292 & 164 & 306 & 190 \\
\hline fst & 184 & 83 & 214 & 165 & 229 & 122 & 302 & 187 & 243 & 255 & 247 & 215 \\
\hline $\mathrm{fzd} 4$ & 500 & 278 & 280 & 205 & 417 & 206 & 483 & 453 & 439 & 237 & 411 & 230 \\
\hline$g b \times 2.1$ & 426 & 187 & 317 & 255 & 320 & 217 & 280 & 228 & 377 & 168 & 428 & 238 \\
\hline hey1 & 126 & 137 & 134 & 123 & 123 & 114 & 115 & 89 & 184 & 141 & 140 & 99 \\
\hline hk_g6pd & 203 & 193 & 168 & 165 & 153 & 154 & 216 & 218 & 152 & 191 & 182 & 165 \\
\hline hk_gapdh & 134 & 126 & 143 & 141 & 154 & 142 & 82 & 94 & 132 & 98 & 99 & 114 \\
\hline hk_H4 & 74512 & 69800 & 80701 & 82824 & 80536 & 78943 & 113028 & 115679 & 109154 & 114673 & 106415 & 108403 \\
\hline hk_ODC & 8462 & 9739 & 8299 & 9223 & 8897 & 9781 & 7555 & 6997 & 7631 & 7482 & 7825 & 7466 \\
\hline hnf1b & 462 & 205 & 361 & 147 & 543 & 220 & 321 & 277 & 445 & 174 & 583 & 155 \\
\hline hoxa1-a & 13 & 1 & 14 & 1 & 38 & 3 & 18 & 5 & 34 & 1 & 34 & 1 \\
\hline hoxa1-b & 166 & 55 & 223 & 57 & 246 & 25 & 191 & 82 & 267 & 91 & 251 & 54 \\
\hline hoxa2-a & 12 & 6 & 17 & 9 & 17 & 8 & 10 & 11 & 12 & 9 & 19 & 9 \\
\hline hoxa3 & 62 & 12 & 62 & 28 & 53 & 48 & -4 & -2 & 30 & 5 & 40 & 17 \\
\hline hoxa5 & 1 & 1 & 1 & 4 & 8 & 1 & 1 & 1 & 6 & 1 & 1 & 1 \\
\hline hoxb1 & 142 & 67 & 175 & 99 & 197 & 112 & 63 & 42 & 168 & 59 & 178 & 119 \\
\hline hoxb3 & 13 & 4 & 11 & 26 & 23 & 14 & -1 & 7 & 15 & -4 & 18 & 9 \\
\hline hoxb4 & 37 & 34 & 45 & 31 & 44 & 40 & 31 & 41 & 53 & 40 & 44 & 27 \\
\hline hoxd1 & 1469 & 948 & 1279 & 989 & 1198 & 644 & 1738 & 1405 & 1823 & 1025 & 1362 & 886 \\
\hline hoxd4 & 106 & 66 & 169 & 112 & 129 & 205 & 25 & 29 & 117 & 65 & 202 & 204 \\
\hline hunk & 695 & 489 & 629 & 562 & 598 & 445 & 642 & 580 & 611 & 410 & 745 & 584 \\
\hline igf3 & 75 & 45 & 97 & 88 & 113 & 140 & 60 & 44 & 140 & 82 & 102 & 108 \\
\hline kiaa0182 & 302 & 209 & 327 & 255 & 271 & 255 & 434 & 377 & 431 & 322 & 458 & 389 \\
\hline kirrel2 & 196 & 142 & 187 & 263 & 245 & 212 & 266 & 330 & 412 & 251 & 368 & 282 \\
\hline Ihx1 & 87 & 76 & 101 & 78 & 97 & 62 & 57 & 48 & 93 & 54 & 80 & 53 \\
\hline m_Chordin & 1461 & 1601 & 1348 & 1691 & 1398 & 1250 & 2055 & 1834 & 1805 & 1706 & 1404 & 1692 \\
\hline m_Xbra-a/b & 1070 & 1447 & 889 & 1036 & 683 & 598 & 1545 & 1645 & 959 & 1049 & 828 & 884 \\
\hline
\end{tabular}




\begin{tabular}{|c|c|c|c|c|c|c|c|c|c|c|c|c|}
\hline \multirow{3}{*}{ gene } & \multicolumn{6}{|c|}{$s 22$} & \multicolumn{6}{|c|}{$s 23$} \\
\hline & \multicolumn{2}{|c|}{ st.12 } & \multicolumn{2}{|c|}{ st.13 } & \multicolumn{2}{|c|}{ st.14 } & \multicolumn{2}{|c|}{ st.12 } & \multicolumn{2}{|c|}{ st.13 } & \multicolumn{2}{|c|}{ st.14 } \\
\hline & CE & BMS & CE & BMS & CE & BMS & CE & BMS & CE & BMS & CE & BMS \\
\hline m_Xwnt8 & 439 & 504 & 448 & 288 & 294 & 303 & 652 & 806 & 439 & 581 & 305 & 352 \\
\hline me_ventx2.1-b & 1490 & 2890 & 918 & 976 & 224 & 645 & 1810 & 1448 & 1392 & 832 & 1415 & 764 \\
\hline meis3-a & 786 & 361 & 650 & 513 & 646 & 560 & 602 & 463 & 730 & 394 & 872 & 525 \\
\hline mespa-a & 25 & 13 & 14 & 25 & 34 & 18 & 20 & 6 & 35 & 16 & 29 & 12 \\
\hline mespb & 214 & 239 & 157 & 210 & 114 & 133 & 256 & 252 & 183 & 212 & 169 & 128 \\
\hline mxi1 & 398 & 263 & 297 & 293 & 352 & 241 & 323 & 207 & 352 & 259 & 350 & 243 \\
\hline myc-a & 92 & 80 & 110 & 92 & 123 & 117 & 67 & 55 & 98 & 48 & 122 & 75 \\
\hline myc-b & 1196 & 1116 & 1384 & 1370 & 1167 & 1491 & 1054 & 1001 & 1507 & 1033 & 1627 & 1586 \\
\hline ndrg1-a & 1 & 1 & 1 & 1 & 1 & 1 & 1 & 1 & 1 & 1 & 1 & 1 \\
\hline ndrg1-b & 1 & 1 & 1 & 1 & 1 & 1 & 1 & 1 & 1 & 1 & 1 & 1 \\
\hline neurog2 & 98 & 68 & 66 & 96 & 56 & 51 & 122 & 123 & 131 & 111 & 67 & 59 \\
\hline nkx6-2 & 99 & 39 & 93 & 81 & 87 & 86 & 110 & 96 & 137 & 86 & 116 & 103 \\
\hline nodal1 & 7 & 5 & 5 & 6 & 5 & 1 & 1 & 1 & 1 & 1 & 1 & 1 \\
\hline nodal2 & 8 & 3 & 0 & 2 & 1 & 1 & 1 & 3 & 1 & 1 & 1 & 1 \\
\hline $\mathrm{nr} 2 \mathrm{f5}$ & 14 & 1 & 73 & 52 & 126 & 117 & 1 & 1 & 76 & 15 & 122 & $7 \varepsilon$ \\
\hline pdgfb & 10 & 5 & 8 & 10 & 17 & 9 & 0 & 3 & 11 & 1 & 7 & 3 \\
\hline pim1 & 189 & 178 & 144 & 176 & 164 & 180 & 241 & 200 & 237 & 131 & 286 & 194 \\
\hline pkdcc.1 & 233 & 260 & 237 & 296 & 289 & 188 & 383 & 364 & 386 & 295 & 353 & 262 \\
\hline prph & 49 & 11 & 65 & 39 & 65 & 44 & 25 & 19 & 96 & 31 & 94 & 52 \\
\hline rara & 54 & 23 & 71 & 60 & 62 & 44 & 27 & 32 & 60 & 51 & 95 & 67 \\
\hline rgs14 & 1 & 1 & 1 & 1 & 1 & 1 & 1 & 1 & 1 & 1 & 1 & 1 \\
\hline rgs2 & 67 & 68 & 157 & 128 & 146 & 183 & 74 & 58 & 100 & 83 & 103 & 64 \\
\hline sox9-a/b & 114 & 110 & 196 & 192 & 205 & 316 & 101 & 79 & 207 & 111 & 243 & 221 \\
\hline spry2 & 320 & 353 & 313 & 369 & 302 & 302 & 381 & 373 & 393 & 375 & 355 & 333 \\
\hline stox2 & 231 & 210 & 147 & 194 & 190 & 186 & 199 & 157 & 191 & 174 & 227 & 178 \\
\hline tdgf1p2-a & 21 & 18 & 28 & 23 & 41 & 43 & 16 & 2 & 25 & 21 & 29 & 16 \\
\hline tdgf1p2-b & 3 & 1 & 1 & 6 & 4 & 1 & 1 & 1 & 1 & 1 & 1 & 1 \\
\hline tmem72 & 110 & 83 & 121 & 131 & 211 & 149 & 106 & 108 & 228 & 131 & 264 & 168 \\
\hline trib1 & 52 & 55 & 36 & 55 & 40 & 46 & 28 & 22 & 51 & 45 & 45 & 37 \\
\hline twist1-a & 235 & 247 & 257 & 414 & 308 & 445 & 189 & 185 & 303 & 254 & 340 & 424 \\
\hline txnip & 253 & 301 & 182 & 324 & 294 & 344 & 72 & 74 & 126 & 81 & 184 & 112 \\
\hline $\mathrm{XI.15091}$ & 1 & 1 & 1 & 1 & 1 & 1 & 1 & 1 & 1 & 1 & 1 & 1 \\
\hline XI.16263 & 1 & 1 & 1 & 1 & 1 & 1 & 1 & 1 & 1 & 1 & 1 & 1 \\
\hline XI.32109 & 5 & 1 & 1 & 1 & 1 & 1 & 1 & 5 & 4 & 1 & 1 & 1 \\
\hline XI.45046 & 358 & 159 & 454 & 333 & 395 & 326 & 380 & 213 & 549 & 214 & 644 & 410 \\
\hline $\mathrm{XI} .47239$ & 66 & 36 & 65 & 43 & 85 & 80 & 26 & 26 & 81 & 40 & 81 & 95 \\
\hline $\mathrm{XI} .4906$ & 673 & 675 & 530 & 681 & 626 & 620 & 862 & 910 & 860 & 657 & 993 & 740 \\
\hline XI.51509 & 67 & 49 & 76 & 54 & 88 & 51 & 32 & 15 & 76 & 35 & 112 & 53 \\
\hline $\mathrm{XI.57027}$ & 32 & 19 & 30 & 33 & 38 & 23 & 20 & 18 & 36 & 13 & 52 & 40 \\
\hline $\mathrm{XI.57926}$ & 255 & 150 & 247 & 160 & 199 & 147 & 320 & 274 & 352 & 232 & 470 & 268 \\
\hline $\mathrm{XI} .58101$ & 1 & 1 & 1 & 1 & 1 & 1 & 1 & 1 & 1 & 1 & 1 & 1 \\
\hline XI.59256 & 1 & 1 & 1 & 1 & 1 & 1 & 1 & 1 & 1 & 1 & 1 & 1 \\
\hline XI.6091 & 4 & 1 & 3 & 7 & 1 & 7 & 0 & 7 & 8 & 8 & 2 & $\epsilon$ \\
\hline $\mathrm{XI.67202}$ & 1 & 1 & 1 & 1 & 1 & 1 & 1 & 1 & 1 & 1 & 1 & 1 \\
\hline XI.68408 & 1 & 1 & 1 & 1 & 1 & 1 & 1 & 1 & 1 & 1 & 1 & 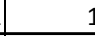 \\
\hline $\mathrm{XI.70850}$ & 1 & 1 & 1 & 1 & 1 & 1 & 0 & 3 & 0 & 1 & 1 & 1 \\
\hline $\mathrm{XI.71159}$ & 679 & 976 & 714 & 870 & 632 & 647 & 706 & 802 & 726 & 814 & 604 & 775 \\
\hline XI.74263 & 1 & 1 & 1 & 1 & 1 & 1 & 1 & 1 & 1 & 1 & 1 & 1 \\
\hline XI.79790 & 26 & 7 & 45 & 44 & 57 & 50 & 25 & 47 & 47 & 47 & 67 & 42 \\
\hline $\mathrm{XI} .80297$ & 64 & 49 & 56 & 63 & 71 & 59 & 72 & 68 & 82 & 71 & 51 & 65 \\
\hline $\mathrm{XI} .85251$ & 1 & 1 & 1 & 1 & 1 & 1 & 1 & 1 & 1 & 1 & 1 & 1 \\
\hline XI.8753 & 1 & 1 & 1 & 1 & 1 & 1 & 1 & 1 & 1 & 1 & 1 & 1 \\
\hline $\mathrm{XI} .9822$ & 8 & 3 & 10 & 10 & 6 & 6 & 5 & 13 & 11 & 1 & 16 & ; \\
\hline \begin{tabular}{|l|}
$X 1.9874$ \\
\end{tabular} & 1 & 1 & 1 & 1 & 1 & 1 & 1 & 1 & 1 & 1 & 1 & \\
\hline znf703 & 2805 & 1489 & 2122 & 1547 & 2119 & 1476 & 2145 & 1533 & 2914 & 1357 & 3248 & $194 \mathrm{C}$ \\
\hline
\end{tabular}




\subsection{Nanostring analysis data for expression characteristics of confirmed RA-responsive genes}

\section{Tab. 6.19 Raw data of expression characteristics}

$(\mathrm{CE}=$ control-embryo; $\mathrm{DE}=$ dorsal endoderm; $\mathrm{VE}=$ ventral endoderm; $\mathrm{D}=$ dorsal tissue; $V=$ ventral tissue)

\begin{tabular}{|c|c|c|c|c|c|c|c|c|c|c|}
\hline \multirow{2}{*}{ gene } & \multicolumn{5}{|c|}{ A } & \multicolumn{5}{|c|}{ B } \\
\hline & CE & $\mathrm{DE}$ & VE & D & $\mathbf{v}$ & CE & $\mathrm{DE}$ & VE & D & $\mathbf{v}$ \\
\hline bhlhe40 & 6 & 10 & 3 & 47 & 21 & 12 & 8 & 8 & 40 & 25 \\
\hline c10orf140 & 1 & 5 & 3 & 2 & 10 & 4 & 7 & 1 & 3 & 10 \\
\hline cass4 & 320 & 329 & 482 & 151 & 305 & 188 & 422 & 400 & 274 & 608 \\
\hline $\mathrm{cd} x 4$ & 480 & 7 & 217 & 522 & 2156 & 723 & 4 & 384 & 517 & 3075 \\
\hline cebpd & 20 & 144 & 44 & 31 & 17 & 37 & 297 & 50 & 77 & 34 \\
\hline cxcr7 & 163 & 105 & 80 & 123 & 110 & 119 & 64 & 49 & 206 & 173 \\
\hline сур26а1 & 724 & 847 & 466 & 922 & 2029 & 973 & 1014 & 379 & 1572 & 1948 \\
\hline dact1-a & 168 & 276 & 95 & 240 & 207 & 165 & 264 & 103 & 420 & 236 \\
\hline dact1-b & 718 & 941 & 692 & 1055 & 926 & 640 & 866 & 404 & 1947 & 1474 \\
\hline dhrs3 & 269 & 745 & 221 & 599 & 286 & 344 & 777 & 248 & 972 & 402 \\
\hline dusp5 & 411 & 326 & 451 & 892 & 903 & 447 & 217 & 280 & 1417 & 1083 \\
\hline dusp6 & 405 & 353 & 317 & 514 & 531 & 295 & 220 & 233 & 644 & 723 \\
\hline e_Cerberus & 2006 & 7729 & 1565 & 1119 & 137 & 1601 & 9325 & 1841 & 2607 & 392 \\
\hline e_darmin & 160 & 859 & 612 & 102 & 13 & 611 & 3674 & 2037 & 225 & 115 \\
\hline e_Sox17alpha & 884 & 2049 & 2028 & 890 & 872 & 799 & 2587 & 2321 & 1121 & 1070 \\
\hline e_Sox17beta & 976 & 1522 & 1953 & 590 & 790 & 814 & 2006 & 2712 & 735 & 1055 \\
\hline e_TGIF2 & 309 & 322 & 416 & 357 & 635 & 218 & 483 & 312 & 889 & 1160 \\
\hline e_vpp1 & 34 & 315 & 2 & 89 & 1 & 65 & 423 & 5 & 85 & 7 \\
\hline ec_Sox2 & 463 & 8 & 22 & 501 & 389 & 603 & 23 & 11 & 1252 & 412 \\
\hline ec_tubb2b & 9 & 25 & 12 & 19 & 7 & 22 & 30 & 25 & 20 & 17 \\
\hline ec_xk81a1 & 293 & 8 & 8 & 6 & 79 & 1685 & 3 & 15 & 17 & 386 \\
\hline em_BMP2-a/b & 320 & 1695 & 551 & 308 & 167 & 265 & 2393 & 518 & 410 & 278 \\
\hline em_BMP4-a/b & 1397 & 920 & 2076 & 878 & 3364 & 1452 & 1031 & 2125 & 783 & 4052 \\
\hline erf & 229 & 170 & 290 & 201 & 248 & 229 & 162 & 240 & 416 & 574 \\
\hline fgfr4-a & 772 & 1241 & 1212 & 472 & 269 & 565 & 1428 & 1167 & 557 & 625 \\
\hline fgfr4-b & 518 & 676 & 583 & 239 & 118 & 396 & 542 & 505 & 364 & 249 \\
\hline foxh1 & 299 & 194 & 264 & 211 & 285 & 304 & 304 & 382 & 613 & 645 \\
\hline fst & 59 & 453 & 119 & 1535 & 56 & 91 & 224 & 66 & 1039 & 50 \\
\hline $\mathrm{fzd} 4$ & 348 & 418 & 280 & 119 & 35 & 296 & 467 & 173 & 302 & 98 \\
\hline$g b \times 2.1$ & 88 & 23 & 18 & 17 & 46 & 64 & 26 & 50 & 59 & 151 \\
\hline hey1 & 64 & 33 & 62 & 102 & 73 & 62 & 42 & 54 & 125 & 195 \\
\hline hk_g6pd & 122 & 100 & 97 & 112 & 133 & 160 & 126 & 113 & 286 & 350 \\
\hline hk_gapdh & 152 & 134 & 146 & 123 & 139 & 146 & 108 & 106 & 297 & 224 \\
\hline hk_H4 & 108920 & 60805 & 63372 & 88804 & 111328 & 83610 & 55389 & 45520 & 135977 & 133543 \\
\hline hk_ODC & 4311 & 3689 & 3620 & 4479 & 4987 & 3902 & 3908 & 3172 & 7514 & 9518 \\
\hline hnf1b & 185 & 650 & 519 & 230 & 96 & 232 & 774 & 590 & 250 & 138 \\
\hline hoxa1-a & 1 & 5 & 1 & 1 & 11 & 6 & 2 & 1 & 14 & 18 \\
\hline hoxa1-b & 33 & 2 & 7 & 54 & 93 & 63 & 3 & 7 & 58 & 188 \\
\hline hoxa2-a & 12 & 7 & 12 & 14 & 15 & 6 & 6 & 7 & 16 & 29 \\
\hline hoxa3 & 2 & 1 & 4 & 6 & 5 & 6 & 4 & 6 & 2 & 10 \\
\hline hoxa5 & 1 & 1 & 1 & 1 & 1 & 3 & 1 & 1 & 2 & 1 \\
\hline hoxb1 & 2 & 2 & 3 & 2 & 12 & 5 & 1 & 2 & 9 & 28 \\
\hline hoxb3 & 9 & 2 & 3 & 14 & 26 & 8 & 1 & 3 & 21 & 29 \\
\hline hoxb4 & 46 & 20 & 17 & 26 & 50 & 23 & 25 & 12 & 45 & 62 \\
\hline hoxd1 & 746 & 24 & 258 & 464 & 1581 & 1015 & 44 & 390 & 669 & 3080 \\
\hline hoxd4 & 3 & 6 & 3 & 9 & 21 & 4 & 6 & 6 & 11 & 25 \\
\hline hunk & 314 & 515 & 562 & 200 & 620 & 707 & 976 & 753 & 1017 & 1844 \\
\hline igf3 & 60 & 53 & 49 & 53 & 43 & 49 & 79 & 32 & 42 & 31 \\
\hline kiaa0182 & 213 & 150 & 199 & 158 & 223 & 310 & 254 & 223 & 519 & 594 \\
\hline kirrel2 & 140 & 387 & 450 & 306 & 344 & 258 & 653 & 746 & 325 & 552 \\
\hline $\ln \times 1$ & 125 & 307 & 260 & 424 & 92 & 80 & 276 & 151 & 611 & 119 \\
\hline $\mathrm{XI} .47239$ & 55 & 28 & 51 & 26 & 55 & 31 & 34 & 40 & 67 & 89 \\
\hline m_Chordin & 1128 & 3549 & 3 & 10256 & 2 & 1432 & 1895 & 2 & 11641 & 5 \\
\hline m_Xbra-a/b & 2321 & 175 & 946 & 4491 & 6800 & 2525 & 133 & 616 & 4320 & 8718 \\
\hline m_Xwnt8 & 1041 & 509 & 2214 & 386 & 3180 & 518 & 246 & 1150 & 395 & 3426 \\
\hline me_ventx2.1-b & 759 & 366 & 1149 & 448 & 1880 & 1207 & 747 & 1965 & 754 & 3757 \\
\hline meis3-a & 266 & 203 & 257 & 244 & 312 & 377 & 254 & 203 & 568 & 453 \\
\hline mespa-a & 13 & 10 & 34 & 12 & 18 & 10 & 10 & 17 & 34 & 40 \\
\hline mespb & 257 & 61 & 579 & 248 & 841 & 194 & 52 & 386 & 316 & 1308 \\
\hline
\end{tabular}




\section{Appendix}

\begin{tabular}{|c|c|c|c|c|c|c|c|c|c|c|}
\hline \multirow{2}{*}{ gene } & \multicolumn{5}{|c|}{ A } & \multicolumn{5}{|c|}{ B } \\
\hline & CE & DE & VE & D & v & $\mathrm{CE}$ & DE & VE & D & v \\
\hline mxi1 & 234 & 98 & 138 & 206 & 178 & 250 & 191 & 179 & 371 & 307 \\
\hline myc-a & 33 & 55 & 58 & 44 & 106 & 38 & 68 & 58 & 64 & 98 \\
\hline myc-b & 454 & 147 & 585 & 583 & 2514 & 922 & 201 & 792 & 490 & 2530 \\
\hline ndrg1-a & 1 & 1 & 1 & 1 & 1 & 2 & 2 & 1 & 1 & 2 \\
\hline ndrg1-b & 1 & 2 & 2 & 1 & 1 & 1 & 3 & 1 & 2 & 4 \\
\hline neurog2-b & 32 & 19 & 12 & 22 & 19 & 30 & 15 & 21 & 59 & 38 \\
\hline nkx6-2 & 24 & 19 & 7 & 355 & 17 & 33 & 11 & 6 & 457 & 15 \\
\hline nodal1 & 57 & 49 & 61 & 103 & 62 & 24 & 24 & 77 & 115 & 142 \\
\hline nodal2 & 117 & 14 & 12 & 108 & 113 & 26 & 15 & 12 & 110 & 131 \\
\hline $\mathrm{nr} 2 \mathrm{f5}$ & 22 & 23 & 27 & 18 & 20 & 6 & 7 & 9 & 2 & 6 \\
\hline pdgfb & 29 & 16 & 18 & 9 & 16 & 10 & 15 & 9 & 21 & 16 \\
\hline pim1 & 304 & 199 & 128 & 230 & 229 & 261 & 228 & 97 & 341 & 410 \\
\hline pkdcc.1 & 196 & 776 & 182 & 407 & 34 & 253 & 674 & 87 & 709 & 33 \\
\hline prph & 16 & 3 & 1 & 19 & 13 & 27 & 1 & 2 & 10 & 15 \\
\hline rara & 13 & 14 & 20 & 26 & 42 & 20 & 11 & 22 & 34 & 54 \\
\hline rgs14 & 4 & 1 & 1 & 1 & 1 & 2 & 7 & 3 & 2 & 5 \\
\hline rgs2 & 24 & 30 & 24 & 140 & 30 & 26 & 8 & 27 & 241 & 129 \\
\hline sox9-a/b & 84 & 89 & 82 & 47 & 187 & 74 & 77 & 57 & 61 & 275 \\
\hline spry2 & 551 & 375 & 229 & 933 & 815 & 434 & 368 & 115 & 1544 & 854 \\
\hline stox2 & 197 & 193 & 127 & 162 & 156 & 175 & 200 & 98 & 259 & 280 \\
\hline tdgf1p2-a & 3 & 10 & 2 & 18 & 1 & 6 & 44 & 7 & 23 & 2 \\
\hline tdgf1p2-b & 1 & 1 & 4 & 3 & 1 & 3 & 4 & 7 & 17 & 2 \\
\hline tmem72 & 33 & 48 & 52 & 63 & 37 & 41 & 111 & 96 & 66 & 46 \\
\hline trib1 & 26 & 24 & 18 & 31 & 14 & 25 & 12 & 11 & 47 & 24 \\
\hline twist1-a & 21 & 99 & 37 & 162 & 17 & 53 & 230 & 14 & 52 & 16 \\
\hline txnip & 72 & 150 & 118 & 71 & 34 & 76 & 222 & 188 & 92 & 87 \\
\hline XI.15091 & 1 & 1 & 1 & 1 & 1 & 1 & 2 & 1 & 1 & 1 \\
\hline XI.16263 & 5 & 1 & 1 & 1 & 3 & 1 & 3 & 2 & 1 & 3 \\
\hline XI.32109 & 10 & 3 & 3 & 8 & 5 & 6 & 7 & 2 & 5 & 10 \\
\hline $\mathrm{XI.45046}$ & 122 & 68 & 168 & 155 & 294 & 157 & 60 & 163 & 271 & 556 \\
\hline $\mathrm{XI.4906}$ & 899 & 368 & 626 & 332 & 686 & 629 & 227 & 214 & 470 & 1003 \\
\hline$X 1.51509$ & 11 & 4 & 5 & 59 & 93 & 24 & 3 & 3 & 39 & 70 \\
\hline XI.57027 & 11 & 57 & 48 & 19 & 56 & 111 & 100 & 62 & 106 & 158 \\
\hline XI.57926 & 290 & 222 & 267 & 641 & 575 & 303 & 137 & 152 & 624 & 918 \\
\hline XI.58101 & 3 & 2 & 2 & 1 & 1 & 1 & 3 & 1 & 2 & 1 \\
\hline XI.59256 & 2 & 3 & 2 & 2 & 1 & 1 & 2 & 1 & 1 & 3 \\
\hline XI.6091 & 19 & 11 & 10 & 26 & 34 & 16 & 6 & 13 & 21 & 32 \\
\hline $\mathrm{XI} .67202$ & 4 & 3 & 1 & 1 & 3 & 1 & 2 & 2 & 1 & 4 \\
\hline XI.68408 & 1 & 1 & 1 & 2 & 1 & 1 & 1 & 1 & 2 & 1 \\
\hline XI.70850 & 6 & 1 & 1 & 3 & 2 & 4 & 1 & 3 & 13 & 17 \\
\hline XI.71159 & 517 & 543 & 361 & 418 & 379 & 409 & 497 & 273 & 462 & 592 \\
\hline$X 1.74263$ & 2 & 1 & 1 & 2 & 4 & 1 & 1 & 1 & 3 & 4 \\
\hline XI.79790 & 16 & 18 & 4 & 7 & 14 & 17 & 31 & 5 & 24 & 11 \\
\hline $\mathrm{XI} .80297$ & 30 & 6 & 5 & 280 & 164 & 63 & 1 & 2 & 424 & 120 \\
\hline XI.85251 & 1 & 2 & 1 & 1 & 3 & 2 & 1 & 2 & 1 & 1 \\
\hline XI.8753 & 1 & 1 & 1 & 2 & 1 & 2 & 1 & 1 & 3 & 2 \\
\hline XI.9822 & 14 & 6 & 9 & 9 & 12 & 19 & 11 & 12 & 30 & 38 \\
\hline XI.9874 & 7 & 1 & 3 & 2 & 1 & 3 & 5 & 1 & 4 & 5 \\
\hline znf703-a/b & 999 & 171 & 492 & 1017 & 2099 & 1334 & 220 & 602 & 1600 & 4659 \\
\hline NEG_A & 1 & 4 & 1 & 2 & 2 & 2 & 2 & 3 & 2 & 3 \\
\hline NEG_B & 2 & 7 & 5 & 4 & 3 & 4 & 3 & 3 & 3 & 7 \\
\hline NEG_C & 1 & 1 & 1 & 1 & 1 & 1 & 2 & 1 & 1 & 1 \\
\hline NEG_D & 2 & 3 & 1 & 1 & 1 & 1 & 1 & 2 & 1 & 3 \\
\hline NEG_E & 6 & 2 & 1 & 7 & 2 & 2 & 5 & 2 & 9 & 4 \\
\hline NEG_F & 7 & 8 & 8 & 5 & 4 & 4 & 7 & 11 & 6 & 10 \\
\hline NEG_G & 1 & 1 & 1 & 1 & 1 & 1 & 1 & 3 & 2 & 1 \\
\hline NEG_H & 1 & 4 & 1 & 1 & 2 & 3 & 1 & 1 & 4 & 1 \\
\hline POS_A & 4707 & 5150 & 4978 & 4878 & 5928 & 5579 & 5222 & 5051 & 6828 & 7020 \\
\hline POS_B & 1824 & 1857 & 1811 & 1786 & 2104 & 2115 & 2239 & 2065 & 2451 & 2944 \\
\hline POS_C & 577 & 652 & 631 & 587 & 742 & 706 & 690 & 647 & 711 & 881 \\
\hline POS_D & 115 & 140 & 125 & 127 & 143 & 134 & 119 & 139 & 175 & 185 \\
\hline POS_E & 24 & 23 & 24 & 29 & 31 & 24 & 24 & 22 & 21 & 27 \\
\hline POS_F & 13 & 10 & 12 & 13 & 19 & 13 & 15 & 5 & 14 & 24 \\
\hline
\end{tabular}


Tab. 6.20 Normalized data of expression characteristics

(Indicated are the Nanostring counts which were normalized to the internal positive controls and Odc and with substracted mean of the negative controls as described in the "Materials and Methods" section). $\mathrm{CE}=$ control-embryo; $\mathrm{DE}=$ dorsal endoderm; $\mathrm{VE}=$ ventral endoderm: $\mathrm{D}=$ dorsal tissue: $\mathrm{V}=$ ventral tissue

\begin{tabular}{|c|c|c|c|c|c|c|c|c|c|c|}
\hline \multirow{2}{*}{ gene } & \multicolumn{5}{|c|}{ A } & \multicolumn{5}{|c|}{ B } \\
\hline & CE & DE & VE & D & v & CE & DE & VE & D & v \\
\hline bhlhe 40 & 1 & 5 & 1 & 47 & 18 & 11 & 4 & 3 & 19 & 3 \\
\hline c10orf140 & 1 & 1 & 1 & 1 & 6 & 1 & 2 & 1 & 1 & 1 \\
\hline cass4 & 378 & 455 & 685 & 168 & 314 & 246 & 554 & 646 & 181 & 322 \\
\hline $\mathrm{cdx} 4$ & 571 & 1 & 304 & 599 & 2244 & 959 & 1 & 620 & 349 & 1670 \\
\hline cebpd & 17 & 194 & 55 & 29 & 14 & 44 & 388 & 72 & 44 & 8 \\
\hline cxcr7 & 189 & 139 & 107 & 135 & 111 & 154 & 78 & 71 & 134 & 84 \\
\hline сур26a1 & 866 & 1185 & 662 & 1063 & 2112 & 1292 & 1342 & 612 & 1079 & 1054 \\
\hline dact1-a & 195 & 380 & 129 & 271 & 212 & 215 & 344 & 159 & 282 & 119 \\
\hline dact1-b & 859 & 1317 & 986 & 1217 & 961 & 848 & 1145 & 653 & 1338 & 795 \\
\hline dhrs3 & 317 & 1041 & 310 & 688 & 294 & 454 & 1027 & 397 & 664 & 209 \\
\hline dusp5 & 488 & 451 & 640 & 1028 & 937 & 591 & 282 & 449 & 972 & 581 \\
\hline dusp6 & 481 & 489 & 448 & 589 & 550 & 388 & 286 & 372 & 437 & 385 \\
\hline e_Cerberus & 2412 & 10886 & 2240 & 1292 & 139 & 2129 & 12401 & 3008 & 1795 & 204 \\
\hline e_darmin & 185 & 1202 & 871 & 111 & 9 & 809 & 4882 & 3330 & 147 & 53 \\
\hline e_Sox17alpha & 1059 & 2879 & 2905 & 1026 & 905 & 1060 & 3435 & 3795 & 767 & 574 \\
\hline e_Sox17beta & 1170 & 2136 & 2798 & 678 & 820 & 1080 & 2662 & 4436 & 500 & 566 \\
\hline e_TGIF2 & 365 & 445 & 590 & 407 & 658 & 286 & 636 & 502 & 606 & 624 \\
\hline e_vpp1 & 33 & 435 & 1 & 96 & 1 & 82 & 556 & 1 & 50 & 1 \\
\hline ec_Sox 2 & 551 & 2 & 24 & 574 & 401 & 799 & 23 & 8 & 857 & 215 \\
\hline ec_tubb2b & 3 & 26 & 10 & 15 & 3 & 25 & 33 & 31 & 5 & 1 \\
\hline ec_xk81a1 & 346 & 2 & 4 & 1 & 78 & 2241 & 1 & 15 & 3 & 201 \\
\hline em_BMP2-a/b & 378 & 2380 & 784 & 350 & 170 & 348 & 3177 & 839 & 275 & 142 \\
\hline em_BMP4-a/b & 1678 & 1288 & 2974 & 1012 & 3504 & 1930 & 1365 & 3474 & 533 & 2204 \\
\hline erf & 269 & 231 & 409 & 226 & 254 & 300 & 208 & 384 & 279 & 303 \\
\hline fgfr4-a & 924 & 1740 & 1733 & 541 & 276 & 748 & 1893 & 1903 & 376 & 331 \\
\hline fgfr4-b & 617 & 944 & 830 & 270 & 119 & 523 & 714 & 818 & 243 & 126 \\
\hline foxh1 & 353 & 265 & 372 & 238 & 293 & 400 & 397 & 617 & 415 & 342 \\
\hline fst & 64 & 630 & 163 & 1775 & 54 & 116 & 291 & 98 & 710 & 17 \\
\hline $\mathrm{fzd} 4$ & 412 & 580 & 395 & 131 & 32 & 390 & 614 & 274 & 200 & 43 \\
\hline$g b \times 2.1$ & 99 & 23 & 18 & 12 & 44 & 80 & 27 & 72 & 32 & 72 \\
\hline hey1 & 70 & 38 & 81 & 111 & 72 & 78 & 49 & 79 & 77 & 96 \\
\hline hk_g6pd & 140 & 132 & 132 & 123 & 135 & 208 & 161 & 176 & 189 & 181 \\
\hline hk_gapdh & 176 & 180 & 202 & 135 & 141 & 190 & 137 & 164 & 196 & 112 \\
\hline hk_H4 & 131375 & 85703 & 91025 & 103093 & 116080 & 111420 & 73695 & 74614 & 94094 & 72950 \\
\hline hk_ODC & 5193 & 5191 & 5192 & 5193 & 5196 & 5195 & 5193 & 5190 & 5191 & 5190 \\
\hline hnf1b & 216 & 907 & 738 & 260 & 96 & 304 & 1023 & 957 & 164 & 65 \\
\hline hoxa1-a & 1 & 1 & 1 & 1 & 7 & 3 & 1 & 1 & 1 & 1 \\
\hline hoxa1-b & 32 & 1 & 2 & 55 & 93 & 79 & 1 & 2 & 31 & 92 \\
\hline hoxa2-a & 7 & 1 & 10 & 9 & 12 & 3 & 1 & 2 & 2 & 6 \\
\hline hoxa3 & 1 & 1 & 1 & 1 & 1 & 3 & 1 & 0 & 1 & 1 \\
\hline hoxa5 & 1 & 1 & 1 & 1 & 1 & 1 & 1 & 1 & 1 & 1 \\
\hline hoxb1 & 1 & 1 & 1 & 1 & 8 & 2 & 1 & 1 & 1 & 5 \\
\hline hoxb3 & 3 & 1 & 1 & 9 & 23 & 6 & 1 & 1 & 5 & 6 \\
\hline hoxb4 & 48 & 19 & 17 & 23 & 48 & 26 & 26 & 10 & 22 & 24 \\
\hline hoxd1 & 892 & 25 & 363 & 531 & 1644 & 1348 & 51 & 630 & 454 & 1672 \\
\hline hoxd4 & 1 & 1 & 1 & 3 & 18 & 1 & 1 & 0 & 1 & 3 \\
\hline hunk & 371 & 717 & 800 & 225 & 642 & 937 & 1292 & 1225 & 695 & 997 \\
\hline igf3 & 65 & 66 & 63 & 54 & 41 & 60 & 98 & 43 & 20 & 7 \\
\hline kiaa0182 & 249 & 202 & 278 & 176 & 228 & 408 & 331 & 356 & 350 & 314 \\
\hline kirrel2 & 161 & 537 & 639 & 348 & 355 & 339 & 862 & 1213 & 216 & 291 \\
\hline Ihx1 & 143 & 424 & 366 & 485 & 92 & 102 & 360 & 238 & 414 & 55 \\
\hline XI.47239 & 59 & 31 & 66 & 23 & 53 & 36 & 38 & 56 & 37 & 38 \\
\hline
\end{tabular}




\begin{tabular}{|c|c|c|c|c|c|c|c|c|c|c|}
\hline \multirow{2}{*}{ gene } & \multicolumn{5}{|c|}{ A } & \multicolumn{5}{|c|}{ B } \\
\hline & CE & $\mathrm{DE}$ & VE & D & $\mathbf{v}$ & CE & DE & VE & D & v \\
\hline m_Chordin & 1353 & 4994 & 1 & 11900 & 1 & 1904 & 2514 & 1 & 8047 & 1 \\
\hline$m \_X b r a-a / b$ & 2792 & 238 & 1351 & 5207 & 7086 & 3360 & 170 & 1000 & 2981 & 4753 \\
\hline m_Xwnt8 & 1248 & 709 & 3173 & 441 & 3312 & 686 & 320 & 1876 & 264 & 1862 \\
\hline me_ventx2.1-b & 908 & 507 & 1643 & 513 & 1956 & 1604 & 987 & 3212 & 513 & 2042 \\
\hline meis3-a & 313 & 277 & 361 & 276 & 321 & 498 & 331 & 323 & 384 & 237 \\
\hline mespa-a & 8 & 5 & 41 & 7 & 15 & 9 & 6 & 18 & 14 & 12 \\
\hline mespb & 302 & 77 & 824 & 281 & 873 & 254 & 62 & 623 & 210 & 704 \\
\hline mxi1 & 275 & 129 & 191 & 232 & 181 & 328 & 247 & 284 & 248 & 157 \\
\hline myc-a & 32 & 69 & 76 & 44 & 106 & 46 & 83 & 85 & 35 & 43 \\
\hline myc-b & 540 & 198 & 833 & 669 & 2617 & 1224 & 260 & 1289 & 330 & 1372 \\
\hline ndrg1-a & 1 & 1 & 1 & 1 & 1 & 1 & 1 & 1 & 1 & 1 \\
\hline ndrg1-b & 1 & 1 & 1 & 1 & 1 & 1 & 1 & 1 & 1 & 1 \\
\hline neurog2-b & 31 & 18 & 10 & 18 & 16 & 35 & 13 & 25 & 32 & 11 \\
\hline$n k x 6-2$ & 21 & 18 & 2 & 405 & 14 & 39 & 8 & 0 & 307 & 1 \\
\hline nodal1 & 61 & 60 & 80 & 112 & 61 & 27 & 25 & 116 & 71 & 67 \\
\hline nodal2 & 134 & 11 & 10 & 118 & 114 & 30 & 13 & 10 & 67 & 61 \\
\hline$n r 2 f 5$ & 19 & 23 & 31 & 14 & 17 & 3 & 2 & 5 & 1 & 1 \\
\hline pdgfb & 27 & 14 & 18 & 3 & 13 & 9 & 13 & 5 & 5 & 1 \\
\hline pim1 & 359 & 272 & 176 & 260 & 235 & 343 & 296 & 149 & 227 & 214 \\
\hline pkdcc. 1 & 229 & 1085 & 254 & 465 & 31 & 332 & 890 & 133 & 482 & 8 \\
\hline prph & 12 & 1 & 1 & 15 & 9 & 31 & 1 & 1 & 1 & 1 \\
\hline rara & 8 & 11 & 21 & 23 & 40 & 22 & 8 & 26 & 14 & 19 \\
\hline rgs14 & 1 & 1 & 1 & 1 & 1 & 1 & 2 & 1 & 1 & 1 \\
\hline rgs2 & 21 & 33 & 27 & 155 & 27 & 30 & 4 & 35 & 158 & 60 \\
\hline sox9-a/b & 94 & 117 & 110 & 47 & 191 & 94 & 95 & 84 & 33 & 140 \\
\hline spry2 & 657 & 520 & 321 & 1076 & 846 & 574 & 483 & 179 & 1059 & 456 \\
\hline stox2 & 230 & 263 & 175 & 181 & 159 & 228 & 259 & 151 & 170 & 143 \\
\hline tdgf1p2-a & 1 & 5 & 1 & 14 & 1 & 3 & 51 & 2 & 7 & 1 \\
\hline tdgf1p2-b & 1 & 1 & 1 & 1 & 1 & 1 & 1 & 2 & 3 & 1 \\
\hline tmem72 & 32 & 59 & 67 & 66 & 34 & 50 & 141 & 148 & 37 & 15 \\
\hline trib1 & 24 & 25 & 18 & 29 & 10 & 29 & 9 & 8 & 23 & 3 \\
\hline twist1-a & 18 & 131 & 45 & 181 & 14 & 66 & 299 & 13 & 27 & 1 \\
\hline txnip & 79 & 202 & 162 & 75 & 31 & 96 & 288 & 298 & 55 & 37 \\
\hline XI.15091 & 1 & 1 & 1 & 1 & 1 & 1 & 1 & 1 & 1 & 1 \\
\hline XI.16263 & 1 & 1 & 1 & 1 & 1 & 1 & 1 & 1 & 1 & 1 \\
\hline XI.32109 & 5 & 1 & 1 & 2 & 1 & 3 & 2 & 1 & 1 & 1 \\
\hline XI.45046 & 140 & 87 & 234 & 173 & 302 & 204 & 73 & 257 & 178 & 294 \\
\hline XI.4906 & 1077 & 510 & 892 & 378 & 711 & 833 & 295 & 341 & 316 & 538 \\
\hline XI.51509 & 6 & 1 & 1 & 61 & 93 & 27 & 1 & 1 & 18 & 28 \\
\hline XI.57027 & 6 & 71 & 61 & 15 & 54 & 143 & 126 & 92 & 64 & 76 \\
\hline XI.57926 & 342 & 304 & 376 & 737 & 595 & 399 & 175 & 239 & 423 & 491 \\
\hline XI.58101 & 1 & 1 & 1 & 1 & 1 & 1 & 1 & 1 & 1 & 1 \\
\hline XI.59256 & 1 & 1 & 1 & 1 & 1 & 1 & 1 & 1 & 1 & 1 \\
\hline $\mathrm{XI} .6091$ & 15 & 7 & 7 & 23 & 31 & 17 & 1 & 12 & 5 & 7 \\
\hline XI.67202 & 1 & 1 & 1 & 1 & 1 & 1 & 1 & 1 & 1 & 1 \\
\hline XI.68408 & 1 & 1 & 1 & 1 & 1 & 1 & 1 & 1 & 1 & 1 \\
\hline XI.70850 & 1 & 1 & 1 & 1 & 1 & 1 & 1 & 1 & 1 & 1 \\
\hline XI.71159 & 616 & 756 & 511 & 478 & 391 & 540 & 654 & 438 & 311 & 313 \\
\hline XI.74263 & 1 & 1 & 1 & 1 & 0 & 1 & 1 & 1 & 1 & 1 \\
\hline XI.79790 & 12 & 16 & 1 & 1 & 10 & 18 & 34 & 1 & 8 & 1 \\
\hline XI.80297 & 29 & 1 & 1 & 318 & 167 & 79 & 1 & 1 & 284 & 55 \\
\hline XI.85251 & 1 & 1 & 1 & 1 & 1 & 1 & 1 & 1 & 1 & 1 \\
\hline $\mathrm{XI} .8753$ & 1 & 1 & 1 & 1 & 1 & 1 & 1 & 1 & 1 & 1 \\
\hline XI.9822 & 9 & 1 & 5 & 3 & 8 & 21 & 8 & 10 & 12 & 11 \\
\hline $\mathrm{XI.9874}$ & 1 & 1 & 1 & 1 & 1 & 1 & 1 & 1 & 1 & 1 \\
\hline znf703-a/b & 1198 & 232 & 699 & 1173 & 2185 & 1773 & 286 & 977 & 1098 & 2535 \\
\hline
\end{tabular}


Tab. 6.21 Calculated mean and standard error of mean (SEM)

Mean and SEM were calculated from the normalized Nanostring counts of two independent experiments

$\mathrm{CE}=$ control-embryo; $\mathrm{DE}=$ dorsal endoderm; $\mathrm{VE}=$ ventral endoderm; $\mathrm{D}=$ dorsal tissue;

$\mathrm{V}=$ ventral tissue

\begin{tabular}{|c|c|c|c|c|c|c|c|c|c|c|}
\hline \multirow{2}{*}{ gene } & \multicolumn{5}{|c|}{ mean } & \multicolumn{5}{|c|}{ SEM } \\
\hline & CE & $\mathrm{DE}$ & VE & D & v & CE & DE & VE & D & v \\
\hline bhlhe40 & 6 & 4 & 2 & 33 & 11 & 5.09 & 0.81 & 1.19 & 14.28 & 7.17 \\
\hline c10orf140 & 1 & 2 & 1 & 1 & 4 & 0.24 & 0.59 & 0.00 & 0.00 & 2.65 \\
\hline cass4 & 312 & 505 & 665 & 174 & 318 & 66.37 & 49.80 & 19.33 & 6.32 & 4.03 \\
\hline $\mathrm{cdx} 4$ & 765 & 1 & 462 & 474 & 1957 & 193.62 & 0.04 & 157.89 & 124.96 & 287.11 \\
\hline cebpd & 31 & 291 & 64 & 36 & 11 & 13.95 & 97.02 & 8.37 & 7.81 & 2.62 \\
\hline cxcr7 & 171 & 109 & 89 & 134 & 97 & 17.66 & 30.51 & 18.30 & 0.96 & 13.14 \\
\hline сур26а1 & 1079 & 1264 & 637 & 1071 & 1583 & 213.04 & 78.57 & 25.05 & 7.90 & 528.76 \\
\hline dact1-a & 205 & 362 & 144 & 276 & 165 & 9.97 & 17.97 & 15.18 & 5.17 & 46.50 \\
\hline dact1-b & 853 & 1231 & 819 & 1278 & 878 & 5.23 & 86.15 & 166.88 & 60.45 & 83.17 \\
\hline dhrs3 & 385 & 1034 & 353 & 676 & 252 & 68.33 & 7.22 & 43.54 & 12.22 & 42.34 \\
\hline dusp5 & 540 & 366 & 545 & 1000 & 759 & 51.32 & 84.47 & 95.42 & 28.32 & 177.99 \\
\hline dusp6 & 435 & 387 & 410 & 513 & 467 & 46.34 & 101.51 & 37.71 & 76.37 & 82.39 \\
\hline e_Cerberus & 2270 & 11643 & 2624 & 1543 & 171 & 141.70 & 757.48 & 383.98 & 251.68 & 32.61 \\
\hline e_darmin & 497 & 3042 & 2101 & 129 & 31 & 311.98 & 1839.84 & 1229.12 & 17.81 & 21.59 \\
\hline e_Sox17alpha & 1059 & 3157 & 3350 & 896 & 740 & 0.60 & 277.93 & 444.88 & 129.58 & 165.39 \\
\hline e_Sox17beta & 1125 & 2399 & 3617 & 589 & 693 & 44.89 & 262.81 & 819.25 & 89.00 & 126.73 \\
\hline e_TGIF2 & 325 & 540 & 546 & 507 & 641 & 39.75 & 95.31 & 44.06 & 99.54 & 17.23 \\
\hline e_vpp1 & 58 & 495 & 1 & 73 & 1 & 24.16 & 60.33 & 0.00 & 23.09 & 0.00 \\
\hline ec_Sox 2 & 675 & 13 & 16 & 716 & 308 & 123.91 & 10.58 & 7.79 & 141.56 & 93.31 \\
\hline ec_tubb2b & 14 & 30 & 20 & 10 & 2 & 10.58 & 3.26 & 10.86 & 4.95 & 1.08 \\
\hline ec_xk81a1 & 1293 & 2 & 9 & 2 & 139 & 947.41 & 0.66 & 5.54 & 0.85 & 61.21 \\
\hline em_BMP2-a/b & 363 & 2779 & 812 & 312 & 156 & 15.06 & 398.36 & 27.84 & 37.76 & 14.18 \\
\hline em_BMP4-a/b & 1804 & 1326 & 3224 & 772 & 2854 & 126.31 & 38.43 & 249.75 & 239.57 & 650.03 \\
\hline erf & 285 & 220 & 396 & 252 & 279 & 15.83 & 11.12 & 12.57 & 26.43 & 24.45 \\
\hline fgfr4-a & 836 & 1817 & 1818 & 459 & 304 & 87.77 & 76.32 & 85.05 & 82.10 & 27.44 \\
\hline fgfr4-b & 570 & 829 & 824 & 256 & 122 & 47.19 & 114.93 & 5.80 & 13.62 & 3.45 \\
\hline foxh1 & 377 & 331 & 494 & 326 & 318 & 23.59 & 66.44 & 122.50 & 88.79 & 24.56 \\
\hline fst & 90 & 460 & 131 & 1242 & 36 & 26.40 & 169.33 & 32.38 & 532.38 & 18.58 \\
\hline $\mathrm{fzd} 4$ & 401 & 597 & 334 & 165 & 38 & 11.30 & 17.01 & 60.31 & 34.58 & 5.47 \\
\hline$g b \times 2.1$ & 90 & 25 & 45 & 22 & 58 & 9.08 & 2.01 & 27.05 & 9.71 & 14.22 \\
\hline hey1 & 74 & 43 & 80 & 94 & 84 & 4.07 & 5.60 & 1.27 & 16.79 & 12.16 \\
\hline hk_g6pd & 174 & 146 & 154 & 156 & 158 & 34.39 & 14.27 & 21.95 & 33.11 & 23.22 \\
\hline hk_gapdh & 183 & 158 & 183 & 166 & 126 & 6.96 & 21.67 & 18.99 & 30.53 & 14.33 \\
\hline hk_H4 & 121398 & 79699 & 82820 & 98594 & 94515 & 9977.92 & 6004.00 & 8205.45 & 4499.58 & 21565.32 \\
\hline hk_ODC & 5194 & 5192 & 5191 & 5192 & 5193 & \begin{tabular}{|l|}
1.35 \\
\end{tabular} & 0.92 & 1.01 & 0.84 & 3.05 \\
\hline hnf1b & 260 & 965 & 848 & 212 & 81 & 44.37 & 57.74 & 109.84 & 47.85 & $15.4 \mathrm{C}$ \\
\hline hoxa1-a & 2 & 1 & 1 & 1 & 4 & 1.09 & 0.00 & 0.00 & 0.18 & 3.17 \\
\hline hoxa1-b & 56 & 1 & 2 & 43 & 93 & 23.43 & 0.00 & 0.30 & 12.11 & 0.18 \\
\hline hoxa2-a & 5 & 1 & 6 & 5 & 9 & 1.88 & 0.03 & 3.89 & 3.43 & 2.95 \\
\hline hoxa3 & 2 & 1 & 1 & 1 & 1 & 1.09 & 0.00 & 0.45 & 0.00 & 0.04 \\
\hline hoxa5 & 1 & 1 & 1 & 1 & 1 & 0.00 & 0.00 & 0.00 & 0.00 & 0.00 \\
\hline hoxb1 & 1 & 1 & 1 & 1 & 7 & 0.42 & 0.00 & 0.00 & 0.00 & 1.65 \\
\hline hoxb3 & 5 & 1 & 1 & 7 & 14 & 1.25 & 0.00 & 0.00 & 1.70 & 8.68 \\
\hline hoxb4 & 37 & 23 & 13 & 22 & 36 & 11.07 & 3.46 & 3.38 & 0.36 & 12.18 \\
\hline hoxd1 & 1120 & 38 & 496 & 493 & 1658 & 227.76 & 13.28 & 133.36 & 38.70 & 14.04 \\
\hline hoxd4 & 1 & 1 & 1 & 2 & 11 & 0.24 & 0.07 & 0.45 & 1.04 & 7.17 \\
\hline \begin{tabular}{|l|} 
hunk \\
\end{tabular} & 654 & 1004 & 1012 & 460 & 820 & 283.07 & 287.29 & 212.56 & 234.97 & 177.43 \\
\hline igf3 & 63 & 82 & 53 & 37 & 24 & 2.18 & 16.12 & 9.97 & 17.07 & 17.00 \\
\hline kiaa0182 & 329 & 267 & 317 & 263 & 271 & 79.45 & 64.19 & 38.85 & 87.03 & 42.95 \\
\hline kirrel2 & 250 & 699 & 926 & 282 & 323 & 88.83 & 162.61 & 287.27 & 66.01 & 31.61 \\
\hline Ihx1 & 123 & 392 & 302 & 449 & 73 & 20.73 & 31.83 & 63.98 & 35.55 & 18.51 \\
\hline XI.47239 & 48 & 34 & 61 & 30 & 46 & 11.16 & 3.80 & 4.85 & 7.25 & 7.41 \\
\hline
\end{tabular}




\begin{tabular}{|c|c|c|c|c|c|c|c|c|c|c|}
\hline \multirow{2}{*}{ gene } & \multicolumn{5}{|c|}{ mean } & \multicolumn{5}{|c|}{ SEM } \\
\hline & CE & $\mathrm{DE}$ & VE & D & V & CE & $\mathrm{DE}$ & VE & D & $\mathbf{v}$ \\
\hline m_Chordin & 1628 & 3754 & 1 & 9973 & 1 & 275.23 & 1239.68 & 0.00 & 1926.31 & 0.00 \\
\hline$m \_$Xbra-a/b & 3076 & 204 & 1176 & 4094 & 5920 & 284.01 & 33.94 & 175.54 & 1113.01 & 1166.81 \\
\hline m_Xwnt8 & 967 & 514 & 2524 & 353 & 2587 & 281.33 & 194.16 & 648.56 & 88.23 & 725.10 \\
\hline me_ventx2.1-b & 1256 & 747 & 2427 & 513 & 1999 & 347.85 & 239.94 & 784.41 & 0.01 & 43.09 \\
\hline meis3-a & 405 & 304 & 342 & 330 & 279 & 92.13 & 26.83 & 19.20 & 54.07 & 41.97 \\
\hline mespa-a & 8 & 6 & 30 & 11 & 13 & 0.18 & 0.52 & 11.49 & 3.96 & 1.51 \\
\hline mespb & 278 & 70 & 724 & 245 & 789 & 24.38 & 7.48 & 100.47 & 35.46 & 84.21 \\
\hline$m x i 1$ & 302 & 188 & 237 & 240 & 169 & 26.81 & 58.92 & 46.60 & 7.96 & 11.99 \\
\hline myc-a & 39 & 76 & 80 & 39 & 75 & 6.77 & 7.39 & 4.87 & 4.23 & 31.54 \\
\hline myc-b & 882 & 229 & 1061 & 500 & 1995 & 341.90 & 31.04 & 228.01 & 169.71 & 622.63 \\
\hline ndrg1-a & 1 & 1 & 1 & 1 & 1 & 0.00 & 0.00 & 0.00 & 0.00 & 0.00 \\
\hline ndrg1-b & 1 & 1 & 1 & 1 & 1 & 0.00 & 0.00 & 0.00 & 0.00 & 0.00 \\
\hline neurog2-b & 33 & 15 & 17 & 25 & 13 & 2.04 & 2.49 & 7.59 & 6.81 & 2.57 \\
\hline nkx6-2 & 30 & 13 & 1 & 356 & 7 & 8.87 & 5.15 & 1.12 & 48.78 & 6.30 \\
\hline nodal1 & 44 & 42 & 98 & 91 & 64 & 17.04 & 17.65 & 18.29 & 20.83 & 3.42 \\
\hline nodal2 & 82 & 12 & 10 & 93 & 88 & 51.89 & 1.03 & 0.21 & 25.47 & 26.18 \\
\hline $\mathrm{nr} 2 \mathrm{f5}$ & 11 & 13 & 18 & 7 & 9 & 7.92 & 10.64 & 13.03 & 6.26 & 7.86 \\
\hline pdgfb & 18 & 13 & 12 & 4 & 7 & 9.47 & 0.38 & 6.56 & 1.20 & 5.77 \\
\hline pim1 & 351 & 284 & 163 & 243 & 224 & 8.08 & 12.35 & 13.43 & 16.36 & 10.44 \\
\hline pkdcc.1 & 281 & 987 & 193 & 473 & 20 & 51.73 & 97.59 & 60.42 & 8.23 & 11.76 \\
\hline prph & 21 & 1 & 1 & 8 & 5 & 9.69 & 0.00 & 0.00 & 6.84 & 4.21 \\
\hline rara & 15 & 9 & 24 & 19 & 29 & 6.84 & 1.63 & 2.66 & 4.17 & 10.19 \\
\hline rgs14 & 1 & 2 & 1 & 1 & 1 & 0.00 & 0.59 & 0.00 & 0.00 & 0.00 \\
\hline rgs2 & 26 & 18 & 31 & 156 & 44 & 4.20 & 14.91 & 3.88 & 1.28 & 16.55 \\
\hline sox9-a/b & 94 & 106 & 97 & 40 & 165 & 0.00 & 10.58 & 13.18 & 7.01 & 25.42 \\
\hline spry2 & 615 & 501 & 250 & 1068 & 651 & 41.77 & 18.55 & 71.22 & 8.17 & 194.67 \\
\hline stox2 & 229 & 261 & 163 & 175 & 151 & 0.85 & 2.05 & 11.89 & 5.26 & 7.89 \\
\hline tdgf1p2-a & 2 & 28 & 1 & 10 & 1 & 1.09 & 23.14 & 0.37 & 3.33 & 0.00 \\
\hline tdgf1p2-b & 1 & 1 & 1 & 2 & 1 & 0.00 & 0.00 & 0.37 & 0.85 & 0.00 \\
\hline tmem72 & 41 & 100 & 107 & 51 & 25 & 8.77 & 40.94 & 40.33 & 14.57 & 9.77 \\
\hline trib1 & 26 & 17 & 13 & 26 & 7 & 2.33 & 8.01 & 4.92 & 2.57 & 3.79 \\
\hline twist1-a & 42 & 215 & 29 & 104 & 7 & 24.00 & 84.16 & 16.11 & 76.88 & 6.30 \\
\hline txnip & 88 & 245 & 230 & 65 & 34 & 8.57 & 42.90 & 68.34 & 10.22 & 2.99 \\
\hline XI.15091 & 1 & 1 & 1 & 1 & 1 & 0.00 & 0.00 & 0.00 & 0.00 & 0.00 \\
\hline XI.16263 & 1 & 1 & 1 & 1 & 1 & 0.00 & 0.00 & 0.00 & 0.00 & 0.00 \\
\hline XI.32109 & 4 & 2 & 1 & 1 & 1 & 0.68 & 0.59 & 0.00 & 0.46 & 0.04 \\
\hline XI.45046 & 172 & 80 & 246 & 176 & 298 & 32.39 & 7.09 & 11.94 & 2.96 & 4.44 \\
\hline XI.4906 & 955 & 402 & 616 & 347 & 624 & 121.72 & 107.43 & 275.22 & 30.93 & 86.71 \\
\hline XI.51509 & 16 & 1 & 1 & 40 & 60 & 10.71 & 0.00 & 0.00 & 21.59 & 32.41 \\
\hline XI.57027 & 74 & 99 & 77 & 39 & 65 & 68.68 & 27.27 & 15.34 & 24.81 & 10.92 \\
\hline XI.57926 & 371 & 240 & 308 & 580 & 543 & 28.35 & 64.40 & 68.19 & 157.01 & 52.06 \\
\hline XI.58101 & 1 & 1 & 1 & 1 & 1 & 0.00 & 0.00 & 0.00 & 0.00 & 0.00 \\
\hline $\mathrm{XI.59256}$ & 1 & 1 & 1 & 1 & 1 & 0.00 & 0.00 & 0.00 & 0.00 & 0.00 \\
\hline XI.6091 & 16 & 4 & 9 & 14 & 19 & 0.55 & 2.85 & 2.47 & 8.67 & 12.03 \\
\hline$X \mid .67202$ & 1 & 1 & 1 & 1 & 1 & 0.00 & 0.00 & 0.00 & 0.00 & 0.00 \\
\hline XI.68408 & 1 & 1 & 1 & 1 & 1 & 0.00 & 0.00 & 0.00 & 0.00 & 0.00 \\
\hline $\mathrm{XI.70850}$ & 1 & 1 & 1 & 1 & 1 & 0.24 & 0.00 & 0.00 & 0.00 & 0.00 \\
\hline $\mathrm{XI.71159}$ & 578 & 705 & 474 & 394 & 352 & 37.93 & 51.13 & 36.52 & 83.62 & 38.93 \\
\hline $\mathrm{XI.74263}$ & 1 & 1 & 1 & 1 & 1 & 0.00 & 0.00 & 0.00 & 0.00 & 0.48 \\
\hline $\mathrm{XI.79790}$ & 15 & 25 & 1 & 4 & 6 & 3.03 & 8.86 & 0.00 & 3.40 & 4.73 \\
\hline XI.80297 & 54 & 1 & 1 & 301 & 111 & 25.24 & 0.00 & 0.00 & 16.66 & 55.77 \\
\hline $\mathrm{XI} .85251$ & 1 & 1 & 1 & 1 & 1 & 0.00 & 0.00 & 0.00 & 0.00 & 0.00 \\
\hline XI.8753 & 1 & 1 & 1 & 1 & 1 & 0.00 & 0.00 & 0.00 & 0.00 & 0.00 \\
\hline XI.9822 & 15 & 4 & 8 & 7 & 9 & 5.57 & 3.26 & 2.36 & 4.32 & 1.08 \\
\hline XI.9874 & 1 & 1 & 1 & 1 & 1 & 0.04 & 0.00 & 0.00 & 0.00 & 0.00 \\
\hline znf703-a/b & 1485 & 259 & 838 & 1136 & 2360 & 287.73 & 26.77 & 139.07 & 37.56 & 175.31 \\
\hline
\end{tabular}




\subsection{Analysis of the Hnf1b-overexpression phenotype}

Tab. 6.22 Quantification of Pdx1 domain in the endoderm

Image $\mathrm{J}$ quantification of $\mathrm{Pdx} 1$ area size (orange dotted line) within the endoderm (green dotted line).
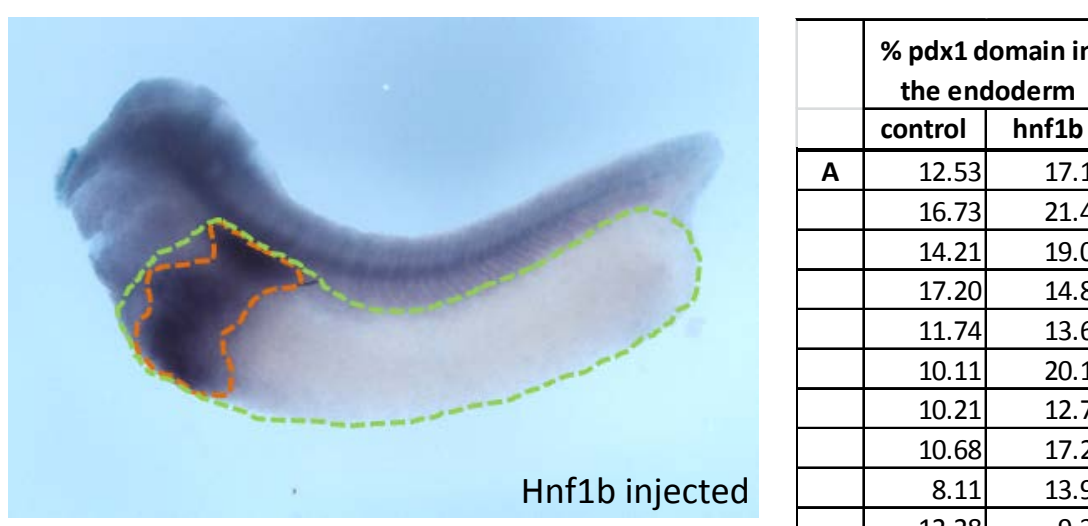

\begin{tabular}{|c|c|c|}
\hline A & 12.53 & 17.16 \\
\hline & 16.73 & 21.40 \\
\hline & 14.21 & 19.05 \\
\hline & 17.20 & 14.84 \\
\hline & 11.74 & 13.68 \\
\hline & 10.11 & 20.14 \\
\hline & 10.21 & 12.70 \\
\hline & 10.68 & 17.27 \\
\hline & 8.11 & 13.95 \\
\hline & 12.28 & 9.31 \\
\hline & 9.75 & 14.98 \\
\hline & 10.38 & 15.40 \\
\hline & 9.47 & 13.82 \\
\hline & 11.45 & 8.39 \\
\hline & 4.84 & 11.78 \\
\hline & 8.83 & 10.69 \\
\hline & 4.54 & 10.90 \\
\hline & 5.31 & 12.81 \\
\hline & 6.91 & 9.79 \\
\hline & 6.58 & 10.71 \\
\hline & 5.67 & 12.66 \\
\hline & 7.03 & 14.98 \\
\hline & 7.67 & 8.71 \\
\hline & 7.04 & 7.69 \\
\hline B & 10.15 & 9.80 \\
\hline & 9.55 & 10.07 \\
\hline & 8.88 & 9.70 \\
\hline & 11.11 & 14.12 \\
\hline & 9.46 & 12.72 \\
\hline & 9.80 & 12.34 \\
\hline & 8.54 & 13.45 \\
\hline & 8.70 & 9.38 \\
\hline & 9.76 & 14.48 \\
\hline & 9.93 & 9.73 \\
\hline & 11.92 & 17.79 \\
\hline & 10.07 & 16.01 \\
\hline & 12.41 & 20.68 \\
\hline & 13.41 & 16.89 \\
\hline & 12.70 & 13.64 \\
\hline & 11.41 & 28.30 \\
\hline & 10.11 & 11.43 \\
\hline & 11.88 & 11.43 \\
\hline & 13.21 & 12.45 \\
\hline & 11.70 & 9.95 \\
\hline & 4.50 & 9.39 \\
\hline & 5.94 & 8.76 \\
\hline & 3.30 & 11.88 \\
\hline & 6.18 & 10.42 \\
\hline & 8.53 & 8.07 \\
\hline & 6.25 & 6.13 \\
\hline & 5.40 & 5.88 \\
\hline & 6.70 & \\
\hline & 4.20 & \\
\hline & 5.88 & \\
\hline
\end{tabular}


Tab. 6.23 Quantification of Ptf1a domain in the endoderm

Image J quantification of Ptf1a area size (orange dotted line) within the endoderm (green dotted line).
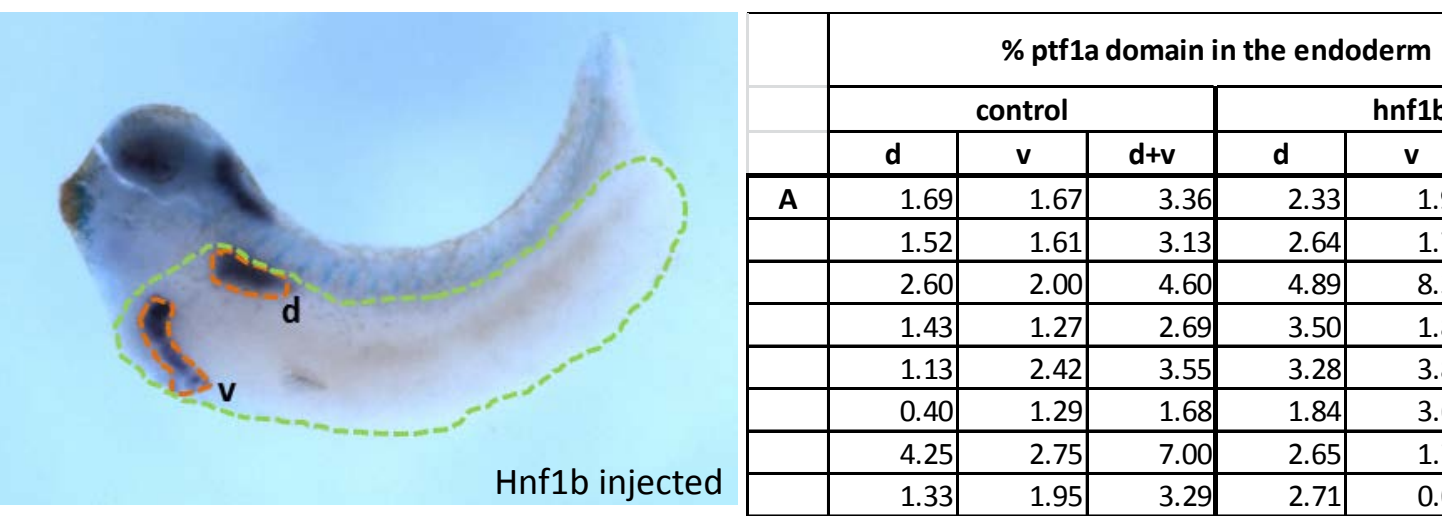

\begin{tabular}{|c|c|c|c|c|c|c|}
\hline \multicolumn{1}{|c|}{} & \multicolumn{3}{c|}{ control } & \multicolumn{3}{c|}{ hnf1b } \\
\cline { 2 - 7 } & $\mathbf{d}$ & $\mathbf{v}$ & $\mathbf{d}+\mathbf{v}$ & $\mathbf{d}$ & $\mathbf{v}$ & $\mathbf{d}+\mathbf{v}$ \\
\hline A & 1.69 & 1.67 & 3.36 & 2.33 & 1.97 & 4.30 \\
\hline
\end{tabular}

\begin{tabular}{|r|r|r|r|r|r|r|}
\hline $\mathbf{A}$ & 1.69 & 1.67 & 3.36 & 2.33 & 1.97 & 4.30 \\
\hline & 1.52 & 1.61 & 3.13 & 2.64 & 1.74 & 4.39 \\
\hline & 2.60 & 2.00 & 4.60 & 4.89 & 8.30 & 13.19 \\
\hline & 1.43 & 1.27 & 2.69 & 3.50 & 1.82 & 5.31 \\
\hline
\end{tabular}

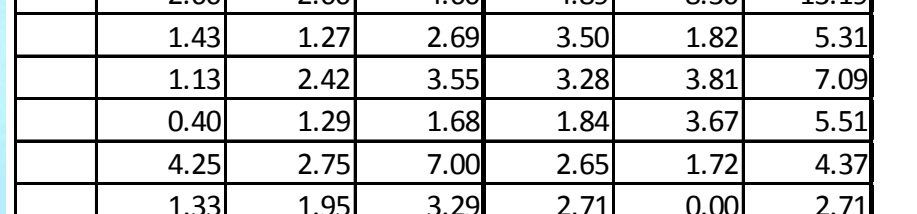

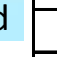

\begin{tabular}{|c|c|c|c|c|c|c|}
\hline & 1.33 & 1.95 & 3.29 & 2.71 & 0.00 & 2.71 \\
\hline & 1.56 & 1.28 & 2.83 & 1.51 & 0.00 & 1.51 \\
\hline & 2.63 & 1.81 & 4.45 & 0.97 & 2.16 & 3.13 \\
\hline & 1.97 & 0.00 & 1.97 & 1.61 & 0.00 & 1.61 \\
\hline & 1.00 & 0.00 & 1.00 & 3.46 & 1.62 & 5.07 \\
\hline & 0.77 & 0.26 & 1.03 & 1.85 & 1.32 & 3.17 \\
\hline & 1.29 & 0.00 & 1.29 & 2.65 & 1.99 & 4.64 \\
\hline & 0.95 & 0.25 & 1.20 & 3.08 & 1.49 & 4.57 \\
\hline & 2.93 & 0.67 & 3.60 & 2.68 & 1.70 & 4.37 \\
\hline & 1.46 & 0.53 & 1.99 & 3.08 & 1.98 & 5.06 \\
\hline & 0.59 & 0.00 & 0.59 & 2.89 & 1.67 & 4.55 \\
\hline & 1.60 & 0.00 & 1.60 & 2.62 & 3.04 & 5.67 \\
\hline & 2.01 & 0.29 & 2.30 & 2.33 & 1.52 & 3.85 \\
\hline & 0.87 & 0.00 & 0.87 & 2.94 & 1.91 & 4.85 \\
\hline B & 3.30 & 0.81 & 4.11 & 2.86 & 1.28 & 4.14 \\
\hline & 3.19 & 1.65 & 4.84 & 3.22 & 2.71 & 5.93 \\
\hline & 2.09 & 1.57 & 3.65 & 3.73 & 1.86 & 5.59 \\
\hline & 2.59 & 0.73 & 3.31 & 3.93 & 1.07 & 5.00 \\
\hline & 3.17 & 0.84 & 4.01 & 3.07 & 1.18 & 4.26 \\
\hline & 1.38 & 0.58 & 1.96 & 3.01 & 2.82 & 5.83 \\
\hline & 3.05 & 1.03 & 4.08 & 4.89 & 2.41 & 7.30 \\
\hline & 2.16 & 0.82 & 2.98 & 3.70 & 3.75 & 7.45 \\
\hline & 3.85 & 1.12 & 4.96 & 3.33 & 1.06 & 4.39 \\
\hline & 2.56 & 1.28 & 3.83 & 2.41 & 1.66 & 4.07 \\
\hline & 3.11 & 1.20 & 4.31 & 2.62 & 1.13 & 3.75 \\
\hline & 3.72 & 1.37 & 5.09 & 1.54 & 1.00 & 2.54 \\
\hline & 2.56 & 2.11 & 4.67 & 3.85 & 2.12 & 5.97 \\
\hline & 3.80 & 1.35 & 5.15 & 0.62 & 1.31 & 1.93 \\
\hline & 2.56 & 1.87 & 4.43 & 1.34 & 1.78 & 3.12 \\
\hline & 3.12 & 1.25 & 4.37 & 3.39 & 3.86 & 7.25 \\
\hline & 3.83 & 2.20 & 6.03 & & & \\
\hline & 3.07 & 1.55 & 4.62 & & & \\
\hline & 3.42 & 1.38 & 4.80 & & & \\
\hline & 1.29 & 0.94 & 2.23 & & & \\
\hline & 1.22 & 1.22 & 2.44 & & & \\
\hline & 0.90 & 0.78 & 1.68 & & & \\
\hline & 0.79 & 0.25 & 1.04 & & & \\
\hline & 0.36 & 0.97 & 1.33 & & & \\
\hline & 1.33 & 0.81 & 2.14 & & & \\
\hline & 1.01 & 0.76 & 1.77 & & & \\
\hline & 1.33 & 0.52 & 1.85 & & & \\
\hline & 2.59 & 1.32 & 3.91 & & & \\
\hline
\end{tabular}


Tab. 6.24 Predicted Fzd4-gRNA off-target sequences in exonic regions of the Xenopus laevis genome

highlighted in green are the tested off-targets; PAM= protospacer adjacent motif

\begin{tabular}{|c|c|c|c|c|c|c|}
\hline Coordinates & strand & MM & target_seq & PAM & gene name & gene id \\
\hline $\begin{array}{l}\text { Scaffold } 149 \\
581: 4344665 \\
4344687\end{array}$ & + & 4 & GGAAGATG[GTGAACCTGAAG] & AGG & utp14a & $\begin{array}{l}\text { XB-GENE- } \\
5954915\end{array}$ \\
\hline $\begin{array}{l}\text { Scaffold230 } \\
826: 3712453 \\
3712475\end{array}$ & - & 5 & AGAGCAGG[TTGATCCTGATG] & AGG & impad1 & $\begin{array}{l}\text { XB-GENE- } \\
5751113\end{array}$ \\
\hline $\begin{array}{l}\text { Scaffold102 } \\
068: 223547- \\
223569\end{array}$ & + & 5 & TGCTCAGT[CTGATCCTGATG] & GGG & kremen2 & $\begin{array}{l}\text { XB-GENE- } \\
866619\end{array}$ \\
\hline $\begin{array}{l}\text { Scaffold139 } \\
113: 827475- \\
827497\end{array}$ & - & 5 & GTTTCATG[ATGGTCCTGATG] & CGG & fzd7 & $\begin{array}{l}\text { XB-GENE- } \\
483735\end{array}$ \\
\hline $\begin{array}{l}\text { Scaffold307 } \\
11: 2325955- \\
2325977\end{array}$ & + & 5 & GCCTCCTG[ATGAGCCTGATG] & AGG & zmym4 & $\begin{array}{l}\text { XB-GENE- } \\
1217387\end{array}$ \\
\hline $\begin{array}{l}\text { Scaffold719 } \\
7: 648108- \\
648130\end{array}$ & - & 5 & GGTACACC[GTTATTCTGATG] & TGG & edc3 & $\begin{array}{l}\text { XB-GENE- } \\
5890837\end{array}$ \\
\hline $\begin{array}{l}\text { Scaffold476 } \\
83: 3850870- \\
3850892\end{array}$ & - & 5 & TATACAAG[GTGATCCTGGTG] & AGG & exog & $\begin{array}{l}\text { XB-GENE- } \\
981842\end{array}$ \\
\hline $\begin{array}{l}\text { Scaffold551 } \\
71: 287242- \\
287264\end{array}$ & + & 5 & GGGAATTG[GTGATCCTGTTA] & GGG & gcdh & $\begin{array}{l}\text { XB-GENE- } \\
1016454\end{array}$ \\
\hline
\end{tabular}




\subsection{Nanostring code sets}

Tab. 6.25 Nanostring Code set used for the confirmation of pancreatic organoid formation

\begin{tabular}{|c|c|c|c|}
\hline Target & Accession & Target Region & Target sequence \\
\hline Actb & $\begin{array}{l}\text { NM_001088953. } \\
1\end{array}$ & $1179-1279$ & $\begin{array}{l}\text { ATGCTTCTAAAGGACAGACCCTTTCAACATGAACAAA } \\
\text { TGTACCTGTGCAGGAAGATCACATTGGCATGGCTTT } \\
\text { ACTCTTTTGTTGGCGCTTGGCTCAGAA }\end{array}$ \\
\hline $\begin{array}{l}\text { Amylase } \\
\text { alpha2A/ } \\
\text { B }\end{array}$ & $\begin{array}{l}\text { NM_001086441. } \\
1\end{array}$ & $555-655$ & $\begin{array}{l}\text { GGATTTGGCAATGGAGAAAGACTATGTTCGTGGGAA } \\
\text { GATTGCCGAATACATGAACAATCTAATTAACATTGGG } \\
\text { GTTGCTGGATTCAGACTGGATGCTGCT } \\
\end{array}$ \\
\hline Chordin & $\begin{array}{l}\text { NM_001088309. } \\
1\end{array}$ & $1455-1555$ & $\begin{array}{l}\text { GACGTCCGCAAAGCATGTCAGGCATAATCACAGTCA } \\
\text { GAAAATCATGTGACACTTTGCAGAGTGTGTTATCGG } \\
\text { GTGGTGACGCTTTAAATCCCACCAAAAC }\end{array}$ \\
\hline Сyp26a1 & $\begin{array}{l}\text { NM_001095469. } \\
1\end{array}$ & $984-1084$ & $\begin{array}{l}\text { TCTTGCACTTCACAAAGATGTTCTGGAAAAGGTCCG } \\
\text { CAAGGAGCTCGAAACACAGGGTCTGTTGTCAACGAA } \\
\text { ACCCGAGGAGAAAAAGGAACTAAGCATG }\end{array}$ \\
\hline Darmin & BC045077.1 & $787-887$ & $\begin{array}{l}\text { TATATTTGCTGAACACGCTTGCAGATGGAAAGGGTC } \\
\text { GCATCCTTGTCCCAGGGATTTATGAGGCCGTAGCAC } \\
\text { CTGTGGGTGAAAATGAAACAGATTTGTA }\end{array}$ \\
\hline Dkk1 & $\begin{array}{l}\text { NM_001085592. } \\
1\end{array}$ & $770-870$ & $\begin{array}{l}\text { GAGATTTTCCAGCGTTGTCACTGCGGTGCCGGACTC } \\
\text { TCGTGCCGGTTACAGAAAGGAGAATTTACAACTGTC } \\
\text { CCTAAAACATCGAGACTTCACACTTGCC }\end{array}$ \\
\hline $\begin{array}{l}\text { Elastase } 2 \\
\text { A }\end{array}$ & $\begin{array}{l}\text { NM_001092506. } \\
1\end{array}$ & $512-612$ & $\begin{array}{l}\text { AATGGCCCAGCACCAGATAAACTGCAGCAGGGGCT } \\
\text { CTTACTGGTGGTCGATTACCCAACTTGTTCCCAGCG } \\
\text { GGACTGGTGGTGGAAAAGCGTAAATACCA }\end{array}$ \\
\hline FoxE1 & $\begin{array}{l}\text { NM_001095544. } \\
1\end{array}$ & $1594-1694$ & $\begin{array}{l}\text { CAGTCGGGCATGTGAAATCACGTTGACTATGGTCTG } \\
\text { CTGATTGTACCCTTAGATGGGCAGCCATGTAAAGGC } \\
\text { AAGATACCATTCTCTTACAGTCATATTG }\end{array}$ \\
\hline FoxE4 & $\begin{array}{l}\text { NM_001085733. } \\
1\end{array}$ & $16-116$ & $\begin{array}{l}\text { GGATCCCAGATGACTTTTGCTGTGTTGGGACTGAGA } \\
\text { GGGTTTTGTTTATGAATTTGGCAAGTTTCCCTTATTAT } \\
\text { TCAGGCATGTGCACCATGACTGCAGA }\end{array}$ \\
\hline G6pd & $\begin{array}{l}\text { NM_001086550. } \\
1\end{array}$ & $862-962$ & $\begin{array}{l}\text { GTGGAGGATACTTTGACGAATTTGGCATCATCCGGG } \\
\text { ATGTCATGCAAAATCACTTGCTCCAAATGATGTGTTT } \\
\text { GATGGCTATGGAGAAGCCGGTCTCCAC }\end{array}$ \\
\hline Gapdh & $\begin{array}{l}\text { NM_001087098. } \\
1\end{array}$ & $773-873$ & $\begin{array}{l}\text { ACCTGCCGCCTGCAGAAGCCGGCCAAGTACGATGA } \\
\text { CATCAAGGCCGCCATTAAGACTGCATCAGAGGGCCC } \\
\text { AATGAAGGGAATCCTGGGATACACACAAG }\end{array}$ \\
\hline GATA-2 & $\begin{array}{l}\text { NM_001090574. } \\
1\end{array}$ & $192-292$ & $\begin{array}{l}\text { CCAGGACTAACGGGGACAACTCACTTTCACTTTTAAC } \\
\text { AGCCGAAGCCTTGCTTATAGGAAGGACACATATATC } \\
\text { TGGCCGATCTCTGGAGAGAACTTTGCT }\end{array}$ \\
\hline $\begin{array}{l}\text { GATA- } \\
4 \mathrm{a} / \mathrm{b}\end{array}$ & $\begin{array}{l}\text { NM_001090629. } \\
1\end{array}$ & $571-671$ & $\begin{array}{l}\text { GCACCCAAACATAGAGTTTTTTGACGATTTTTCCGAG } \\
\text { GGCCGAGAATGTGTCAACTGTGGAGCAATGTCAACC } \\
\text { CCACTTTGGAGGCGGGATGGAACAGGC }\end{array}$ \\
\hline Glucagon & $\begin{array}{l}\text { NM_001085673. } \\
1\end{array}$ & $385-485$ & $\begin{array}{l}\text { AGCAATTGGATGAAAAAGCAGCCAAAGAATTTATTGA } \\
\text { CTGGCTAATAAATGGAGGACCATCAAAAGAAATCATT } \\
\text { TCAAGACGCAATGCAGAATTTGAGAG }\end{array}$ \\
\hline $\mathrm{H} 4$ & $\begin{array}{l}\text { NM_001094457. } \\
1\end{array}$ & $129-229$ & $\begin{array}{l}\text { GGAGAGGGGGAGTCAAGCGCATCTCTGGCCTCATC } \\
\text { TATGAGGAGACTCGTGGGGTCCTCAAGGTTTTCCTG } \\
\text { GAGAATGTCATCCGGGACGCCGTCACCTA }\end{array}$ \\
\hline Hes1-a & $\begin{array}{l}\text { NM_001087927. } \\
1\end{array}$ & $557-657$ & $\begin{array}{l}\text { GAAGGGGTCAACACAGATGTCCGGACCCGACTCCT } \\
\text { GGGGCATCTTGCCAACTGCATGAACCAGATCAATGG } \\
\text { CATGAACTACCCTACCCAGCCCCAGATGC }\end{array}$ \\
\hline $\begin{array}{l}\text { HNF6 } \\
\text { gene2 }\end{array}$ & DC109227.1 & $141-241$ & $\begin{array}{l}\text { TGTGTCCCAGTTGGCAATGTTATTGGAAGCTTTACTC } \\
\text { TTATGAGAGAGGACAGAGGTTTGGGGCCACCAAGTA } \\
\text { ACTTTTACAGTCACTATCCCAAAGACA }\end{array}$ \\
\hline Hoxa1-b & X62053.1 & $1149-1249$ & $\begin{array}{l}\text { TGAGATGATGGAATGGTAAAAAAGCTCAATGTTTTCT } \\
\text { AGCACAGGAGAAACAGCAGTGTTTGCTGACCGCATA } \\
\text { CCTCAGTAATCTCTCTCATTTACTAGG }\end{array}$ \\
\hline Insulin-a & $\begin{array}{l}\text { NM_001085882. } \\
1\end{array}$ & $608-708$ & $\begin{array}{l}\text { AGGTGACAGGGCAGGTTACATTTATTAAGTTCAGAT } \\
\text { GTAAAAAAAAAAAAAATCTGGAAGAAAAAGAGCCACC } \\
\text { CCAATTGTTAGTCCCATCTTGCTTTTA }\end{array}$ \\
\hline Insulin-b & $\begin{array}{l}\text { NM_001085881. } \\
1\end{array}$ & $165-265$ & $\begin{array}{l}\text { CTAGTATGTGGGGATAGAGGCTTCTTCTACTACCCTA } \\
\text { AGATCAAACGGGACATCGAACAAGCACAGGTCAATG } \\
\text { GACCCCAGGACAACGAGTTAGATGGAA }\end{array}$ \\
\hline
\end{tabular}




\begin{tabular}{|c|c|c|c|}
\hline Target & Accession & Target Region & Target sequence \\
\hline$|s|-1$ & $\begin{array}{l}\text { NM_001110718. } \\
1\end{array}$ & $1102-1202$ & $\begin{array}{l}\text { TACAGAGTGACATTGATCAGCCGGCCTTCCAGCAAC } \\
\text { TGACCTGTTTCTCACAGGTTAATTTTTCAGAAGGAGG } \\
\text { ACCTGGCTCTAACTCTACAGGGAGTGA }\end{array}$ \\
\hline Ngn3 & $\begin{array}{l}\text { NM_001134785. } \\
1\end{array}$ & $216-316$ & $\begin{array}{l}\text { AAAGAAGCAAAGGGTCAAGCGAATGAGATCAAAAGT } \\
\text { AAAGAGCGACGGCGTCGTCGTAAAACAGAGGAGAA } \\
\text { ATCGCAGGGTGAAAGCCAATGACAGAGAG }\end{array}$ \\
\hline Nkx2.1 & $\begin{array}{l}\text { NM_001085624. } \\
1\end{array}$ & $1392-1492$ & $\begin{array}{l}\text { TTCAGACTCTCTATGGTGTCACCAACAAGATGCTTGT } \\
\text { ACAGTACACAGGGCTTTACTGGAGATCTGGGGATAA } \\
\text { GATGCTACTGAAACCTTCAGTCCTCAA }\end{array}$ \\
\hline Nkx6.1 & $\begin{array}{l}\text { NM_001099916. } \\
1\end{array}$ & $172-272$ & $\begin{array}{l}\text { AATACATCATTCCCTCTGCAGCTTGAACTGATTTAAA } \\
\text { CCTCGGCCATCCAACACTACAGCCCAGCGTCGGTAG } \\
\text { GATATAGTGATTCACCCTTGGAGTTGT }\end{array}$ \\
\hline Nkxx2.2 & $\begin{array}{l}\text { NM_001085622. } \\
1\end{array}$ & $133-233$ & $\begin{array}{l}\text { AGAATTGGGTGCTTTAGCCAGACCCTCAGCTAAACC } \\
\text { TGACTTTGGCATTTTTCCCTGGAGTTACAAACTATAG } \\
\text { GAATAGCGCTTGGTGCTCCCTCAACTG }\end{array}$ \\
\hline Noggin & $\begin{array}{l}\text { NM_001085644. } \\
1\end{array}$ & $561-661$ & $\begin{array}{l}\text { ACCCAACTCTTATTTTGTGCAGCTGTGTGCAGCATG } \\
\text { GATCATTCCCAGTGCCTTGTGACTATATATGCTCTGA } \\
\text { TGGTCTTCTTGGGACTTAGAATAGACC }\end{array}$ \\
\hline Odc & $\begin{array}{l}\text { NM_001086698. } \\
1\end{array}$ & $855-955$ & $\begin{array}{l}\text { GGATATAATTGGTGTGAGTTTCCATGTTGGCAGTGG } \\
\text { CTGCACTGATCCACAGACTTATGTACAAGCTGTCTCA } \\
\text { GATGCACGATGTGTCTTTGACATGGGG }\end{array}$ \\
\hline Pax6-b & $\begin{array}{l}\text { NM_001172195. } \\
1\end{array}$ & $1339-1439$ & $\begin{array}{l}\text { CTTACAGTGCTTTGCCACCTATGCCTAGTTTTACGAT } \\
\text { GGGCAACAATCTACCTATGCAAGTCTCATTTCCCCTG } \\
\text { GAGTGTCAGTCCCAGTTCAAGTACCC } \\
\end{array}$ \\
\hline PDia2 & $\begin{array}{l}\text { NM_001090179. } \\
1\end{array}$ & $881-981$ & $\begin{array}{l}\text { TGCTGCCCAGATCCCAAACCACTTGTTGCTGTTTATC } \\
\text { AATAAGAGTGACGATTCCCAACTGGTGCTGCTGGAA } \\
\text { CATTTCCGCAAAGCAGCTCCTGACTTT }\end{array}$ \\
\hline Pdx1 & $\begin{array}{l}\text { NM_001172211. } \\
1\end{array}$ & $162-262$ & $\begin{array}{l}\text { CCAGGCAGTCCTCCAGACATCTCACCGTATGAAGTG } \\
\text { CCTCCCATATCTGAAGAGCCCATTGTTCCTCACCTCC } \\
\text { ATCACCATCACTACCATCATCACCACC }\end{array}$ \\
\hline Ptf1a-a/b & AY372268.3 & $149-249$ & $\begin{array}{l}\text { CATTCCTCTAGGGACGCCCTGGACGCAGACGACTTT } \\
\text { TTGGAAGACGATGTAGACTTCTTGGCCGGTCAGATC } \\
\text { CAAGACTATTACAGAGACAGCCGAGTGC }\end{array}$ \\
\hline RALDH2 & NM_001090775. & $966-1066$ & $\begin{array}{l}\text { TGGACTATGCAGTTGAACAAGCACACCAAGGTGTGT } \\
\text { TCTTTAACCAAGGACAGTGCTGTACTGCTGGCTCGC } \\
\text { GGACATTCGTAGAAGATTCCATTTATGA }\end{array}$ \\
\hline $\begin{array}{l}\text { Somatost } \\
\text { atin }\end{array}$ & $\begin{array}{l}\text { NM_001089250. } \\
1\end{array}$ & $178-278$ & $\begin{array}{l}\text { CAGAAATCACTCGCAGCGGCAGGGAAACAGGAGTT } \\
\text { GGCCAAGTATTTCCTGGCAGAGTTGCTATCAGACCC } \\
\text { TTCCCAGACAGAGAATGAAGCATTGGAAT }\end{array}$ \\
\hline Sox1 & NM_001095674. & $1663-1763$ & $\begin{array}{l}\text { CTGTGCTCATCCTTTTAGAGACAATGCGTCCAGCCT } \\
\text { GCTCATCAAGGATCGGGCAGCACACTTGCCTCCACT } \\
\text { TTGCCACAACTGCCCTCTCTTTTTCTTT }\end{array}$ \\
\hline Sox17a & NM_001088162. & $567-667$ & $\begin{array}{l}\text { TGCTGGGCGCAGATGGAAGGATGTGTCTAGAGAACT } \\
\text { TCAGCCTGGGTTATCATGAGCAGACTTACTCCCACG } \\
\text { GCCAGGTTCCTCAGAGCAGCCACTACAG }\end{array}$ \\
\hline Sox17b & $\begin{array}{l}\text { NM_001088164. } \\
1\end{array}$ & $163-263$ & $\begin{array}{l}\text { ATGGACCGAACCCCTGACCGTGTTTCAGGACCTCAA } \\
\text { ACCCAAGAGGGATGAAGGATCTGCCGATTCCAGAAG } \\
\text { CAAAGCCGAGGGTCGGATCCGCCGACCC }\end{array}$ \\
\hline Sox2 & $\begin{array}{l}\text { NM_001088222. } \\
1\end{array}$ & $901-1001$ & $\begin{array}{l}\text { CCGGGCATGTCTCTGGGATCCATGGGCTCGGTAGT } \\
\text { CAAGTCGGAATCCAGCTCCAGTCCACCTGTAGTCAC } \\
\text { CTCTTCTTCCCATTCGCGGGCTCCGTGCC }\end{array}$ \\
\hline Sox3 & $\begin{array}{l}\text { NM_001090679. } \\
1\end{array}$ & 998-1098 & $\begin{array}{l}\text { GCTCACTCACATATAACACTTTGTGCCCTTTGCTAAA } \\
\text { GACGCTTTACTTGCCTGCTGGCAACTATCAGACTGC } \\
\text { CGCATAAAACATTTAAAAAAAAAAATC }\end{array}$ \\
\hline SP-C & $\begin{array}{l}\text { NM_001096721. } \\
1\end{array}$ & $18-118$ & $\begin{array}{l}\text { CCACCTGTCTATTCAGAGTTGCCAATCCCATGTTTCG } \\
\text { GTGGGGTTAAGAAGCTTGTCTGTGTGGTCCTGGTGG } \\
\text { TGGTGGTCCTTGTGCTGGTTCTGGTTG }\end{array}$ \\
\hline TGIF2 & $\begin{array}{l}\text { NM_001094168. } \\
1\end{array}$ & $2638-2738$ & $\begin{array}{l}\text { TTTGAGATATTTGTGAAGTGTGCCTTGGGAAGCAGC } \\
\text { ACATTTCCCGGTAACACGAGTCCTTAGACTGACCTTT } \\
\text { TTGAGTACTAGGACAAAAGTAACCTGA }\end{array}$ \\
\hline Tm4sf3 & NM_001087390. & $133-233$ & $\begin{array}{l}\text { TTTGGGTGTCTCAATTTGGGTCCGAGTCAGCAAAAAT } \\
\text { GTGCAAAAAGAACTGAACATTGAAGGAGGAAGCTTG } \\
\text { CTTGCAGCAGTTGATCTCATGATTGCA }\end{array}$ \\
\hline $\begin{array}{l}\text { Trypsinog } \\
\text { en/trypsin }\end{array}$ & ВС056068.1 & $390-490$ & $\begin{array}{l}\text { GCCCTCGGCCTGTGCTTCTGCCGGCACCAACTGCCT } \\
\text { GATCTCTGGCTGGGGGAACACCCTGAGCAGCGGCA } \\
\text { CCAATTACCCAGATCTCCTGCAGTGCCTG }\end{array}$ \\
\hline
\end{tabular}




\begin{tabular}{|l|l|l|l|}
\hline Target & Accession & Target Region & \multicolumn{1}{c|}{ Target sequence } \\
\hline $\begin{array}{l}\text { Ventx2.1- } \\
\text { b }\end{array}$ & $\begin{array}{l}\text { NM_001087981. } \\
1\end{array}$ & $1075-1175$ & $\begin{array}{l}\text { TTCCACAGGACAAAAAATTGCACTGAATGTTGCTATT } \\
\text { GGCAAGATGATTACAGAATAGCTGGCTACTATTGGC } \\
\text { CTATTTGTATGTATCTTACATGATT }\end{array}$ \\
\hline Xpp1 & $\begin{array}{l}\text { NM_001258387. } \\
1\end{array}$ & $177-277$ & $\begin{array}{l}\text { CATCTCTGGTGCAATGTCAGTCATTCCTCAGCCGGG } \\
\text { GGATTTGAGTGGTCTGTTTCCCGGCACATTCTCCT } \\
\text { TCCCAGAAACTCCAGATCATCAGCTCCA }\end{array}$ \\
\hline xCRABP1 & $\begin{array}{l}\text { NM_001090578. } \\
\text { EU816559.1 }\end{array}$ & $534-634$ & $\begin{array}{l}\text { AGCCCACTGGATGAAAGATCCTGTCTCTTTAGCAAA } \\
\text { GTCAAACTTACAAACAAAATGAATGGTGAGGCAG } \\
\text { ATTATGTTAAACTCTTTGCACAAGTAT }\end{array}$ \\
\hline xCRABP2 & $\begin{array}{l}\text { NM_001085780. } \\
1\end{array}$ & $\begin{array}{l}\text { TGACGAACTTCTGAAAGCTCTAGGTGTTATGCTATG } \\
\text { CTTAGGAAAGTGGCTGTAGCAGCAGCTTCTAAGCCT } \\
\text { CATGTTGAAATCCGTCAAAATGGGGAC }\end{array}$ \\
\hline Xhex & $\begin{array}{l}\text { NM_001085590. } \\
1\end{array}$ & $\begin{array}{l}\text { CTGACCATGACTGCTGATGATGTTGTCTGCACACGG } \\
\text { ATTACATCAGGGACTAATTTACAGAACTCTATGGGA } \\
\text { TAAGAGGCACCATCCTCATCCTTCCCA }\end{array}$ \\
\hline
\end{tabular}


Tab. 6.26 Nanostring Code set for the verification of RA-responsive candidates

\begin{tabular}{|c|c|c|c|}
\hline Target & Accession & Target region & Target sequence \\
\hline Bhlhe40 & ВС073563.1 & $350-450$ & $\begin{array}{l}\text { GAAAGACCTGTTGCCGGAGCACCTCAAACTGACTACTTTG } \\
\text { GGTCACCTTGAGAAAGCCGTGGTTCTGGAGCTGACCTTGA } \\
\text { AACACGTGCAGTCTTTGTCC }\end{array}$ \\
\hline BMP2-a/b & $\begin{array}{l}\text { NM_001085 } \\
884.1\end{array}$ & $1290-1390$ & $\begin{array}{l}\text { GTTGCCCCACCTGGGTATCATGCCTTTTACTGCCACGGGG } \\
\text { AATGTCCTTTTCCACTGGCAGACCATTTAAACTCTACAAAC } \\
\text { CATGCAATCGTACAAACTT }\end{array}$ \\
\hline BMP4-a/b & $\begin{array}{l}\text { NM_001088 } \\
032.1\end{array}$ & $1146-1246$ & $\begin{array}{l}\text { GAGATTGTCCATTTCCCTTGGCTGATCACCTAAACTCAACT } \\
\text { AACCATGCTATTGTACAAACTCTGGTAAACTCTGTTAACGC } \\
\text { AAGCATCCCAAAAGCGTG }\end{array}$ \\
\hline C10orf140 & DR716716.1 & $783-883$ & $\begin{array}{l}\text { CTGTGCTCTTCACTCCAGTATCATGCGCTGTGACGTATCCT } \\
\text { GGGATATCTCACAATCGTGAACTGAAAAGCAACTCTCTCCT } \\
\text { GATACAGTACCACTCTCA }\end{array}$ \\
\hline Cass4 & $\begin{array}{l}\text { NM_001091 } \\
015.1\end{array}$ & $2773-2873$ & $\begin{array}{l}\text { AGGTTGCCCTGCTAGCTGTTCAAGAGTTCTTTGAAGGTGTT } \\
\text { TAGGTGGCACACAGGAACTGTAGCCTGTAGCCATGTAGGT } \\
\text { GCCTTTTAATCAAGTGCAT }\end{array}$ \\
\hline $\mathrm{Cdx} 4$ & $\begin{array}{l}\text { NM_001087 } \\
251.1\end{array}$ & $834-934$ & $\begin{array}{l}\text { TGCAAGCATGTCACTCTGTCCCAATGAACTACTAGCCTTGG } \\
\text { CAGTATTAAGTGAACACTGATCACTCAGACATGAGACCTAC } \\
\text { AAGTCATCACCATAGTTG }\end{array}$ \\
\hline Cebpd & $\begin{array}{l}\text { NM_001089 } \\
609.1\end{array}$ & $1695-1795$ & $\begin{array}{l}\text { ACTCAGGATAAGGGTTCATTGATGCCACATTTGTAATCCTA } \\
\text { TTAACCTGTTGGATGTATGCCAAGCTGCAGCCATACTCCCT } \\
\text { TACTGTTTCATTCATCTC }\end{array}$ \\
\hline Cerberus & $\begin{array}{l}\text { NM_001088 } \\
331.1\end{array}$ & $754-854$ & $\begin{array}{l}\text { CTGGATCTAAGAATGTAGTAAAGGTTGTCATGATGGTAGAG } \\
\text { GAATGCACGTGTGAAGCTCATAAGAGCAACTTCCACCAAAC } \\
\text { TGCACAGTTTAACATGGA }\end{array}$ \\
\hline Chordin & $\begin{array}{l}\text { NM_001088 } \\
309.1\end{array}$ & $1455-1555$ & $\begin{array}{l}\text { GACGTCCGCAAAGCATGTCAGGCATAATCACAGTCAGAAA } \\
\text { ATCATGTGACACTTTGCAGAGTGTGTTATCGGGTGGTGAC } \\
\text { GCTTTAAATCCCACCAAAAC }\end{array}$ \\
\hline Cxcr7 & $\begin{array}{l}\text { NM_001088 } \\
767.1\end{array}$ & $812-912$ & $\begin{array}{l}\text { GGGGACCAAGAGAGGAGAATCAGTGGAAGGCTTATTGTTT } \\
\text { CTTATGTAGTTGTGTTTATGGTTTGTTGGCTGCCATACCAT } \\
\text { GCCATGGTCATACTAGACG }\end{array}$ \\
\hline Сyp26a1 & $\begin{array}{l}\text { NM_001095 } \\
469.1\end{array}$ & 984-1084 & $\begin{array}{l}\text { TCTTGCACTTCACAAAGATGTTCTGGAAAAGGTCCGCAAGG } \\
\text { AGCTCGAAACACAGGGTCTGTTGTCAACGAAACCCGAGGA } \\
\text { GAAAAAGGAACTAAGCATG }\end{array}$ \\
\hline Dact1-a & $\begin{array}{l}\text { NM_001088 } \\
772.1\end{array}$ & $41-141$ & $\begin{array}{l}\text { CACGTTGTGCAGAAGTCGGGCTTCGGTTCTTGGTTGGGAA } \\
\text { TAGCGAGGCGGATTCTCATTGGAGCCCCGTGTACGTGAGT } \\
\text { TGCAGCCGCTGCAGATGCTC }\end{array}$ \\
\hline Dact1-b & $\begin{array}{l}\text { NM_001090 } \\
441.1\end{array}$ & 95-195 & $\begin{array}{l}\text { GCGCAGGAGACGCTAAGTCGCAGAAGTTGATGCATTGTAT } \\
\text { CCGCTGATAAGTACTGGGAGTTTGGTTTCTTGCGGTATAGT } \\
\text { GTGAGGATAAAGATCCCCG }\end{array}$ \\
\hline Darmin & BC045077.1 & $787-887$ & $\begin{array}{l}\text { TATATTTGCTGAACACGCTTGCAGATGGAAAGGGTCGCATC } \\
\text { CTTGTCCCAGGGATTTATGAGGCCGTAGCACCTGTGGGTG } \\
\text { AAAATGAAACAGATTTGTA }\end{array}$ \\
\hline Dhrs3 & $\begin{array}{l}\text { NM_001092 } \\
373.1\end{array}$ & $817-917$ & $\begin{array}{l}\text { ATGGCCACATTGTTTGTATAAACTCTGTGCTGGCCTTATCT } \\
\text { GCCATCCCTGGTGCCATTGACTATTGCACTTCCAAATCGTC } \\
\text { CTCCTTTGCCTTCATGGA }\end{array}$ \\
\hline Dusp5 & $\begin{array}{l}\text { NM_001092 } \\
854.1\end{array}$ & $581-681$ & $\begin{array}{l}\text { TGTTGAGATCCTTCCGTTCCTATATCTCGGCAGCGCCTATC } \\
\text { ACGCTTCCAAATGCGAGTTCCTTGCGAATCTGCACATCACC } \\
\text { GCCTTACTCAATGTCTCG }\end{array}$ \\
\hline Dusp6 & $\begin{array}{l}\text { NM_001089 } \\
787.1\end{array}$ & $686-786$ & $\begin{array}{l}\text { TGACAGGGATCCCAGCAGTGCAACAGATTCAGACGGTAGT } \\
\text { CCTCTATCCAACCCTCAGCCATCATTTCCCGTTGAGATCTT } \\
\text { ACCCTACCTTTACCTCGGT }\end{array}$ \\
\hline Erf & $\begin{array}{l}\text { NM_001096 } \\
253.1\end{array}$ & $225-325$ & $\begin{array}{l}\text { GCGTCTGTGGGGCGTTCGGAAATGCAAACCGCAGATGAAT } \\
\text { TATGATAAACTGAGCCGGGCACTCAGGTATTATTATAACAA } \\
\text { GAGGATTCTCCATAAAACC }\end{array}$ \\
\hline Fgfr4-a & $\begin{array}{l}\text { NM_001087 } \\
718.1\end{array}$ & $230-330$ & $\begin{array}{l}\text { TTGCTTTCGGTTCGGGGATTTATTTTGTGCCGCTGAGGAAC } \\
\text { GGAGACTAGTTCTTATGAATGGGATCACGAGTAACCTTCTA } \\
\text { ACGGCACCCGGAGATCCG }\end{array}$ \\
\hline Fgfr4-b & $\begin{array}{l}\text { NM_001088 } \\
550.1\end{array}$ & $2808-2908$ & $\begin{array}{l}\text { TTTATTTCCCCGGCCCCAAGAGATGGGCAAAAGTAGCACT } \\
\text { GTGATCCCACTTGCCAGGGCACATGAACTTCTTAGCAACG } \\
\text { GAGATAAGTTCACAGCTAAG }\end{array}$ \\
\hline Foxh1 & $\begin{array}{l}\text { NM_001088 } \\
351.1\end{array}$ & $563-663$ & $\begin{array}{l}\text { GGGTAACTTCTGGACGGTGGATGTTAGCCGGATTCCTCTG } \\
\text { GATGCGATGAAGCTGCAGAACACTGCGTTGACCCGAGGTG } \\
\text { GATCAGACTACTTTGTCCAG }\end{array}$ \\
\hline Fst & $\begin{array}{l}\text { NM_001090 } \\
590.1\end{array}$ & $355-455$ & $\begin{array}{l}\text { ATGTAAAATGAACAAGAAGAACAAGCCGAGGTGTGTCTGC } \\
\text { GCTCCGGATTGTTCCAACATTACTTGGAAAGGTTCAGTGTG } \\
\text { CGGAATTGATGGCAAAACC }\end{array}$ \\
\hline Fzd4 & $\begin{array}{l}\text { NM_001090 } \\
453.1\end{array}$ & $1046-1146$ & $\begin{array}{l}\text { CATCATGAGATTGGTGGATGCCGATGAATTGACTGGCCTTT } \\
\text { GCTACGTGGGCAACCAAAACATAGACGCGCTCACGGGCTT } \\
\text { TGTGGTCGCGCCTCTTTTT }\end{array}$ \\
\hline
\end{tabular}




\begin{tabular}{|c|c|c|c|}
\hline Target & Accession & Target region & Target sequence \\
\hline G6pd & $\begin{array}{l}\text { NM_001086 } \\
550.1\end{array}$ & $862-962$ & $\begin{array}{l}\text { GTGGAGGATACTTTGACGAATTTGGCATCATCCGGGATGT } \\
\text { CATGCAAAATCACTTGCTCCAAATGATGTGTTTGATGGCTA } \\
\text { TGGAGAAGCCGGTCTCCAC }\end{array}$ \\
\hline Gapdh & $\begin{array}{l}\text { NM_001087 } \\
098.1\end{array}$ & $773-873$ & $\begin{array}{l}\text { ACCTGCCGCCTGCAGAAGCCGGCCAAGTACGATGACATCA } \\
\text { AGGCCGCCATTAAGACTGCATCAGAGGGCCCAATGAAGGG } \\
\text { AATCCTGGGATACACACAAG }\end{array}$ \\
\hline Gbx2.1 & $\begin{array}{l}\text { NM_001090 } \\
431.1\end{array}$ & 1593-1693 & $\begin{array}{l}\text { GTAGTTTTACGACAGTAATAGGGCCATAATATGGTCATCCC } \\
\text { TGGACAATGAACAAATTAGCCACGGGGTCAGACAATGCAG } \\
\text { GTTGTTGGTTGCCAATAAC }\end{array}$ \\
\hline $\mathrm{H} 4$ & $\begin{array}{l}\text { NM_001094 } \\
457.1\end{array}$ & $129-229$ & $\begin{array}{l}\text { GGAGAGGGGGAGTCAAGCGCATCTCTGGCCTCATCTATGA } \\
\text { GGAGACTCGTGGGGTCCTCAAGGTTTTCCTGGAGAATGTC } \\
\text { ATCCGGGACGCCGTCACCTA }\end{array}$ \\
\hline Hey1 & $\begin{array}{l}\text { NM_001090 } \\
457.1\end{array}$ & $1605-1705$ & $\begin{array}{l}\text { ATCAACAGCAATGCCTTCAGTAAAGCTGTGCATGGAAGGG } \\
\text { GTGTGTGCTGAAAAGTGGGGCCACAAGCATTCAGAGGGTT } \\
\text { AAACATAACACTGAGTGCAC }\end{array}$ \\
\hline Hnf1b & $\begin{array}{l}\text { NM_001089 } \\
811.1\end{array}$ & $1626-1726$ & $\begin{array}{l}\text { CACAGCAGCCATTTATGGCGACTGTGACTCAGCTACAGAAT } \\
\text { TCACACATGTATGCGCACAAGCAAGAGCCTCCCCAGTATTC } \\
\text { CCACACATCTCGTTTTCC }\end{array}$ \\
\hline Hoxa1-a & $\begin{array}{l}\text { NM_001085 } \\
719.1\end{array}$ & $168-268$ & $\begin{array}{l}\text { CAACTTTCCAGTCGTGCGCAGTCAGTGCTAATAACTGCAAC } \\
\text { GGCGACGATCGTTTTGTGGTCGGACGAGGGGTGCAGATAA } \\
\text { GTTCTCACACCCATCACCA }\end{array}$ \\
\hline Hoxa1-b & X62053.1 & $1149-1249$ & $\begin{array}{l}\text { TGAGATGATGGAATGGTAAAAAAGCTCAATGTTTTCTAGCA } \\
\text { CAGGAGAAACAGCAGTGTTTGCTGACCGCATACCTCAGTA } \\
\text { ATCTCTCTCATTTACTAGG }\end{array}$ \\
\hline Hoxa2-a & $\begin{array}{l}\text { NM_001085 } \\
750.1\end{array}$ & $130-230$ & $\begin{array}{l}\text { GGCTGAGTTGTTTAAAGGGCTTGGAGGAGAGGGCCATGAA } \\
\text { TTACGAATTTGAGCGAGAGATTGGTTTTATCAATAGTCAGC } \\
\text { CGTCGCTTGCTGAGTGCCT }\end{array}$ \\
\hline Hoxa3 & $\begin{array}{l}\text { NM_001086 } \\
824.1\end{array}$ & $277-377$ & $\begin{array}{l}\text { CATTGGAGGAGGAACGCCACGTGACAGAGGGGTGCCAAT } \\
\text { GTTATTCTTTACGGGTGTCAAGACCCTGTCAGTTTGTGAAA } \\
\text { TAAATATTGGGAAACAACGA }\end{array}$ \\
\hline Hoxa5 & $\begin{array}{l}\text { AC236444_r } \\
\text { a.1 }\end{array}$ & 298-398 & $\begin{array}{l}\text { ACTCTTCTCTGTGACTTCTGTGAAGATACCCTCCTCCCCAA } \\
\text { TCTCTTGTCCACCCTTTATCCTAGCAATGAGCCTTTTAAACT } \\
\text { GGGTGAAATAGCTTCAA }\end{array}$ \\
\hline Hoxb1 & FJ422584.1 & $486-586$ & $\begin{array}{l}\text { CTCCTGCCCTCCAGATCAAGCTCTACCCAACCACACCTTTG } \\
\text { ACTGGATGAAAGTAAAGAGAAATCCTCCCAAGACAACCAAA } \\
\text { CCAATGGACTATGGACCC }\end{array}$ \\
\hline Hoxb3 & M91588.1 & 8-108 & $\begin{array}{l}\text { AGCGCGCACCGCTTACACGAGCGCACAACTGGTGGAACTG } \\
\text { GAGAAGGAGTTTCACTTTAATCGCTACCTGTGCCGCCCCA } \\
\text { GGAGGGTGGAGATGGCCAAC }\end{array}$ \\
\hline Hoxb4 & $\begin{array}{l}\text { NM_001096 } \\
264.1\end{array}$ & $217-317$ & $\begin{array}{l}\text { GCAATAGCAGCAGCAGCGCGTCCTACTCCTCCTGCCAAGG } \\
\text { ATCTGTGCGTCAAAGCGCAAGGCTGCCTCACTCATCTGGA } \\
\text { CTTGTCCCAGGCGAGAAAGC }\end{array}$ \\
\hline Hoxd1 & $\begin{array}{l}\text { NM_001090 } \\
566.1\end{array}$ & $58-158$ & $\begin{array}{l}\text { CGGCCAGAGAAGTCTCCCTAGAGATGAATTCCTACCTAGA } \\
\text { ATACACTTCTTGCGGGGATGTTTTAGCTTTTTCACCCAAGTT } \\
\text { CTGCCGCAGCGACCACAG }\end{array}$ \\
\hline Hoxd4 & $\begin{array}{l}\text { NM_001173 } \\
995.1\end{array}$ & $1013-1113$ & $\begin{array}{l}\text { CAGGAAATAATGACTCCAGGGCAGTCAATTTCATAGGTGAT } \\
\text { GCTGTGTAAAGGATTTCTATGGCAATAAGGGACTATTCAGT } \\
\text { TCAGAGGCACAACCAACA }\end{array}$ \\
\hline Hunk & $\begin{array}{l}\text { NM_001091 } \\
243.1\end{array}$ & $1214-1314$ & $\begin{array}{l}\text { CCACATGTCTGAGAAACTTGGCTACAAACACAGCGATGTGA } \\
\text { TCAACGTCATTCTCTCCAATCGAGCTTGCCATACCCTGGCC } \\
\text { GTCTACTTCCTCCTAAAC }\end{array}$ \\
\hline $\operatorname{lgf3}$ & $\begin{array}{l}\text { NM_001088 } \\
668.1\end{array}$ & $389-489$ & $\begin{array}{l}\text { CTATCCTCGAGTCTTACTGTGCGGCTCCAGTCACTAACTTC } \\
\text { ACTGGCAGAGAAGAGCAAAAGTCCTAAGATCATTCTCCAAG } \\
\text { ACCTATGCCACCAGTTTA }\end{array}$ \\
\hline Kiaa0182 & $\begin{array}{l}\text { NM_001097 } \\
124.1\end{array}$ & $2037-2137$ & $\begin{array}{l}\text { GAGTTGGCAGAGAAGTATCAATCACAAAGAGAGTCTTCAG } \\
\text { CCATGGAACATGCAGGTTACACGCATACTCCATTCTTAGCT } \\
\text { GAGCTAGAAAAGTCAACTC }\end{array}$ \\
\hline Kirrel2 & $\begin{array}{l}\text { NM_001086 } \\
488.1\end{array}$ & $690-790$ & $\begin{array}{l}\text { CCCAGTCGCCAATGTCCTGCTTGATGTACCCTACAATCTCA } \\
\text { CCTGTCTTGCTTCCGTCGCTAAGCCTGCTGCAGAGATTACC } \\
\text { TGGTTCCGTGATGGAAAG }\end{array}$ \\
\hline Lhx1 & $\begin{array}{l}\text { NM_001090 } \\
659.1\end{array}$ & $21-121$ & $\begin{array}{l}\text { TCTATTCTCCTAATCCGCCCATTCCTCTAAAATCCCAAATAA } \\
\text { CCAAAGGCAATGGTTCACTGTGCTGGATGCGAAAGGCCCA } \\
\text { TTCTGGACCGTTTCTTGT }\end{array}$ \\
\hline $\begin{array}{l}\text { LOC10015 } \\
8377\end{array}$ & $\begin{array}{l}\text { NM_001127 } \\
821.1\end{array}$ & $392-492$ & $\begin{array}{l}\text { GTCTATAAGGTGACACAGGGTATTGGATCTAAGTCGATGGT } \\
\text { GGTGAAAATTTACAAAAACGATGTCGATGAGGAGGGAATTG } \\
\text { TCCGAGAGATCACTTTGC }\end{array}$ \\
\hline Meis3-a & $\begin{array}{l}\text { NM_001088 } \\
397.1\end{array}$ & $1517-1617$ & $\begin{array}{l}\text { CTATGATTGACCAATCTAATCGCACAGGGCAAGGAGGTGC } \\
\text { TCCCTACAGTCCAGATGGTCAGAACATGGGAGGATATGTC } \\
\text { ATGGATGGACAACAACACAT }\end{array}$ \\
\hline Mespa-a & $\begin{array}{l}\text { NM_001085 } \\
581.1\end{array}$ & $1570-1670$ & $\begin{array}{l}\text { TGTGAAAGGCTATGGCATTAAATATACTGTCTTCTGAAAGA } \\
\text { ATCTTGGCTTCCTAGTGCTTTCTGTGAAACAATAGGTAATG } \\
\text { GCTGCAAATAAAAGGTCC }\end{array}$ \\
\hline Mespb & $\begin{array}{l}\text { NM_001135 } \\
226.1\end{array}$ & $725-825$ & $\begin{array}{l}\text { TATTAAAGCAGGACATGACCTCCCCACAGTACAATAGCACA } \\
\text { GTAGCTACGCCTGTCAGTCAACAGTATGTGACAGGGCTCC }\end{array}$ \\
\hline
\end{tabular}




\begin{tabular}{|c|c|c|c|}
\hline Target & Accession & Target region & $\begin{array}{r}\text { Target sequence } \\
\end{array}$ \\
\hline & & & AACACACGATTCACTGTAA \\
\hline Mxi1 & $\begin{array}{l}\text { NM_001095 } \\
701.1\end{array}$ & $2418-2518$ & $\begin{array}{l}\text { GCTGCTGCAGGTCATTATCATTCTGCAATCTGTGTTTGGGT } \\
\text { GGACTTCCCCTTTAATCTGTTACAAACCAGAAATGACACAT } \\
\text { CCCAGAATCCTCAGCTCC }\end{array}$ \\
\hline Myc-a & $\begin{array}{l}\text { NM_001085 } \\
896.1\end{array}$ & $523-623$ & $\begin{array}{l}\text { TCTGTCTTCTTCTTCTCCGTGTCCGAGTCAACCACCACCGA } \\
\text { GCCCGCTTAAGTCTCCCTCGTGTCATGGGAGCCTGAGTCT } \\
\text { GGGAGGGACCCACAGGAGC }\end{array}$ \\
\hline Myc-b & $\begin{array}{l}\text { NM_001090 } \\
653.1\end{array}$ & $76-176$ & $\begin{array}{l}\text { AGCTTGTGATACAACGAGAGGAGAGGGAGGGAGTCAAAGC } \\
\text { GTCGGCTCAGCCTGTGGATTTACAAGCGCGACAGCTCTCA } \\
\text { TTAGGAAACCTTGGACTTGT }\end{array}$ \\
\hline Ndrg1-a & $\begin{array}{l}\text { NM_001094 } \\
390.1\end{array}$ & $1428-1528$ & $\begin{array}{l}\text { AAAGACCTGCTTGCCAATTGGAAACATCCTACCCTTCCCGC } \\
\text { TATCTGGGTTACTCCACCAGCAAATGGGGGGTTATGTTTTG } \\
\text { ATATAATCACTGTGAACT }\end{array}$ \\
\hline Ndrg1-b & $\begin{array}{l}\text { NM_001087 } \\
158.1\end{array}$ & 1429-1529 & $\begin{array}{l}\text { GAAGACCTGCTTGCTAATTGGAAACATCTTAACCTCTTCGC } \\
\text { TGTCTGGGTTACTCCACCAGCAAACGAAGGGGGAGGCTAC } \\
\text { GTTTTGATATAACTGTGAA }\end{array}$ \\
\hline Neurog2-b & $\begin{array}{l}\text { NM_001088 } \\
335.1\end{array}$ & $1172-1272$ & $\begin{array}{l}\text { CATCGTTAGCTATGTGTATTAGGAAACTGTCTATCCCTCAT } \\
\text { CTGCACCTGTTAGACTACAGCTACCAACTTCCTGTTACCAG } \\
\text { GGGGCTACTGGGTAATGT }\end{array}$ \\
\hline Nkx6-2 & $\begin{array}{l}\text { NM_001096 } \\
886.1\end{array}$ & $28-128$ & $\begin{array}{l}\text { TGTTTGTCGCCGCTGCATGTGTGGGATCATTACTGCCCTGA } \\
\text { AACTTGCGCTGATCGCCGGGAAGTTGCGACAGAAGCCGTT } \\
\text { TGCCTCGGGGACTTCTCTC }\end{array}$ \\
\hline Nodal1 & $\begin{array}{l}\text { NM_001085 } \\
796.1\end{array}$ & $781-881$ & $\begin{array}{l}\text { CTCTGCCAACCATATTGGCTTTCCCAGCCTCATCAAGACAG } \\
\text { CAGAGTCATCCAAATACGTTGACATAGAGAAAGCTAGTAGA } \\
\text { GTGCCTGGTATTAGGAGA }\end{array}$ \\
\hline Nodal2 & $\begin{array}{l}\text { NM_001087 } \\
967.1\end{array}$ & $776-876$ & $\begin{array}{l}\text { GACAAGCCCACTGCTAGTCATTTTGGATCTCCTAGTCTCAT } \\
\text { CCACACTGTGGAGTCTTCCAAGTATGTCATGAGTGAAAACA } \\
\text { CAGTCAGGGTGACAGATA }\end{array}$ \\
\hline $\mathrm{Nr} 2 \mathrm{f5}$ & $\begin{array}{l}\text { NM_001101 } \\
759.1\end{array}$ & $301-401$ & $\begin{array}{l}\text { AGTTCACCTGTGAAGGGTGCAAGAGTTTCTTTAAGAGGTCG } \\
\text { GTGAGGAGGAACCTAACCTACACATGTAGGAGCAACAGAG } \\
\text { ACTGTCCTATAGATCAACA }\end{array}$ \\
\hline Odc & $\begin{array}{l}\text { NM_001086 } \\
698.1\end{array}$ & $855-955$ & $\begin{array}{l}\text { GGATATAATTGGTGTGAGTTTCCATGTTGGCAGTGGCTGCA } \\
\text { CTGATCCACAGACTTATGTACAAGCTGTCTCAGATGCACGA } \\
\text { TGTGTCTTTGACATGGGG }\end{array}$ \\
\hline Pdgfb & $\begin{array}{l}\text { NM_001094 } \\
466.1\end{array}$ & $345-445$ & $\begin{array}{l}\text { CGGGTCATTCGCAGCTTAGATGCAGAACAAGCCGTCCTTG } \\
\text { CAGAGTGCAAACCCCGCGTCGAAGTGTTTGAGATTTCTCG } \\
\text { CAAGTTAGTGGACCCCACTA }\end{array}$ \\
\hline Pim1 & $\begin{array}{l}\text { NM_001095 } \\
150.1\end{array}$ & $1270-1370$ & $\begin{array}{l}\text { AGACATGTGGTGACCTCAGACTGTGATACTTACCTTGGTGG } \\
\text { TTCCTCCTCCTTTCTAAAAGATGGACTTTGAAGGTGTGAGG } \\
\text { AGAGATGAGAAGGGTTTG }\end{array}$ \\
\hline Pkdcc.1 & $\begin{array}{l}\text { NM_001097 } \\
762.1\end{array}$ & $1330-1430$ & $\begin{array}{l}\text { TCCCGTGTTTACATGAAAAAACCAAGCAACCTCAAAAAGTG } \\
\text { ATGCGGATGGGAAGAGTCACGCTCGCTTATATGATGCACT } \\
\text { GAAGTACTAATTGGCTTTA }\end{array}$ \\
\hline Prph & $\begin{array}{l}\text { NM_001087 } \\
060.1\end{array}$ & $1440-1540$ & $\begin{array}{l}\text { GACCCAGTTCCATTGCAATACCTCTCCATGGACGGAACCTC } \\
\text { CTGTGCTTTTCTAATTGATGGATACTGAGGGTCATCTCCAC } \\
\text { TAACACACTCCTTTATCT }\end{array}$ \\
\hline Rara & $\begin{array}{l}\text { NM_001090 } \\
254.1\end{array}$ & $2435-2535$ & $\begin{array}{l}\text { ACCTGAACAGTCTAAAGGGTTTGGGGAGCAGTTAAAGGGG } \\
\text { AAATAAAGAGAGGAGATGTGAGTGCTTTGTGCTTGGGTGT } \\
\text { GTGATGGAAGTGAAGAGGAA }\end{array}$ \\
\hline Rgs14 & $\begin{array}{l}\text { NM_001092 } \\
238.1\end{array}$ & $752-852$ & $\begin{array}{l}\text { AAGGACATTGCAAAGAAACTTTAGACAGCCGCGAACTCTCA } \\
\text { TCTCGCCGGGAATCATGGGTATCACTTAACTCCAATAACAG } \\
\text { TCTGGAGCTGAGTCTCAA }\end{array}$ \\
\hline Rgs2 & $\begin{array}{l}\text { NM_001095 } \\
045.1\end{array}$ & $24-124$ & $\begin{array}{l}\text { CTCTCTTACTAGCATCACTGACTAATCCAGAACAAGCAGAA } \\
\text { TCGAGCACAGGGACTTATACAAATCCTTGGAGGGAAATTAT } \\
\text { GCAGAGCGCAATGTTTCT }\end{array}$ \\
\hline Sox17alpha & $\begin{array}{l}\text { NM_001088 } \\
162.1\end{array}$ & $567-667$ & $\begin{array}{l}\text { TGCTGGGCGCAGATGGAAGGATGTGTCTAGAGAACTTCAG } \\
\text { CCTGGGTTATCATGAGCAGACTTACTCCCACGGCCAGGTT } \\
\text { CCTCAGAGCAGCCACTACAG }\end{array}$ \\
\hline Sox17beta & $\begin{array}{l}\text { NM_001088 } \\
164.1\end{array}$ & $163-263$ & $\begin{array}{l}\text { ATGGACCGAACCCCTGACCGTGTTTCAGGACCTCAAACCC } \\
\text { AAGAGGGATGAAGGATCTGCCGATTCCAGAAGCAAAGCCG } \\
\text { AGGGTCGGATCCGCCGACCC }\end{array}$ \\
\hline Sox2 & $\begin{array}{l}\text { NM_001088 } \\
222.1\end{array}$ & $901-1001$ & $\begin{array}{l}\text { CCGGGCATGTCTCTGGGATCCATGGGCTCGGTAGTCAAGT } \\
\text { CGGAATCCAGCTCCAGTCCACCTGTAGTCACCTCTTCTTCC } \\
\text { CATTCGCGGGCTCCGTGCC }\end{array}$ \\
\hline Sox9-a/b & $\begin{array}{l}\text { NM_001090 } \\
807.1\end{array}$ & $441-541$ & $\begin{array}{l}\text { GAAGTTCCCCGTGTGCATCAGAGAAGCGGTCAGCCAGGTG } \\
\text { TTGAAGGGATATGATTGGACCCTGGTACCGATGCCAGTCA } \\
\text { GAGTTAATGGATCCAGCAAG }\end{array}$ \\
\hline Spry2 & $\begin{array}{l}\text { NM_001088 } \\
769.1\end{array}$ & $157-257$ & $\begin{array}{l}\text { TTTCCCTCACACTTCATTTAGTGGATACAAGGATCGCGCTG } \\
\text { GATTGGGGGAGCCTGGAACCCTGACACATGTTTTCTGTGT } \\
\text { CCCTTCCTATCGGGCTTGG }\end{array}$ \\
\hline Stox2 & $\begin{array}{l}\text { NM_001093 } \\
369.1\end{array}$ & $162-262$ & $\begin{array}{l}\text { TTTTTTCCAGGTGTACCCACTCCAAGTCCAGAGGTACTTCG } \\
\text { GCATACACTTAACATGCTTGTGCGAGAGAGAAAGATCTACC } \\
\text { CTACTCCCGACGGATATT }\end{array}$ \\
\hline
\end{tabular}




\begin{tabular}{|c|c|c|c|}
\hline Target & Accession & Target region & Target sequence \\
\hline Tdgf1p2-a & $\begin{array}{l}\text { NM_001095 } \\
665.1\end{array}$ & $843-943$ & $\begin{array}{l}\text { CACCAGAGAAAACAAGGCTGCATTATCGTAGAAGATCCTG } \\
\text { CATAGAAAAAAATCTCACTGCTCTATAAGCATAAATATCTAC } \\
\text { ATTAACTCTTCCCATGGA }\end{array}$ \\
\hline Tdgf1p2-b & $\begin{array}{l}\text { NM_001127 } \\
822.1\end{array}$ & $1958-2058$ & $\begin{array}{l}\text { GGAAGGCCAAATTGGACAGTAATGCTCATATACAAATGCAA } \\
\text { GCGCAAATCGCTATAATGGATTAAAAAAATCAGCACCCATT } \\
\text { GACACTGTTCAGAAAGCT }\end{array}$ \\
\hline TGIF2 & $\begin{array}{l}\text { NM_001094 } \\
168.1\end{array}$ & $2638-2738$ & $\begin{array}{l}\text { TTTGAGATATTTGTGAAGTGTGCCTTGGGAAGCAGCACATT } \\
\text { TCCCGGTAACACGAGTCCTTAGACTGACCTTTTTGAGTACT } \\
\text { AGGACAAAAGTAACCTGA }\end{array}$ \\
\hline Tmem72 & BC133254.1 & $204-304$ & $\begin{array}{l}\text { TGTGCTTAGGCAAAACAGGACGCATGGGAGGATTTCAAAA } \\
\text { ATTTGTGGGATATGGCCTGCTATCAGTGGCTTGCTTTTTGC } \\
\text { ACCCAGTTTTGGTCTGGCA }\end{array}$ \\
\hline Trib1 & $\begin{array}{l}\text { NM_001095 } \\
725.1\end{array}$ & $3019-3119$ & $\begin{array}{l}\text { GCACTAAACGTCCTTGCGGTTCGGTGATTACTGTATCTGTG } \\
\text { ATACAATGGAGCGACTCTGTAAATAGTGACGCGCATTCGCT } \\
\text { CAGAAGAAACTGATTGCA }\end{array}$ \\
\hline Tubb2b & $\begin{array}{l}\text { NM_001086 } \\
064.1\end{array}$ & $900-1000$ & $\begin{array}{l}\text { TGCACTTTTTTATGCCAGGCTTTGCCCCATTAACAAGTCGT } \\
\text { GGCAGCCAACAATACCGAGCCCTGACAGTGCCAGAACTAA } \\
\text { CACAGCAAATGTTTGATTC }\end{array}$ \\
\hline Twist1-a & $\begin{array}{l}\text { NM_001085 } \\
883.1\end{array}$ & $619-719$ & $\begin{array}{l}\text { AGGGAGCCTGGTCCATGTCTGCATCTCACTAACAGCAATG } \\
\text { CCACTACAGCTCAGGCCACACACAAAGATTATACTTATACC } \\
\text { AATGAATGGGAAAAAAAAC }\end{array}$ \\
\hline Txnip & $\begin{array}{l}\text { NM_001093 } \\
153.1\end{array}$ & $1515-1615$ & $\begin{array}{l}\text { TTTGACACTTGCCTTAAACCTACATCACCTGATGATGTGCC } \\
\text { ATGAAGAGAAAGAGTTGTCTGTTTCCCACCATACAGTATGT } \\
\text { GGACAGGAATCTGTGGCC }\end{array}$ \\
\hline Ventx2.1-b & $\begin{array}{l}\text { NM_001087 } \\
\text { 981.1 }\end{array}$ & $1075-1175$ & $\begin{array}{l}\text { TTCCACAGGACAAAAAATTGCACTGAATGTTGCTATTGGCA } \\
\text { AGATGATTACAGAATAGCTGGCTACTATTGGCCTATTTGTT } \\
\text { ATGTATCTTTACATGATT }\end{array}$ \\
\hline Vpp1 & $\begin{array}{l}\text { NM_001258 } \\
387.1\end{array}$ & $177-277$ & $\begin{array}{l}\text { CATCTCTGGTGCAATGTCAGTCATTCCTCAGCCGGGGGAT } \\
\text { TTGAGTGGTCTGTGTTCCCGGCACATTCTCCTTCCCAGAAA } \\
\text { CTCCAGATCATCAGCTCCA }\end{array}$ \\
\hline Xbra-a/b & $\begin{array}{l}\text { NM_001090 } \\
578.1\end{array}$ & $1529-1629$ & $\begin{array}{l}\text { TGTAGGCCTCCAAAACAACTTAAAGATGTGCTTAGGCAAGT } \\
\text { TATATCAGTGTTTACCTGCTTCTAAAGACTTCATGGGCCCA } \\
\text { ACCAGGTGTGGGTGGTCT }\end{array}$ \\
\hline Xk81a1 & $\begin{array}{l}\text { NM_001086 } \\
376.1\end{array}$ & $323-423$ & $\begin{array}{l}\text { AAGTGAGAGCCTTGGAAGCCGCCAATAACGACCTGGAAGG } \\
\text { GAAGATCCGTAACTGGTACGATAAGCAATCAGATGCAGGC } \\
\text { ATTGGTGCTGGGTCTAAAGA }\end{array}$ \\
\hline XI.15091 & BI443651.1 & $131-231$ & $\begin{array}{l}\text { TACAAATGATTTGGAGAGAAACATTCCTTTCCAAAAATAGGT } \\
\text { TTCAATCTTTGGCAGTGGGGAAATCGAAGTGAGATTTTGTG } \\
\text { AAATGGCTAGGATGAAA }\end{array}$ \\
\hline XI.16263 & DC025247.1 & $247-347$ & $\begin{array}{l}\text { ACGTTAGGACATTACAGGAAGGGGCATTGTTAATCTCATAA } \\
\text { ACCACTGATTGTTGAAGACACAAAGGCATTGAAGGCATAAA } \\
\text { GACCTGCGTATTTCTACA }\end{array}$ \\
\hline XI.32109 & BP735133.1 & $170-270$ & $\begin{array}{l}\text { GGGTTGAAATTGCCCCTTTAGTAGGCCAATCCAGTCCTGTA } \\
\text { AACAACAGGTAGCACAGCAAGGTGTCTGCCATCAACCTTA } \\
\text { GAGTCTCTCCAGCTTTCTT }\end{array}$ \\
\hline XI.45046 & CF286593.1 & $284-384$ & $\begin{array}{l}\text { ACTGGAAAAAGGGGAGTTTTAGTTCTGCAACATTGGCTGTA } \\
\text { CCACATGCTGCCTGTCACCTGTATATTTCAAGCTGGCCATA } \\
\text { CACACGAAGCAATACAAT }\end{array}$ \\
\hline$X I .4906$ & DC050951.1 & $373-473$ & $\begin{array}{l}\text { TCCAGAGAAGCCTGGCCCACAAGTGCCCAGTGGTTCCTCG } \\
\text { TCCAACCCCAACATCCCAGTCCAGCAGCAACATCTCCGTC } \\
\text { GAGGACCATTTCTCCAAAGC }\end{array}$ \\
\hline XI.51509 & DY570900.1 & 86-186 & $\begin{array}{l}\text { CTTACAAGGGAATGGGGGCGCCTATGTGTCACCCAATATG } \\
\text { CAAGCAAGTCCGGTGTACGTAGGGGGTAACTATGTGGATT } \\
\text { CTGTGCCAGCACAAGGCCCT }\end{array}$ \\
\hline XI.57926 & $\begin{array}{l}\text { DQ096998. } \\
1\end{array}$ & $670-770$ & $\begin{array}{l}\text { ACCGCACCAAAACATTTAACCTCTTCCATGCCAGTCCAATA } \\
\text { CACCCCAAAGCAGCCCGTTACAGCTGCACATGTAATCTCC } \\
\text { AGTTACCCTCATGCTTGTA }\end{array}$ \\
\hline XI.58101 & BJ089218.1 & $113-213$ & $\begin{array}{l}\text { TCCAAAAAGAGGTTACAACCTTTGGCAGTGGGGAATTTGAA } \\
\text { GAGAGAATTTGTGAAATGGCTTTGGCTGAAAACTTGTCATT } \\
\text { GGAAGCAGTTGTGCGGTC }\end{array}$ \\
\hline XI.59256 & DR729445.1 & $601-701$ & $\begin{array}{l}\text { TTTAACCGGAAGTTCCTTAATACCCAAGGTGTCTTGCCTAT } \\
\text { GGACACAATGGCATAAAGCAAGGGATGCAAACTAGGGCCA } \\
\text { ATCTTTCCAAGTTACAGTT }\end{array}$ \\
\hline XI.6091 & DC024574.1 & $286-386$ & $\begin{array}{l}\text { AACTGAATCTGGCCCAGTATATCTCATTGCTTATGGTGTGC } \\
\text { ATCAATTATTTTTTGCAGTACAGTAGCACATACATGGGGTA } \\
\text { CCCTGAGAAATATGGCGC }\end{array}$ \\
\hline XI.67202 & DC016425.1 & $34-134$ & $\begin{array}{l}\text { ACAAATGTTATGTTTGAGTCATTAAAAACTGAGGAATGAGA } \\
\text { GCTGATTGCACATGGATACAGGGCAGTGATTGGCTGTTCC } \\
\text { TTTTAGGGTTGCACCGAAT }\end{array}$ \\
\hline XI.68408 & DC067179.1 & $265-365$ & $\begin{array}{l}\text { GGATCAGACAGTGCTCCTTTGCATGTTGATACCTGAGGAAT } \\
\text { GTGGTAAAAGATATGAATCTTAGTTGGGCAGCCCAAAGGAT } \\
\text { GAGCCCCAATTTACATCA }\end{array}$ \\
\hline XI.70850 & BJ622644.1 & $207-307$ & $\begin{array}{l}\text { CCCATAGAGAGTCGCCGACCCCTGGGAGCCGCATACAGA } \\
\text { GAAGATATTTATATACTGCTCATTGTGGCTCTAACTTAGGG }\end{array}$ \\
\hline
\end{tabular}




\begin{tabular}{|c|c|c|c|}
\hline Target & Accession & Target region & Target sequence \\
\hline & & & CCACACATAGTGCTACACAG \\
\hline$X I .71159$ & $\begin{array}{l}\text { NM_001086 } \\
068.1\end{array}$ & $26-126$ & $\begin{array}{l}\text { GTGCTGAGCTATTGTGTATCGGCAATTCGGAGAACTGAGG } \\
\text { TGAAACTTTCCAGAGCCCAGAAAGAGCCTCGTTACTAGTGT } \\
\text { CAGCGTCCAGTTGATCTGT }\end{array}$ \\
\hline $\mathrm{XI} .74263$ & BJ631099.1 & $266-366$ & $\begin{array}{l}\text { CTAAGGATCTCTCTGCAAAGTCTATTGTCCAAATTGGGGAA } \\
\text { CATGTCAGGCAGCAGGACAAAAAAATTGTGGGATATTCGG } \\
\text { AAAATGCTTGAAGGACACC }\end{array}$ \\
\hline $\mathrm{XI} .79790$ & DR715148.1 & $408-508$ & $\begin{array}{l}\text { GGCAATGAGCCCCGATTTCTGGCCGCCTGGTTCGGATTGG } \\
\text { CCCAACTGGGGGTGGTGTCCGCCTTCCTCAACACCAACGT } \\
\text { GCGGAAAGGGGCCCTAATGC }\end{array}$ \\
\hline $\mathrm{XI} .80297$ & Bl449170.1 & $41-141$ & $\begin{array}{l}\text { GTGTCCATCTGCCCATCAATGAGCATTGTCTTCTAAGAGGA } \\
\text { TCCAAAACCATAAAGAATTGGATACAGCTGTTTCGGAACTG } \\
\text { GATTTTCCAGCATTTCCC }\end{array}$ \\
\hline XI.85251 & EE318610.1 & $705-805$ & $\begin{array}{l}\text { TCGAACTTGATGGTTTGACTACACGTTGATCTAGTCAGCCT } \\
\text { AATCTACCTCTACAATCAGAGGTGTGGCAATGCACTGACCC } \\
\text { GTTATCTTTTCTCGCTCC }\end{array}$ \\
\hline XI.8753 & BC057717.1 & $143-243$ & $\begin{array}{l}\text { GCACGTTTAAACCACCAACTCACCCTCTGATGCCCGTTCCG } \\
\text { GTGTTGTTTCACAGAAGATCACAGGCTGGTGACATCATGCT } \\
\text { GGTATCGGCATTACCGTC }\end{array}$ \\
\hline XI.9822 & $\begin{array}{l}\text { NM_001096 } \\
102.1\end{array}$ & $3424-3524$ & $\begin{array}{l}\text { GGACGGTAGCGTATCAGTGTGAAGGCTCTGTGACTCTTCT } \\
\text { GATTTTACTGAACTACTAATCCCAGCAGTCCCACAGATCCT } \\
\text { GCTGTTGGTACAGTATGAA }\end{array}$ \\
\hline XI.9874 & DC012344.1 & $489-589$ & $\begin{array}{l}\text { AGCAGCATATGATGATAACTCAAGGTCACAAATCAGGCTTC } \\
\text { ATGTGACAGATAGCCTCCCCTGAAAAGTTCAATCTTGTTCA } \\
\text { ATGCACAACCACCGGCTT }\end{array}$ \\
\hline Xwnt8 & $\begin{array}{l}\text { NM_001088 } \\
168.1\end{array}$ & $125-225$ & $\begin{array}{l}\text { GTCAGTCAATAACTTTCTGATGACAGGACCCAAGGCATATC } \\
\text { TGACATACTCAGCGAGTGTTGCCGTGGGTGCGCAGAATGG } \\
\text { AATTGAGGAGTGTAAATAT }\end{array}$ \\
\hline Znf703-a/b & BC046863.1 & $1582-1682$ & $\begin{array}{l}\text { ATCCTACTACTCCCCATACGCATTATATGGACAGAGGCTAA } \\
\text { CATCAGCTTCAGCGCTAGGATACCAGTAAATACGACTCCCA } \\
\text { AACTCATAGACTGTATAT }\end{array}$ \\
\hline
\end{tabular}




\section{Abbreviations}

${ }^{\circ} \mathrm{C}$

$\mu \mathrm{g}$

$\mu \mathrm{g}$

$\mu l$

$\mu \mathrm{M}$

A

AP

BCIP

$\mathrm{BMB}$

bp

C

cDNA

$\mathrm{dH}_{2} \mathrm{O}$

DIG

DNA

dNTP

DTT

E. coli

EDTA

et al.

G

GFP

h

hCG

LB

M

mg

$\min$

$\mathrm{ml}$

$\mathrm{mM}$ degrees celsius

microgram

microgram

microliter

micromolar

Adenine

Alkaline phosphatase

5-Bromo-4-Chloro-3-Indolyl-Phosphate

Bohringer Mannheim blocking reagent

Base pairs

Cytosine

complementary DNA

distilled water

Digoxygenin

deoxyribonucleic acid

deoxynucleoside triphosphate

Dithiothreitol

Escherichia coli

ethylene diamine tetraacetic acid

et alii

Guanine

green fluorescent protein

hour(s)

human chorionic gonadotropin

Luria-Bertani

molar

milligram

minute(s)

milliliter

millimolar 
mRNA

NBT

ng

$\mathrm{nl}$

PBS

PCR

$\mathrm{pH}$

RNA

rpm

RT

$\mathrm{sec}$

$\mathrm{T}$

T7E1

$\mathrm{Taq}$

$T_{m}$

Tris

U

UTR

$w / v$

WMISH

X-Gal messenger RNA

Nitro-Blue Tetrazolium

nanogram

nanoliter

phosphate buffered saline

polymerase chain reaction

negative decade logarithm of hydrogen ion concentration

ribonucleic acid

revolutions per minute

reverse transcriptase

second(s)

Thymine

T7 endonuclease I

Thermus aquaticus

melting temperature

Tris(Hydroxymethyl)Aminomethane

uracil / unit(s)

untranslated region

weight per volume

whole mount in situ hybridization

5-Bromo-4-Chloro-3-Indolyl- $\beta-d-G a l a c t o s i d e$ 


\section{List of figures}

Fig. 1.1 Pancreas organogenesis in Xenopus laevis.

Fig. 1.2 Model for dorsal endoderm patterning, mesoderm induction and organizer formation in Xenopus

Fig. 1.3 Fate maps of Xenopus endoderm from gastrula to early somite stage and overview of signals involved in pancreas specification. .20

Fig. 1.4 Overview of pancreas organogenesis and lineage decisions .23

Fig. 1.5 Paracrine RA-signaling and expression of RA-metabolizing enzymes during Xenopus gastrulation 26

Fig. 1.6 Therapeutic potential of in vitro generated organoids. 30

Fig. 3.1 RA-dependent induction of pancreatic marker genes in Vegt/Nogginprogramed explants

Fig. 3.2 Formation of pancreatic organoids that recapitulate the process of pancreas development.

Fig. 3.3 Formation of pancreatic organoids from RA-programed explants (WMISH)

Fig. 3.4 Direct RA-target gene Cyp26a1 is induced within one hour

Fig. 3.5 Identification, verification and expression characteristics of early RAresponsive genes in the context of pancreas specification

Fig. 3.6 Hnf1b is directly induced by RA and required for pancreas specification in pancreatic organoids.

Fig. 3.7 Hnf1b is required for pancreas specification in vivo

Fig. 3.8 Hnf1b is not sufficient to substitute for RA in pancreas specification in ectodermal explants.

Fig. 3.9 Fzd4 and Fzd4s are directly induced by RA and required for pancreas specification in pancreatic organoids

Fig. 3.10 Wnt-signaling in programed explants .93

Fig. 4.1 Model of pancreas specification involving direct RA-targets, Fzd4 and Hnf1b, in Xenopus embryos. 
Fig. 6.1 Detection of endodermal, mesodermal and neuro-ectodermal marker genes . 129

Fig. 6.2 Detection of marker genes for pancreatic structures 130

Fig. 6.3 Detection of early direct and indirect RA-target genes by RNA-sequencing 131

Fig. 6.4 Confirmation of RA-responsiveness 132

Fig. 6.5 Expression of RA-responsive genes at gastrula stage by WMISH 133

Fig. 6.6 Expression characteristics of endodermal, mesodermal and ectodermal markers at gastrula stage by Nanostring analysis. 134

Fig. 6.7 Expression characteristics of RA-responsive genes at gastrula stage by Nanostring analysis 135

Fig. 6.8 RA-responsive expression of Hnf1b in the dorsal endoderm 136

Fig. 6.9 Knockdown of Hnf1b through loss of exon 2 137

Fig. 6.10 Fzd4 has an alternative splice variant Fzd4s 138

Fig. 6.11 Fzd4/Fzd4s expression analysis 139

Fig. 6.12 RA-responsive expression of Fzd4/Fzd4s 140

Fig. 6.13 Mutation analysis of CRISPR/Cas treated pancreatic organoids by DNA sequencing 


\section{List of tables}

Tab. 2.1 Provided constructs for sense RNA …............................................

Tab. 2.2 Provided constructs for anti-sense RNA …........................................35

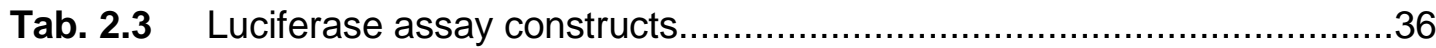

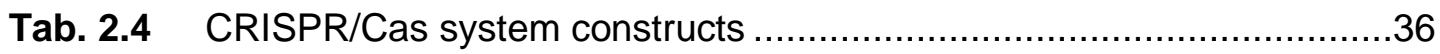

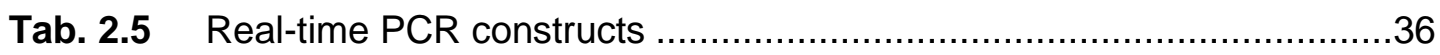

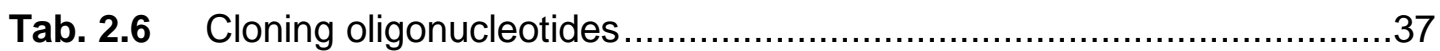

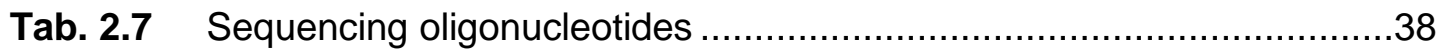

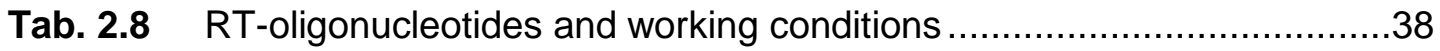

Tab. 2.9 Real-time PCR oligonucleotides .................................................

Tab. 2.10 Morpholino oligonucleotides ......................................................40

Tab. 6.1 Normalized data of two independent experiments ...........................143

Tab. 6.2 Calculated mean and standard error of mean (SEM) .......................144

Tab. 6.3 Summary of 102 differentially expressed genes in the absence of $\mathrm{CHX}$.

Tab. 6.4 Summary of differentially expressed genes in the presence of CHX ...146

Tab. 6.5 Comparison of differentially expressed genes absence versus presence of $\mathrm{CHX}$ (putative direct RA-targets).

Tab. 6.6 RNA-sequencing of explants $1 \mathrm{~h}$ after RA addition in the absence of

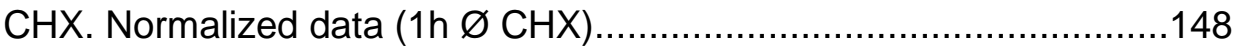

Tab. 6.7 RNA-sequencing of explants $2 \mathrm{~h}$ after RA addition in the absence of $\mathrm{CHX}$. Normalized data $(2 \mathrm{~h} \varnothing \mathrm{CHX})$.

Tab. 6.8 RNA-sequencing of explants $1 \mathrm{~h}$ after RA addition in the presence of $\mathrm{CHX}$. Normalized data $(1 \mathrm{~h}+\mathrm{CHX})$.

Tab. 6.9 RNA-sequencing of explants $2 \mathrm{~h}$ after RA addition in the presence of

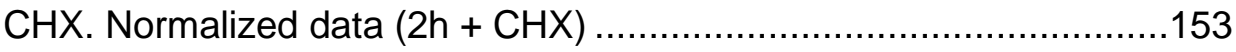

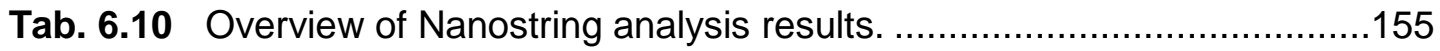

Tab. 6.11 Raw data of RA-inducibility in explants .......................................157

Tab. 6.12 Normalized data of RA-inducibility in explants ...............................159 
Tab. 6.13 Raw data of RA-inducibility in whole embryos ............................... 161

Tab. 6.14 Normalized data of RA-inducibility in whole embryos ....................... 163

Tab. 6.15 Raw data of RA-dependency in dorsal tissue ................................ 165

Tab. 6.16 Normalized data of RA-dependency in dorsal tissue ........................ 166

Tab. 6.17 Raw data of RA-dependency in whole embryos ............................167

Tab. 6.18 Normalized data of RA-dependency in whole embryos ….................. 169

Tab. 6.19 Raw data of expression characteristics ....................................... 171

Tab. 6.20 Normalized data of expression characteristics............................... 173

Tab. 6.21 Calculated mean and standard error of mean (SEM) ....................... 175

Tab. 6.22 Quantification of Pdx1 domain in the endoderm ............................ 177

Tab. 6.23 Quantification of Ptf1a domain in the endoderm ............................ 178

Tab. 6.24 Predicted Fzd4-gRNA off-target sequences in exonic regions of the Xenopus laevis genome ................................................................ 179

Tab. 6.25 Nanostring Code set used for the confirmation of pancreatic organoid formation

Tab. 6.26 Nanostring Code set for the verification of RA-responsive candidates...... 


\section{Acknowledgements}

I would like to express my appreciation to Prof. Tomas Pieler for providing me such an interesting research project. I am grateful for his guidance and constructive feedback throughout my studies.

I thank my thesis committee members Prof. Herbert Jäckle and Prof. Andreas Wodarz for their continuous interest in my work and for the helpful discussions.

Furthermore, I would like to thank Dr. Kristine Henningfeld for her support and reasonable suggestions.

I also want to thank Prof. Ahmed Mansouri, Prof. Ernst Wimmer and Prof. Matthias Dobbelstein for their participation as extended thesis committee members.

Moreover, I have to thank all my colleagues in the department of Developmental Biochemistry, especially Dr. Juliane Melchert, Dr. Patrick Berndt, Sven Richts, Dr. Juliane Pfennig, Dr. Diana, Bauermeister, Katja Ditter and Ilona Wunderlich for the nice working atmosphere and support.

My special thanks to Patrick Becker for his patience, interest in my work and continuous support. 


\title{
Curriculum Vitae
}

\author{
Name: \\ Maja B. Gere \\ Date of Birth: \\ 27.02.1980 \\ Place of Birth: \\ Hoyerswerda, Germany \\ Address: \\ Pfalz-Grona-Breite 45, 37081 Göttingen, Germany \\ e-Mail: \\ maja.gere@med.uni-goettingen.de, mgere@web.de
02/2012 - 03/2016 Doctoral studies: "Characterization of the retinoic acid- induced gene network responsible for pancreas specification in Xenopus laevis"
Dept. of Developmental Biochemistry, Georg August University of Göttingen, Supervisor: Prof. T. Pieler
$01 / 2011-01 / 2012$
Research assistant
Dept. of Chromatin Biochemistry, MPI for Biophysical Chemistry, Göttingen, Dr. W. Fischle
08/2010 - 12/2010 Research assistant
Dept. of Developmental Biology, Georg August University of Göttingen, Dr. N. Prpic-Schäper
09/2005 - 08/2010 Diploma studies: "RNA-binding proteins as interaction partner of HP1"
Dept. of Developmental Biology, Georg August University of Göttingen, Supervisor: Prof. S. Hoyer-Fender
09/2000-09/2005 Biological technical assistant
Dept. of Virology, Charité Campus Benjamin Franklin, Berlin, Prof. R. Heilbronn, Prof. T. Cathomen
08/1998-07/2000 Apprenticeship as Biological technical assistant
OSZ Werder (an der Havel)
08/1992 - 07/1998 Abitur
Konrad-Zuse Gymnasium, Hoyerswerda

